Novel in vitro and in silico tools for the development of mesoporous silica formulations with optimal precipitation inhibitors

Dissertation zur Erlangung des Doktorgrades der Naturwissenschaften

vorgelegt beim Fachbereich Biochemie, Chemie und Pharmazie der Johann Wolfgang Goethe -Universität in Frankfurt am Main

von

Daniel Joseph Price

aus Liverpool

Frankfurt 2020 


\section{i. Dedication}

Molly: my best friend, wife and partner. Without you, none of this is possible.

Rosella, Daisy and Atlas: my three wonderful children, you keep me motivated, positive and joyful- this is for you.

My Parents: You got me to where I needed to be, I will be forever grateful for your sacrifice and support.

My Family: All of you. Thank you for everything you have given me, your support, your faith and encouragement.

\section{ii. Acknowledgements}

First and foremost, my thanks and gratitude are extended to Prof. Dr. Jennifer Dressman, Prof. Dr. Christoph Saal, Prof. Dr. Martin Kuentz and Dr. Anita Nair for their support, guidance and supervision throughout the duration of my PhD studies. It has been a pleasure to work together and I hope to continue doing so in the future.

I feel fortunate to have been part of a multi-cultural, multi-disciplinarian PhD consortium. Special thanks are extended to all of my PEARRL colleagues. It was a pleasure to progress through this journey together and I hope our paths continue to cross in the future. In the same light, thanks are extended to all of the beneficiaries, partners, supervisors and funding bodies that made PEARRL a reality.

Finally, I would like to thank all of my colleagues at Merck KGaA, Frankfurt Goethe University and BfArM for enabling me to achieve everything I have in the duration of my studies. Access to cutting edge technologies, equipment and expertise has been a big contributor to my progress these past four years. 


\section{TABLE OF CONTENTS}

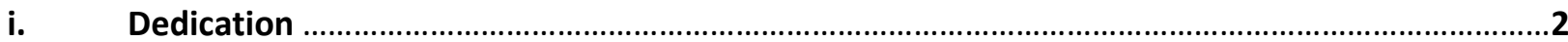

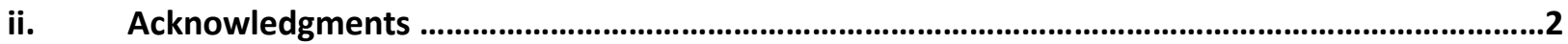

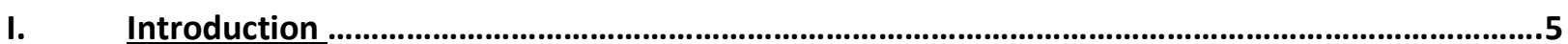

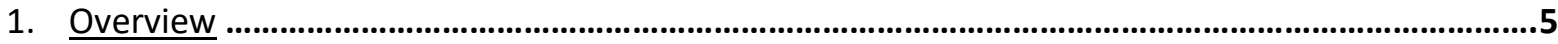

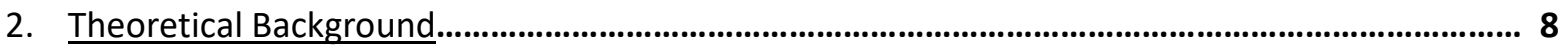

2.1 Factors Impacting Oral Drug Absorption .............................................................................8

2.1.1 Solubility .............................................................................................................................. 8

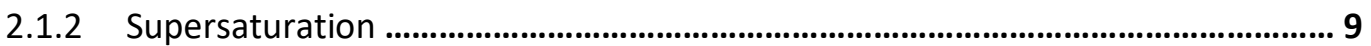

2.1.3 Impact of Solubility and Supersaturation on Oral Absorption ................................ 10

2.1.4 Dissolution and Impact on Oral Absorption ............................................................. 12

2.2 Approaches to Improve Drug Solubility, Dissolution and Oral Absorption .......................... 14

2.2.1 Formulation Technologies to Tackle Poor Drug Solubility.......................................14

2.2.2 Preventing Precipitation from the Supersaturated State.......................................18

3. Recent Advances in Supersaturating Formulations........................................................................... 26

3.1 Mesoporous Silica: An Emerging Formulation Technology ................................................26

3.2 Mesoporous Silica and Precipitation Inhibitors....................................................................... 38

3.3. Investigating the Importance Drug-Polymer Interactions in Precipitation Inhibition...... 39

3.3.1 UV-vis Spectroscopy ....................................................................................................... 39

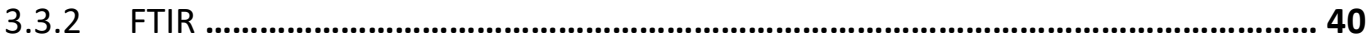

3.3.3 Raman Spectroscopy …............................................................................................... 42

3.3.4 Nuclear Magnetic Resonance (NMR) Spectroscopy ..............................................44

3.3.5 Differential Scanning Calorimetry (DSC) ...............................................................50

3.3.6 Modelling Precipitation Inhibition...................................................................................51

3.4. Bridging the Gap to Systematic and Facile Precipitation Inhibitor Screening....................57

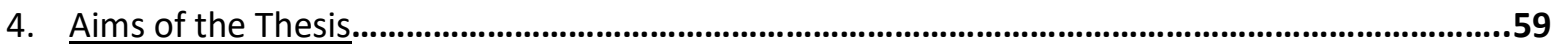




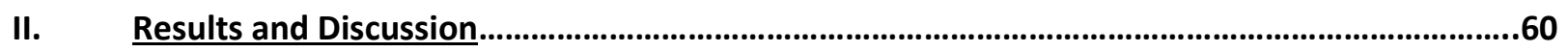

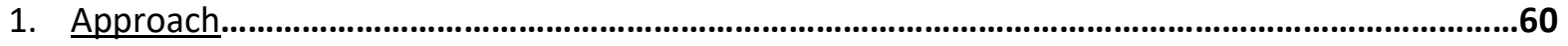

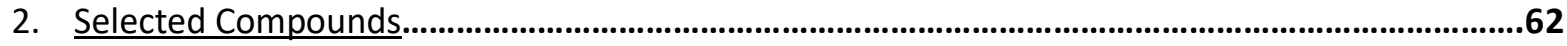

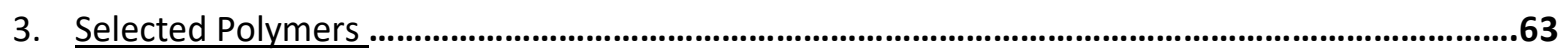

4. Co-Incorporation: A New Method to Combine Precipitation Inhibitors with Mesoporous Silica...65

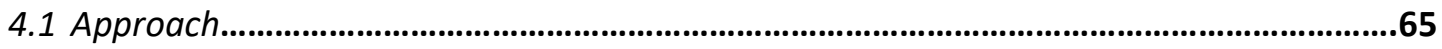

4.2 Solid State of Formulations...........................................................................................................66

4.3 Experimental Considerations for Dissolution Selection .........................................................68

4.4 Dissolution of Simple Blends: The Importance of Precipitation Inhibitors..........................69

4.5 Co-Incorporating HPMCAS in the Loading Method: Precipitation and Dissolution...........70

4.6 Co-Incorporated Formulations: Just a Solid Dispersion? .........................................................74

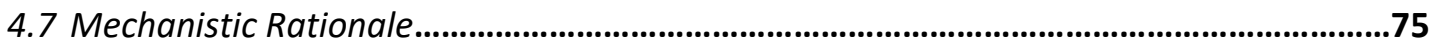

5. In Silico Pharmaceutics: A New Method to Select Precipitation Inhibitors for Mesoporous Silica.77

5.1 The Role of Molecular Interactions: Quality by Selection .......................................................77

5.2 COSMO-RS: Combining Quantum Chemistry and Thermodynamics...................................78

5.3 COSMO-RS for Precipitation Inhibitor Screening: Theory.....................................................82

5.4 Incorporation of Precipitation Inhibitors with Mesoporous Silica Formulations..............84

5.5 COSMO-RS for Precipitation Inhibitor Screening: Output...................................................84

5.6 Correlation between COSMO-Rank and Formulation Performance.....................................87

5.7 Limitations of the Approach .............................................................................................................90

5.8 Implications of the COSMO-RS Approach................................................................................91

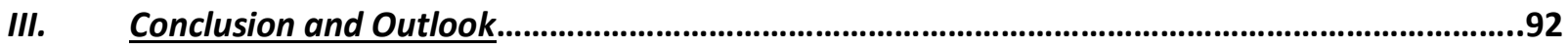

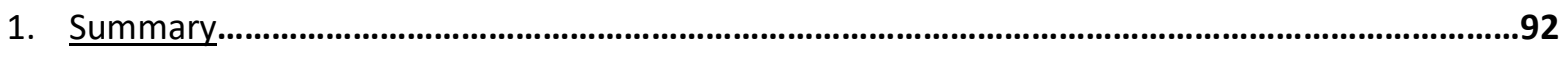

2. Co-incorporation: A New Method to Combine Precipitation Inhibitors with Mesoporous Silica..93

3. In Silico Pharmaceutics: A New Method to Select Precipitation Inhibitors for Mesoporous Silica.93

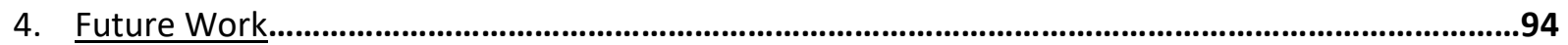

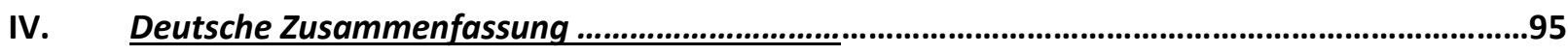

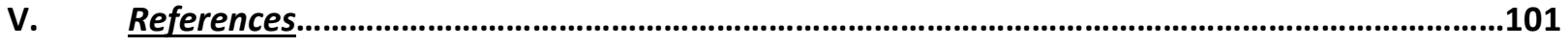

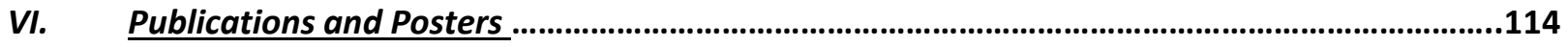

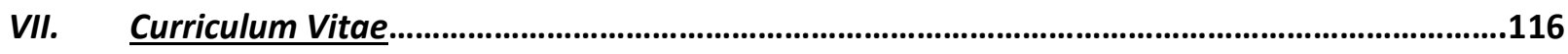

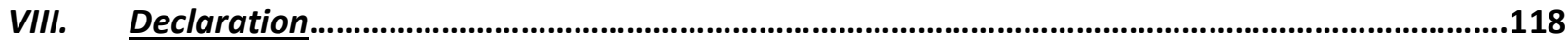

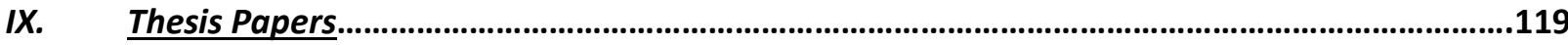




\section{Introduction}

\section{Overview}

Due to benefits around convenience, patient compliance and cost-effectiveness, the oral drug delivery route is by far the most favored for administration of medications (Krishnaiah, et al. 2010). For an orally delivered drug to exert its biological effect, it must first pass through the gastrointestinal tract (GI-tract) and into the systemic circulation. Therefore, the pharmacological effect of an orally delivered drug is related to its bioavailability, the percentage of the administered amount of drug reaching systemic circulation. The bioavailability of a drug is related to its ability to cross the barriers from the GI-tract, particularly passage from the GI milieu via the intestinal membrane (Zheng, et al. 2010). To achieve this, a drug must be sufficiently soluble in the fluids of the Gl-tract and permeable to the cell membrane of the intestine. It is on these two parameters that the Biopharmaceutics Classification System (BCS) is based, which groups drugs into classes based on solubility and permeability (Amidon, et al. 1995).

In order to develop a framework for understanding the molecular properties that demonstrate a bioavailability risk, Lipinski proposed his 'rules of five (Lipinski, et al. 2000). These rules propose that, for good oral bioavailability, a drug must have no more than five hydrogen bond donors and not more than 10 hydrogen bond acceptors, a molecular weight less than $500 \mathrm{Da}$ and a log $\mathrm{P}$ of less than 5 . Over the years, there has been an exponential increase in compounds not passing the rule of 5, with poor physicochemical properties becoming more common (Gardner, et al. 2004). As a result, there is a higher incidence of drugs with low solubility in compound development pipelines, resulting in reduced bioavailability. Such low bioavailability significantly increases the risk of drug attrition, representing a loss in both therapeutic and economic potential. For example, although it is reported that $60 \%$ of approved drugs are poorly soluble, it is hypothesized that up to $90 \%$ of candidates in the development pipeline will fall under this category (Taylor, et al. 2016). This highlights the big challenge of poor drug solubility to the pharmaceutical industry and demonstrates the importance of avoiding the low bioavailability risks 
associated with poor aqueous solubility. In response, chemists and formulation scientists have developed a toolkit of strategies that can improve the solubility and subsequent bioavailability of these poorly soluble candidates. These approaches include chemical modifications that can be incorporated into synthesis such as salt formation and prodrugs; or formulation modification approaches such as micelle systems, cosolvents, particle size reduction, complexation and amorphous technologies (Timpe, et al. 2007).

Amorphous formulations are especially appealing due to the significant improvement in solubility the amorphous form can provide (Timpe, et al. 2007). By definition, an amorphous solid does not have a longrange order. This is in contrast to a crystalline solid. The absence of long-range order leads to a less dense packing of molecules in an amorphous, solid-state form and decreased intermolecular interaction (Leuner, et al. 2000). Due to this, amorphous solids tend to have substantially higher solubilities than their crystalline counterparts. However, amorphous solids are usually physically unstable or metastable due to the high energy associated with this solid-state form. Re-crystallization can drive an amorphous solid-state form to decrease its energy. Due to this tendency, it is unusual for a drug candidate to be used as a pure amorphous solid (Timpe, et al. 2007). Instead, formulation scientists can modify and stabilize the solidstate form of the poorly soluble drug molecule using various approaches. Most common amongst these approaches involve immobilizing the drug in the amorphous form in a polymeric matrix (Chokshi, et al. 2007). The two main methods to produce such polymeric amorphous solid dispersions are hot melt extrusion and spray drying.

One under-utilized route to amorphous solids is the use of mesoporous materials as drug carriers. A mesoporous material is defined by the International Union of Applied and Pure Chemists (IUPAC) as "any material containing pores of dimensions between 2 and 50nm" (McCarthy, et al. 2016). In recent years, mesoporous silica has emerged as a novel formulation option for stabilization and deliver of amorphous molecules. Mesoporous silica is a silicon dioxide excipient that possesses a highly porous network. Upon impregnation of the silica with a concentrated drug solution, the drug can be molecularly adsorbed onto 
the surface of the silica. Due to the size of the pores, which have an approximate mean diameter of $6 \mathrm{~nm}$, the molecularly adsorbed molecule is locally and sterically confined, preventing recrystallization (Ditzinger, 2018). Upon administration of mesoporous silica formulations to the body, there is a displacement of individual drug molecules from the surface of the silica into solution, which generates supersaturation with regards to the crystalline drug. Mesoporous silica is therefore an example of a supersaturating formulation. As the supersaturated solution in GI-fluids usually is not stable, it requires incorporation of a precipitation inhibitor to sustain the supersaturated concentrations for physiologically relevant timescales (Guzman, et al. 2007).

The combination of supersaturating formulation technology with a precipitation inhibitor is commonly referred to as "The Spring and Parachute" model. Here, the spring is the technology that generates a high concentration of the drug in solution and supersaturation. The precipitation inhibitor is the "parachute" that sustains supersaturated drug concentrations and slows the inevitable return towards thermodynamic concentrations (Guzmann, et al. 2007).

Given that mesoporous silica is a relatively novel formulation technology, little work has been done to understand how to most effectively select and formulate precipitation inhibitors for silica formulations. Therefore, the aim of this thesis is to update the state of the art of precipitation inhibitor theory, selection and design and to propose new experimental and in silico methods for the screening, selection and incorporation of precipitation inhibitors with mesoporous silica formulation technology. 


\section{Theoretical Background}

\subsection{Factors impacting oral drug absorption}

\subsubsection{Solubility}

Solubility is the ability of a substance, or solute, to dissolve in a solvent to form a homogenous mixture of the two (Grant, et al. 1990). The thermodynamic solubility is the equilibrium state where dissolution rate is equal to precipitation rate, i.e. the point at which no change is observed in the concentration in the system (Yalkowsky, et al. 1999).

Solubility of a solute in a solvent is governed by a number of factors, which, when simplified, can be broken down into three main components. First, the individual solute molecule must be separated from the solid material. This requires the breaking of solute-solute intermolecular interactions, or the crystal lattice in case of crystalline solids. Next, a cavity must be created within the solvent, this is referred to as cavitation. Finally, the solute molecule must be inserted into the newly created cavity. Therefore, the process of solvation is dictated by the energy costs or gains associated with each individual step in the process (Eq. 1) (Yalkowsky, et al. 1999).

\section{$S=f(c r y s t a l$ lattice energy + cavitation energy + solvation energy) (Equation 1)}

It is generally accepted that the cavitation energy is negligible. Therefore, the solvation energy can be seen as a function of the affinity of the drug for the solvent and the crystal lattice energy of the drug. In the case of oral drug delivery, the solvents in question are the media of the GI tract. The affinity of the drug for the solvent is usually a molecular property relating to the hydrophilicity, lipophilicity and hydrogen-bonding capability of the molecule. The crystal lattice energy is related to the strength of the intermolecular interactions in the crystal lattice (Yalkowsky, et al. 1999). 


\subsubsection{Supersaturation}

Supersaturation can be defined from a physicochemical perspective as a system in which the free energy of the solute in solution is higher than the free energy of a crystalline or amorphous solid phase at equilibrium (Figure 1) (Taylor, et al. 2016). Essentially, this refers to a solution in which the concentration of drug is higher than the thermodynamic solubility, representing an excess energy system.

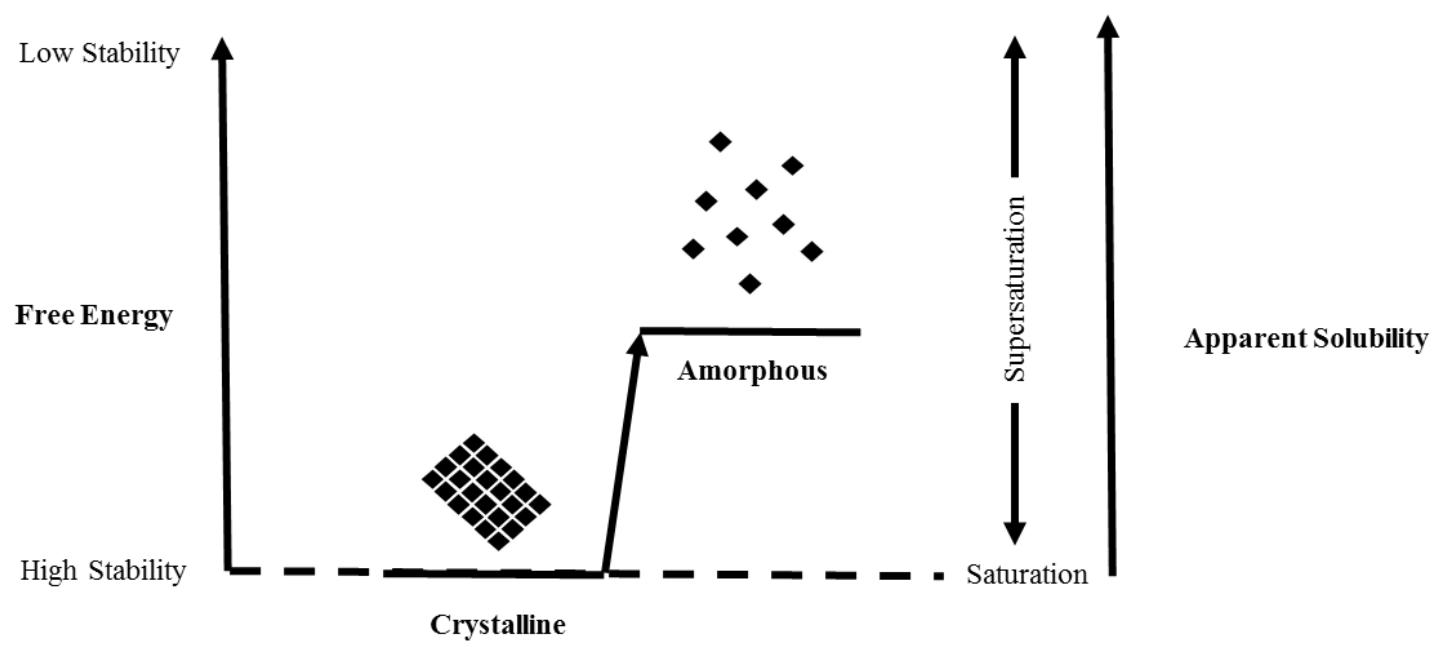

Figure 1. Energy diagram showing the advantages and disadvantages of crystalline and amorphous solidstate form from a stability and solubility perspective. Reproduced with permission from the publisher (Price, et al. 2018)

During a dissolution process yielding a supersaturated solution of a drug, the concentration of the drug in solution increases until its thermodynamic solubility is exceeded (Taylor, et al. 2016) (Yalkowsky, et al. 1999). Starting from this point supersaturation is established. Given the excess energy of the supersaturated state, this system exists in a metastable state. Ultimately, this metastability acts as a driving force for precipitation of the solute in either an amorphous or a crystalline form. This precipitation behavior must be considered if the goal of the generation of supersaturation is to increase absorption across the intestinal membrane (Price, et al. 2018). 
Supersaturation can be triggered either by delivering the drug in a pre-solubilized form (e.g. SEDDS or lipidbased formulations) or in a rapidly dissolving, metastable solid-state form (e.g. amorphous solid solution, amorphous dispersion, metastable polymorphs, nanosized particles, or even pharmaceutical salts or cocrystals and prodrugs) (Timpe, et al. 2007).

\subsubsection{Impact of Solubility and Supersaturation on Oral Absorption}

For an orally delivered drug to reach its physiological target, it must first pass through the stomach, into the intestine and through the intestinal membrane to the systemic circulation. Therefore, absorption of the drug from the intestine is a critical stage in the action of a drug (Zheng, et al. 2012).

This adsorption through the cell membrane is referred to as flux $(F)$ and is related to the concentration gradient through the membrane and the innate permeability of the molecule. (Equation 2).

$F=\frac{D K A}{h}\left(C_{\text {lumen }}-C_{\text {basolateral }}\right) \quad$ (Equation 2)

$C_{\text {lumen }}$ is the drug concentration adjacent to the membrane, while $C_{\text {basolateral }}$ is the concentration of drug absorbed through the membrane, resulting in the concentration gradient. $\mathrm{K}$ is the partition coefficient of drug between the aqueous intestinal fluid and membrane, $h$ is the width of the membrane, $D$ is the diffusion coefficient of the drug and $A$ is the surface area of the intestinal membrane, which collectively represent the permeability of the drug through the membrane (Zheng, et al. 2012).

One can assume, based on the circulation of blood away from the point of absorption, that the concentration of drug in the intestinal lumen is significantly higher than the concentration of drug at the basolateral membrane, so $C_{\text {lumen }}$ can be neglected. Furthermore, D, K, A and $\mathrm{h}$ are fixed values that are related to the drug's permeability through the cell membrane, and can be collectively referred to as a permeability constant, $\mathbf{P}$. Therefore, the maximum flux of the drug through the intestinal membrane is usually considered to be a product of the intestinal constant, $\mathbf{P}$, and the thermodynamic solubility of the 
drug in the intestine, $C_{\text {lumen. }}$ As a result, Eq 1. can be re-written (Equation 3). However, in the case of a supersaturating formulation, this would be the apparent solubility upon generation of supersaturation.

\section{$F=P . C_{\text {lumen }} \quad$ (Equation 3)}

In other words, absorption of the drug through the intestinal membrane into systemic circulation is a function of permeability and solubility or apparent solubility. A low thermodynamic solubility is expected to result in reduced absorption from the GI tract. However, if the intestinal concentration can be enhanced via the generation of supersaturation, oral absorption of the drug can be substantially improved (Ditzinger, et al. 2018).

The importance of solubility and permeability in oral drug absorption is underpinned in the Biopharmaceutics Classification System (BCS), which groups compounds into various classes related to these two properties (Amidon, et al. 1995).

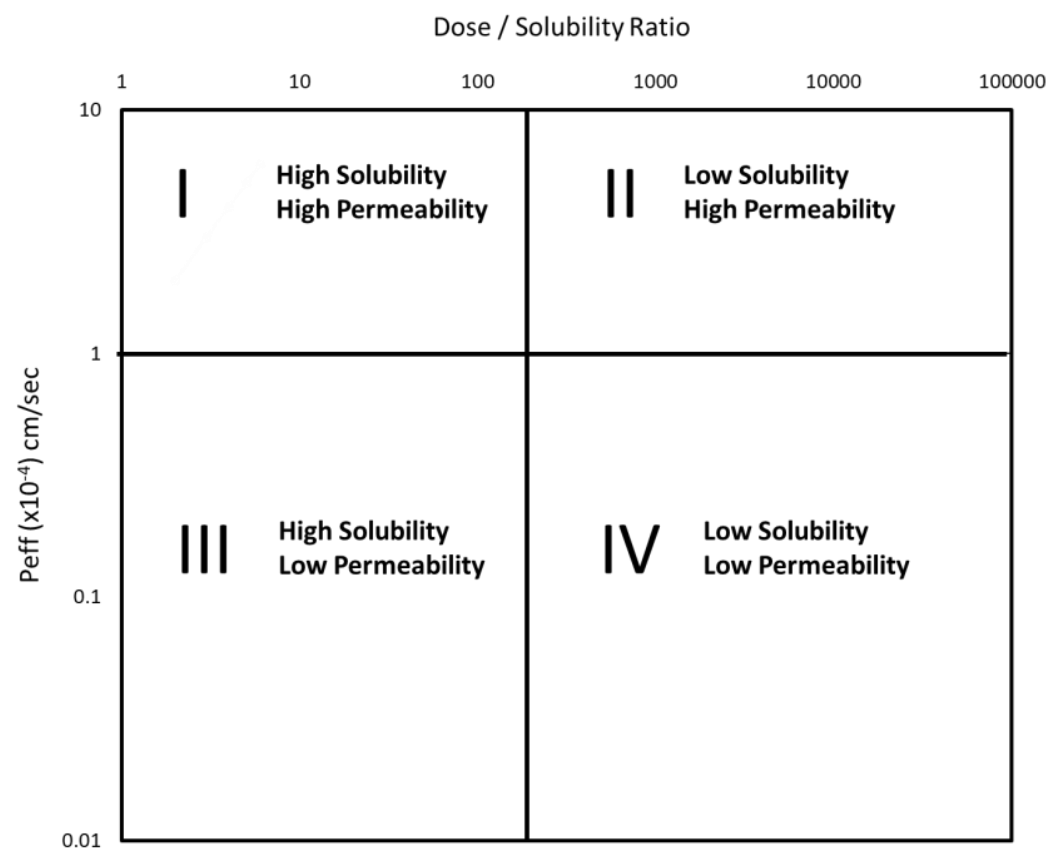

Figure 2. The Biopharmaceutical Classification System. Reproduced with permission from the publisher (Price, et al. 2018) 
As per the BCS, a drug is counted as "highly soluble" if the highest dose strength is soluble in $250 \mathrm{~mL}$ of aqueous media across physiological $\mathrm{pH}$ range $(\mathrm{pH} 1.2-7.5)$. A drug is considered to be highly permeable if the extent of absorption through a cell-membrane is $90 \%$ or more based on mass-balance determination (Amidon, et al. 1995). This can be assessed in in vitro permeability assays or in in vivo studies relative to an intravenous dose.

\subsubsection{Dissolution and Impact on Oral Absorption}

Although solubility and permeability are important components of drug absorption in the GI tract, another important consideration is dissolution (Dokoumetzidis, et al. 2006). Thermodynamic solubility, by definition, is an equilibrium value and contains no information about the rate at which the system reaches this equilibrium. Therefore, it is also crucial to consider the dissolution rate of the compound, to ensure that the equilibrium concentrations will be realized before the compound is cleared from the body via waste, with typical small intestinal transit times of between 2 and 5 hours being reported (Dokoumetzidis, 2006).

According to the film theory of dissolution, in order for a drug particle to dissolve, a concentration gradient must exist between the drug in solution (C) and the drug concentration at equilibrium $\left(C_{s}\right)$, the two of which are separated by a layer of unstirred aqueous phase (h). This gradient is the driving force for dissolution, and the rate of dissolution is therefore related to the rate of diffusion through the unstirred boundary layer (Noyes and Whitney, 1897).

This rate of diffusion across the unstirred boundary layer can be considered by simple diffusion kinetics (Equation 4):

$\frac{d c x}{d t}=\frac{D A}{h}\left(C_{s}-C\right) \quad$ (Equation 4)

where $\boldsymbol{D}$ is the diffusion rate of the drug and $\boldsymbol{A}$ is the surface area of the dissolving solid. 
Practically speaking, the dissolution rate of a drug is related to its surface area, or particle size, and its equilibrium solubility.

Given the importance of both solubility and dissolution, it is clear that the BCS class of a molecule may be misleading, if the rate of dissolution is not known. Recently, the BCS has been refined and expanded in a framework referred to as the "developability classification system" (DCS). This system, developed by Butler and Dressman, was designed to facilitate the appropriate formulation of BCS II compounds (Figure 3) (Butler, et al. 2010). Key changes in the DCS include the measurement of solubility in biorelevant media, rather than compendial buffers. Furthermore, the volume of solvent which is used for the assessment is changed from $250 \mathrm{~mL}$ to $500 \mathrm{~mL}$. Perhaps the most impactful change is in the separation of BCS II into two subcategories: DCS Ila and IIb, depending on whether the absorption of the compound is dissolution limited (Ila) or solubility limited (IIb). This is a significant distinction that can aid pharmaceutical scientists in developing formulations for DCS II compounds, as dissolution limited solubility can be overcome with simple techniques such as particle size reduction which accelerates dissolution, in accordance with the Noyes Whitney equation based on offering a higher specific surface (Dokoumetzidis, et al. 2006). However, using the same approach for DCS IIb drugs would be ineffective, as here it would be key to increase concentration of the dissolved drug, e.g. by realizing a supersaturated system in the GI tract. 


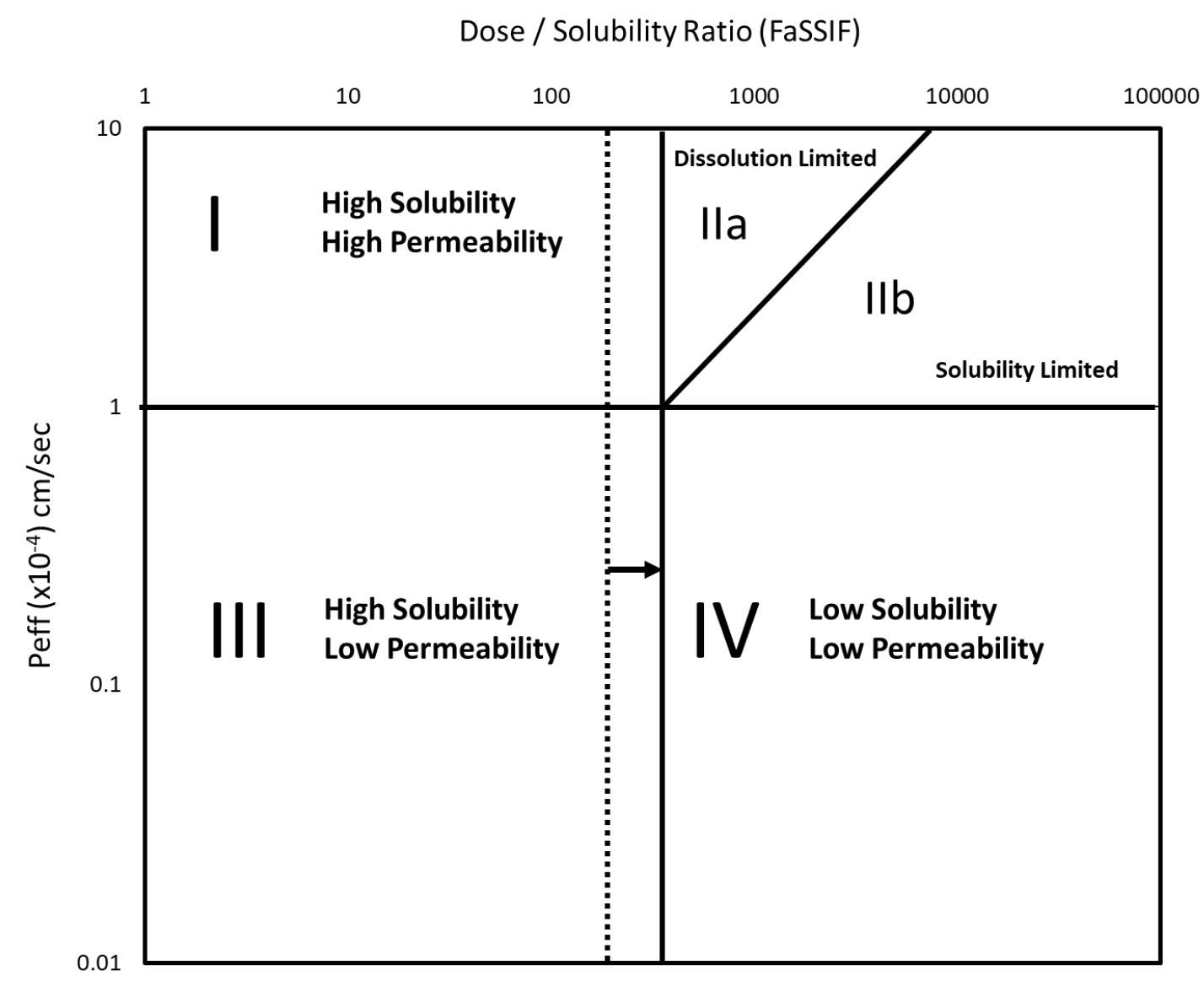

Figure 3. The Developability Classification System (Butler, et al. 2010). Reproduced with permission from the publisher (Price, et al. 2018).

\subsection{Approaches to Improve Drug Solubility, Dissolution and Oral Absorption}

\subsubsection{Formulation Technologies to Tackle Poor Druq Solubility}

$60 \%$ of approved drugs meet the BCS criteria for low solubility. However, it has been reported that $90 \%$ of drug candidates in pharmaceutical R\&D pipelines meet this definition (Zheng, et al. 2008). Therefore, approaches to overcome the biopharmaceutical barriers for poorly soluble compounds are an increasingly important component of a successful drug development campaign. For this purpose, several formulation options exist. 


\section{Lipid-Based Formulations}

Lipid-based formulations (LBFs) are a relatively common approach to overcome solubility limited (DCS IIb) oral absorption (Ditzinger, 2018). They are defined as delivery systems, in which the drug is dissolved or suspended in a lipidic system. LBFs can consist of triglyceride oils, partial glycerides, surfactants or cosurfactants and co-solvents (Pouton, et al. 2000). Generally, solubilized LBFs are more common, and the bioavailability enhancement is related to generation of solubilized and/or supersaturated drug upon the dispersion and digestion of the lipid in the GI tract. As previously discussed, in the case of supersaturation being generated in LBFs, there is a risk of precipitation due to the metastable nature of the supersaturated state, which may require a precipitation inhibitor to sustain concentrations and thus improve oral drug absorption. A wide variety of drugs have been formulated in LBF (Ditzinger, et al. 2018). Drugs that are especially suited to LBF are the so-called "grease-ball" molecules, those with a high lipophilicity represented by a high log P. Furthermore, such compounds may also have relatively weak crystal lattices, improving the likelihood of complete solubilization into the lipid phase. For "brick-dust" molecules, those with a relatively high crystal lattice energy, solubilization generally will be limited in the lipid phase and LBFs are not as useful for formulation of these drugs. However, recent work has been carried out to demonstrate the possibility of supersaturating such "brick-dust" molecules in lipid formulations using heat-cool cycles (Koehl, et al. 2019). Of course, such formulations may encounter long-term stability issues, which could limit practical application as commercially viable formulations, but they are an attractive option for pre-clinical studies where dose-escalation requires high concentrations in easily deliverable formulations, e.g. for toxicological studies (Koehl, et al. 2019).

\section{Complexation}

Complexation in the context of drug solubilization generally refers to formulation with a class of excipients known as cyclodextrins and is a common strategy for improving performance of drugs with solubility 
limitations (DCS IIb) (Brewster, et al. 2007). Cyclodextrins are cyclic oligosaccharides that are derived from starch and are composed of $\alpha-(1,4)$-linked $\alpha$-D-glucopyranose units. The classification of CDs is related to the number of glucose units, with $\alpha, \beta, \gamma$ CDs consisting of six, seven or eight units, respectively. CDs have a distribution of polarity throughout the molecule, with polar hydroxyl groups on the outer rim of the characteristic "torus" shape and less polar oxygen and hydrogen groups on the inside (Brewster, et al. 2007). As a result, hydrophobic drugs can complex with the hydrophobic inner core whilst the outer hydrophilic groups facilitate the aqueous solubility of the entire complex, thus potentially enhancing oral drug absorption. A critical factor for enhanced bioavailability with CDs is the drug's off rate from the complex. If a drug is bound too strongly to the hydrophobic inner core, the solubilized drug substance will pass through the $\mathrm{GI}$ tract staying bound to the CD (Brewster, et al. 2008). As the molecular weight of the $\mathrm{CD}$-drug complex is much higher than that of the drug substance, it cannot be absorbed through the gut wall and the drug would be excreted as the complex with the feces (Ditzinger, et al. 2019).

\section{Particle Size Reduction}

In accordance with the Noyes-Whitney equation, reduction of particle size can increase dissolution rate of a drug (Noyes and Whitney, et al. 1897) Therefore, especially for DCS Ila drugs, reduction of particle size is a viable and often used technique to overcome limited oral absorption. Historically, particle size reduction has referred to micronization of drugs, in which drug particles are deconstructed into smaller particles via mechanical activation with milling approaches including, but not limited to ball milling, wet milling and cryo-milling. Recent technologies enable the production of particles on the nanoscale (Khadka, et al. 2014). Such nanosizing approaches can be described as either "top-down" or "bottom-up" depending on the method employed. The top down approach is analogous to the micronization approaches, in which specialized milling approaches such as pearl milling or high-pressure homogenization are applied to deconstruct micron-sized particles into nanoparticles that enhance dissolution and thus improve absorption (Khadka, et al. 2014). The "top-down" approach, by contrast, engineers the particle 
properties during the precipitation process, such that nanoprecipitates are generated (Khadka, et al. 2014). For compounds whose high crystal lattice energy is limiting to solvation (e.g. brickdust molecules) nano-suspensions are attractive alternatives to solubilization techniques (Koehl, et al. et al. 2019). This is especially the case for those compounds whose solubility is limited in both polar and apolar solvents.

A key difference between micronization and nano-sizing is the necessity to stabilize nanoparticles to prevent agglomeration and a return to micron-sized particles. Typically, surfactants or polymers can be incorporated to ensure particles remain on the nanoscale (Ditzinger, et al. 2019). Long-term stability can be an issue with nano-suspensions, which limit their application. However, they are especially useful in pre-clinical trials where large doses of drug candidates are required but have also found application in approved formulations.

\section{Formulation Technologies for Amorphous Systems}

Solubility and dissolution of a poorly soluble drug can be enhanced by altering the solid-state form of a drug substance to the amorphous form. The amorphous state is defined by a lack of long-range order, in contrast to a crystal structure (Williams, et al. 2013). Without the need to disturb a crystal lattice, the energy required to break the intermolecular interactions in the amorphous phase is significantly reduced. Therefore, creating a solution of an amorphous solid is inherently energetically more favorable than for the corresponding crystalline solid.

Energetically speaking, the amorphous form is in a state of higher energy (Figure 4) and is therefore unstable or metastable and unlikely to remain amorphous over the duration of the shelf-life of the product (Taylor, et al. 2016). Amorphous drugs are therefore generally not used directly in drug products without an additional stabilizing component (Williams, et al. 2013). 


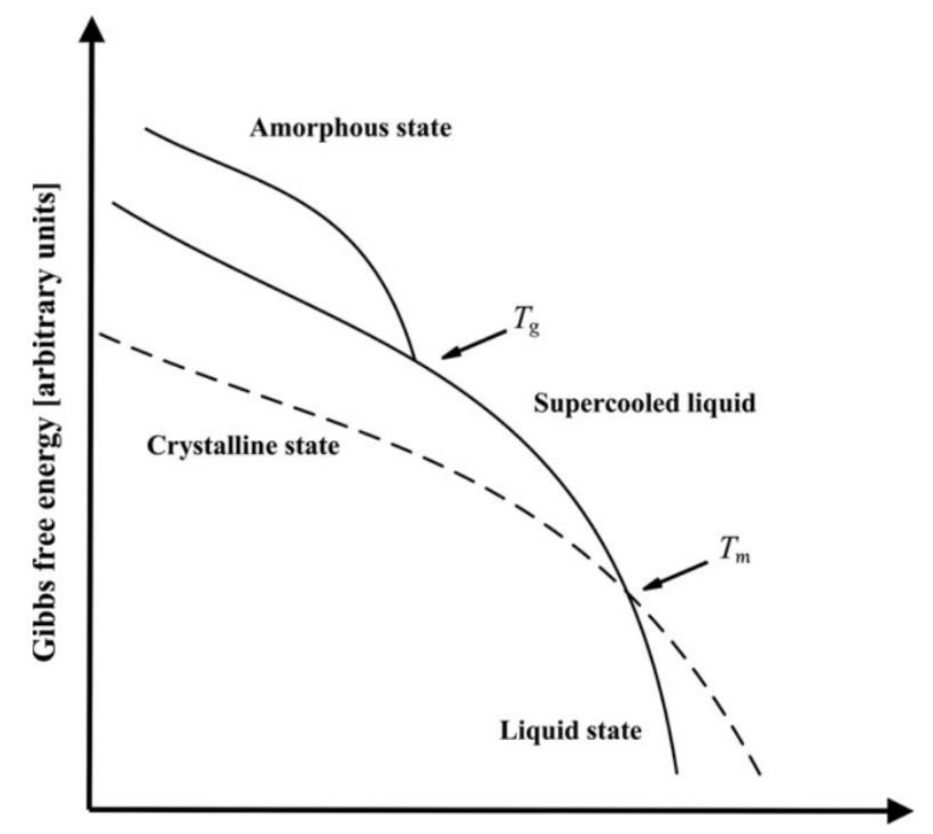

Figure 4. Gibbs Free Energy curve demonstrating the excess energy present in the amorphous state. Reproduced with permission from the publisher (Price, et al. 2018).

Therefore, the critical issue for successful amorphous formulation is stabilization of the amorphous form. Historically, this has been achieved through formulation in amorphous solid dispersions (ASD) (Ditzinger, et al. 2018). According to definition, an ASD is any formulation in which amorphous drug substance is distributed throughout a carrier, typically a polymer. For optimal stability, it is desirable that the drug is distributed homogenously throughout the carrier, allowing stabilization of the amorphous form in the polymer matrix (Williams, et al. 2013).

\subsubsection{Preventing Precipitation from the Supersaturated State}

Collectively, the combination of a supersaturating formulation technology with a precipitation inhibitor is known as the "Spring and Parachute" model. The formulation generates the "spring" of supersaturation leading to a fast and high increase of drug concentration in solution, while the precipitation inhibitor acts 

et al. 2004) (Figure 5).

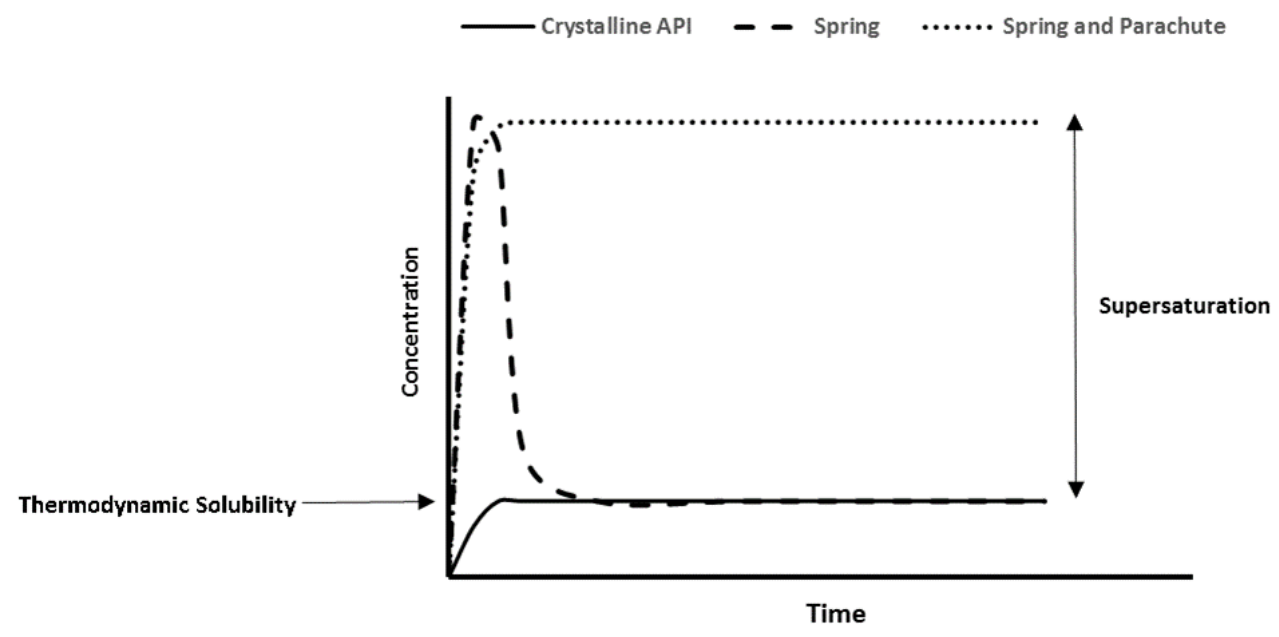

Figure 5. The Spring and Parachute model. A common formulation approach where a 'spring' generates supersaturation and a 'parachute' prevents precipitation. Reproduced with permission from the publisher (Price, et al. 2018).

Ultimately, the success of supersaturating formulations is related to the appropriate selection and combination of precipitation inhibitors, i.e. the "parachute", with the selected "spring" formulation technology. To ensure this, a thorough understanding of the underpinnings of precipitation and precipitation inhibition is essential.

\section{Precipitation}

Precipitation or crystallisation of supersaturated drug from solution is an energy driven process related to the excess energy of a supersaturated solution that has reached a critical labile point where phase separation of solute molecules occurs instantaneously and without external influence (Mullin, et al. 2000). This process is a function of concentration, temperature and composition of the solution. A higher 
concentration of solute increases the lability of the system, whereas an increase in temperature decreases the lability of the system (Figure 6).

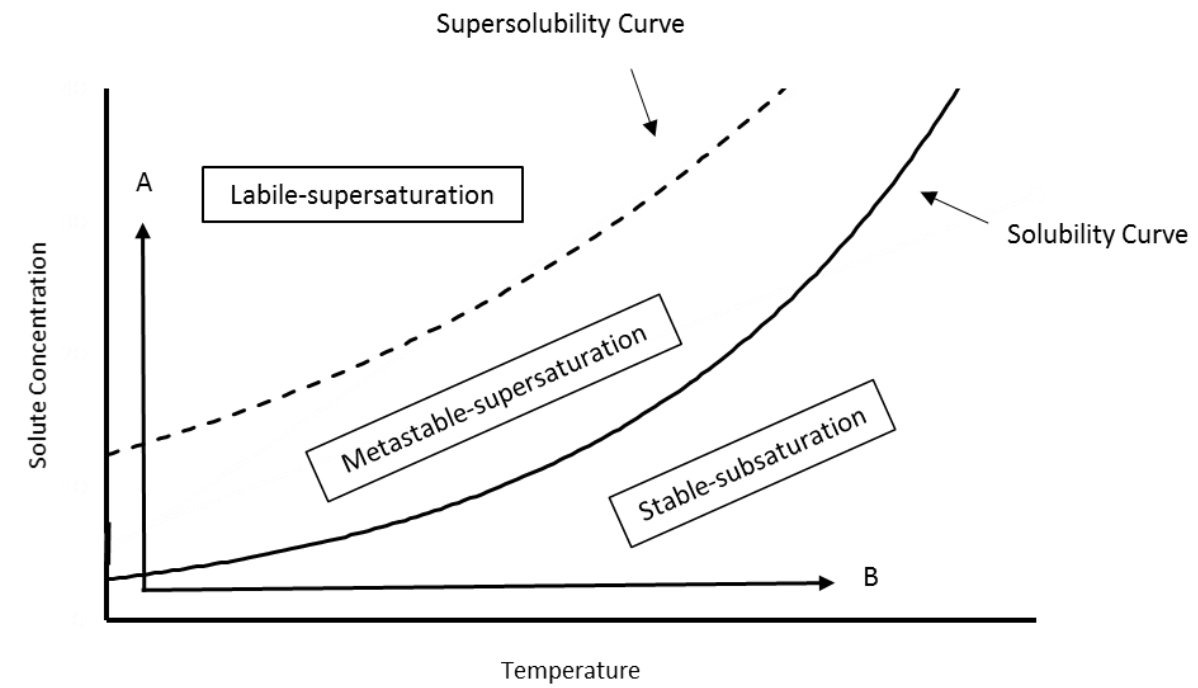

Figure 6. Solubility-supersolubility diagram (Mullin, 2007). A more labile solution is obtained with a higher drug concentration (arrow A). A more stable solution is obtained by the increase in temperature (arrow B). Reproduced with permission from the publisher (Price, et al. 2018).

The process of crystallization or precipitation occurs in two key stages: a) nucleation and b) crystal or particle growth (Mullin, et al. 2000). For crystal and particle growth, a critical nucleus consisting of solute molecules must first be attained. Nucleation and particle growth can occur at the same time and at different rates, depending on the system and stage of precipitation (Warren, et al. 2010). The exact mechanism of nucleation remains unclear but can occur spontaneously or can be induced. It can be induced with or without seed crystals and in the presence or absence of a cluster surface, e.g. foreign particles or surface defects. In any case, nucleation can be described mathematically by classical nucleation theory (CNT) (Mullin, et al. 2000) (Equation 5).

$$
J=A \exp \left(-\frac{B}{\ln ^{2} S}\right) \quad \text { (Equation 5) }
$$


Where $J$ is the rate of nucleation, $S$ is the supersaturation, $A$ is a nucleation constant, $B$ is a thermodynamic value related to the energy cost of nucleation. Both A and B are considered fixed for practical purposes, which underscores the importance extent of supersaturation play in the precipitation processes.

Crystal growth can occur once critical nuclei are formed. Various mechanistic hypotheses have been proposed for crystal growth, but the predominant model is the Adsorption Layer Theory (Mullin, et al. 2000). In this model, it is assumed that a layer of adsorbed molecules exists on the growing crystal or precipitate. This layer exists in equilibrium with the bulk solution. The model states that the solid precipitate will only grow if the solute molecules in solution can adsorb to a site on the growing nucleus or particle which represents an energy minimum (Warren, et al. 2010). These contacts are typically found at imperfections or kinks on the growing crystal or particle surface. This process results in the formation of the thermodynamically favourable polymorph over time, but kinetic forms may arise first. This phenomenon is known as Ostwald's rule of stages, which indicates that the fastest forming polymorph does not represent the thermodynamic stable polymorph but rather that polymorphs are obtained in the order of decreasing energy (Nyvlt, et al. 1995).

For supersaturating formulations, it is critical to prevent both nucleation and crystal growth to allow for increased absorption.

\section{Precipitation Inhibition}

Typically, inhibition of precipitation from the supersaturated state is achieved by incorporating polymers into the formulation (Warren, et al. 2010). These polymeric "precipitation inhibitors" have seen widespread usage in the literature, with the most common polymers used including cellulose derivatives, methacrylates and polyvinylpyrrolidones (Warren, et al. 2010). This kinetic effect is related to polymeric interference with nucleation and crystal growth through interaction with both the supersaturated drug and the aqueous environment ( $\mathrm{Xu}$, et al. 2013). These interactions generally prevent the adsorption of 
drug molecules onto the growing crystal face and therefore precipitation is arrested. As previously alluded to, this is a kinetic effect and usually has no impact on the thermodynamic saturation concentrations of the system. In some systems, the polymer may also have a co-solvent effect, thus contributing to both a thermodynamic and kinetic stabilization of the drug in solution (Warren, et al. 2010).

For kinetic inhibition, interaction between the drug and polymer is key. Specifically, polymers can interact with the drug via hydrogen bonds, polar, or dispersion forces, to varying degrees (Price, et al. 2018; Price, et al. 2019; Warren, et al. 2010; Brouwers et al. 2009; Gao, 2 et al. 2012) Although extensive work has been carried out on the application and screening of precipitation inhibition, the exact mechanism of interaction remains unclear. In any case, it is likely to vary from system to system. More broadly, PI effectiveness has been related to properties such as temperature, molecular weight, polarity and hydrogen bonding capabilities of both the drug and polymer (Warren, et al. 2010). In addition to the more general discussion about potential properties relating to effective inhibition, some modes of action have also been proposed. For example, the extent of polymer adsorption onto the surface of the growing crystal is often discussed as one of the critical factors for successful precipitation inhibition. This adsorption introduces steric hindrance, which disrupts the diffusion of supersaturated drug through the solid-liquid boundary layer to the surface of the growing crystal (Brouwers, et al. 2009). In addition, the crystal conformation can be disrupted by polymers in solution. Also discussed, is the effect of polymers on the surface tension and viscosity of the solution, which can reduce the diffusion of the drug to the crystal growth sites (Warren, et al. 2010). Thus, an important factor to consider is the effect of the polymer on solution viscosity. The effect of the viscosity is two-fold: first, an increased viscosity results in a slower solute diffusion coefficient and second, an increased viscosity is often related to an increased number of potential binding sites for polymer-drug interaction. However, even considering the potential PI mechanism, there are still differing conclusions in the literature. Some studies show polymer hydrophobicity to be a critical property in precipitation inhibition (Prasad, et al. 2016), some suggest that 
hydrogen bond interactions play a pivotal role (Warren, et al. 2010), while yet others propose that polymer surface coverage is an important factor (Schram, et al. 2015). Ultimately, although many precipitation inhibition mechanisms have been proposed, it remains unclear to what extent these factors effect precipitation inhibition. In all likelihood, multiple precipitation inhibition mechanisms contribute to the observed effect, with the balance depending on the specific properties of both the drug substance and the precipitation inhibitor.

\section{Selection of Precipitation Inhibitors}

Given the large number of substances that can act as PIs reported in the literature, various screening methods to select drug-PI combinations have been developed. Invariably, these screening methods involve the generation of supersaturation in combination with a variety of analytical techniques that can determine the rate and extent of precipitation of a drug over time in many samples. A wide variety of methods to generate supersaturation are reported in the literature, including use of amorphous solids, shifts in temperature or $\mathrm{pH}$, use of pharmaceutical salts or solvent shifts. (Warren, et al. 2010) Of these techniques, the most common is the solvent shift method. This involves dissolving the drug in high concentrations in a favourable organic solvent which is miscible with water (e.g. DMSO). A small volume of this solution is added to an aqueous phase to generate a supersaturated state. Analytical techniques such as UV spectroscopy, HPLC or nephelometry can be used to assess drug concentration or amount of precipitate over time, which in turn gives information about the efficiency of the inhibitor being studied. (Warren, et al. 2010)

For example, during a drug development campaign, two Johnson and Johnson drugs, $\mathrm{A}$ and $\mathrm{B}$, required addition of PIs to a surfactant-based bioenabling formulation that generated supersaturation but did not itself prevent precipitation (Creasey, et al. 2011). In order to select an appropriate PI candidate, supersaturation was generated in the presence of a range of potential PIs for both compounds, and then 
HPLC was employed to determine residual drug concentration in solution after 24 hours. This screening platform identified Pluronic F127 as the most efficient PI (Creasey, et al. 2010; Li, et al. 2012). This type of experimental set-up is particularly attractive when pursuing surfactant-based formulations, as the methodology can simultaneously screen surfactant systems as well as PIs. In this respect, one can simultaneously assess the extent of the supersaturation generated by the surfactant and how sustained the profile is in the presence of PIs (Li, et al. 2012). The main drawback of the given experimental design is that barely any information is obtained about the kinetics of drug precipitation in the presence of polymer when using a single time point.

An alternative screening platform which utilises off-line chromatography was reported by Petrusevska and co-workers (Petrusevska, et al. 2013). In this study, supersaturation was generated for fenofibrate and carbamazepine, in the presence of various potential PIs, using a solvent shift from DMSO into aqueous buffer. Experiments were carried out in 96 well plates. The plates were sealed and incubated, with samples being taken at 30, 90, 180 and 360 minutes, filtered and analysed with UPLC for drug content. This method provides more information about which $\mathrm{PI}$ is the most efficient over physiologically relevant time scales. In this study, it was found that surfactants such as Tween ${ }^{\circledR}$ and Cremophor ${ }^{\circledR}$ were most efficient for fenofibrate, whereas for carbamazepine cellulose derivatives such as HPMC and HPMCAS were the optimal systems (Petrusevska, et al. 2013).

Despite the limited time-resolution that such 'off-line' methods can provide, they can be very reliable when it comes to predicting performance of the final formulation. Yamashita and colleagues performed a similar high-throughput screen for a range of surfactants, oils and polymers in combination with itraconazole. The screen demonstrated that HPMCAS was the most efficient PI. When itraconazoleHPMCAS spray-dried dispersions were manufactured and compared to the commercially available Sporanox ${ }^{\circledR}$ HPMC-based dispersions, the HPMCAS-based formulations significantly outperformed the commercial product in dissolution tests (Yamashita, et al. 2011). 
Analytical techniques that offer in situ analysis are very appealing as they can provide a real-time picture of supersaturation-precipitation behaviour. It was demonstrated by Warren and colleagues that utilising in situ nephelometry, a technique that uses light-scattering to measure particle concentration, can provide an indirect measure of drug concentration in a high-throughput screen (Warren, 2010). In this instance, the nephelometer measures light scattering of the samples, which directly relates to the total concentration of particular matter in suspension and drug in solution. A large number of species was screened for precipitation inhibition using a plate reader, according to which the researchers were able to sort the PIs into three distinct groups. (Warren, et al. 2010)

Chauan and co-workers expanded upon this technique by utilising an in-situ UV probe that provided timeresolved information about the concentration of drug in solution (Chauhan, et al. 2014). This method was used to assess the interactions between indomethacin and a wide range of polymers. After a solvent-shift to generate supersaturation, the turbidity and drug concentration were measured using an in-situ UVprobe. The time-resolved data so obtained enable further calculation and processing. Chauan and colleagues subsequently used these data to calculate the precipitation induction time (time delay between supersaturation and precipitation) as well as the rate of precipitation, which indicated that PVP, HPMC and Eudragit E100 increased induction time and decreased the precipitation rate. Subsequently, successful solid dispersions of indomethacin-PVP, indomethacin-HPMC and indomethacin-Eudragit ${ }^{\circledast}$ E100 were developed (Chauhan, 2014). A similar study with dipyridamole was also carried out (Chauhan, et al. 2013). The downside of using nephelometry as a screening tool is the difficulty of screening systems that are insoluble. For example, some supersaturating formulations, e.g. mesoporous silica, contain insoluble excipients that would interfere with the light scattering and make analysis difficult.

Recent advances in PI screening have seen the introduction of smaller scale dissolution techniques, such as the $\mu$ DISS profiler ${ }^{T M}$ apparatus, produced by Pion. The $\mu$ DISS ${ }^{\mathrm{TM}}$ utilises in situ UV in combination with liquid handling and can be used to efficiently study supersaturation and precipitation in real-time. 
Palmelund and co-workers studied six different poorly soluble drugs in combination with HPMC or PVP at different degrees of supersaturation using this technique (Palmelund, et al. 2016). They were able to successfully discriminate between innate solubility enhancements of the polymers vs. precipitation inhibition. For the BCS IV drug, aprepitant, for example, both polymers increased solubility by approximately $150 \%$, with the solubility being the same in both polymer systems. There were distinct differences in the curves observed in the real-time data display, with HPMC showing a more pronounced effect on the dissolution profile than PVP. Therefore, for this system, HPMC acted as a more effective PI than PVP (Palmelund, et al. 2016). According to the authors, the $\mu$ DISS profiler ${ }^{\mathrm{TM}}$ is particularly appealing as the experimental protocols can be easily standardised to reduced inter- and intra-lab variability. (Palmelund, 2016). The $\mu$ DISS profiler ${ }^{\mathrm{TM}}$ has also been applied to investigate the effect of prandial state and PIs on the precipitation of supersaturated zafirlukast (Madsen, et al. 2016).

\section{Recent Advances in Supersaturating Formulations}

\subsection{Mesoporous Silica: An Emerging Formulation Technology}

Mesoporous silica is a relatively novel formulation option for poorly soluble drugs, with no examples of approved drug products and only one proof of concept study in man (Bukara, 2016). However, there has been a dramatic increase in interest around mesoporous silica-based formulations in the past decade. A summary of all mesoporous silica formulations reported to date is shown in Table 1.

Mesoporous silica refers to a variety of materials synthesized to produce a $\mathrm{SiO}_{2}$ mesoporous structures. (Limnell et al., 2011) Mesoporous silica can be ordered or non-ordered (Barbe et al. 2008; Kresse et al. 1992). The former include classic structures such as SBA-15 and MCM-41, (Mellaerts et al. 2010) whilst the latter include novel, proprietary excipients manufactured by drug delivery specialists, such as Parteck SLC ${ }^{\circledast}$ (Merck Milipore) (Laine et al., 2016; O’Shea et al. 2017) and Neusilin ${ }^{\text {TM }}$ (Fuji Chemical) (Khanfar and Al-Nimry, 2017). Such materials have a wide range of applications, including tissue engineering 
(Vallet-Regi et al., 2010), catalysis (Saad, et al. 2017), chromatography (Majors, et al. 1972), adsorbents in environmental sciences (Bhatnagar et al., 2010) and drug delivery for poorly soluble drugs (Wang, et al. 2009). With respect to drug delivery applications, it has been widely reported that mesoporous silica can act as a dissolution enhancer by 'trapping' drug in the amorphous form within the mesoporous network (O’Shea, et al. 2017; Laine, et al. 2016; Van Speybroeck, et al. 2010; Vialpando, et al. 2011; Dressman, et al. 2010; Xu, et al. 2013).

For ordered mesoporous silica, a template synthesis process is carried out, in which pores are constructed with consistent pore size and geometry. For unordered mesoporous silica, pore size and geometry are not controlled. An exhaustive comparison of the advantages and disadvantages of ordered vs. unordered mesoporous silica is lacking in the literature. However, the costs of producing ordered mesoporous silica limits their applicability from an economic perspective. Mesoporous silica, by definition, are porous in nature with pores from between 2-50 nm in diameter (McCarthy, et al. 2016). However, in line with their usual intended application, pore sizes on the lower end of this range are more common. This porosity results in a high surface area, up to $1000 \mathrm{~m}^{2} / \mathrm{g}$, which is important for loading drugs inside the porous network.

There are various methods of loading crystalline drugs onto mesoporous silica. The methods can be grouped into three categories: solvent-based (e.g. Laine et al. 2016), mechanical activation (e.g. Qian and Bogner, 2011) and vapour-mediated (e.g. Gignone, et al. 2014). A thorough overview of the loading methods of poorly soluble drug onto mesoporous silica is beyond the scope of this thesis, but the interested reader is referred to a number of publications that provide a good overview of the different routes to drug-loaded silica (McCarthy, et al. 2016; Wantanabe, et al. 2000; Qian and Bogner, 2011; Hillerstrom, et al. 2014; Limnell, et al. 2011). 
Of the methods described in the literature, the solvent-based approach is most commonly employed. This can be attributed to the poor loading efficiencies and time-consuming processes involved in the solventfree loading methods (Qian and Bogner, 2011). The solvent approaches can be grouped into two main categories: solvent impregnation and incipient wetness. During the solvent impregnation loading approach, the drug is dissolved in an organic solvent and the solution is added to mesoporous silica. Adsorption of the drug onto the silica is initiated through mechanical agitation or sonication of the slurry. Finally, the solvent is removed, which can be achieved using a number of methods including vacuum drying, spray drying, lyophilization or rotary evaporation. (Laine, et al. 2016; Wei, et al. 2017; Limnell, et al. 2011; Meer, et al. 2013). Incipient wetness involves the steady addition of small volumes of a concentrated solution of the drug in an organic solvent onto the heated silica, such that the full amount of solvent is adsorbed into the network and then rapidly evaporated, leaving the drug within the pores (O'Shea 2017; Dressman, et al. 2015). Both methods result in a drug-loaded silica, in which the previously crystalline drug substance is now amorphous or molecularly dispersed on the surface of the silica. This can be confirmed with analytical methods such as DSC or PXRD.

The theoretical maximum drug content that can be loaded onto mesoporous silica is dependent on the surface area (Vallet-Regi, et al. 2007), pore volume (Wang et al. 2012) and pore geometry of the silica (Heikkila, et al. 2007) with potential values as high as $75 \%$ (w/w drug/silica) reported in the literature (Wei, et al. 2017). Experimentally, however, such high drug loadings are not achievable, with residual crystallinity often observed in drug-loads exceeding 50\% (wt/wt) (Wei, et al. 2017). Indeed, most cases of drug-loaded silica in the literature do not exceed $40 \%$ loading, in line with other amorphous formulation technologies (Table 1). 
Table 1.

Drugs for which formulations based on mesoporous silica have been elaborated and characterized

\begin{tabular}{|c|c|c|c|c|c|c|c|c|c|c|c|}
\hline Compound & Ref. & Mwt & BCS Class & $\log P$ & $\operatorname{Tg}(\mathrm{K})$ & Ref. & $\operatorname{Tm}(\mathrm{K})$ & $\mathrm{Tm} / \mathrm{Tg}$ & pKa (acid) & pKa (base) & Loading (\%) \\
\hline Atorvastatin & $\begin{array}{c}\text { Maleki } \\
\text { et al. (2016) }\end{array}$ & 558.64 & II & 5.39 & 685.325 & $\begin{array}{l}\text { Shete } \\
\text { et al. (2010) }\end{array}$ & 433.5 & 0.63 & 4.33 & - & 20 \\
\hline Valsartan & $\begin{array}{l}\text { Biswas } \\
\text { (2017) }\end{array}$ & 435.519 & III & 5.27 & 349.5 & $\begin{array}{l}\text { Skotnicki } \\
\text { et al. (2013) }\end{array}$ & 390 & 1.12 & $4 . .37$ & - & 43.25 \\
\hline Carvedilol & $\begin{array}{c}\mathrm{Hu} \\
\text { et al. (2012) }\end{array}$ & 406.482 & II & 3.42 & 312.53 & $\begin{array}{l}\text { Wyttenbach } \\
\text { et al. (2016) }\end{array}$ & 388 & 1.24 & - & 8.74 & 41.6 \\
\hline Furosemide & $\begin{array}{l}\text { Ambrogi } \\
\text { et al. (2012) }\end{array}$ & 330.745 & IV & 1.75 & 374.7 & $\begin{array}{l}\text { Nielsen } \\
\text { et al. (2015) }\end{array}$ & 479.5 & 1.28 & 4.25 & - & 30 \\
\hline Glibenclamide & $\begin{array}{c}\text { Van Speybroeck } \\
\text { et al. (2011) }\end{array}$ & 494.004 & II & 3.79 & 345.4 & Bartsch et al. (2005) & 442.5 & 1.28 & 4.32 & - & 30 \\
\hline Ezetimibe & $\begin{array}{l}\text { Kiekens et al. } \\
\text { (2012) }\end{array}$ & 409.4 & II & 4.56 & 336.35 & Knapik et al. (2014) & 436.5 & 1.30 & - & - & 20 \\
\hline
\end{tabular}




\section{Table 1. (contd.)}

Drugs for which formulations based on mesoporous silica have been elaborated and characterized

\begin{tabular}{|c|c|c|c|c|c|c|c|c|c|c|c|}
\hline Compound & Ref. & Mwt & BCS Class & $\log P$ & $\operatorname{Tg}(\mathrm{K})$ & Ref. & $\operatorname{Tm}(\mathrm{K})$ & $\mathrm{Tm} / \mathrm{Tg}$ & pKa (acid) & pKa (base) & Loading (\%) \\
\hline Celecoxib & $\begin{array}{l}\text { Laine } \\
\text { et al. (2016) }\end{array}$ & 381.373 & II & 4.01 & 331.1 & $\begin{array}{l}\text { Wyttenbach } \\
\text { et al. (2016) }\end{array}$ & 431.5 & 1.30 & - & - & 33 \\
\hline Felodipine & $\begin{array}{c}\mathrm{Hu} \\
\text { et al. (2015) }\end{array}$ & 384.259 & II & 3.44 & 317.9 & $\begin{array}{l}\text { Wyttenbach } \\
\text { et al. (2016) }\end{array}$ & 418.5 & 1.32 & - & 5.39 & 25 \\
\hline Itraconazole & $\begin{array}{l}\text { Mellaerts } \\
\text { et al. (2008) }\end{array}$ & 705.64 & II & 7.31 & 332.7 & $\begin{array}{l}\text { Wyttenbach } \\
\text { et al. (2016) }\end{array}$ & 439.7 & 1.32 & - & 3.7 & 25 \\
\hline Indomethacin & $\begin{array}{c}\text { Wang } \\
\text { et al. (2010) }\end{array}$ & 357.79 & II & 3.53 & 319.6 & $\begin{array}{l}\text { Wyttenbach } \\
\text { et al. (2016) }\end{array}$ & 424.5 & 1.33 & 3.8 & - & 20 \\
\hline Curcumin & $\begin{array}{c}\text { Hartono } \\
\text { et al. (2016) }\end{array}$ & 368.38 & II & 3.28 & 343 & $\begin{array}{c}\text { Pawar } \\
\text { et al. (2012) }\end{array}$ & 456.5 & 1.33 & - & - & 25 \\
\hline
\end{tabular}




\section{Table 1. (contd.)}

Drugs for which formulations based on mesoporous silica have been elaborated and characterized

\begin{tabular}{|c|c|c|c|c|c|c|c|c|c|c|c|}
\hline Compound & Ref. & Mwt & BCS Class & $\log P$ & $\operatorname{Tg}(\mathrm{K})$ & Ref. & $\operatorname{Tm}(\mathrm{K})$ & $\mathrm{Tm} / \mathrm{Tg}$ & pKa (acid) & pKa (base) & Loading (\%) \\
\hline Telmisartan & $\begin{array}{l}\text { Aftab Alam } \\
\text { et al. (2013) }\end{array}$ & 514.617 & II & 6.04 & 401.4 & $\begin{array}{c}\text { Lepek } \\
\text { et al. (2013) }\end{array}$ & 535.5 & 1.33 & 3.65 & 6.13 & 37.5 \\
\hline Ketoprofen & $\begin{array}{l}\text { Abd-Elrahman } \\
\text { et al. (2016) }\end{array}$ & 254.281 & II & 3.61 & 270.58 & $\begin{array}{l}\text { Wyttenbach } \\
\text { et al. (2016) }\end{array}$ & 367.5 & 1.36 & 3.88 & - & 20 \\
\hline Griseofulvin & $\begin{array}{l}\text { Salonen } \\
\text { et al. (2005) }\end{array}$ & 352.766 & II & 2.17 & 361.5 & $\begin{array}{l}\text { Zhu } \\
\text { et al. (2010) }\end{array}$ & 493.5 & 1.37 & - & - & 16.5 \\
\hline Fenofibrate & $\begin{array}{c}\text { Bukara } \\
\text { et al. (2016) }\end{array}$ & 360.831 & II & 5.28 & 254.8 & $\begin{array}{l}\text { Wyttenbach } \\
\text { et al. (2016) }\end{array}$ & 354 & 1.39 & - & - & 30 \\
\hline Nifedipine & $\begin{array}{c}\text { Xu } \\
\text { et al. (2013) }\end{array}$ & 346.335 & II & 1.82 & 320.3 & $\begin{array}{l}\text { Wyttenbach } \\
\text { et al. (2016) }\end{array}$ & 446.5 & 1.39 & - & 5.33 & 20 \\
\hline Cinnarizine & $\begin{array}{l}\text { Van Speybroeck } \\
\text { et al. (2009) }\end{array}$ & 368.514 & $\|$ & 5.88 & 281.1 & $\begin{array}{l}\text { Wyttenbach } \\
\text { et al. (2016) }\end{array}$ & 392.5 & 1.40 & - & 8.4 & 20 \\
\hline
\end{tabular}




\section{Table 1. (contd.)}

Drugs for which formulations based on mesoporous silica have been elaborated and characterized

\begin{tabular}{|c|c|c|c|c|c|c|c|c|c|c|c|}
\hline Compound & Ref. & Mwt & BCS Class & $\log P$ & $\operatorname{Tg}(K)$ & Ref. & $\operatorname{Tm}(\mathrm{K})$ & $\mathrm{Tm} / \mathrm{Tg}$ & pKa (acid) & pKa (base) & Loading (\%) \\
\hline Phenylbutazone & $\begin{array}{l}\text { Van Speybroeck } \\
\text { et al (2009) }\end{array}$ & 308.374 & II & 4.14 & 270.75 & $\begin{array}{l}\text { Wyttenbach } \\
\text { et al. (2016) }\end{array}$ & 378.5 & 1.40 & 5.13 & - & 20 \\
\hline Danazol & $\begin{array}{l}\text { Van Speybroeck } \\
\text { et al. (2009) }\end{array}$ & 337.5 & II & 3.46 & 352 & $\begin{array}{l}\text { Alhalaweh } \\
\text { et al. (2015) }\end{array}$ & 498.5 & 1.42 & - & - & 20 \\
\hline Flurbiprofen & $\begin{array}{c}\text { Tozuka } \\
\text { et al. (2005) }\end{array}$ & 244.261 & II & 3.94 & 268.7 & $\begin{array}{l}\text { Wyttenbach } \\
\text { et al. (2016) }\end{array}$ & 384 & 1.43 & 4.42 & - & 30 \\
\hline Hesperidin & $\begin{array}{c}\text { Wie } \\
\text { et al. (2017) }\end{array}$ & 610.565 & II & 2.68 & 346.9 & $\begin{array}{l}\text { Shete } \\
\text { et al. (2014) }\end{array}$ & 501 & 1.44 & - & - & 28.6 \\
\hline Etravirine & $\begin{array}{l}\text { Mellaerts } \\
\text { et al. (2013) }\end{array}$ & 435.28 & IV & 5.54 & 372.5 & $\begin{array}{c}\text { Weuts } \\
\text { et al. (2010) }\end{array}$ & 538.5 & 1.45 & - & 4.13 & 20 \\
\hline
\end{tabular}


Table 1. (contd.)

Drugs for which formulations based on mesoporous silica have been elaborated and characterized

\begin{tabular}{|c|c|c|c|c|c|c|c|c|c|c|c|}
\hline Compound & Ref. & Mwt & BCS Class & $\log P$ & $\operatorname{Tg}(K)$ & Ref. & $\operatorname{Tm}(\mathrm{K})$ & $\mathrm{Tm} / \mathrm{Tg}$ & pKa (acid) & pKa (base) & Loading (\%) \\
\hline Carbamazepine & $\begin{array}{c}\text { Wang } \\
\text { et al. (2012) }\end{array}$ & 236.269 & ॥ & 2.77 & 329.5 & $\begin{array}{c}\mathrm{Li} \\
\text { et al. (2000) }\end{array}$ & 478.5 & 1.45 & - & - & 20 \\
\hline Lovastatin & $\begin{array}{c}\text { Khafar } \\
\text { et al. (2017) }\end{array}$ & 404.54 & II & 3.9 & 301.5 & $\begin{array}{c}\text { Elder } \\
\text { et al. (1990) }\end{array}$ & 448 & 1.49 & - & - & 36.26 \\
\hline Acetaminophen & $\begin{array}{c}\text { Hacene } \\
\text { et al. (2016) }\end{array}$ & 151.163 & III & 0.91 & 298.1 & $\begin{array}{l}\text { Wyttenbach } \\
\text { et al. (2016) }\end{array}$ & 443.5 & 1.49 & - & - & 18.8 \\
\hline Aceclofenac & $\begin{array}{c}\text { Kumar } \\
\text { et al. (2014) }\end{array}$ & 353.0216 & II & 3.88 & 284.8 & $\begin{array}{l}\text { Wyttenbach } \\
\text { et al. (2016) }\end{array}$ & 424.5 & 1.49 & 3.44 & - & 50 \\
\hline Ibuprofen & $\begin{array}{c}\text { Zhang } \\
\text { et al. (2011) }\end{array}$ & 206.29 & ॥ & 3.84 & 230.7 & $\begin{array}{l}\text { Wyttenbach } \\
\text { et al. (2016) }\end{array}$ & 349.5 & 1.51 & 4.85 & - & 32.6 \\
\hline
\end{tabular}




\section{Table 1. (contd.)}

Drugs for which formulations based on mesoporous silica have been elaborated and characterized

\begin{tabular}{|c|c|c|c|c|c|c|c|c|c|c|c|}
\hline Compound & Ref. & Mwt & BCS Class & $\log P$ & $\operatorname{Tg}(\mathrm{K})$ & Ref. & $\operatorname{Tm}(\mathrm{K})$ & $\mathrm{Tm} / \mathrm{Tg}$ & pKa (acid) & pKa (base) & Loading (\%) \\
\hline Pretomanid & $\begin{array}{c}\text { Xia } \\
\text { et al. (2014) }\end{array}$ & 359.258 & - & 3.11 & - & - & 140 & N/A & - & - & 40.4 \\
\hline Cilostazol & $\begin{array}{c}\text { Wang } \\
\text { et al (2014) }\end{array}$ & 369.46 & II & 3.31 & - & - & 160 & N/A & - & - & 25 \\
\hline Resveratrol & $\begin{array}{l}\text { Summerlin } \\
\text { et al. (2016) }\end{array}$ & 228.247 & II & 3.4 & - & - & 254 & N/A & - & - & 20 \\
\hline Mefloquine & $\begin{array}{l}\text { Letchmanan } \\
\text { et al. (2017) }\end{array}$ & 378.312 & II & 4.11 & - & - & 243 & N/A & - & 9.46 & 33 \\
\hline Atazanivir & $\begin{array}{c}\text { Xia } \\
\text { et al. (2012) }\end{array}$ & 704.856 & II & 4.54 & - & - & 195 & N/A & - & 4.42 & 32.8 \\
\hline
\end{tabular}


The loading process can be considered from an energetic perspective: the surface of the silica has high energy and a large area. Therefore, the loading and uptake of drug onto the surface allows the system to lose excess energy, thus decreasing the Gibbs energy of the system. At the moment, there is insufficient information in the literature to state definitively how the drug is taken up within the porous network, and the mechanism is likely to vary with the chemical structure of the drug substance. Some hypotheses include: hydrogen bond interactions (Wang, et al. 2010; Kinnari, et al. 2011), hydrophobic interactions (Xu, et al. 2013), capillary action (Yanagihara, 2013) and ionic interactions (Atkin, et al. 2003; Turku, et al. 2007), although the latter depends on the silol groups on the surface of the silica to be in the anionic state, which may not be the case under physiological or ambient conditions.

One of the key differentiators of mesoporous silica from alternative amorphous formulation approaches is the superior stability that can be introduced via nanoconfinement within the mesoporous structure. High stability of the amorphous or molecularly dispersed drug is generally obtained once it is adsorbed into the porous network of the silica. The work of Muller and co-workers demonstrated amorphous stability at ambient and accelerated conditions for 30 different mesoporous silica formulations (Muller, et al. 2013).

This enhanced stability is especially attractive for so-called "GFA-1" or glass-forming ability 1 drugs. The glass forming ability of a drug is based on the overall propensity of a molecule to re-crystallize. First described by Avramov in 2003 as "the propensity of a liquid to vitrify upon cooling" (Avramov, et al. 2003), the GFA of a molecule can be determined experimentally based on a DSC method developed by Baird et al. (Baird, et al. 2010). In this approach, drug is placed in a DSC pan and subjected to a melt-cool-melt cycle. In this cycle, the DSC curves are examined for any crystallization behaviour. For GFA-3 compounds, no crystallization is observed at any point, indicating good stability in the amorphous form. For GFA-2 compounds, no crystallization is observed in the first melt-cool cycle, but when additional energy is supplied to the system in the second melt cycle, crystallization is observed, indicating a moderate stability 
in the amorphous form. For GFA-1 compounds, crystallization is observed in the first melt-cool cycle, indicating that even when the system is decreasing in energy, crystallization is favoured. This so-called "cold crystallization" is indicative of poor stability in the amorphous form. GFA-1 compounds are therefore very challenging to formulate in the amorphous form. A recent study by Wyttenbach and Kuentz confirmed the level of challenge, as only $6.25 \%$ of commercially available amorphous formulations fall into this class (Wyttenbach et al. 2017).

Recent work has demonstrated that mesoporous silica can successfully stabilize such compounds whereas traditional polymer-based technologies are unsuccessful (Ditzinger and Price, 2019). Furthermore, it has been shown that molecular mobility is significantly reduced, regardless of moisture or temperature, which is a critical consideration when carrying out the required ICH Q1 stability studies during drug development. Brás and colleagues demonstrated how adsorption and nanoconfinement of ibuprofen onto mesoporous silica resulted in a significant reduction of all known types of molecular mobility (Brás et al., 2014). Most interestingly, the Johari-Goldstein relaxation, a type of molecular flexibility which has been related to physical instability of the amorphous form, was reduced (Brás et al., 2014; Mehta et al., 2016). Similar to the work by Mehta and colleagues, this work focused on a good glass forming drugs (GFA-3). However, follow-on work also demonstrated reduction in molecular mobility for the successful stabilization of the very poor glass former Menthol (GFA-1), which has a glass transition temperature of $-54.3{ }^{\circ} \mathrm{C}$ (Cordeiro et al., 2017). In the study, Cordeiro and colleagues successfully stabilized amorphous menthol due to nanoconfinement within mesoporous silica. This stabilization was related to a decrease in molecular mobility associated with $\alpha$ and $\beta$ relaxation, which correspond to free-range mobility and molecular flexibility, respectively, and are critical for crystallization. Furthermore, a new type of molecular mobility, S-type, was observed, which was related to hindered mobility and nanoconfinement in the pore and was significantly slower than standard molecular mobility events (Cordeiro et al., 2017). As a result, drugloaded silica can often be stored in open containers and at elevated temperature and/or humidity. 
Upon delivery to an aqueous medium, such as the GI tract, the drug is displaced from the mesoporous network and has the potential to generate a supersaturated solution. Although yet to be extensively studied in the literature, the release mechanism can likely be described using diffusion kinetics. Simplified, upon administration to an aqueous environment, there is a diffusion of water into the porous network via capillary action. This in turn solubilizes the loaded drug, which generates a concentration gradient between the inside of the silica and the external medium. This in turn drives release of drug via diffusion along said concentration gradient, generating supersaturation and potentially increased absorption (Xu, et al. 2012).

Broadly speaking, this kind of release behavior can be described by the classical Higuchi Equation (Equation 6), which was proposed by Higuchi in 1961 to explain the release behavior of drug from thin ointment films (Higuchi, et al. 1961). Further work and modifications allowed this equation to be expanded to describe the release of any drug adsorbed or entrapped within an inorganic and insoluble matrix into an external medium via diffusion (Siepmann and Peppas, 2011) as follows:

$$
M_{t}=A \sqrt{2 c_{i n i} D c_{s} t} \quad \text { (Equation 6) }
$$

where $M_{t}$ is the amount of drug released from the inorganic carrier, $A$ is the surface area available for release, $c_{\text {ini }}$ is initial drug concentration in the carrier, $D$ the diffusion coefficient of drug in the insoluble carrier and $c_{s}$ is the maximum (saturation) concentration of the drug in the carrier.

Based on the Higuchi equation, the release of drug from mesoporous carriers can be described as a diffusion-driven process that, similarly to the loading process, is dependent on pore surface area, pore volume and pore morphology. Indeed, there have been a number of examples in the literature where Higuchi diffusion kinetics successfully predicted and modelled release of ibuprofen from mesoporous materials (Andersson, et al. 2004; Horcajada, et al. 2004; Zhao, et al. 2008).

Although the Higuchi diffusion model is a good starting point to understand the release behavior of a drug from mesoporous materials, it is not the whole picture. Indeed, in most studies that have attempted to 
model release, it has been over-predicted using Higuchi diffusion kinetics (Bathfield, et al. 2016; Bouchoucha, et al. 2016; Kinnari, et al. 2011). The Higuchi equation treats the diffusion process of a drug from the inorganic carrier as a one-step diffusion limited process. In reality, the release of most drugs from mesoporous silica likely follows a two-step dissociation-diffusion process: (1) Dissociation of the drug from the silica surface, which involves breaking interactions between the drug and the silica surface, and creating interactions between the diffused water and the silica surface (hydrogen bond and dipolar interactions), and (2) Diffusion out of the pore. For example, Kinnari and co-workers demonstrated that release of itraconazole from porous silica can be described with the Higuchi equation for about $80 \%$ of drug release, with a significant deviation for the remaining $20 \%$. In this instance, the first $80 \%$ accounted for physically entrapped drug within the pores, which diffused out of the carrier as previously described.

The remaining $20 \%$ accounted for itraconazole that was interacting with the carrier via $\mathrm{H}$-bonding. Therefore, the release of the final $20 \%$ proceeded via the two-step dissociation-diffusion process and did not align with the Higuchi equation (Kinnari, et al. 2011).

\subsection{Mesoporous Silica and Precipitation Inhibitors}

Like other formulations that generate supersaturation, it is essential to prevent precipitation from the supersaturated state for physiological time frames in order to increase oral absorption (Price, et al. 2018). The key difference between mesoporous silica and other bio-enabling formulations such as polymeric ASDs is that the silica - unlike excipients used in ASDs - itself does not inhibit precipitation from the supersaturated state (Laine, 2016). Therefore, it is essential to incorporate an additional polymeric excipient to function as a precipitation inhibitor (PI) in mesoporous silica formulations. One crucial difference between precipitation inhibition in mesoporous silica formulations and polymer ASDs is that one is less confined by the formulation process in the selection of the precipitation inhibitor for mesoporous silica formulations. For example, for an HME formulation, the polymer must be able to act as a precipitation inhibitor and 
meet the restrictive requirements for successful processing with HME including heat resistance and accessible melting point (Timpe, et al. 2007). The same is true for spray-dried dispersions, which require the polymer to be soluble in organic solvents and resistant to the friction and heat generated in the spray-dry process (Timpe, et al. 2007). For mesoporous silica, however, the process of loading is simple and requires basic lab equipment, and the available polymer space for PI selection is broader, increasing the likelihood of good performance (Laine, et al. 2016). This characteristic of mesoporous silica formulations could be especially beneficial when considering recent advances in understanding precipitation inhibition, where optimal precipitation inhibitors could be combined with mesoporous silica formulations that would be unsuitable for formulation with either spray dry dispersion or hot melt extrusion.

\subsection{Investigating the Importance of Drug-Polymer Interactions in Precipitation Inhibition}

Recently, there has been an increased interest in understanding the process of precipitation inhibition from a fundamental mechanistic perspective (Warren, et al. 2011; Price, et al. 2018). Specifically, advanced analytical tools have been used to study the interaction between dissolved, supersaturated drug and precipitation inhibitor on a molecular level. Interactions between drug and polymer has been shown to be the most critical success factor for a precipitation inhibitor system (Price, et al. 2018). The following techniques have been used to gain insight into such interactions:

\subsubsection{UV-vis Spectroscopy}

UV-vis-spectroscopy has been used more recently to investigate in detail kinetics of precipitation and drug-polymer interactions during precipitation inhibition.

Patel and co-workers applied second-derivative UV-spectroscopy to model the supersaturation and precipitation of indomethacin from supersaturated conditions in a solvent-shift experiment [Include reference]. This approach was able to extract a large amount of information from the system, especially relating to mechanism of precipitation. They found that precipitation of indomethacin was related to diffusion towards the growing crystal face, and that incorporation of a precipitation inhibitor 
could interfere with the crystal binding domains of the growing crystal, due to favourable hydrogen bond interactions between the polymers and indomethacin (Patel, et al. 2014).

Nie and colleagues used UV-vis spectroscopy as an orthogonal technique to support their mechanistic hypothesis for clofazimine-HPMCP interaction (Nie, et al. 2014). This was especially useful as clofazimine is red in both the crystalline and amorphous forms, but a colour shift to purple occurred in the presence of HPMCP in solid dispersions. Qualitatively, this was also observed for mixtures of drug and carboxylic acid analogues (e.g. glacial acetic acid), but not for polymers without carboxylic acids, such as HPMC. It was concluded that the bathochromic shift was associated with a proton-transfer from the carboxylic acid functional group of HPMCP (Nie, et al. 2014). In combination with principal component analysis, the drug: PI (w: w) ratio below which no interaction was observed was calculated to be 1:0.5. Using the same approach, it was concluded that the drug: PI ratio at which full protonation of the imine occurs, i.e. the strongest interaction, was at 1:1.5 (Nie, et al. 2014). Such information can be valuable in the design and development of PI-based formulations. A similar study was conducted by Misic and co-workers in the investigation of acid-base interactions between the poorly soluble drugs, loratadine and carvedilol, and oleic acid (Misic, et al. 2014).

Patel and co-workers also utilized UV-vis spectroscopy in combination with mathematical modelling. This study involved the combination of online second-derivative UV-spectroscopy and modelling using the diffusion reaction model in order to give real-time concentration values and mechanistic insight for indomethacin in supersaturated solutions. This methodology was able to provide a large amount of information about the precipitation behavior, including that at high degrees of supersaturation the precipitation is diffusion limited, which fits in well with the diffusion-reaction model (Patel, et al. 2011).

\subsubsection{FTIR Spectroscopy}

Fourier-Transform Infrared (FTIR) spectroscopy is one of the most commonly employed spectroscopic techniques and can provide functional group information based on a molecule's vibrational response to IR light absorption. FTIR spectroscopy is highly attractive from a drug development setting as it can 
be employed to study interactions between compounds in complex mixtures. Due to this, FTIR spectroscopy is a good prospect for the investigation of drug-polymer interactions (Gaffney, et al. 2012)

For example, Nie and co-workers performed an experiment to determine interactions between clofazimine and HPMCP, which has been previously reported to have very high drug loading capacity in solid dispersions (Nie, et al. 2015). IR spectra were analysed in order to identify changes in the vibrational modes of clofazimine and HPMCP in a solid dispersion (Nie, et al. 2016). A new peak, at $3310 \mathrm{~cm}^{-1}$ was observed in the IR spectrum of the solid dispersion, which corresponded to the stretching mode of the ionised imine in the clofazimine, protonated by the carboxylic acid groups in the phthalate substituent of the HPMCP due to in situ formation of a polymeric, amorphous salt with the drug. Furthermore, a sensitivity analysis showed that at ratios less than 1:0.5 w/w (drug: polymer), this effect was not observed. Additionally, the intensity of the peak increased with increasing HPMCP concentration. This acid-base interaction between HPMCP and clofazimine was further supported by the appearance of peaks at $1540 \mathrm{~cm}^{-1}$ and $1395 \mathrm{~cm}^{-1}$, which both correspond with the formation of a carboxylate group (Nie, et al. 2016). Knowledge about solid-state interactions such of these can be directly correlated to solid-state stability and loading capacity as well as to enhanced precipitation inhibition and supersaturation. Indeed, the combination of HPMCP and clofazimine in a solid dispersion resulted in a 10-fold increase in apparent clofazimine solubility (Nie, et al. 2015).

Petrusevska and colleagues also employed FITR spectroscopy to investigate the mechanism of interactions between a successful drug-PI formulation, sirolimus and HPMC, which was developed during a commercial screening platform. Solid dispersions of HPMC and sirolimus demonstrated significant variation from the neat samples of amorphous sirolimus as well as a physical blend. Specifically, the sirolimus peaks at $1680-1640 \mathrm{~cm}^{-1}(\mathrm{C}=\mathrm{C})$ and $1760-1670 \mathrm{~cm}^{-1}(\mathrm{C}=0)$ in the solid dispersion were significantly broader than the pure drug, which the authors concluded was a result of interaction between the two species (Petrusevska, et al. 2013b). This interaction was suggested to be 
partly responsible for the 2 -fold increase in supersaturation and dissolution in the sirolimus-HPMC solid dispersion versus the commercially available Rapamune nanoparticles. Finally, this formulation was used in a clinical pharmacokinetics trial, in which the novel formulation significantly outperformed the commercial formulation, Rapamune. This effect was attributed to the enhanced precipitation inhibition properties of HPMC in the novel formulation (Petrusevska, et al. 2013b). This study is a good demonstration of how FTIR can be employed to aid in the development of a precipitation inhibitor formulation from screening to clinical testing.

Another application of FTIR spectroscopy is the characterization of precipitates, which can provide information about the effect of precipitation inhibitors. This was carried out in a recent study by Chavan, in which IR spectroscopy was used to ensure that a range of polymers being studied for precipitation inhibitors did not affect the solid-state phase behaviour (polymorphism) of the drug, nifedipine (Chavan, et al. 2016). In this instance, the FTIR spectra of the precipitates for all three polymers (HPMC, PVP and HPC) aligned well with crystalline nifedipine, indicating no polymorphic change was induced by the polymers. This is an important consideration as when the drug does precipitate from the supersaturated condition (precipitation being just a kinetic effect) a change in polymorphism could have unintended pharmacokinetic effects.

\subsubsection{Raman Spectroscopy}

Raman spectroscopy is a technique that uses inelastic (Raman) scattering from laser light sources to study vibrational transitions. This information can be used to ascertain structural information about a sample. Raman spectra are obtained by recording the light scattered by a sample, which can be difficult as a majority of this light will be of similar frequency as the excitation source, so-called Rayleigh scattering. Only a small proportion of light scattered will be shifted based on vibrational transitions in the sample. This is called Stokes- and anti-Stokes-scattering. It is the intensity of the Stokes-shifted light that is plotted against Stokes-shift to create Raman spectra (Rostron, et al. 2016). Raman spectroscopy is a technique that is complementary to IR spectroscopy, and has certain advantages. 
Similar to IR spectroscopy, Raman spectra can be interpreted to ascertain information about interactions between two species. Unlike IR, this can be carried out in aqueous solution, which has particular advantages when considering drug polymer interactions, where water often plays an essential role (Paudel, et al. 2015).

Raut and co-workers utilized an in-situ Raman probe, placed inside a dissolution set-up, in order to investigate the precipitation inhibition effect of Vitamin E TPGS on two model drugs, probucol and indomethacin, in self-emulsifying drug delivery systems compared to labrasol formulations. In order to achieve this, the formulations were added to a solution at $\mathrm{pH} 1.2$, followed by a $\mathrm{pH}$ shift to $\mathrm{pH} 6.8$. Insertion of a Raman probe into these solutions allowed the collection of time-resolved Raman spectra for both the solid precipitate and the species in solution, which were analyzed for molecular interactions between the drug and excipients. For probucol, Raman peaks were observed at 540 and $1164 \mathrm{~cm}^{1}$, corresponding to the hydroxyl groups in the molecules. However, in the presence of Vitamin E TPGS, this peak dropped significantly in intensity, with the peak at $1164 \mathrm{~cm}^{-1}$ disappearing completely. This was due to the interaction of the probucol hydroxyl groups with carbonyl groups of the precipitation inhibitor. These interactions had a profound effect on precipitation, with no precipitation being observable in the presence of Vitamin E TPGS, in spite of the system being supersaturated to 100-fold of the thermodynamic solubility of Probucol (Raut, et al. 2015).

Similar observations were made for indomethacin, in which a shift was observed from the typical $1689 \mathrm{~cm}^{-1}$ carbonyl peak to a peak at $1680 \mathrm{~cm}^{-1}$ in the presence of Vitamin E TPGS, indicating a hydrogen bonded carbonyl group of indomethacin is present. Indeed, this moiety was essential for the precipitation inhibition effect of Vitamin E TPGS as this peak was not observed in the formulations where precipitation occurred. In addition, this effect was enhanced due to the presence of further interactions between the hydroxyl groups of indomethacin with the carbonyl groups of Vitamin E TPGS, indicated by diminished hydroxyl intensities in the presence of the inhibitor. Interestingly, in the case of indomethacin, it was observed that interactions were only evident whenever a certain 
"supersaturation threshold" was obtained, below which interactions were not observable, and precipitation occurred. This is an important factor to bear in mind: although a drug and polymer may theoretically interact strongly, the formulation in question must generate a particular concentration before interactions will be present. For Labrasol formulations of both compounds, however, there were no discernible interaction motifs in the Raman spectra, and the subsequent formulations performed poorly, with all samples precipitating (Raut, et al. 2015). Although not all drugs and formulations would be suitable for this methodology, model drugs, such as the ones mentioned, can be used in combination with a range of polymeric PIs to gain fundamental information about the important binding sites of polymers. Such information could be used to fine-tune the selection process. In addition to probing interactions in solution, Raman spectroscopy is a useful tool for investigating short-range interactions in the solid state. This can be particularly beneficial in the development of solid dispersion formulations, such as hot melt extrusion (HME) and spray-dried dispersions (SDD), where both drug-polymer miscibility and the precipitation inhibition performance of the polymer is based on these interactions. Chauhan and co-workers utilized this technique, among a wide range of spectroscopic tools, to develop solid dispersions of dipyridamole (Chauhan, et al. 2013). The team found that the most successful formulations consisted of drug-HPMC and drug-Eudragit $E 100^{\circledR}$, which performed significantly better than all other polymers screened. Further investigation, utilizing solidstate Raman spectroscopy, revealed that interactions were present between the drug and HPMC and Eudragit $\mathrm{E} 100^{\circledR}$ (Chauhan, et al. 2013).

\subsubsection{Nuclear Magnetic Resonance (NMR) Spectroscopy}

Nuclear magnetic resonance (NMR) spectroscopy is a spectroscopic technique that exploits the electromagnetic emission of nuclei in a magnetic field to gain structural information about the sample (Gunther, et al. 2013). Proton NMR is the most commonly utilised, but other nuclei can be studied, e.g. carbon, fluorine, silicon and lithium (Gunter, et al. 2013). NMR spectroscopy has wide application in 
drug development and can be a particularly useful in formulation design (Garido and Beckman, et al. 2014).

\section{One-dimensional (1D) NMR Spectroscopy}

Ueda and colleagues utilised 1D NMR spectroscopy as a tool to assess the impact of HPMCAS substitution patterns on the precipitation behaviour of carbamazepine (Ueda, et al. 2014). In the study, it was observed that HPMCAS successfully inhibited the precipitation of carbamazepine, depending on the ratio of succinyl and acetate groups in the polymer. Highest degrees of carbamazepine supersaturation were sustained in the presence of HPMCAS grades with low succinyl and high acetyl substitutions. This observation was explained by the hypothesis that the more hydrophobic the polymer, the higher the affinity for the growing crystal surface (see section 4.6. Polymer Surface Coverage). In order to expand upon this theory, the group utilised $1 \mathrm{H}$ NMR spectroscopy to provide information about the molecular mobility of carbamazepine in solution, with a range of HPMCAS variants. A good correlation was observed between precipitation inhibition and the molecular mobility. A lower molecular mobility, associated with an increased interaction between the drug and the polymer, corresponded to a more successful precipitation inhibition. It was hypothesized that this interaction was the insertion of HPMCAS into growing aggregates that have not yet reached the critical nuclei size for crystal growth to occur, which prevents the formation of the crystal lattice (Ueda, et al. 2014). Thus, 1D NMR spectroscopy can be a useful tool to assess the effect of polymers on drug mobility, which can provide information about potential precipitation inhibition effects.

In a recent study by Prasad and co-workers, 1D 1H NMR spectroscopy was utilised to probe the inhibitory effect of a range of polymers on indomethacin precipitation after the generation of supersaturation. It was hypothesized that interactions between the polymers and the carboxylic acid functionality of indomethacin was essential for precipitation inhibition. Therefore, focus was placed on studying this interaction. The chemical shift of the carboxylic acid group, at $3.70 \mathrm{ppm}$, was closely monitored for changes in chemical shift, which could indicate a change in the chemical environment 
surrounding the protons and therefore may indicate an interaction mechanism between the drug and the polymer. Eudragit ${ }^{\circledast}$ E100 and PVP, when combined with the drug in solution, shifted the carboxylic acid peak to a lower value, due to shielding effects (Prasad, et al. 2016). The investigators utilised this shift to quantify the strength of drug-polymer interaction and subsequent precipitation inhibition effect. Eudragit E100 resulted in a larger down shift than PVP. For both polymers this shift was directly proportional to the concentration of the polymer. Additionally, it was observed that when the formulation was changed from a binary system, with drug and one polymer, to a ternary system, with both polymers, this shift was even more pronounced. This provided evidence for a synergistic contribution of the two polymers to the precipitation inhibition. This NMR data supported the dissolution performance of the drug in the presence of polymers, where a larger and more sustained supersaturation was generated with Eudragit E100 in a binary system.

\section{Two-dimensional (2D) NMR Spectroscopy: NOESY and DOSY}

There are a range of 2D NMR spectroscopic techniques that can give information about correlations of different atoms through space such as Nuclear Overhauser effect spectroscopy (NOESY) and diffusion ordered spectroscopy (DOSY).

NOEs occur when there is an enhancement of an NMR signal following cross relaxation and magnetic transfer in systems where there are dipolar spin interactions (Kwan and Huang, et al. 2008). NOEs are observable when two nuclei are close in space. This can be exploited to determine if two different molecules (for example a drug and a polymer) are close enough to each other for an interaction to take place which will have an influence on precipitation behaviour. Furthermore, the resultant NOESY spectra can be interpreted to determine which part of the drug molecule is interacting with which part of the precipitation inhibitor, based on the standard 1D structural NMR spectra.

Prior to their work surrounding the importance of substituent ratios for HPMCAS precipitation inhibition, which utilised 1D NMR for the calculation of molecular mobility, Ueda and colleagues first established the mechanism of interaction between HPMCAS and carbamazepine in solution utilising

$$
\text { P a g e } 46 \mid 286
$$


NOESY (Ueda, 2013). During this experiment, it was observed that HPMCAS-HF (a grade of HPMCAS, relating to the ratio of acetyl to succinyl substituents) had cross-peak interactions with the aromatic protons and amide protons of carbamazepine, suggesting the possibility of both hydrogen bond interactions and hydrophobic interactions. After further inspection of the intensities of the crosspeaks, it was concluded that the more predominant effect was a hydrophobic interaction between the HPMCAS acetyl substituents and the aromatic region of carbamazepine (Ueda, et al. 2013). This interaction appeared to be essential for successful precipitation inhibition.

NOESY has also been used in combination with High Resolution Magic-Angle Spinning (HR-MAS) NMR spectroscopy to understand the interactions between the poorly soluble drug mefenamic acid with Eudragit $^{\oplus}$ EPO in supersaturated solutions (Higashi, et al. 2014). Although MAS was originally developed to study solids in NMR, HR-MAS NMR spectroscopy can also offer improved resolution for the study of highly viscous solutions and has been used in the pharmaceutical industry to detect and quantify drug in formulations such as gels and creams (Marzarati, et al. 2013). Higashi and co-workers were able to significantly improve the recorded NMR spectra for mefenamic acid-Eudragit EPO solution under MAS conditions. This allowed cross-peaks to be observed during the NOESY experiments. These cross-peaks showed evidence of multiple points of interaction between the drug and the polymer, indicating two different motifs. Firstly, a hydrophobic interaction between the aromatic portion of the drug and the EPO backbone and, secondly, a hydrophilic hydrogen-bond interaction between the amino alkyl part of EPO and the carbonyl groups of mefenamic acid. Furthermore, it was observed that the intensities of the two sets of cross-peaks were similar, leading the authors to conclude that both interactions play an important role in precipitation inhibition (Higashi, et al. 2014).

DOSY is a tool that allows the calculation of diffusion coefficients based on how quickly a species moves in a given time. In order to obtain this information, DOSY excites the species with multiple pulses and then measures the distance travelled between pulses, from which diffusion coefficients can be obtained. These diffusion coefficients are directly related to the size and molar mass of the species. 
Therefore, based on the presence of signals specific for the drug and the polymer, DOSY spectra can be used to distinguish whether one is observing the drug on its own or in a complex with a polymer, which would have a different diffusion coefficient based on the increased size (Johnson, et al. 1998). For the study of drug-polymer interactions, DOSY can be used alongside NOESY to offer an orthogonal confirmation of drug-polymer interactions.

DOSY was an essential part of a study to assess a novel spray dried dispersion matrix, HPMCAS and dodecyl (C12) poly(N-isopropylacrylamide) (PNIPAm), for suitability to enhance the delivery of the poorly soluble drug, phenytoin (Li, et al. 2017). After dissolution of the solid dispersion, the C12PNIPAm polymers formed micelles with the dodecyl groups at their core, which successfully sustained the supersaturated state of phenytoin generated by the SDD. Furthermore, a novel observation was recorded in that the C12-PNIPAm inhibited precipitation of the supersaturated phenytoin by inclusion of the drug within the corona of the micelles, as opposed to the core. It was also concluded that the HPMCAS in the formulation had little effect on sustaining the supersaturation compared to $\mathrm{C} 12$ PNIPAm, and instead, was responsible for the enhanced dissolution of the drug from the SDD (Li, et al. 2017). These conclusions were backed by both NOESY and DOSY data. The NOESY spectra of the novel formulation showed cross-peak interactions between the phenyl groups of the phenytoin and the isopropyl groups of the PNIPAm polymer. Conversely, NOESY spectra revealed no cross-peaks for phenytoin combined with HPMCAS. On application of DOSY as an orthogonal approach, no reduction in diffusion coefficient for phenytoin was observed in HPMCAS. Conversely, the diffusion coefficient decreased dramatically, in a concentration-dependent manner, in the presence of C12PNIPAm. This provided the researchers with strong evidence that the C12-PNIPAm was responsible for the remarkable sustained supersaturation that was observed upon dissolution of this novel SDD, and that this mechanism was taking place within the corona of the micelles (Li, et al. 2017). 


\section{Solid State NMR Spectroscopy}

Solid state (SS) NMR spectroscopy can be utilised to gain structural information about solids. One key distinguishing property of solids from an NMR perspective is the presence of anisotropy, that is, directionally dependent interactions. These anisotropic effects have a significant impact on the magnetic interactions of nuclei, and so are of critical importance for the structural elucidation of solids via this technique. Furthermore, these effects result in very broad NMR signals if no magic angle spinning (MAS) conditions are applied. Therefore, SS NMR spectroscopy is generally carried out in combination with MAS, which reduces these effects and results in narrower peak width and better resolved spectra. SS NMR spectroscopy has been utilised in the literature for determining interactions between drugs and precipitation inhibitors in the solid state, such as in solid dispersions or hot melt extrudates. Chauhan and colleagues utilised $13 \mathrm{C}$ cross-polarization magic angle spinning SS NMR spectroscopy to investigate the interactions between indomethacin and polymers in solid dispersions (Chauhan, et al. 2014). Three different polymers, Eudragit ${ }^{\circledR}$ S100, PVP and HPMCAS, were screened for indomethacin interactions using this method. Chemical shift changes of the indomethacin signals were recorded in the presence of the polymers, with a larger chemical shift change indicating a stronger interaction. For the aromatic region, a slight chemical shift change was observed for all three polymers, with the biggest shift occurring in the PVP solid dispersions. This correlated well with the observed performance of the SDD during biorelevant dissolution, which outperformed all other polymers due to enhanced precipitation inhibition (Chauhan, et al. 2014).

NMR spectroscopy can provide definitive details about the molecular interactions between drug and precipitation inhibitors. Such mechanistic detail can be valuable when considering the selection of precipitation inhibitors. Therefore, NMR spectroscopy is a valuable tool to be applied by the pharmaceutical scientist in the development of supersaturating formulations. The use of NMR spectroscopy for this purpose remains 'exotic' in the literature, with key examples coming from just a few research groups. This may be due to the difficulties associated with obtaining meaningful spectra 
from drug-polymer systems, which make up most examples in the literature. Firstly, the drugs used in supersaturating formulations are innately poorly soluble, which can make preparing NMR samples with high enough concentrations for analysis difficult. Secondly, polymers themselves often have very broad and poorly resolved spectral peaks, which can be difficult to interpret. Thirdly, a 'catch-22' exists between resolved spectral data and strong interactions: the stronger the interactions between drug and polymer, the broader and less resolved the peaks become, which also leads to difficulties. For these reasons, the MAS design described by Higashi and co-workers (Higashi, et al. 2014) is a very attractive prospect, due to the increased resolution that can be provided.

\subsubsection{Differential Scanning Calorimetry (DSC)}

Differential scanning calorimetry (DSC) is a thermal analysis technique that sees wide application in the pharmaceutical sciences (Sheng, et al. 2016). DSC records the amount of heat required to increase the temperature of a sample compared to a reference material (e.g. indium). This produces a DSC curve, from which a wide range of information can be obtained about key thermal events such as melting, crystallization, glass formation and decomposition. One particularly useful application of DSC is in the identification of amorphous materials, which will exhibit a glass-transition instead of a melting thermal event in a DSC curve (Sheng, et al. 2016). Although not as common, DSC can also be used to investigate interactions between two different species. Traditional DSC cannot achieve this and instead, modulated DSC (MDSC) is utilised. MDSC differs from traditional DSC in that it operates using two simultaneous heating rates, in contrast to the single linear heating rate used in DSC. MDSC utilises both a linear heating rate and a modulated heating rate that allows simultaneous measurement of the heat capacity of the sample. Such an approach allows the analysis of more complex mixtures due to its higher sensitivity and resolution.

Chauhan and co-workers used MDSC to learn about the mechanism of interaction between dipyridamole and a range of precipitation inhibitors. Previously, MDSC has been used to assess miscibility between a drug and polymer, based on a change in melting event. Expanding on this idea, 
in this study, the melting temperatures of dipyridamol and dipyridamol precipitates in the presence of polymers were essential to determining whether any drug-polymer interaction was taking place. For Eudragit ${ }^{\circledast}$ E100, Eudragit ${ }^{\circledast}$ S100 and HPMC, additional melting and glass endotherms were observed in the MDSC curves. The authors of the study reasoned that this shift occurred due to interaction of the drug with the polymers in solution, which corresponded with an increased precipitation inhibition. Indeed, of the six polymers studied, only those polymers where a change in melting temperature of the precipitates was observed were successful precipitation inhibitors (Chauhan, et al. 2013). But the authors also offered a word of caution, saying that, although melting point changes were present, this is not definitive proof of interaction. Certain polymers, e.g. PEG, can dissolve a drug and therefore alter the melting temperature. Rather, the authors suggest that MDSC is a useful tool to determine if there are no interactions present, as was the case with the unsuccessful precipitation inhibitors, Eudragit S100, Eudragit L100 and PEG 8000 (Chauhan, et al. 2013). To state that an interaction is definitely present, MDSC should be used with complementary analytical techniques.

\subsubsection{Modelling Precipitation Inhibition}

\section{Adsorption Modelling}

Initial work on adsorption modelling for precipitation inhibition used the well-established adsorption isotherms, including the Langmuir (Langmuir, et al. 1918), and Freundlich models (Skopp, et al. 2009), often combined with crystallization models such as the Kubota-Mullin model (Kubota, et al. 1995; Kubota, et al. 1997; Kubota, et al. 2000) to predict the adsorption of additives on crystal surfaces and the effect on crystal growth. The Kubota-Mullin model proposes a monolayer by monolayer crystal growth, in which precipitation inhibition depends on the surface coverage, $\vartheta$, and space between polymers absorbed onto the growing crystal surface, $L$, where $I$ is the free energy of the unit length

(Equation 7). (Kubota, et al. 1995; Kubota, et al. 1997; Kubota, et al. 2000)

$$
\theta=\frac{L}{l}
$$

(Equation 7) 
Schram and colleagues adapted the model in order to predict polymer performance from the experimentally obtained surface coverage values (Schram, et al. 2015b). In this case, polymer effectiveness, $R_{p} / R_{0}$, is the ratio of crystal growth in the presence $\left(R_{p}\right)$ and absence of polymer $\left(R_{0}\right)$ and depends on the fractional surface coverage, $\vartheta$; the relative supersaturation, $\sigma$; the edge free energy per unit length, $\gamma$; the size of a growth unit, $a$; the temperature, $T$, the Boltzman constant, $k$, and the average distance between absorbed polymers, I (Equation 8)

$$
\frac{R_{p}}{R_{0}}=1-\frac{\gamma a}{k T \sigma(\theta l)} \theta \quad \text { (Equation 8) }
$$

The average distance between polymers is specific for each system and depends on the amount of polymer adsorbed to the surface. Thus, I was proportional to the experimental polymer surface coverage determined by AFM. Consequently, a correlation was established, with which / could be determined from the polymer surface coverage. This correlation enables the calculation of the fractional surface coverage in the Kubota-Mullin model and subsequently the theoretical effectiveness of crystal growth inhibitors. In this study, the theoretical effectiveness calculations were in good agreement with the experimental values (Schram, et al. 2015b).

This approach was also adopted by Alonzo and co-workers to determine the effect of polymers on crystal growth and nucleation of felodipine (Alonzo, et al. 2012). The team observed experimentally that the efficiency of HPMC inhibiting precipitation of felodipine from supersaturated solution was dependent on the extent of supersaturation generated. This was supported using a Langmuir adsorption model that indicated at high degrees of supersaturation the fractional surface coverage of the growing crystals was decreased, due to the higher propensity to re-crystallize at such high supersaturation (Alonzo, et al. 2012).

The use of adsorption models to study precipitation inhibition of indomethacin was extensively studied by Patel and co-workers (Patel, et al. 2015; Patel, et al. 2014; Patel, et al. 2011). Using second- 
derivative UV spectroscopy in combination with a first-order crystal growth model, Patel and Anderson investigated growth rates of indomethacin in the presence of various PIs. For HP- $\beta-C D$, precipitation inhibition was modelled using diffusion layer theory (Patel, et al. 2011). This model successfully predicted that, at high degrees of supersaturation, HP- $\beta-C D$ inhibition could be related to the reversible complexation between the two species at the diffusion layer (Patel, et al. 2011).

Furthermore, it was observed experimentally that HPMC and PVP significantly outperformed HP- $\beta-C D$, in agreement with the inhibition models (Patel, et al. 2011).

In a further study by Patel and co-workers, molecular weight of PVP and its effect on precipitation inhibition was studied using Langmuir isotherms generated with the solution depletion method. In this method, PVP with varying molecule weight was mixed with solution of the model drug, indomethacin. These systems were then allowed to equilibrate before being passed through a size exclusion column. The sample weight was then determined through the column to determine the concentration of the adsorbed polymer on the surface of the growing crystal. Adsorption isotherms based on surface coverage were then constructed, which showed that adsorption potential for PVP was directly proportional to PVP molecular weight. This was also validated experimentally, with higher molecular weight PVP inhibiting the precipitation of indomethacin most effectively, due to an increased surface coverage (Patel, et al. 2014).

\section{Molecular Modelling}

Many different types of molecular models have been applied to mechanistically study crystallization and precipitation, ranging in complexity from Monte Carlo methods to molecular dynamics simulations and quantum mechanics (Myerson, et al. 1999).

Mandal and co-workers developed a coarse-grained (CG) model for crystal growth based on force fields obtained from simulators (Mandal et al. 2016). Coarse graining allows the simulation of complex systems without the need for extensive computation time, due to the use of simplified atomistic representations. Such an approach is often used to model the interaction of proteins and small 
molecules (Levitt, et al. 1975). There are many software packages that can carry out the CG process, such as MARTINI (Levitt, et al. 1975), however, such software packages often oversimplify the molecules, leading to the danger that information important to understand crystallisation behaviour may be lost. In order to improve upon these established CG processes, Mandal and colleagues utilised a CG model based on the radial distribution functions of the molecules, which were obtained from atomic simulations carried out in the crystalline state (Mandal et al. 2016). As a result, the CG model developed was able to simulate crystal growth of the organic molecule, phenytoin, in the absence and presence of the polymer HPMCAS (Mandal et al. 2017). Furthermore, the simulation was able to correctly predict that inhibition of phenytoin by HPMCAS is highly dependent on the substitution of the polymer, with an increased acetate substitution slowing crystal growth most effectively. The results of this simulation robustly predicted the experimental data (Mandal et al. 2017).

\section{Thermodynamic and Kinetic Modelling}

The interplay between drug, polymer and the aqueous environment is complex from a thermodynamic perspective. In order to consider such a system in its entirety, a thermodynamic model would need to assess the polymer, drug and water in a ternary system. In this system, both liquid-liquid phase separation and solid-liquid equilibrium would need to be considered (Taylor, et al. 2016; Paus, et al. 2016). Although such an approach is attractive due to the high level of detail of information that could be obtained, it remains a difficult task. Emerging statistic thermodynamic models such as the perturbed-chain statistical associating fluid theory (PC-SAFT) (Paus, et al. 2016) could handle the required complexity of such a system. However, as yet, there have been no examples of such an approach in the literature and it remains unlikely due to the difficulties associated with establishing such a model. Therefore, currently, application of thermodynamic models is limited, and instead kinetic approaches have been adopted to describe the interaction between polymers and drugs in precipitation inhibition. Specifically, the interaction parameter $\chi$ of the Flory-Huggins (FH) theory has often been employed (Flory, et al. 1953; Flory, et al. 1942; Chen, et al. 2014; Baghel, et al. 2016). 
The Flory-Huggins solution equation (Equation 9) is used to describe the behavior of polymers in solution. It is an adaption of the standard Gibbs energy equation incorporating an extra term to modify entropy to consider molecules of different sizes. In the Flory-Huggins equation the enthalpic portion of the Gibbs equation is represented by the Chi parameter, $\chi$ :

$$
\Delta G=R T\left[n_{1} \ln \varphi_{1}+n_{2} \ln \varphi_{2}+n_{1} \varphi_{2} \chi_{1,2}\right]
$$

\section{(Equation 9)}

where $R$ is the ideal gas constant and $T$ is the absolute temperature. $n_{1}, n_{2}$ and $\phi_{1}$ and $\varphi_{2}$ are the number of moles and volume fraction, respectively, for component 1 and 2 of the system, and $\chi$ is the interaction enthalpy upon association of component 1 and 2 .

Using the Flory-Huggins equations one can consider mixtures of drug and polymers, whereby the enthalpic contribution becomes $\chi$. Specifically, it is hypothesized that the interaction related to the $\chi$ parameter in the equation can be related to the interaction between drug and polymer in solution, which is essential for inhibition of precipitation. Specifically, this is related to the hydrophobic, polar or hydrogen bond interactions between the drug (D) and polymer (P) (Warren, et al. 2011). The $\chi$ parameter can be determined experimentally by combining the Flory-Huggins equation with experimental DSC measurements (Marsac, et al. 2009).

In addition, it is possible to utilize in silico predictions of the $\chi$ parameter, which reduces the number of experiments required, thus allowing experimental work to focus on the most promising formulations. This can be achieved in several ways. One common method is to calculate the drug, $\delta_{D}$, and polymer, $\delta_{p}$, in relation to the molar volume of the drug, $V_{m}$, the temperature, $T$, and the ideal gas constant, $R$ (Rubenstein, et al. 2003) (Equation 10):

$$
\chi_{D P}=\frac{V_{m}\left(\delta_{D}-\delta_{P}\right)^{2}}{R T}
$$


The extended Hansen solubility parameters, $\delta_{D}$, and $\delta_{P}$, can be predicted based on chemical structure alone, using group contribution methods, quantitative structure property relationships (QSRP), molecular dynamics or quantum approaches such as the Continuum Order Solvation model for Real Solvents (COSMO-RS) (Van Krevelen, et al. 2009; Tian, et al. 2015; Gharagheizi, et al. 2011; Gharagheizi, et al. 2015; Tantishaiyakul, et al. 2006; Niederquel, et al. 2018). In addition to group contribution methods, it has been shown that solubility parameters can be predicted from quantitative structure property relationships (QSPR) (Gharagheizi, et al. 2011; Gharagheizi, et al. 2015; Tantishaiyakul, et al. 2006). A first calculation option is to simulate the internal energy change due to vaporization, $\Delta E_{v}$ (Gupta, 2011), which can then be incorporated into the cohesive energy density equation along with $V_{m}$, the molar volume (Hilderbrand, et al. 1950) (Equation 11):

$$
\delta=(C E D)^{1 / 2}=\left[\frac{\Delta E_{v}}{V_{m}}\right]^{1 / 2}
$$

In this instance, the total energy difference for isolated molecules and for the bulk system with periodic boundary conditions provides an estimate of $\Delta E_{v}$ (Hilderbrand, et al. 1950).

Although there has been a lot of work in determining experimental and in silico methods to determine the $\chi$ parameter, there are only a few examples of this parameter being used to understand inhibition of precipitation. Baghel and co-workers studied solid dispersions of dipyridamole and cinnarizine with PVP and polyacrylic acid (PAA) (Baghel et al. 2016). It was found that the combinations capable of forming hydrogen bonds (dipyridamol-PVP; dipyridamol-PAA and cinnarizine-PAA) in the solution state were more effective at keeping the drug in a supersaturated state than those not able to hydrogen bond (cinnarizine-PVP). In this instance, cinnarizine-PVP had the highest predicted $\chi_{D P}$ parameter, suggesting weakest interactions, in line with the observed precipitation inhibition results. However, it was noted that, despite their significantly different supersaturation performance, the difference 
between the $\chi_{D P}$ parameters of cinnarizine-PVP and cinnarizine-PAA was not great, and that other aspects such as the hydrophilicity of the polymer should also be considered.

Similar findings were also reported by Chen and co-workers who compared solid dispersions of griseofulvin, felodipine, and ketoconazole with PVP vinyl acetate (PVP-VA) and HPMC-AS (Chen et al., 2014). Although felodipine interacted much more effectively with PVP-VA in the solid-state $\left(\chi_{D P}=-1.9\right)$ than with HPMC-AS $\left(\chi_{D P}=-0.21\right)$, this behavior was not replicated in aqueous dispersions, where the HPMCAS solid-dispersion generated higher and more sustained supersaturation profiles upon dissolution. This was likely due to the hydrophilic interactions of PVP-VA with water upon exposure to an aqueous environment, which may have reduced or negated the favorable interactions with felodipine.

\subsection{Bridging the Gap to Systematic and Facile Precipitation Inhibitor Screening}

There has been an exponential increase in the number of research papers addressing precipitation inhibition from a mechanistic perspective. This effort, combined with the use of advanced analytical tools, has led to a deeper understanding of drug-polymer interactions. Techniques to investigate experimental drug-polymer interactions are time-consuming and not ideally suited to early stage pharmaceutical development, where such decisions must be made. Furthermore, although the de novo design of Pls to maximize interaction with the drug and water in a balanced way is an exciting prospect, there are some key hurdles to this technology becoming widely applicable. For example, from a regulatory perspective such an approach could be very costly and restrictive, since additional safety studies would be required to demonstrate an absence of polymer-related toxicity, if new PIs are used. Therefore, a rather more interesting and applicable approach would be "de novo selection" of PIs based on an understanding of interaction between the drug and polymer. Such an approach would retain some of the advantages of a bespoke PI selection process whilst avoiding the additional regulatory burden associated with creating a novel excipient, designed based solely on optimal 
interactions. For excipient selection, in silico tools are an attractive prospect, as they can limit the time required by reduce the experimental screening requirements.

However, the in-silico tools that have recently been employed in the design and selection of precipitation inhibitors are currently costly and time-consuming, making them less attractive for "de novo selection" of a precipitation inhibitor from a large database. Therefore, there exists a gap between the current understanding of drug-polymer interactions and a quick and efficient screening tool for the selection of precipitation inhibitors that are already approved for pharmaceutical use. A simplified computational approach that can predict interactions between drugs and pharmaceutically relevant polymers would therefore be of great value to the development of supersaturating formulations in the pharmaceutical industry. 


\section{Aims of the Thesis}

The aim of the thesis studies was to develop and optimize a new workflow to formulate mesoporous silica with precipitation inhibitors, leading to the design of robust supersaturating formulations for the oral delivery of poorly soluble drugs. This involved five key activities:

1) Review of the current state of the art of mesoporous silica formulations with respect to the importance of drug loading and interactions to the release profile. In addition, subsequent supersaturation and precipitation on release were examined and reviewed.

2) Identification of the critical parameters involved in precipitation inhibition, including recent advances in the use of advanced analytical tools for understanding and selecting precipitation inhibitors.

3) Optimization of the formulation method for combining precipitation inhibitors with mesoporous silica. This approach shifted away from the standard approach of physically combining precipitation inhibitors post-loading. The number of formulation steps required was reduced and the efficiency of precipitation inhibition upon dissolution was improved.

4) Resolution and rationalization of a mechanistic physical analysis of co-incorporated drug/PI mesoporous silica formulations, closely examining the importance of drugpolymer interaction.

5) Application of new-found knowledge regarding the importance of drug-polymer interactions to develop a quantum-chemical based in silico screening tool for the selection of optimal precipitation inhibitors for mesoporous silica formulations.

These goals were achieved by combining advanced and novel analytical tools and in silico models identified from an extensive literature review. 


\section{Results and Discussion}

\section{Approach}

Precipitation inhibitors, and understanding of how they function, are essential components of modern enabling formulations, which is reflected in the large body of work on this topic that has been published in the past decade. To form a basis for the thesis studies, a review article covering fundamental considerations of supersaturation and precipitation as well as recent innovative efforts combining modelling and analytical tools to understand the importance of drug-polymer interaction in effectively maintaining supersaturated drug concentrations was prepared (Price et al. 2018). The review revealed that formulation scientists can now incorporate top-level analytical tools such as advanced NMR spectroscopy techniques to determine the optimal precipitation inhibitor for a given drug.

While advances in PI selection were identified in the literature review, experimental incorporation precipitation inhibitors into supersaturating formulations remains at a basic level, with simple physical mixtures being the most common approach. To rectify this situation, a new processing method for combining precipitation inhibitors with mesoporous silica was developed: the co-incorporation approach. This development avoids having to add the PI as a physical mixture post-loading by instead incorporating the precipitation inhibitor at the very first step i.e. in the solvent-mediated loading of drugs onto mesoporous silica (Price et al. 2020). This approach significantly improved the precipitation inhibition effect of HPMCAS when combined with mesoporous silica for formulations of celecoxib and glibenclamide. One of the key reasons for this enhancement was the generation of drug-polymer interactions in the formulation itself, rather than first being generated when the formulation is dissolved (which is the case when the drug-loaded silica id physically blended with the PI) (Laine, 2016; Price, 2018). The importance of drug-polymer interactions was highlighted in both the review article and the co-incorporation studies. Despite this success, there remains a gap between our knowledge around the importance of drug-polymer interactions and the practicalities of incorporating this consideration in standard formulation development. Although the use of 2D NMR spectroscopy to 
determine drug-polymer interaction is described in the literature, its routine use is not realistic during the early stage of pharmaceutical development where efficiency and time are key. Instead, precipitation inhibitors are still routinely selected using trial-and-error based approaches, noting that these are also inefficient from a time and resource perspective. Therefore, the next stage of this work was to bridge this efficiency-knowledge gap by developing a quick and easy in silico protocol to calculate drug-polymer enthalpy using minimal resources (Price et al. 2019). This was achieved using the COSMO-RS model, which combines quantum mechanics and thermodynamics to calculate the drug-polymer excess enthalpy of interaction. Combining the new approaches described in this body of work will enable development of optimal mesoporous silica formulations in the pharmaceutical industry (Figure 7).

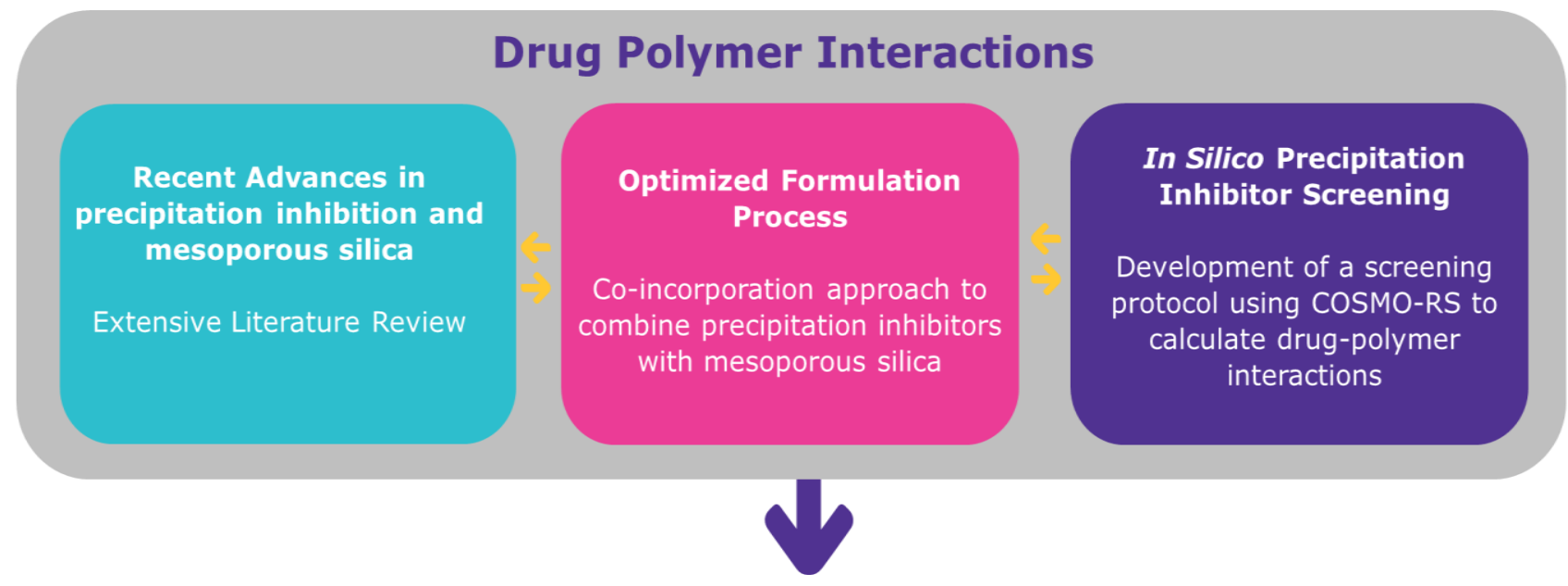

\section{Optimized Mesoporous Silica + Precipitation inhibitor formulations}

Figure 7. Overview of the thesis 


\section{Selected Compounds}

Three model BCS II compounds, with a broad range of physicochemical properties were selected.

\section{Glibenclamide}

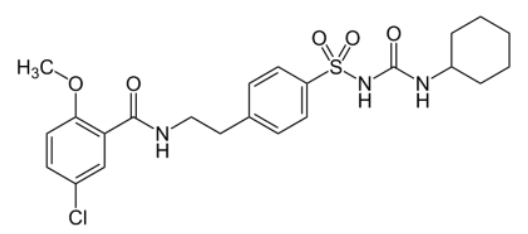

Figure 8. Glibenclamide

Glibenclamide is an orally administered hypoglycemic drug used for treatment of diabetes, via stimulation of pancreatic beta cells to secrete insulin (Furman, et al. 1977). Glibenclamide is a BCS II compound, indicating that the highest dose of the drug is not soluble in $250 \mathrm{~mL}$ of water across physiological pH range (Amidon, et al. 1995). Glibenclamide has been utilized in a range of supersaturating drug formulations, including mesoporous silica (Van Speybroeck, et al. 2011).

\section{Dipyridamole}

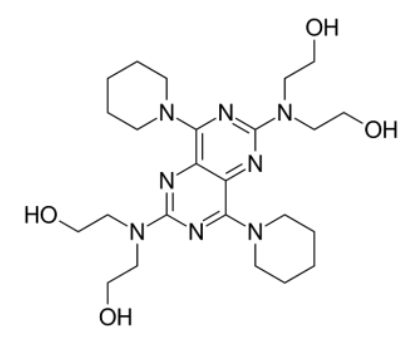

Figure 9. Dipyridamole

Dipyridamole is an anticoagulant that inhibits clot formation via blood vessel dilation and interaction and inhibition of phosphodiesterase (Brown, et al. 2015). Dipyridamole is also a BCS II compound. Dipyridamole has previously been formulated with amorphous technologies such as spray-dried dispersions and hot melt extrusion (Peng, et al. 2018). 


\section{Fenofibrate}<smiles>CC(C)OC(=O)C(C)(C)Oc1ccc(C(=O)c2ccc(Cl)cc2)cc1</smiles>

Figure 10. Fenofibrate

Fenofibrate is the pro-drug form of fibric acid. Fibric acid is used in the treatment of elevated blood lipid levels (Staels, et al. 1998). Like glibenclamide and dipyridamole, fenofibrate is a BCS II compound. Fenofibrate has been utilized in a wide range of supersaturating drug formulations, including mesoporous silica (Dressman et al. 2015).

Table 2. Physicochemical Property Comparison of the Model Drugs

\begin{tabular}{cccccc} 
Compound & Log P & pKa (acid) & pKa (basic) & Molecular Weight & FaSSIF Solubility $(\mu \mathrm{g} / \mathrm{mL})$ \\
\hline Glibenclamide & 5.3 & 4.3 & - & 494.0 & 14.0 \\
Dipyridamole & 4.7 & - & 6.4 & 504.6 & 8.1 \\
Fenofibrate & 1.5 & - & - & 360.8 & 20.0
\end{tabular}

\section{Selected Polymers}

A wide range of polymers was selected for the polymer database. Initially, polymers were identified from the pool of polymers which have been proposed as PIs in the literature. This included those typically used in precipitation inhibition and amorphous solid dispersion applications. Expanding upon these typical polymers, further polymers approved for pharmaceutical use were chosen from the literature and from online databases.

For input into COSMO-RS, it was necessary to convert polymer structures into smiles notation. Due to the restrictions associated with quantum chemistry, it is not possible to consider structures larger than 
$50000 \mathrm{Da}$. Therefore, polymer structures were represented as trimers in the in-silico screening. This was not deemed counterproductive to the study, as the hypothesis was related to local molecular interactions, which are assumed to be sufficiently captured by trimer sequences of the polymer.

The following polymers were included in the database, along with lactose and sorbitol:

Alginic acid gum

Cellulose acetate phthalate (CAP)

Chitosan

Eudragit EPO

Eudragit L100

Eudragit RL100

Hydroxyethyl methyl cellulose (HEMC)

Hydroxypropyl methyl cellulose (HPMC)

Hydroxypropyl methyl cellulose acetate succinate

(HPMCAS)

Hydroxyethyl cellulose (HEC)

Lactose

Locust bean gum

Methyl cellulose

Polyethylene glycol (PEG)

Polyglycolide (PGA)
Polyethylene oxide (PEO)

Polylactic acid (PLA)

Polylactide-co-polyglycolide (PLGA)

Poly (methyl methacrylate) (PMMA)

Polyvinyl acetate (PVAC)

Polyacetylene

Polyether polyol

Polyethylene Imine

Polyvinyl acetate-co-poly (methyl methacrylate)

(PVAC-PMMA)

Polypropylene glycol (PPG)

Poly (vinyl alcohol) (PVA)

Poly (vinyl alcohol)-co-polyvinylpyrrolidone

(PVA-PVP)

Polyvinylpyrrolidone (PVP)

Sodium carboxymethyl cellulose (SCMC)

Sorbitol 


\section{Co-incorporation: A New Method to Combined Precipitation Inhibitors with Mesoporous Silica}

\subsection{Approach}

To date no systematic study of how best to incorporate precipitation inhibitors into mesoporous silica formulations has appeared in the scientific literature. The current standard practice involves combining inhibitors in a physical mixture with the drug-loaded silica, either with a mortar and pestle or by overhead stirring. Due to the lack of a standard protocol, there is uncertainty about how reliably the precipitation inhibitor is combined with the drug-loaded silica on a batch to batch basis. In addition to the practical limitations of the approach, the incorporation of inhibitor post-loading represents a potentially unnecessary and not insignificant step in the formulation procedure. By contrast, coincorporating the $\mathrm{PI}$ alongside the drug not only abbreviates the formulation manufacturing procedure but can also improve the dissolution performance of the formulation. Previous work by the author demonstrated how incorporation of HPMCAS alongside celecoxib onto mesoporous silica substantially improved both in vitro and in vivo performance of the poorly soluble drug (Laine et al. 2016). Still, to gain more traction in the field, further evidence was needed a) to demonstrate that a co-incorporation approach can significantly improve the performance of drug-loaded silica formulations, and b) to resolve the mechanisms behind this formulation performance enhancement via robust and extensive analytical characterization of the co-incorporated formulation.

Glibenclamide was the drug selected for these studies. One of the key concerns of the co-incorporation approach is whether the accessibility of larger molecules to the porous network is hindered by the presence of a polymer. This was addressed with glibenclamide, which is a relatively large molecule with a molecular weight of $494 \mathrm{Da}$. Glibenclamide is also optimal from an analytical perspective due to the presence of fluorine in the molecule, which enables it to be tracked using energy dispersive $\mathrm{x}$-ray scanning electron microscopy (EDX SEM). This tool could then be leveraged to track the location of the drug within the co-incorporated formulations, to help build a mechanistic rationale for the coincorporated formulation performance. 
HPMCAS was the polymer selected for the mechanistic study. HPMCAS is a well-established PI and has a track record in the literature of successfully sustaining supersaturated solutions for a range of drugs (Warren et al. 2010; Price et al. 2018; Laine et al. 2016). For example, Laine and co-workers demonstrated the successful precipitation inhibition of supersaturated celecoxib with HPMCAS in both single-medium FaSSIF dissolution and transfer dissolution (Laine et al. 2016). HPMCAS has been regularly combined with mesoporous silica formulations to successfully sustain supersaturated drug concentrations. For glibenclamide, HPMCAS has the capacity to interact with the drug via hydrogen bond interactions, which have been shown to be crucial for successful precipitation inhibition. (Price et al. 2018; Price et al. 2019) Additionally, it has been demonstrated that HPMCAS successfully sustained supersaturated concentrations of glibenclamide in a solvent-shift-based precipitation inhibitor experimental screen (Ueda et al. 2015).

\subsection{Solid State of Formulations}

Loading of glibenclamide onto mesoporous silica, both with and without HPMCAS, resulted in successful shift from a crystalline to an amorphous solid-state form. Encouragingly, the inclusion of HPMCAS in this process did not have an impact on the ability of the drug to enter the pores, in line with earlier findings in which HPMCAS was successfully co-incorporated during the loading of celecoxib onto mesoporous silica without any impact on the final solid-state form of the formulation (Laine et al. 2016). This work paralleled attempts at incorporating mesoporous silica in HME platforms, in which Hanada and co-workers demonstrated that silica could be successfully utilized alongside HPMC to yield a high drug-load, ternary drug-silica-polymer system (Hanada et al. 2018). 
(a)

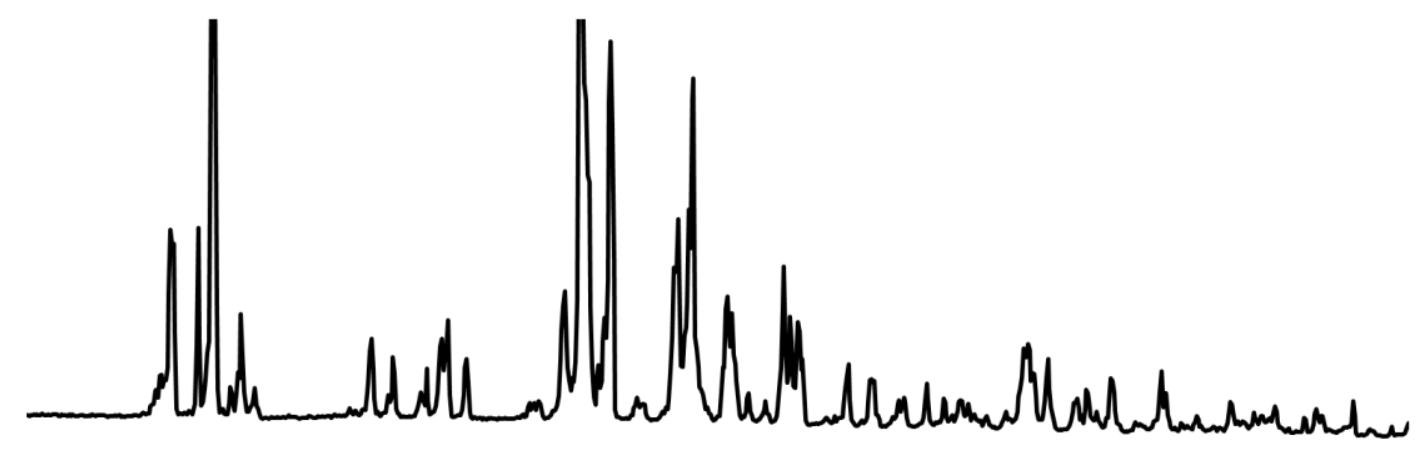

(b)

(c)

(d)

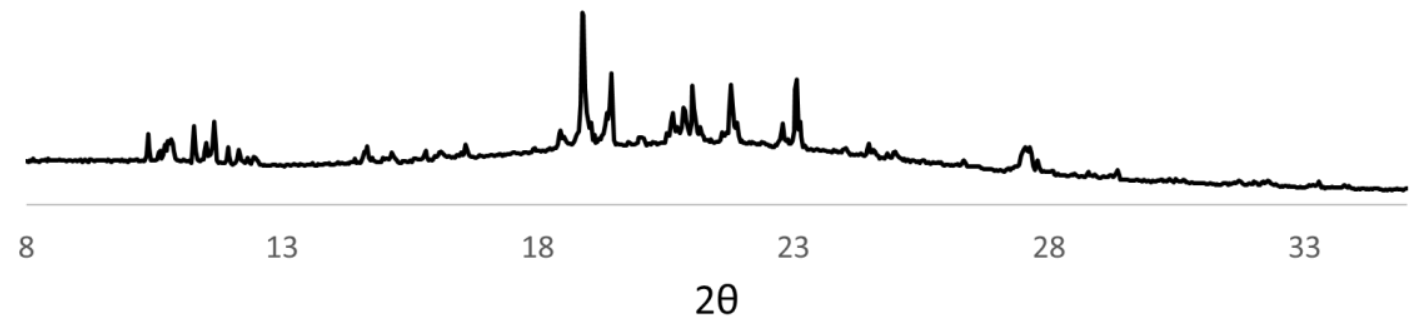

Figure 11. XRPD pattern for crystalline glibenclamide (GB) (a), glibenclamide loaded silica (b), coincorporated GB/HPMCAS loaded silica (c) and GB and HPMCAS prepared by rotary evaporation (d).

One significant difference, however, was the appearance of the particles when HPMCAS was incorporated during the loading process. The particles in the co-incorporated sample looked significantly bigger and different in shape than the simple drug-loaded silica samples (Figure 12). Upon further investigation, it was observed that these large particles consisted of polymer plates upon which drug-loaded silica was dispersed. This is analogous to a standard polymeric amorphous solid dispersion and is believed to be the first example of a solid dispersion of loaded silica to be described in the literature. 


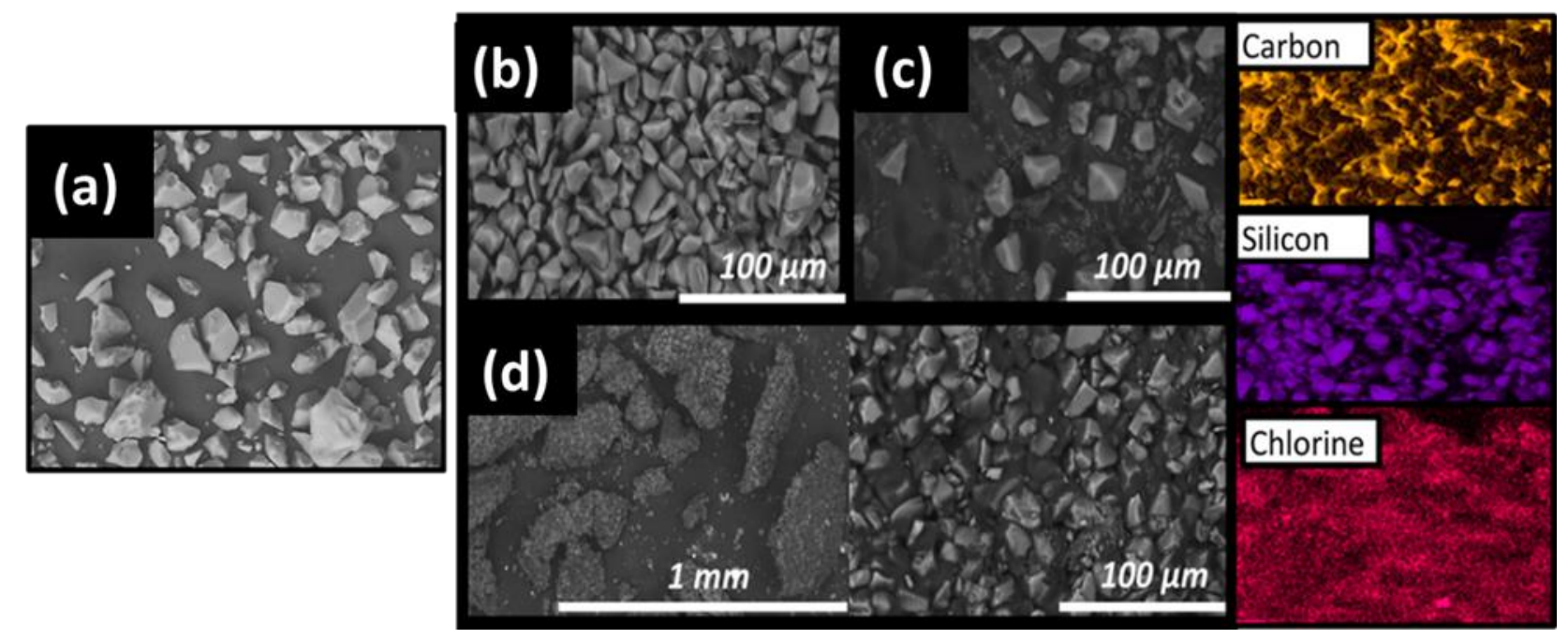

Figure 12. Left: SEM of unloaded mesoporous silica (a), glibenclamide loaded silica (b), glibenclamide loaded silica + HPMCAS blend (c) and co-incorporated glibenclamide/HPMCAS loaded silica (d). Right: SEM EDS of co-incorporated glibenclamide/HPMCAS loaded silica showing carbon (yellow), silicon (purple) and chlorine (pink) atoms are highlighted. Chlorine is a marker for glibenclamide.

\subsection{Experimental Considerations for Dissolution Selection}

Recently, there has been a shift away from traditional sink dissolution tests towards non-sink dissolution for supersaturating formulations (Augustijns et al. 2012; Sun et al. 2015). The reason for this shift is that supersaturation and precipitation are unlikely to be detected und sink conditions. Most supersaturating formulations are now routinely characterized in non-sink dissolution tests. However, unless the effect of transfer of the formulation out of the stomach into the small intestine can be considered, a robust in vitro-in vivo correlation can be hard to obtain. Although supersaturation results in single-medium (e.g. FaSSIF) dissolution tests may seem promising, the full extent of precipitation can often only be realized by generating transfer dissolution data. Supersaturation or a higher thermodynamic solubility of the drug in gastric fluid may trigger the generation of seeds when the solution is transferred to the intestine. In some cases, this results in significantly poorer dissolution performance of the formulation in the FaSSIF portion of the experiment, with no enhancement versus the crystalline drug substance (Kambayashi, et al. 2019). During investigation into the co-incorporation 
approach, biorelevant transfer dissolution experiments were utilized to capture the full precipitation inhibition picture, and to help elucidate a mechanistic rationale.

\subsection{Dissolution of Simple Blends: The Importance of Precipitation Inhibitors}

Based on the dissolution of silica loaded with pure glibenclamide (Figure 13), it is clear that a $\mathrm{PI}$ is required to sustain the supersaturation over a physiologically relevant timescale. Although a simple blend with HPMCAS did have a marked effect on the dissolution compared to pure glibenclamideloaded silica, it did not successfully sustain supersaturation, with concentrations falling towards that of the crystalline drug substance by the end of the experiment.
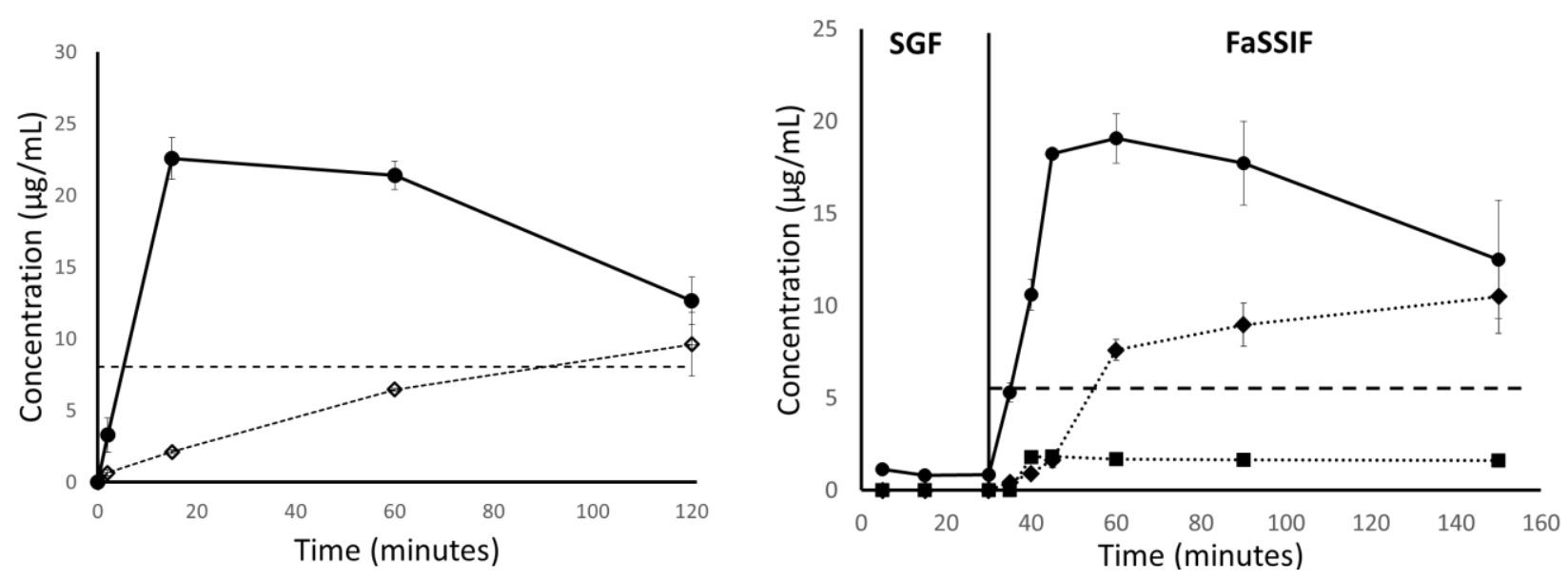

Figure 13. (left) FaSSIF mini-dissolution (37c, pH 6.5) and (right) transfer dissolution of glibenclamide (diamonds), glibenclamide loaded silica (crosses) and glibenclamide loaded silica + HPMCAS blend (circles). Equilibrium solubility is shown by the dashed lines. Equilibrium solubility in SGF is below limit of detection.

XRPD on the solid residues from both experiments revealed drug crystallinity (Figure 14), suggesting the precipitation inhibition effect of the polymer in the simple blend was only temporary. For the transfer dissolution, the crystallinity observed in the post-SGF residue was especially important, as the formation of seed crystals early in the experiment has the potential to increase the precipitation rate in the intestinal portion of the experiment, suggesting that the full formulation potential had not been realized. 
The effect of seed crystals on the precipitation of supersaturated drugs is well-known. Patel and colleagues demonstrated the increased precipitation rate of supersaturated indomethacin in the presence of seed crystals, compared to in their absence (Patel et al. 2013). This effect is related to first principles of nucleation and crystallization, in which a solid precipitate can act as a trigger for heterogeneous nucleation, reducing the critical nuclei induction time (Price et al. 2018). Therefore, although blending glibenclamide loaded silica with HPMCAS improved the formulation performance relative to the loaded silica alone, the precipitation inhibition is not optimal, and significant precipitation still occurs during dissolution.

(a)

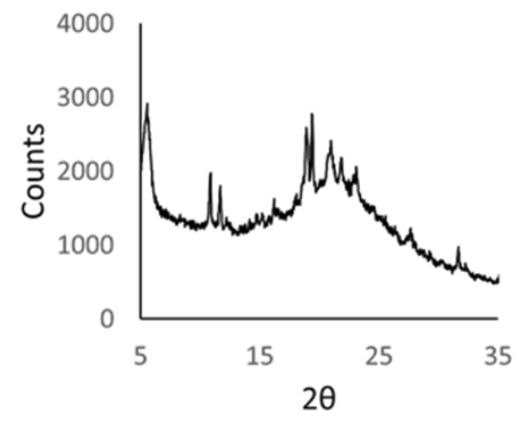

(b)

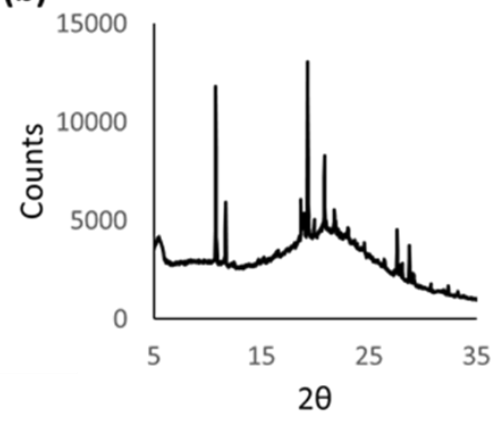

Figure 14. XRPD patterns for post-FaSSIF dissolution residues for (a) glibenclamide loaded silica and (b) glibenclamide loaded silica + HPMCAS blend

\subsection{Co-Incorporating HPMCAS in the Loading Method: Precipitation and Dissolution}

In contrast to the simple HPMCAS blend, incorporating HPMCAS during the loading step resulted in a substantial and sustained supersaturation in the single medium-FaSSIF dissolution assay (Figure 15). Furthermore, the post-dissolution residue for the co-incorporated formulation was amorphous, suggesting the co-incorporated HPMCAS was able to more effectively inhibit precipitation than when simply blended with the formulation (Figure 15). 


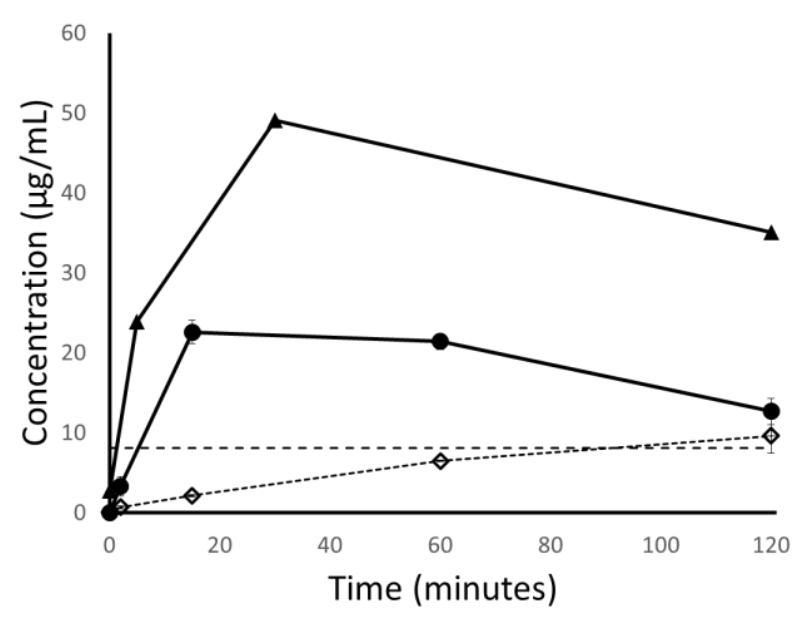

(a)

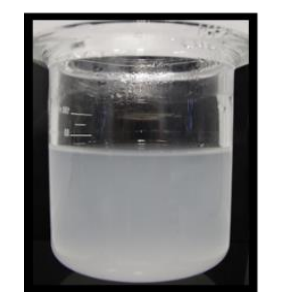

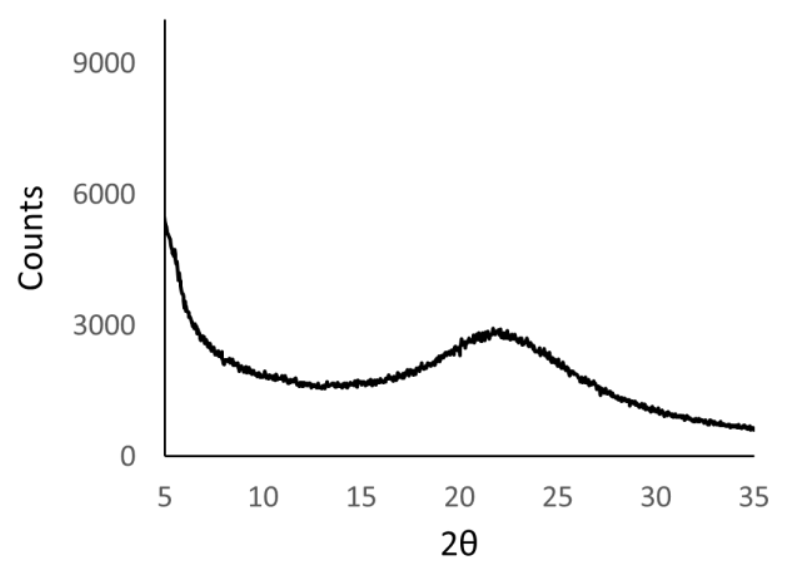

(c)

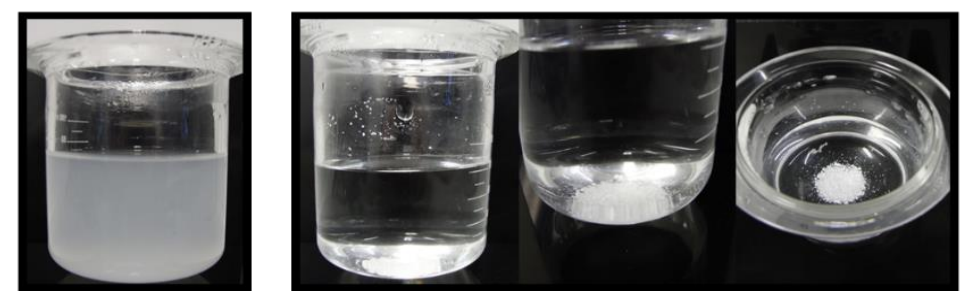

Figure 15. Left: FaSSIF mini-dissolution $\left(37^{\circ} \mathrm{C}, \mathrm{pH} 6.5\right)$ of glibenclamide (diamonds), glibenclamide loaded silica + HPMCAS blend (circles) and co-incorporated HPMCAS/GB loaded silica (triangles). Thermodynamic solubility is shown by the dashed line. Right: XRPD residue of co-incorporated HPMCAS/GB loaded silica post-FaSSIF dissolution. Bottom: Appearance of glibenclamide loaded silica (a), glibenclamide loaded silica + HPMCAS blend (b) and co-incorporated GB/HPMCAS loaded silica (c) dispersed in simulated gastric fluid.

In line with the previous discussion on the importance of transfer dissolution tests for supersaturating formulations, it is in this experiment that the true potential of the co-incorporation approach can be observed. In contrast to both the simple, drug-loaded silica and its physical blend with the PI, no drug was released from the co-incorporated sample in the SGF portion of the assay, and the residue remained amorphous. 

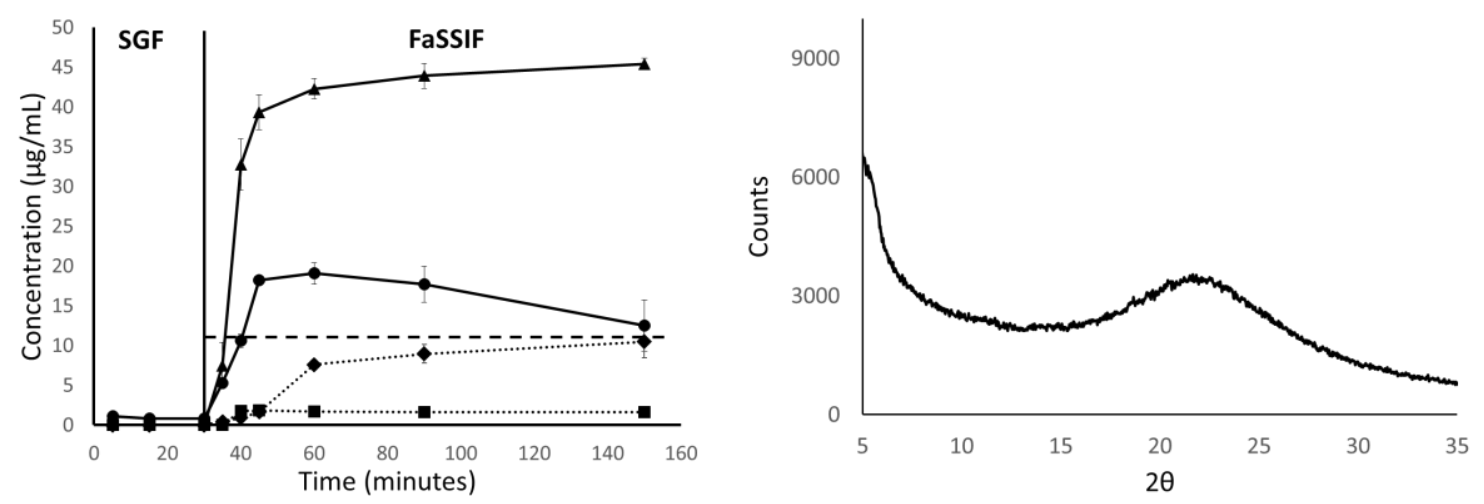

Figure 16. Left: Biorelevant transfer dissolution of glibenclamide loaded silica (squares), crystalline glibenclamide (diamonds), glibenclamide loaded silica + HPMCAS blend (circles) and co-incorporated glibenclamide/HPMCAS loaded silica (triangles). Thermodynamic solubility is shown by the dashed line. Thermodynamic solubility in SGF was less than the limit of detection. Right: XRPD pattern of coincorporated GB/HPMCAS loaded silica post-transfer dissolution.

The mechanism for this significant benefit is that the HPMCAS plates remain intact and thus the drugloaded silica remains immobilized under gastric conditions, preventing drug release from the silica and the formation of seed crystals.-These results demonstrate clearly that changing the manufacturing process, without changing the qualitative and quantitative composition of the formulation, can introduce new properties to the formulation. By incorporating the HPMCAS alongside the drug in the loading process instead of merely physically blending it in, enteric properties were easily introduced without the addition of extra excipients, coating processes or special capsules, which are typically required to prevent drug release in the stomach (Qiu, 2017). The enteric property should be especially advantageous in the delivery of poorly soluble weakly basic compounds, for which premature release in the stomach and subsequent supersaturation followed by precipitation in the small intestine could be avoided by this approach.

Combination of weak bases with mesoporous silica has been investigated as a potential formulation approach. For example, Van Speybroeck and colleagues described the improved oral absorption of itraconazole loaded silica in rats. However, the authors found that HPMCAS-based silica formulations 
were unsuccessful in preventing the release and precipitation of the drug in the stomach and therefore absorption was not optimized (Van Speybroeck et al. 2010). It is reasonable to assume from the glibenclamide studies described above that a HPMCAS-based co-incorporated formulations would be a suitable way forward to improving the in vivo absorption of poorly soluble weak bases by avoiding release in the stomach. However, further work is needed to verify the advantages of the coincorporation approach in a range of poorly soluble weak bases.

A critical factor for effective precipitation inhibition is the presence of drug-polymer interactions (Price et al., 2018). Specifically, hydrogen bond interaction, hydrophobic interactions and Van de Waals interactions have all been shown to play a critical role in the inhibition of precipitation (Prasad, et al. 2016; Schram, et al. 2015; Warren et al. 2015; Price et al. 2018). Therefore, one hypothesis for the improved precipitation inhibition of the co-incorporated formulation was the 'preformation' of drug-polymer interactions in the solid state. This was confirmed by solid-state NMR data, which showed a peak-shift indicative of interaction in the co-incorporated sample, but not in the simple blend (Figure 17).

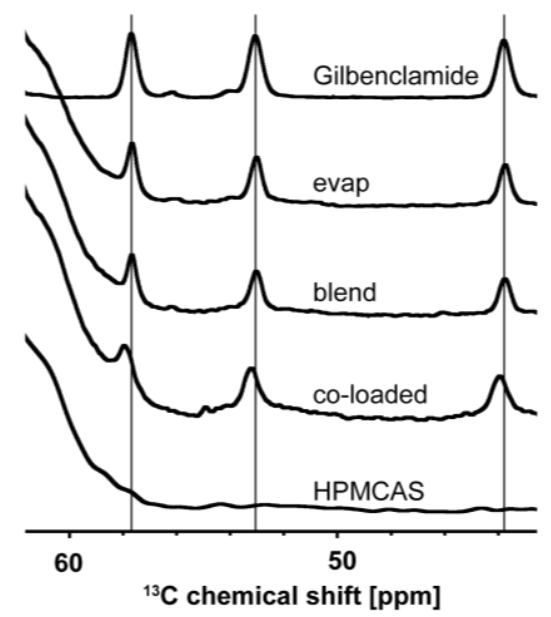

Figure 17. ${ }^{13} \mathrm{C}$ NMR spectra for different formulations studied in the co-incorporation study, showing a distinct spectral shift for the co-incorporated samples, indicative of a molecular interaction. 
SS-NMR spectroscopy was carried out on all samples (Figure 17). The $13 \mathrm{C}$ peaks for the drug were identical in all samples except the co-incorporated formulation. In the co-incorporated formulation, a low field shift of $0.2-0.5 \mathrm{ppm}$ for all drug peaks was observed. For example, the characteristic drug peak at $53 \mathrm{ppm}$ was observable in all samples except the co-incorporated formulation, in which the peak shifted to $53.5 \mathrm{ppm}$. This is indicative of an interaction taking place between the drug and the polymer in the solid state, which can take place once the drug is immobilized in the silica and subsequently in the HPMCS plate. The results suggest that solid-state drug-polymer interactions and hence dissolution performance can be altered by changing the method used to manufacture the formulation. Demonstration of a solid-state interaction between the HPMCAS and the drug in the coincorporated formulations provided further rational for the importance of such interactions on the effective performance of a precipitation inhibitor.

\subsection{Co-incorporated Formulations: Just a Solid Dispersion?}

Polymeric amorphous solid dispersions are the most common amorphous formulation technology applied. In particular, ASDs produced by spray-drying from organic solution are regularly used in commercial formulations (Timpe, et al. 2007). Therefore, it was important to rule out the possibility of a polymeric ASD being formed during the removal of solvent in the rotary evaporator step, with the silica having no impact on the overall performance. To assess this, a control sample consisting of HPMCAS-glibenclamide, i.e. without mesoporous silica, was prepared by evaporation from an organic solvent. Visually, the control sample appeared similar to the co-incorporated formulation, with platelike particle observed in SEM (Figure 18). However, EDS data showed that the drug substance was no longer only confined within the polymer platelets, and instead was distributed throughout the entire sample (Figure 18). 


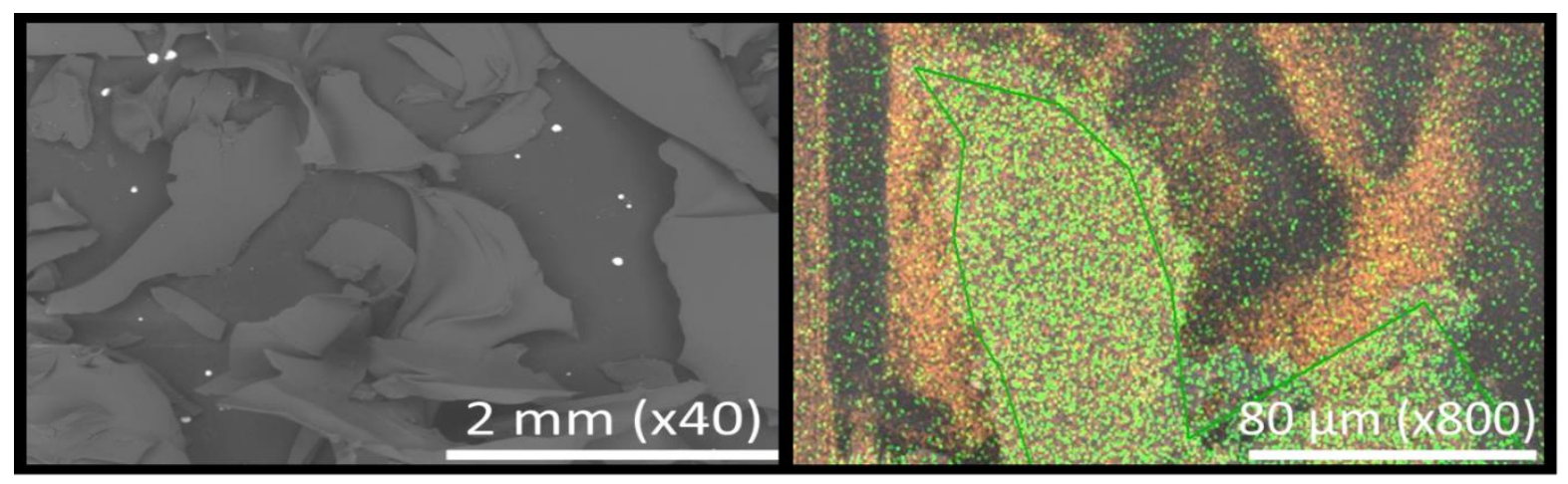

Figure 18. SEM (left) and EDS (right) images of glibenclamide and HPMCAS prepared by solvent evaporation shows the same particle size and morphology as the co-incorporated samples. However, in this sample the drug (indicated by green) is no longer confined within the polymer plate and is freely distributed throughout the sample.

Without the nano-confinement effects of the silica (Ditzinger, et al. 2018; Ditzinger/Price, et al. 2019), the drug was able to re-crystallize, which was observed in the XRPD (Figure 11). Ultimately, this resulted in the control sample showing no improvement in FaSSIF dissolution versus crystalline drug (Figure 19). These results are in stark contrast to the co-incorporation formulation, where the drug was confined within the mesoporous silica particles, which in turn were immobilized in the polymer platelets and released only when the formulation was subjected to intestinal conditions.

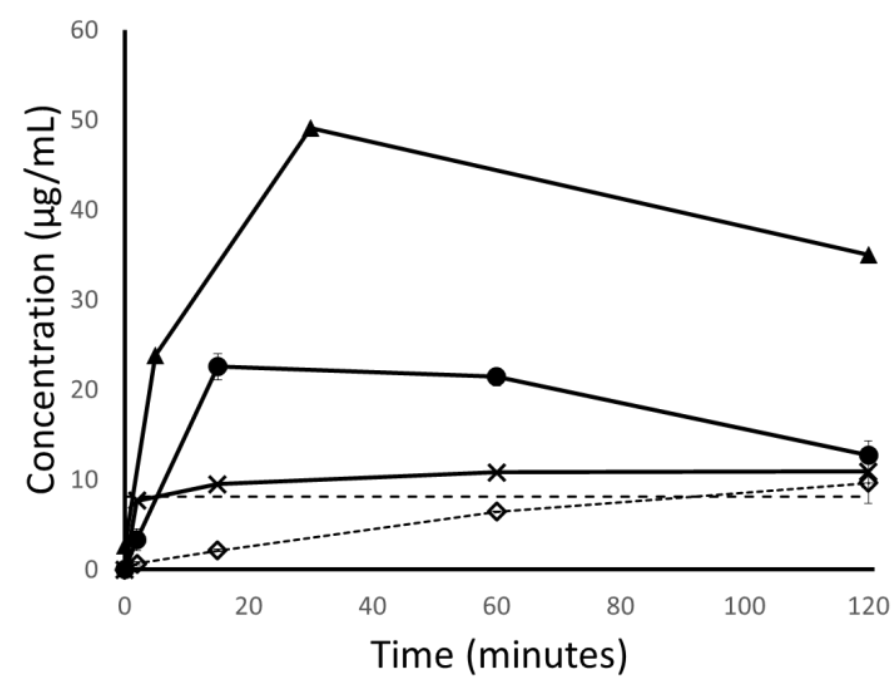


Figure 19. FaSSIF Mini dissolution (37c, pH 6.5) of the control sample, GB/HPMCAS prepared via rotary evaporator (X) compared to co-incorporated GB/HPMCAS loaded silica (triangles), GB loaded silica + HPMCAS blend (circles), and crystalline glibenclamide (X).

Finally, even if a portion of the sample was able to remain amorphous in the polymer platelets, the lack of drug-polymer interaction (as indicated by the solid-state NMR spectra from the control sample) would lead to a reduction in the precipitation inhibition effect of the polymer (Figure 17). Thus, it can be concluded that the silica and the polymer played a synergistic role in the co-incorporated formulation, which out-performed both the physical blend and the control.

\subsection{Mechanistic Rationale}

Incorporating the precipitation inhibitor alongside the drug when loading onto mesoporous silica substantially improves formulation performance compared to a simple physical blend. Both dissolution and supersaturation were improved in both single-medium FaSSIF dissolution and biorelevant transfer dissolution. Furthermore, the co-incorporation approach allowed the removal of a time-consuming and inefficient blending step. To provide a physical mechanistic basis for the improved performance, a range of spectroscopic tools was utilized. It was concluded that the improved dissolution performance is a synergistic effect related to two key factors: formation of drug-polymer interactions in the solid state, and lack of release and premature precipitation under gastric conditions due to the immobilization of drug-loaded silica particles within the enteric HPMCAS plates (Figure 20). Crucially, both properties are absent in a simple blend of HPMCAS with the drug-loaded silica. The incorporation of precipitation inhibitors with the drug during loading onto mesoporous silica formulations has the potential to improve both the process and formulation efficiency in the development of poorly soluble drugs and underlines the importance of effective drug-polymer interactions in inhibiting precipitation from mesoporous silica formulations. 


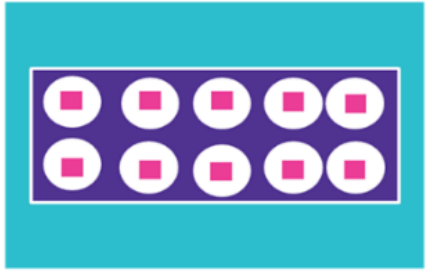

Solid State and Stomach

$\checkmark$ Formation of drug-polymer interactions

$\checkmark$ Loaded silica confined to polymer plate

$\checkmark$ No release in stomach

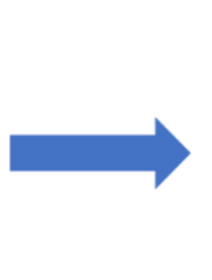

Intestines

$\checkmark$ Breakdown of polymer plate

$\checkmark$ Release of supersaturated API

$\checkmark$ Improved precipitation inhibition
Active pharmaceutical ingredient

Precipitation inhibitor

Mesoporous silica

Figure 20. Mechanistic rationale for enhanced performance of co-incorporated $\mathrm{Pl} / \mathrm{drug}$ loaded silica formulations.

\section{In Silico Pharmaceutics: A New Method to Select Precipitation Inhibitors for Mesoporous Silica}

\subsection{The Role of Molecular Interaction: Quality by selection}

The mechanistic rationale for improved formulation performance with the co-incorporation approach reaffirms the importance of drug-polymer interactions in successful precipitation inhibition. However, the polymer selected, HPMCAS, demonstrated solid-state interactions only when it was coincorporated rather than simply blended into the formulation. Although this was a positive outcome, it suggests that there is still further room for improvement by selecting a precipitation inhibitor that interacts optimally with the drug, i.e. able to generate an effective and strong precipitation inhibition effect while avoiding an overly strong interaction with a slow off-rate. Such an approach, "quality by selection", could enable optimization of the formulation process for supersaturating systems. There have been previous attempts at rationalizing drug-polymer interactions in precipitation inhibition, but those approaches are mostly complicated and therefore not practicable for screening in early pharmaceutical development (Price et al. 2018). On the other hand, current empirical experimental screening approaches can be intensive both from a time and labor perspective, as well as a resource perspective in terms of the amount of drug required, which may be difficult due to paucity of available compound in the early stages of development (Price et al. 2018). Therefore, an approach that can 
incorporate understanding of the drug-polymer interactions with a quick and efficient screening process would be very useful. For this purpose, the Conductor like Screening Model for Real Solvent (COSMO-RS), which was developed by Klamt (Klamt, et al. 1993; 1995; 2003), is a highly interesting prospect.

\subsection{COSMO-RS: Combining Quantum Chemistry and Thermodynamics}

The conductor like screening model (COSMO) is a quantum mechanical solvent-based theory that is used to generate sigma surface profiles for molecules of interest (Klamt, et al. 1993; 1995; 2003). This is achieved via conventional quantum chemistry, which treats molecules in isolation at $\mathrm{T}=0 \mathrm{~K}$ within a cavity inside a dielectric continuum (Figure 21) (Klamt, et al. 1993; 1995; 2015). The polar surface distribution, or $\sigma$ profile, of this cavity is solved using the Schrodinger equation. The $\sigma$ profile describes the distribution of charge throughout the cavity surrounding a molecule and, as such, the charges counteract that of the molecule. For example, the electron withdrawing ability of oxygen results in a small $\delta$ - charge on the oxygen atom, this is counteracted with a positive $\sigma$ charge in the molecular cavity (Klamt, et al. 1993).

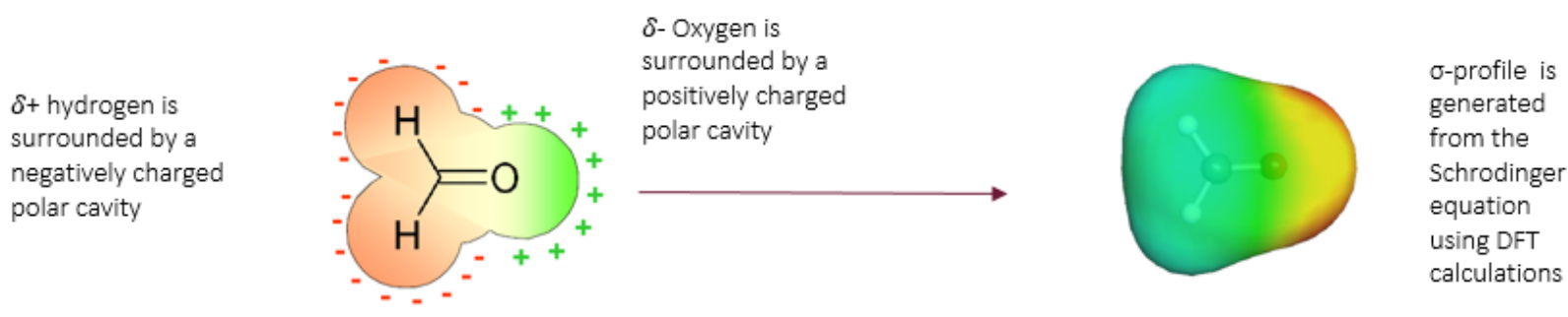

Figure 21. COSMO applies quantum mechanical methods to calculate the polar surface cavity of a molecule in isolation at $0 \mathrm{~K}$. The charge distribution in the polar cavity counteracts the charge distribution of the molecule. Taken from the COSMOlogic website, with permission.

The chemical potential, $\mu$, can be derived from sigma profiles. This is very useful as chemical potential can be used to calculate thermodynamic parameters of interest. However, due to the assumptions 
applied by COSMO, this process is unreliable for all but the most neutral compounds (Klamt, et al. 1995)

Thus, COSMO-RS (Conductor like Screening Model for Real Solvents), was developed in order to extend applicability of this theory to real-world problems. COSMO-RS implements COSMO as highlighted above to generate $\sigma$ profiles, however, it then assumes that the sigma profile segments are in close contact and can interact with one another in a pair-wise fashion (Klamt, et al. 1995) (Figure 22).

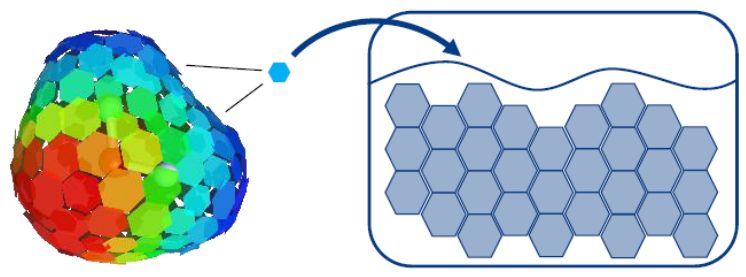

Figure 22. COSMO-RS is a statistical thermodynamic theory that assumes sigma profile surface segments can come in contact and interact in a pairwise fashion

Application of COSMO-RS allows the calculation of the sigma potential of a mixture of two systems (Klamt, et al. 1995). The sigma potential is calculated by considering the energy requirements of combining sigma segments of one system within the sigma surface of the other (Klamt, et al. 1998). Assuming a system consisting of Compound A and Compound B: First, a segment must be removed from $A$ to create a cavity for the incorporation of segment $B$; this requires energy associated with removing pre-existing contacts between A-segments, $-\boldsymbol{\mu}_{s}(A)$. Second, the new B-segment must be added to the cavity in sigma surface $A$; this involves forming new interactions between $A$ and $B$, with related energy costs and gains associated with the two segments interacting, $\boldsymbol{E}(\boldsymbol{B}, \boldsymbol{A})$, referred to as the COSMO-RS energy. Taken together, these two energy terms describe the thermodynamic cost of combining one B sigma-segment with a one cavity in the sigma-surface of A. To solve the energy cost for the whole system, this calculation is carried out iteratively for all possible combinations of $A$ and $B$ sigma-segments (Equation 12). This value is referred to as the Sigma Potential of A and B. 


$$
\mu_{s}(A, B)=-k T \ln \int p_{s}(\sigma \mathrm{A}) \exp \left\{\frac{E(\sigma \mathrm{A}, \sigma \mathrm{B})-\mu_{s}(\sigma \mathrm{B})}{R T}\right\} \mathrm{dA} \sigma
$$

This equation has high real-world applicability as the COSMO-RS energy term, $\boldsymbol{E}(\boldsymbol{B}, \boldsymbol{A})$, is calculated such that all binding modes (electrostatic, hydrogen bonding, van der Waals and combinatorial) are considered in the equation (Equations 13-15).

\section{Electrostatic interaction:}

$$
E_{\text {coloumb }}(\sigma)=\frac{\alpha}{2}\left(\sigma+\sigma^{\prime}\right)^{2}
$$

where $\alpha$ is an adjustable parameter that is calculated in situ via parameterization, and $\sigma$ and $\sigma^{\prime}$ are the solute and solvent segment, respectively.

\section{Hydrogen bond interactions:}

$$
E_{h b}(\sigma)=c_{\mathrm{hb}} T \max \left[0, \sigma_{a c c}-\sigma_{h b}\right] \min \left[0, \sigma_{d o n}+\sigma_{h b}\right]
$$

(Equation 14)

where $\sigma_{a c c}$ and $\sigma_{d o n}$ are the sigma profile densities of the hydrogen bond acceptor and donor, respectively. $c_{\mathrm{hb}}$ and $\sigma_{h b}$ are the adjustable parameters corresponding to the hydrogen bond prefactor and the hydrogen bond threshold, respectively. This equation is constructed with minimum and maximum thresholds to ensure that the screening charges exceed the required values for hydrogen bonding to occur.

\section{Van der Waals interactions:}

$$
E_{v d w}=\sum_{k} \gamma_{k} A_{k}
$$

where the dispersion energy, $E_{v d w}$, is related to the surface area of the contact point, A, on the specific element, $k$, and on an adjustable pre-factor, $\gamma$.

From the sigma potential of $A, B$ the chemical potential can be derived, from which a wide range of thermodynamic parameters can be calculated.

\section{Chemical Potential A, B:}


The full implementation of COSMO and COSMO-RS can be carried out using the software COSMOtherm with both quantum mechanics and thermodynamics being carried out by the software. The rate determining step in this process is, by far, the quantum mechanical calculations. Depending on the complexity of the molecules in question, these calculations can take days or weeks and require a large amount of computing power. This is especially problematic for screening purposes, which should be as quick and efficient as possible. Of course, there is the option of bypassing the need for quantum mechanics calculations if the $\sigma$ profile of the molecule of interest is already in the master database. This, is, however, especially unlikely for complicated organic molecules, especially drug candidates in development.

To avoid this time intensive step, one can create additive sigma surfaces based on pre-calculated fragments stored in a database. This database is available in the software package COSMOquick (Loschen, et al. 2012). In short, when a molecule is entered into COSMOquick the database is searched for molecules with similar chemical structures. The relevant parts of these sigma profiles are then either 'switched on' or 'switched off' and combined to form a new sigma profile for the molecule in question (Figure 23). This additive sigma profile can then be used in combination with COSMO-RS in the same way described above. (Loschen, et al. 2012)

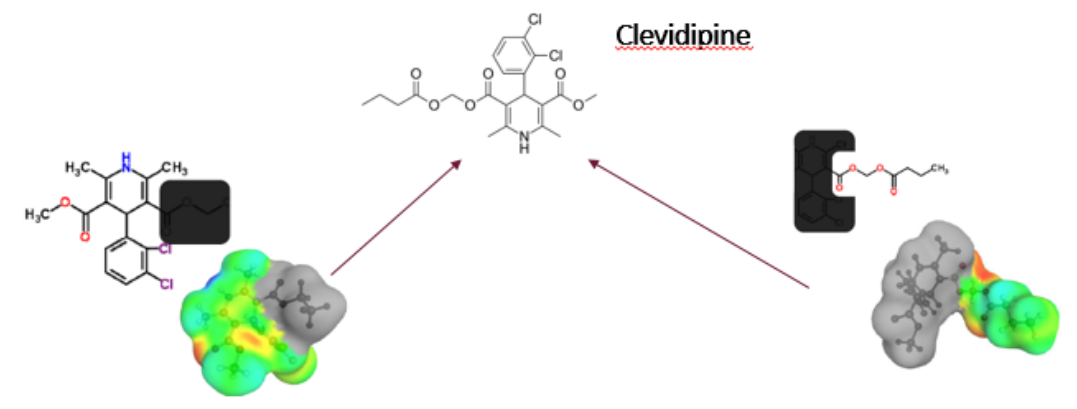

Figure 23. COSMOquick applies a fragmentation-based approach to predict the sigma profile of unknown molecules. This avoids the use of time-consuming quantum mechanical calculations. Taken from the COSMOlogic website, with permission. 
This equation is constructed with minimum and maximum thresholds to ensure that the screening charges exceed the required values for hydrogen bonding to occur.

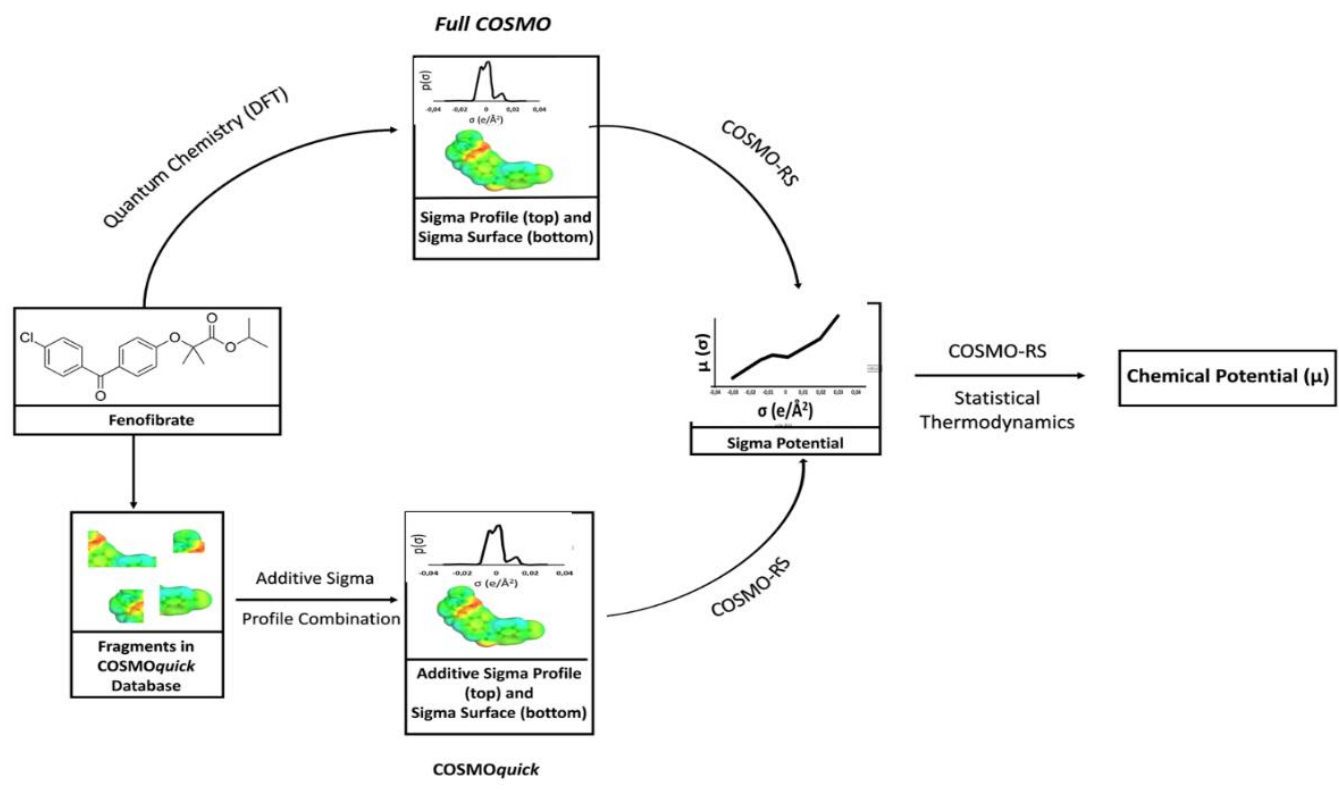

Figure 24. Chemical potential, and in turn a wide-range of thermodynamic properties, can be derived from sigma profiles using COSMO-RS theory. Sigma profiles can be obtained by two ways, either through de novo quantum chemical calculations or through an additive combination of previously calculated molecular fragments stored in a large database. The former approach is applied in the full COSMO calculation, whilst the latter is applied in the software package COSMOquick (bottom).

\subsection{COSMO-RS for Precipitation Inhibitor Screening: Theory}

As previously discussed, the interaction between drug and polymer is essential for effective precipitation inhibition (Price et al. 2018; Price et al. 2020). The interaction is complex, essentially involving a ternary system where drug, polymer and water all interact to various degrees. Current methods, such as PC-SAFT, would be able to capture such a system but would involve a large amount of time and computing intensity (Paus, et al. 2015). We propose a simplified approach, in which the mixing (or excess) enthalpies of drug and excipient are calculated using COSMOquick. This estimated enthalpy is then used to rank potential precipitation inhibitors based on the strength of their molecular 
interaction with the drug substance. It is hypothesized that this novel in silico protocol can be used to screen potential precipitation inhibitors, allowing for a more focused selection to be carried out and thus significantly reducing the experimental burden of screening many potential inhibitors by trial and error, whilst ensuring the selection of an optimal inhibitor.<smiles>CC(C)OC(=O)C(C)(C)Oc1ccc(C(=O)c2ccc(Cl)cc2)cc1</smiles>

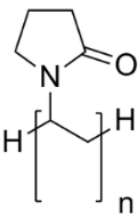

API

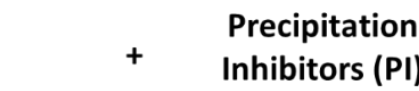

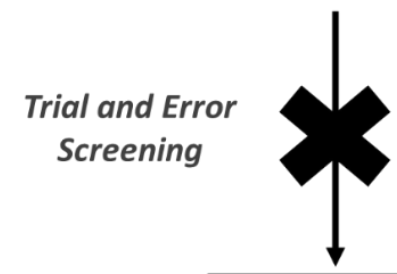

PI Selection

\section{Focused Screening}

\section{COSMO-RS}

Drug-polymer

mixing energy

PI Ranking

Figure 25. Workflow to determine optimal precipitation inhibitors for supersaturating formulations using the in silico computational tool COSMO-RS

COSMOquick (COSMOlogic, Germany, Version 1.6) was used to calculate excess enthalpy of interaction between drug and polymer. Polymer structures were approximated as trimers, since the quantum chemical calculations cannot capture the full complexity of large molecules like polymers. Furthermore, this was not deemed critical to the study as the hypothesis was related to local molecular interactions, which are assumed to be sufficiently captured by trimer forms of the polymer. Ratio of drug: PI was set at 1:3 to align with the ratios used in the formulations, and the temperature was set at $37^{\circ} \mathrm{C}$. With this approach, 52 calculations can be carried out in just 5 minutes. 


\subsection{Incorporation of Precipitation Inhibitors with Mesoporous Silica Formulations}

Although incorporating precipitation inhibitors alongside the drug in mesoporous silica formulations was a main theme of the doctoral studies, this formulation approach was not included in the development of the in-silico screening approach. This was to ensure that any relationship between the calculated drug-polymer interactions and the overall formulation performance was related only to the innate precipitation inhibition potential of the polymer, and not to processing techniques.

\subsection{COSMO-RS for Precipitation Inhibitor Screening: Output}

Using the COSMO-RS screening protocol, excess enthalpy of interaction upon mixing drug and polymer can be calculated for a given drug in combination with $\sim 50$ potential precipitation inhibitors in as little as five minutes. This represents a significant time saving versus traditional experimental screening. All potential inhibitors can be assessed using enthalpy of interaction as a rank-order parameter, which is designated the "COSMO Rank" (Figure $\mathbf{5}$ a-c). This is related to the hypothesis that the more negative the enthalpy, the higher the chance of successful precipitation inhibition based on interactions between drug and PI.

The output from all three polymer screenings is shown in Figure 26. Without consideration of the dissolution data, the initial observations point towards a correlation between predicted performance and experimental performance. For example, Chauhan and co-workers reported that Eudragit EPO and HPMC were the most successful polymers to inhibit precipitation of dipyridamole vs. a selection of alternative polymers (Eudragit S100, Eudragit RL100, PEG and PVP) (Chauhan et al. 2014). Importantly, the initial COSMO-RS calculations were able to predict the poor ability of PVP to inhibit dipyridamole precipitation experimentally, since a positive enthalpy of interaction was predicted. This was observed experimentally in the same study by Chauhan and co-workers. 


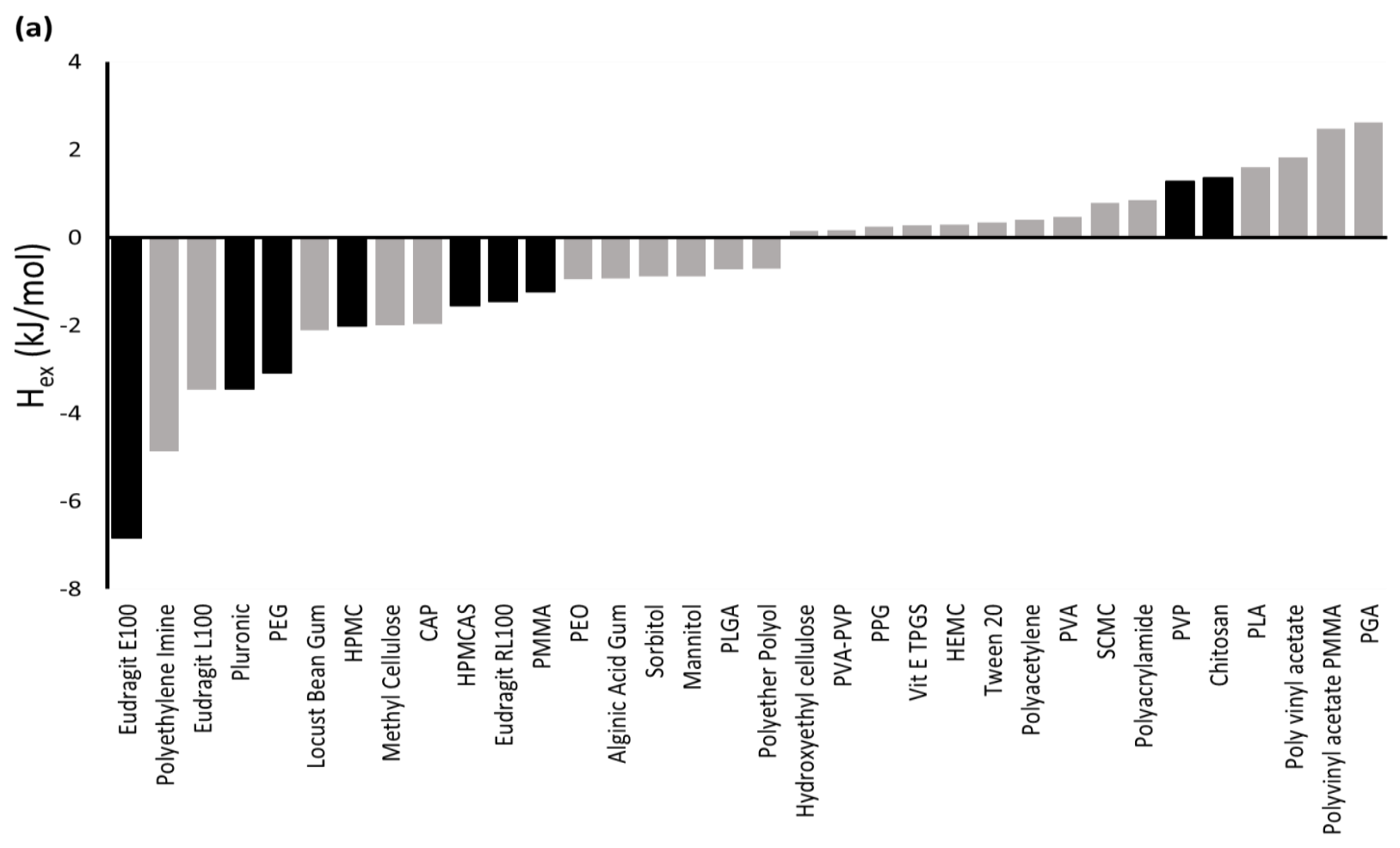

(b)

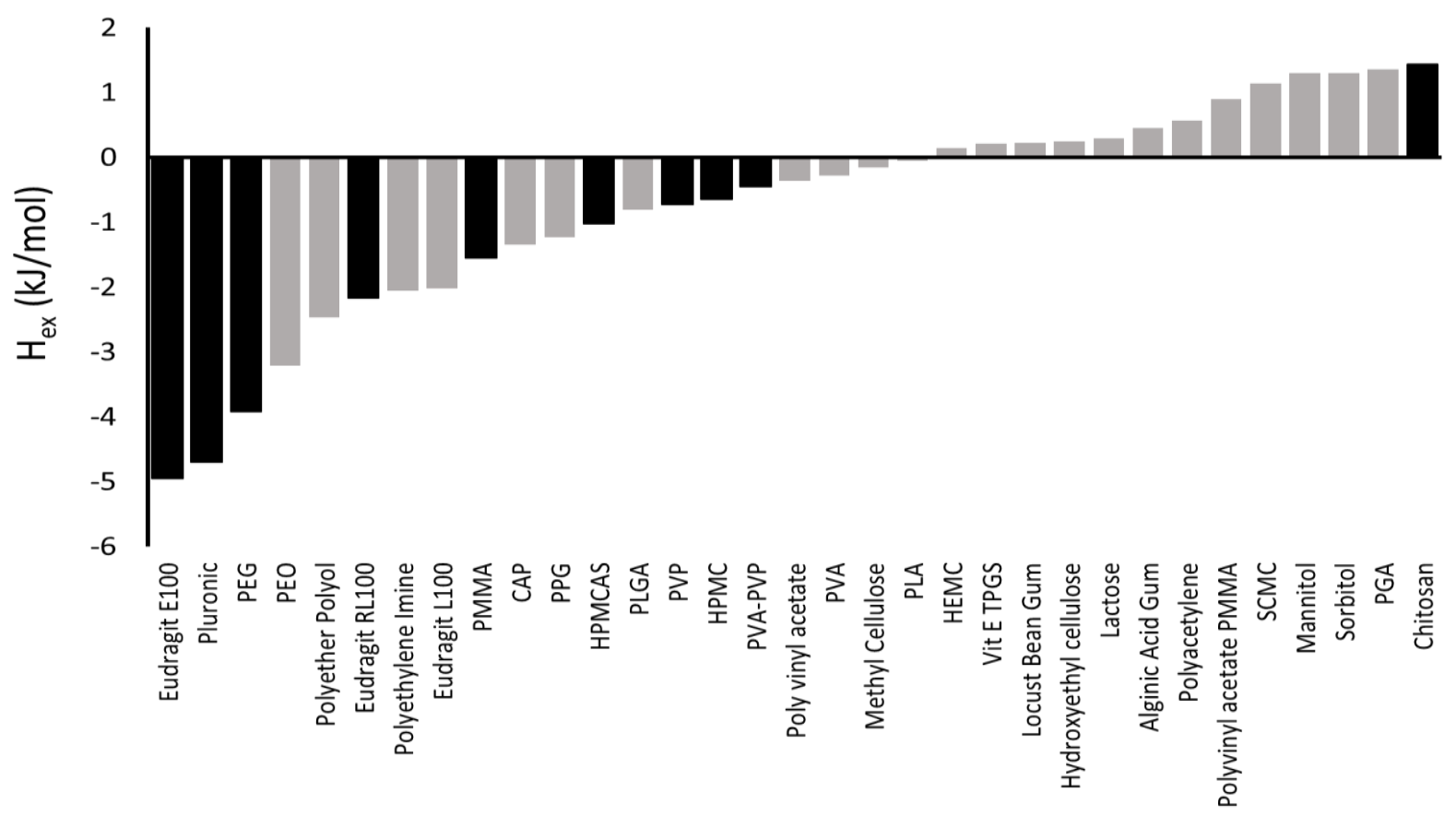


(c)

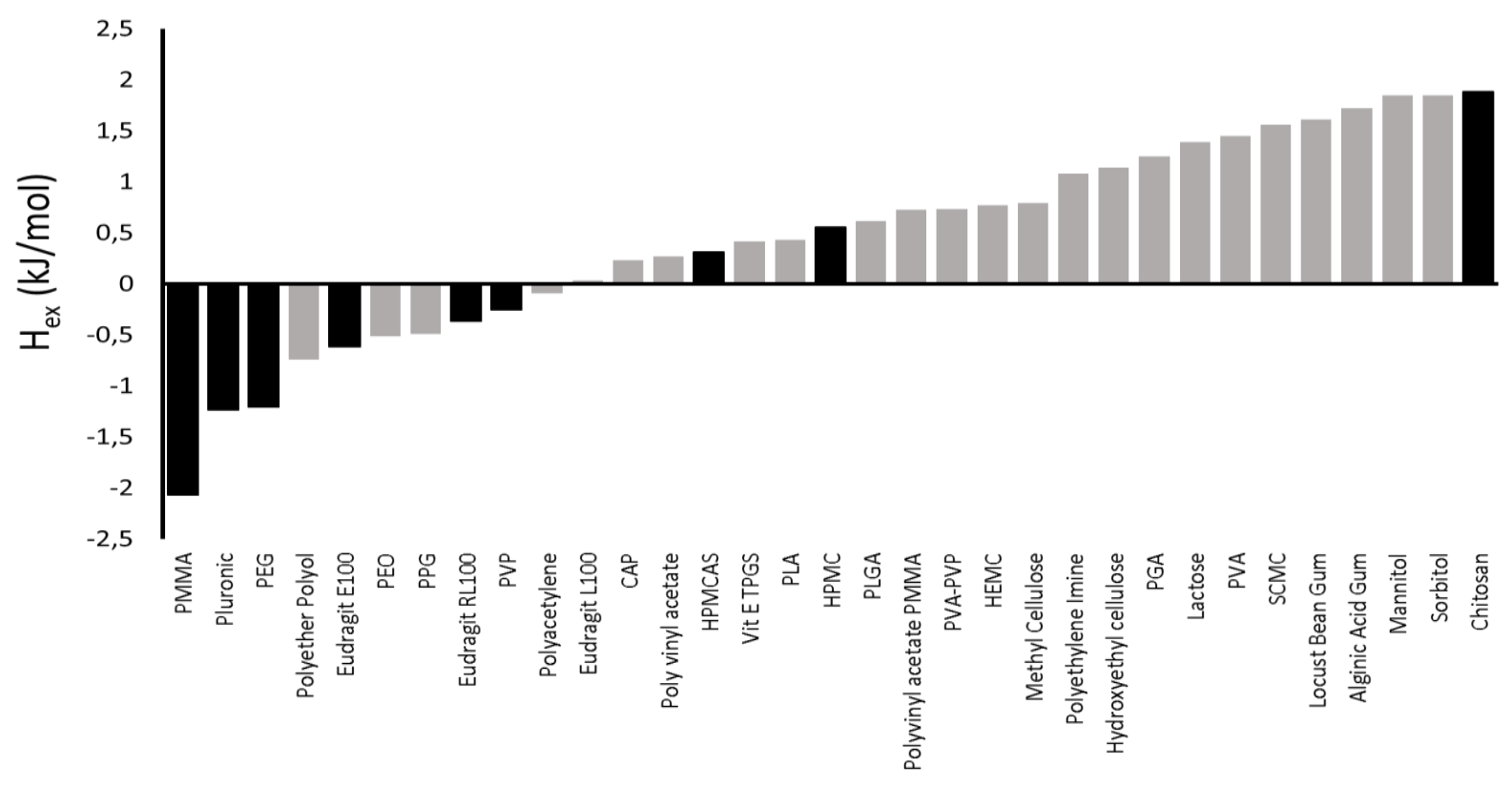

Figure 26. COSMO-RS Screen: calculated excess enthalpy of interaction between dipyridamole (a), glibenclamide (b) and fenofibrate (c) with a range of potential precipitation inhibitors. Polymers studied experimentally to test the correlation are highlighted as dark bars: Eudragit EPO, Pluronic (PLR), PEG, HPMCAS, PVP, HPMC, Eudragit RLPO, PMMA and Chitosan.

The advantages of the COSMO-RS screening method can be readily seen by comparing it to traditional screening approaches. For example, Petrusevska et al. employed an experimental high-throughput screening to arrive at the conclusion that cellulose-based polymers were most effective in inhibiting precipitation of carbamazepine, in contrast to fenofibrate, which performed best in the presence of surfactant. (Petrusevska et al. 2013). This was achieved using a combination of solvent-shift method and off-line chromatography. There are two main limitations to this experimental approach. First, inclusion of organic solvents in order to generate supersaturated concentrations provides some uncertainty due to the potential impact of the solvent on the dissolution, interaction and supersaturation of the drug-polymer mixtures. Second, although there have been significant advances in throughput, there is a still a practical limit to the number of polymers that can be reasonably screened using these experimental platforms. Illustratively, the top-performing polymers indicated by 
the in-silico screening protocol were not included in the Petrusevska studies. This shows that experimental screening limits the polymer selection space and reduces the likelihood that the best polymer will be found. Instead, from a selection of standard polymeric precipitation inhibitors such as PVP, HPMCAS and HPMC a "good enough" polymer will be identified. This approach introduces a large amount of uncertainty as to whether the most efficient formulation has been realized and, as observed in our theoretical calculations, these polymers are often mediocre compared to the extended polymer toolbox (He, et al. 2010; O'Shea, et al. 2017; Laine, et al. 2016; Vora, et al. 2016)

\subsection{Correlation between COSMO-Rank and Formulation Performance}

The correlation between the rank order predicted by COSMO and the rank order observed in the dissolution experiments was determined to be $0.91(0.001, p<0.05), 0.81(0.01, p<0.05)$ and 0.61 (0.076, $p<0.5)$ for dipyridamole, glibenclamide and fenofibrate, respectively (Table 3-5). For dipyridamole and glibenclamide, this indicated a very strong positive correlation between COSMO prediction and formulation performance. This demonstrates that the COSMO-RS screening protocol can be used to select the most optimal precipitation inhibitors whilst avoiding the costly and timeconsuming experimental screening. For fenofibrate, a positive correlation was observed between the prediction and results, but to a lesser extent, and was especially impacted by the poor performance of PEG (see section 6.5). 
Table 3. Spearman's rank correlation coefficient analysis: dipyridamole results

\begin{tabular}{|c|c|c|c|c|}
\hline Polymer & $\begin{array}{l}\text { Calculated Enthalpy } \\
(\mathrm{kJ} / \mathrm{mol})\end{array}$ & $\begin{array}{l}\text { AUC ( } \quad \mu g \cdot m g \\
\left.m L^{-1}\right)\end{array}$ & $\begin{array}{l}\text { COSMO } \\
\text { Rank }\end{array}$ & $\begin{array}{l}\text { Dissolution } \\
\text { Rank }\end{array}$ \\
\hline Eudragit EPO & -6.84 & 29000 & 1 & 1 \\
\hline$P L R$ & -3.45 & 14000 & 2 & 3 \\
\hline PEG & -3.08 & 8600 & 3 & 5 \\
\hline HPMC & -2.12 & 19000 & 4 & 2 \\
\hline HPMCAS & -2.01 & 13000 & 5 & 4 \\
\hline Eudragit RLPO & -1.55 & 6600 & 6 & 6 \\
\hline PMMA & -1.46 & 5800 & 7 & 7 \\
\hline$P V P$ & -1.23 & 5300 & 8 & 8 \\
\hline Chitosan & 1.28 & 5100 & 9 & 9 \\
\hline $\begin{array}{l}\text { Spearman's Rank } \\
\text { Correlation Coefficient }\end{array}$ & 0.91 & Significance & $\begin{array}{l}0.001 \\
P<0.05\end{array}$ & \\
\hline
\end{tabular}

Table 4. Spearman's rank correlation coefficient analysis: glibenclamide results

\begin{tabular}{|c|c|c|c|c|}
\hline Polymer & $\begin{array}{l}\text { Calculated Enthalpy } \\
(\mathrm{kJ} / \mathrm{mol})\end{array}$ & $\begin{array}{l}\text { AUC ( } \mu \mathrm{g} \cdot \mathrm{mg} \\
\mathrm{mL}^{-1} \text { ) }\end{array}$ & $\begin{array}{l}\text { COSMO } \\
\text { Rank }\end{array}$ & $\begin{array}{l}\text { Dissolution } \\
\text { Rank }\end{array}$ \\
\hline Eudragit EPO & -4.96 & 11000 & 1 & 1 \\
\hline$P L R$ & -4.70 & 7200 & 2 & 2 \\
\hline$P E G$ & -3.92 & 960 & 3 & 7 \\
\hline$H P M C$ & -2.17 & 6000 & 4 & 4 \\
\hline HPMCAS & -1.55 & 6100 & 5 & 3 \\
\hline Eudragit RLPO & -1.03 & 2100 & 6 & 5 \\
\hline PMMA & -0.72 & 2000 & 7 & 6 \\
\hline$P V P$ & -0.64 & 600 & 8 & 8 \\
\hline Chitosan & 1.44 & 100 & 9 & 9 \\
\hline $\begin{array}{lr}\text { Spearman's Rank } \\
\text { Correlation Coefficient }\end{array}$ & 0.81 & Significance & $\begin{array}{l}0.01 \\
P<0.05\end{array}$ & \\
\hline
\end{tabular}


Table 5. Spearman's rank correlation coefficient analysis: fenofibrate results

\begin{tabular}{|c|c|c|c|c|}
\hline Polymer & Calculated Enthalpy $(\mathrm{kJ} / \mathrm{mol})$ & $\begin{array}{l}\text { AUC }(\mu g \cdot m g \\
\left.m^{-1}\right)\end{array}$ & $\begin{array}{l}\text { COSMO } \\
\text { Rank }\end{array}$ & $\begin{array}{l}\text { Dissolution } \\
\text { Rank }\end{array}$ \\
\hline PMMA & -2.07 & 10000 & 1 & 3 \\
\hline PLR & -1.24 & 9000 & 2 & 4 \\
\hline PEG & -1.21 & 5200 & 3 & 7 \\
\hline Eudragit EPO & -0.62 & 19000 & 4 & 1 \\
\hline Eudragit RLPO & -0.37 & 13000 & 5 & 2 \\
\hline PVP & -0.26 & 5400 & 6 & 5 \\
\hline HPMCAS & 0.31 & 5300 & 7 & 6 \\
\hline HPMC & 0.55 & 4200 & 8 & 8 \\
\hline Chitosan & 1.88 & 1700 & 9 & 9 \\
\hline $\begin{array}{l}\text { Spearman's Rank } \\
\text { Correlation Coefficient }\end{array}$ & 0.63 & Significance & $\begin{array}{l}\mathbf{0 . 0 8} \\
P<0.05\end{array}$ & \\
\hline
\end{tabular}

From a mechanistic perspective, the COSMO approach is highly attractive as all potential modes of interaction are considered in the calculation. Specifically, when considering the total energy required to combine sigma contacts of the drug and polymer molecules, the sigma potential is calculated (ps $\left.\sigma^{\prime}\right)$. One key component of this energy term is the COSMO-RS energy co-efficient $E\left(\sigma, \sigma^{\wedge^{\prime}}\right)$, which considers all major modes of molecular interaction including hydrogen bond, coloumbic and Van der Waals interactions. All these modes of interaction have been shown to have a pivotal impact on the overall precipitation inhibition performance of a polymer (Price, et al. 2018). The most common interactions are hydrogen bond and Van de Waals interactions (Warren, et al. 2010). Eudragit EPO is a good example, being the best performing polymer in both the calculation screening and experimental dissolution. There are several publications reporting that the superior inhibitory potential of Eudragit EPO is related to strong hydrogen bonding and hydrophobic interactions (Price, et al. 2018; Higashi, et al. 2014). These authors recorded hydrophobic interactions between the aromatic portion of the drug and the EPO polymer backbone as well as a hydrophilic hydrogen bond interaction between 
the amino alkyl groups of EPO and the carbonyl groups of the drug in solid state NMR spectroscopy. Such strong interactions between Eudragit EPO and all the drugs studied are possible, hence the good performance of this polymer in both the in silico and experimental screenings.

Furthermore, considering the precipitation inhibitors that did not perform well, one can relate the calculation, dissolution performance and potential points of interaction from a mechanistic perspective. One of the interesting cases here is the lack of successful inhibition of dipyridamole precipitation by PVP. As previously mentioned, PVP is one of the polymers most commonly used as a precipitation inhibitor. However, PVP has been shown to be ineffective in sustaining dipyridamole in solution. Chauhan and colleagues demonstrated that no interaction takes place between PVP and dipyridamole in the solid state or in solution (Chauhan et al. 2013). This was successfully identified by the COSMO screen and is reflected in the dissolution performance of the formulation in this study.

Ultimately, these robust mechanistic calculations increase the successful prediction of drug-PI interaction and thus precipitation inhibition, as reflected in the strong positive correlations achieved between the COSMO-rank and the final formulation performance.

\subsection{Limitations of the Approach}

COSMO-RS does not take into consideration the impact of water on the interaction between the drug and PI. It has been widely reported that for a precipitation inhibitor to successful sustain drug into solution, it must interact with both the drug and the water in the medium or GI tract (Ting, et al. 2016; Schram, et al. 2015; Price, et al. 2018). This simplification becomes especially problematic when considering polymers that have very high hydrophobicity or hydrophilicity, as demonstrated by Schram and co-workers (Schram, et al. 2015; Schram, et al. 2016). From the data presented in this study, the COSMO prediction for PEG does not correlate to the overall dissolution performance, with PEG being the major deviation in the correlation for all three drugs. This is likely related to the hydrophilicity issue. PEG is very hydrophilic and will bind and interact preferentially with water, this reduces the interaction with the drug and therefore in failure to realize the desired precipitation inhibitor performance. When 
the Spearman's rank correlation coefficient analysis was repeated without PEG, the correlation between COSMO-rank and dissolution-rank significantly improved to $0.98(0.0004, p<0.05)$ for dipyridamole and glibenclamide. Furthermore, the fenofibrate correlation, which was previously weaker and did not pass significance improves to $0.8(0.022, \mathrm{p}<0.05)$ and passes the significance test.

These results show that the COSMO-RS protocol should be applied with the foresight that outliers and exceptions may be possible for very hydrophobic and hydrophilic inhibitors. To improve the model, $\log \mathrm{P}$ could be calculated for all of the trimers within the inhibitor database, and with further work, an upper and lower logP threshold could be incorporated to remove any false positives related to this assumption.

Another limitation is the lack of information regarding molecular weight and viscosity of the inhibitors for incorporation into the calculations. Although conflicting values for these parameters can be found in the literature, both play an important role in precipitation inhibition (Price, et al. 2018). Two main hypotheses have been put forward to explain why these parameters should play an important role. The first, and lesser reported, states that molecular weight and viscosity affect precipitation inhibition via changes in the diffusion kinetics of both the drug and polymer in solution (Price, et al. 2018; Warren, et al. 2010). This is something that is not and cannot be considered in the COSMO-RS protocol. The second, and more widely reported, hypothesis relates to an increasing number of binding sites when molecular weight and viscosity are increased (Price, et al. 2018; Warren, et al. 2010). Given that the COSMO-RS protocol considers in its calculations the interaction between a PI trimer and a drug, this parameter is already considered and therefore, any effect of viscosity between different PIs should be minimized. When considering different viscosities of the same inhibitor, however, the COSMO-RS protocol cannot guide selection of a particular viscosity grade.

\subsection{Implications of the COSMO-RS Approach}

In silico tools are an attractive option for bridging the gap between our current understanding of precipitation inhibitors and practical selection of inhibitors to be used in supersaturating formulations 
in pharmaceutical development. In this work, the COSMO-RS model was applied as a novel in silico screening tool to successfully predict the formulation performance of a wide range of precipitation inhibitors in formulations of glibenclamide, fenofibrate and dipyridamole. Specifically, it was hypothesized that free enthalpy of mixing (drug-polymer) could be used as a parameter for ranking inhibitors, from highest potential for successful precipitation inhibition to lowest, based on the theoretical interaction between the inhibitor and the drug. For all three compounds a strong positive correlation was observed between the rank assigned based on the calculated free enthalpy of mixing and the overall dissolution performance of the formulation. Conceptually, such an approach can be applied for any formulation that requires precipitation inhibitors. Given the high-throughput and highspeed nature of the in-silico calculations, the screening protocol can be carried out for a large number of drug-PI combinations in very short timeframes. Ultimately, this study highlights how in silico tools can be used to improve efficiency of PI selection as well as the likelihood that the most optimal formulation can be realized.

\section{Conclusion and Outlook}

\section{Summary}

This body of work aimed to improve the processes underpinning the design and development of mesoporous silica with precipitation inhibitors. First, this involved two extensive literature reviews in the areas of solubility enhancement formulation technologies and precipitation inhibition. Second, a mechanistically rational experimental approach was developed to improve the formulation of precipitation inhibitors with mesoporous silica. The "co-incorporation" approach significantly improved process efficiency and formulation performance. Finally, combining insights from the aforementioned reviews with learnings from the mechanistic analysis of the "co-incorporation" approach, an in silico screening protocol was developed to calculate the enthalpy of interaction between drug and polymer, and thus identify the most optimal precipitation inhibitor for a given formulation. Ultimately, these activities have resulted in a substantially optimized workflow for 
selecting and combining precipitation inhibitors in mesoporous silica formulations, resulting in more efficient and effective results.

\section{Co-incorporation: A New Method to Combine Precipitation Inhibitors with Mesoporous Silica}

A novel co-incorporated formulation of glibenclamide and the precipitation inhibitor, HPMCAS, onto mesoporous silica was described. By co-incorporating the precipitation inhibitor, the formulation significantly outperformed the commonly applied simple physical blend, regarding improved supersaturation and dissolution in both single-medium FaSSIF and transfer dissolution assays. Furthermore, the co-incorporation approach allows the removal of a time-consuming and inefficient blending step. To provide a physical mechanistic basis for the improved performance the coincorporated formulation, a range of spectroscopic tools were utilized. It was concluded that the improved dissolution performance is a synergistic effect related to two key factors: formation of drugpolymer interactions in the solid state, and lack of release and premature precipitation under gastric conditions due to the immobilization of API-loaded silica particles within the enteric HPMCAS plates. Crucially, both of these properties are absent in a simple HPMCAS blend. Ultimately, the coincorporation of precipitation inhibitors with the API on mesoporous silica formulations has the potential to improve both the process and formulation efficiency in the development of poorly soluble drugs. Furthermore, the mechanistic analysis of the formulation performance underlines the importance of drug-polymer interactions for successful precipitation inhibition and performance of mesoporous silica formulations.

\section{In Silico Pharmaceutics: A New Method to Select Precipitation Inhibitors for Mesoporous Silica}

Building on the extensive review article and the critical parameters identified during the coincorporation study, drug-polymer interactions were identified as a critical factor in determining formulation success in supersaturating formulations. Building on this, and the general absence of any technique or screening approach that is suitable for pharmaceutical screening, a novel in silico screening protocol for the selection of precipitation inhibitors for supersaturating formulations was developed. The protocol used the COSMO-RS model to calculate excess enthalpy of interaction between API and precipitation inhibitors, which was then applied as a rank-order parameter to select potential precipitation inhibitors. Conceptually, such an approach may be applied for any enabling formulation that requires precipitation inhibitors, for example HME or SDD, but further work is required to validate this cross-formulation applicability. Despite the simplifications and assumptions in the COSMO-RS protocol, strong positive correlations were obtained between the rank-order prediction and formulation performance for the APIs studied. Furthermore, given the high- 
throughput and high-speed nature of the in-silico calculations, the screening protocol is very attractive as a score-card approach for the design of enabling formulations for poorly soluble APIs in the pharmaceutical industry. Ultimately, this study highlights how in silico tools can be used to improve efficiency of precipitation inhibitor selection as well as the likelihood that the most optimal formulation will be realized.

\section{Future Work}

Looking forward, further work will be carried out to combine the co-loading approach with the COSMO-RS screening protocol to assess if the correlations observed using the blending method also apply to the co-loading approach. Furthermore, there a potentially further applications of the COSMO-RS model in pharmaceutical screening, including, but not limited to screening of polymers for spray dried dispersion of hot melt extrusion formulations, assessment of co-crystal or coamorphous excipients and assessment of excipients for effective lipid formulation. Further down the line, it is expected that computation approaches will have a transformative impact on the efficiency and outcome of pharmaceutical development, with tools such as artificial intelligence and machine learning already on the horizon to assist in the design and development of oral solid dosage form formulations. 


\section{Deutsche Zusammenfassung}

\section{Einführung}

Damit ein oral verabreichtes Arzneimittel seine biologische Wirkung entfalten kann, muss es zunächst durch den Magen-Darm-Trakt (GI-Trakt) in den systemischen Kreislauf gelangen. Um dies zu erreichen, muss ein Medikament in den Flüssigkeiten des GI-Trakts ausreichend löslich sein. In den letzten Jahren gab es in den Entwicklungspipelines vermehrt Arzneimittel mit geringer Löslichkeit, was zu einer verringerten Bioverfügbarkeit und einem erhöhten Risiko des Scheiterns während des Entwicklungsprozesses führte. Als Reaktion darauf haben Chemiker und Formulierer Strategien entwickelt, die die Löslichkeit und die daraus resultierende Bioverfügbarkeit dieser schwer löslichen Kandidaten verbessern können. Zu diesen Ansätzen gehören chemische Modifikationen in der Synthese, wie Salzbildung und Prodrugs oder Formulierungsmodifizierungsansätze wie mizellare Systeme, Co-Solventie, Partikelgrößenreduzierung, Komplexbildung und Technologien mit amorphen Phasen (Timpe, et al. 2007). Amorphe Formulierungen sind besonders attraktiv aufgrund der deutlichen Verbesserung der Löslichkeit, die die amorphe Form bieten kann (Timpe, et al. 2007). Amorphe Feststoffe haben wesentlich höhere Löslichkeiten im Vergleich zu Formulierungen, die die entsprechenden kristallinen Phasen verwenden. Allerdings sind amorphe Feststoffe aufgrund der hohen Energie, die mit dieser Festkörperform verbunden ist, in der Regel instabil oder metastabil. Daher müssen spezielle Formulierungstechnologien eingesetzt werden, um die amorphe Form des schwer löslichen Arzneimittels zu stabilisieren. Die häufigste dieser Methoden besteht darin, den Wirkstoff in der amorphen Form in einer polymeren Matrix zu immobilisieren (Chokshi, et al. 2007). Amorphe Formulierungen erzeugen in Lösung Konzentrationen, die höher sind als die thermodynamische Löslichkeit. Dies wird als Übersättigung bezeichnet. Übersättigung ist ein energetisch ungünstiger Zustand. Deshalb müssen solche Formulierungen auch sicherstellen, dass eine Ausfällung aus diesem Zustand verhindert wird. Typischerweise können polymere Hilfsstoffe als Fällungsinhibitoren eingesetzt werden. 


\section{Die Bedeutung von Arzneistoff-Polymer-Wechselwirkungen bei der Fällungsinhibierung}

Polymere Präzipitationsinhibitoren haben in der Literatur eine weite Verbreitung gefunden, wobei die am häufigsten verwendeten Polymere Cellulosederivate, Methacrylate und Polyvinylpyrrolidone beinhalten (Warren, et al. 2010). Die Wirkung von Polymeren zur Verhinderung von Ausfällungen beruht auf der Interferenz mit der Keimbildung und dem Kristallwachstum durch Wechselwirkung sowohl mit dem in übersättigter Lösung vorliegenden Molekülen des Arzneimittels als auch mit der wässrigen Umgebung (Xu, et al. 2013). Diese Wechselwirkungen verhindern die Adsorption von Wirkstoffmolekülen an der wachsenden Kristallfläche, so dass die Präzipitation gestoppt wird. Für die kinetische Hemmung ist die Wechselwirkung zwischen dem Arzneimittel und dem Polymer von entscheidender Bedeutung. Polymere können insbesondere über Wasserstoffbrückenbindungen, polare oder Dispersionskräfte in unterschiedlichem Ausmaß mit dem Arzneimittel wechselwirken (Price, et al. 2018; Price, et al. 2019; Warren, et al. 2010; Brouwers et al. 2009; Gao, et al. 2012). In der Literatur wurde über viele Techniken berichtet, bei denen Technologien wie die NMR-Spektroskopie zum Einsatz kommen. Der derzeitige Stand der Technik zur Untersuchung der Wechselwirkungen zwischen Wirkstoff und als Präzipitationsinhibitor eingesetztem Polymer ist jedoch für das Screening nicht geeignet, da hohe API-Mengen und ein hoher zeitlicher Aufwand benötigt werden, die für die pharmazeutische Entwicklung in einem frühen Stadium nicht verfügbar sind.

\section{Mesoporöse Kieselsäure: Eine neue Formulierungstechnologie}

Eine neue Technologie zur Formulierung amorpher Feststoffe ist die Verwendung mesoporöser Materialien als Träger für den Wirkstoff. Mesoporöses Siliziumdioxid ist ein Siliziumdioxid-Hilfsstoff, der ein hochporöses Netzwerk besitzt. Mit Beladung des Siliziumdioxids mit einer konzentrierten Lösung des Wirkstoffs wird dieser molekular an der Oberfläche des Siliciumdioxids adsorbiert. Aufgrund der Größe der Poren, die einen mittleren Durchmesser von etwa $6 \mathrm{~nm}$ haben, ist das molekular adsorbierte Molekül des Wirkstoffs lokal und sterisch fixiert, wodurch eine Rekristallisation verhindert wird (Ditzinger, et al. 2018). Bei der Verabreichung mesoporöser 
Siliziumdioxidformulierungen kommt es zu einer Verdrängung einzelner Wirkstoffmoleküle von der Oberfläche des Siliziumdioxids in Lösung, wodurch eine Übersättigung gegenüber dem kristallinen Wirkstoff entsteht, die mit Präzipitationsinhibitoren stabilisiert werden muss (Guzman, et al. 2007). Da es sich bei mesoporösem Siliziumdioxid um eine neue Formulierungstechnologie handelt, ist bisher wenig darüber bekannt wie Fällungsinhibitoren am effektivsten ausgewählt und mit mesoporösen Siliziumdioxidformulierungen kombiniert werden können.

\section{Ziele der Dissertation}

Das Ziel der Arbeit war die Entwicklung und Optimierung eines neuen Arbeitsablaufs zur Auswahl von Präzipitationsinhibitoren in mesoporösen Siliziumdioxidformulierungen. Hierzu wurde zunächst der aktuelle Stand der Technik von mesoporösen Siliziumdioxidformulierungen und Präzipitationsinhibitoren betrachtet. Danach wurde die Methode zur Kombination von Präzipitationsinhibitoren mit mesoporösem Siliziumdioxid analysiert und verbessert, indem ein CoInkorporationsansatz entwickelt wurde. Drittens wurde eine physikalisch-mechanistische Rationale des neu entwickelten Ansatzes entwickelt. Schließlich wurden die Erkenntnisse hieraus in Kombination mit dem oben erwähnten Stand der Technik, zur Entwicklung eines neuen computergestützten Arbeitsablaufs zur Auswahl der optimalen Präzipitationsinhibitoren für ein gegebenen Wirkstoff verwendet. Dieser basierte auf der Kombination von Quantenmechanik und Thermodynamik zur Berechnung der Mischungsenthalpie von Arzneistoff und Polymeren als Prädiktor für eine wirksame Inhibition der Präzipitation.

\section{Eine neue Methode zur Kombination von Präzipitationsinhibitoren mit mesoporösem}

\section{Siliziumdioxid}

Es gibt keine systematische Studie darüber, wie Präzipitationsinhibitoren am besten in mesoporösen Siliziumdioxidformulierungen verwendet werden können. Die derzeitige Praxis beinhaltet die Verwendung von Präzipitationsinhibitoren in einer physikalischen Mischung mit dem mit dem Wirkstoff beladenen Siliciumdioxid. Der Mischvorgang erfolgt entweder durch Pistill und Mörser oder 
durch einfaches Rühren. Da es keine fest definierte Methode hierfür gibt, besteht Unsicherheit darüber, wie zuverlässig der Präzipitationsinhibitor mit dem mit dem Wirkstoff beladenen Siliziumdioxid kombiniert wird, so dass eine konstante Qualität der Chargen erhalten wird. Zusätzlich zu den praktischen Einschränkungen des Ansatzes stellt die Einarbeitung des Präzipitationsinhibitors nach der Beladung des Siliziumdioxids einen unnötigen und nicht unbedeutenden Schritt im Formulierungsprozess dar. Das Auflösen des Präzipitationsinhibitors zusätzlich zum Wirkstoff in die zur Beladung des Siliziumdioxid verwendeten Lösung kann das Auflösungsverhalten und die Verhinderung der Präzipitation verbessern. In dieser Arbeit wurde eine neuartige Formulierung von Glibenclamid und dem Präzipitationsinhibitor HPMCAS auf mesoporösem Siliciumdioxid beschrieben. Durch die gemeinsame Einarbeitung des Präzipitationsinhibitors übertraf die Formulierung die physikalische Mischung deutlich. Darüber hinaus ermöglicht der Co-Inkorporationsansatz das Weglassen eines zeitaufwändigen und ineffizienten Mischungsschritts. Um ein physikalisch-mechanistisches Verständnis für die verbesserte Leistung der Formulierung zu schaffen, wurde eine Reihe von spektroskopischen Werkzeugen eingesetzt. Daraus resultiert, dass das verbesserte Auflösungsverhalten und die bessere Inhibition der Präzipitation ein synergistischer Effekt ist, der mit zwei Faktoren zusammenhängt: Wirkstoff-Polymer-Wechselwirkungen in der festen Phase und Verhinderung der Freisetzung unter den Bedingungen des Magens aufgrund der Immobilisierung von API-beladenen Siliziumdioxidpartikeln im enterischen HPMCAS. Entscheidend ist, dass diese beiden Eigenschaften bei einer einfachen HPMCAS-Mischung fehlen. Letztlich hat die Co-Inkorporation von Präzipitationsinhibitoren mit dem Wirkstoff auf mesoporösen Siliziumdioxidformulierungen das Potenzial, sowohl die Prozess- als auch die Formulierungseffizienz bei der Entwicklung schwerlöslicher Arzneimittel zu verbessern. 


\section{Eine neue Methode zur Auswahl von Präzipitationsinhibitoren für mesoporöse}

\section{Siliziumdioxidformulierungen mittels in-silico Verfahren}

Das mechanistische Verständnis für eine verbesserte Formulierungsleistung mit dem CoInkorporationsansatz bestätigt die Bedeutung von Wirkstoff-Polymer-Interaktionen für eine erfolgreiche Präzipitationsinhibition. Die Bedeutung dieser wurde schon in der Literaturrecherche identifiziert. Daher wäre ein Ansatz, der das Verständnis der Wirkstoff-Polymer-Wechselwirkungen mit einem schnellen und effizienten Screening-Verfahren verbinden kann, sehr nützlich. Zu diesem Zweck wurde das "Conductor like Screening Model for Real Solvent" (COSMO-RS), das von Klamt (Klamt, et al. 1993; 1995; 2003) entwickelt wurde, identifiziert. COSMO-RS ist ein quantenmechanisches Modell, mit dessen Hilfe thermodynamische Eigenschaften abgeleitet werden können (Klamt, et al. 1993; 1995; 2003). Aufbauend auf diesem theoretischen Ansatz schlugen wir vor, die Mischungsenthalpien von Wirkstoff und Polymer mit Hilfe der COSMO-RS-Theorie zu berechnen. Nach der Entwicklung des in-silico Ansatzes zur Berechnung dieser Eigenschaft wurde die WirkstoffPolymer-Mischungsenthalpie verwendet, um potenzielle Präzipitationsinhibitoren auf der Grundlage der Stärke der molekularen Wechselwirkung mit dem Wirkstoff zu klassifizieren. Es wurde die Hypothese aufgestellt, dass dieses neuartige in-silico Protokoll für das Screening potenzieller Fällungsinhibitoren verwendet werden kann. Dies ermöglicht eine gezieltere Auswahl und reduziert somit den experimentellen Aufwand für das Screening vieler potenzieller Präzipitationsinhibitoren erheblich. Dieser neue Ansatz wurde beim Screening von Präzipitationsinhibitoren für drei Modellverbindungen angewandt: Glibenclamid, Dipyridamol und Fenofibrat, die mit mesoporösem Siliziumdioxid formuliert wurden. Für alle drei Wirkstoffe wurde eine starke positive Korrelation zwischen dem Rang, der aufgrund der berechneten freien Mischungsenthalpie vergeben wurde, und der gesamten Formulierungsleistung beobachtet. Letztendlich zeigt diese Studie auf, wie in-silico Werkzeuge eingesetzt werden können, um die Effizienz der Auswahl des Präzipitationsinhibitors zu verbessern. 


\section{Schlussfolgerung und Ausblick}

Diese Arbeit zielte darauf ab, die Prozesse zu verbessern, die dem Design und der Entwicklung von mesoporösem Siliziumdioxidformulierungen mit Präzipitationsinhibitoren zugrunde liegen. Dazu gehören zunächst zwei umfangreiche Literaturrecherchen auf dem Gebiet der löslichkeitsverbessernden Formulierungstechnologien und der Präzipitationsinhibition. Zweitens wurde ein mechanistisch-rationaler experimenteller Ansatz zur Verbesserung der Formulierung von Präzipitationsinhibitoren mit mesoporösem Siliziumdioxid entwickelt, wobei der "Co-Inkorporations Ansatz" die Formulierungsleistung deutlich verbesserte. Schließlich wurde ein in-silico ScreeningProtokoll zur Berechnung der Wechselwirkung zwischen Wirkstoff und Polymer entwickelt, um den optimalen Präzipitationsinhibitor für eine Formulierung zu identifizieren. Dazu wurden die Erkenntnisse aus der Litertaturrecherche und die Erkenntnisse aus der mechanistischen Analyse des "Co-Inkorporations Ansatzes" kombiniert. Diese Aktivitäten haben zu einem optimierten Arbeitsablauf für die Auswahl und Kombination von Präzipitationsinhibitoren in mesoporösen Siliziumdioxidformulierungen geführt. In Zukunft werden weitere Arbeiten durchgeführt, um den „CoLoading-Ansatz" mit dem COSMO-RS-Screening-Protokoll zu kombinieren. Ziel ist zu beurteilen, ob die mit der Mischmethode beobachteten Korrelationen auch auf den "Co-Loading-Ansatz" zutreffen. Darüber hinaus gibt es mögliche weitere Anwendungen des COSMO-RS-Modells beim Screening, einschließlich, aber nicht beschränkt auf: Screening von Polymeren für sprühgetrocknete Dispersion von hot-melt Extrudaten, Bewertung von co-kristallinen oder co-amorphen Formulierungen und Bewertung von Lipidformulierung. Für die Zukunft wird erwartet, dass in-silico Ansätze einen Einfluss auf die Effizienz und Leistung in der pharmazeutischen Entwicklung haben werden, wobei Werkzeuge wie künstliche Intelligenz und maschinelles Lernen bereits in Sichtweite sind, um Design und Entwicklung von oralen festen Dosierungsformulierungen zu unterstützen. 


\section{References}

Abd-Elrahman, AA. et al. Ketoprofen mesoporous silica nanoparticles SBA-15 hard gelatin capsules: preparation and in vitro/in vivo characterization. Drug Delivery. 2016; 23(9): 3387-3398

Alhalaweh, A. et al. Physical stability of drugs after storage above and below the glass transition temperature: Relationship to glass-forming ability. International Journal of Pharmacy. 2015; 495(1): 312-317

Alonzo, DE. et al. Characterizing the impact of hydroxypropylmethyl cellulose on the growth and nucleation kinetics of felodipine from supersaturated solutions. Crystal Growth \& Design. 2012; 12(3): 1538-1547

Ambrogi, V. et al. Use of SBA-15 for furosemide oral delivery enhancement. European Journal of Pharmaceutical Science. 2012; 46(1-2): 43-48

Amidon, GL. et al. A theoretical basis for a biopharmaceutic drug classification: the correlation of in vitro drug product dissolution and in vivo bioavailability. Pharmaceutical Research. 1995; 12(3)

Andersson, J. et al. Influences of material characteristics on ibuprofen drug loading and release profiles from ordered micro- and mesoporous silica matrices. Chemistry of Materials. 2004; 16: 41604167

Atkin, R. et al. Mechanism of cationic surfactant adsorption at the solid-aqueous interface. Advanced Colloid and Interface Sciences. 2003; 103(3): 219-304

Augustijns, P. and Brewster, ME. Supersaturating drug delivery systems: fast is not necessarily good enough. Journal of Pharmaceutical Sciences. 2012; 101(1): 7-9

Avramov, I. et al. Glass-forming ability versus stability of silicate glasses. II. Theoretical demonstration. Journal of Non-Crystalline Solids. 2003; 320: 9-20

Baghel, S. et al. Theoretical and experimental investigation of drug-polymer interaction and miscibility and its impact on drug supersaturation in aqueous medium. European Journal of Pharmaceutics and Biopharmaceutics. 2016; 107, 16-31.

Baghel, S. et al. Theoretical and experimental investigation of drug-polymer interaction and miscibility and its impact on drug supersaturation in aqueous medium. European Journal of Pharmaceutics and Biopharmaceutics. 2016; 107, 16-31.

Baird, JA. et al. Classification System to Assess the Crystallization Tendency of Organic Molecules from Undercooled Melts. Journal of Pharmaceutical Sciences. 2010; 99: 3787-3806.

Barbe, C. et al. Silica particles: A Novel Drug Delivery System. Advanced Materials. 2004; 16: 1959-1966.

Bartsch, S. et al. Physicochemical properties of the binary system glibenclamide and polyethylene glycol 4000. Journal of Thermal Analysis and Calorimetry. 2005; 77(2): 1321-1337

Bathfield, M. et al. Thermosensitive and Drug-Loaded Ordered Mesoporous Silica: A Direct and Effective Synthesis Using PEO-b-PNIPAM Block Copolymers. Chemistry of Materials. 2016; 28(10): 3374-3384 
Bhatnagar, A and Sillanpaa, M. Utilization of Agro-industrial and Municipal Waste Materials as Potential Adsorbents for Water Treatment- a Review. Chemical Engineering Journal. 2010; 157: 277296.

Biswas N. Modified mesoporous silica nanoparticles for enhancing oral bioavailability and antihypertensive activity of poorly water soluble valsartan. European Journal of Pharmaceutical Science. 2017; 99: 152-160

Bouchoucha, M. et al. Size-Controlled Functionalized Mesoporous Silica Nanoparticles for Tunable Drug Release and Enhanced Anti-Tumoral Activity. Chemistry of Materials. 2016; 28(12): 4243-4258

Brás, AR. et al. 2014. Influence of Nanoscale Confinement on the Molecular Mobility of Ibuprofen. Journal of Physical Chemistry C. 2014; 118: 13857-13868

Brewster, ME. et al. Supersaturating Drug Delivery Systems: Effect of Hydrophilic Cyclodextrins and other Excipients on the Formation and Stabilisation of Supersaturated Drug Solutions. Archiv der Pharmazie. 2008, 63(3), 217-220

Brewster, ME. et al. The Utility of Cyclodextrins for Enhancing Oral Bioavailability. Journal of Controlled Release. 2007, 123, 78-99.

Brouwers, J. et al. Supersaturating drug delivery systems: The answer to solubility-limited oral bioavailability? Journal of pharmaceutical science. 2009; 98(8): 2549-2572.

Brown, DG. et al. A review of traditional and novel oral anticoagulant and antiplatelet therapy for dermatologists and dermatologic surgeons. Journal of the American Academy of Dermatology. 2015; 72 (3): 524-534.

Bukara, K. et al. Ordered mesoporous silica to enhance the bioavailability of poorly watersoluble drugs: Proof of concept in man. European Journal of Pharmaceutics and Biopharmaceutics. 2016; 108: 220-225.

Butler, JM. And Dressman, JB. The developability classification system: application of biopharmaceutics concepts to formulation development. Journal of Pharmaceutical Sciences. 2010; 99(12).

Chauhan, H. et al. Correlating the behaviour of polymers in solution as $\mathrm{PI}$ to its amorphous stabilization ability in solid dispersions. Journal of Pharmaceutical Sciences. 2013; 102(6): 1924-1935

Chauhan, H. et al. Correlation of inhibitory effects of polymers on indomethacin precipitation in solution and amorphous solid crystallization based on molecular interaction. Pharmaceutical Research. 2014; 31(2): 500-515.

Chavan, B. et al. Evaluation of the inhibitory potential of HPMC, PVP and PC polymers on nucleation and crystal growth. RSC Advances. 2016; 6: 77569-77567

Chen, Y. et al. Drug-polymer-water interaction and its implication for the dissolution performance of amorphous solid dispersions. Molecular Pharmaceutics. 2014; 12: 576-589.

Chen, Y. et al. Drug-polymer-water interaction and its implication for the dissolution performance of amorphous solid dispersions. Molecular Pharmaceutics. 2014; 12: 576-589. 
Chokshi, RJ. et al. Improving the Dissolution Rate of Poorly Soluble Drugs by Solid Dispersion and Solid Solution: Pros and Cons. Drug Delivery. 2007; 14(1): 33-45

Cordeiro, T. et al. Stabilizing Unstable Amorphous Menthol through Inclusion in Mesoporous Silica Hosts. Molecular Pharmaceutics. 2017; 14: 3164-3177.

Creasey, AA. et al. Inhibiting the Precipitation of Poorly Water-Soluble Drugs from Labrasol Formulations. Pharmaceutical Technology. 2011; 35(6)

Ditzinger, F. and Price, DJ. et al. Opportunities for Successful Stabilization of Poor GlassForming Drugs: A Stability-Based Comparison of Mesoporous Silica Versus Hot Melt Extrusion Technologies. Pharmaceutics. 2019; 11(577).

Ditzinger, F. et al. Lipophilicity and hydrophobicity considerations in bio-enabling oral formulations approaches-a PEARRL review. Journal of Pharmacy and Pharmacology. Epub ahead of print

Dokoumetzidis, A. and Macheras, P. A Century of Dissolution Research: From Noyes and Whitney to the Biopharmaceutics Classification System. International Journal of Pharmaceutics. 2006, 321(1-2): 1-11.

Dressman, B. et al. Mesoporous silica-based dosage forms improve release characteristics of poorly soluble drugs: case example fenofibrate. Journal of Pharmacy and Pharmacology. 2015; 68(5): 634-645

Elder, JP. A new accelerated oxidative stability test for glass-forming organic compounds. Thermochimica Acta. 1990; 166: 199-206

Flory, PJ. Principles of Polymer Chemistry. Cornell University Press. 1953

Flory, PJ. Thermodynamics of high-polymer solutions. Journal of Chemical Physics. 1942; 10: 51-61.

Furman, BL. et al. Studies on the hypoglycaemic action of gliclazide, a sulphonyl-urea drug. Journal of Pharmacy and Pharmacology. 1977; 55.

Gaffney, JS. et al. Fourier Transform Infrared (FTIR) Spectroscopy. In: Characterization of Materials. $2^{\text {nd }}$ edn. Hoboken, NJ, USA, Wiley, 2012

Gao, P. and Shi, Y. Characterization of supersaturating formulations for improved absorption of poorly soluble drugs. The AAPS journal. 2012; 5: 1-11.

Gardner, CR. et al. Drugs as Materials: Valuing Physical Form in Drug Discovery. Nature Reviews: Drug Discovery. 2004; 3: 926-943.

Garido, L. and Beckmann, N. New Applications of NMR in Drug Discovery and Development. $1^{\text {st }}$ edn. London, UK, Royal Society of Chemistry, 2014

Gharagheizi, F. et al. Group contribution-based method for determination of solubility parameter of nonelectrolyte organic compounds. Journal of Industrial Engineering and Chemistry Research. 2011; 50: 10344-10349. 
Gharagheizi, F. QSPR studies of solubility parameter by means of genetic algorithm-based multivariate linear regression and generalized regression neutral network. QSAR and Combinatorial Sciences. 2008; 27(2): 165-170.

Gignone, A. et al. Incorporation of clotrimazole in Ordered Mesoporous Silica by supercritical CO2. Microporous and Mesoporous Materials. 2014; 200: 291-296 1990

Grant, D. and Higuchi, T. Solubility Behaviour of Organic Compounds. John Wiley and Sons.

Gunther, H. NMR Spectroscopy: Basic Principles, Concepts and Applications in Chemistry. $3^{\text {rd }}$ edn. Hoboken, NJ, USA, Wiley, 2013

Gupta, J. et al. Prediction of solubility parameters and miscibility of pharmaceutical compounds by molecular dynamics simulations. Journal of Physical Chemistry B. 2011; 115, 2014-2023

Guzmán, HR. et al. A "spring and parachute" approach to designing solid celecoxib formulations having enhanced oral absorption. The AAPS Journal. 2004; 6(1): T2189

Hacene. YC. et al. Drug loaded and ethylcellulose coated mesoporous silica for controlled drug release prepared using a pilot scale fluid bed system. International Journal of Pharmacy. 2016; 506: $132-147$

Hanada, M. et al. Enhanced Dissolution of a Porous Carrier-Containing Ternary Amorphous Solid Dispersion System Prepared by a Hot Melt Method. J Pharm Sci. 107(1), (2018), 362-371

Hartono, SB. et al. Amine functionalized cubic mesoporous silica nanoparticles as an oral delivery system for curcumin bioavailability enhancement. Nanotechnology. 2016; 27(50)

He, H. et al. In vitro and in vivo evaluation of fenofibrate solid dispersion prepared by hotmelt extrusion. Drug Dev Ind Pharm. 2010; 36(6): 681-687.

Heikkila, T. et al. Evaluation of mesoporous TCPSi, MCM-41, SBA-15, and TUD-1 materials as API carriers for oral drug delivery. Drug Delivery. 2007; 14(6): 337-347

Higashi, K. et al. Insights into Atomic-level Interaction between Mefenamic Acid and Eudragit ${ }^{\circledR}$ EPO in a Supersaturated Solution by High Resolution Magic-Angle Spinning NMR Spectroscopy. Molecular Pharmaceutics. 2014; 11(1): 351-357.

Higuchi, T. Rate of Release of Medicaments from Ointment Bases Containing Drugs in Suspension. Journal of Pharmaceutical Sciences. 1961; 50(10): 874-875

Hildebrand, J. and Scott, R. 1950. Solubility of Nonelectrolytes. Reinhold Pub Co., New York

Hillerstrom, A. et al. Solvent strategies for loading and release in mesoporous silica. Colloid and Interface Science Communications. 2014; 3: 5-8

Horcajada, P. et al. Influence of pore size of MCM-41 matrices on drug delivery rate. Microporous and Mesoporous Materials. 2004; 68: 105-109

Hu, L. et al. Multilayer encapsulated mesoporous silica nanospheres as an oral sustained drug delivery system for the poorly water-soluble drug felodipine. Materials Science and Engineering $C$. 2015; 47: 313-324 
$\mathrm{Hu}, \mathrm{Y}$. et al. 3D cubic mesoporous silica microsphere as a carrier for poorly soluble drug carvedilol. Microporous and Mesoporous Materials. 2012; 147(1): 94-101.

Johnson, CS. Diffusion ordered nuclear magnetic resonance. Progress in Nuclear Magnetic Resonance Spectroscopy. 1999, 34, 203-256

Kambayashi, A. et al. Predicting the Changes in Oral Absorption of Weak Base Drugs Under Elevated Gastric pH Using an In Vitro-In Silico-In Vivo Approach: Case Examples- Dipyridamole, Prasugrel, and Nelfinavir. Journal of Pharmaceutical Sciences. 2019; 108: 584-591

Khadka, P. et al. Pharmaceutical particle technologies: An approach to improve drug solubility, dissolution and bioavailability. Asian Journal of Pharmaceutical Sciences. 2014; 9(6): 304316.

Khanfar, M. and Al-Nimry, S. Stabilization and Amorphization of Lovastatin Using Different Types of Silica. AAPS PharmSciTech. 2017; 6: 3321-3343

Khanfar, M. and Al-Nimry, S. Stabilization and Amophization of Lovastatin Using Different Types of Silica. AAPS PharmSciTech. 2017; 18: 2358-2367

Kiekens, F. et al. Use of ordered mesoporous silica to enhance the oral bioavailability of ezetimibe in dogs. Journal of Pharmaceutical Science. 2012; 101: 1136-1144

Kinnari, P. et al. Comparison of mesoporous silicon and non-ordered mesoporous silica materials as drug carriers for itraconazole. International Journal of Pharmaceutics. 2011; 414: 148-156

Kinnari, P. et al. Comparison of mesoporous silicon and non-ordered mesoporous silica materials as drug carriers for itraconazole. International Journal of Pharmaceutics. 2011; 414: 148-156

Klamt, A. and Schuurmann, G. COSMO: a new approach to dielectric screening in solvents with explicit expressions for the screening energy and its gradient. Journal of the Chemical Society. $1993 ; 2(5)$ : 799-805

Klamt, A. Conductor-like Screening Model for Real Solvents: A New Approach to the Quantitative Calculation of Solvation Phenomena. Journal of Physical Chemistry. 1995; 99(7): 22242235.

Klamt, A. et al. A Comprehensive Comparison of the IEFPCM and SS(V)PE Continuum Solvation Methods with the COSMO Approach". Journal of Chemical Theory and Computation. 2015; 11(9): 4220-4225.

Klamt, A. Refinement and Parametrization of COSMO-RS. Journal of Physical Chemistry. 1998; 102(26): 5074-5085.

Knapik, J. et al. Physical stability of the amorphous anticholesterol agent (ezetimibe): the role of molecular mobility. Molecular Pharmaceutics. 2014; 11(11): 4280-4290

Koehl, NK. Et al. New Insights into Using Lipid Based Suspensions for 'Brick Dust' Molecules: Case Study of Nilotinib. Pharmaceutical Research. 2019; 36(56) 
Kresse, CT. et al. Ordered Mesoporous Molecular Sieves Synthesized by a Liquid-crystal Template Mechanism. Nature. 1992; 359: 710-712.

Krishnaiah, YSR. Pharmaceutical Technologies for Enhancing Oral Bioavailability of Poorly Soluble Drugs. Journal of Bioequivalence and Bioavailability. 2010; 2: 28-36.

Kubota, $\mathbf{N}$ and Mullin, JW. A kinetic model for crystal growth from aqueous solution in the presence of impurity. Journal of Crystal Growth. 1995; 152(3): 203-208

Kubota, N. et al. Supersaturation dependence of crystal growth in solutions in the presence of impurity. Journal of Crystal Growth. 1997; 182 (1-2): 86-94.

Kubota, N. et al. The combined influence of supersaturation and impurity concentration on crystal growth. Journal of Crystal Growth. 2000; 212 (3-4): 480-488.

Kumar, D. et al. Impact of surface area of silica particles on dissolution rate and oral bioavailability of poorly water soluble drugs: a case study with aceclofenac. International Journal of Pharmacy. 2014; 461(1-2): 459-468

Kwan, EE. and SG. Huang. Structural Elucidation with NMR Spectroscopy: Practical Strategies for Organic Chemists. European Journal of Organic Chemistry. 2008; 4: 671-2688

Laine, AL. et al. Enhanced oral delivery of celecoxib via the development of a supersaturable amorphous formulation utilising mesoporous silica and co-loaded HPMCAS. International Journal of Pharmaceutical Sciences. 2016; 8(1):118-25

Langmuir, I. The Adsorption of Gases on Plane Surfaces of Glass, Mica and Platinum. Journal of the American Chemical Society. 1918; 40(9): 1361-1403

Letchmanan, K. et al. Dissolution and physicochemical stability enhancement of artemisinin and mefloquine co-formulation via nano-confinement with mesoporous SBA-15. Colloids and Surfaces B Biointerfaces. 2017; 1(155): 560-568.

Leuner, $\mathbf{C}$ and Dressman, J. Improving Drug Solubility for Oral Delivery Using Solid Dispersions. European Journal of Pharmaceutical Science. 2000; 351: 209-218. 698

Levitt, M. and Warshel, A. Computer simulation of protein folding. Nature. 1975; 253: 694-

Li, S. et al. Enhanced bioavailability of a poorly water-soluble weakly basic compound using a combination approach of solulilization agents and PIs: a case study. Molecular Pharmaceutics. 2012; 9(5): 1100-1108

Li, Y. et al. In situ dehydration of carbamazepine dihydrate: a novel technique to prepare amorphous anhydrous carbamazepine. Pharmaceutical Development Technology. 2000; 5(2): 257266

Li, Z. et al. Enhanced Performance of Blended Polymer Excipients in Delivering a Hydrophobic Drug through the Synergistic Action of Micelles and HPMCAS. Langmuir. 2017; 33(11): 2837-2848 
Limnell, T. et al. Drug Delivery Formulations of Ordered and Nonordered Mesoporous Silica: Comparisons of Three Drug Loading Methods. Journal of Pharmaceutical Sciences. 2011; 100(8): 32943306.

Lipinski, CA. Drug-like Properties and the Causes of Poor Solubility and Permeability. Journal of Pharmacological and Toxicological Methods. 2000; 44: 235-249.

Loschen, C. and Klamt, A. COSMOquick: A Novel Interface for Fast $\sigma$-Profile Composition and Its Application to COSMO-RS Solvent Screening Using Multiple Reference Solvents. Industrial Engineering and Chemical Resarch. 2012; 51(43): 14303-14308.

Vialpando, JA. et al. Use of ordered mesoporous silica for oral delivery of poorly soluble drugs. Therapeutic Delivery. 2011; 2(8): 1079-1091.

Madsen, CM. et al. Supersaturation of zafirlukast in fasted and fed state intestinal media with and without precipitation inhibitor. European Journal of Pharmaceutical Sciences. 2016; 91: 31-39

Majors, RE. High Performance Liquid Chromatography on Small Particle Silica Gel. Analytical Chemistry. 1972; 44: 1722-1726.

Maleki, A. et al. Dissolution enhancement of a model poorly water-soluble drug, atorvastatin, with ordered mesoporous silica: comparison of MSF with SBA-15 as drug carriers. Expert Opinion Drug Delivery. 2016; 13(2): 171-181

Mandal, T. et al. A framework for multi-scale simulation of crystal growth in the presence of polymers. Soft Matter. 2017; 13: 1904-1914

Mandal, T. et al. Coarse-grained modeling of crystal growth and polymorphism of a model pharmaceutical molecule. Soft Matter. 2016; 12(39): 8246-8255

Marsac, PJ. et al. Estimation of drug-Polymer miscibility and solubility in amorphous solid dispersions using experimentally determined interaction parameters. Pharmaceutical Research. 2009; 26: 139-151.

Marzarati, M. et al. Feasibility of $1 \mathrm{H}$-high resolution-magic angle spinning NMR spectroscopy in the analysis of viscous cosmetic and pharmaceutical formulations. Analytical Chemistry. 2013; 85(8): 3822-3827

McCarthy, CA. Mesoporous silica formulations strategies for drug dissolution enhancement. Expert Opionion Drug Delivery. 2016; 13(1): 93-108

Meer, T. et al. Solubility modulation of bicalutamide using porous silica. Journal of Pharmaceutical Investigation. 2013; 43

Mehta, M. et al. Effect of Water on Molecular Mobility and Physical Stability of Amorphous Pharmaceuticals. Molecular Pharmaceutics. 2016; 13, 1339-1346.

Mellaerts, R. et al. Aging Behaviour of Pharmaceutical Formulations of Itraconazole on SBA-15 Ordered Mesoporous Silica Carrier Material. Microporous and Mesoporous Materials. 2010; 180(1-3): 154-161.

Misic, Z. et al. Understanding the interactions of oleic acid with basic drugs in solid lipids on different biopharmaceutical levels. Journal of Excipients and Food Chemistry. 2014; 5(2): 113-134. 
Mohd AA. et al. Commercially bioavailable proprietary technologies and their marketed products. Drug Discovery Today. 2013; 18.

Müller, RH. et al. CapsMorph: >4 Years long-term stability of industrially feasible amorphous drug formulations. International Controlled Release Symposium 40. 2013; Honolulu/Hawai.

Mullin, JW. Crystallisation. Butterworth-Heinemann, 2001.

Myerson, EAS. Molecular Modeling Applications in Crystallisation. Cambridge University Press. 1999

Nie, H. et al. Investigating the interaftion pattern and structural elements of a drug.polymer complex at the molecular level. Molecular Pharmaceutics. 2015; 12(7): 2459-2468

$\mathrm{Nie}, \mathbf{H}$. et al. Solid-State Spectroscopic Investigation of Molecular Interactions between Clofazimine and Hypromellose Phthalate in Amorphous Solid Dispersions. Molecular Pharmaceutics. 2016; 13(11): 3964-3975

Niederquell, A. et al. New prediction methods for solubility parameters based on molecular sigma profiles using pharmaceutical materials. International Journal of Pharmaceutics. 2018; 546(12): 137-144.

Nielsen, LH. et al. Stabilisation of amorphous furosemide increases the oral drug bioavailability in rats. International Journal of Pharmacy. 2015; 490(1-2): 334-340

Noyes, AA. And Whitney, WR. 1897. The rate of solution of solid substances in their own solutions. Journal of the American Chemical Society. 1897; 19: 930-934.

Nyvlt, J. The Ostwald Rule of Stages. Crystal Research and Technology. 1995; 30(4): 443-449

O'Shea, JP et al. Mesoporous silica-based dosage forms improve bioavailability of poorly soluble drugs in pigs: case example fenofibrate. Journal of Pharmacy and Pharmacology. 2017 (epub ahead of print)

Palmelund, H. et al. Studying the propensity of compounds to supersaturate: A practical and broadly applicable approach. Journal of Pharmaceutical Sciences. 2016; 105: 3021-3029

Patel, DD and Anderson BD. Adsorption of Polyvinylpyrrolidone and its Impact on Maintenance of Aqueous Supersaturation of Indomethacin via Crystal Growth Inhibition. Journal of Pharmaceutical Sciences. 2015; 104(9): 2923-2933

Patel, DD. and Anderson, BD. Effect of precipitation inhibitors on indomethacin supersaturation maintenance: mechanisms and modeling. Molecular Pharmaceutics. 2014; 11(5): 1489-1499.

Patel, DD. et al. Maintenance of Supersaturation I: Indomethacin Crystal Growth Kinetic Modeling Using an Online Second-derivative Ultraviolet Spectroscopic Method. Journal of Pharmaceutical Sciences. 2011; 100(7): 2623-2641

Paudel, A. et al. Raman spectroscopy in pharmaceutical product design. Advanced Drug Delivery Reviews. 2015; 89, 3-20 
Paus, R. et al. Predicting the solubility advantage of amorphous pharmaceuticals: A novel thermodynamic approach. Molecular Pharmaceutics. 2015; 12: 2823-2833.

Pawar, Y B. et al. Phase behavior and oral bioavailability of amorphous Curcumin. European Journal of Pharmaceutics and Biopharmaceutics. 2012; 47(1): 56-64

Peng, T. et al. Influence of Polymers on the Physical and Chemical Stability of Spray-dried Amorphous Solid Dispersion: Dipyridamole Degradation Induced by Enteric Polymers. AAPS PharmSciTech. 2018; 19: 2620-2628.

Petrusevska, M. et al. Evaluation of a high-throughput screening method for the detection of the excipient-mediated precipitation inhibition of poorly soluble drugs. Assay Drug Development Technology. 2013a; 11(2): 117-129

Petrusevska, M. et al. Hyrodypropyl methylcellulose mediated preciptiation inhibition of sirolimus: from a screening campaign to a proof-of-concept human study. Molecular Pharmaceutics. 2013b; 10: 2299-2310.

Pouton, CW. Lipid Formulations for Oral Administration of Drugs: Non-emulsifying, Selfemulsifying and "Self-Microemulsifying" Drug Delivery Systems. European Journal of Pharmaceutical Sciences. 2000, 11: S93-S98.

Prasad, D. et al. Role of molecular interactions for synergistic precipitation inhibition of poorly soluble drug in supersaturated drug-polymer-polymer ternary solution. Molecular Pharmaceutics. 2016; 13: 756-765

Prasad, D. et al. Role of molecular interactions for synergistic precipitation inhibition of poorly soluble drug in supersaturated drug-polymer-polymer ternary solution. Molecular Pharmaceutics. 2016; 13: 756-765

Price, DJ. et al. Approaches to increase mechanistic understanding and aid in the selection of precipitation inhibitors for supersaturating formulations - a PEARRL review. Journal of Pharmacy and Pharmacology. 2018; 71, 483-509.

Price, DJ. et al. Calculation of drug-polymer mixing enthalpy as a new screening method of precipitation inhibitors for supersaturating pharmaceutical formulations. European Journal of Pharmaceutical Sciences. 2019; 132: 132-142

Price, DJ. et al. Incorporation of HPMCAS during loading of glibenclamide onto mesoporous silica improves dissolution and inhibits precipitation. European Journal of pharmaceutical Sciences. 2020; 141: 105-113.

Qian KK, and Bogner RH. Spontaneous crystalline-to-amorphous phase transformation of organic or medicinal compounds in the presence of porous media, part 1: Thermodynamics of spontaneous amorphization. Journal of Pharmaceutical Science. 2011; 100(7): 2801-2815.

Qiu, Y. and Lee, PI. Rational Design of Oral Modified-release Drug delivery systems. In: Developing Solid Oral Dosage Forms. Pharmaceutical Theory and Practice. 2017, 519-554

Raut Desai, S. Investigating the mechanism of supersaturation and precipitation inhibition of poorly soluble drugs from self-emulsifying drug delivery systems (SEDDS). 2013 Ph.D. Thesis, Massachusetts College of Pharmacy and Health Sciences. 
Rostron, P. et al. Raman Spectroscopy, Review. International Journal of Engineering and Technical Research. 2016; 6(1)

Rubenstein, M. and Colby, R. 2003. Polymer Physics. Oxford University Press, Oxford, UK. Saad, A. Triazole/Triazine-Functionalized Mesoporous Silica As a Hybrid Material Support for Palladium Nanocatalyst. Langmuir. 2017 (epub ahead of print)

Salonen, J. et al. Mesoporous silicon in drug delivery applications. Journal of Pharmaceutical Sciences. 2008; 97(2)

Schram, CJ. et al. Influence of Polymers on the Crystal Growth Rate of Felodipine: Correlating Adsorbed Polymer Surface Coverage to Solution Crystal Growth Inhibition. Langmuir. 2015b; 31(41): 11279-11287

Schram, CJ. et al. Influence of Polymers on the Crystal Growth Rate of Felodipine: Correlating Adsorbed Polymer Surface Coverage to Solution Crystal Growth Inhibition. Langmuir. 2015b; 31(41): 11279-11287

Sheng, Q. Thermal Analysis of Pharmaceuticals. In: Analytical Techniques in the Pharmaceutical Sciences. Ed: Mullertz, A. et al. Springer, New York City, USA; 2016

Shete, G. et al. Solid State Characterization of Commercial Crystalline and Amorphous Atorvastatin Calcium Samples. AAPS PharmSciTech. 2010; 11(2): 598-609

Shete, G. Molecular Relaxation Behavior and Isothermal Crystallization above Glass Transition Temperature of Amorphous Hesperetin. Journal of Pharmaceutical Sciences. 2014; 103(1): 167-178

Siepmann, J. et al. Higuchi equation: Derivation, applications, use and misuse. International Journal of Pharmaceutics. 2011; 418(1): 6-12

Skopp, J. Derivation of the Freundlich Adsorption Isotherm from Kinetics. Journal of Chemical Education. 2009; 86(11): 1341

Skotnicki, M. et al. Thermal behavior and phase identification of Valsartan by standard and temperature-modulated differential scanning calorimetry. Drug Development and Industrial Pharmacy. 2013; 39(10): 1508-1514

Staels, B. et al. Mechanism of action of fibrates on lipid and lipoprotein metabolism. Cardiovascular Drugs. 1998; 98: 2088-2093.

Summerlin, N. et al. Colloidal mesoporous silica nanoparticles enhance the biological activity of resveratrol. Colloids and Surfaces B: Biointerfaces. 2016; 144: 1-7

Sun, DD. and Lee, PI. Haste Makes Waste: The Interplay Between Dissolution and Precipitation of Supersaturating Formulations. AAPS Journal. 2015; 17(6): 1317-1326

Tantishaiyakul, V. et al. Prediction of solubility parameters using partial least square regression. International Journal of Pharmaceutics. 2006; 325: 8-14. 
Taylor, L. and Zhang, GGZ. Physical chemistry of supersaturated solutions and implications for oral absorption. Advanced Drug Delivery Review. 2016; 101: 122-142

Tian, B. et al. Theoretical prediction of phase diagram for solid dispersion. Pharmaceutical Research. 2015; 32, 840-851.

Timpe, C. Strategies for Formulation Development of Poorly Water Soluble Candidates- A Recent Perspective. American Pharmaceutical Reviews. 2007; 10(3): 104-109.

Ting, M. et al. High-Throughput Excipient Discovery Enables Oral Delivery of Poorly Soluble Pharmaceuticals. ACS Central Science. 2016; 2(10): 748-755

Tozuka, Y. et al. Effect of pore size of FSM-16 on the entrapment of flurbiprofen in mesoporous structures. Chemical and Pharmaceutical Bulletin. 2005; 53: 974-977

Turku, et al. Thermodynamics of tetracycline adsorption on silica. Environmental Chemistry Letters. 2007; 5(4): 225-228

Ueda, K. et al. Inhibitory effect of hydroxypropyl methylcellulose acetate succinate on drug recrystallization from a supersaturated solution assessed using nuclear magnetic resonance measurements. Molecular Pharmaceutics. 2013; 10(10): 3801-3811

Ueda, K. et al. The effect of HPMCAS functional groups on drug crystallisation from the supersaturated state and dissolution improvement. International Journal of Pharmaceutics. 2014; 464: 205-213

Vallet-Regi M. Ordered Mesoporous Materials in the Context of Drug Delivery and Tissue Engineering. Chemistry: A European Journal. 2010; 27: 5593-5604.

Van Krevelen, DW. and Te Nijenhuis, K. Properties of Polymers: Their Correlation with Chemical Structure; Their Numerical Estimation and Prediction from Additive Group Contributions. Elsevier. New York, 2009.

Van Speybroeck, M. et al. Enhanced absorption of the poorly soluble drug fenofibrate by tuning its release rate from ordered mesoporous silica. European Journal of Pharmaceutical Sciences. 2010; 41(5): 623-630

Van Speybroeck, M. et al. Ordered mesoporous silica material SBA-15: a broad-spectrum formulation platform for poorly soluble drugs. Journal of Pharmaceutical Science. 2009; 98: 2648-2658

Van Speybroeck, M. et al. Preventing release in the acidic environment of the stomach via occlusion in ordered mesoporous silica enhances the absorption of poorly soluble weakly acidic drugs. Journal of Pharmaceutical Sciences. 2011; 100(11): 4864-4876

Vora, C. et al. Preparation and characterization of dipyridamole solid dispersions for stabilization of supersaturation: effect of precipitation inhibitors type and molecular weight. Pharmaceutical Development Technologies. 2016; 21(7): 847-855

Wang, S. Ordered Mesoporous Materials for Drug Delivery. Microporous Materials, 2009; 117: $1-9$. 
Wang. Y. et al. The investigation of MCM-48-type and MCM-41-type mesoporous silica as oral solid dispersion carriers for water insoluble cilostazol. Drug Development and Industrial Pharmacy. 2014; 40(6): 819-828

Warren, DB et al. Using polymeric precipitation inhibitors to improve the absorption of poorly water-soluble drugs: A mechanistic basis for utility. Journal of Drug Targetting. 2010; 18(10): 704-731.

Watanabe $\mathrm{T}$, et al. Solid state radical recombination and charge transfer across the boundary between indomethacin and silica under mechanical stress. Journal of Solid State Chemistry. 2000; 164(1): 27-33.

Wei, Q. et al. Oral hesperidin-Amorphization and improved dissolution properties by controlled loading onto porous silica. International Journal of Pharmacy. 2017; 518(1-2): 253-263

Weuts, I. et al. Physicochemical Properties of the Amorphous Drug, Cast Films, and Spray Dried Powders to Predict Formulation Probability of Success for Solid Dispersions: Etravirine.

Pharmaceutical Technology. 2010; 100(1)

Wie, Q. et al. Oral hesperidin-Amorphization and improved dissolution properties by controlled loading onto porous silica. International Journal of Pharmaceutics. 2017; 518 (1-2): 253-263.

Williams, H.D. et al. Strategies to Address Low Drug Solubility in Discovery and Development. Pharmacological Reviews. 2013, 65, 3

Wyttenbach, N. et al. Theoretical Considerations of the Prigogine-Defay Ratio with Regard to the Glass-Forming Ability of Drugs from Undercooled Melts. Molecular Pharmaceutics. 2016; 13: $241-250$

Wyttenbach, N. and Kuentz, M. Glass-forming ability of compounds in marketed amorphous drug products. European Journal of Pharmaceutics and Biopharmaceutics. 2017; 112: 204-208.

Xia, X. et al. Encapsulation of Anti-Tuberculosis Drugs within Mesoporous Silica and Intracellular Antibacterial Activities. Nanomaterials. 2014; 4(3): 813-826

Xia, X. et al. In vivo enhancement in bioavailability of atazanavir in the presence of protonpump inhibitors using mesoporous materials. ChemMedChem. 2012; 7: 43-48

Xu, S. and Dai, W. G. Drug Pls in supersaturating formulations. International journal of Pharmaceutics. 2013; 453(1): 36-43.

Xu, W. et al. Mesoporous systems for poorly soluble drugs. International Journal of Pharmaceutics. 2013; 453(1): 181-197

Yalkowsky, SH. Solubility and Solubilization in Aqueous Media. Oxford University Press. 1999.

Yamashita, T. et al. Solvent shift method for anti-precipitant screening of poorly soluble drugs using biorelevant medium and dimethyl sulfoxide. International Journal of Pharmaceutics. 2011; 419(12): $170-174$

Yanagihara, H. et al. Adsorption-Desorption and Transport of Water in Two-Dimensional Hexagonal Mesoporous Silica. The Journal of Physical Chemistry C. 2013; 117(42): 21795-21802. 
Zhang, H. et al. Synthesis of novel mesoporous silica nanoparticles for loading and release of ibuprofen. Journal of Controlled Release. 2011; 152; 38-39.

Zhao, et al. Uniform mesoporous carbon as a carrier for poorly water soluble drug and its cytotoxicity study. European Journal of Pharmaceutics and Biopharmaceutics. 2012; 80: 535-543

Zheng, W. et al. Selection of Oral Bioavailability Enhancing Formulations during Drug Discovery. Drug Development and Industrial Pharmacy. 2012; 38(2): 235-247

Zhu, L. et al. Fast surface crystallization of amorphous griseofulvin below Tg. Pharmaceutical Research. 2010; 27(8): 1558-1567 


\section{Publications}

1) Price, DJ. et al. Approaches to increase mechanistic understanding and aid in the selection of precipitation inhibitors for supersaturating formulations - a PEARRL review. Journal of Pharmacy and Pharmacology. 2019. 71(4); 483-509. doi: 10.1111/iphp.12927

Personal Contribution: lead author, project management, editing of chapters, collation of final manuscript.

2) Price, DJ. et al. Calculation of drug-polymer mixing enthalpy as a new screening method of precipitation inhibitors for supersaturating pharmaceutical formulations. European Journal of Pharmaceutical Sciences. 2019. 132; 142-156. doi: 10.1016/i.ejps.2019.03.006

Personal Contribution: lead author, concept development, experimental design, data collection

3) Price, DJ. et al. Incorporation of HPMCAS during loading of glibenclamide onto mesoporous silica improves dissolution and inhibits precipitation. European Journal of Pharmaceutical Sciences. 2020. 141; 105-113. doi:10.1016/i.ejps.2019.105113.

Personal Contribution: lead author, concept development, experimental design, data collection

4) Price, DJ. et al. Opportunities for Successful Stabilization of Poor Glass-Forming Drugs: A StabilityBased Comparison of Mesoporous Silica Versus Hot Melt Extrusion Technologies. Pharmaceutics. 2019. 11(11); 577. doi: 10.3390/pharmaceutics11110577

Personal Contribution: co-lead author, concept development, experimental design, data collection

5) Ditzinger, FS. and Price, DJ. et al. Lipophilicity and hydrophobicity considerations in bio-enabling ora formulations approaches - a PEARRL review. Journal of Pharmacy and Pharmacology. 2019. 71(4); 464-482. doi: 10.1111/iphp.12984

Personal Contribution: co-author and editing the final manuscript

6) Jancovic, S. and Price, DJ. et al. Application of the solubility parameter concept to assist with oral delivery of poorly water-soluble drugs - a PEARRL review. Journal of Pharmacy and Pharmacology. 2019. 71(4); 441-463. doi: 10.1111/jphp.12948

Personal Contribution: co-author and editing the final manuscript

7) Laine, AL. and Price, DJ. et al. Enhanced oral delivery of celecoxib via the development of a supersaturable amorphous formulation utilising mesoporous silica and co-loaded HPMCAS. International Journal of Pharmaceutics. 2016. 512(1); 118-125. doi: 10.1016/i.ijpharm.2016.08.034

Personal Contribution: concept development, experimental design, data collection, co-lead author

8) Koehl, N. and Price, DJ. et al. In silico, in vitro and in vivo evaluation of precipitation inhibitors in supersaturated lipid based formulations of venetoclax. Molecular Pharmaceutics. Under Review

Personal Contribution: concept development, computation pharmaceutics calculations, data analysis, co-author 


\section{$\underline{\text { Posters }}$}

1. Price, DJ. et al. A Novel In Silico Screening Protocol For The Selection Of Optimized Precipitation Inhibitor Systems For Supersaturable Formulations. Poster presented at: AAPS Annual Meetingg; 2017 November 12-15; San Diego, CA. USA.

2. Price, DJ. et al. API and Precipitation Inhibitor Co-Loaded Mesoporous Silica: A Novel Synergistic Formulation Type with Advantageous Dissolution Performance. Poster presented at: PharmSci 360; 2018 October $26^{\text {th }}$ - November $5^{\text {th }}$; Washington DC, USA.

3. Price, DJ. et al. Molecular-level insight into mesoporous silica formulations: Effect of surface interaction on formulation performance. Poster presented at: PBP European Meeting; 2019 March 23-25; Bologna, Italy. 

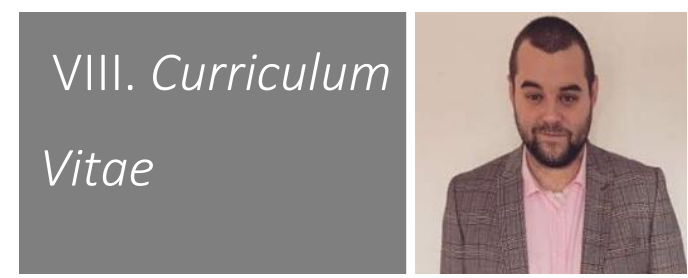

\section{Daniel Joseph Price}

E-mail: danjosephprice@gmail.com/daniel-joseph.price@merckgroup.com Mobile: +4917645623482 Born: 31/01/94, U.K.

\section{EDUCATION AND QUALIFICATIONS}

PhD in Pharmaceutical Technology:

October 2016- Current Goethe University Frankfurt in collaboration with Merck KGaA

- Thesis title: Novel in vitro and in silico tools for the development of mesoporous silica formulations with optimal precipitation inhibitors

Master and Bachelor of Science in Industrial Medicinal Chemistry: $1^{\text {st }}$ Class Honours

September 2012- May 2016, University of Leeds

- Master's thesis title: "Controlled release of doxorubicin with novel polymer architects"

- Bachelor thesis title: "Improving the bioavailability of poorly soluble drugs with mesoporous silica"

- Industrial Research Prize for highest graded bachelor thesis carried out in industry.

- Craig Jordan Prize for top graduating student (Masters and Bachelors).

- Master's research prize for top graded thesis.

- Salters' Graduate Prize, nationwide prize for the top 5 chemistry graduates in the UK, based on academic achievement, leadership and potential for impacting the global chemical industry.

International Baccalaureate Diploma: Top 5\% in China

August 2010-May 2012, Dulwich College Shanghai, Shanghai, China

RELEVANT EXPERIENCE

April 2019-Present

Technical Product Manager, Merck Group: Darmstadt, Germany

- Responsible for marketing strategy and execution for a portfolio of solubility enhancing functional excipients within Merck Life Science.

- Project management and long-term strategy planning for innovation projects.

- Co-ordinating and leading marketing activities on product development and launch.

- Influencing strategic business decisions within solid formulation.

- Interface between commercial and R\&D organizations.

October 2016-April 2019

PhD Researcher, Merck Group: Darmstadt, Germany

- Marie Cure Fellow investigating supersaturating drug formulations for poorly soluble drugs, specifically mesoporous silica. This was part of the Horizon 2020 project PEARRL (www.pearrl.eu).

- Increased understanding of the fundamental mechanistic details of mesoporous silica technology.

- Development of an in silico protocol that calculated API-polymer mixing enthalpy.

- Led a team of 10 authors to publish an extensive review article on the selection of precipitation inhibitors in early development. This article was designed and executed to act as a formulation guide for industrial pharmaceutical scientists developing poorly soluble drug formulations.

- Delivered training courses on the use of Parteck SLC ${ }^{\circledR}$ mesoporous silica. 

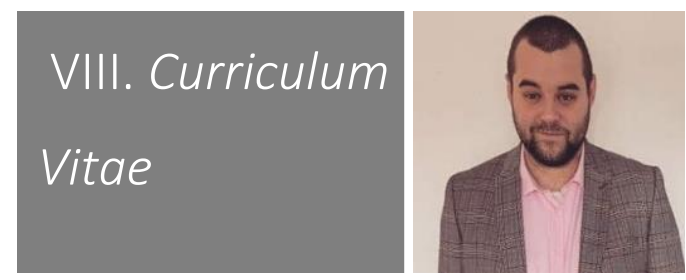

\section{Daniel Joseph Price}

February 2018-May 2018

Visiting Scientist, BfArM: Bonn, Germany

- Observed the EMA Quality Working Party and contributed to training on „mathematical modelling in regulatory submissions".

- Attended scientific advice meetings with companies visiting BfArM gave me an understanding of the interactions that take place between industry and the regulatory bodies.

- Completed a mock assessment of a marketing authorization application (MAA), which gave me an insight into the key requirements for submission of new APIs and excipients to the EMA.

September 2015-May 2016

Research Student, University of Leeds School of Chemistry: Leeds, UK

- Investigated the synthesis of novel biodegradable polymers for controlled-release drug delivery.

- Demonstrated how O-carboxyanhydride and $\mathrm{N}$-carboxyanydride ring-opened co-polymers could successfully deliver doxorubicin directly to tumour cells via acid-based hydrolysis.

September 2014-September 2015

Formulation Scientist, Pfizer: Cambridge, UK

- Investigated Parteck SLC ${ }^{\circledR}$ mesoporous silica for increasing the bioavailability of poorly soluble drugs.

- Regularly presented data within the company and in collaboration with Merck Millipore to a high standard of presentation and professionalism, which spurred an increased interest in Parteck SLC ${ }^{\circledR}$.

- Instilled a legacy for the use of Parteck SLC ${ }^{\circledR}$ mesoporous silica in Pfizer for pre-clinical candidates.

July 2012-August $2012 \quad$ Research Intern, Shanghai Institute of Materia Medica: Shanghai, China

- Gained first-hand exposure to a Chinese research environment during a molecular biology-based lab project, during which I contributed to elucidation efforts for GPCR LGR-4.

\section{SKILLS AND HOBBIES}

- Living and studying in China for my high school education instilled in me a passion for Chinese culture, people and language.

- Prior to moving to China, I was enrolled in theatre school full-time. This has resulted in a strong confidence in communication, public speaking and effective presentation.

- My hobbies include rugby, acting, singing and playing piano.

PERSONAL DETAILS

D.O.B: $31 / 01 / 1994$

Nationality: British

Marital Status: Married

Current Address: Franklinstrasse 25, 64285, Darmstadt, Hessen, Germany

Current Employer: Merck KGaA, Darmstadt, Germany 


\section{Declaration}

I hereby declare that this thesis has not been and will not be submitted in whole or in part to another University for the award of any other degree. Except where stated otherwise by reference or acknowledgment, the work presented was generated by myself under the supervision of my advisors during my doctoral studies.

Darmstadt, 2010

Daniel Joseph Price 


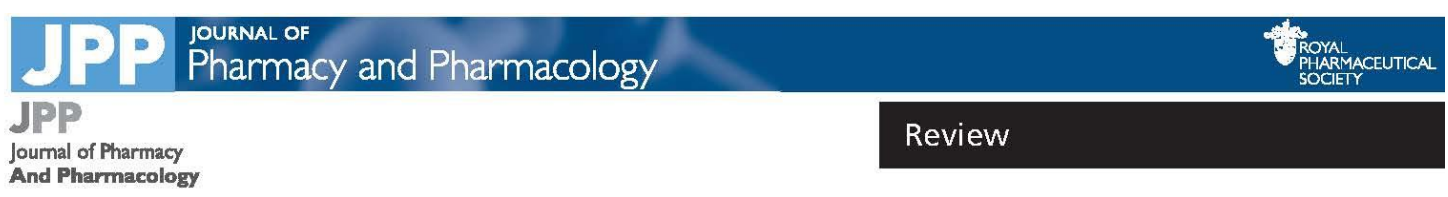

\section{Approaches to increase mechanistic understanding and aid in the selection of precipitation inhibitors for supersaturating formulations - a PEARRL review}

Daniel J. Price ${ }^{a, b}(D)$, Felix Ditzinger, ${ }^{c, d}\left(\mathbb{D}\right.$, Niklas J. Koehl ${ }^{e}$, Sandra Jankovic $c^{c, d}\left(\mathbb{D}\right.$, Georgia Tsakiridou $^{f, g}$, Anita Nair ${ }^{\mathrm{a}}$, René Holm ${ }^{\mathrm{h}}$, Martin Kuentz ${ }^{\mathrm{d}}$, Jennifer B. Dressman ${ }^{\mathrm{b}}$ and Christoph Saal ${ }^{\mathrm{a}}$

${ }^{a}$ Merck KGaA, Darmstadt, ${ }^{b}$ Frankfurt Goethe University, Frankfurt, Germany, ${ }^{C}$ Department of Pharmaceutical Sciences, University of Basel, Basel, "Institute of Pharma Technology, University of Applied Sciences and Arts Northwestern Switzerland, Muttenz, Switzerland, "School of Pharmacy, University College Cork, Cork, Ireland, ${ }^{\mathrm{f}}$ Pharmathen SA, Product Design \& Evaluation, ${ }^{9}$ Department of Pharmacy, University of Athens, Athens, Greece and hDrug Product Development, Janssen Research and Development, Johnson and Johnson, Beerse, Belgium

Keywords

controlled and sustained release systems: development of novel analytical techniques; dosage form design and characterisation; pharmaceutical analysis; pharmaceutics and drug delivery

\section{Correspondence}

Christoph Saal, Merck KGaA, 250

Frankfurter Strasse, 64293 Darmstadt

Hessen, Germany.

E-mail: christoph.saal@merckgroup.com

Received January 3,2018

Accepted April 16, 2018

do: $10.1117 /$ jphp. 12927

\begin{abstract}
Objectives Supersaturating formulations hold great promise for delivery of poorly soluble active pharmaceutical ingredients (APIs). To profit from supersaturating formulations, precipitation is hindered with precipitation inhibitors (PIs), maintaining drug concentrations for as long as possible. This review provides a brief overview of supersaturation and precipitation, focusing on precipitation inhibition. Trial-and-error PI selection will be examined alongside established PI screening techniques. Primarily, however, this review will focus on recent advances that utilise advanced analytical techniques to increase mechanistic understanding of PI action and systematic PI selection.

Key findings Advances in mechanistic understanding have been made possible by the use of analytical tools such as spectroscopy, microscopy and mathematical and molecular modelling, which have been reviewed herein. Using these techniques, PI selection can be guided by molecular rationale. However, more work is required to see widespread application of such an approach for PI selection.

Summary Precipitation inhibitors are becoming increasingly important in enabling formulations. Trial-and-error approaches have seen success thus far. However, it is essential to learn more about the mode of action of PIs if the most optimal formulations are to be realised. Robust analytical tools, and the knowledge of where and how they can be applied, will be essential in this endeavour.
\end{abstract}

\section{Introduction}

Among the various routes of administration for drugs, oral administration is the most commonly employed. It is costeffective and convenient for the patient, leading to a very high patient compliance. ${ }^{[1]}$ Various dosage forms are available for oral delivery including solid formulations such as capsules and tablets, as well as liquid formulations such as solutions, suspensions and syrups. For the active pharmaceutical ingredient (API) to exert its pharmacological effect, it must be released from the dosage form and absorbed from the gastrointestinal (GI) tract into the systemic circulation, where it can be transported to its physiological target. Thus, the bioavailability of a drug relies, among other parameters, on its capability to dissolve in the GI milieu and pass through the intestinal membrane. ${ }^{[2]}$ It is from these two parameters (solubility and permeability) that the Biopharmaceutics Classification System arose, a system which groups drugs into four classes, based on solubility and permeability. ${ }^{[3]}$

Due to recent scientific advances such as high-throughput screening in combination with combinatorial chemistry; X-ray diffraction of target proteins and computational chemistry, we now have an increased understanding of how small molecules bind to targets. Therefore, it has become easier to identify 'hits' that have therapeutic 


\section{Thesis Papers}

potential. In parallel, there has been a large increase in the number of druggable targets which have been discovered and validated using a broad spectrum of novel methods, such as proteomics, genomics and even gene editing. $\left.{ }^{[2,4} 7\right]$ On the other hand, it is recognised that use of such discovery tools often results in the identification of a higher proportion of lipophilic, high molecular weight and poorly soluble molecules, which do not adhere to Lipinski's rules of $5 .{ }^{[5]}$ Albeit positive for the industry, these shifts have also increased the number of drug candidates with poor physicochemical profiles (low solubility, high $\log P$, high molecular weight, poor solubility) appearing in research and development pipelines. As a result, there is a higher risk of attrition during pharmaceutical research and development due to insufficient oral bioavailability, which represents a loss in therapeutic and economic potential. It has been reported that approximately $40 \%$ of all commercial drugs are classified as poorly soluble. ${ }^{[8]}$ Extending this trend to those compounds still in the development pipeline, it has been reported that anywhere between $80 \%$ and $90 \%$ are 'not highly soluble., ${ }^{[8]}$ Therefore, the need for effective formulation approaches for these compounds, to avoid low bioavailability due to poor aqueous solubility, has never been greater.

Faced with these challenges, pharmaceutical scientists have developed a toolkit of strategies, which use physicochemical knowledge of solvation and dissolution to enhance solubilisation and to overcome poor oral bioavailability. ${ }^{[7]}$ These approaches can involve modifying the chemical form of an API for example with (1) salt formation, ${ }^{[9]}$ (2) co-crystals ${ }^{[10]}$ or (3) prodrugs. ${ }^{[11]}$ Or, alternatively, formulation approaches such as (4) solvents, co-solvents and lipids $\left.{ }^{[2} 15\right] ;$ (5) micelle systems ${ }^{[16}{ }^{18]} ;(6)$ particle size reduction ${ }^{[19]}$; (7) complexation ${ }^{[20]}$; and (8) solid amorphous dispersions, produced by techniques such as hot melt extrusions (HMEs), spray-dried dispersions (SDDs), co-precipitates or mesoporous silica. $\left.{ }^{[21} 26\right]$

In recent years, formulations that generate and stabilise a supersaturated state in vivo have come to the forefron when considering the delivery of poorly soluble drugs. ${ }^{[8,27,28]}$ Supersaturation is a state in which the concentration of a solute exceeds the thermodynamic (equilibrium) solubility of the molecule. Such a state is highly attractive for compounds with low aqueous solubility as artificially high API concentrations can be generated in the GI tract. This increases the absorptive flux, which can subsequently increase absorption and bioavailability. ${ }^{[27,29]}$ Such an approach must, however, be considered from an energetic perspective as well. ${ }^{[30]}$ The free energy of the supersaturated state is significantly higher than that of the saturated solution, and there is a strong driving force for the system to return to its thermodynamically stable state via crystallisation and precipitation processes. ${ }^{[8]}$ Therefore, successful supersaturating formulations should not only generate increased API concentrations in solution, but should also be able to stabilise the supersaturated state. Often, this stabilising factor takes the form of a precipitation inhibitor (PI), which prevents the recrystallisation and precipitation process. Therefore, PIs are an integral part of supersaturating formulations, and a robust understanding of the mechanisms behind precipitation inhibition is essential for effective formulation design. ${ }^{[8]}$ This review offers a brief overview of the physical chemistry underpinning supersaturation and precipitation before examining recent work utilising cutting-edge analytical techniques and methods that have led to an increased understanding of precipitation inhibition mechanisms, and how such understanding can be used in the selection of optimal PI systems for supersaturating formulations. To the best of our knowledge, this review is the first to consider precipitation inhibition and precipitation inhibitor selection from this perspective.

\section{Supersaturating formulations}

In order to understand the process of precipitation inhibition, it is important to have an overview of the physical-chemical underpinnings of drug supersaturation. As a complete treatment of supersaturated solutions is beyond the scope of this review, the interested reader is referred to an excellent review recently published by Taylor and Zhang. ${ }^{[8]}$

Supersaturation, from a physicochemical perspective, is generally defined as a system in which the free energy of the solute in solution is higher than that of the crystal form or amorphous solid phase of the drug at equilibrium (Figure 1) ${ }^{[8,31]}$ Practically speaking, this is any system in which the concentration of drug in solution exceeds the thermodynamic solubility of the pure API. Increasing the concentration in the GI tract via supersaturation can increase the overall absorption of the drug. As a result, supersaturating formulations are highly appealing for drugs with low thermodynamic solubility, which often exhibit poor bioavailability. ${ }^{[3]}$

The gap between the free energy of the supersaturated state and the equilibrium state can lead to stability issues. ${ }^{[8,30]}$ Therefore, the absorption advantages previously mentioned are not always realised, as this instability can lead to precipitation of amorphous or crystalline material from the supersaturated solution. ${ }^{[27,32]}$ Any precipitated drug would have to redissolve in the GI tract in order become available for absorption and, therefore, the potential absorption advantage is typically diminished. ${ }^{[33]}$ Consequently, an effective supersaturating formulation needs not only to generate high supersaturation, but also to maintain this for a physiologically relevant time. With typical upper GI transit time, this would be $2-4 \mathrm{~h}$, the time during which 


\section{Thesis Papers}
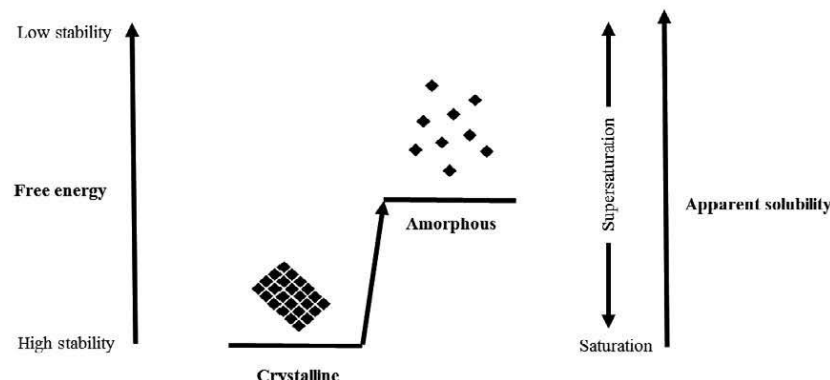

Figure 1 Energy diagram showing crystalline and amorphous solid states of drug substance from a stability and solubility perspective.

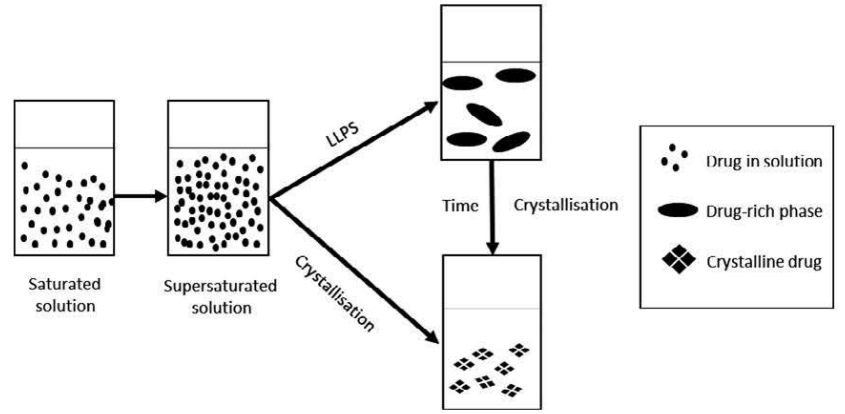

Figure 2 Liquid liquid phase separation (LLPS) generates drug-rich phases in solution, which eventually crystallise. Effective use of precip tation inhibition can prolong the supersaturation lifetime post-LLPS.

most drug absorption takes place after gastric emptying when the drug is taken in the fasted state. ${ }^{[34]}$ In order to successfully develop such a formulation, it is important to have a good understanding of the physical basis of supersaturation, and how this knowledge can be exploited to maximal effect.

During the dissolution of supersaturating formulations, drug concentration increases until the crystalline saturation solubility is exceeded. After this point, supersaturation is achieved, which exists in a metastable state. ${ }^{[35]}$ This metastability is the driving force of nucleation and thus precipitation of either amorphous or crystalline solids. ${ }^{[3,37]}$ The rates of nucleation and precipitation, in relation to the dissolution rate of the formulation, are the key parameters when considering whether the supersaturated state will be maintained.

Successful supersaturating formulations may also exceed the so-called amorphous solubility, a metastable state in which amorphous drug exists in a pseudo-equilibrium with the dissolution medium, eventually returning to the crystalline form. ${ }^{[31,3841]}$ 'Amorphous solubility' has been calculated theoretically based on the crystalline saturation solubility and by considering the amorphous material as a supercooled liquid. ${ }^{[42,43]}$ High drug concentrations of slowly crystallising drugs are typically limited by a kinetically favoured liquid-liquid phase separation (LLPS), (Figures 2 and 3 ) in which separation of drug molecules into drug-rich 'droplets' (100-500 nm) from the aqueous phase occurs via spinodal decomposition. ${ }^{\mid 8,44} 48 \mid$

At, or approaching, the theoretical amorphous solubility the LLPS state is at a lower energy than the supersaturated solution, and so is thermodynamically favoured. LLPS proceeds via quick spinodal decomposition and so can only occur when fast crystallisation does not occur. Drug molecules in the drug-rich regions exist in the amorphous state and usually tend to fall back to the more stable crystalline state over time. ${ }^{[4,50]}$ Thus, the process of LLPS extends the lifetime of the molecule in solution, such that high concentrations can be achieved over longer periods of time, compared to the unstable supersaturated state. Typically, precipitation inhibition would aim to avoid any precipitation from the supersaturated state. However, given that 


\section{Thesis Papers}

precipitation inhibition is concerned with the biopharmaceutical performance of a drug, the effect of a PI on sustaining LLPS is also relevant to the current body of work. In this instance, LLPS can be considered a 'reservoir' of supersaturation such that the initially unstable supersaturation results in a plateau of supersaturation at the LLPS due to the separation of API in droplets. ${ }^{[8]}$ This is a direct analogy to the 'Spring and Parachute' model proposed by Guzman et al. ${ }^{[51]}$ (Figure 4) and has also been referred to as a 'Spring and Plateau' approach. ${ }^{[8]}$ Generally, the spring effect is generated either by delivering the drug in a presolubilised form (e.g. SEDDS or lipid-based formulations) or in a rapidly dissolving form (e.g. amorphous, less stable polymorphs, particle size engineering, amorphous dispersion, solid solutions and prodrugs). ${ }^{[27]}$ To complete the model, PIs act as 'parachutes' by hindering nucleation and arresting precipitation ${ }^{[33,52]}$ or, maintaining the lifetime of the droplet state after LLPS. Both of these processes increase the concentration and lifetime of the supersaturated drug in solution.
The success of supersaturating formulations is dependent on appropriate selection of 'Spring-Parachute' combinations. Consequently, mechanistic understanding of the role of the PI in supersaturating formulations is imperative for the educated choice of successful PI-drug combinations.

\section{Precipitation inhibition: theory and practice}

From a pharmaceutical perspective, precipitation is a process whereby a solid phase separates from a liquid phase. This can yield either amorphous or crystalline materials, or mixtures of both. Although precipitation in the amorphous form is possible from supersaturated solutions, the vast majority of work has focussed on crystallisation theory. Fortunately, when considering inhibition, classical crystallisation theory can be applied to the precipitation of both crystalline and amorphous solids. ${ }^{[27,28,54]}$ In the following section, a short overview of the current theories of crystallisation is presented. For more details, the reader is referred

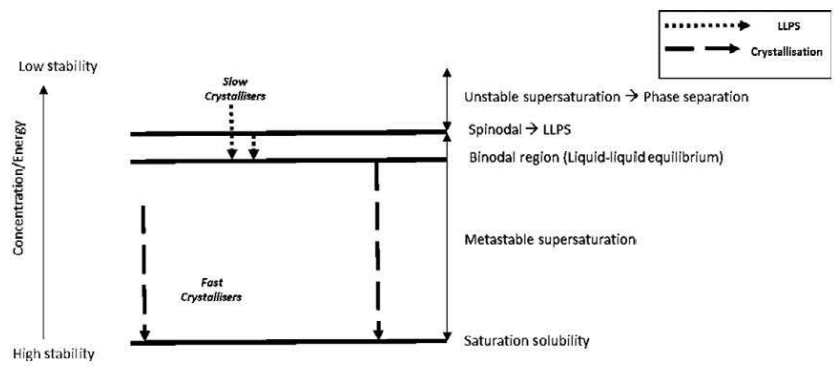

Figure 3 The fate of a drug molecule in a highly supersaturated solution depends on the kinetics of crystallisation, with slow crystallizers (dotted arrows) often undergoing liquid liquid phase separation. Fast crystallizers (dashed arrow) do not undergo liquid liquid phase separation. Adapted from Taylor and Zhang. ${ }^{[8]}$

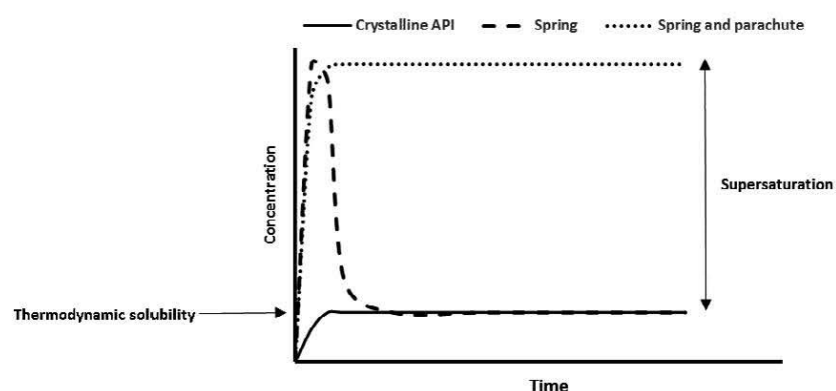

Figure 4 The Spring and Parachute model. A common formulation approach where a 'spring' generates supersaturation and a 'parachute' prevents precipitation. Collectively, this can improve the absorption of a poorly soluble API. ${ }^{[51,53]}$ 


\section{Thesis Papers}

to the book by Mullin on 'Crystallisation' ${ }^{\text {[30] }}$ and to an excellent review by Warren et al. ${ }^{[54]}$

\section{Crystallisation and precipitation}

Crystallisation is an energy-driven process whereby a molecule in solution at supersaturated concentrations precipitates to a solid, crystalline material. ${ }^{[55]}$ As supersaturation concentrations approach a critical level, the system becomes labile and 'precipitation is self-influenced instantaneously without any external influence. ${ }^{[30]}$ Before this critical point, the supersaturation is said to be metastable and precipitation may not occur instantaneously and/or spontaneously, but can be easily induced via mechanical activation or the addition of seed crystals. ${ }^{[30,56]}$ Crystallisation from a supersaturated solution is a function of the concentration, temperature and pressure of the solution. Practically speaking, however, it is generally only temperature and concentration that are considered (Figure 5). A higher concentration of solute increases the lability of the system, whereas an increased temperature decreases lability. For concentration, the higher the concentration of a solute in solution, the higher the degree of supersaturation, which increases excess energy of the system. Conversely, as temperature increases, the saturation concentration usually also increases, and the degree of supersaturation is decreased (Figure 5).

Crystallisation occurs in two key stages: nucleation and crystal growth. Simply put, solute molecules must come together (nucleate) until a critical size of a nucleus is reached, after which crystal growth can occur. During crystallisation, both nucleation and crystal growth are occurring simultaneously at different rates, depending on the stage of the crystallisation process and the conditions of the system ( $v i s$ a vis solute concentration and solvent temperature). ${ }^{[54]}$ Nucleation can occur spontaneously or

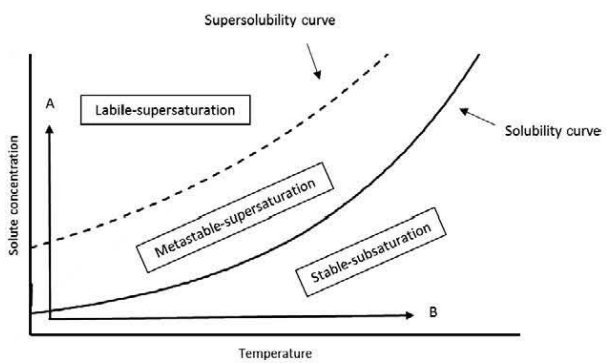

Figure 5 Solubility supersolubility diagram amended from Mullin. ${ }^{[30}$ A more labile solution is obtained with a higher orug concentration (arrow A) A more stable solution is obtained by the increase in temperature (arrow B). artificially, ${ }^{[30]}$ for example via agitation, mechanical shock, $\mathrm{pH}$ change or dilution. The exact process of nucleation is still unclear, although several mechanisms have been proposed in the literature, none have been fully validated. ${ }^{[57]}$ Nevertheless, nucleation can occur in a number of ways, either with or without seed crystals (secondary and primary nucleation, respectively) or in the presence or absence of a cluster surface, for example foreign particles or container defects (heterogenous/2D and homogenous/3D crystallisation, respectively). ${ }^{[30]}$ The nucleation rate $(J)$ can be described by the classical nucleation theory (CNT) (Equation 1). ${ }^{[58]}$

$$
J=A \exp \left(-\frac{B}{\ln ^{2} S}\right)
$$

$S$ is the supersaturation of the system. The pre-exponential factor $A$ is a kinetic measure that described how the molecules come together to form nuclei. Given the random nature of this process, with a wide range of accessible clusters, which can grow or decay in multiple directions, the pre-exponential factor is often calculated using Monte Carlo simulations. The thermodynamic factor, $B$, describes the free energy barrier for the formation of a nucleus, which is also referred to as 'nucleation work'. For practical purposes, $A$ and $B$ are considered to be constant in systems with fixed temperatures. This equation underscores the importance and significance that supersaturation, $S$, plays in the nucleation process. ${ }^{[58,59]}$

After stable nuclei have been formed, they increase in size and form visible crystals via crystal growth. ${ }^{[30]}$ The process of crystal growth has been extensively described in the literature. ${ }^{[30,37,60,61]}$ Various models have been put forward to provide a theoretical mechanism for the process of crystal growth including (1) surface energy theory, (2) diffusion theory and (3) adsorption layer theory. ${ }^{[30]}$ All three models have significant failings; however, the most widely accepted and quoted model is the adsorption layer theory. ${ }^{[30]}$

Adsorption layer theory is based on the thermodynamic assumption that there is a layer of adsorbed molecules on the surface of a growing crystal, which is in an equilibrium state with the bulk solution surrounding the layer. The crystal will grow if the adsorbed molecules on the surface find a position that is thermodynamically favourable and exhibits high attraction forces between the adsorbed molecule and the crystal, enabling extension of the lattice. ${ }^{[30]}$ This occurs at imperfections and kinks on the surface of the growing crystal face. ${ }^{[30,54]}$ Despite this process, the resultant crystal may not be the thermodynamically most favourable. It is common for metastable polymorphs to form, ${ }^{[62]}$ based on the quick access of surface adsorbed solutes to energetically stable, but not the most energetically stable sites. This phenomenon, whereby the first crystal formed is simply kinetically favoured and leads via different stages to a more 


\section{Thesis Papers}

stable solid, was first suggested by Wilhelm Ostwald and is now referred to as 'Ostwald's rule?. ${ }^{[63]}$ That being said, if left for a sufficient time period, the thermodynamically most stable polymorph should form in most cases. ${ }^{[61]}$

For supersaturating formulations, nucleation of the generated supersaturated solution would be highly probable if the formulation was not stabilised by addition of PIs. ${ }^{[33]}$ If the process of precipitation is arrested for physiologically relevant time scales, the supersaturated state can be maintained for long enough to allow for increased absorption. ${ }^{[54]}$ Polymers, surfactants and cyclodextrins have been investigated as excipients that can maintain the supersaturated state. ${ }^{[33]}$ Another consideration is the effect that endogenous molecules (e.g. bile salts) in the body can have on precipitation inhibition. ${ }^{\text {[64 } 65]}$

\section{Polymers as precipitation inhibitors}

The most common PIs employed in the pharmaceutica industry are polymers. Table 1 lists a large range of polymers, including cellulose derivatives, polyvinylpyrrolidones and methacrylates, which have been evaluated as precipitation inhibitors in oral drug products.

Polymers function by slowing down the process of nucleation and crystal growth through interaction with the dissolved API molecules and interaction with and adsorption onto growing crystals. ${ }^{[33,54]}$ Given the kinetic nature of this effect, the thermodynamic equilibrium solubility is usually not affected by the polymer, except in a number of limited cases, where polymers can have a co-solvent effect as well. $\left.{ }^{[54,66}{ }^{68}\right]$ Kinetic inhibition of nucleation relies on molecular interactions between the polymer and the drug, that is hydrogen bonds, polar, or dispersion forces. ${ }^{[27,28,54]}$ Each of these interactions may contribute to varying degrees, especially when considering the impact of water. This is an area that has seen increasing interest in the literature lately $\left.{ }^{[65,68} 70\right]$ but more work is required to resolve the exact contributions and nature of the PI-API interaction. More generally, such interactions can be influenced by temperature, molecular weight, polarity and hydrogen bonding capabilities of both the drug and the polymer. One potential mode of action is the adsorption of the polymer onto the growing crystal surface, blocking the access of the solute to the surface. Furthermore, polymers can disrupt the growth rate on crystal surfaces by binding onto imperfection sites, thus flattening the surface and removing the interaction point. This is highly dependent on the balance of interactions between the solid and the polymer and the interaction between the liquid and the polymer. ${ }^{[71]}$ The adsorption of the polymer onto the surface of the particle also introduces steric hindrance, which disrupts the diffusion of the molecules at the solid-liquid interface. ${ }^{[72}{ }^{74]}$ It has previously been reported that the hydrophobicity of the polymer
Table 1 Examples of PIs studied in the literature

\begin{tabular}{|c|c|}
\hline Inhibitor name & Reterence(s) \\
\hline Eudragit $^{\mathbb{E}} 5100$ & [91] \\
\hline Eudragit ${ }^{\mathrm{E}}$ E100 & {$[91,92]$} \\
\hline $\begin{array}{l}\text { Foly(ethylene oxide)-b-poly(propylene)-b- } \\
\text { poly(ethylene oxide) (Poloxamer) (Pluronic }\end{array}$ & {$[93,94]$} \\
\hline Foly(ethylene glycol) (PEG) & {$[91,95]$} \\
\hline Foly(ethylene imine) $\langle\mathrm{PEI})$ & {$[54,72]$} \\
\hline Eudragit RL100 & {$[54,96]$} \\
\hline Foly(ether)-co-poly(o) (PEPO) & {$[72]$} \\
\hline Foly(propylene glyco) (PPG) & {$[54,97,98]$} \\
\hline Foly(styrene) sulfonic acid (PSS) & {$[99,100]$} \\
\hline $\begin{array}{l}\text { Foly(vinylpyrrolidone) (PVP) (Povidone) } \\
\text { (Copovidone) }\end{array}$ & {$[69,10110$} \\
\hline Foly(vinyl acetate)-co-poly/viny pyrrolidone) & {$[106,107]$} \\
\hline
\end{tabular}

(PVA-PVP)

$\begin{array}{ll}\text { Hydroxyethyl cellulose (HEC) } & {[93,94]} \\ \text { Poly(methyl methacrylate) (PMMA) } & {[54,98]}\end{array}$

Foly(methyl methacrylate) (PMMA) [54,98]

Foly(lactic acid) (PLA) [91]

Poly(vinyl acetate) phthalate (PVAP) [54]

Hydroxypropyl methyl cellulose acetate $\quad[23,24,68$,

succinate (HPMCAS) 108113

Cellulose acetate phthalate (CAP) [113]

Hydroxypropyl methyl cellulose (HPMC) $\quad[74,114117$

Foly(vinyl alcohol) (PVOH) $\quad[28,54]$

Poly(acrylic acid) (PAA) $\quad[28,54,718]$

Foly(acetylene) $\quad[28,54,116]$

Methyl cellulose
$[28,54]$

Foly(lactid-co-glycolid) (PLGA) [54]

Sodium carboxymethyl cellulose (SCMC) [54]

Chitosan [54]

Foly(urethane) (PUR) [119]

Mannitol [54]

Poly(glycolide) (PGA) [54]

Locust bean gum $\quad[54,119129]$

Alginic acid gum [54]

Hydroxy propyl- $\beta$-cyclodextrin (HPBCD) $\quad[20,54,115$,

(Captisol ${ }^{6}$ )

Sodium dodecy/ sulphate (SDS) [54]

FEG-40 hydrogenated castor oil [54]

(Cremophor ${ }^{\mathrm{E}}$ )

Poly(ethylene glycol) sorbitan

monolaurate (Tween $\left.{ }^{\circledR} 20\right)$

Sorbitol

Sodium cholate $\quad[65]$

Sodium deoxycholate [65]

Sodium chenodeoxycholate [65]

Sodium lithocholate $\quad[65]$

Sodium ursodeoxycholate [65]

Sodium hyodeoxycholate [65]

Sodium taurocholate

Sodium glycocholate [65]

Sodium glycodeoxycholate $[65]$

Sodium glycochenodeoxycholate [65]

Sodium glycoursodeoxycholate [65]

Sodium taurodeoxycholate [65]

Sodium taurochenodeoxycholate [65] 


\section{Thesis Papers}

influences this balance. ${ }^{[71,75]}$ Additionally, the $\mathrm{pH}$ of the solvent affects ionisable polymers and ionisable APIs. ${ }^{[58,76}$ Another potential mode of action for polymeric PIs is via alteration of the solid-liquid interface, which can cause a change in surface energy and hinders the diffusion of new molecules to the crystal surface. Furthermore, the solubility and surface tension in the bulk solution can undergo changes and therefore may contribute to the precipitation inhibition. ${ }^{[72,77]}$ However, for this purpose, it is not currently understood to what extent the polymer needs to be in a colloidal state vs solubilised as random coils. ${ }^{[54]}$ Another important factor to consider is viscosity; as the viscosity of the solution increases, the molecular mobility of the drug in solution decreases. This increases the energy required for the diffusion of drug through the solution and can have a profound effect on both nucleation and crystal growth, both of which depend on diffusion of drug to another solute molecule or to the growing crystal, respectively.

For a given polymer, a high molecular weight is associated with an increase in viscosity as well as an increase in the number of binding sites. For this reason, it is often unclear which factor is responsible for any increased inhibition when considering a range of molecular weight polymers. For example, Chavan et al. ${ }^{[78]}$ concluded that viscosity was an important polymer characteristic in the precipitation inhibition of nifedipine from supersaturated solution by HPMC, with higher viscosity samples of HPMC delaying the induction time of crystallisation for the longest periods of time. However, viscosity is not the only determinant of precipitation inhibition by polymers, other reports in the literature were unable to show a significant impact of polymer viscosity. ${ }^{[69]}$ In any case, it is likely that the mechanisms(s) of interaction and the biggest contributor to the inhibitory effect will vary with the individual system.

\section{Surfactants and cyclodextrins as precipitation inhibitors}

Similar to polymers, surfactants and cyclodextrins have also been reported to sustain drugs in solution kinetically through molecular interactions. ${ }^{[79,80]}$ However, they also have the potential to inhibit precipitation by increasing the solubility of the API, thereby reducing supersaturation. For surfactants and cyclodextrins, this can occur via micellar solubilisation and complexation, respectively. ${ }^{[79}$ 82] Thus, surfactants and cyclodextrins have often been called thermodynamic precipitation inhibitors. $\left.{ }^{[20,54,79} 88\right]$ This distinction relates to the mechanism of action of the inhibitory affect. For conventional polymer PIs, the drug is sustained in solution only temporarily, and precipitation will eventually occur. Therefore, polymers have a kinetic effect on drug precipitation. For surfactants and cyclodextrins, however, increasing the solubility (i.e. reduction in supersaturation) is a more sustained effect. The classification of cyclodextrins and surfactants as precipitation inhibitors is therefore somewhat tentative, and the distinction of kinetic inhibition vs solubilisation effects has typically not been addressed in the literature. Therefore, further mechanistic work surrounding the mode of action of surfactants and cyclodextrins on precipitation inhibition will be required to better understand the relevance of these compounds as PIs. For a list of all surfactants and cyclodextrins reported in the literature to act as PIs, please refer to Table $\mathbf{1}$

\section{Endogenous precipitation inhibitors}

Endogenous surfactants in the GI tract can also inhibit the precipitation of supersaturating formulations during dissolution in vivo. In theory, surfactants such as bile salts and lecithin have the potential to inhibit crystallisation via the mechanisms mentioned in the previous sections. For example, Chen et al. ${ }^{[89]}$ showed that sodium taurocholate was able to extend nucleation time significantly (up to 11 -fold) for a group of 11 structurally diverse compounds. This may partly explain why many in vitro dissolution tests overestimate the precipitation of supersaturated API. ${ }^{[90]}$ Further work by Li et al. ${ }^{[65]}$ expanded the precipitation screen to 13 different bile salts. It was observed that most of the 13 bile salts investigated inhibited precipitation of celecoxib, nevirapine and flibanserin, with varying degrees. Further, it was concluded that van de Waal and hydrogen bond interactions between the inhibitor and the molecule in solution were the key factors determining PI effects. ${ }^{[65]}$ In this respect, there are clear similarities with formulation-based PIs. Although more work is required, it is clear already that it is important to understand and take into account the effect of endogenous molecules on supersaturating formulation, especially in the design of in vitro dissolution tests.

\section{Precipitation inhibitor screening methods}

Given the large number of PIs reported in the literature, (Table 1) various screening methods to select API-PI combinations have been developed. Invariably, these screening methods involve the generation of supersaturation in combination with a variety of analytical techniques that can determine the rate and extent of precipitation of a drug over time in a large number of samples. A wide variety of methods to generate supersaturation are reported in the literature, including use of amorphous solids, shifts in temperature or $\mathrm{pH}$, use of salts or solvent shifts. ${ }^{[54]}$ Of these techniques, the most common is solvent shift, which involves dissolving the API in high concentrations in a favourable solvent (e.g. DMSO), a small volume of which is then added an aqueous phase to generate a supersaturated 


\section{Thesis Papers}

state. Analytical techniques such as UV spectroscopy, HPLC or nephelometry can then be used to assess API con centration or concentration of precipitate over time, which in turn gives information about the efficiency of the inhibi tor being studied ${ }^{(5 s)}$

For example, during a drug development regime, two Johm son and Johnson trugs, $A$ and $B$, required aditition of $\mathrm{PIs}$ to $\mathrm{a}$ surfartant based bioenabling formulation that generated supersaturation but did not itself prevent precipitation. ${ }^{[120]}$ To select an appropriate PI candidate, spersaturation was generated in the presence of a range of potential PIs for both compounds, and then, HPLC was employed to determine residual drug concentration after $24 \mathrm{~h}$. This sareening platform identified Pluronic F 27 as the most efficient PI. ${ }^{[96,120]}$ As a side note, this type of experimental $s$ et up is particularly attrar tive when pursuing surfartant based formulations, as the methodologr can simultaneously screen surfartant systems as well as PIs. In this respect, one can simultaneously assess the extent of the supersaturation generated by the surfartant and how sustained the profile is in the presence of PIs. ${ }^{[9]}$ The main drawback of the given experimental design is that barely any information is obtained about the kinetics of drug precipitation in the presence of polymer as only a single time point is used.

An alternative screening plafform, which utilised off line chromatography, was reported by Petrusevska ot $a l^{[125)}$ In this study, supersaturation was generated for fenofibrate and carbamazepine, in the presence of PIs, using a solvent shift from DMSO into aqueous buffer. The plates were sealed and incubated, with samples being taken at 30, 50, 180 and $360 \mathrm{~min}$, filtered and analysed with UPLC for APY content. This method provides more information about which PI is the most efficient over physiologically relevant timescales. In this study, it was found that surfartants such as Tween and Cremophor ${ }^{\circ}$ were most efficient for fenofi brate, whereas for carbamazepine, cellulose derivatives such as HPMC and HPMCAS were the optimal systems. ${ }^{[125]}$

In spite of the limited time resolution that such 'off line' methods can provide, they can often be very reliable when it comes to predicting performance of the final formula tion. Yamashita and colleagues performed a similar high throughput screen for a range of surfartants, oils and polymers in combination with itraconazole. ${ }^{[120]}$ The screen demonstrated that HPMCAS was the most efficient 'anti precipitator'. When itraconazole HPMCAS spray dried dispersions were manufactured and compared to the com mertally available Sopranox HPMC based dispersions, the HPMCAS based formulations significantly outper formed the commerial product in dissolution tests. ${ }^{[126]}$

Analytical techniques that offer in situ analysis are very appealing as they can provide a real time picture of super saturation precipitation behaviour. It was demonstrated by Warren and colleagues that utilising in situ nephelometry, a technique that uses light scartering to measure particle con centration, can provide an indirect measure of API concen tration in a high throughput sareen. ${ }^{[50]}$ In this instance, the nephelometer measures light scartering of the samples, which directly relates to the total concentration of particu lar marter in suspension and APY ín solution. A large num ber of species were sareened for precipitation inhibition using a plate reader, after which the researchers were able to sort the Pls into three distinct groups based on the neph elometry data (Figure 6$)^{[S a)}$

Chauan et al. ${ }^{(0)}$ expanded upon this technique by utilis ing an in situ UV probe that provided time resolved infor mation about the concentration of API in solution. This

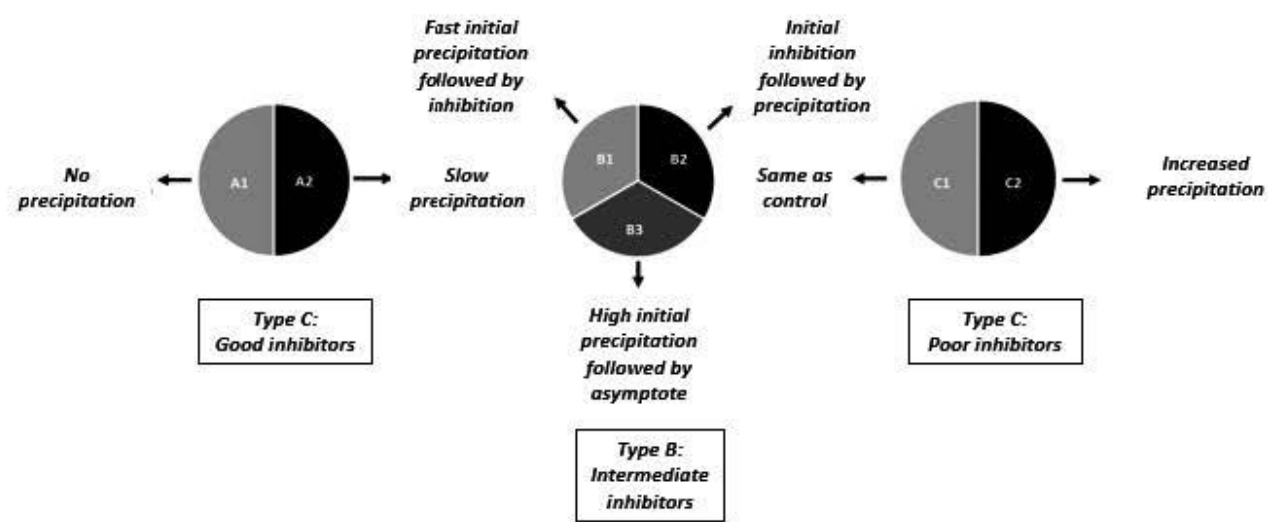

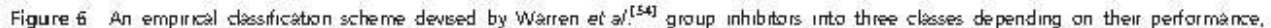
developed using high-throughput PI screening. 


\section{Thesis Papers}

method was used to assess the interactions between indomethacin and a wide range of polymers. After a solvent shift to generate supersaturation, the turbidity and API concentration were measured using an in situ UV probe. The timeresolution of the data is highly useful and allows further calculation and processing to be carried out. Chauan and colleagues subsequently used their data to calculate precipitation induction time (time delay between supersaturation and precipitation) as well as rate of precipitation, in which they showed that PVP, HPMC and Eudragit E100 increased induction time and decreased the rate. Subsequently, successful solid dispersions of indomethacin-PVP, indomethacin-HPMC and indomethacin-Eudragit ${ }^{\circledR}$ E100 were developed. ${ }^{[70]} \mathrm{A}$ similar study, with dipyridamole, was also carried out. ${ }^{[91]}$ The downside of using nephelometry as a screening tool is the difficulty of screening any systems that are insoluble. For example, some supersaturating formula tions, for example mesoporous silica, contain insoluble excipients that would interfere with the light scattering and make analysis difficult.

Recent advances in PI screening have seen the introduction of smaller scale dissolution techniques, such as the $\mu$ DISS profiler ${ }^{\text {TM }}$ apparatus, produced by Pion. The $\mu$ DISS utilises in situ UV in combination with liquid handling and can be used to efficiently study supersaturation and precipitation in real-time. Palmelund et al ${ }^{[127]}$ were able to study six different poorly soluble drugs in combination with HPMC or PVP at different degrees of supersaturation. This method was successful in discriminating between innate solubility enhancements of the polymers vs precipitation inhibition. For the BCS IV drug, aprepitant, for example, both polymers increased solubility by approximately $150 \%$, with the solubility being the same in both polymer systems. That being said, there were distinct differences in the curves observed in the real-time data display, with HPMC showing a more pronounced effect on the dissolution profile than PVP. Therefore, for this system, HPMC acted as a more effective PI than PVP. ${ }^{[70]}$ The $\mu$ DISS profiler ${ }^{\text {TM }}$ is particularly appealing as the experimental protocols can be easily standardised to reduce inter- and intralaboratory variability. ${ }^{[127]}$ The $\mu$ DISS profiler ${ }^{\text {TM }}$ has also been applied to investigate the effect of prandial state and PIs on the precipitation of supersaturated zafirlukast. ${ }^{[129]}$ Further methods have also been employed in small-scale precipitation testing. ${ }^{128}$ 130]

\section{Approaches for precipitation inhibitor selection and increased mechanistic understanding}

Although screening, and especially small-scale screening, of PIs is attractive from a throughput perspective, it adds little to the mechanistic understanding of precipitation inhibition. Detailed mechanistic investigations usually require a comparatively larger testing scale that employs advanced analytical methods. In recent years, such studies have come to the forefront as the need for precipitation inhibitors has become greater. This section offers an overview of these recent advances as well as a more general overview of the analytical tools that are important, and will continue to be of importance, in the design and selection of PI systems for supersaturating formulations. Also important to the development of PI formulations is their in vivo performance. Although this has been covered extensively in the literature, it is beyond the scope of this review. The interested reader is referred to a number of recent papers that study the effect of PI selection on in vivo performance of supersaturating formulation. ${ }^{[24,115,131]}$

\section{Experimental approaches}

Recently, there have been a wide range of novel experimental approaches applied to precipitation inhibitor selection. In addition, these approaches often offer a wealth of mechanistic detail. For a summary of the experimental approaches described in this section, please see Table 2 .

\section{NMR spectroscopy}

Nuclear magnetic resonance (NMR) spectroscopy is a spectroscopic technique that exploits the electromagnetic emission of a nuclei in a magnetic field to gain structural information about the sample. ${ }^{[132]}$

Ueda and colleagues utilised ID NMR spectroscopy as a tool to assess the impact of HPMCAS substitution pattern on the precipitation behaviour of carbamazepine. ${ }^{[133]}$ In their study, it was observed that HPMCAS successfully inhibited the precipitation of carbamazepine, depending on the ratio of succinyl and acetate groups in the polymer. Specifically, it was observed that the highest degrees of carbamazepine supersaturation were sustained in the presence of HPMCAS grades with low succinyl and high acetyl substitutions. The increased acetyl substitution was concluded to be essential for precipitation inhibition, in line with the idea that the more hydrophobic the polymer, the higher the affinity for the growing crystal surface as discussed above. In order to expand upon this assumption, the group utilised ${ }^{1} \mathrm{H}$ NMR spectroscopy to provide information about the molecular mobility of carbamazepine in solution, with a range of HPMCAS variants. A good correlation was observed between precipitation inhibition and the molecular mobility. A lower molecular mobility corresponded to a more successful precipitation inhibition, due to increased interaction between the drug and the polymer. It was hypothesised that this interaction consisted of insertion of HPMCAS into growing aggregates before nucleation, which prevents the formation of the crystal lattice. ${ }^{[133]}$ 


\section{Thesis Papers}

Table 2 summary of experimental techniques recently applied for selection and increased understanding of precipitation inhibition

\begin{tabular}{|c|c|c|c|c|c|}
\hline Method & Type & Theory & Application & Limitations & Ref. \\
\hline $\begin{array}{l}\text { 1D nuclear } \\
\text { magnetic } \\
\text { resonance } \\
\text { (NMR) } \\
\text { spectroscopy }\end{array}$ & Spectroscopic & $\begin{array}{l}\text { - Individual nuclei have unique } \\
\text { response to magnetic fields } \\
\text { - Molecules have a distinct } \\
\text { NMR spectrum based } \\
\text { on their response } \\
\text { - Changes to the environment } \\
\text { of the nuclei can affect } \\
\text { the response }\end{array}$ & $\begin{array}{l}\text { - Structural information } \\
\text { - Interaction between } \\
\text { two species } \\
\text { - Mobility }\end{array}$ & $\begin{array}{l}\text { - Molecule must be } \\
\text { sufficiently soluble } \\
\text { - weaker interactions } \\
\text { cannot be studied } \\
\text { - No information about } \\
\text { intermolecular bonding }\end{array}$ & {$\left[\begin{array}{ll}131 & 133\end{array}\right]$} \\
\hline $\begin{array}{l}\text { Nuclear } \\
\text { Overhauser } \\
\text { Effect } \\
\text { Spectroscopy } \\
\text { (NOESY) }\end{array}$ & Spectroscopic & $\begin{array}{l}\text { - Cross-relaxation and magnetic } \\
\text { transfer during spin } \\
\text { interactions } \\
\text { lead to NOE effects } \\
\text { - For NOEs to occur, } \\
\text { the two nuclei must } \\
\text { be close in space }\end{array}$ & $\begin{array}{l}\text { - Determination of } \\
\text { intermolecular } \\
\text { interactions }\end{array}$ & $\begin{array}{l}\text { - Molecule must be } \\
\text { sufficiently soluble } \\
\text { - Atoms of interest must } \\
\text { not have overlapping } \\
\text { spectra } \\
\text { - Resolution can be poor }\end{array}$ & [134] \\
\hline $\begin{array}{l}\text { Diffusion- } \\
\text { ordered } \\
\text { spectroscopy } \\
\text { (DOSY) }\end{array}$ & Spectroscopic & $\begin{array}{l}\text { - Uses pulses to measure the } \\
\text { speed of travelling complexes } \\
\text { - Diffusion coefficients can be } \\
\text { calculated }\end{array}$ & $\begin{array}{l}\text { - DOESY diffusion coefficients } \\
\text { can be correlated to size of } \\
\text { interacting species } \\
\text { - Can differentiate between } \\
\text { API in solution and an } \\
\text { API-PI complex } \\
\text { - Orthogonal confirmation } \\
\text { of NOE } \\
\text { interactions }\end{array}$ & $\begin{array}{l}\text { - Molecule must be } \\
\text { sufficiently soluble } \\
\text { - Atoms of interest } \\
\text { must not } \\
\text { have overlapping } \\
\text { spectra } \\
\text { - Resolution can be poor }\end{array}$ & [135] \\
\hline $\begin{array}{l}\text { Solid-state } \\
\text { (SS) NMR } \\
\text { spectroscopy }\end{array}$ & Spectroscopic & $\begin{array}{l}\text { - NMR spectroscopy applied } \\
\text { to solids } \\
\text { - Magic angle spinning } \\
\text { (MAS) is used to limit } \\
\text { the effects of } \\
\text { directionally dependent } \\
\text { interactions, also known } \\
\text { as anisotropy }\end{array}$ & $\begin{array}{l}\text { - MAS conditions can } \\
\text { be applied } \\
\text { to liquids } \\
\text { - This can be used to } \\
\text { improve } \\
\text { resolution of interactions }\end{array}$ & $\begin{array}{l}\text { - Not common } \\
\text { - Equipment can be hard } \\
\text { to access }\end{array}$ & [136] \\
\hline $\begin{array}{l}\text { FTIR } \\
\text { spectroscopy }\end{array}$ & 5pectroscopic & $\begin{array}{l}\text { - Infrared light absorption } \\
\text { produces a different } \\
\text { vibrational response } \\
\text { depending on chemical } \\
\text { environment }\end{array}$ & $\begin{array}{l}\text { - Structural elucidation } \\
\text { - Determination of } \mathrm{i} \\
\text { ntermolecular } \\
\text { interactions }\end{array}$ & $\begin{array}{l}\text { - Difficult to carry out in } \\
\text { solution due to water's } \\
\text { individual } \\
\text { response to IR light } \\
\text { - Weak molecular } \\
\text { interactions } \\
\text { cannot be resolved }\end{array}$ & [137] \\
\hline $\begin{array}{l}\text { UV Vis } \\
\text { spectroscopy }\end{array}$ & Spectroscopic & $\begin{array}{l}\text { - Absorption of light in } \\
\text { the UV-visible range } \\
\text { produces a different } \\
\text { vibrational response } \\
\text { depending on chemical } \\
\text { environment }\end{array}$ & $\begin{array}{l}\text { - Concentration } \\
\text { concentration } \\
\text { quantification by } \\
\text { Beer-Lambert law } \\
\text { - Determination of } \\
\text { intermolecular } \\
\text { interactions } \\
\text { - Dissolution kinetics } \\
\text { - Precipitation kinetics }\end{array}$ & $\begin{array}{l}\text { - Weak molecular } \\
\text { interactions } \\
\text { cannot be resolved } \\
\text { - Molecule must absorb } \\
\text { in the UVNis range }\end{array}$ & [138] \\
\hline $\begin{array}{l}\text { Raman } \\
\text { Spectroscopy }\end{array}$ & Spectroscopic & $\begin{array}{l}\text { - Absorption of inelastic } \\
\text { Raman light from lasers } \\
\text { produces a different } \\
\text { vibrational response } \\
\text { depending on } \\
\text { chemical environment }\end{array}$ & $\begin{array}{l}\text { - Structural elucidation } \\
\text { - Determination of } \\
\text { intermolecular interactions } \\
\text { - Analysis in solution }\end{array}$ & $\begin{array}{l}\text { - Only a small proportion } \\
\text { of light will be in the } \\
\text { Raman range (ca. 10-8\%) } \\
\text { - Weak interactions } \\
\text { cannot be resolved }\end{array}$ & {$\left[\begin{array}{ll}144 & 145\end{array}\right]$} \\
\hline
\end{tabular}




\section{Thesis Papers}

\begin{tabular}{|c|c|c|c|c|c|}
\hline Method & Type & Theory & Application & Limitations & Ref. \\
\hline $\begin{array}{l}\text { Fluorescence } \\
\text { Spectroscopy }\end{array}$ & Spectroscopic & $\begin{array}{l}\text { - Measured fluorescence } \\
\text { after adsorption of light }\end{array}$ & $\begin{array}{l}\text { - Determination of } \\
\text { intermolecular interactions } \\
\text { - Highlight changes in } \\
\text { hydrophobicity and } \\
\text { hydrophilicity } \\
\text { - Demonstration of phase } \\
\text { change behaviour }\end{array}$ & $\begin{array}{l}\text { - Required a fluorescent } \\
\text { probe }\end{array}$ & [49] \\
\hline $\begin{array}{l}\text { Differential Scanning } \\
\text { Calorimetry (DSC) }\end{array}$ & Thermal & $\begin{array}{l}\text { - Measures the heat input } \\
\text { required to raise the } \\
\text { temperature of a sample }\end{array}$ & $\begin{array}{l}\text { - Determination of the glass } \\
\text { transition temperature } \\
\text { - Solid state characterization } \\
\text { - Determination of } \\
\text { intermolecular } \\
\text { interaction }\end{array}$ & $\begin{array}{l}\text { - Does not consider } \\
\text { the effect of water } \\
\text { - Cannot measure weak } \\
\text { interactions }\end{array}$ & [150] \\
\hline Synchrotron & Diffractometry & $\begin{array}{l}\text { - Electromagnetic radiation } \\
\text { emitted } \\
\text { from charged particles } \\
\text { accelerated } \\
\text { in a curved path } \\
\text { - synchrotrons can be } \\
\text { used as an x-ray source } \\
\text { - Enhances the flux } \\
\text { of } x \text {-rays, which improves } \\
\text { the resolution }\end{array}$ & $\begin{array}{l}\text { - Detection of early } \\
\text { stage crystals }\end{array}$ & $\begin{array}{l}\text { - Expensive } \\
\text { - Equipment is not } \\
\text { widely available }\end{array}$ & {$\left[\begin{array}{ll}147 & 149\end{array}\right]$} \\
\hline Video Microscopy & Microscopy & $\begin{array}{l}\text { Video microscopes can be } \\
\text { combined with image } \\
\text { analysis to record } \\
\text { precipitation in progress }\end{array}$ & $\begin{array}{l}\text { - Image analysis can } \\
\text { calculate precipitation } \\
\text { initiation time } \\
\text { - Detection of early } \\
\text { crystallization events }\end{array}$ & $\begin{array}{l}\text { - Complicated set up } \\
\text { - Not well-established }\end{array}$ & [118] \\
\hline $\begin{array}{l}\text { Atomic Force } \\
\text { Microscopy } \\
\text { (AFM) }\end{array}$ & Microscopy & $\begin{array}{l}\text { - High-resolution microscopy } \\
\text { - Cantilever, or tip, interacts } \\
\text { with the surface of the } \\
\text { sample } \\
\text { - This interaction deflects } \\
\text { an electron beam } \\
\text { - The pattern of the electron } \\
\text { bean can provide } \\
\text { information about } \\
\text { the sample down to the } \\
\text { nanometer scale }\end{array}$ & $\begin{array}{l}\text { - Imagine of crystal } \\
\text { surfaces } \\
\text { - Can be used to study } \\
\text { polymer surface } \\
\text { coverage }\end{array}$ & $\begin{array}{l}\text { - Expensive } \\
\text { - Equipment is not } \\
\text { common place }\end{array}$ & [49] \\
\hline
\end{tabular}

In a recent study by Prasad et al., ${ }^{[92]} 1 \mathrm{D}{ }^{1} \mathrm{H}$ NMR spectroscopy was utilised to probe the interactions behind the inhibitory effect of a range of polymers on indomethacin precipitation after the generation of supersaturation. It was hypothesised that interactions between the polymers and the carboxylic acid functionality of indomethacin were essential for precipitation inhibition. Thus, the chemical shift of the carboxylic acid functional group, at $3.70 \mathrm{ppm}$, was closely monitored for changes that could indicate that the chemical environment surrounding the protons had been altered. Eudragit ${ }^{\circledR}$ E100 and PVP, when combined with drug in solution, shifted the carboxylic acid peak to a lower value, due to shielding effects. ${ }^{\left[{ }^{[92]}\right.}$ The investigators utilised this shift to quantify the strength of drug-polymer interaction and subsequent precipitation inhibition effect. Eudragit E100 resulted in a larger downward shift than PVP, and for both polymers, this shift was directly proportional to the concentration of the polymer. Additionally, it was observed that when the formulation was changed from a binary system (drug/polymer), to a ternary system (drug/ polymer 1/polymer 2 ), the shift was even more pronounced. This provided evidence for a synergistic contribution of both polymers to the precipitation inhibition. Dissolution performance of the drug in the presence of 


\section{Thesis Papers}

polymers revealed that a larger and more sustained supersaturation was generated with Eudragit E100 than with PVP, while the ternary system gave the best results. ${ }^{[92]}$ Therefore, the change in chemical shift was shown to be a useful parameter when assessing the effect of polymers on indomethacin precipitation.

Although use of 1D NMR spectroscopy to gain information about molecular mobility and chemical shift variations can be a useful tool when assessing the impact of PIs, it is somewhat limited in its ability to detect weaker intermolecular interactions, which are likely to play a highly important role in precipitation inhibition. Furthermore, typical ID NMR spectroscopy gives information about atoms that are chemically bonded and can only provide limited information about intermolecular or supramolecular effects. However, there is a range of 2D NMR spectroscopic techniques that can yield information about correlations of different atoms through space such as nuclear Overhauser effect spectroscopy (NOESY) and diffusion-ordered spectroscopy (DOSY). ${ }^{[134,135]}$

Prior to their work surrounding the importance of substituent ratios for HPMCAS precipitation inhibition, which utilised ID NMR spectroscopy for the calculation of molecular mobility, Ueda and colleagues first established the mechanism of interaction between HPMCAS and carbamazepine in solution by utilising NOESY. ${ }^{[145]}$ During this experiment, it was observed that HPMCAS-HF (a particular grade of HPMCAS, relating to the ratio of acetyl to succinyl substituents) had cross-peak interactions with the aromatic protons and amide protons of carbamazepine, suggesting the possibility of both hydrogen bond interactions and hydrophobic interactions. After further inspection of the intensities of the cross-peaks, it was concluded that the more predominant effect was a hydrophobic interaction between the HPMCAS acetyl substituents and the aromatic region of carbamazepine. ${ }^{[145]}$ This interaction was concluded to be essential for successful precipitation inhibition.

Nuclear Overhauser effect spectroscopy has also been used in combination with high-resolution magic angle spinning (HR-MAS) NMR spectroscopy, to understand the interactions between the poorly soluble drug mefenamic acid and Eudragit ${ }^{\circledR}$ EPO in supersaturated solutions. ${ }^{[136]}$ HR-MAS NMR spectroscopy is an enhanced spectroscopic technique that can offer improved resolution for the study of complex solutions and has been used in the pharmaceutical industry to detect and quantify API in complex formulations such as gels and creams. ${ }^{[137]}$ Higashi et al. were able to significantly improve the NMR spectra for mefenamic acid-EPO solutions under MAS conditions. This allowed cross-peaks to be observed during the NOESY experiments. These cross-peaks showed evidence of multiple points of interaction between the API and the polymer, indicating two different interactions: first, a hydrophobic interaction between the aromatic part of the API and the EPO backbone, and, second, a hydrophilic hydrogen bond interaction between the aminoalkyl groups of EPO and the carbonyl groups of mefenamic acid was described. Furthermore, it was observed that the intensities of the two sets of cross-peaks were similar, leading the authors to conclude that both interactions played an important role in precipitation inhibition. ${ }^{[36]}$

Diffusion-ordered spectroscopy was an essential part of a study assessing the suitability of a novel spray-dried dispersion matrix, HPMCAS and dodecyl $\left(\mathrm{C}_{12}\right)$ poly( $\mathrm{N}$-isopropylacrylamide) (PNIPAm), for the system's suitability to enhance the delivery of a poorly soluble drug, phenytoin. ${ }^{[146]}$ After dissolution of the solid dispersion, the $\mathrm{C}_{12}$ PNIPAm polymers formed micelles with the dodecyl groups, which successfully sustained the supersaturated state of phenytoin generated by the spray-dried dispersion (SDD). Furthermore, it was observed that the $\mathrm{C}_{12}$-PNIPAm inhibited precipitation of the supersaturated phenytoin by inclusion of the drug within the corona of the micelles, rather than the core. It was also concluded that the HPMCAS in the formulation had little effect on sustaining the supersaturation compared to $\mathrm{C}_{12}$-PNIPAm, which instead, was responsible for the enhanced dissolution of the drug from the SDD. ${ }^{[146]}$ These conclusions were reached using both NOESY and DOSY data. The NOESY spectra of the novel formulation showed cross-peak interactions between the phenyl groups of the phenytoin and the isopropyl functionality on the PNIPAm polymer. Conversely, NOESY spectra revealed no cross-peaks for phenytoin combined with HPMCAS. On application of DOSY as an orthogonal approach, no reduction in diffusion coefficient for phenytoin was observed in HPMCAS or $\mathrm{C}_{2}$-PNIPAm. Conversely, the diffusion coefficient decreased dramatically in the presence of $\mathrm{C}_{12}$-PNIPAm, in a concentration-dependent manner. This provided the researchers with strong evidence that the $\mathrm{C}_{12}$-PNIPAm was responsible for the remarkable sustained supersaturation that was observed upon dissolution of this novel SDD, as well as the mechanism taking place within the corona of the micelles, which was not present in the $C_{2}$ variant. ${ }^{[146]}$

\section{Solid-state NMR spectroscopy}

Chauhan et al. ${ }^{[0]}$ utilised carbon cross-polarisation magic angle spinning SS-NMR spectroscopy to investigate the interactions between indomethacin and polymers in solid dispersions. Three different polymers: Eudragit ${ }^{\circledR}$ S100, PVP and HPMCAS were screened for indomethacin interactions using this method. Chemical shift changes in the indomethacin signal were recorded in the presence of the polymers, with a larger chemical shift change indicating a stronger interaction. For the aromatic region, a slight 


\section{Thesis Papers}

chemical shift change was observed for all three polymers, with the biggest shift occurring in the PVP SDD: this correlated well with the observed performance of the SDD during biorelevant dissolution, which outperformed all other polymers due to enhanced precipitation inhibition. ${ }^{\text {[70] }}$

\section{IR/FTIR spectroscopy}

FTIR spectroscopy is highly attractive in a drug development setting as it can be employed to study interactions between compounds, even in complex mixtures. ${ }^{[137]} \mathrm{Nie}$ et al. ${ }^{[147,148]}$ performed an experiment to determine interactions between clofazimine and hypromellose phthalate (HPMCP), which has been previously reported to have very high drug loading capacity in solid dispersions. IR spectra were analysed to identify changes in the vibrational modes of clofazimine and HPMCP in a solid dispersion. ${ }^{[147]} \mathrm{A}$ new peak, at $3310 \mathrm{~cm}^{-1}$, was observed in the IR spectrum of the solid dispersion, which corresponded to the stretching mode of the ionised imine in the clofazimine. This was present due to protonation by the carboxylic acid groups in the phthalate substituent of the HPMCP. A sensitivity analysis showed that the effect was no longer observed at ratio less than 1:0.5 w/w (API/polymer). Additionally, the intensity of the peak increased with increasing HPMCP concentration. This acid-base interaction between HPMCP and clofazimine was further supported by the appearance of peaks at 1540 and $1395 \mathrm{~cm}^{-1}$, which both correspond to the formation of a carboxylate group. ${ }^{[147]}$ Knowledge about solid-state interactions can be directly correlated to solidstate stability and loading capacity, that is drug to polymer ratio, as well as to enhanced precipitation inhibition and supersaturation. Indeed, the combination of HPMCP and clofazimine in a solid dispersion resulted in a 10 -fold increase in apparent clofazimine solubility. ${ }^{[147]}$

Petrusevska and colleagues also employed FTIR spectroscopy to investigate the mechanism of interaction between a successful API-PI formulation: sirolimus and HPMC. ${ }^{[149]}$ Solid dispersions of HPMC and sirolimus demonstrated significant variation from the neat samples as well as a physical blend. Specifically, the sirolimus peaks at $1680-1640 \mathrm{~cm}^{-1}(\mathrm{C}=\mathrm{C})$ and $1760-1670 \mathrm{~cm}^{-1}(\mathrm{C}=\mathrm{O})$ in the solid dispersion were far broader than those from the pure drug, which the authors concluded was a result of interaction between the two species. ${ }^{[149]}$ This interaction was suggested to be partly responsible for the two-fold increase in supersaturation and dissolution in the sirolimus-HPMC solid dispersion vs the commercially available Rapamune ${ }^{\circledR}$ nanoparticles. In a subsequent human pharmacokinetic study, the novel formulation significantly outperformed the commercial formulation. This effect was attributed to the enhanced precipitation inhibition properties of HPMC in the novel formulation. ${ }^{[149]}$
Another application of FTIR spectroscopy is the characterisation of precipitates. In a recent study by Chavan, IR spectroscopy was used to verify that potential polymeric PIs did not affect the solid-state phase behaviour (polymorphism) of the drug, nifedipine. ${ }^{[143]}$ In this instance, the FTIR spectra of the precipitates for all three polymers (HPMC, PVP and HPC) aligned well with crystalline nifedipine, indicating that no polymorphic change was induced by the polymers.

\section{UV-Vis spectroscopy}

UV-Vis spectroscopy enables quantitative analysis of any molecule that absorbs light in the UV-Vis range, which make it a very useful spectroscopic technique, arguably, one of the most widely used in pharmacentical sciences. ${ }^{[138]}$ This section will focus on instances where UV-Vis spectroscopy has been applied for more advanced studies, such as in the determination of interactions between drug and polymer or in-depth studies looking at precipitation kinetics during dissolution of supersaturating formulations.

Nie and colleagues used UV-Vis spectroscopy as an orthogonal technique to support their mechanistic hypothesis for clofazimine-HPMCP interaction. ${ }^{[147]}$ This was especially useful as clofazimine is red in both crystalline and amorphous forms, but a colour shift to purple occurred in the presence of HPMCP in solid dispersions. Qualitatively, this was also observed for mixtures of drug and carboxylic acid analogues (e.g. glacial acetic acid), but not for polymers without carboxylic acids, such as HPMC. It was concluded that the bathochromic shift was associated with a proton transfer from the carboxylic acid functional group of HPMCP. ${ }^{[147]}$ In combination with principal component analysis, the API : PI ratio at which no interaction was observed was calculated to be at ratios below $1: 0.5$. Using the same approach, it was concluded that the API : PI ratio at which full deprotonation of the imine occurs, that is the strongest interaction, was at $1: 1.5^{[147,148]}$ Such information can be valuable in the design and development of PI-based formulations. A similar study was conducted by Misic et al..$^{[150]}$ in the investigation of acid-base interactions between the poorly soluble drugs, loratadine and carvedilol, and oleic acid.

Patel et at. ${ }^{[151]}$ also utilised UV-Vis spectroscopy in combination with mathematical modelling. This study involved the combination of online second-derivative UV spectroscopy and modelling using the diffusion-reaction model to give real-time concentration values and mechanistic insight for indomethacin in supersaturated solutions. This methodology was able to provide a large amount of information about the precipitation behaviour, including that at high degrees of supersaturation, the precipitation 


\section{Thesis Papers}

was bulk diffusion limited, which fits in well with the diffusion-reaction model. ${ }^{[151]}$

UV-Vis spectroscopy can also be employed to increase the understanding of phase behaviour of supersaturated solutions, which can aid in the selection of PIs. Jackson et al ${ }^{[48]}$ utilised two techniques, UV-Vis spectroscopy and fluorescence spectroscopy to determine the phase change behaviour of danazol during LLPS into drug-rich amorphous or drug-rich crystalline phases in the presence and absence of PIs. During phase separation (e.g. LLPS), danazol scatters light, which increases UV extinction. Therefore, it is possible to use UV-Vis spectroscopy to determine the concentration at which LLPS occurs in supersaturated solution. ${ }^{[47]}$ It was reported that LIPS onset occurred at $13 \mu \mathrm{g} / \mathrm{ml}$, a value which could be decreased to varying degrees in the presence of polymers. Furthermore, it was determined that apparent decrease in the LLPS induction time in the presence of polymers correlated well with the ultimate performance of the polymer, with HPMCAS and HPMC showing both the biggest decrease on LIPS induction time (both to $8 \mathrm{~s})$ and the biggest increase in precipitation induction time (277 and $163 \mathrm{~s}$, respectively). ${ }^{[48]}$ Therefore, in-line UV spectroscopy could be a valuable tool in the assessment of precipitation inhibition efficiency via the study of LLPS induction times from supersaturated solutions. This is a particularly attractive posibility as LLPS induction time can be measured relatively easily using this method in a highthroughput experimental set-up.

\section{Raman spectroscopy}

Raut et al. ${ }^{[3]}$ utilised an in situ Raman probe, placed inside a dissolution set-up, to investigate the precipitation inhibition effect of vitamin E TPGS on two model compounds, probucol and indomethacin, in a self-emulsifying drug delivery systems. To achieve this, the formulations were added to a solution at $\mathrm{pH} 1.2$, followed by a $\mathrm{pH}$ shift to $\mathrm{pH}$ 6.8. The Raman probe enabled the collection of timeresolved Raman spectra for both the solid precipitate and the species in solution, which were analysed for molecular interactions between the drug and excipients. For probucol, Raman peaks were observed at 540 and $1164 \mathrm{~cm}^{-1}$, corresponding to the hydroxyl groups in the molecules. How ever, in the presence of vitamin E TPGS, this peak dropped significantly in intensity, with $1164 \mathrm{~cm}^{-1}$ disappearing completely. This was attributed to the interaction of the probucol hydroxyl groups with carbonyl groups of the PI. These interactions had a profound effect on precipitation, with no precipitation observed in the presence of vitamin $\mathrm{E}$ TPGS, in spite of the system being supersaturated to 100 fold of the thermodynamic solubility of probucol. ${ }^{[2]}$ Similar observations were made for indomethacin. Interestingly, in the case of indomethacin, it was observed that interactions were only evident whenever a certain 'supersaturation threshold' was obtained, ${ }^{[82]}$ below which interactions were not observable and precipitation occurred. This is an important factor to bear in mind: although a drug and polymer may theoretically interact strongly, the formulation in question must generate a particular concentration before interactions will occur.

In addition to probing interactions in solution, Raman spectroscopy is a useful tool for investigating short-range interactions in the solid state. This can be particularly beneficial in the development of solid dispersion formulations, such as hot melt extrusion (HME) and spray-dried dispersions (SDD), where both drug-polymer miscibility and the precipitation inhibition performance of the polymer are based on these interactions. Chauhan et al. ${ }^{[91]}$ utilised this technique, among a wide range of spectroscopic tools, to develop solid dispersions of dipyridamole. The team found that the most successful formulations consisted of drugHPMC and drug-Eudragit E100 ${ }^{\circledR}$, which performed significantly better than all other polymers screened. Utilising solid-state Raman spectroscopy, it was revealed that interactions were present between the drug and HPMC and Eudragit E100 ${ }^{\oplus}$. 91$]$

Raman spectroscopy can also be applied to study the extent of drug precipitation because dissolved and solid drug generally differ in their spectra. Both types of spectra can be used in a multivariate calibration. Thus, in-line dispersive Raman spectroscopy has been used to monitor drug precipitation from supersaturated dipyridamole solutions using a transfer test ${ }^{[152]}$; and this spectroscopic approach has also been reported to study drug precipitation from lipid-based formulations in the course of digestion. ${ }^{[153]}$

\section{Fluorescence spectroscopy}

Fluorescence spectroscopy can be used to determine interaction mechanisms, changes in hydrophobicity and phase change behaviour. This is achieved by utilising fluorescence probes, such as pyrene. ${ }^{[48]}$ Pyrene is a good probe for hydrophobicity, as when it preferentially partitions into hydrophobic microenvironments, a change in its fluorescence absorption bands, $I_{1}$ and $I_{3}$, occurs. ${ }^{[48]}$ Jackson et al. exploited this phenomenon in order to assess the concentration at which LLPS occurred in the presence of danazol and to determine whether a crystalline or non-crystalline phase was formed. For LLPS, it was determined that no significant change in the $I_{1} / I_{3}$ ratio in the pyrene fluorescence spectra occurred below danazol concentrations of $13 \mu \mathrm{g} / \mathrm{ml}$ in the pure sample. Above this, the change in $I_{1} / I_{3}$ aligned well with the formation of a non-crystalline LLPS, suggesting an onset of LLPS at $13 \mu \mathrm{g} / \mathrm{ml}$. Additionally, it was observed that incorporation of a polymeric PI decreased the LLPS onset concentration, with HPMCAS and HPMC 


\section{Thesis Papers}

having the biggest impact, decreasing LLPS onset concentration to 8 and $9 \mu \mathrm{g} / \mathrm{ml}$, respectively. Observing the changes in $I_{1} / I_{3}$ can provide information about the induction time of precipitation as, generally speaking, the $I_{1} / I_{3}$ ratio returns to normal after recrystallisation. This is due to the disappearance of the drug-rich phase and the expulsion of pyrene from the crystal lattice and back into the aqueous phase. ${ }^{[154]}$ In the study, all three polymers showed a sustained $I_{1} / I_{3}$ ratio for at least $15 \mathrm{~min}$. These data correlated with the induction times, with HPMCAS showing the longest induction time of 4 h. ${ }^{[48]}$

Creasey et al. utilised this technique to assess the interaction between Pluronic and Labrasol in a formulation being developed for two Johnson and Johnson compounds. Pyrene solution was added to solutions of Labrasol, Pluronic and mixtures of the two. Fluorescence spectra for pyrene were then collected to record the effect of Pluronic on the micropolarity of the Labrasol formulations. ${ }^{[124]}$ In this instance, the $I_{1} / I_{3}$ ratio in the mixture of Labrasol and Pluronic was significantly lower than for Labrasol alone, which indicates a more pronounced hydrophobic microenvironment in the sample. It was hypothesised by the researchers that this increased hydrophobicity was the key factor for inhibiting precipitation, allowing the drug to be held more tightly within the microstructures formed by the surfactant. Finally, this mechanism had a profound effect on both compounds with a 500 - and 200 -fold increase in concentration compared to no excipients added. ${ }^{[124]}$

\section{Differential scanning calorimetry}

Differential scanning calorimetry (DSC) is a thermal analysis technique that sees wide application in pharmaceutical sciences, for example in solid-state characterisation. ${ }^{[141]}$ Although not as common, DSC can also be used to investigate interactions between two species. However, traditional DSC cannot achieve this and instead, modulated DSC (MDSC) must be used. MDSC differs from traditional DSC in that it operates using two simultaneous heating rates, in contrast to the single linear heating rate used in DSC. MDSC utilises both a linear heating rate and a modulated heating rate that allows simultaneous measurement of the heat capacity of the sample.

Chauhan et al ${ }^{[91]}$ used MDSC to investigate the mechanism by which dipyridamole (DPD) interacts with a range of PIs. Previously, MDSC has been used to assess miscibility between a drug and polymer, based on changes in melting events. Expanding on this idea, the melting temperatures of the DPD and DPD precipitates in the presence of polymers were used to identify whether any drug-polymer interaction was taking place. For Eudragit ${ }^{\circledR}$ E100, Eudragit ${ }^{\circledR}$ S100 and HPMC, additional melting endotherms were observed in the MDSC curves. The authors of the study reasoned that this change in the thermal behaviour occurred due to interaction of the drug with the polymers in solution, which corresponded with an increased precipitation inhibition. Indeed, out of the six polymers studied, only those polymers where a change in melting temperature of the precipitates was observed were successful PIs. ${ }^{[91]}$ Moreover, the authors also cautioned that, although melting point changes were present, this was not a definitive proof of interaction as certain polymers, for example PEG, can dissolve a drug and therefore alter the melting temperature. Rather, DSC is a useful tool to determine lack of interaction, as was the case with the unsuccessful PIs, Eudragit \$100, Eudragit L100 and PEG 8000 . To state that an interaction is definitely present, MDSC should be used with complementary analytical techniques.

\section{Synchrotron radiation}

Synchrotron radiation is the electromagnetic radiation emitted from charged particles that are accelerated in a curved fashion, for example in a circular particle accelerator. ${ }^{[142]}$ For diffraction studies, synchrotron radiation enhances the flux of X-ray radiation, ${ }^{[144]}$ which leads to diffraction patterns with higher resolutions, obtained in a shorter period of time. The technique is a highly sophisticated analytical tool, and practical usage is limited due to the fact that the equipment is not common and is highly expensive. Therefore, synchrotron light sources exist at dedicated sites, of which there are only a few around the world (a list of dedicated sites can be found at: http:// www.lightsources.orgflight-source-facility-information). ${ }^{\text {[143] }}$

It was reported by Van Eerdenbrugh and colleagues that synchrotron radiation could be utilised to study the precipitation and crystallisation behaviour of API in a supersaturated solution. ${ }^{[155]}$ This was achieved using wide-angle $\mathrm{X}$-ray scattering (WAXS) with synchrotron X-ray beams. The research group concluded that the method could detect crystalline particles at a minimum suspension concentration of $2.6 \mathrm{mg} / \mathrm{ml}$ for all samples in their 52-sample study, including a large proportion of poorly soluble model drugs, ${ }^{[156]}$ which translates to a sensitivity of around $0.26 \%$ $\mathrm{w} / \mathrm{w}$ drug in aqueous suspension. This sensitivity is significantly higher than all other conventional methods for detecting crystallinity and is also in agreement with previous studies, which showed that crystals with a size of $<1 \mathrm{~nm}$ could be detected. ${ }^{[155]}$

Further work by this group incorporated PIs into the same experimental design, to study mechanistically the effect of PIs on the crystallisation time of a range of compounds. ${ }^{[157]}$ In this study, the solvent-shift method was applied to generate supersaturated solutions in the presence of a particular amount of predissolved polymer. The onset time of crystallisation was examined using several 


\section{Thesis Papers}

measurements over $24 \mathrm{~h}$, to determine the delay in onset of crystallisation. Combining the results with polarised light microscopy demonstrated the presence of LLPS, which was prolonged in the presence of some polymers. Similar to other reports in the literature, ${ }^{[73]}$ the most hydrophobic and hydrophilic polymers were not effective PIs, supporting the hypothesis that an effective PI must interact with both the aqueous medium and API. ${ }^{[158]}$

Due to the high costs and low availability of the synchrotron methods and equipment, it is unlikely that this approach will ever find widespread adoption for screening of precipitation inhibitors, and certainly not in routine drug development. Additionally, although it is significantly more sensitive than standard methods, there is still a limit to how easily one can detect the early crystallisation events taking place during precipitation.

\section{Video microscopy}

For screening of PIs, microscopy is an attractive approach as the efficiency of inhibitors can be visualised, offering insight into early crystallisation events that would not be typically observable via conventional methods such as detection by UV spectroscopy. However, the use of manual microscopy is not highly prevalent in the literature, probably due to the time and labour requirements. To circumvent this, various studies have combined conventional microscopy with video analysis. ${ }^{[14]}$

Recent work by Christfort et al. utilised such a system to investigate supersaturated solutions of tadalafil in the presence and absence of a PI, HPMC. Samples were viewed under video light microscopes, and multiparticle analysis was carried out on the videos to assess total precipitation as a function of time. ${ }^{[114]}$

\section{Atomic force microscopy}

Atomic force microscopy (AFM) is a type of high-resolution microscopy that can image on the nanometre scale. Due to this high resolution, AFM has been applied to study the effect of polymer surface coverage on growing crystals, and how this relates to precipitation inhibition. According to the adsorption layer model, before a solute molecule can join a growing crystal lattice, it must adsorb and interact with the surface at a site with high attraction forces. This interaction can be hindered or disrupted with the use of polymers, which can bind the solute in solution or adsorb onto the growing crystal surface. For the latter mechanism, it has been hypothesised that understanding the conformation of polymers on the surface of the growing lattice is key to inhibition of crystal growth. ${ }^{[75,158]}$

Expanding upon this idea Schram et al ${ }^{[76]}$ studied the effect of the $\mathrm{pH}$ on the adsorption of HPMCAS on felodipine crystals and the subsequent effect on precipitation inhibition, using AFM and in situ UV spectroscopy, respectively. It was observed during the AFM experiments that at $\mathrm{pH} 3.0$ the polymer was adsorbed as small aggregates and at $\mathrm{pH} 6.8$ the whole surface was covered with a monolayer. The space between two polymer aggregates at $\mathrm{pH} 3.0$ ranged from 25 to $50 \mathrm{~nm}$, compared to $0.9 \mathrm{~nm}$ at $\mathrm{pH} 6.8$. Therefore, at a higher $\mathrm{pH}$, there was a greater polymer surface coverage and greater inhibition of crystal growth. Indeed, the ratio of crystal growth (crystal growth rate in the absence of an inhibitor/crystal growth rate in the

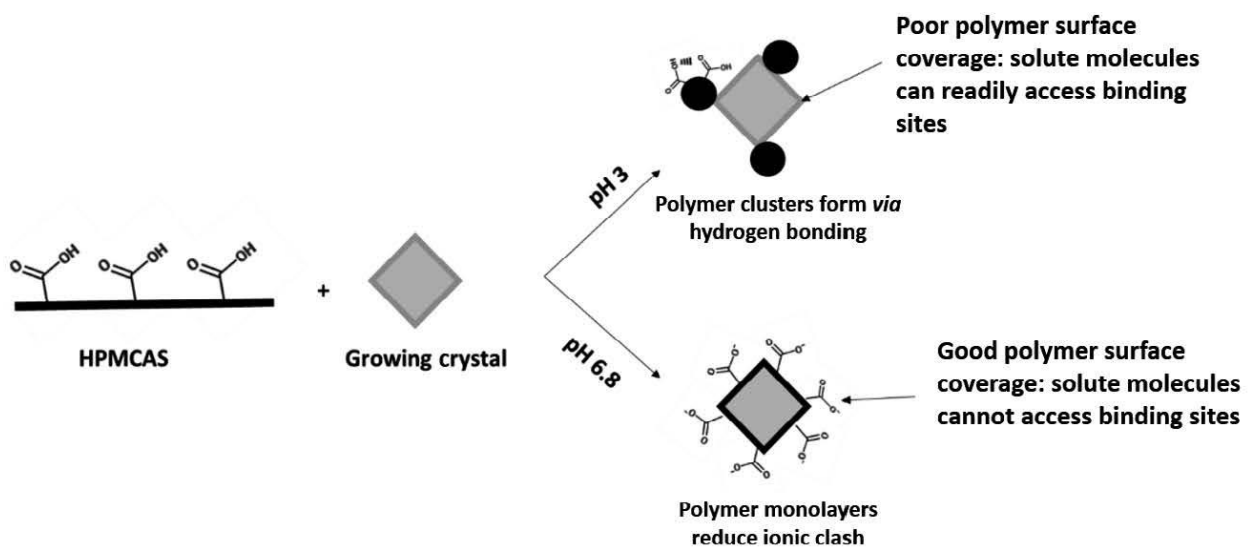

Figure 7 schematic diagram showing the conclusions of $5 \mathrm{chram}^{[75]}$ : polymer surface coverage in relevant physiological environment is a crucial factor to be considered in the selection of polymeric precipitation inhibitors. 


\section{Thesis Papers}

presence of inhibitor) was 1.28 and 2.29 at $\mathrm{pH} 3$ and 6.8 , respectively. This was explained by the intra- and intermolecular hydrogen bonding of HPMCAS at the different pHs investigated. ${ }^{[75]}$ With a pKa of 5.5, HPMCAS is unionised at $\mathrm{pH}$ 3. As a result, intermolecular interaction between the succinyl groups can occur, which allows the polymer to form coils and aggregates. Conversely, at $\mathrm{pH}$ 6.8 , the polymer is ionised, and self-repulsion results in the molecules forming a monolayer on the surface with no interaction between the succinyl groups. These observations led Schram and colleagues to conclude that the conformation of a polymer, and more importantly the total surface coverage of a polymer, is crucial when considering the observed inhibitory effect (Figure 7). ${ }^{[75]}$

Additional work by Schram and colleagues was dedicated to establishing a correlation between polymer surface coverage and precipitation inhibition. ${ }^{[158]}$ For this, the group again employed atomic force microscopy (AFM) to study the interaction between a range of polymers and felodipine crystals. The measurements were made in suspension, with height and phase contrast images recorded simultaneously. The phase contrast in the images is dependent on the surface characteristics of the sample, and therefore, difference between surface properties can be detected between different samples. ${ }^{[159]}$ The images were then analysed using 'Imagej', a software that can detect changes in digital images based on pixel changes, ${ }^{[160]}$ and thus could be used to calculate total surface coverage of the polymer on the growing crystal. In the study, it was reported that polyacrylic acid (PAA), a hydrophilic polymer, was not adsorbed to the crystal surface, while PVP, another hydrophilic polymer, formed aggregates to decrease the surface area exposed to the crystal surface to $8 \pm 0.8 \%$ coverage. At the other end of the scale, it was observed that hydrophobic polymers like polyvinyl acetate (PVAc) also minimised their exposed surface area to the liquid by forming aggregates adsorbed on the surface of the crystal (14 $\pm 0.7 \%$ coverage). ${ }^{[158]}$ By contrast, polymers with both properties (hydrophobicity and hydrophilicity), like HPMC, resulted in the best surface coverage by adsorbing to the surface of a crystal in a more monolayer-like fashion ( $54 \pm 0.8 \%$ coverage). ${ }^{[158]}$ A linear correlation between surface area coverage and polymer effectiveness (based on the aforementioned crystal growth ratio) was established, showing that a good surface coverage can predict good precipitation inhibition.

Atomic force microscopy is a useful tool for studying polymer surface coverage and conformation, essentia parameters when considering the effectiveness of a PI in supersaturating formulation. In combination with knowledge of the PI conformations throughout the physiological $\mathrm{pH}$ range, such parameters have the potential to be utilised routinely as part of a selection protocol for PIs. More work is required to bridge the gap between these proof-of- concept studies towards a more widespread application which could operate in industrial settings. Particular emphasis should be placed on demonstrating correlations with different API-PI systems, and on the development and validation of the in silico models to predict polymer performance based on polymer surface coverage, which could offer a very attractive avenue in the prescreening phase of PI selection. One issue may be the accessibility and complexity of AFM, which is not often commonly used in early-stage development.

\section{Computational approaches}

\section{Mathematical modelling}

Mathematical modelling of physical, physicochemical and physiological processes can be a useful tool in drug devel-

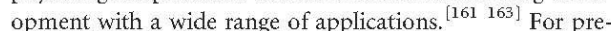
cipitation inhibition, models that are related to adsorption, crystallisation and dissolution have been very valuable in a number of key studies. However, the use of mathematical models to aid in the understanding of precipitation behaviour, and the effect of inhibitors, has not yet been adopted widely.

Adsorption modelling. Early work in the literature utilised well-established adsorption isotherms, including the Langmuir ${ }^{[164]}$ and Freundlich models, ${ }^{[161]}$ to predict the adsorption of additives on crystal surfaces and consequently to estimate the crystal growth kinetics inhibition through mathematical modelling. This approach is often used in combination with crystallisation models such as the Kubota-Mullin model, which models crystal growth. ${ }^{[163,165,166]}$

The Kubota-Mullin model applies a step-growth model, which assumes that a crystal grows monolayer by monolayer. The growth inhibition depends on the PI and the distance between the adsorbed species on the surface. In the model, crystal growth is linked to fractional surface coverage, $\theta$, which is the ratio of the average distance between the potential adsorption sites, $L$, and the average distance between the adsorbed species, $l$; and the free energy of the unit length (Equation 2). ${ }^{[163,165,166]}$

$$
\theta=\frac{L}{l}
$$

Expanding on their work using AFM to probe the polymer surface coverage of a range of PIs on felodipine crystals, Schram and colleagues adapted the model to predic polymer performance from the experimentally obtained surface coverage values. ${ }^{[158]}$ In this case, polymer effectiveness, $R_{\mathrm{p}} / R_{0}$, is the ratio of crystal growth in the presence $\left(R_{p}\right)$ and absence of polymer $\left(R_{0}\right)$ and depends on the 


\section{Thesis Papers}

fractional surface coverage, $\theta$; the relative supersaturation, $\sigma$; the edge free energy per unit length, $\gamma$; the size of a growth unit, $a$; the temperature, $T$; the Boltzmann constant, $k$; and the average distance between absorbed polymers, $l$ (Equation 3)

$$
\frac{R_{\mathrm{p}}}{R_{0}}=1-\frac{\gamma a}{k T \sigma(\theta l)} \theta
$$

The average distance between polymer molecules is specific for each system and depends on the amount of polymer adsorbed to the surface. Thus, $l$ was proportional to the experimental polymer surface coverage determined by AFM Consequently, a correlation was established, with which $l$ could be determined from the polymer surface coverage. This allows the calculation of the fractional surface coverage in the Kubota-Mullin model (Equation 2) and subsequently the theoretical effectiveness of crystal growth inhibitors (Equation 3). In this study, the theoretical effectiveness calculations were in good agreement with the experimental values. ${ }^{[158]}$

Alonzo et al. ${ }^{[52]}$ also adopted the Kubota-Mullin model to study the effect of HPMC on crystal growth and nucleation of felodipine in a supersaturated solution. In combination with the Langmuir adsorption model, the research team explored the effect of HPMC on felodipine crystallisation. Based on the model, it was predicted that the effect of HPMC on the crystallisation of felodipine crystals was highly dependent on the extent of supersaturation, with greater supersaturation reducing the effect of the inhibitor. The theoretical predictions were in agreement with the experimental crystal growth rate in the presence of HPMC It was also predicted that polymers successfully interrupting the nucleation process would be more advantageous than those that affect the crystal growth rate, which was supported experimentally. ${ }^{[52]}$ Further work utilising the Kubota-Mullin model was carried out by Ilevbare et al., ${ }^{[4]}$ who were able to successfully estimate the crystallisation rate of the poorly soluble drug, ritonavir, in the presence and absence of a polymer.

One of the most extensive studies on the use of adsorption and crystallisation models was carried out by Pate et al. ${ }^{[69,103,151]}$ They studied the precipitation behaviour of indomethacin in the presence of PIs. The effect of molecular weight on the inhibition potential of PVP and of $\mathrm{N}$-vinylpyrrolidone on indomethacin crystallisation ${ }^{[69]}$ was investigated using adsorption isotherms generated via the solution depletion method. Using a wide range of molecular weights, the two inhibitors were combined with indomethacin in an aqueous suspension and allowed to equilibrate for a set amount of time. The samples were then filtered and analysed using size exclusion chromatography to determine the concentration of the adsorbed polymer, which provided a value of the extent of inhibitor surface adsorption. These values were then used to construct adsorption isotherms based on the Langmuir model. It was shown that the adsorption potential for PVP was greater than N-vinylpyrrolidone. Further, the isotherms demonstrated that the adsorption capacity for PVP was directly proportional to PVP molecular weight. These model predictions were then validated experimentally, with PVP significantly outperforming $\mathrm{N}$-vinylpyrrolidone in the inhibition of indomethacin precipitation, with increasing molecular weight of PVP also correlating to a higher degree of sustained supersaturation. ${ }^{[103]}$

Further work by Patel and Anderson employed the previously developed second-derivative UV method in combination with a first-order crystal growth model to investigate the growth rates of indomethacin in the presence of various PIs. ${ }^{\text {[151] }}$ HPMC and PVP significantly outperformed $H P \beta C D$, in agreement with the inhibition models. For HPBCD, precipitation inhibition was modelled using diffusion layer theory, based on the assumption that HP $\beta C D$ complexes with indomethacin in the diffusion layer of crystal growth. This model successfully predicted that, at high degrees of supersaturation, HP $\beta C D$ inhibition could be related to the reversible complexation between the two species at the diffusion layer. ${ }^{[151]}$

Molecular modelling. Many types of molecular modelling can be used in simulation of crystallisation or precipitation, from quantum mechanical approaches, to Monte Carlo methods to molecular dynamics simulations. ${ }^{[167]}$ Molecular simulations of crystallisation and precipitation are of high interest as they can offer a high throughput that can be applied to a wide range of systems. Mandal et al. ${ }^{[162]}$ developed a framework that enabled the simulation of crystallisation behaviour of a range of organic molecules in the presence of inhibitors. In this study, a coarse-grained (CG) model for crystal growth ${ }^{[168]}$ based on force fields obtained from simulators was applied to a range of molecules. Coarse-graining is an approach that allows the simulation of complex systems without using extensive computation time due to the use of simplified atomistic representations. Such an approach is often used to model the interaction of proteins and small molecules. ${ }^{[169]}$ There are many software packages that can carry out the CG process, such as MARTINI ${ }^{[68]}$; however, such software packages often oversimplify the molecules such that information important to understand crystallisation behaviour may be lost. To improve upon these established CG processes, Mandal and colleagues utilised a CG model based on the radial distribution functions of the molecules, which were obtained from atomic simulations carried out in the crystalline states. ${ }^{[162]}$ As a result, the CG model developed was able to simulate crystal growth of the organic molecule, phenytoin, in the absence and presence of the polymer HPMCAS. ${ }^{[162,168]}$ 


\section{Thesis Papers}

Furthermore, the simulation was able to correctly predict that inhibition of phenytoin by HPMCAS is highly dependent on the substitution of the polymer, with an increased acetate substitution slowing crystal growth most effectively. This simulation proved to be very robust in its prediction as these observations were also demonstrated experimentally. ${ }^{[162]}$

\section{Chi parameter and interaction enthalpy}

A rigorous thermodynamic treatment of PI selection would have to consider the drug and PI in water as a ternary system, where both the solid-liquid equilibrium of solubility and liquid-liquid phase separation of amorphous solubility are considered. ${ }^{[8,40]}$ Such a pursuit is very attractive given the high degree of information one can learn about the system. An example of such a theoretical model is the perturbed-chain statistical associating fluid theory (PC-SAFT), which bears much promise but is also demanding, due to the various parameters that are required to fully describe a system. ${ }^{[0]}$ Such highly parameterised methods still have to be adopted in the area of precipitation inhibition to enable an early in silico screening of excipients. Moreover, a focus on equilibrium thermodynamics may not be the most descriptive way to model drug precipitation due to the importance of kinetics. Non-equilibrium thermodynamics is an even more challenging approach, and so far, attempts have only been made to consider interactions parameters of simpler thermodynamic models for empirical kinetic considerations.

To consider the kinetics of precipitation, the interaction parameter $\chi$ of the Flory-Huggins $(\mathbf{F H})$ theory ${ }^{[170,171]}$ can be employed. ${ }^{[172,173]}$ The Flory-Huggins solution equation (Equation 4) describes the thermodynamic behaviour of polymers in solution. The equation is an adaption of the standard Gibbs energy equation, introducing extra terms to adjust the entropy portion to account for the dissimilarity of molecular sizes. In the Flory-Huggins equation, the enthalpic portion of the Gibbs equation is represented by the chi parameter, $\chi$ :

$$
\Delta G=R T\left[n_{1} \ln \varphi_{1}+n_{2} \ln \varphi_{2}+n_{1} \varphi_{2} \chi_{1,2}\right]
$$

In the Flory-Huggins equation, $R$ is the ideal gas constant and $T$ is the absolute temperature. $n_{1}, n_{2}$ and $\varphi$ are the number of moles and volume fraction, respectively, for components 1 and 2 of the system, while $\chi$ is the interaction enthalpy upon association between components 1 and 2.

Expanding upon the Flory-Huggins equation, one can consider mixtures of drug and polymers, whereby the enthalpic contribution becomes, $\chi_{\mathrm{DPP}}$. In this approach, it is assumed that the interactions considered in $\chi_{\mathrm{DP}}$ are also relevant for the kinetics of sustaining drug supersaturation. This is based on the understanding that some of the mechanisms by which a polymer can inhibit precipitation are dependent on energetic interactions such as hydrophobic, polar or hydrogen bond interactions between the drug and PI. ${ }^{[54]}$ The $\chi_{\mathrm{DP}}$ parameter can be determined experimentally by combining the Flory-Huggins equation with experimental DSC measurements. ${ }^{\text {[17] }}$ This parameter has been applied to other areas of drug development, for example in the assessment of drugpolymer miscibility in the screening of candidate formulations. ${ }^{[175]}$ It is possible to utilise in silico predictions of the $\chi_{D P}$ parameter, which reduces the number of experiments required, thus allowing more focus on the most promising formulations. This can be achieved in a number of ways, with one key strategy combining the total solubility parameters of the drug, $\delta_{\mathrm{D}}$, and polymer, $\delta_{\mathrm{P}}$, in relation to the molar volume of the drug, $V_{m}$, the temperature, $T$, and the ideal gas constant, $R$ (Equation 5). [176]

$$
\chi_{\mathrm{DP}}=\frac{V_{\mathrm{m}}\left(\delta_{\mathrm{D}}-\delta_{\mathrm{P}}\right)^{2}}{R T}
$$

The extended Hansen solubility parameters, $\delta_{\mathrm{D}}$, and $\delta_{\mathrm{P}}$, can be predicted based on chemical structure alone, using group contribution methods. ${ }^{[177]}$ Indeed, this determination of $\chi_{D P}$ via partial solubility parameters has been used to construct entire phase diagrams for solid dispersions based on the Flory-Huggins theory. ${ }^{[178]}$

In addition to group contribution methods, it has been shown that solubility parameters can be predicted based on quantitative structure-property relationships (QSPR). ${ }^{[19}$ 181] With rising computational power, there is also the option to use molecular dynamic (MD) simulations to calculate solubility parameters and hence to determine $\chi_{\mathrm{DP}}$. A first calculation option is to simulate internal energy change due to vaporisation, $\Delta E_{\mathrm{v}}{ }^{[182]}$ This $\mathrm{MD}$ approach utilises the original definition of the solubility parameter as a cohesive energy density (CED) (Equation 6) ${ }^{[183] .}$.

$$
\delta=(\mathrm{CED})^{1 / 2}=\left[\frac{\Delta E_{\mathrm{v}}}{V_{\mathrm{m}}}\right]^{1 / 2}
$$

In this instance, total energy difference for isolated molecules and for the bulk system with periodic boundary conditions provides an estimate of $\Delta E_{\mathrm{v}}{ }^{[183]}$ That being said, calculation of the cohesive energy difference and conversion of the solubility parameter into $\chi_{\mathrm{Dp}}$ are not the only way to obtain the Flory-Huggins interaction parameter by means of molecular simulations. Fan et al. ${ }^{[184]}$ developed a molecular simulation method to derive phase diagrams of binary mixtures (Equations 7 and 8 ). 


\section{Thesis Papers}

$$
\begin{gathered}
\chi_{1,2}=\frac{z \Delta w_{1,2}}{R T} \\
\Delta w_{1,2}=w_{1,2}-\frac{1}{2}\left(w_{1,1}+w_{2,2}\right)
\end{gathered}
$$

The interaction parameter between two molecules, $\left(\chi_{1,2}\right)$, is obtained directly from the corresponding pairwise interactions, $w$, and hence the energies $w_{1,1}, w_{2,2}, w_{1,2}$ (Equations 7 and 8 ). This can be achieved by averaging large number of configurations using a Monte Carlo approach, taking into consideration the number of neighbouring molecules (i.e. coordination number, $z$ ) in combination with the ideal gas constant, $R$, and temperature, $T$.

There are numerous routes to obtain the Floryy-Huggins $\chi_{\text {DP }}$. That being said, there are only a few examples of this parameter being used to understand precipitation inhibition. Baghel et al. ${ }^{[173]}$ studied solid dispersions of dipyridamole (DPY) and cinnarizine (CNZ) with polyvinylpyrrolidone (PVP) and polyacrylic acid (PAA). It was found that the combinations capable of forming hydrogen bonds (DPY-PVP; DPY-PAA and CNZ-PAA) in the solution state were more effective at keeping the drus in supersaturation than those not able to hydrogen bond (CNZ-PVP). In this instance, CNZ-PVP had the highest predicted $\chi_{\mathrm{DP}}$ parameter, suggesting a less stable interaction, in line with the observed precipitation inhibition results. However, it was noted that, despite their significantly different supersaturation performance, the difference between the $\chi_{\mathrm{D}}$ parameters of CNZ-PVP and CNZ-PAA was not great, and that other aspects such as the hydrophilicity of the polymer should also be considered.

Similar findings were also reported by Chen et al. ${ }^{[172]}$ who compared solid dispersions of griseofulvin, felodipine and ketoconazole with PVP vinylacetate (PVP-VA) and HPMCAS. Although felodipine interacted much more effectively with PVP-VA in the solid state $\left(\chi_{\mathrm{DP}}=-1.9\right)$ than with HPMCAS $\left(\chi_{\mathrm{DP}}=-0.21\right)$, this behaviour was not replicated in aqueous dispersions, where the HPMCAS solid dispersion generated higher and more sustained supersaturation profiles upon dissolution. This was likely due to the hydrophilic interactions of PVP-VA with water upon exposure to an aqueous environment, which may have reduced or negated the favourable interactions with felodipine.

\section{Mechanistic design of precipitation inhibitors}

The development and use of modern analytical techniques have provided an increased understanding of PI interactions. Utilising knowledge of potential binding modes with the drug, it has become possible to select-or even design - PIs on a drug-by-drug basis.
The first example of such an approach was published by Ting et al., ${ }^{[68]}$ who synthesised co-polymers inspired by HPMCAS using reversible addition-fragmentation chain transfer (RAFT) polymerisation. These novel polymers were then used in SDDs and studied for interactions with probucol, danazol and phenytoin, to determine which structural motifs were important for inhibition of precipitation. For probucol, the most efficient polymers were those with only succinyl and acetyl functionality, improving dissolution up to 180 -fold. Conversely, for danazol and phenytoin, polymers that exhibited a higher prevalence of hydroxyl groups were able to better sustain supersaturation. This was supported by FTIR spectroscopy. In the FTIR spectra, $-\mathrm{OH}$ stretching absorptions of danazol shifted and broadened in the presence of polymers with high proportions of $-\mathrm{OH}$ functionality, indicating the presence of hydrogen bond interactions. ${ }^{[68]}$

Mosquera-Giraldo et al. ${ }^{[185]}$ also utilised a mechanistic design approach to fine-tune inhibition of telaprevir precipitation by cellulosic polymers. In this approach, a large number of chemically diverse cellulosic polymers were synthesised containing different combinations of key functional group substituents such as alcohols, amides, esters, ethers, carboxylic acids and sulphides. The novel polymers were grouped into two distinct chemical groups: the $\omega$-carboxyalkanoates, which were cellulosic polymers with a wide variety of hydrocarbon chain lengths capped with a terminal carboxylic acid, and a second group which included the aforementioned chemically diverse functional groups. The solubility parameters for the novel polymers were calculated, and they were also screened using an in situ UV probe to determine the onset of precipitation in supersaturated telaprevir solutions generated with the solvent-shift approach. It was found that for the $\omega$-carboxyalkanoates, both the solubility parameter range and the carboxylic acid functionality were important for precipitation inhibition. Similar conclusions were reached in the second group of polymers, in which it was found that the only functional group that appeared to influence precipitation was the terminal carboxylic acid, with all other functionality showing little to no effect on precipitation inhibition. Furthermore, there was also a direct correlation with hydrocarbon chain length and ultimate PI performance. Ultimately, it was concluded that both a terminal carboxylic group and long chain length were essential for effective inhibition, with the carboxylic acid providing hydrophilicity for the drug to remain in solution, while the hydrocarbon chain was essential for hydrophobic interactions with the growing crystal surface. [185]

Expanding on the aforementioned studies, Ting et al. ${ }^{[186]}$ were the first to demonstrate a de novo design of polymeric inhibitors based on molecular interactions with phenytoin. A high-throughput excipient design process that 


\section{Thesis Papers}

could yield a wide range of chemically diverse polymeric fragments, referred to as 'synthons,' was applied. These synthons were selected based on the known binding interactions of phenytoin in the formation of the crystal lattice, namely the hydrogen bond interaction between carbonyl oxygen and cyclin imines. The fragments selected were $\mathrm{N}$-isopropylacrylamide (NIPAm), diethyl amide (DEA) and isopropyl methacrylate (IPMA), which could insert themselves into the growing crystal lattice via chemical interaction with the phenytoin molecule, thereby disrupting its internal hydrogen bond interactions and thus arrest crystallisation. Hydrophilic partner fragments, dimethyl amide (DMA), amide (AM) and hydroxyethyl methacrylate (HEMA), which can interact with water, were also included in the screen. The resultant polymers thus had the ability to bind to the growing crystal, inhibit crystallisation and maintain the drug in solution. ${ }^{[186]}$ Based on this chemical library of six synthons, a controlled, and high-throughput RAFT polymerisation was used to synthesise a large library of distinct novel polymers, 60 in total. These polymers were then screened for precipitation inhibition of phenytoin in a high-throughput screen utilising the solvent-shift method in combination with UV spectroscopy. Poly (NIPAm70-coDMA30) was able to maintain phenytoin supersaturation at $>1000 \mu \mathrm{g} / \mathrm{ml}$ for over $3 \mathrm{~h}$. This was a significant improvement relative to the commercially available excipient, HPMCAS, which is able to sustain concentrations at only $100 \mu \mathrm{g} / \mathrm{ml}$ for $3 \mathrm{~h}^{[192]}$

Nuclear Overhauser effect spectroscopy and DOSY spectroscopy were used to further assess the potential interaction of the drug phenytoin with the precipitation inhibitor synthons. NOESY data showed that the best performing polymer, poly(NIPAm70-co-DMA30), exhibited strong cross-peaks with the aromatic portion of phenytoin and the isopropyl portion of the polymer, coupled with complementary hydrophilic interactions offered by the DMA. Further, the DOSY data showed a significant decrease in reduced diffusion coefficients for phenytoin in the presence of the polymer. Together, the two spectroscopic techniques were able to elucidate the mechanistic interaction between poly(NIPAm70-co-DMA30) and phenytoin and explain the highly improved precipitation inhibition. ${ }^{\text {[192] }}$

Although the de nowo design of PI to maximise interaction with API is an exciting prospect, there are some key hurdles for this technology to be widely applicable. For example, from a regulatory perspective, such an approach could be very costly and restrictive, as additional safety studies would be required to demonstrate an absence of polymer-related toxicity. Adopting a similar approach that is based on selection of polymers already approved by health authorities such as the FDA for use in pharmaceuticals might be more efficient. Such an approach would retain some of the advantages of a bespoke PI selection process while avoiding any additional regulatory burden.

\section{Concluding remarks}

In recent years, focus has been placed on understanding the stabilisation of the supersaturated state and the importance of PI selection. Although classical crystallisation theory has been well described and applied to in vitro crystallisation and precipitation inhibition, the relative importance of a wide range of PI properties including molecular weight, viscosity and number of hydrogen bond donors/acceptors is not yet entirely clear, with different studies reaching different conclusions. Ultimately, it is accepted that precipitation inhibition is not a 'one-size-fits-all' process and that each API will have different dependencies, which can make a priori PI selection difficult. Although a diverse set of highthroughput screening methods are available which can be used to identify suitable precipitation inhibitors, such an approach does not provide any mechanistic information about how precipitation inhibitors function. With this in mind, huge strides have been made in recent years towards elucidating the precipitation inhibition process for a wide range of drug-inhibitor systems, as covered in this review.

Spectroscopic techniques such as NMR, IR, Raman, UVVis and fluorescence spectroscopy can be employed to study interaction mechanisms between API and polymer as well as to gain understanding about the phase behaviour of supersaturated API solutions. Further work in this area, particularly on developing new techniques to improve sensitivity of detection, would be valuable in order to allow application to a wider range of systems. From a thermal perspective, $\mathrm{mDSC}$ has seen limited application in detecting subtle changes in melting points of drug-polymer mixtures, which can be indicative of molecular interaction. Additional thermal tools, such as isothermal calorimetry, could be investigated to gain insights into additive effects on nucleation and growth inhibition using various excipients. Microscopic techniques, such as atomic force microscopy, have been pivotal in building a picture of how polymers interact with the growing surface. This has been particularly important when understanding the effect of $\mathrm{pH}$ and polarity on polymer surface coverage, which can have a dramatic effect on PI performance. Furthermore, video microscopy has emerged recently as an interesting technique that, when coupled with image analysis, can visualise the crystallisation and nucleation behaviour of supersaturated API. This can be achieved both in the presence and absence of polymer and can allow the detection of early-stage precipitation events that may not be detectable by conventional methods. Another approach which can achieve this high sensitivity is synchrotron radiation, which has been used to study nucleation in detail. It is clear that the application of this technology is not feasible on a larger scale, so more work 


\section{Thesis Papers}

should be carried out on alternative approaches that can provide increased sensitivity for detection of crystallinity. Further progress has also been made on understanding the precipitation/inhibition interplay from a theoretical perspective, using thermodynamic modelling and molecular simulations. In particular, the molecular simulation of atomistic detail in crystallisation and inhibition is a highly interesting area. Such simulations can simultaneously decrease the amount of experimental work required in the development of the formulations while increasing the amount of understanding yielded about the systems studied, which could help to lower the risk when working with the development of supersaturating formulations.

The combination of increased understanding of precipitation inhibition processes with advanced analytics has the potential to completely reshape how PIs are selected in drug development in the future. The possibility of bespoke polymer design or at least bespoke PI selection for each individual supersaturating formulation has also become possible. In this approach, it will be possible to decrease the number of experiments required and perhaps increase the absorption performance of the final formulation, which may in turn reduce the required dose. This would have a downstream impact on the overall efficiency and cost of the development of poorly soluble drug candidates. These savings could ultimately be passed on to the patient or reinvested in innovative drug discovery and development and could lead to earlier access to breakthrough therapies for patients. Therefore, the need for increased mechanistic understanding in the selection of PIs has never been greater.

\section{Declarations}

\section{Conflict of interest}

The Authors declare that they have no conflict of interests to disclose.

\section{Funding}

This project has received funding from the European Union's Horizon 2020 Research and Innovation Programme under grant agreement No. 674909.

\section{References}

1. Krishnaiah YSR. Pharmaceutical tech nologies for enhancing oral bioavail ability of poorly soluble drugs. $J$ Bioequiv Bioavailab 2010; 2: 28-36.

2. Zheng $W$ et al Selection of oral bioavailability enhancing formulations during drug discovery. Drug Dev Ind Pharm 2012; 38: 235-247.

3. Amidon GL et al. A theoretical basis for a biopharmaceutic drug classification: the correlation of in vitro drug product dissolution and in vivo bioavailability. Pharm Res 1995; 12 : 413-420.

4. Lipinski CA. Poor aqueous solubility an industry-wide problem in drug discovery. Am Pharm Rev 2002; 5: 82-85.

5. Lipinski CA. Drug-like properties and the causes of poor solubility and permeability. I Pharmacol Toxicol Methods 2000; 44: 235-249.

6. Gardner CR et al. Drugs as materials: valuing physical form in drug discovery. Nat Rev Drug Discov 2004; 3: 926-943.

7. Yu LX. An integrated model for determination of poor oral drug absorption. Pharm Res 1999; 16: 1883-1887.
8. Taylor L, Zhang GGZ. Physical chemistry of supersaturated solutions and implications for oral absorption. Adv Drug Deliv Rev 2016; 101: $122-$ 142 .

9. Serajuddin ATM. Salt formation to improve drug solubility. Adv Drug Deliv Rev 2007; 59: 603-616.

10. Childs SL et al. Formulation of a danazol cocrystal with controlled supersaturation plays an essential role in improving bioavailability. $\mathrm{Mol}$ Pharm 2013; 10: 3112-3127.

11. Kumpulainen RJ et al. Prodrugs: design and clinical applications. Nat Rev Drug Discov 2008; 7: 255-270.

12. Timpe C. Strategies for formulation development of poorly water soluble candidates - a recent perspective. Am Pharm Rev 2007; 10: 104-109.

13. Porter CJ et al. Lipids and lipidbased formulations: optimizing delivery of lipophilic drugs. Nat Rev Drug Discov 2007; 6: 231-248.

14. Strickley $\mathrm{R}$ et al. Solubilizing vehicles for oral formulation development. In: Augustijins P et al. ed. Solvent Systems and Their Selection in Pharmaceutics and Biopharmaceutics, Biotechnology: Pharmaceutical Aspects,
Vol. VI. New York, NY: Springer, 2007: 257-308.

15. Pole DL. Physical and biological considerations in the use of nonaqueous solvents in oral bioavailability enhancement. I Pharm Sci 2008; 97: 1071-1088.

16. Nakano M. Places of emulsions in drug delivery. Adv Drug Deliv Rev 2000; 45: 1-4.

17. Pouton CW. Lipid formulations for oral administration of drugs: non-emulsifying, self-emulsifying and "self-microemulsifying" drug delivery systems. Eur I Pharm Sci 2000; 11: 9398.

18. Gursoy RN, Benita S. Self-emulsifying drug delivery systems (SEDDS) for improved oral delivery of lipophilic drugs. Biomed Pharmacother 2004; 58: 173-182.

19. Date A et al. Current strategies for engineering drug nanoparticles. Curr Opin Colloid Interface Sci 2004; 9: 222-235.

20. Brewster ME et al. The utility of cyclodextrins for enhancing oral bioavailability. I Controlled Release 2007; 123: 78-99.

21. Leuner C, Dressman J. Improving drug solubility for oral delivery using solid dispersions. Eur J Pharm Sci 2000; 351: 209-218. 


\section{Thesis Papers}

22. Chokshi RJ et at. Improving the dissolution rate of poorly soluble drug by solid dispersion and solid solution: pros and cons. Drug Deliv 2007 14: $33-45$.

23. Laine AL et al. Enhanced oral delivery of celecoxib via the development of a supersaturable amorphous for mulation utilising mesoporous silica and co-loaded HPMCAS. Int Pharm 2016; 512: 118-125.

24. O'Shea JP et al. Mesoporous silicabased dosage forms improve bioavail ability of poorly soluble drugs in pigs case example fenofibrate. I Pharm Pharmacol 2017; 69: 1284-1292.

25. McCarthy CA. Mesoporous silica formulations strategies for drug dissolution enhancement. Expert Opin Drus Deliv 2016; 13: 93-108.

26. Wilson $\mathrm{M}$ et al. Hot-melt extrusion technology and pharmaceutical application. Ther Deliv 2012; 3: 787-797.

27. Brouwers $\mathrm{J}$ et al. Supersaturating drug delivery systems: the answer to solubility-limited oral bioavailability? J Pharm Sci 2009; 98: 2549-2572.

28. Gao P, Shi Y. Characterization of supersaturating formulations for improved absorption of poorly soluble drugs. AAPS J 2012; 5: 1-11.

29. Gao P, Morozowich W. Developmen of supersaturating SEDDS (S-SEDDS) formulations for improving the oral absorption of poorly soluble drugs. Expert Opin Drug Deliv 2005; 3: $97-110$

30. Mullin IW. Crystallisation. Oxford Butterworth-Heinemann, 2001

31. Yalkowsky SH. Solubility and Solubilization in Aqqueous Media. New York: Oxford University Press, 1999.

32. Mer VKL. Nucleation in phase tran sitions. Ind Eng Chem Res 1952; 44 1270-1277.

33. Xu S, Dai WG. Drug PIs in supersaturating formulations. Int $I$ Pharm 2013; 453: 36-43

34. Degen LP, Phillips SF. Variability of gastrointestinal transit in healthy women and men. Gut 1996; 39: 299-305.

35. Holzbach RT. Metastability behaviour of supersaturated bile. Hepatol ogy 1984; 4: 155S-158S.
36. Mersmann A ed. Crystallisation Technology Handbook. Florida, USA: CRC Press, 2001.

37. Kashchiev D, Van Rosmalen GM. Nucleation in solutions revisited. Cryst Res Technol 2003; 38: 555-574.

38. Hancock BC, Parks M. What is the true solubility advantage for amorphous pharmaceuticals? Pharm Re 2000; 17: 397-404

39. Murdande SB et al. Solubility advantage of amorphous pharmaceuticals: II. Application of quantitative thermodynamic relationships for predic tion of solubility enhancement in structurally diverse insoluble phar maceuticals. Pharm Res 2010; 27 2704-2714

40. Paus $\mathrm{R}$ et al. Predicting the solubility advantage of amorphous pharmaceuticals: a novel thermodynamic approach. Mol Pharm 2015; 12: 2823-2833.

41. Hoffman ID. Thermodynamic driv ing force in nucleation and growt processes. I Chem Phys 1958; 29: 1192-1193.

42. Almeida e Sousa L et al. Assessment of the amorphous "solubility" of group of diverse drugs using new experimental and theoretical approaches. Mol Pharm 2014; 12: 484-495.

43. Mosquera-Giraldo LI, Taylor LS Glass-liquid phase separation in highly supersaturated aqueous solutions of telaprevir. Mol Pharm 2015 12: 496-503

44. Maeda $\mathrm{K}$ et al Novel phenomena of crystallisation and emulsification of hydrophobic solute in aqueous solution. I Colloid Interface Sci 2001;234: $217-222$

45. Lafferrère L et al. Study of liquid-liquid demixing from drug solution. Cryst Growth 2004; 269: 550-557.

46. Tung H. Critical Issues in Crystallisa tion Practice. Crystallisation of Organic Compounds: An Industria Perspective. Hoboken, NJ: John Wiley \& Sons Inc, 2008: 101-116.

47. Ilevbare GA et al. Liquid-liquid phase separation behaviour in highly supersaturated aqueous solutions of poorly water-soluble drugs: implications for solubility enhancin formulations. Cryst Growth Des 2013; 13: $1497-1509$.

48. Jackson MJ et al. Dissolution of danazo amorphous solid dispersions: supersaturation and phase behavior as a function of drug loading and polymer type. Mol Pharm 2015; 13: 223-231.

49. Bonnett PE et al. Solution crystallisation via a submerged liquid-liquid phase boundary: oiling out. Chem Commun 2003; 6: 698-699.

50. Deneau E, Steele G. An in-line study of oiling out and crystallisation. Org Process Res Dev 2005; 9: 943-950.

51. Guzmán HR et al. A "spring and parachute" approach to designing solid celecoxib formulations having enhanced oral absorption. AAPS $J$ 2004; 6: T2189.

52. Alonzo DE et al. Characterizing the impact of hydroxypropylmethyl cellulose on the growth and nucleation kinetics of felodipine from supersaturated solutions. Cryst Growth Des 2012; 12: 1538-1547.

53. Guzmán HR et al. Combined use of crystalline salt forms and precipitation inhibitors to improve ora absorption of celecoxib from solid oral formulations. $J$ Pharm Sci 2007 96: 2686-2702.

54. Warren $\mathrm{DB}$ et al. Using polymeric precipitation inhibitors to improve the absorption of poorly water-soluble drugs: a mechanistic basis for utility. $J$ Drug Target 2010; 18: 704-731.

55. Lima-de-Faria I, Buerger MJ. Historical Atlas of Crystallography. Dordrecht Boston: Published for Internationa Union of Crystallography by Kluwer Academic Publishers, 1990

56. Boistelle R, Astier JP. Crystallisation mechanisms in solution. I Cryst Growth 1988; 90: 14-30.

57. Kalikmanov VI. Nucleation Theory Lecture Notes in Physics 860 Springer, 2013.

58. Davey RJ et al. Nucleation of organic crystals - a molecular perspective. Angew Chem Int Ed 2013; 52: 21662179.

59. Kashchiev D. Nucleation: Basic The ory with Applications. Oxford, Boston: Butterworth Heinemann, 2000. 


\section{Thesis Papers}

60. Rodriguez-Hornedo N, Murphy D. Significance of controlling crystallisation mechanisms and kinetics in pharmaceutical systems. J Pharm Sci 1999; 88: 651-660.

61. Lindfors $\mathrm{L}$ et al. Nucleation and crystal growth in supersaturated solutions of a model drug. I Colloids Interface Sci 2008; 325: 404-413.

62. Dunitz JD, Bernstein J. Disappearing polymorphs. Acc Chem Res 1995; 28: 193-200.

63. Nyvlt J. The Ostwald rule of stages. Cryst Res Technol 1995; 30: 443-449.

64. Kostewicz ES et al. Predicting the precipitation of poorly soluble weak bases upon entry in the small intestine. J Pharm Pharmacol 2004; 56: 43-51.

65. Li N et al. A comparison of the crystallisation inhibition properties of bile salts. Cryst Growth Des 2016; 16 7286-7300.

66. Loftsson T. The effect of water-soluble polymers on açueous, solubility of drugs. Int I Pharm 1996; 127: 293-296.

67. Usui $F$ et al. Inhibitory effects of water-soluble polymers on precipitation of RS-8359. Int J Pharm 1997; 154: 59-66.

68. Ting JM et al. Deconstructing HPMCAS: excipient design to tailor polymer-drug interactions for oral drug delivery. Acs Biomater Sci Eng 2015; 1: 978-990.

69. Patel DD, Anderson BD. Effect of precipitation inhibitors on indomethacin supersaturation maintenance: mechanisms and modeling Mol Pharm 2014; 11: 1489-1499.

70. Chauhan $\mathrm{H}$ et al. Correlation of inhibitory effects of polymers on indomethacin precipitation in solution and amorphous solid crystallisation based on molecular interaction. Pharm Res 2014; 31: 500-515.

71. Somasundaran P, Krishnakumar S. Adsorption of surfactants and polymers at the solid-liquid interface. Colloids Surf A 1997; 123: 491-513.

72. Machefer $S$ et al. Effect of polymer admixtures on the growth habit of ionic crystals. Study on crystal growth kinetics of potassium dihydrogen phosphate in water/ polyol mixtures. J Cryst Growth 2008; 310: 5347-5356.

73. Gao $\mathrm{P}$ et al. Characterization and optimization of AMG 517 supersaturating self-emulsifying drug delivery system (S-SEDDS) for improved oral absorption. I Pharm Sci 2009; 98: 516-528.

74. Dinunzio JC et al. Amorphous compositions using concentration enhancing polymers for improved bioavailability of itraconazole. Mol Pharm 2008; 5: 968-980.

75. Ilevbare $\mathrm{G}$ et al. Understanding polymer properties important for crystal growth inhibition-impact of chemically diverse polymers on solution crystal growth of ritonavir. Cryst Growth Des 2012; 12: 3133-3143.

76. Schram CJ et al. Impact of polymer conformation on the crystal growth inhibition of a poorly water-soluble drug in aqueous solution. Langmuir 2015; 31: 171-179.

77. Pellett $\mathrm{M}$ et al. Supersaturated solutions evaluated with an in vitro stratum corneum tape stripping technique. Int J Pharm Sci 1997b; 151: 91-98.

78. Chavan B et al. Evaluation of the inhibitory potential of HPMC, PVP and $\mathrm{PC}$ polymers on nucleation and crystal growth. RSC Adv 2016; 6: 77569-77576.

79. Saal $W$ et al. The quest for exceptional drug solubilization in diluted surfactant solutions and consideration of residual solid state. Eur J Pharm Sci 2018; 111: 96-103.

80. Dai WG et al. Evaluation of drug precipitation of solubility-enhancing liquid formulations using milligram quantities of a new molecular entity (NME). J Pharm Sci 2007; 96: 2957-2969.

81. Rosen MJ, Kunjappu JT. Surfactant and Interfacial Phenomena, 4th edn. Hoboken, NJ: Wiley, 2012

82. Vogt $\mathrm{M}$ et al. Dissolution improvement of four poorly water soluble drugs by cogrinding with commonly used excipients. Eur $J$ Pharm Biopharm 2008; 68: 330-337.

83. Raut Desai S. Investigating the mechanism of supersaturation and precipitation inhibition of poorly soluble drugs from self-emulsifying drug delivery systems (SEDDS). 2013 Ph.D. Thesis, Massachusetts College of Pharmacy and Health Sciences.

84. Brewster ME et al. Comparative interaction of 2-hydroxypropyl- $\beta$-cyclodextrin and sulfobutylether- $\beta$-cyclodextrin with itraconazole: phasesolubility behavior and stabilization of supersaturated drug solutions. Eur J Pharm Sci 2008; 32: 94-103.

85. Iervolino $\mathrm{M}$ et al. Membrane penetration enhancement of ibuprofen using supersaturation. Int $J$ Pharm 2000; 198: 229-238.

86. Loftsson $\mathrm{T}$ et al. Effects of cyclodextrins on drug delivery through biological membranes. J Pharm Sci 2007; 96: 2532-2546.

87. Fine-Shamir $\mathrm{N}$ et al. Toward successful cyclodextrin based solubility-enabling formulations for oral delivery of lipophilic drugs: solubility-permeability trade-off, biorelevant dissolution, and the unstirred water layer. Mol Pharm 2017; 14: 2138-2146.

88. Westerberg G, Wiklund L. b-cyclodex trin reduces bioavailability of orally administered [3H]-benzo[a]pyrene in the rat. J Pharm Sci 2005; 94: 114-119.

89. Chen $\mathrm{J}$ et al. Bile salts as crystallization inhibitors of supersaturated solutions of poorly water-soluble compounds. Cryst Growth Des 2015; 15: 2593-2597.

90. Carlert $S$ et al. Predicting intestinal precipitation: a case example for a basic BCS class II drug. Pharm Res 2010; 27: 2119-2130.

91. Chauhan $\mathrm{H}$ et al. Correlating the behaviour of polymers in solution as PI to its amorphous stabilization ability in solid dispersions. I Pharm Sci 2013; 102: 1924-1935.

92. Prasad D et al. Role of molecular interactions for synergistic precipitation inhibition of poorly soluble drug in supersaturated drug-polymer-polymer ternary solution. Mol Pharm 2016; 13: 756-765.

93. Li S et al. Enhanced bioavailability of a poorly water-soluble weakly basic compound using a combination approach of solubilization agents and PIs: 


\section{Thesis Papers}

a case study Mot pharm 2012. 9: 1100-1108.

94. Dai WG et al. Combination of pluronic/vitamin E TPGS as a potential inhibitor of drug precipitation. Int J Pharm 2008; 255: 31-37.

95. Overhoff KA et al. Effect of stabilizer on the maximum degree and extent of supersaturation and oral absorption of tacrolimus made by ultra-rapid freezing. Pharm Res 2008; 25: 167-175.

96. Patra $\mathrm{C}$ et al. Pharmaceutical significance of Eudragit: a review. Future Pharm Sci 2017; 3: 33-45.

97. Lee DR et al. A polyvinylpyrrolidone-based supersaturating selfemulsifying drug delivery system for enhanced dissolution of cyclosporine A. Polymers 2017; 9: 123

98. Wen $\mathrm{H}$ et al. Hydrogen bonding interactions between adsorbed polymer molecules and crystal surface of acetaminophen. $J$ Colloid Interface So 2005; 290: 325-335.

99. Martinez-Cruz $\mathrm{N}$ et al. Effect of molecular weight of polystyrene sulfonic acid sodium salt polymers on the precipitation kinetics of sodium bicarbonate. Cryst Growth 2004; 270: 573-581.

100. Kawaguchi $\mathrm{H}$ et al. Crystallisation of inorganic compounds in polymer solutions. 1. Control if shape and form of calcium carbonate. Colloid Polym Sci 1992; 270: 1176-1181.

101. Marsden CM et al. Supersaturation of zafirlukast in fasted and fed state intestinal media with and without PIs. Eur I Pharm Sci 2016; 25: 31-39

102. Vora $\mathrm{C}$ et al. Preparation an characterization of dipyridamole solid dispersions for stabilization of supersaturation: effect of precipita tion inhibitors type and molecula weight. Pharm Dev Technol 2016; 21 847-855.

103. Patel DD, Anderson BD. Adsorption of polyvinylpyrrolidone and its impact on maintenance of acueous supersaturation of indomethacin via crystal growth inhibition. $J$ Pharm Sci 2015; 104: 2923-2933.

104. Edwards $\mathrm{F}$ et al. Using droplet-based microfluidic technology to study the precipitation of a poorly water- soluble weakly basic drug upon pH-shift. Analyst 2013; 138: 339-345.

105. Knopp MM et al. Effect of polyme type and drug dose on the in vitro and in vivo behavior of amorphous solid dispersions. Eur J Pharm Biopharm 2016; 105: 106-114

106. Janssens $S$ et al. Characterization of ternary solid dispersions of itraconazole, PEG 6000, and HPMC 2910 E5. J Pharm Sci 2008; 96: 2110-2120.

107. Vandecruys $R$ et al. Use of a screening method to determine excipient which optimize the extent and stability of supersaturated drug solution and application of this system to solid formulation design. Int $J$ Pharm 2007; 342: 168-175.

108. Lang $B$ et al. Dissolution enhancement of itraconazole by hot-mel extrusion alone and the combination of hot-melt extrusion and rapic freezing - effect of formulation and processing variables. Mol Pharm 2014; 11: 186-196

109. Pereira JM et al. Interplay of degradation, dissolution and stabilization of clarithromycin and its amorphous solid dispersions. Mol Pharm 2013, 10: 4640-4653.

110. Shan $\mathrm{N}$ et al. Improved human bioavailability of vemurafenib, practically insoluble drug, using an amorphous polymer-stabilized soli dispersion prepared by a solventcontrolled coprecipitation process. Pharm Sci 2013; 102: 967-981.

111. Huang Y, Dai W-G. Fundamenta aspects of solid dispersion technolog for poorly soluble drugs. Acta Pharm $\sin B$ 2014; 4: 18-25.

112. Tajarobi $\mathrm{F}$ et al. The influence of crystallisation inhibition of HPMC and HPMCAS on model substance dissolution and release in swellable matrix tablets. Eur J Pharm Biopharm 2011; 78: 125-133.

113. Curatolo $W$ et al. Utility of hydroxypropyl methylcellulose acetate succinate (HPMCAS) for initiation and maintenance of drug supersaturation in the GI milieu. Pharm Res 2009 ; 26: 1419-1431.

114. Christfort JF et al. Development of video-microscopic tool to evaluate the precipitation kinetics of poorlywater soluble drugs: a case study with tadalafil and HPMC. Mol Pharm 2017; 14: 4154-4160.

115. Ruff $\mathrm{A}$ et al. Evaluating the predictability of the in vitro transfer model and in vivo rat studies as a surrogate to investigate the supersaturation and precipitation behaviour of different Albendazole formulations for humans. Eur I Pharm Sci 2017; 105: 108-118.

116. Pygall $S$ et al. Extended release of flurbiprofen from tromethamine-buffered HPMC hydrophilic matrix tablets. Pharm Develop Technol 2017. https:// doi.org/ 10.1080/10837450.2017. 1301470. [Epub ahead of print]

117. Kourentas A et al. Evaluation of the impact of excipients and an albendazole salt on albendazole concentrations in upper small intestine using an in vitro biorelevant gastrointestinal transfer (BioGIT) system. I Pharm Sci 2016; 105: 2898-2903.

118. Gift $\mathrm{AD}$ et al. Influence of polymeric excipients on crystal hydrate formation kinetics in aqueous slurries. I Pharm Sci 2008; 97: 5198-5211.

119. Chandy $T$ et al. Inhibition of in vitro calcium phosphate precipitation in presence of polyurethane via surface modification and drug delivery. Appl Biomater 1994; 5: 245-254.

120. Dionisio $\mathrm{M}$ et al. Locust bean gum: exploring its potential for biopharmaceutical applications. I Pharm Bioallied Sci 2012; 4: 175-185.

121. Wu $\mathrm{Z}$ et al. Absorption and tissue tolerance of ricobendazole in the presence of hydroxypropyl-beta-cyclodextrin following subcutaneous injection in sheep. Int I Pharm 2010; 397: 96 102 .

122. Brouwers $\mathrm{J}$ et al. Early identification of availability issues for poorly water-soluble microbicide candidates in biorelevant media: a case study with saquinavir. Antiviral Res 2011, 91: $217-223$.

123. Peeters $\mathrm{J}$ et al. Characterization of the interaction of 2-hydroxypropylbeta-cyclodextrin with itraconazole at pH 2, 4, and 7.J Pharm Sci 2002; 91 $1414-1422$ 


\section{Thesis Papers}

124. Creasey AA et al. Inhibiting the precipitation of poorly water-soluble drugs from labrasol formulations. Pharm Technol 2011; 23: 30-34.

125. Petrusevska $M$ et al. Evaluation of a high-throughput screening method for the detection of the excipientmediated precipitation inhibition of poorly soluble drugs. Assay Drug Dev Technol 2013a; 11: 117-129.

126. Yamashita $T$ et al. Solvent shiff method for anti-precipitant screening of poorly soluble drugs using biorelevant medium and dimethyl sulfoxide. Int J Pharm 2011; 419: 170-174.

127. Palmelund $\mathrm{H}$ et at. Studying the propensity of compounds to supersaturate: a practical and broadly applicable approach. $J$ pharm Sci 2016; 105: 3021-3029.

128. Madsen CM et al. Supersaturation of zafirlukast in fasted and fed state intestinal media with and without precipitation inhibitor. Eur $J$ Pharm Sci 2016; 91: 31-39.

129. Kuentz M. Analytical technologies for real-time drug dissolution and precipitation testing on a small scale. Pharm Pharmacol 2014; 67: 143-159.

130. Quan $G$ et al. Supersaturable solid self-microemulsifying drug delivery system: precipitation inhibition and bioavailability enhancement. Int I Nanomed 2017; 12: 8801-8811.

131. Gunther H. NMR Spectroscopy: Basic Principles, Concepts and Application in Chemistry, 3rd edn. Hoboken, NJ Wiley, 2013.

132. Ohno A et al. Application of NMR spectroscopy in medicinal chemistry and drug discovery. Curr Top Med Chem 2011; 11: 68-73.

133. Garido L, Beckmann N. New Applico tions of NMR in Drug Discovery and Development, 1st edn. London, UK: Royal Society of Chemistry, 2014

134. Kwan EE, Huang SG. Structural elucidation with NMR spectroscopy practical strategies for organic chemists. Eur J Organic Chem 2008; 2018: 2671-2688.

135. Johnson CS. Diffusion ordered nuclear magnetic resonance. Prog Nucl Magn Reson Spectrosc 1999; 34: 203-256.
136. Higashi $\mathrm{K}$ et al Insights into atomiclevel interaction between mefenamic acid and Eudragitei EPO in a supersaturated solution by high resolution magicangle spinning NMR spectroscopy. Mol Pharm 2014; 11:351-357.

137. Gaffney IS et al. Fourier Transform Infrared (FTIR) spectroscopy. In: Kaufman EN, ed. Characterization of Materials, 2nd edn. Hoboken, NJ Wiley, 2012: 1104-1135.

138. Watson D. Pharmaceutical Analysis, 4th edn. Amsterdam, the Netherlands: Elsevier, 2016.

139. Rostron P et al. Raman spectroscopy, review. Int J Eng Tech Res 2016; 6: 5064.

140. Paudel A et al. Raman spectroscopy in pharmaceutical product design. Adv Drug Deliv Rev 2015; 89: 3-20.

141. Sheng $\mathrm{Q}$. Thermal analysis of pharmaceuticals. In: Mullertz A et al. ed. Analytical Techniques in the Pharmaceutical Sciences. New York City, NY Springer, 2016: 363-387.

142. Williams GP. A general review of synchrotron radiation, its uses and special technologies. Vacuum 1982 32: 333-345.

143. Light Source. Light source facility information. http://www.lightsources. org/light-source-facility-information. Accessed July 21st, 2017.

144. Holmes KC et al. Synchrotron radiation as a source for $\mathrm{X}$-ray diffraction the beginning. In: Mandelkow E, ed. Synchrotron Radiation in Chemistry and Biology III. Berlin, Heidelberg: Springer Berlin Heidelberg, 1989: 1-7.

145. Ueda $\mathrm{K}$ et al. Inhibitory effect of hydroxypropyl methylcellulose acetate succinate on drug recrystallization from a supersaturated solution assessed using nuclear magnetic resonance measurements. Mol pharm 2013; 10: 3801-3811.

146. Li $Z$ et al. Enhanced performance of blended polymer excipients in delivering a hydrophobic drug through the synergistic action of micelles and HPMCAS. Langmuir 2017; 33: $2837-$ 2848 .

147. Nie H et al. Solid-state spectroscopic investigation of molecular interactions between dofazimine and hypromellose phthalate in amorphous solid dispersions. Mol Pharm 2016; 13: 3964-3975.

148. Nie $\mathrm{H}$ et al. Investigating the interaction pattern and structural elements of a drug-polymer complex at the molecular level. Mol Pharm 2015; 12 : 2459-2468.

149. Petrusevska $M$ et al. Hydroxypropyl methylcellulose mediated precipitation inhibition of sirolimus: from a screening campaign to a proof-ofconcept human study. Mol Pharm 2013b; 10: 2299-2310.

150. Misic $Z$ et al. Understanding the interactions of oleic acid with basic drugs in solid lipids on different biopharmaceutical levels. I Excip Food Chem 2014; 5: 113-134.

151. Patel DD et al. Maintenance of supersaturation I: indomethacin crystal growth kinetic modeling using an online second-derivative ultraviolet spectroscopic method. J Pharm Sci 2011; 100: 2623-2641.

152. Arnold YE et al. Advancing in-vitro drug precipitation testing: new process monitoring tools and a kinetic nucleation and growth model. I Pharm Pharmacol 2011; 63: 333-341.

153. Stillhart $\mathrm{C}$ et al. Insights into drug precipitation kinetics during in vitro digestion of a lipic-based drug delivery system using in-line Raman spectroscopy and mathematical modeling. Pharm Res 2013; 30: 3114-3130.

154. Aisha AFA et al. Solid dispersion of alpha-mangostin improve its aqueous solubility through self-assembly of nanomicelles. I Pharm Sci 2011;101: 815-825.

155. Van Eerdenbrugh $B$ et al. Classification of the crystallization behavior of amorphous active pharmaceutical ingredients in acueous environments. Pharm Res 2014; 31: 969-982.

156. Dong YD et al. Applications of X-ray scattering in pharmaceutical science. Int J Pharm 2011; 417: 101-111.

157. Raina SA et al. Trends in the precipitation and crystallisation behavior of supersaturated aqueous solutions of poorly water-soluble drugs assessed using synchrotron radiation. $J$ Pharm Sci $2015 ; 104: 1981-1992$ 


\section{Thesis Papers}

158. Schram CJ et al. Influence of polymers on the crystal growth rate of felodipine: correlating adsorbed polymer surface coverage to solution crystal growth inhibition. Langmuir 2015b; 31: 11279-11287.

159. Garcia R, Tamayo J. Deformation, contact time, and phase contrast in tapping mode scanning force microscopy. Langmuir 1996; 12: 44304435 .

160. Abramoff $\mathrm{MD}$ et al. Image processing with ImageJ. Biophoton Int 2004, 11: $36-42$.

161. Skopp J. Derivation of the Freundlich adsorption isotherm from kinetics. J Chem Educ 2009; 86: 1341.

162. Mandal $T$ et al. A framework fo multi-scale simulation of crystal growth in the presence of polymers Soft Matter 2017; 13: 1904-1914.

163. Kubota N, Mullin JW. A kinetic model for crystal growth from aqueous solution in the presence of impurity. I Cryst Growth 1995; 152: 203-208.

164. Langmuir I. The adsorption of gase on plane surfaces of glass, mica and platinum. I Am Chem Soc 1918; 40: 1361-1403.

165. Kubota $\mathrm{N}$ et al. Supersaturation dependence of crystal growth in solutions in the presence of impurity. J Cryst Growth 1997; 182: $86-$ 94.

166. Kubota $\mathrm{N}$ et al. The combined influence of supersaturation and impurity concentration on crystal growth. $J$ Cryst Growth 2000; 212: 480-488.

167. Myerson EAS. Molecular Modeling Applications in Crystallisation. Cam bridge: Cambridge University Press, 1999
168. Mandal T et al Coarse-grained modeling of crystal growth and polymorphism of a model pharmaceutical molecule. Soft Matter 2016; 12: 8246-8255.

169. Levitt $M$, Warshel A. Computer simulation of protein folding. Nature 1975; 253: 694-698.

170. Flory PJ. Principles of Polymer Chemistry. Ithaca, NY: Cornell University Press, 1953.

171. Flory PJ. Thermodynamics of high polymer solutions. I Chem Phys 1942; 10: 51-61.

172. Chen $Y$ et al. Drug-polymer-water interaction and its implication for the dissolution performance of amorphous solid dispersions. Mo Pharm 2014; 12: 576-589.

173. Baghel $S$ et al. Theoretical and experimental investigation of drug-poly mer interaction and miscibility and its impact on drug supersaturation in acueous medium. Eur $I$ Pharm Biopharm 2016; 107: 16-31.

174. Marsac PJ et al. Estimation of crugpolymer miscibility and solubility in amorphous solid dispersions using experimentally determined interac tion parameters. Pharm Res 2009; 26 139-151.

175. Qian F et al. Drug-polymer solubility and miscibility: stability consideration and practical challenges in amorphous solid dispersion development. J Pharm Sci 2010; 99: 29412947.

176. Rubenstein M, Colby R. Polyme Physics. Oxford, UK: Oxford Univer sity Press, 2003.

177. Van Krevelen DW, Te Nijenhuis K. Properties of Polymers: Their Correla tion with Chemical Structure; Thei Numerical Estimation and Prediction from Additive Group Contributions. New York, NY: Elsevier, 2009

178. Tian B et al. Theoretical prediction of phase diagram for solid dispersion. Pharm Res 2015; 32: 840-851.

79. Gharagheizi $\mathrm{F}$ et al. Group contribution-based method for determination of solubility parameter of nonelectrolyte organic compounds. I Ind Eng Chem Res 2011; 50: 1034410349

180. Gharagheizi F. QSPR studies of solubility parameter by means of genetic algorithm-based multivariate linear regression and generalized regression neutral network. QSAR Comb Sc 2008; 27: 165-170

181. Tantishaiyakul $\mathrm{V}$ et al. Prediction of solubility parameters using partial least square regression. Int $J$ Pharm 2006; 325: 8-14.

182. Gupta $\mathrm{J}$ et al. Prediction of solubility parameters and miscibility of pharmaceutical compounds by molecular dynamics simulations. I Phys Chem B 2011; 115: 2014-2023.

183. Hildebrand I, Scott R. Solubility of Nonelectrolytes. New York, NY: Reinhold Pub Co., 1950.

184. Fan CF et al. Application of molecular simulations to derive phase diagrams of binary mixtures. Macromolecules 1992; 25: 3667-3676.

185. Mosquera-Giraldo LI et al. Mechanistic design of chemically diverse polymers with applications in ora drug delivery. Biomacromol 2016; 17 3659-3671.

186. Ting $\mathrm{M}$ et at. High-throughpu excipient discovery enables oral delivery of poorly soluble pharmaceuticals. ACS Cent Sci 2016; 2: 748 755. 
X. Thesis Papers 
European Journal of Pharmaceutical Sciences 132 (2019) 142-156

Contents lists available at ScienceDirect

European Journal of Pharmaceutical Sciences

journal homepage: www.elsevier.com/locate/ejps

\section{Calculation of drug-polymer mixing enthalpy as a new screening method of precipitation inhibitors for supersaturating pharmaceutical formulations}

Daniel J. Price ${ }^{a, b}$, Anita Nair ${ }^{a}$, Martin Kuentz ${ }^{c}$, Jennifer Dressman ${ }^{b}$, Christoph Saal ${ }^{a, *}$

a Merck KGaA, Darmstadt, Germany

${ }^{b}$ Frankfurt Goethe University, Frankfurt, Germany

'University of Applied Sciences and Arts Northwestern Switzerland, Institute of Pharma Technology, Muttenz, Switzerland

\section{A R T I C L E IN FO}

Keywords:

Precipitation inhibition

Supersaturation

Bioenabling formulations

in silico tools

Enthalpy

Screening
A B S T R A C T

Supersaturating formulations are widely used to improve the oral bioavailability of poorly soluble drugs. However, supersaturated solutions are thermodynamically unstable and such formulations often must include a precipitation inhibitor (PI) to sustain the increased concentrations to ensure that sufficient absorption will take place from the gastrointestinal tract. Recent advances in understanding the importance of drug-polymer interaction for successful precipitation inhibition have been encouraging. However, there still exists a gap in how this newfound understanding can be applied to improve the efficiency of PI screening and selection, which is still largely carried out with trial and error-based approaches. The aim of this study was to demonstrate how drugpolymer mixing enthalpy, calculated with the Conductor like Screening Model for Real Solvents (COSMO-RS), can be used as a parameter to select the most efficient precipitation inhibitors, and thus realize the most successful supersaturating formulations. This approach was tested for three different Biopharmaceutical Classification System (BCS) II compounds: dipyridamole, fenofibrate and glibenclamide, formulated with the supersaturating formulation, mesoporous silica. For all three compounds, precipitation was evident in mesoporous silica formulations without a precipitation inhibitor. Of the nine precipitation inhibitors studied, there was a strong positive correlation between the drug-polymer mixing enthalpy and the overall formulation performance, as measured by the area under the concentration-time curve in in vitro dissolution experiments. The data suggest that a rank-order based approach using calculated drug-polymer mixing enthalpy can be reliably data suggest that a rank-order based approach using calculated drug-polymer mixing enthalpy can be reliably
used to select precipitation inhibitors for a more focused screening. Such an approach improves efficiency of precipitation inhibitor selection, whilst also improving the likelihood that the most optimal formulation will be realized.

\section{Introduction}

A large proportion of active pharmaceutical ingredients (APIs) currently in development are classified as poorly soluble, with numbers quoted for the total percentage varying from $40 \%$ to $90 \%$ (Loftsson and Brewster, 2010). Given that drugs must be sufficiently solubilized in the gastrointestinal (GI) tract to be absorbed into systemic circulation, poorly soluble drugs represent a challenge for successful oral delivery (Brouwers et al., 2009). In response, a wide range of formulation techniques have been developed to enhance the apparent solubility of the API in the intestinal lumen (Zheng et al., 2012). These options can improve absorption due to the generation of a supersaturated solution of the API in the GI tract, i.e. above the equilibrium solubility, which increases the driving force for absorption through the GI mucosa into the systemic circulation (Brouwers et al., 2009; Taylor and Zhang, 2016). Supersaturation is an energetically unfavorable state, and is at best metastable (Taylor and Zhang, 2016). Due to this, there is an innate tendency for the supersaturated solution to return to a lower energy state, through precipitation. Therefore, to derive maximum benefit from supersaturation of the API, such formulations often include a precipitation inhibitor to sustain the period over which the API remains in solution (Price et al., 2018). This formulation approach is often re-

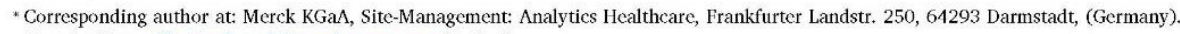

E mail address: Christoph.saal@merckgroup.com (C. Saal).
}

https://doi.org/10.1016/j.ejps.2019,03.006

Received 15 December 2018; Received in revised form 8 February 2019; Accepted 10 March 2019

Available online 12 March 2019

0928-0987/ (C) 2019 Elsevier B.V. All rights reserved. 


\section{Thesis Papers}

ferred to as the "spring and parachute" model (Guzman et al., 2007). In this model, the 'parachute' is the precipitation inhibitor that inhibits or slows the precipitation of the API from the supersaturated 'spring' generated by the formulation.

Currently, the mechanistic details of precipitation inhibition are not fully understood, and the specific molecular properties that yield efficient precipitation inhibitors have not yet been clarified. Given this uncertainty, a variety of hypotheses for the molecular mechanisms of precipitation inhibition have been proposed (Warren et al., 2010; Price et al., 2018). Additionally, it appears that precipitation inhibition is also an API-specific process. Therefore, there may be no single mechanism that describes all cases of precipitation inhibition. For example, some studies show polymer hydrophobicity to be a critica property in precipitation inhibition (Prasad et al., 2016), some sugges that hydrogen bond interactions play a pivotal role (Warren et al. 2010), whilst yet others propose that polymer surface coverage is an important factor (Schram, 2015). In all likelihood, multiple precipitation inhibition mechanisms may contribute to the observed effect, with the balance depending on the specific properties of both the API and the precipitation inhibitor.

The current lack of clear mechanistic understanding makes selection of precipitation inhibitors rather inefficient and time consuming, due to overreliance on 'trial and error' based experimental approaches. Typically PI screening is carried out by generating supersaturation with a solvent-shift, in which API is dissolved in high concentrations in favorable solvent ( $e . g$. DMSO), which is then added to an aqueous phase to generate supersaturation. Analytical techniques such as UV spectroscopy, HPLC or nephelometry are then applied to track changes in concentration of API in solution or precipitation over time. These concentration time profiles are then used to provide a measure of $P$ effectiveness (Price et al., 2018). One of the recent developments in this area is the $\mu \mathrm{DISS}{ }^{\mathrm{Ta}}$ profiler, which applies in situ UV probes in combination with liquid handling to study supersaturation and precipitation in real time. Using this approach, Palmelund and colleagues assessed the precipitation of 6 different BCS II drugs in combination with two polymers, PVP and HPMC (Palmelund et al., 2016). But despite recent advances in this area, the experimental selection of precipitation in hibitors remains both lengthy and costly.

In addition to the time and cost resources involved, this approach is unlikely to lead to certain identification of the most effective precipitation inhibitor, but rather to "one that works". To date, most supersaturating formulations incorporate one of a standard set of polymers, such as hydroxypropyl methyl cellulose acetate succinate (HPMCAS), polyvinylpyrrolidone (PVP) or hydroxypropyl methyl cellulose (HPMC) (Warren et al., 2010; Price et al., 2018). To overcome these drawbacks, there have recently been various attempts to establish a more mechanistic rational for precipitation inhibitor selection, as reviewed by Price et al. (2018). One of the more recent advances is the use of experimental tools that can design new precipitation inhibitor based on a mechanistic understanding of the specific interaction be tween the precipitation inhibitor and the API on a structural, molecula basis (Mosquera-Giraldo et al., 2016; Ting et al., 2016). Unfortunately, such de novo processes often require complex syntheses that yield novel excipients, which are impractical due to the need for toxicological studies to qualify them for use in pharmaceuticals. Therefore, mone work is required to establish an efficient and rational precipitation in hibitor selection process using excipients that are already approved for pharmaceutical use.

To bridge the gap between mechanistic understanding of pre cipitation inhibition and the realities of selecting precipitation in hibitors during pharmaceutical development, a more practical and robust approach is required. Such an approach, that can incorporate understanding of the importance drug-polymer interactions with a quick and efficient screening process, would be very useful. For thi purpose, the Conductor like Screening Model for Real Solvent (COSMORS), which was developed by Klamt (1995), is a highly interesting prospect. COSMO-RS combines quantum mechanical molecular calculations with fluid-phase thermodynamics. The first step is to calculate screening charge distributions of a molecule of interest in a continuum, based on density functional theory (DFT) (Kohn and Sham, 1965). The so-called 'sigma profiles' that are obtained from these calculations are then used in COSMO-RS, where statistical thermodynamics is applied to estimate the chemical potential and further characteristics of the system, such as solubility and partition coefficients. COSMO-RS has previously been used as a screening tool to calculate the solubility of early-development APIs in a database of excipients for pre-clinical formulations (Pozarska et al., 2013). However, further pharmaceutical applications have been limited, possibly due to the fact that the rate determining step, the quantum chemical calculations, are computationally very intensive. From a screening perspective, this is a sig nificant drawback. A practical alternative to facilitate application of COSMO-RS theory for screening purposes is the software package COSMOquick. COSMOquick removes the need for the time-consuming quantum chemical calculations of molecular surface charges, whilst still carrying out the remaining COSMO-RS calculations to derive chemical potential. This is achieved by additively combining fragments of pre viously calculated sigma profiles stored in a database to compute a new sigma profile for molecules of interest (Fig. 1) (Hornig and Klamt 2005). The COSMOquick approach has been recently applied pharmaceutically by the Kuentz group for the calculation of solubility parameters for a wide-range of molecules (Niederquell et al., 2018). Another application of the COSMOquick software is in co-crystal screening approaches, which uses the calculated excess enthalpy of interaction between and API and a co-former to assess the likelihood of co-crystal formation (Loschen and Klamt, 2012).

To consider precipitation inhibition fundamentally, it is assumed that an interaction between API and polymer must be present. This interaction could take many forms (e.g. London, dipolar, hydrogen bonding and Coulombic forces) or combinations thereof. It can be hypothesized that the more efficient the interaction between the drug and polymer, the more effective the inhibition process (Price et al., 2018). However, this may be difficult to calculate, since the API and precipitation inhibitor must interact in a complex aqueous environment We propose a simplified approach, in which the mixing (or excess) enthalpies of drug and excipient are calculated using COSMOquick. This estimated enthalpy is then used to rank potential precipitation in hibitors based on the strength of their molecular interaction with the API. It is hypothesized that this novel in silico protocol can be used to screen potential precipitation inhibitors allowing for a more focused selection to be carried out, thus significantly reducing the experimental burden of screening inhibitors by trial and error and ensuring the selection of an optimal inhibitor. 


\section{Thesis Papers}

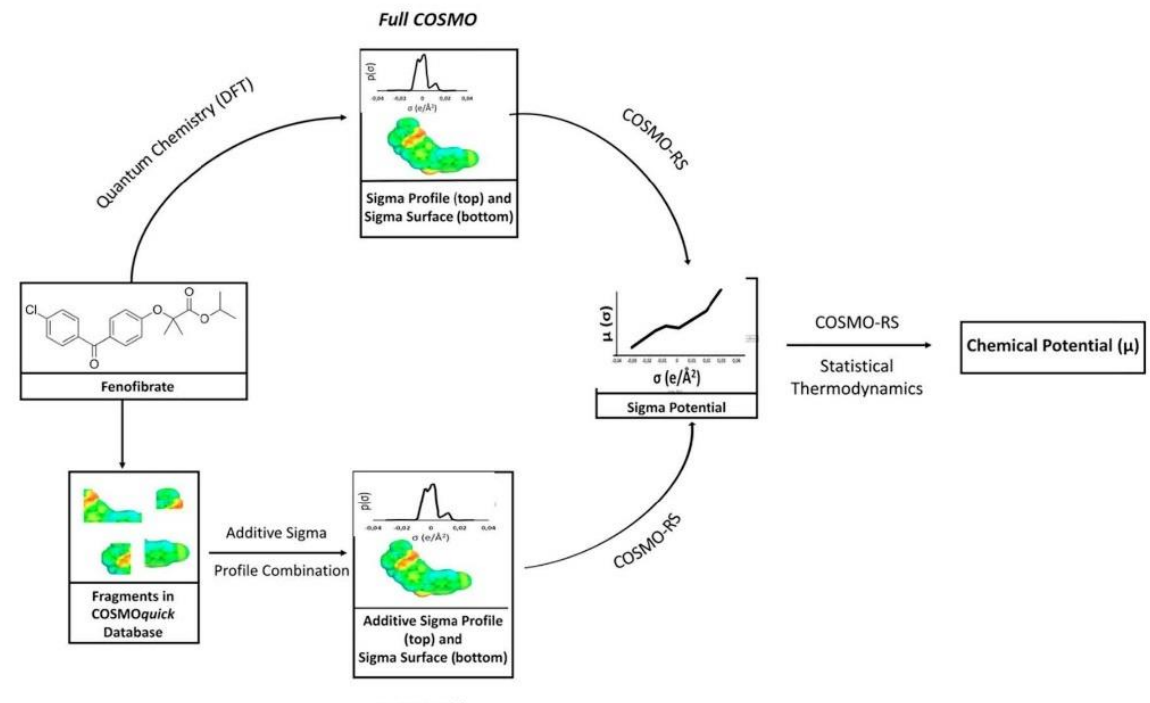

cosmoquick

Fig. 1. Chemical potential, and in turn a wide-range of thermodynamic properties, can be derived from sigma profiles using COSMO-RS theory. Sigma profiles can be obtained by two ways, either through de novo quantum chemical calculations or through an additive combination of previously calculated molecular fragments stored in a large database. The former approach is applied in the full COSMO calculation, whilst the latter is applied in the software package COSMOquick (bottom).

\section{Materials and methods}

\subsection{Materials}

Crystalline dipyridamole (DPD), crystalline glibenclamide (GB), crystalline fenofibrate (FF) (thermodynamic polymorphs were all purchased from MilliporeSigma (St Louis, MO), (Table 1). Poyl(ethylene glycol) (PEG), poly(methyl methacrylate) (PMMA), Pluronic ${ }^{\circ}$ (PLR), HPMC, PVP, chitosan (CH), reagent grade acetone, HPLC grade acetonitrile and HPLC grade methanol were all purchased from MilliporeSigma (St Louis, MO, USA). AOOAT (HPMCAS-MF) was purchased from ShinEtsu (Japan). Parteck ${ }^{\oplus}$ SLC was a gift sample from Merck

Table 1

Selected APIs and their relevant properties.

\begin{tabular}{|c|c|c|c|c|c|}
\hline API & MWt $[\mathrm{g} / \mathrm{moll}$ & $\operatorname{cog} \mathrm{P}^{\mathrm{a}}$ & $\mathrm{cpKa}^{a}$ & Number of $\mathrm{H}$-bond donors & Number of H-bond acceptors \\
\hline & 505 & 1.5 & 6.6 (basic) & 4 & 12 \\
\hline & 361 & 5.3 & - & 0 & 5 \\
\hline & 494 & 4.7 & 4.3(acidic) & 3 & 9 \\
\hline & & & & & \\
\hline
\end{tabular}

a Calculated with ChemAxon.
KGaA (Germany). Eudragit (Eu) RL and EPO were obtained from Evonik (Germany). Powder to make biorelevant dissolution medium, Fasted Simulated Intestinal Fluid (FaSSIF), was purchased from Biorelevant.com (UK).

\subsection{Experimental}

2.2.1. Thermodynamic solubility

API $(2-3 \mathrm{mg})$ was accurately weighed into a Uniprep ${ }^{*}$ syringeless filter $(5 \mathrm{~mL} ; 0.45 \mu \mathrm{m}) .2 \mathrm{~mL}$ of FaSSIF (Galia et al., 1998), composed of simulated intestinal fluid powder (FaSSGF, FaSSIF \& FeSSIF Powder) dissolved in a $\mathrm{pH} 6.5$ phosphate buffer, was added and the samples 


\section{Thesis Papers}

agitated at $450 \mathrm{rmm}$ for $24 \mathrm{~h}$ at $37^{\circ} \mathrm{C}$. The $\mathrm{pH}$ was checked at $7 \mathrm{~h}$ and adjusted with $0.1 \mathrm{~N} \mathrm{NaOH}$ or $0.1 \mathrm{~N} \mathrm{HCl}$, if deviation greater than $0.05 \mathrm{pH}$ units were observed. The final $\mathrm{pH}$ was also recorded after $24 \mathrm{~h}$

Samples were filtered after $24 \mathrm{~h}$ and the filtrates were diluted with acetonitrile and water (1:4) to avoid precipitation from the saturated solution. Samples were analyzed with UPLC (Thermo Dionex Ultimate 3000 , Thermo Fisher, MA, USA) to determine the API concentration API concentrations were determined by comparing the peak area to a standard calibration curve of nine standard concentrations (Eq. (1)) Three quality control samples of known concentrations were also prepared and used to check the robustness of the calibration curve. The analysis was carried out in duplicate.

$$
\begin{aligned}
& C\left[\mu \mathrm{gml}^{-1}\right]=\frac{a(A) F(a)}{m} \\
& C=\text { concentration of sample } \\
& \mathrm{a}(\mathrm{A})=\text { peak area for analyte } \mathrm{mL} \\
& \mathrm{m}=\text { gradient of the calibration curve } \\
& \mathrm{F}(\mathrm{A})=\text { dilution factor for analyte. }
\end{aligned}
$$

2.2.2. Parteck SLC ${ }^{\varpi}$ loading procedure

All API-loaded silica samples were prepared using the solvent impregnation rotary evaporator method (Laine et al., 2016) as follows: A solution $\left(10 \mathrm{mg} \mathrm{mL}^{-1}\right)$ of API in acetone was added to Parteck SLC (1:2 w/w API/Parteck SLC ${ }^{\circledR}$ ) under magnetic stirring for $15 \mathrm{~min}$. The suspension was then transferred to a rotary evaporator, and solvent was removed under reduced pressure at $40{ }^{\circ} \mathrm{C}$. After complete removal of solvent, the powder was left to dry in the rotary evaporator under reduced pressure for $2 \mathrm{~h}$.

\subsubsection{Loading content determination}

To determine the $\%(\mathrm{w} / \mathrm{w})$ of API in the mesoporous silica, the loaded samples were dispersed in acetone. Samples were taken after $1 \mathrm{~h}$, centrifuged, and filtered before being quantified with UPLC. The percentage API content was calculated relative to the mass of loaded samples dispersed within the acetone. The study was performed in triplicate.

2.2.4. Combination of API loaded silica with precipitation inhibitor

API loaded silica was combined with precipitation inhibitors as a physical mixture using a mortar and pestle in the mass ratio of $1: 1$. Thi results in an API:silica:PI ratio of 1: 2: 3.

\subsubsection{FaSSIF mini-dissolution experiment}

Around $5 \mathrm{mg}$ of API (or the equivalent of API-loaded silica) was weighed accurately into a glass vial. To this, $5 \mathrm{~mL}$ of FaSSIF was added. The vials were agitated at $37{ }^{\circ} \mathrm{C}$ for $2 \mathrm{~h}$. Samples were taken at $2,15,60$ and $120 \mathrm{~min}$, filtered, diluted if appropriate, and analyzed with UPLC (Thermo Dionex Ultimate 3000, Thermo Fisher, MA, USA). Residues were collected via centrifugation and analyzed for crystallinity with powder X-ray diffraction (PXRD). This was carried out on API, API + polymer samples, API loaded silica and API loaded silica + polymers. The mini-dissolution trials were conducted in duplicate for all samples.

\subsection{Powder X-ray diffaction (PXRD)}

Samples were prepared between X-ray amorphous films and measured in transmission mode using Cu-Ka1-radiation and a Stoe StadiP 611 KL diffractometer equipped with Dectris Mythen1K PSD. Th measurements were evaluated with the software WinXPow 3.03 by Stoe, Crystallographica Search/Match Version 3.1.0.2 and the ICDD
PDF-4 + 2014 Database and Igor Pro Version 6.34 by Wavemetrics Inc. Finger/Cox/Jephcoat. Angular range: $1-65^{\circ} 2 \theta$; PSD-step width: $2^{\circ} 2 \theta$ angular resolution: $0.015^{\circ} 2 \theta$; measurement time: $15 \mathrm{~s} / \mathrm{step}, 0.25 \mathrm{~h}$ overall.

\subsubsection{COSMO-RS calculations}

COSMOquick (COSMOlogic, Germany, Version 1.6) was used to calculate excess enthalpy of interaction between API and polymer. APIs and PIs were entered in smiles notation. Polymer structures were approximated as trimers, since the quantum chemical calculations cannot capture the full complexity of large molecules like polymers. Furthermore, this was not deemed critical to the study as the hypothesis was related to local molecular interactions, which are assumed to be sufficiently captured by trimer forms of the polymer. Ratio of API:PI was set at 1:3 to align with the ratios used in the formulations, and the temperature was set at $37^{\circ} \mathrm{C}$.

COSMOquick calculates sigma profiles of the API and precipitation inhibitor molecules based on an additive-combination approach against a database of previously calculated quantum chemical sigma profiles (Loschen and Klamt, 2012). Once sigma profiles are generated, several equations are performed to derive the energy required to combine the sigma profile of the API and PI. First, a sigma surface segment of the PI must be removed from the surface in order to make room for a new API segment, this requires energy associated with removing pre-existing contacts between precipitation inhibitor segments, $-\mu \mathrm{s}\left(\sigma^{\prime}\right)$. Second, a new API segment must be added to the precipitation inhibitor sigma surface, this involves forming new interactions between API and PI, with related energy costs and gains associated with the two segments interacting, $\mathrm{E}(\sigma, \sigma)$. This value is called the COSMO-RS interaction energy and, importantly, is calculated such that all binding modes (electrostatic, hydrogen bonding, van der Waals and combinatorial) are considered in the equation. (Appendix 3, Eqs. (1)-(3)). Once a value for the sigma potential is reached, thermodynamic calculations provide the chemical potential of mixing the API and PI (Appendix 3, Eq. (4)). From the chemical potential, a wide range of further thermodynamic properties can be calculated. In the current approach enthalpy of interaction, $H_{e x}$ also referred to as the enthalpy change of mixing. $\Delta H_{\text {mixo }}$ was calculated as a rank order parameter to assess the propensity of the drug to interact with excipient. As addressed in the introduction, this approach represents a substantial simplification of the more complex solid-liquid equilibrium in aqueous medium. A similar approach has been previously applied to screen co-formers in co-crystal selection and details can be inferred from the literature (Abramov et al., 2012).

In this study, the excess enthalpy of interaction between API and precipitation inhibitor is referred to as the "COSMO-Rank". According to the working hypothesis, the more negative the calculated excess enthalpy of interaction, the higher the COSMO-Rank and thus the better the inhibition of precipitation of the API. For a full description of the calculations carried out within the software package, readers are re ferred to Loschen and Klamt (2012).

\subsubsection{Speamar's rank correlation coefficient}

Spearman's rank correlation coefficient is a non-parametric method that allows statistical rank correlation to be carried out between two sets of rankings. In this instance, Spearman's rank correlation coefficient analysis was applied to the COSMO-rank and the rank order of the formulation performance. For the latter, $\mathrm{AUC}_{0-120}$ of the dissolution profiles was selected as the best overall descriptor of formulation performance and was calculated using Eq. (2). 


\section{Thesis Papers}

Table 2

\begin{tabular}{cccc}
\multicolumn{2}{l}{ UPLC gradient and flow rates. } \\
\hline Time (mins) & $\begin{array}{l}\text { Flow rate (mL/ } \\
\text { min) }\end{array}$ & $\begin{array}{l}\text { \% (v:v) mobile phase } \\
\text { A }\end{array}$ & $\begin{array}{l}\% \text { (v:v) mobile phase } \\
\text { B }\end{array}$ \\
\hline 0 & 0.83 & 90 & 10 \\
0.83 & 0.83 & 10 & 90 \\
1.2 & 1.5 & 90 & 10 \\
2 & 1.5 & 90 & 10 \\
2.01 & 0.83 & 90 & 10 \\
\hline
\end{tabular}

Table 3

Thermodynamic solubilities of dipyridamole, glibenclamide and fenofibrate in FasSIF, pH 6.5 at $37^{\circ} \mathrm{C}$

\begin{tabular}{lc}
\hline Compound & FasSIF thermodynamic solubility $(\mu \mathrm{g} / \mathrm{mL})$ \\
\hline Dipyridamole & $19.6 \pm 0.7$ \\
Glibenclamide & $8.1 \pm 0.1$ \\
Fenofibrate & $14.0 \pm 0.3$ \\
\hline
\end{tabular}

$A U C_{0-120}=\sum\left(\frac{C_{n}+C_{n+1}}{2} \times\left(t_{n+1}-t_{n}\right)\right)$

$C=$ concentration $(\mu \mathrm{g} / \mathrm{mL})$

$T=$ time (minutes)

$\mathrm{n}=$ sampling point at time, $\mathrm{t}$

Spearman's rank correlation coefficient was calculated by Rstudio

( $R$ version 2.15.12) using the code

corr $<$ - cor.test $(x=$ FILENAME\$VARIABLE1, $y=$ FILENAME\$VARIABLE2, method $=$ 'spearman')
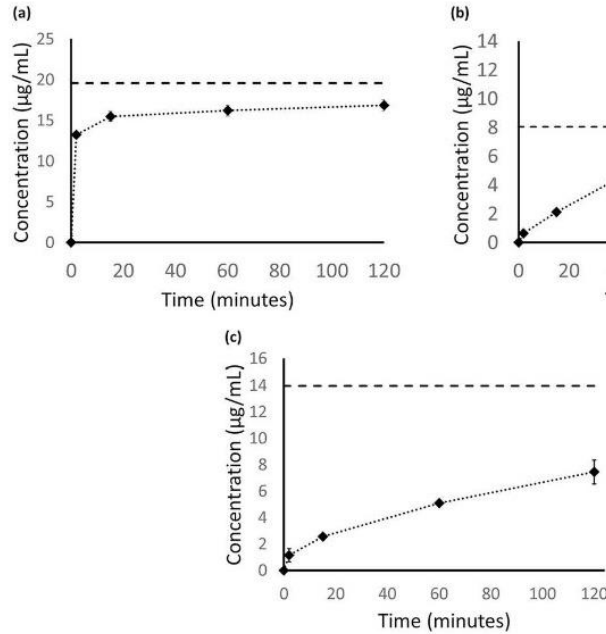

Fig. 2. FaSSIF dissolution profiles ( $2 \mathrm{~h}$ ) of diypridamole (a), glibenclamide (b) and fenofibrate (c). The thermodynamic solubility of the respective API is represented by the dashed line in each figure.
2.2.9. UPLC method

UPLC analysis was performed using a Thermo Dionex Ultimate 3000 (Thermo Fisher, MA, USA) equipped with a diode array detector (Thermo Fisher, MA, USA). Chromatographic separation was achieved on an Acquity UPLC BEH column C $8(2.1 \times 50 \mathrm{~mm}, 1.7 \mu \mathrm{m}$, Waters, MA, USA). The mobile phases A and B consisted of water:formic acid 99:1 (v:v) and acetonitrile:formic acid 99:1 (v:v), respectively. Gradient and flow rate is shown in Table 2. System management, data acquisition and processing were performed with the Chromeleon ${ }^{\text {TM }}$ software package, version 7.2 (Thermo Fisher, MA, USA).

\section{Results}

3.1. Dissolution profile of crystalline API

The thermodynamic solubilities of dipyridamole, glibenclamide and fenofibrate are shown in Table 3. All three compounds are classified as "poorly soluble" in FaSSIF, pH 6.5 (Amidon et al., 1995).

For all three compounds, the thermodynamic solubility was approached over the duration of the FaSSIF dissolution test (Fig. 2). The differences in particle size and morphology. The average concentration of glibenclamide slightly exceeded the measured solubility after $2 \mathrm{~h}$ of dissolution, but this was not statistically significant. Furthermore, some variation in glibenclamide FaSSIF thermodynamic solubility has been recorded in the literature, with values ranging from 8 to $10 \mu \mathrm{g} / \mathrm{mL}$. This is in accordance with the observed dissolution behavior (Fagerberg et al., 2010; Fagerberg et al., 2012; Wie et al., 2006).

\subsection{Loading onto mesoporous silica}

Successful loading of APIs onto mesoporous silica was confirmed

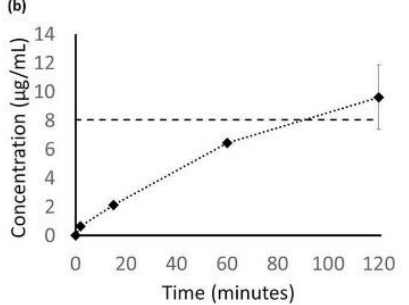
rate of approach differed among the APIs, which may be related to 


\section{Thesis Papers}
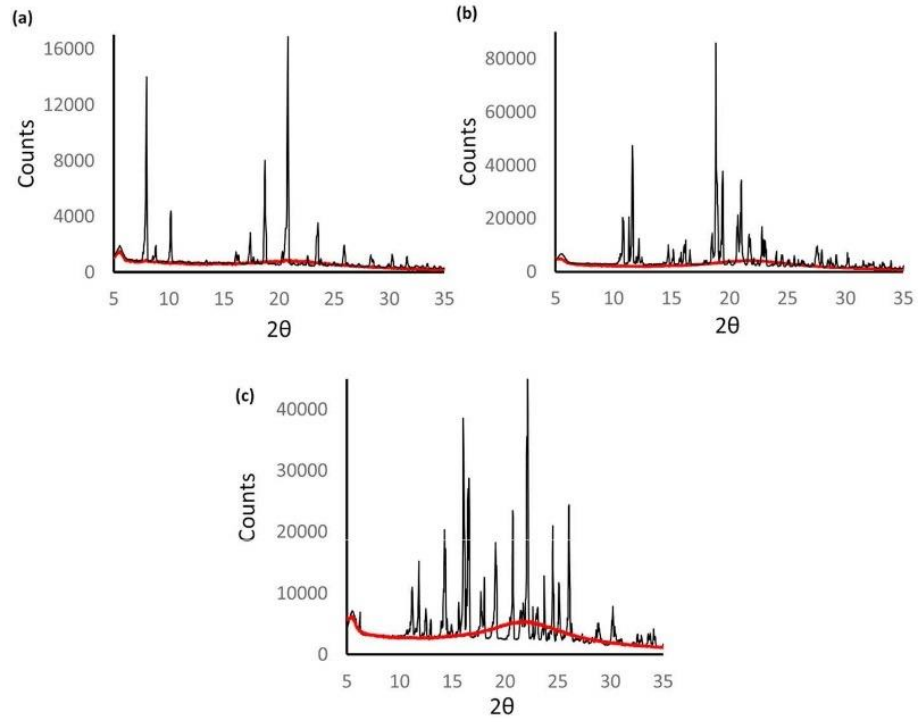

Fig. 3. PXRD diffraction patterns of the pure API (black) and API loaded onto mesoporous silica (red) for dipyridamole (a), glibenclamide (b) and fenofibrate (c). (For interpretation of the references to color in this figure legend, the reader is referred to the web version of this article.)

Table 4

API loaded silica total API content.

\begin{tabular}{ll}
\hline API & \% loading of API onto mesoporous silica (w/w \%) \\
\hline Dipyridamole & $30.1 \pm 0.1$ \\
Glibenclamide & $29.4 \pm 0.1$ \\
Fenofibrate & $29.7 \pm 0.3$ \\
\hline
\end{tabular}

with PXRD by showing a successful shift from the crystalline (pure API) to the amorphous solid-state form after loading onto the mesoporous silica. The absence of Bragg diffraction patterns is indicative of an amorphous material (Fig. 3)

Loading content was similar $(\sim 30 \% \mathrm{w} / \mathrm{w})$ for all three compounds, as determined by UPLC (Table 4 ).

Delivering the API in the amorphous form significantly improved the dissolution performance in FaSSIF for all three compounds (Fig. 4) As seen in Fig. 4, dipyridamole, glibenclamide and fenofibrate showed 4,25 or 3 -fold supersaturation relative to the thermodynamic solubility, respectively. However, all three profiles also show precipitation and a decrease in concentration towards the thermodynamic solubility within around $30 \mathrm{~min}$.

3.3. Precipitation inhibitor screening: calculation of excess enthalpy

The COSMO-RS in silico screening protocol was based on calculation of the enthalpy of interaction between each of the APIs with different potential precipitation inhibitors. Results are summarized in Fig. 5. According to the hypothesis, the more negative the calculated enthalpy of interaction, the higher the 'COSMO Rank'. A COSMO rank of 1 thus indicates the best potential for successful precipitation inhibition. By contrast, the more positive the enthalpy of interaction, the less likely the polymer is to be of use as a precipitation inhibitor. Of the inhibitors studied experimentally, Eudragit EPO, was predicted to be the best precipitation inhibitor for both dipyridamole and glibenclamide, whereas for fenofibrate, PMMA was assigned the highest COSMO rank. For all three compounds, chitosan was assigned COSMO rank 9, reflecting its high calculated enthalpy of interaction, which was hypothesized to translate into poor precipitation inhibition performance.

\subsection{Dissolution data for API loaded onto mesoporous silica with} precipitation inhibitor added

Each of the loaded mesoporous silica samples were physically combined with a selection of polymers (HPMCAS, HPMC, PVP, PEG, Eudragit EPO, Pluronic, PMMA, Eudragit RLPO and Chitosan) such that the final ratio of API:PI was 1:3, w/w (API:PI:Silica; $1: 3: 2, \mathrm{w} / \mathrm{w}$ ). Therefore, Table 5 shows the final \% API content in the formulations after combination with the precipitation inhibitors. The final API concentrations are similar to conventional supersaturating formulations that require precipitation inhibitors.

To assess the predictive power of the COSMO calculation, dissolution in FaSSIF was carried out for each of the API-loaded mesoporous 


\section{Thesis Papers}

(a)

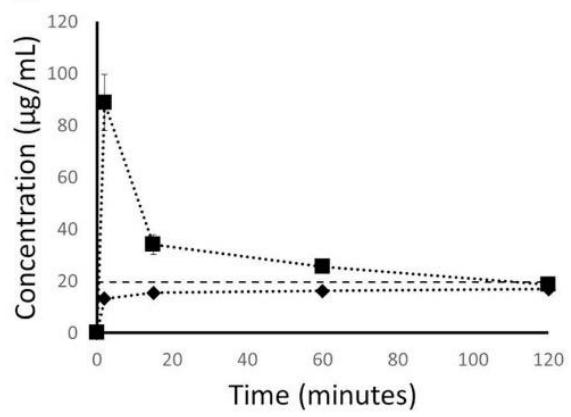

(b)

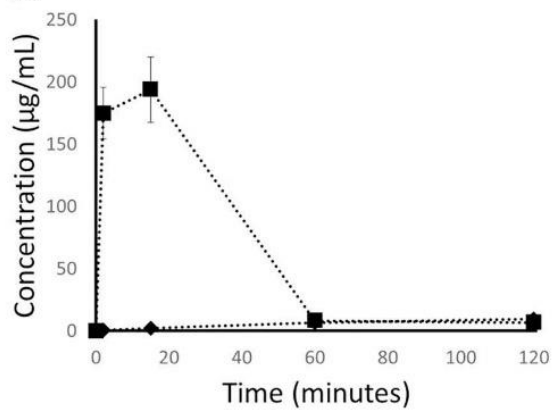

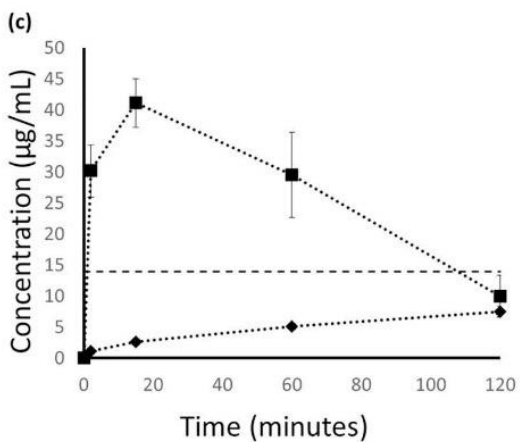

Fig. 4. Dissolution profiles of pure API $(\diamond)$, and API loaded onto mesoporous silica at $37^{\circ} \mathrm{C}$.

silica samples in combination with each of the selected precipitation inhibitors. (Figs. 6-8).

\subsection{Spring-parachute plots}

The performance of the precipitation inhibitor is rated in terms of it ability to sustain supersaturation. To reflect this, the data in Fig. 9 indicates the maximum concentration ('Spring') achieved compared to the concentration at the end of the assay ("Parachute").

\subsection{Spearman's rank correlation analysis}

The overall formulation performance was assessed by calculating the AUC of the dissolution profiles for each system (Eq. (2)). Statistical analysis was then carried out to determine the correlation between COSMO-rank and formulation performance, with a higher AUC indicating a better formulation performance (Tables 6-8).

Given that dissolution performance for PEG formulations appears to consistently deviate from the correlation of COSMO rank with dissolution performance for all three APIs, the statistical analysis was re- run without PEG. For all samples, the correlation improved, with the Spearman's rank correlation coefficients for dipyridamole and glibenclamide both increased to $0.98(0.0004, \mathrm{p}<0.05)$, indicating a strong positive correlation. For fenofibrate, removing PEG from the set also improved the Spearman's rank coefficient to $0.8(0.022$, $\mathrm{p}<0.05$ ) and passes the significance criterion. The results suggest that PEG may behave as an outlier in the COSMO calculations.

\section{Discussion}

In silico tools are an attractive option for bridging the gap between our current understanding of precipitation inhibitors and practical selection of inhibitors to be used in supersaturating formulations in pharmaceutical development. In this work, we applied the COSMO-RS model as a novel in silico screening tool to successfully predict the formulation performance of a wide range of precipitation inhibitors in formulations of glibenclamide, fenofibrate and dipyridamole. Specifically, it was hypothesized that free enthalpy of mixing (APIpolymer) could be used as a parameter for ranking inhibitors, from highest potential for successful precipitation inhibition to lowest, based 

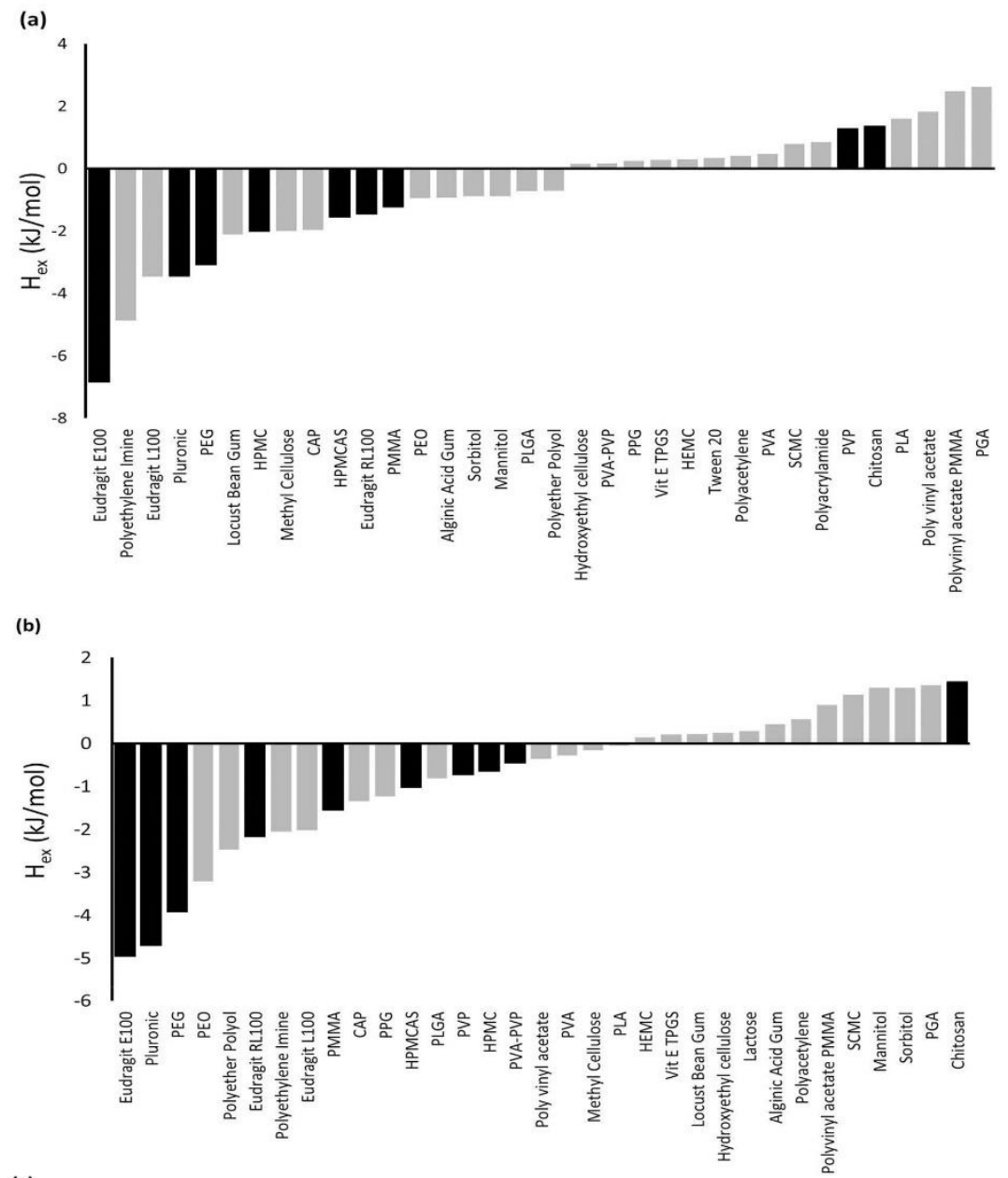

(c)

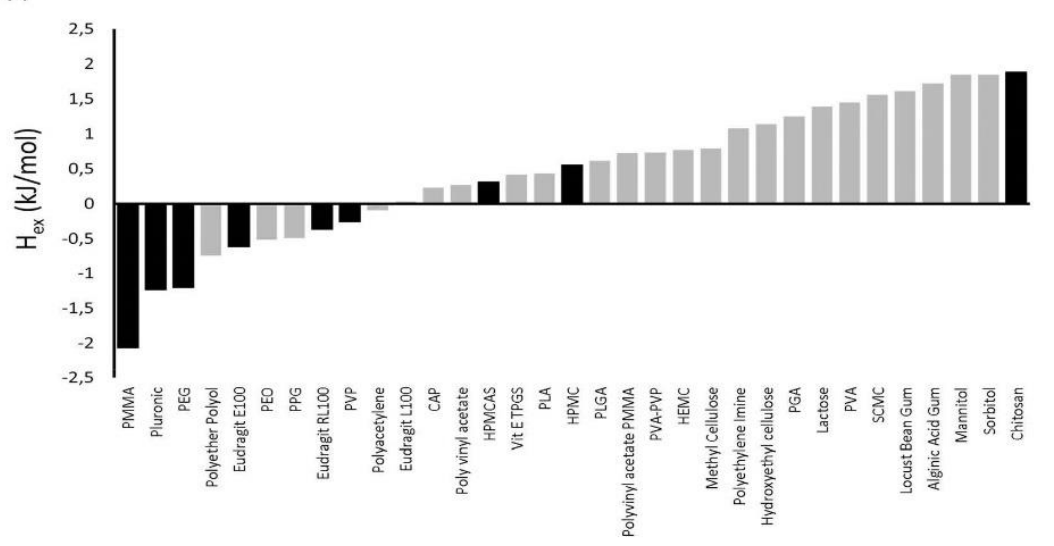

(caption on next page) 


\section{Thesis Papers}

Fig. 5. COSMO-RS Screen: calculated excess enthalpy of interaction between dipyridamole (a), glibenclamide (b) and fenofibrate (c) with a range of potential precipitation inhibitors. Polymers studied experimentally to test the correlation are highlighted as dark bars: Eudragit EPO, Pluronic (PLR), PEG, HPMCAS, PVP, HPMC, Eudragit RLPO, PMMA and Chitosan.

Table 5

Final API content $(\%, w / w)$ in the formulations after incorporation of precipitation inhibitors.

\begin{tabular}{lll}
\hline API & $\begin{array}{l}\text { \% Loading of API onto mesoporous silica } \\
(\mathrm{w} / \mathrm{w} \%)\end{array}$ & $\begin{array}{l}\text { Final } \mathrm{API} \text { content } \\
(\%)\end{array}$ \\
\hline $\begin{array}{l}\text { Dipyridamole } \\
\text { Glibenclamide }\end{array}$ & $30.1 \pm 0.1$ & 15.1 \\
Fenofibrate & $29.4 \pm 0.1$ & 14.7 \\
\hline
\end{tabular}

(a)

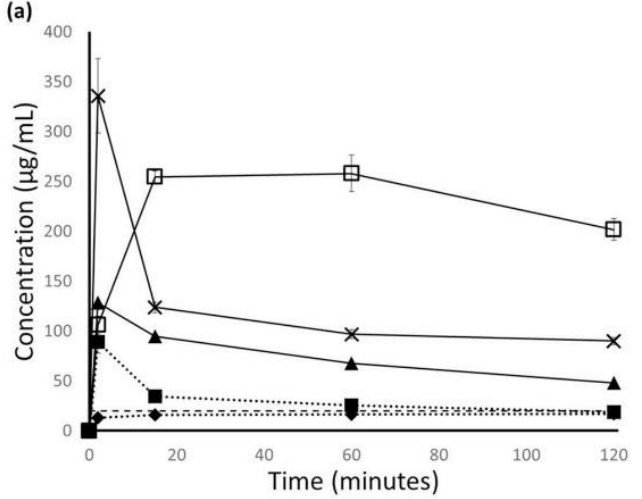

(c)

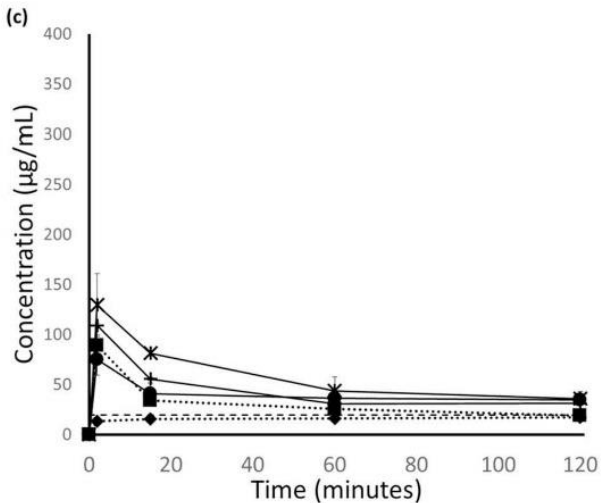

on the theoretical interaction between the inhibitor and the API. For all three compounds a strong positive correlation was observed between the rank assigned based on the calculated free enthalpy of mixing and the overall formulation performance.

4.1. The Importance of enabling formulations

The experimental thermodynamic solubility values for dipyridamole, glibenclamide and fenofibrate in FaSSIF are in line with values previously reported in the literature (Table 2) (Leigh et al., 2013;

(b)

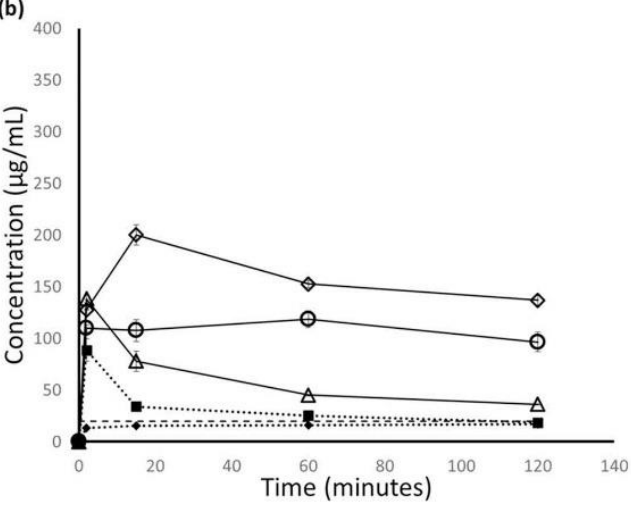

Fig. 6. Dissolution profiles in FaSSIF for dipyridamole $(\diamond)$, dipyridamole loaded mesoporous silica $(\boldsymbol{\square})$ and dipyridamole loaded mesoporous silica with precipitation inhibitors selected from the COSMO-RS screen. (a): Eudragit EPO $(\square)$, Pluronic $(X)$, PEG $(\mathbf{\Delta})$; (b): HPMC $(\diamond)$, HPMCAS $(O)$ Fudragit RLPO $(\triangle)$ and $(c)$ : PMMA (), PVP (⿶) and Chitosan ( + ). The order of the listed inhibitors corresponds to the rank order in the COSMO screen (i.e. Eudragit EPO COSMO rank \#1 Chilosan COSMO rank \#9). 


\section{Thesis Papers}

(a)

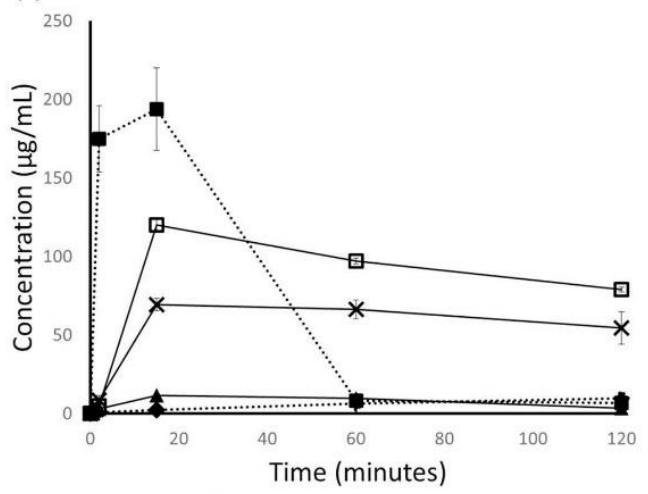

(b)

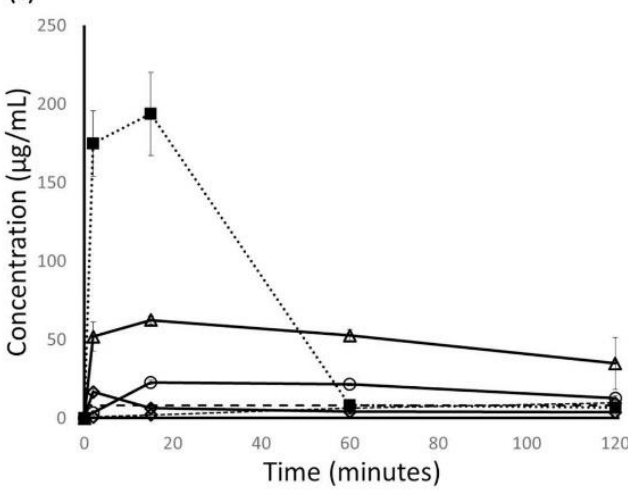

(c)

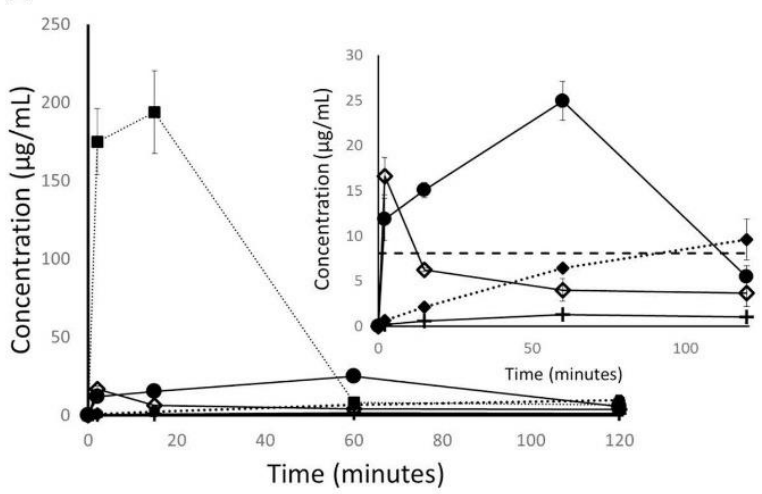

Fig. 7. Dissolution profiles in FaSSIF for glibenclamide $(\bullet)$, glibenclamide loaded mesoporous silica $(\mathbf{D})$ and glibenclamide loaded mesoporous silica with precipitation inhibitors selected from the COSMO-RS screen. (a): Eudragit EPO $(\square)$, Pluronic $(X)$, PEG $(\mathbf{\Delta})$; (b): HPMCAS $(O)$, HPMC $(\vartheta)$, Eudragit RLPO $(\triangle)$ and $(c)$ : PMMA (), PVP $(\bullet)$ and Chitosan $(+)$. The order of the listed inhibitors corresponds to the rank order in the COSMO screen (i.e. Eudragit EPO COSMO rank $\# 1$ Chitosan COSMO rank \#9).

Fagerberg et al., 2012and Buch et al., 2010). All three compounds represent Biopharmaceutical Classification System (BCS) II APIs (Amidon et al., 1995). This low solubility, coupled with the incomplete dissolution of the drugs in biorelevant media (Fig. 2), signals the potential for oral absorption and bioavailability risks during development (Zheng et al., 2012). The selected APIs represent a typical range of molecular weights, charge, hydrophobicity and number of hydrogen bond donors and acceptors typical for poorly soluble drugs. Therefore, all three API are model candidates for absorption enhancement by formulation with enabling formulations. Indeed, there are already many examples in the literature of combining each of these APIs with enabling formulation such as lipid-based formulations, addition of surfactants to the formulation and formulations containing high-energy forms of the API such as solid dispersions (Thongnopkoo and Puttipipatkchachorn, 2016; Guo et al., 2011; Zecevic et al., 2018; Theil et al., 2017; Taniguchi et al., 2013).

\subsection{Mesoporous silica: a model supersaturating formulation}

In the current approach, mesoporous silica was selected as a mode supersaturating formulation. Mesoporous silica stabilizes the amorphous API via nano-encapsulation in the pores, which have an ap proximate mean diameter of $4 \mathrm{~nm}$. Upon contact with an aqueous environment, the drug is released in a molecularly dispersed fashion and thus supersaturation is generated (Ditzinger et al., 2019). Both fenofibrate and glibenclamide have previously been formulated with mesoporous silica in the literature. Van Speybroeck and co-workers reported that, after successfully loading onto ordered mesoporous silica, glibenclamide was completely released within $30 \mathrm{~min}$, compared to just $60 \%$ release at 120 min for the commercial formulation, Daonil (Van Speoybroeck et al., 2011). Furthermore, the improved in vitro dissolution performance translated into an improved in vivo performance in rats, with the extent of absorption fourfold higher for glibenclamide 


\section{Thesis Papers}

(a)

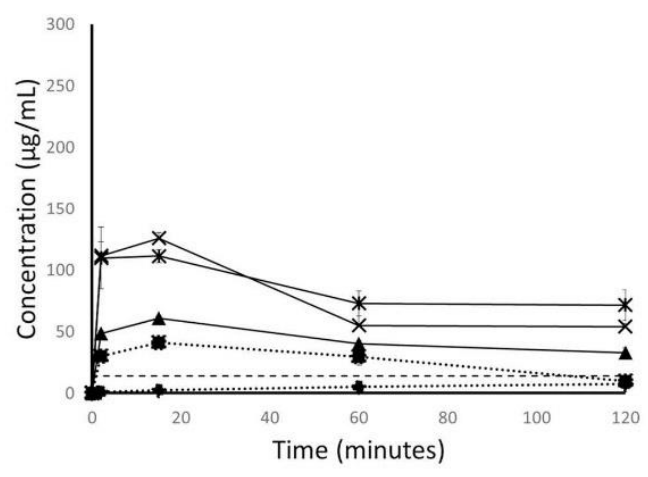

(c) (b)

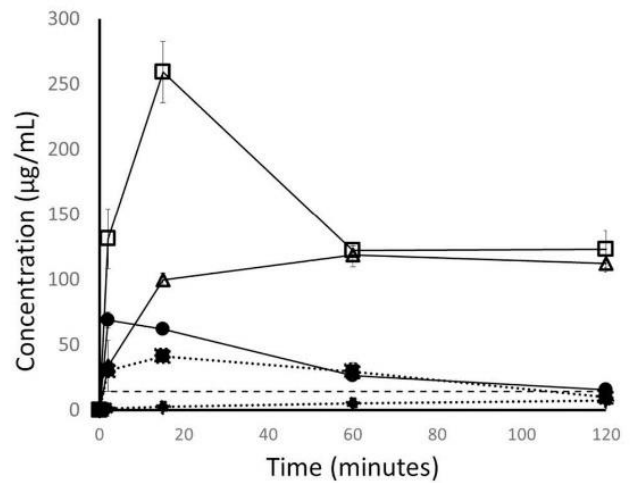

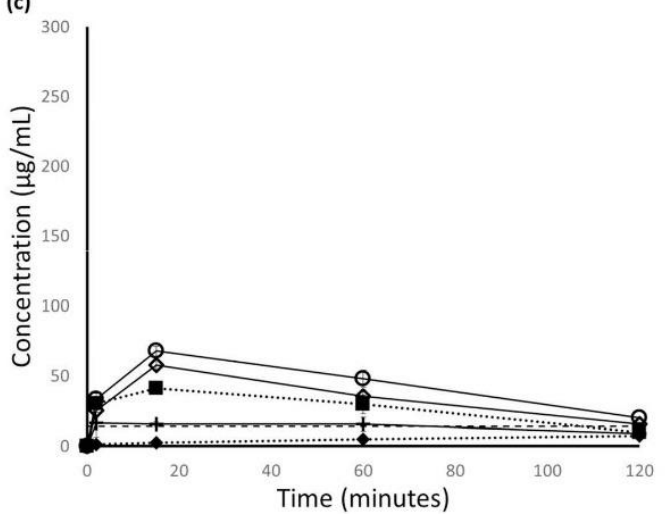

Fig. 8. Dissolution profiles in FaSSIF for fenofibrate $(\diamond)$, fenofibrate loaded mesoporous silica $(\boldsymbol{\square})$ and fenofibrate loaded mesoporous silica with precipitation inhibitors selected from the COSMO-RS screen. (a): PMMA $($ ), Pluronic $(X)$, PFG $(\mathbf{A})$; (b): Eudragit F.PO $(\square)$, Eudragit RI.PO $(\triangle)$, PVP $(\bullet)$ and $(c)$ : HPMCAS $(O)$, HPMC $(0)$ and Chitosan $(1)$. The order of the listed inhibitors corresponds to the rank order in the COSMO screen (i.e. PMM $\triangle$ COSMO rank \#1 - Chitosan COSMO rank \#9).

loaded silica than the commercial formulation (Van Speoybroeck et al., 2011).

Mesoporous silica has also been demonstrated to improve both in vitro and in vivo performance of fenofibrate versus commercially available products (Van Speybroeck et al., 2010; Ahern et al., 2013; Uejo et al., 2013; Hong et al., 2016; Bukara et al., 2016; O'Shea et al., 2017; Dressman et al., 2016). Furthermore, fenofibrate was the API chosen for the first proof of concept clinical trial of mesoporous silica in man (Bukara et al., 2016). In that study, which compared fenofibrate loaded silica with the commercially available micronized Lipanthyl ${ }^{0}$ formulation, a $77 \%$ increase in $\mathrm{C}_{\max }$, a reduced $\mathrm{t}_{\max }$ and a $54 \%$ increase $\mathrm{AUC}$ ${ }_{24 h}$ /dose for fenofibrate loaded silica was observed, demonstrating that loading this API onto mesoporous silica can increase both the rate and extent of absorption.

Given this pre-established success with mesoporous silica for glibenclamide and fenofibrate, successful loading onto silica, as demonstrated by the solid-state shift from crystalline to amorphous, was expected for both compounds (Fig. 3). Furthermore, the loading efficiency and extent of supersaturation was in line with previous examples in the literature (Table 4; Fig, 4) (O'Shea et al., 2017; Van Speoybroeck et al., 2011). In contrast, for dipyridamole, which has been formulated with other enhancing approaches such as solid dispersions, there have not yet been any published reports of formulation with mesoporous silica. For this molecule, a successful loading was also confirmed by the change in solid-state from crystalline to amorphous (Fig. 3). Ad ditionally, supersaturation was generated, and the observed loading efficiency obtained was in line with typical loading values reported in the literature (Table 4; Fig. 4) (McCarthy et al., 2016).

For all three APIs, loading onto mesoporous silica resulted in the typical 'spring' profile in the FaSSIF dissolution curves (Fig. 4). First reported by Guzman (Guzman, 2007), the presence of short-lived supersaturation followed by precipitation is typical for delivery of the compound in the amorphous form, such as with mesoporous silica. This is related to the metastable nature of the supersaturated state, which is returned to a thermodynamically more favorable state via the precipitation of the supersaturated API (Taylor and Zhang, 2016). The 
D.J. Price, et al.

(a)

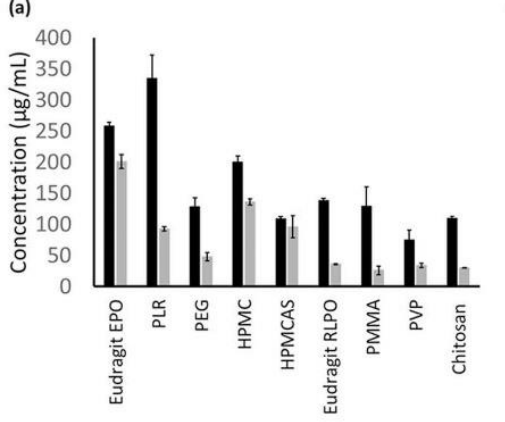

(b)

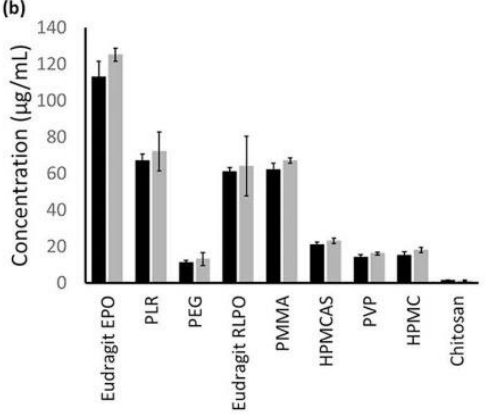

Fig. 9. Maximum concentration, 'Spring' (dark bars) versus the concentration at the (n) of the dissolution experinent at 120 min, 'Parache', (light bers) for idamole (a), glibenclamide (b) and fenofibrate (c) loaded mesoporous silica in combination with a range of precipitation inhibitors during FaSSIF dissolution, with decreasing COSMO-rank for the respective API from left to right.

(c)

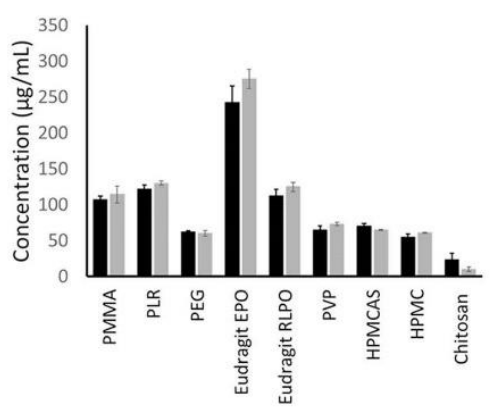

Table 6

Spearman's rank correlation coefficient analysis: dipyridamole.

\begin{tabular}{ccccc}
\hline Polymer & $\begin{array}{l}\text { Calculated } \\
\text { enthalpy } \\
(\mathrm{kJJ} / \text { mol })\end{array}$ & $\begin{array}{l}\text { AUC } \\
\left(\mu g m g \mathrm{~mL}^{-1}\right)\end{array}$ & COSMO rank & Dissolution rank \\
\hline Eudragit EPO & -6.81 & 29,000 & 1 & 1 \\
PL.R & -3.45 & 14,000 & 2 & 3 \\
PEG & -3.08 & 8600 & 3 & 5 \\
HPMC & -2.12 & 19,000 & 4 & 2 \\
HPMCAS & -2.01 & 13,000 & 5 & 4 \\
Eudragit RL.PO & -1.55 & 6600 & 6 & 6 \\
PMMA & -1.46 & 5800 & 7 & 7 \\
PVP & -1.23 & 5300 & 8 & 8 \\
Chitosan & 1.28 & 5100 & 9 & 9 \\
Spearman's rank & 0.91 & Significance & \multicolumn{2}{c}{0.001} \\
correlation & \multicolumn{5}{|c|}{$\mathrm{p}<0.05$} \\
coefficient & \multicolumn{5}{|l}{} \\
\hline
\end{tabular}

dissolution profiles of the three API-loaded silica confirm the need for a precipitation inhibitor, or a "parachute", to maintain the API concentration in solution over physiologically relevant time scales.

4.3. Precipitation inhibitor selection with COSMO-RS

Given the current lack of understanding surrounding structure-inhibition relationships, selection of precipitation inhibitors has typically been empirical, requiring a large amount of experimental screening (Price et al., 2018). Such time-consuming experimental approaches have been reported in the literature for fenofibrate. Petrusevska et al.
Table 7 Spearman's rank correlation coefficient analysis: glibenclamide.

\begin{tabular}{|c|c|c|c|c|}
\hline Polymer & $\begin{array}{l}\text { Calculated } \\
\text { enthalpy } \\
(\mathrm{kJ} / \mathrm{mol})\end{array}$ & $\begin{array}{l}\text { AUC } \\
\left(\mu g \cdot \mathrm{mg} \mathrm{mL}^{-1}\right)\end{array}$ & COSMO rank & Dissolution rank \\
\hline Eudragit EPO & -1.96 & 11,000 & 1 & 1 \\
\hline PL.R & -4.70 & 7200 & 2 & 2 \\
\hline PEG & -3.92 & 960 & 3 & 7 \\
\hline HPMC & -2.17 & 6000 & 4 & 4 \\
\hline HPMCAS & -1.55 & 6100 & 5 & 3 \\
\hline Eudragit RLPO & -1.03 & 2100 & 6 & 5 \\
\hline PMMA & -0.72 & 2000 & 7 & 6 \\
\hline PVP & -0.64 & 600 & 8 & 8 \\
\hline Chitosan & 1.44 & 100 & 9 & 9 \\
\hline $\begin{array}{c}\text { Spearman's rank } \\
\text { correlation } \\
\text { coefficient }\end{array}$ & 0.81 & Significance & \multicolumn{2}{|c|}{$\begin{array}{c}0.01 \\
p<0.05\end{array}$} \\
\hline
\end{tabular}

employed a high-throughput screening protocol that coupled a solventshift approach with off-line chromatography to screen a wide-range of precipitation inhibitors for fenofibrate (Petrusevskra et al., 2013). In the study, it was determined that surfactant-based inhibitors were the most optimal for fenofibrate, as opposed to the cellulosic polymers, which proved to be optimal for carbamazepine (Petrusevskra et al., 2013). Interestingly, the top performing polymers highlighted in the current study were not included in the high-throughput screen, which further indicates that, in addition to the large resource costs involved in experimental screening, the most optimal inhibitors may be missed due 


\section{Thesis Papers}

Table 8

$\underline{\text { Spearman's rank correlation coefficient analysis: fenofibrate }}$

\begin{tabular}{ccccc}
\hline Polymer & $\begin{array}{l}\text { Calculated } \\
\text { enthalpy }(\mathrm{kJ} / \mathrm{mol})\end{array}$ & $\begin{array}{l}\text { AUC } \\
\left(\mu \mathrm{g} \mathrm{mg} \mathrm{mL}^{-1}\right)\end{array}$ & COSMO rank & $\begin{array}{l}\text { Dissolution } \\
\text { rank }\end{array}$ \\
\hline PMMA & -2.07 & 10,000 & 1 & 3 \\
PLR & -1.24 & 9000 & 2 & 4 \\
PEG & -1.21 & 5200 & 3 & 7 \\
Eudragit EPO & -0.62 & 19,000 & 4 & 1 \\
Eudragit PLPO & -0.37 & 13,000 & 5 & 2 \\
PVP & -0.26 & 5400 & 6 & 5 \\
HPMCAS & 0.31 & 5300 & 7 & 6 \\
HPMC & 0.55 & 4200 & 8 & 8 \\
Chitosan & 1.88 & 1700 & 9 & 9 \\
Spearnan's rank & 0.63 & Significance & \multicolumn{2}{c}{0.08} \\
correlation & & & \multicolumn{2}{|c|}{$\mathrm{p}<0.05$} \\
coefficient & & & \multicolumn{2}{|l}{} \\
\hline
\end{tabular}

to practical limitations on the number of inhibitors that can be screened experimentally. This problem is especially pronounced when, instead of conducting a high throughput experimental precipitation inhibito screen, two to three "usual suspects" are selected for experimental screening. In this approach, polymers such as PVP, HPMCAS or HPMC are often selected and individually screened in combination with the supersaturating formulation of interest, after which the most successful polymer of the three is selected. This approach introduces a large amount of uncertainty as to whether the most efficient formulation has been realized (He et al., 2010; O'Shea et al., 2017; Laine et al., 2016; Vora et al., 2016).

To reduce the resources required for precipitation inhibito screening, it would be highly desirable to replace experimental with insilico tools. Using the COSMO-RS screening protocol described herein, drug-polymer mixing enthalpy can be calculated for an API in combination with $\sim 50$ potential precipitation inhibitors in as little as two minutes. This represents a significant time-saving versus traditional experimental screening. During early pharmaceutical development, time can be the most critical factor in the success of failure of a project, so such savings are highly attractive. Furthermore, there is no limit to the number of molecules that can be included in the database. All potential inhibitors can be assessed using enthalpy of interaction as a rank-order parameter, which is designated the "COSMO Rank" (Fig. $5 \mathrm{a}-\mathrm{c}$ ). This is related to the hypothesis that the more negative the enthalpy, the higher the chance of successful precipitation inhibition based on interaction between API and PI. This hypothesis is links back to the principle that interactions between the API and PI are essential to efficient precipitation inhibition, which has been demonstrated in many studies (Price et al., 2018).

Even without considering the dissolution data generated in these studies, the rank order proposed by the COSMO-RS screen already points towards promising results. For example, in a study by Chauhan and co-workers, it was reported that Eudragit EPO and HPMC were able to sustain supersaturated solutions of dipyridamole significantly longer than all other polymers studied (Eudragit S100, Eudragit RL100, PEC and PVP). Both the in silico prediction and the dissolution studies car ried out in our studies reflect these findings. Interestingly, PVP, which is often used as a first-line candidate in precipitation inhibitor selection, did not perform well as a precipitation inhibitor in the study by Chauhan et al. (2013). The CoSMo calculation for dipyridamole (Fig. 5a) was able to identify that PVP would not be a suitable polymer $(\Delta \mathrm{H}=+1.28 \mathrm{~kJ} / \mathrm{mol})$ for dipyridamole.

\subsection{Correlation of COSMO-rank with dissolution performance}

In addition to the general observation that the COSMO-RS calculated excess enthalpy of interaction appears to be a useful rank-order parameter for the selection of precipitation inhibitors, a statistical analysis of the correlation between the COSMO-RS rank and the dis solution data was also conducted (Tables 4-6). Comparing these two parameters with Spearman's rank correlation coefficient analysis, the correlation between the rank order predicted by COSMO-RS and the rank order observed in the dissolution experiments was determined to be $0.91(0.001, \mathrm{p}<0.05), 0.81(0.01, \mathrm{p}<0.05)$ and $0.61(0.076$ $\mathrm{p}<0.5)$ for dipyridamole, glibenclamide and fenofibrate, respec tively. For dipyridamole and glibenclamide, the very strong positive correlation between COSMO prediction and formulation performance demonstrated that the COSMO-RS screening protocol can be used to select the most optimal precipitation inhibitors whilst avoiding the costly and time-consuming experimental screening. For fenofibrate, the correlation observed between the predictions and the results was lower. Furthermore, the significance in the Spearman's rank correlation coefficient analysis was $>0.05$ for fenobibrate, which introduces un certainty as to the conclusions that can be drawn based on the analysis However, when PEG was excluded from the analysis, all correlations improved substantially and the correlation coefficient for fenofibrate $(0.8)$ reached statistical significance $(\mathrm{p}<0.05)$. It thus seems that $\mathrm{PEG}$ may be an outlier in terms of the COSMO predictions, although this would have to be tested with more APIs to be sure. We note that a strong positive correlation was independently achieved for each API, which suggests that the presented approach can be applied robustly and reliably.

In addition to the area under the curve of the dissolution profile, one can also consider how well the API sustains the API in solution by ad dressing the differences between the peak, 'spring', concentration and the final, 'parachute', concentration. As shown in Fig. 9, there is a good correlation between how well an inhibitor sustains the initial 'spring' concentrations and the COSMO-rank. By combining both the AUC of the dissolution profiles and spring-parachute behavior of an inhibitor, a broad landscape of precipitation inhibitor performance can be seen which aligns well with the COSMO-RS predictions.

From a mechanistic perspective, the COSMO approach is highly attractive. Specifically, to calculate the energy required to combine the quantum sigma surfaces of the API and PI, the sigma potential (pso') the energy of forming new contacts between the two must be considered, this is reflected in the COSMO-RS energy term, $E\left(\sigma, \sigma^{\wedge}\right)$. This term significantly improves the mechanistic applicability of the approach, as all potential modes of interaction between API and PI are considered: hydrogen bond interactions, coulombic interactions and van de Waals interactions (see Appendix 3).

As recently reviewed by our group (Price et al., 2018) the majority of precipitation inhibitors sustain supersaturated API in solution via interactions. Although varying from system to system, the most common interactions are hydrogen bond interactions and hydrophobic interactions. For example, in accord with our findings, there have been a number of papers that show the successful precipitation inhibition of supersaturated APIs by Eudragit EPO, this is based on its ability to in teract strongly via both hydrogen bonding and hydrophobic interac tions. For example, Higashi and co-workers studied the effect of Eudragit EPO in combination with the poorly soluble drug mefenamic acid with 2D NOESY NMR (Higashi et al., 2014). The team found that the successful precipitation inhibition of Eudragit EPO was related to hydrophobic interactions between the aromatic portion of the API and the EPO polymer backbone; as well as a hydrophilic hydrogen bond in teraction between the aminoalkyl groups of EPO and the carbonyl groups of the API. Such interactions are also possible with dipyridamole, fenofibrate and glibenclamide.

Furthermore, considering the precipitation inhibitors that did not perform well, one can relate the calculation, dissolution performance and potential points of interaction from a mechanistic perspective. One of the interesting cases here is the lack of successful inhibition of dipyridamole precipitation by PVP. As previously mentioned, PVP is one of the polymers most commonly used as a precipitation inhibitor However, PVP has been shown to be ineffective in sustaining 


\section{Thesis Papers}

dipyridamole in solution, this was also identified by the COSMO-screen and is reflected in the dissolution performance of the formulation in this study. Chauhan and colleagues demonstrated that no interaction takes place between PVP and dipyridamole in the solid state (Chauhan et al. 2013), this is in line with the results in the current study as well as th fact that the COSMO-calculated enthalpy of interaction was positive and thus unfavorable.

Ultimately, these robust mechanistic calculations increase the suc cessful prediction of API-PI interaction and thus precipitation inhibition, as reflected in the strong positive correlations achieved between the COSMO-rank and the final formulation performance. For a full overview of the COSMO-RS equations, see Appendix 3.

4.5. Assumptions and limitations of the proposed in silico screening protocol

Our approach, which utilized excess enthalpy calculations to scree precipitation inhibitors, does not take into consideration the impact of water on the interaction between the API and PI. It has been repeatedly suggested that for a precipitation inhibitor to successfully sustain drus in solution, it must interact with both the API and the water in the medium or GI tract (Ting et al., 2016; Schram, 2015; Price et al., 2018). To exclude consideration of water's role in mediating API interaction with the precipitation inhibitor becomes especially problematic when considering polymers that have very high hydrophobicity or hydro philicity, as demonstrated by Schram and co-workers (Schram, 2015; Schram et al., 2016). From the data presented in this study, it is clea that the COSMO prediction for PEG did not correlate to the overal dissolution performance. Furthermore, $\mathrm{PEG}$ was the only clear outlier in the correlation for all three samples. This may be due to the aforementioned potential problem: $\mathrm{PEG}$ is very hydrophilic and is expected to bind and interact preferentially with water. This reduces the direct interaction with the API and therefore the desired precipitation inhibitor performance is not realized. The effect of removing PEG from the dataset is pronounced for all three compounds, such that when the Spearman's rank correlation coefficient analysis was repeated withou PEG, the correlation between COSMO-rank and dissolution-rank significantly improved to $0.98(0.0004, \mathrm{p}<0.05)$ for dipyridamole and glibenclamide, and to $0.8(0.022, \mathrm{p}<0.05)$ for fenofibrate. Due to this, the COSMO-RS protocol should be applied with the foresight that outliers and exceptions may be possible for very hydrophobic and hy drophilic inhibitors.

Another limitation of the COSMO-RS in-silico approach is the focus on local molecular interactions, whereas any supramolecular effects are neglected. Although factors such as molecular weight, viscosity and diffusivity of the precipitation inhibitors play an important role in precipitation inhibition (Warren et al, 2010; Price et al., 2018), insufficient information regarding these factors is available for many of the polymers. There are two main hypotheses with respect to the importance of these parameters to precipitation inhibition. The first, an lesser reported, states that molecular weight and viscosity affect pre cipitation inhibition via changes in the diffusion kinetics of both the drug and polymer in solution (Warren et al., 2010). Such effects cannot be taken into account by the COSMO-RS approach at the moment. The second, and more widely reported hypothesis, relates to an increasing number of binding sites when molecular weight and viscosity are increased (Warren et al., 2010). Such binding sites increase when a polymer is at least to some extent swollen in aqueous medium, but such swelling and the theta condition of the polymer in aqueous medium is an aspect that was not considered in the presented in silico screening. I would thus be beyond the scope of this approach to determine the effect of viscosity or molecular weight with the current COSMO-RS protocol. Finally, to complement the enthalpic considerations of interaction of PI with API, there are also entropic considerations that are not easily considered using the current COSMO-RS approach, therefore, for some API-precipitation inhibitor combinations, where the interaction is entropically unfavorable, the COSMO-RS approach may fail to predict the experimental result. Of course, it is also possible that the interaction is entropically favorable e.g. in the case of disruption of supramolecular structuring of polymers in aqueous solution, as previously reported (Schram et al., 2015a, 2015b).

\section{Conclusions}

In this work, we describe a novel in silico screening protocol for the selection of precipitation inhibitors for supersaturating formulations. The protocol uses the COSMO-RS model to calculate excess enthalpy of interaction between API and precipitation inhibitors, which is then used as a rank-order parameter to select potential precipitation inhibitors. Conceptually, such an approach may be applied for any enabling formulation that requires precipitation inhibitors, for example HME or SDD, but further work is required to validate this cross-formulation applicability. Despite the simplifications and assumptions in the pre sented COSMO-RS protocol, strong positive correlations were obtained between the rank-order prediction and formulation performance for the APIs studied. Furthermore, given the high-throughput and high-speed nature of the in-silico calculations, the screening protocol is very at tractive as a score-card approach for the design of enabling formulations for poorly soluble APIs in the pharmaceutical industry. Ultimately, this study highlights how in-silico tools can be used to improve efficiency of precipitation inhibitor selection as well as the like lihood that the most optimal formulation will be realized.

\section{Acknowledgements}

We would like to thank Axel Becker and Michael Lange (Merck KGaA, Darmstadt) for their helpful discussions and input into developing understanding of COSMO-RS and the applications currently applied.

\section{Funding}

This work has received funding from the European Union's Horizon 2020 research and innovation programme under grant agreement No. 674909 (PEARRL).

\section{Appendix A. Supplementary data}

Supplementary data to this article can be found online at https:// doi.org/10.1016/i.ejps.2019.03.006.

\section{References}

Abramov, Y.A., et al, 2012. Rational coformer or solvent selection for pharmaceutical cocrystallization or desolvation. J. Pham. Sci. 101 (10), 3687-3697. https://doi.org $10.1002 / \mathrm{jps} .23227$

Ahern, J.A., et al., 2013. Comparison of fenofibrate-mesoporous silica drug-loading doi.org/10.1016/j.ejps.2013.08.026.

Amidon, G.L., et al., 1995. A theoretical basis for a biophamaceutic drug classification: the correlation of in viftro drug product dissolution and in vivo bioavallablity. Pharn Res. 12 (3), 413-420.

Brouwers, J., et al, 2009. Supersaturating drug delivery systems: the answer to solubilitylimited oral bioavailability? J. Pharm. Sci. 98 (8), 2549-2572. https://doi.org/10. $1002 / \mathrm{jps} .21650$

Buch, P., et al., 2010. IVIV in oral absorption for fenofibrate immediate release tablets using dissolution and dissolution permeation methods. Pharmazie 65, 723-728.

kara, K., et al., 2016. Ordered mesoporous silica to enhance the bioavailability of $220-225$. https://doi.org $10.1016 /$ /.ejpb. 2016.08 . 0202 .

Chauhan, H., et al., 2013. Correlating the behavior of polymers in solution as precipitation inhibitor to its amorphous stabilization ability in solid dispersions. J. Pharm. Sci.

102 (6), 1924-1935.
Ditzinger, F., et al, 2019 . Lipophilicity and hydrophobicity considerations in bio-enabling oral formulations approaches-a PEARRL review. J. Pham. Pharnacol. 71, 464-48 Dressman, J.B., et al, 2016. Mesoporous silica-based dosage forms improve release
characteristics of poorly soluble drugs: case example fenofibrate. J. Pharm. 


\section{Thesis Papers}

Pharmacol. 68 (5), 634-645. hitps://doi.org/10.1111/jphp.12465.

Ferberg, J.H., et al., 2010. Dissolution rate and apparent solubility of pocrly soluble drugs in blorelevant dissolution media. Mol. Pharm. 7 (5), 1419-1430. https://dol

agerberg, J.H., et al., 2012. Ethanol effects on apparent solubility of poorly soluble drugs in simulated mp 2006467.

Galia, E., et al., 1998. Evaluation of various dissolution media for predicting in vivo performance of class I and II drugs. Pharm. Res. 15 (5), 698-705.

Guo, F., et al., 2011. Self-microemulsifying drug delivery system for improved oral bioavailability of dipyridamole: preparation and evaluation. Arch. Pharm. Res. 34 (7), 1113-1123. https://doi.org/10.1007/s12272-011-0709-8.

Guznan, HR., et al., 2007. Combined use of crystalline salt forms and precipitation inhibitors to improve oral absorption of celecoxib from solid oral formulations. J

He, H., et al., 2010. In vitro and in vivo evaluation of fenofibrate solid dispersion prepared by hot-melt extrusion. Drisg

Higashi, $\mathrm{K}$, et al., 2014. Insights into atomic-level interaction between mefenamic acid and eudragit" $\mathrm{EPO}$ in a supersaturated solution by hich-resolution magic-angle and eudragit ${ }^{\oplus}$ EPO in a supersaturated solution by high-rest
spinning NMR spectroscopy. Mol. Pharm. 11 (1), 351-357.

Hong, S., et al., 2016. High dug load, stable, manufacturable and bicavailable fenofibrat formulations in mesoporous silica: a comparison of spray drying versus solvent impregnation methoss. Drug Deliv 23 (1), 316-327. https://doi.org/10.3109/
10717544.2014.913323.

Homig, M., Klamt, A., 2005. COSMOfrag: a novel tool for high-throughput ADME property prediction and similarity screening based on quantum chemistry. J. Chen Inf. Model 45 (5), 1169-117\%. https / doi.org/10.1021/ci0501948.

Clamt, A., 1995. Conductor-like screening model for real solvents: a new approach to the quantitative calculation of solvation $\mathrm{p}$

Fohn, WW Sham, L J 1065 , Self concistent equations including exchange and correlation effects. Phys. Rev. 140

Laine, A.L., et al., 2016. Enhanced oral delivery of celecoxib via the development of a supersaturable amorphous formulation utilising mesoporous silica and co-loaded
HPMCAS. Int. J. Pharm. 512 (1), 118-125. https://doi.org/10.1016/j.jpharm. 2016 .

08.034 .
Leigh, M., et al., 2013. Comparison of the solubility and dissolution of drugs in fasted state biorelevant media (FaSSIF and FaSSIF-V2). Dissolution Tech. https://doi. org 10.14227/DT200313P44.

Loftsson, T., Brewster, M.E., 2010. Phamnaceutical applications of cyclodextrins: basic cience and product development. J. Pha

Loschen, C., Klant, A., 2012. CoSMOquick: a novel interface for fast $\sigma$-profile compostion and its application to COSMO-RS solvent screening using multiple reference tion and its application to COSMO-PS solvent screening using multiple reference solvents. In
ie 3023675 .

McCarthy, C.A., et al., 2016. Mesoporous silica formulation strategies for drug dissolution enhancement: a review. Exp Op Drug Deliv 13, 93-108. https://doi.org/10.1517/ 17425247.2016.1100165.

Mosquera-Giraldo, et al., 2016. Mechanistic design of chemically diverse polymers with applications in oral drug delivery. Biomacromol. 17 (11), 3659-3671. https://doi. org/10.1021/acs.biomac.6b01156.

Niederquell, A., et al., 2018. New prediction methods for solubility parameters based on molecular sigma profiles using pharmaceutical materials. Int. J. Pharm. 546 (1-2)

Shea, J., et al., 2017. Mesoporous silica-based dosage forms improve bioavailability of poorly soluble drugs in pigs: case example fenofib

palmelund, H. et al., 2016. Studying the propensity of compounds to supersaturate: a practical and broadly applicable approach. J. Pharm. Sci. 105, 3021-3029. Petrusevskra, M., et al., 2013. Evaluation of a high-throughput screening method for the detection of the excipient-mediated precipitation inhibition of poorly soluble drugs.
Assay Drug Dev Technol 11 (2), 117-129. hitps://dol.org/10.1089/adt.2012.466. Assay Drug Dev Technol 11 (2), $117-129$.
pazarska, A., et al, 2013. Application of COSMO-RS as an excipient ranking tool in early formulation development. Burr.

Prasad, D., et al., 2016. Role of molecular interactions for synergistic precipitation inhibition of poorly soluble drug in supersaturated drug-polymer-polymer ternary solution.
5 b0065.

Price, D.J., et al., 2018. Approaches to increase mechanistic understanding and aid in the selection of precipitation inhibitors for supersaturating formulations - a PEARRL review. J. Pharm. Pharmacol. https://doi.org/10.1111/jphp.12927. (epub ahead of

print).
Schram, C.J, et al., 2015a. Impact of polymer conformation on the crystal growth inhibition of a poorly water-soluble drug in aqueous solution. Langmuir 31 (1) 171-179. https://doi.org/10.1021/la503644r

chram, C.J., et al., 2015b. Influence of polymers on the crystal growth rate of felodipine: correlating adsorbed polymer surface coverage to solution crystal growth inhibition

Schram, $\mathrm{CJ}$ et al 2016 . Polymer inhibition of crystal growth by surface poisoning. Crystal Growth and Design 16 (4), 2094-2103. https://doi org /10.1021/acs.cgd $5 \mathrm{~b} 01779$.

Taniguchi, C., et al., 2013. New dipyridamole salt with improved dissolution and oral bioavailability under hypochlorhydric conditions. Drug Metab Pharmacokinet 28 (5) 383-390.

Taylor, L.S., Zhang, G.G.Z., 2016. Physical chemistry of supersaturated solutions and implications for oral absorption. Adv. Drug Deliv. Rev. 101, 122-142. https://doi org/10.1016/j.addr. 2016.03.006.

Theil, $F$., et al., 2017. Extraordinary long-term-stability in kinetically stabilized amorphous solid dispersions of fenofibrate. Mol. Pharm. 14 (12), 4636-4647. https://do org/10.1021/acs.molpharmaceut.7b00735.

2016. New metastable form of glibenclamide from ternary solid dispersions containing polyvinylpy $70-79$.

Ting, M., et al., 2016. High-throughput excipient discovery enables oral delivery of poorly cscentsci.6b00268.

Uejo, F., et al., 2013. Dissolution improvement of fenofibrate by melting inclusion in mesoporous silica. Asia J Pharm Sci 8 (6), 329-335. https://doi.org/10.1016/j.ajps 2013.11.001

Van Speoybroeck, M., et al., 2011. Preventing release in the acidic environnent of the stomach via occlusion in ordered mesoporous silica enhances the absorption of poorly soluble weakly acidic drugs. J. Pharm. Sci. 100 (11), 4864-4876. hitps://dof.

Speybroeck, M., et al., 2010. Enhanced absorption of the poorly soluble drug fenoits release rate from ordered mesoporous silica. Eur. J. Pharm. Sci. 41 (5), 623-630. https://doi.org/10.1016/j.ejps. 2010.09.003

Vora, C., et al. 2016. Preparation and characterization of dipyridamole solid dispersions for stabilization of supersaturation: effect of precipitation inhibit ors type and mole cular weight. Pharm. Dev. Technol. 21 (7), 847-855.

Warren, D.B., et al., 2010. Using polymeric precipitation inhibitors to improve the absorption of poorly water-soluble drugs: a mechanistic basis for utility. J Drug Targ 18 (10), 704-731. https://doi.or: / 10.3109/1061186X.2010.525652.

Wie, H., et al., 2006. Biorelevant dissolution media as a predictive tool for glyburide a class II drug. Eur. J. Pharm. Sci. 29 (1), 45-52. https://doi.org/10.1016/j.ejps. 2006

Zecevic, D.E., et al., 2018. From benchtop to pilot scale-experimental study and computational assessment of a hot-melt extrusion scale-up of a solid dispersion of di$1016 /$ i. iipharm 2017.12 .033 .

Zheng, W., et al, 2012. Selection of oral bioavailability enhancing formulations during drug discovery. Drug Dev. Ind. Pharm. 38 (2), 235-247. https://doi.org/10.3109/ 03639045.2011 .602406 


\section{$\mathrm{X}$. Thesis Publications}

European Journal of Pharmaceutical Sciences 141 (2020) 105113

Contents lists available at ScienceDirect

European Journal of Pharmaceutical Sciences

journal homepage: www.elsevier.com/locate/ejps

\section{Incorporation of HPMCAS during loading of glibenclamide onto mesoporous silica improves dissolution and inhibits precipitation}

Daniel J. Price ${ }^{\mathrm{a}, \mathrm{b}}$, Anita Nair ${ }^{\mathrm{a}}$, Johanna Becker-Baldus ${ }^{\mathrm{c}}$, Clemens Glaubitz ${ }^{\mathrm{c}}$, Martin Kuentz ${ }^{\mathrm{d}}$, Jennifer Dressman ${ }^{\mathrm{b}}$, Christoph Saal ${ }^{\mathrm{a}, *}$

"Merck KGaA, Darmstadt, Germany

Institute of Pharmaceutical Technology, Goethe University, Frankfurt, Germany

Werte for Biomolecular Magnetic Resonance, Goethe University, Frankfurt, Germany

"University of Arts and Applied Sciences Norlhwestern Swizzerland, Basel, Swizzerland

A R T I CLE I N F O

\section{Keywords:}

Mesoporous silica

Supersaturation

Precipitation inhibition

Solid dispersion

Mechanistic

\begin{abstract}
A B S T R A C T
Mesoporous silica has emerged as an enabling formulation for poorly soluble active pharmaceutical ingredients (APIs). Unlike other formulations, mesoporous silica typically does not inhibit precipitation of supersaturated API therefore, a suitable precipitation inhibitor (PI) should be added to increase absorption from the gastrointestinal (Gi) tract. However, there is limited research about optimal processes for combining PIs with silica formulations. Typically, the PI is added by simply blending the API-loaded silica mechanically with the selected PI. This has the drawback of an additional blending step and may also not be optimal with regard to release of drug and PI. By contrast, loading PI simultaneously with the API onto mesoporous silica, i.e. co-incorporation, is attractive from both a performance and practical perspective. The aim of this study was to demonstrate the utility of a co-incorportion approch for combining PIs with silica forme utility of a rationale for improvement of the performance of silica formulations using the co-incorporation approach. The
results indicate that co-incorporating HPMCAS with glibenclamide onto silica significantly improved the extent and duration of drug supersaturation in single-medium and transfer dissolution experiments. Extensive spectroscopic characterization of the formulation revealed that the improved performance was related to the formation of drug-polymer interactions already in the solid state; the immobilization of API-loaded silica on HPMCAS plates, which prevents premature release and precipitation of API; and drug-polymer proximity on disintegration of the formulation, allowing for rapid onset of precipitation inhibition. The data suggests that coincorporating the PI with the API is appealing for silica formulations from both a practical and formulation performance perspective.
\end{abstract}

1. Introduction

Among the various administration routes for drugs, oral administration is the most commonly employed. It is cost-effective and convenient for the patient, leading to a very high patient compliance (Krishnaiah, 2010). APIs must be absorbed to become orally bioavailable, a process which relies in turn on sufficient solubility and permeability of the API (Zheng, 2012). In recent years there has been an exponential increase in drugs exhibiting poor solubility: it is reported that approximately $60 \%$ of all drugs on the market are poorly soluble (Taylor and Zhang 2016). It has been suggested that anywhere between 80 and $90 \%$ of compounds in development also demonstrate low solubility (Loftsson and Brewster, 2010). These estimates highlight the need for effective formulation approaches to avoid low bioavailability associated with poor aqueous solubility.

To overcome these challenges, formulators have developed a series of promising formulation strategies (Ditzinger, 2018). These approaches include: (i) solvents, co-solvents and lipids; (ii) micelle systems; (iii) particle size reduction; (iv) complexation; and (v) amorphous technologies (Zheng, 2012). One of the most common approaches for improving bioavailability is via the generation of supersaturated solutions in the GI-tract, which can drive improved absorption (Zheng, 2012). However, these systems are metastable due to the energetic propensity of the compound to precipitate (Price, 2018) Therefore, precipitation inhibitors (PIs) are often used to sustain the supersaturated state by inhibiting or slowing down precipitation of

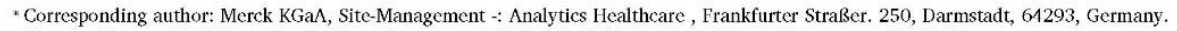

E mail addresses: daniel-joseph.price@merckgroup.com (D.J. Price), Christoph.saal@merckgroup.com (C. Saal).

https://doi.org/10.1016/j.ejps.2019.105113

Received 6 June 2019; Received in revised form 16 October 2019; Accepted 18 October 2019

Available online 23 October 2019

0928-0987/@ 2019 Elsevier B.V. All rights reserved. 


\section{Thesis Publications}

drug (Warren, 2010). Successful PI systems can sustain drug super saturation over physiologically relevant time-scales by interfering with the crystallization process (Price, 2018). Precipitation inhibitors can kinetically prevent re-crystallization via a number of mechanisms, including: viscosity, co-solvency and drug-polymer interactions, with the latter widely being reported to being especially importan (Warren, 2010; Price, 2018). Recent advances in precipitation inhibi tion design and selection include de novo precipitation inhibitor design (Ting, 2017) and in silico calculation of drug-polymer mixing enthalpies for precipitation inhibitor selection (Price et al., 2019).

One under-utilized formulation technology to generate drug super saturation is mesoporous silica. Mesoporous silica is a silicon dioxide excipient that has a highly porous network, consisting of mesopores between 2 and $50 \mathrm{~nm}$ in diameter (Ditzinger, 2018). These materials have very high specific surface areas and are used in catalysis, en vironmental clean-up, chromatography, and drug deliver (McCarthy, 2016). Poorly soluble APIs can become molecularly adsorbed on the surface of the silica and sterically confined such that recrystallization cannot occur (Knapik, 2016). Indeed, this is one of the most widely reported advantages of mesoporous silica, in its enhance stabilization capabilities due to nanoconfinement in the porous network (McCarthy, 2016). Mesoporous silica-based 'spring and parachute' for mulations have been widely demonstrated in the literature, from both an in vitro and in vivo perspective (Ditzinger, 2018; McCarthy, 2016). Van Speybroeck and colleagues originally described how such pre cipitation inhibitors, including HPMC and HPMCAS, can enhance the oral absorption of itraconazole released from mesoporous silica in rats (Van Speybroeck, 2010). This was also demonstrated in pigs, with O'Shea and colleagues using the precipitation inhibitor HPMCAS to improve the oral absorption of fenofibrate released from mesoporou silica (O'Shea, 2016). Recent work on precipitation inhibitors for me soporous silica has also taken place, Price and co-workers developed of an in silico screening approach which calculates drug-polymer mixing enthalpy for the optimized selection of precipitation inhibitors for mesoporous silica formulations (Price et al., 2019). In spite of these recent advances in mesoporous silica and precipitation inhibition, the method of combining precipitation inhibitors with mesoporous silica remains relatively inefficient. Typically, PIs are mechanically blended with the API-loaded silica formulations after the drug is loaded (usually with a mortar and pestle). However, it has recently been shown that incorporating the PI into the API loading process itself can dramatically improve both in vitro and in vivo performance of a celecoxib loaded silica formulation (Laine, 2016). In light of this proof of concept, there is a need for further mechanistic research. This study aims to demonstrate the utility of a co-incorporation approach for combining PIs with silica formulations, and to develop a mechanistic rationale to explain the improvement in performance of silica formulations using the coincorporation approach.

\section{Experimental}

\subsection{Materials}

Crystalline glibenclamide (GB), reagent grade acetone, HPLC grade acetonitrile and HPLC grade methanol were all purchased from MilliporeSigma (St Louis, MO, USA). AQOAT (HPMCAS-MF) was purchased from ShinEtsu (Japan). Parteck ${ }^{\circledR}$ SLC was a gift sample from Merck KGaA (Germany). FaSSGF/FaSSIF/FeSSIF powder to make biorelevant dissolution medium, Fasted Simulated Intestinal Fluid (FaSSIF), was obtained from Biorelevant.com (UK).

\subsection{Methods}

2.2.1. Determination of thermodynamic solubility

FaSSIF was prepared by weighing $45 \mathrm{mg}$ of FaSSGF/FaSSIF/FeSSIF powder into $45 \mathrm{~mL}$ of phosphate buffer (pH 6.5) (Galia et al., 1998).
SGF ( $\mathrm{pH}$ 1.2) was prepared according to USP monographs. Glib enclamide $(2-3 \mathrm{mg})$ was accurately weighed into a Uniprep ${ }^{2}$ syringeless filter ( $5 \mathrm{~mL} ; 0.45 \mu \mathrm{m}$ ). $2 \mathrm{~mL}$ of either FaSSF ( $\mathrm{pH}$ 6.5) or SGF (pH 1.2) was added and the samples were agitated at $450 \mathrm{rpm}$ for $24 \mathrm{~h}$ at $37^{\circ} \mathrm{C}$. The $\mathrm{pH}$ was checked at $7 \mathrm{~h}$ and adjusted with $0.1 \mathrm{~N} \mathrm{NaOH}$ or 0.1 $\mathrm{N} \mathrm{HCl}$, if a deviation greater than $+/-0.05 \mathrm{pH}$ units was observed. The final $\mathrm{pH}$ was also recorded after $24 \mathrm{~h}$.

Samples were filtered with PTFE $0.45 \mu \mathrm{m}$ Whatman filters after $24 \mathrm{~h}$. Filtrates were diluted with acetonitrile and water (1:4) to avoid precipitation from the saturated solution. Samples were analyzed with ultra-high performance liquid chromatography (UPLC) (Thermo Dionex Ultimate 3000, Thermo Fisher, MA, USA) to determine the API concentration. API concentration was determined based on a standard calibration curve of nine standard concentrations $(50,30,10,5,3,1,0.5$, $0.3,0.1 \mu \mathrm{g} / \mathrm{mL}$ ). Three quality control samples of known concentrations $(30,3,0.3 \mu \mathrm{g} / \mathrm{mL})$ were prepared and used to check the robustness of the calibration curve. The determination was carried out in duplicate.

\subsubsection{UPLC method}

UPLC analysis was performed using a Thermo Dionex Ultimate 3000 (Thermo Fisher, MA, USA) equipped with a diode array detector at $240 \mathrm{~nm}$ (Thermo Fisher, MA, USA). Chromatographic separation was achieved on an Acquity UPLC BEH column C8 $(2.1 \times 50 \mathrm{~mm}, 1.7 \mu \mathrm{m}$, Waters, MA, USA). The mobile phases A and B consisted of water: formic acid 99:1 (v:v) and acetonitrile : formic acid 99:1 (v: v), re spectively. Gradient and flow rate is shown in Table 2 . System man agement, data acquisition and processing were performed with the Chromeleon ${ }^{\text {Tx }}$ software package, version 7.2 (Thermo Fisher, MA, USA)

2.2.3. Parteck SLC ${ }^{\oplus}$ standard loading procedure and standard PI incorporation

Glibenclamide loaded silica was prepared using the solvent impregnation rotary evaporator method (Laine, et al 2016) as follows: A solution $(10 \mathrm{mg} / \mathrm{mL})$ of API in acetone was added to Parteck SLC (1:2 w/w API/Parteck SLC $\left.{ }^{\circledast}\right)$ under magnetic stirring for $30 \mathrm{~min}$. The suspension was then transferred to a rotary evaporator, and the solvent was removed under reduced pressure at $40^{\circ} \mathrm{C}$. After complete removal of the solvent, the powder was left to dry in the rotary evaporator under reduced pressure for a further $2 \mathrm{~h}$. The formulation was then physically combined with HPMCAS (API: Parteck SLC ${ }^{\oplus}$ HPMCAS 1: 2: 3 w/w) using a pestle and mortar

\subsubsection{Parteck SLC ${ }^{\circledast} A P I / P I$ co-incorporation procedur}

Glibenclamide/HPMCAS co-incorporated Silica samples were pre pared using the solvent impregnation rotary evaporator method. A solution of API $(10 \mathrm{mg} / \mathrm{mL})$ and HPMCAS $(30 \mathrm{mg} / \mathrm{mL})$ in acetone was added to Parteck SLC (1:2:3 API: Parteck SLC ${ }^{\varpi}$ HPMCAS) under magnetic stirring, which was continued for $30 \mathrm{~min}$. The suspension was transferred to a rotary evaporator, and the solvent was removed under reduced pressure at $40^{\circ} \mathrm{C}$. After complete removal of the solvent, the powder was left to dry in the rotary evaporator under reduced pressure for a further $2 \mathrm{~h}$

2.2.5. Preparation of an API-HPMCAS sample as control

A control sample consisting of only of API and HPMCAS was also prepared. A solution with the same concentrations of API $(10 \mathrm{mg} / \mathrm{mL})$ and HPMCAS ( $30 \mathrm{mg} / \mathrm{mL}$ ) as described above for the API - silica - PI system in acetone was prepared under magnetic stirring for $15 \mathrm{~min}$. The solution was then transferred to a rotary evaporator, and the solvent was removed under reduced pressure at $40^{\circ} \mathrm{C}$. After complete removal of the solvent, the powder was left to dry in the rotary evaporator under reduced pressure for a further $2 \mathrm{~h}$. Residual solvent concentration was recorded with $2 \mathrm{D}{ }^{1} \mathrm{H}$ NMR to ensure residual solvent was below the ICH limit of $0.5 \%$ (data not shown). 


\section{$\mathrm{X}$. Thesis Publications}

2.2.6. Determination of glibenclamide loading onto mesoporous silica

To determine the $\%(w / w)$ of API in the mesoporous silica, the loaded samples were dispersed and stirred in DMSO as this solvent is known to dissolve glibenclamide readily. Samples were taken after $1 \mathrm{~h}$, centrifuged, filtered and diluted before being quantified by UPLC, according to the method described in 2.2.2. The API content was calculated relative to the mass of loaded samples dispersed within the DMSO. The study was performed in triplicate.

\subsubsection{Powder X-ray diffraction (PXRD)}

Samples were prepared between X-ray amorphous films and measured in transmission mode using $\mathrm{Cu}-\mathrm{K} \alpha 1$-radiation and a Stoe StadiP 611 KL diffractometer equipped with Dectris Mythen1K PSD. The measurements were evaluated with the software WinXPow 3.03 by Stoe, Crystallographica Search/Match Version 3.1.0.2, the ICDD PDF$4+2014$ Database and Igor Pro Version 6.34 by Wavemetrics Inc. Finger/Cox/Jephcoat. Angular range: $1-65^{\circ} 2 \theta$; PSD-step width: $2^{\circ} 2 \theta$ angular resolution: $0.015^{\circ} 2 \Theta$ measurement time: $15 \mathrm{~s} / \mathrm{step}, 0.25 \mathrm{~h}$ overall.

\subsubsection{FaSSIF mini-dissolution experiment}

Around $5 \mathrm{mg}$ of API (or the equivalent of API-loaded silica) was weighed accurately into a glass vial. $5 \mathrm{~mL}$ of FaSSFF was added. The vials were agitated at $37^{\circ} \mathrm{C}$ and $450 \mathrm{rpm}$ in a shaker for $2 \mathrm{~h}$. Samples were taken at 2, 15, 60 and 120 min, filtered (0.45 PTFE Whatman filters), diluted, and analyzed by UPLC. Solid residues at the end of the experiment were collected via centrifugation and analyzed for crystallinity with powder X-ray diffraction (PXRD). This was carried out on the following samples: API, API + polymer, API loaded silica and API loaded silica + PI. The mini-dissolution trials were conducted in duplicate for all samples.

\subsubsection{Biorelevant transfer experiments}

The experimental set-up for the transfer experiments is demonstrated in Fig. 1 .

Around $150 \mathrm{mg}$ of API or equivalent was accurately weighed in $100 \mathrm{~mL}$ stoppered flask - the exact sample masses varied dependent on the formulation (see Table 1)

$25 \mathrm{~mL}$ of simulated gastric fluid ( $\mathrm{pH} 1.2$ ) prepared according to USP monographs was added to the flask and agitated at $450 \mathrm{rpm}$ and $37^{\circ} \mathrm{C}$. $50 \mathrm{~mL}$ of FaSSIF were added to a separate flask, which was also agitated at $450 \mathrm{rpm}$ and $37^{\circ} \mathrm{C}$. After $30 \mathrm{~min}$, the API suspension in SGF pH 1.2 was transferred at a zero-order rate of $0.85 \mathrm{~mL} / \mathrm{min}$ using a peristaltic pump, until the complete gastric contents were transferred ( $\sim 30 \mathrm{~min})$ into the FaSSF compartment. Samples were withdrawn from the in testinal compartment at regular time points using a $1 \mathrm{~mL}$ syringe to a sampling tube fitted with a pre-filter of $10 \mu \mathrm{m}$ and filtered again using a $0.45 \mu \mathrm{m}$ PTFE Whatman syringe filter and diluted. Samples were then

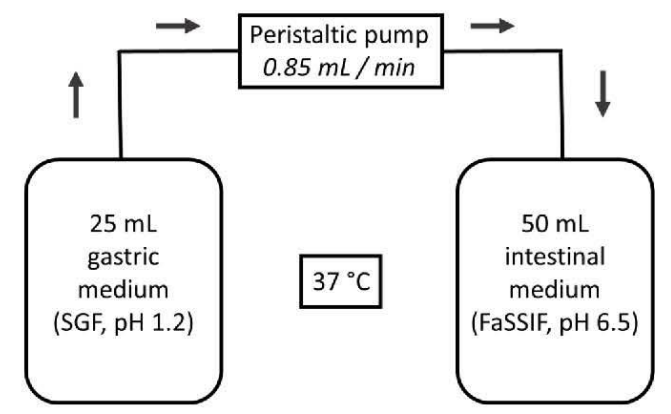

Fig. 1. Experimental diagram showing mini-transfer set-up
Table 1

Transfer dissolution sample preparation

\begin{tabular}{lll}
\hline Formulation & Weighed mass (mg) & API mass (mg) \\
\hline GB loaded silica & 450 & 150 \\
GB loaded silica HPMCAS Blend & 900 & 150 \\
GB and HPMCAS co-incorporated silica & 900 & 150 \\
\hline
\end{tabular}

Table 2

UPLC gradient and flow rates

\begin{tabular}{llll}
\hline Time (mins) & $\begin{array}{l}\text { Flow rate (mL/ } \\
\text { min) }\end{array}$ & $\begin{array}{l}\text { \% (v:v) Mobile phase } \\
\text { A }\end{array}$ & $\begin{array}{l}\%(v: v) \text { Mobile phase } \\
\text { B }\end{array}$ \\
\hline 0 & 0.83 & 90 & 10 \\
0.83 & 0.83 & 10 & 90 \\
1.2 & 1.5 & 90 & 10 \\
2 & 1.5 & 90 & 10 \\
2.01 & 0.83 & 90 & 10 \\
\hline
\end{tabular}

analyzed by UPLC for API content. The post-dissolution residues were then collected and analyzed for crystallinity with XRPD.

2.2.10. Single medium SGF dissolution assay (in tandem to transfer assay)

Around $150 \mathrm{mg}$ of API or equivalent was accurately weighed into a $100 \mathrm{~mL}$ stoppered flask. The exact sample masses varied dependent on the formulation (see Table 1). $25 \mathrm{~mL}$ of SGF (pH 1.2, gastric compartment) was added to the flask and the contents agitated at $450 \mathrm{rpm}$ and $37^{\circ} \mathrm{C}$. Samples were withdrawn at regular time points using a $1 \mathrm{~mL}$ syringe to a sampling tube fitted with a pre-filter of $10 \mu \mathrm{m}$ and filtered again using a $0.45 \mu \mathrm{m}$ PTFE Whatman syringe filter and suitably di luted. Samples were then analyzed with UPLC for API content. The postdissolution residues were directly collected and analyzed for crystallinity with PXRD.

2.2.11. Scanning electron microscopy (SEM) and energy-dispersive $X$-ray spectroscopy (EDX)

Samples were prepared on copper tape and imaged using a Hitachi TM3000 Tabletop Microscope, $W$ cathode, low vacuum, accelerating voltage $5 \mathrm{kV}$ and $15 \mathrm{kV}$, 4-Quadrant BSE detector, magnification $15 \mathrm{x}-$ $30,000 x$. For the energy dispersive X-ray spectroscopy (EDX) data, a standard-less quantitative analysis was performed by using the ZAF correction, considering the correction for light elements standardless element coefficient factors (SEC).

2.2.12. Solid-state nuclear magnetic resonance (NMR) spectroscopy

Solid-state NMR experiments were performed under magic-angle sample (MAS) spinning using a Bruker $4 \mathrm{~mm}$ MAS HXY probe in double resonance mode in combination with a Bruker Avance $600 \mathrm{MHz}$ wide bore NMR spectrometer (Bruker). The sample spinning frequency was $10 \mathrm{kHz}$, and the readout on the probe thermocouple was set to $290 \mathrm{~K}$ ${ }^{13} \mathrm{C}$-CP experiments were performed using a contact time of $1 \mathrm{~ms}$ and $100 \mathrm{kHz}$ high power proton decoupling following the SPINAL64 scheme was applied during acquisition. The recycle delay was $3 \mathrm{~s}$. The spectra were indirectly referenced to DSS via the $\mathrm{CH} 2$ signal of Adamantane at $40.49 \mathrm{ppm}$. Solid-state NMR measurements were repeated on multiple batches to ensure reliability of the interpretation.

3. Results

3.1. Solid-state form of glibenclamide in formulations

The glibenclamide powder used in this work is crystalline in the solid-state as shown by XRPD (Fig, 2a). Succesful loading of glibenclamide onto mesoporous silica was demonstrated by the absence of distinct Bragg peaks in XRPD patterns, which indicated a shift from the 


\section{Thesis Publications}

(a)

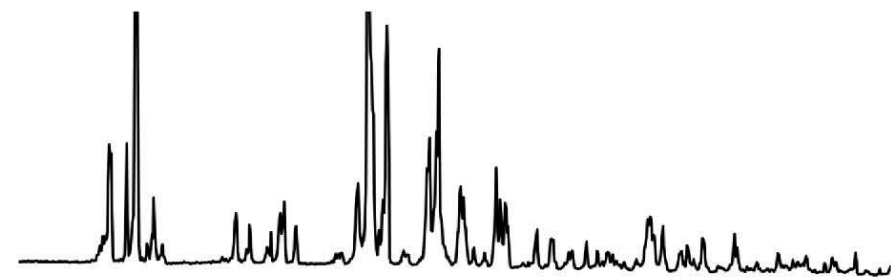

(b)

(c)

(d)

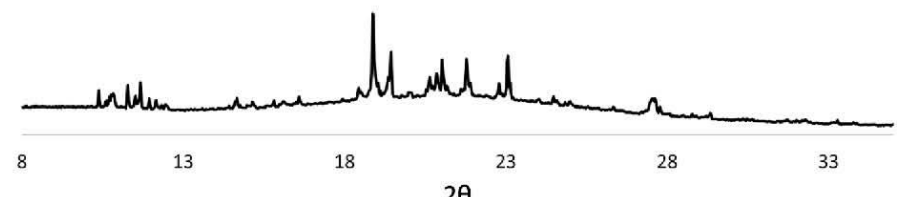

$2 \theta$

Fig. 2. XRPD pattern for crystalline glibenclamide (GB) (a), glibenclamide loaded silica (b), GB and HPMCAS co-incorporateded silica (c) and GB and HPMCAS prepared by rotary evaporation (d)

crystalline to the amorphous state (Fig. 2b). The co-incorporation process did not interfere with the solid-state conversion of glibenclamide: the co-incorporated sample exhibited the same shift from crystalline to amorphous post-loading ( $F i g .2 c$ ). However, the control sample, which consisted of HPMCAS/GB prepared by solvent evaporation, showed partial crystallinity, which aligned with the XRPD patter for the unmodified crystalline glibenclamide (Fig. $2 d$ ).

\subsection{Loading content of glibenclamide in mesoporous silica formulations}

The \% loading of glibenclamide determined by UPLC is shown in Table 3. The final glibenclamide content in the final mesoporous silica formulations was around $15 \%$, irrespective of whether the drug was first loaded onto the silica and then combined with HPMCAS, or the HPMCAS was incorporated during drug loading. Drug loading levels are modest, which could be a limitation for drugs that are administered at high doses. However, they are in line with usual supersaturating drug formulations that require precipitation inhibitors (Price et al., 2019; Ditzinger, 2018)

3.3. Scanning electron microscopy (SEM) and electron dispersive $X$-ray spectroscopy (EDX)

SEM images for glibenclamide loaded silica, glibenclamide loaded silica + HPMCAS Blend and SEM and EDX images for glibenclamide and HPMCAS co-incorporated silica are shown in Fig. 3. The unloaded

Table 3

API loaded silica total API content

\begin{tabular}{lll}
\hline Formulation & $\begin{array}{l}\text { Theoretical } \\
\text { loading } \% \text { ) }\end{array}$ & $\begin{array}{l}\text { Actual loading } \\
\text { (UPLC) (\%) }\end{array}$ \\
\hline $\begin{array}{c}\text { Glibenclamide loaded silica } \\
\text { (without HPMCAS) }\end{array}$ & 30 & $30.1 \pm 0.1$ \\
$\begin{array}{c}\text { Glibenclamide and HPMCAS } \\
\text { co-incoporated silica }\end{array}$ & 15 & $15.9 \pm 0.2$ \\
$\begin{array}{c}\text { Glibenclamide loaded } \\
\text { silica + HPMCAS blend }\end{array}$ & - & 15.1 \\
\hline
\end{tabular}

silica is shown in Fig. 3a. In glibenclamide loaded silica, the char acteristic silica particles are also present (Fig. $3 b$ ). This is also the case for glibenclamide loaded silica + HPMCAS physical mixture (Fig. $3 c$ ), where the particles are simply 'diluted' by the addition of the polymer, which is depicted as the dark texture in between the silica particles. However, large platelet particles were observed when HPMCAS was incorporated during the loading step onto the silica (Fig. 3d). The EDX images show that the platelet particles are carbon based and therefore likely composed of HPMCAS. The silica particles appear to be embedded in the HPMCAS plate, as when the image was zoomed to the same resolution as Fig. 3, the images looked similar to characteristic silica particles. Chlorine, used as a marker for glibenclamide, was observed within the silica particles on the HPMCAS plate, with no API observable outside of the platelets. These observations suggest that the formulation is a solid dispersion of API loaded silica in HPMCAS (Fig. 3 bottom).

Based on this potential combination of silica loading and classical solid dispersion, it was important to assess what role the silica plays in the formulations. As can be seen in Fig. 4, the particles produced in the solvent evaporation of glibenclamide and HPMCAS (control sample) are similar to the particles produced when the HMPCAS in incorporated during the drug loading step onto the silica. However, EDX analysis shows a key diffence between the formulations, in that the drug marker, chlorine, is no longer confined within the polymer (plates), but is freely distributed in the control sample.

\subsection{FaSSIF mini-dissolution}

In FaSSIF mini-dissolution experiments, the concentration of the pure drug approached the thermodynamic solubility value of $8.1 \mu \mathrm{g} / \mathrm{mL}$ (Appendix 1) (Fig. 5a). From the glibenclamide loaded silica formulations, a significant improvement in dissolution was observed in the FaSSIF mini-dissolution experiments, reaching a 25 -fold supersaturation (Fig. 5). However, due to the metastable nature of the supersaturation, these extremely high concentrations were short-lived and the concentration reverted to the thermodynamic solubility within 60 min (Fig. 5). 


\section{Thesis Publications}

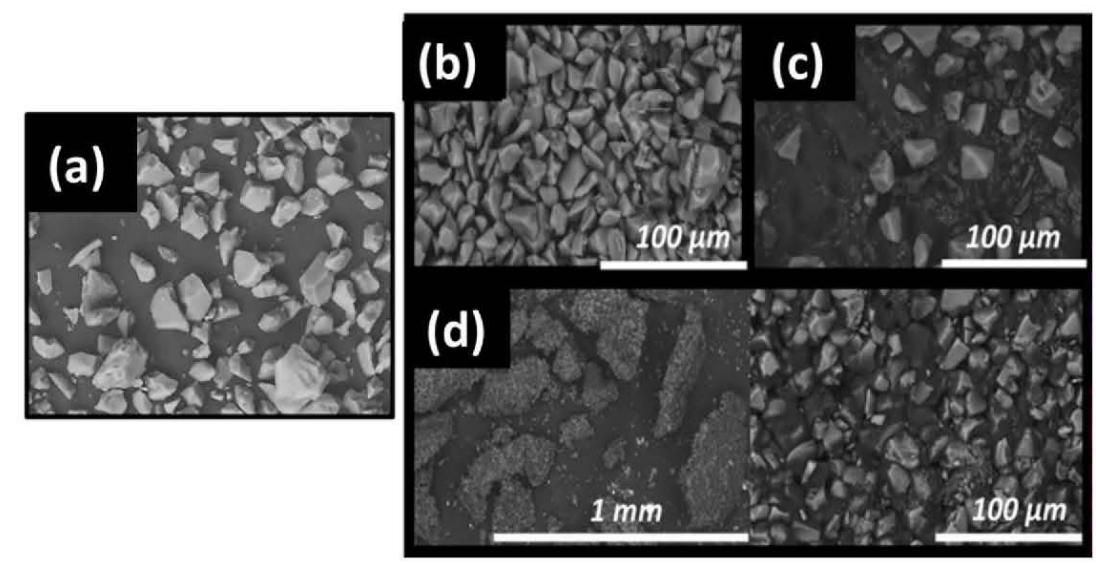

\section{(a)}

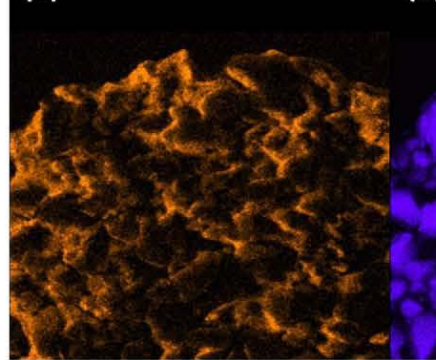

(b)

(c)

Fig. 3. Top: SEM (left) of unloaded mesoporous silica (a), glibenclamide loaded silica (b), glibenclamide loaded silica + HPMCAS physical mixture (c) and HPMCAS incorporated during loading of glibenclamide onto silica (d). Bottom: SEM EDX of HPMCAS incorporated during loading of glibenclamide onto silica. Carbon (a), silicon (b) and chlorine (c) atoms are highlighted. Chlorine is a marker for glibenclamide.

Physically blending the glibenclamide loaded silica with HPMCAS prolonged the duration of supersaturation to at least $2 \mathrm{~h}$, although the degree of supersaturation was lower (about 3-fold) (Fig. 6). Co-incorporating the HPMCAS with the glibenclamide onto the silica further improved the dissolution and precipitation performance, with higher supersaturation (about 6-fold) achieved over the time course of the experiment (Fig. 6). Finally, the control sample, which used the same process as the co-incorporated in the absence of silica, showed almost no improvement in the FaSSIF mini-dissolution relative to the crystalline API. This result is in agreement with the partial crystallinity observed in the XRPD (Fig. 6).

Post-dissolution residues were collected for each of the samples and analyzed by XRPD. Crystalline glibenclamide precipitated in all samples except the co-incorporated formulation, in which the solid residue at the end of the experiment was amorphous (Appendix 2).

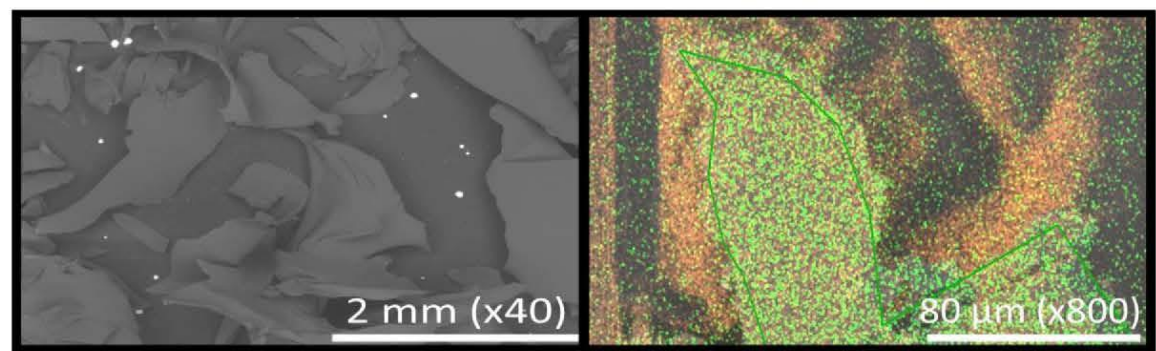

Fig. 4. SEM (left) and EDX (right) images of glibenclamide and HPMCAS prepared by solvent evaporation shows the same particle size and morphology as the coincorporated samples. However, in this sample the drug (indicated by green) is no longer confined within the polymer plate and is freely distributed throughout the sample. 


\section{$\mathrm{X}$. Thesis Publications}

D.J. Price, et al.

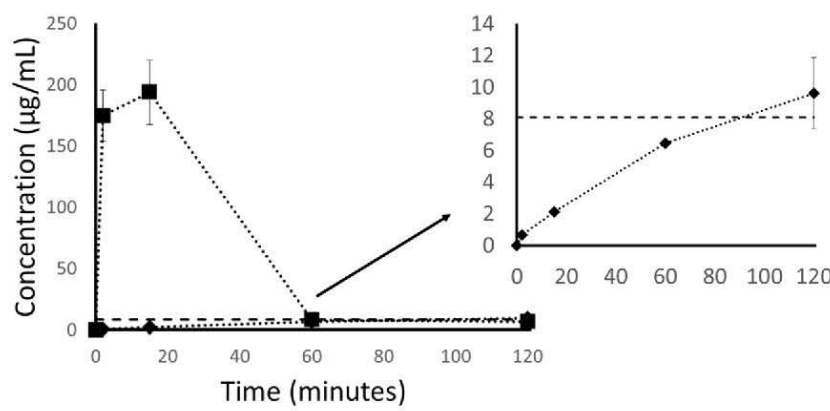

European Journal of Pharmaceutical Sciences 141 (2020) 105113

Fig. 5. Mini-dissolution profiles of glibenclamide ( $\bullet$, and glibenclamide loaded silica ( $(\boldsymbol{\omega})$ in FaSSIF, $\mathrm{pH} 6.5$ at $37^{\circ} \mathrm{C}$ $(n=2)$. Mean Glibenclamide thermodynamic solubility in FassIF is represented by the dashed horizontal line. In the insert on the right, the dissolution of crystalline glibenclamide has been magnified for better comparison.

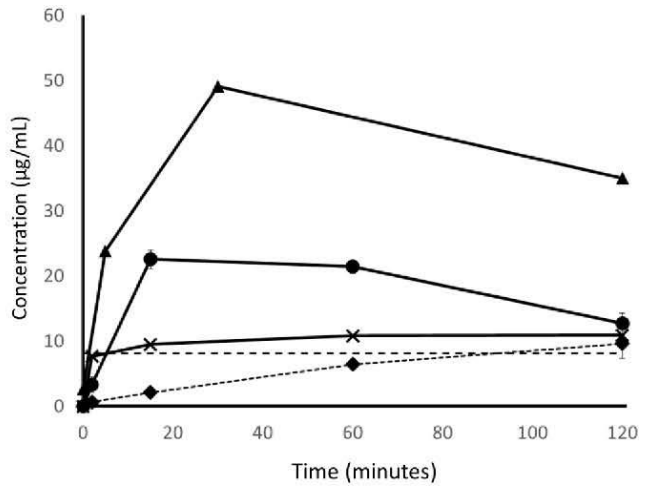

Fig. 6. Mini-dissolution profiles of glibenclamide ( $\bullet$, glibenclamide loaded silica + HPMCAS blend ( and glibenclamide and HPMCAS Co-incorporated silica $(\boldsymbol{\Lambda})$ and control: glibenclamide/HPMCAS prepared by solvent evapora tion (X) in FaSSIF, $\mathrm{pH} 6.5$ at $37^{\circ} \mathrm{C}(n=2)$.

\subsection{Transfer model experiments}

During transfer model dissolution experiments with pure glibenclamide, no concentrations were detected in the SGF portion of the assay (Fig. 7). This is in line with the thermodynamic solubility results, which indicated that the solubility of glibenclamide was under the limit of detection of the UPLC method (Appendix 1). After transfer into the FaSSIF portion of the experiment, the concentration profile closely overlapped with the mini-dissolution profile, suggesting that the dissolution of crystalline glibenclamide was largely unaffected by prewetting in SGF (Fig. 7).

Comparison of results from transfer model and mini-dissolution experiments of glibenclamide loaded silica in the absence of any precipitation inhibitors suggests that single-medium dissolution may lead to different expectations of formulation performance. In the transfer model experiments with glibenclamide loaded silica (Fig. 7); the performance of the loaded silica formulation was even poorer than the unmodified, crystalline parent.

Crystallinity was observed in the post-SGF dissolution residues for glibenclamide loaded silica (Appendix 3). This suggests that, although no release was detectable in SGF, the drug did indeed release but then rapidly precipitated to the crystalline form.

Combination of glibenclamide loaded silica with HPMCAS significantly improved the transfer dissolution performance, with the formulation generating supersaturation in the intestinal phase of the assay (Fig. 7). It was also possible to detect glibenclamide in the SGF

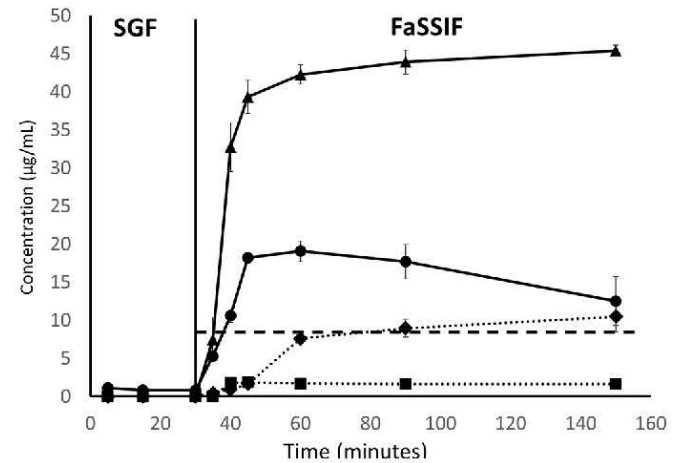

Fig. 7. Biorelevant transfer dissolution of glibenclamide loaded silica ( crystalline glibenclamide $(\uparrow)$, glibenclamide Loaded Silica + HPMCAS Blend $(\bullet)$ and glibenclamide and HPMCAS co-incorporated silica $(\boldsymbol{\Delta})(n=2)$. Transfer from SGF to FaSSIF occurred at $30 \mathrm{~min}$. N.B. no API was detectable dransfer from SGF to FaSSIF occurred at 30 min. N.B. no API was detectable glibenclamide. FaSSIF thermodynamic solubility is shown by the dotted line.

portion of the assay, suggesting that supersaturation occurred in this medium. Similarly to the glibenclamide loaded silica, crystallinity was observed in the post-SGF residue for the sample contining a physical mixture of HPMCAS with the drug loaded silica (Appendix 3). This finding was in agreement with the XRPD patterns obtained post-FaSSIF mini-dissolution (Appendix 2).

The transfer dissolution of the sample where HPMCAS was in corporated during the drug loading step is shown in Fig. 7. Unlike th sample where HPMCAS was added post-loading, no release of glibenclamide was observed in the SGF of the portion of the assay. This is likely explained by the immobilization of the drug loaded silica onto the HPMCAS platelets, which do not disintegrate in the gastric environ ment. As observed in the mini-dissolution experiments, (i) the supersaturation of glibenclamide during the FaSSIF portion of the experiment was significantly greater and more sustained from the co-incorporated formulation compared to the blend. In this case (unlike the pure drug ) similar concentrations were achieved in the mini-dissolution and transfer model experiments.

Visually, the transfer dissolution of the sample in which HPMCA was incorporated during the drug loading step was also quite different from the glibenclamide loaded silica and physical mixture of glib enclamide loaded silica with HPMCAS samples. For glibenclamide loaded silica with and without post-loading addition of HPMCAS, the powder was immediately dispersed in the dissolution vessel, creating suspension. Conversely, no such dispersion was observed within the 


\section{$\mathrm{X}$. Thesis Publications}

D.J. Price, et al.

(a)

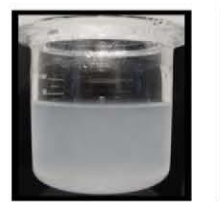

(b)

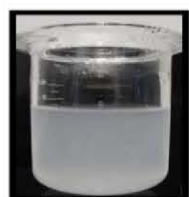

(c)

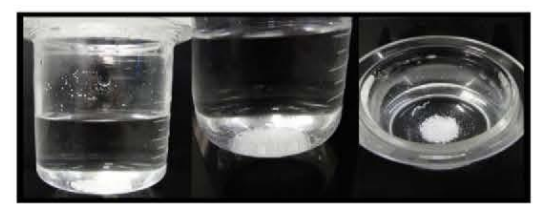

(d)

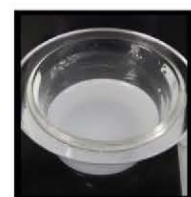

Fig. 8. Images of glibenclamide loaded silica (a) and glibenclamide loaded silica + HPMCAS (b) dispersed in SGF; and glibenclamide and HPMCAS co-incorporated silica dispersed in SGF (c) and FaSSIF (d)

sample in which HPMCAS was incorporated in the drug loading step and the dispersion remained clear (Fig. 8).

Unlike the other silica formulations, the post-SGF dissolution residue for the co-incorporated formulation remained amorphous (Appendix 3).

The control sample was not investigated during the transfer dis solution as it was fully crystalline and behaved identically to pure crystalline glibenclamide during dissolution in FaSSIF. Furthermore, given that the thermodynamic solubility of crystalline glibenclamide is $<$ LOD it was not anticipated that any useful observations could be made from the control sample during the SGF portion of the transfer dissolution.

\subsubsection{Post-dissolution SEM}

To examine the physical behavior of the formulation with HPMCAS incorporated during the drug loading step, the post-SGF and postFaSSIF residues were characterized with SEM. Post-SGF dissolution, the large platelets (Fig. 3) were unchanged (Fig. 9). Increasing the magnification, one can still observe the loaded silica particles immobilized within the polymer platelets. Conversely, in post-FaSSIF dissolution, the only observable particles are of silica, suggesting the polymer platelets had dissolved, allowing the drug to be released from the silica (Fig. 9)

\subsubsection{Solid-state NMR spectroscopy}

SS-NMR spectroscopy was carried out on all samples (Fig. 10). The full spectra are provided in Appendix 4. The ${ }^{13} \mathrm{C}$ peaks for the API were identical in all samples except the co-incorporated formulation. In the co-incorporated formulation, a low field shift of $0.2-0.3 \mathrm{ppm}$ for all API peaks was observed. For example, the characteristic API peak at $53 \mathrm{ppm}$ was observable in all samples except the co-incorporated formulation, in which the peak shifted to $53.5 \mathrm{ppm}$. This is indicative of an interaction taking place between the drug and the polymer in the solidstate, which can take place once the drug is immobilized in the silica

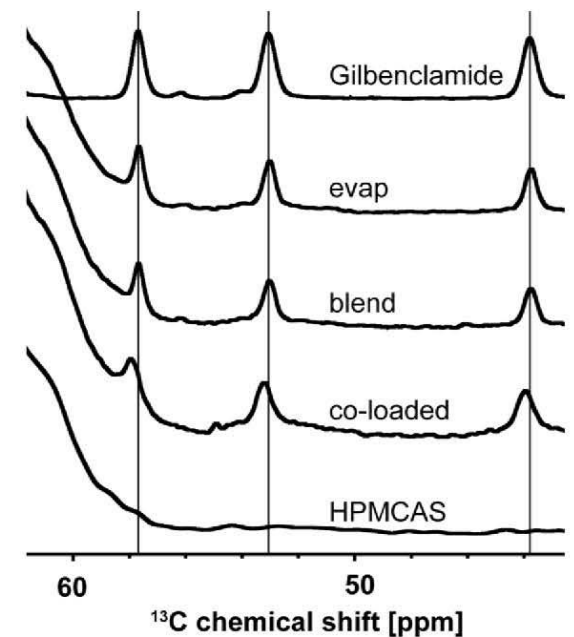

Fig. 10. A section of the ${ }^{13} \mathrm{CNMR}$ spectra for all samples showing characteristic peaks for API ${ }^{13} \mathrm{C}$ atoms at $43.5,53$ and $58 \mathrm{ppm}$. Analysis was carried out on multiple batches $(n=2)$ of co-incorporated formulation and in all cases a $0.03 \mathrm{ppm}$ peak $0.2-03$ pon peaks at $44,53.5$ and $58.5 \mathrm{ppm}$. Given that the spectra were unchanged for different batches and repeats, only one dataset is show. Full spectra are available in Appendix 4.
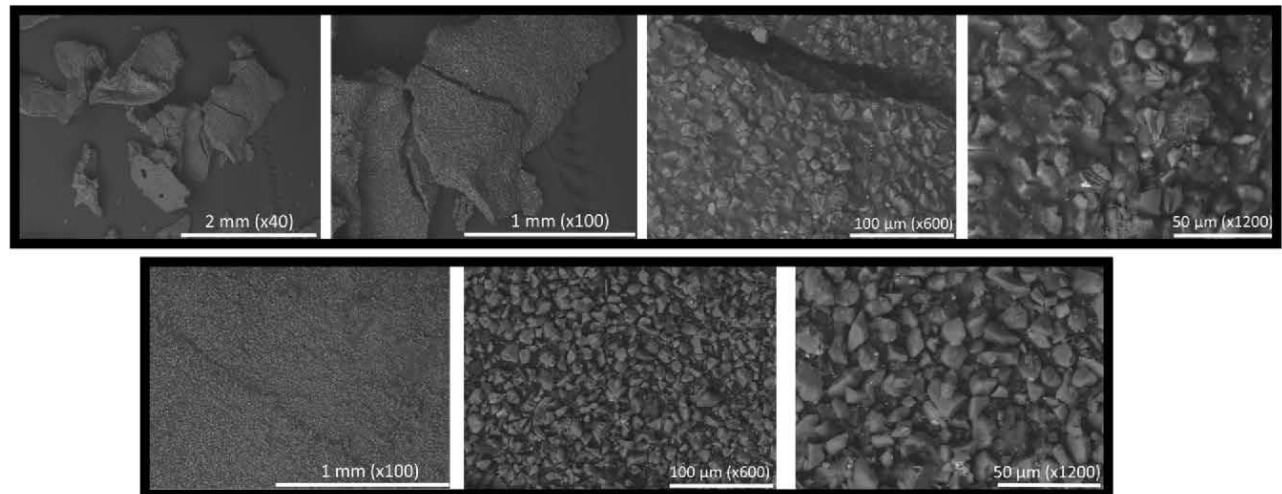

Fig. 9. SEM images of glibenclamide and HPMCAS co-incorporated silica after dissolution in SGF (top) and FaSSIF (bottom) 


\section{Thesis Publications}

and subsequently in the HPMCS plate. By contrast, no peak shift was observed in the control sample, GB/HPMCAS, which was prepared by rotary evaporation. The results suggest that solid-state drug-polymer interactions and hence dissolution performance can be altered by changing the method used to manufacture the formulation.

\section{Discussion}

Mesoporous silica is an emerging oral delivery technique to formulate low soluble drugs. Upon impregnation of the silica with concentrated API solution, drug can be molecularly adsorbed onto the surface of the silica. Due to the size of the pores, which have an approximate mean diameter of $4 \mathrm{~nm}$, the molecularly adsorbed API is locally and sterically confined, preventing recrystallization (Ditzinger, 2018; Price, 2018). More understanding is required to fully resolve the relative importance of the various considerations in the design and development of mesoporous silica formulations. Particularly critical is incorporation of precipitation inhibitors in the final formulation, since without such additives, the supersaturated state of the API is barely stabilized.

To date there has been no systematic study of how best to incorporate precipitation inhibitors in mesoporous silica formulations. Current practice for preparation on a small scale involves combining PI in a physical mixture with the API loaded silica, either by mortar and pestle or overhead stirring. Due to the lack of a standard protocol, there is uncertainty about the reliability of this approach and how well the PI is blended with the loaded silica. In addition to the practical limitation of incorporating the PI post-loading, it represents a further step in product manufacture. By contrast, incorporation of the PI during the loading step removes these limitations while maintaining improvement in dissolution of the API. Laine and co-workers demonstrated that in corporation of HPMCAS during loading of celecoxib onto mesoporous silica substantially improved both the in vitro and in vivo performance of this poorly soluble API (Laine, 2016). In the current study, we have not only demonstrated a marked improvement in dissolution of the BCS II compound, glibenclamide, by the co-incorporation approach, but have additionally proposed a mechanistic hypothesis of how this enhanced performance is achieved.

4.1. Understanding the effect of adsorption onto mesoporous silica on release in a transfer experiment

In the current study, a successful conversion of glibenclamide to the amorphous form after loading onto mesoporous silica was confirme with XRPD. This conversion led to 25 -fold supersaturation during FaSSIF mini-dissolution (Figs. $2 b$ and 5). Given the instability of the FaSSIF mini-dissolution (Figs. $2 b$ and 5 ). Given the instability of the
supersaturated state, the system rapidly precipitated and returned to its thermodynamic solubility, in line with previous studies with meso porous silica (McCarthy, 2016; Laine, 2016; Price et al., 2019). Although precipitation was observed in the single-medium FaSSIF dissolution test, the full effect of precipitation on the overall performance was only realized by considering transfer dissolution data. In thes experiments, no dissolution of crystalline glibenclamide (i.e. pure API) was observed in SGF, because its thermodynamic solubility is below the limit of detection at this $\mathrm{pH}$. By contrast, in the transfer dissolution of the supersaturating silica formulation, API was detected in the SGF phase, suggesting that supersaturation occurred (Fig. 7). This supersaturation of API in the SGF portion of the assay allowed precipitatio to commence, along with the generation of seed crystals. This resulted in significantly poorer dissolution performance of the API-silica formulation in the FasSIF portion of the experiment, relative to the singlemedium approach (Fig. 7). Therefore, one should consider the effect of transfer from the stomach to the intestine when assessing the dissolution performance of supersaturating formulations, especially mesoporous silica-based formulations.
4.2. Application of HPMCAS as a precipitation inhibitor: blending vs. coincorporation

For the current study, HPMCAS was selected as model precipitation inhibitor. HPMCAS is a well-established PI and has a track record in the literature of successfully sustaining supersaturated solutions for a range of APIS (Warren, 2010; Price, 2018; Laine, 2016; Udea, 2015).

From a practical perspective, the co-incorporation of precipitation inhibitor in the same formulation step is appealing, however, one potential concern for the co-incorporation approach is the accessibility of the pores for the API so that adsorption and nanoconfinement can still occur (Laine, 2016). Encouragingly, co-incorporating HPMCAS with glibenclamide onto mesoporous silica successfully converted the solidstate form of the API from the crystalline to the amorphous phase. This is in line with previous experience with celecoxib (Laine, 2016). Previous literature, which describes the incorporation of a polymer into the loading process as a "co-load" might infer the adsorption of the polymer inside the porous network. However, the molecular weight of the HPMCAS polymer used is approximately $18,000 \mathrm{Da}$. This is 36 -times larger than the API, glibenclamide, which has a molecular weight of $484 \mathrm{Da}$. Given the very small size of the pore, $6 \mathrm{~nm}$ in diameter, it is highly unlikely that the polymer is actually co-loaded inside the pore. Further, the particles in samples where HMPCAS has been incorporated into the formulation appear to be larger and different in shape than APIloaded silica samples without HPMCAS (Fig. 3) data confirmed that these plate-like particles were composed of carbon and, therefore, it was concluded that the plate-like particles were comprised of HPMCAS.

The next important consideration, on the location of the API within the formulation, was addressed with EDX spectroscopy. EDX is a useful tool to envisage the distribution of a drug within a formulation. In the samples where HMPCAS was incorporated during the drug loading step, it was observed that drug was adsorbed onto the mesoporous silica particles and partly within the HPMCAS plate. Crucially, there was no API observed outside of these newly present HPMCAS plates. Therefore, it was concluded that co-incorporating the PI resulted in a solid dispersion of glibenclamide as the loaded silica. This appears to be the first example of such a solid dispersion in the literature. Given the novelty of this system, further work should be carried out to investigate the solid state stability of the amorphous API in the system, which is an essential consideration for amorphous formulations (Ditzinger, 2018). Specifically, future work is planned to assess the amorphous stability of the API in the formulation, in line with the ICH O1 conditions for ac celerated stability.

Neither the formulation in which HPMCAS was incorporated during the loading step nor the sample where it was added post-loading was able to capture the extremely high 25 -fold supersaturation generated by simply loading the drug onto the silica. However, it has often been observed that the efficiency of precipitation inhibition is not able to capture the full supersaturation potential generated by the enabling formulation alone (Price, 2018; Price et al., 2019). In spite of this, it was observed that when HMPCAS was incorporated during rather than after the drug loading step, the dissolution profile was much higher. Addition of HPMCAS post-loading improved the performance of glib enclamide loaded silica during both single-medium and transfer dis solution experiments, but there was some evidence of re-crystallization, suggesting that in a simple physical mixture HPMCAS is not able to completely inhibit precipitation. Indeed, incorporating the HPMCAS during the drug loading step demonstrated a 3 -fold enhancement in dissolution performance compared to the simple physical mixture (Figs. 6 and 7). Such an improved precipitation inhibition effect could be related to the formation of drug polymer interactions already in the solid-state, which appears to be crucial for maximum precipitation in hibition (Price, 2018). This was supported by solid-state NMR data, in which a peak-shift was observed for co-incorporated formulations but not for other samples (Fig. 10). Although the peak-shift was small ( 0.2 $0.3 \mathrm{ppm}$ ), it was consistently observed for different batches. Alternative 


\section{Thesis Publications}

methods for obtaining information about drug-polymer interaction, for example 2D NOESY NMR, were unsuccessful because sufficiently concentrated solutions of drug-polymer could not be achieved. Another potential mechanism for enhanced precipitation inhibition in the formulation in which HPMCAS was incorporated during the drug loading step is the generation of an increased viscosity in the microenvironment surrounding the dissolving plates in FaSSIF. Such an increased viscosity would decrease the diffusion time out of the formulation and allow drus and polymer to remain in close proximity, both of which have been shown to be crucial factors in nucleation time in the presence of pre cipitation inhibitors (Price, 2018; Warren, 2010). However, further work would be required to fully confirm this hypothesis.

During the transfer experiment, it was observed that the HPMCAS plates do not disperse in SGF (Fig. 8). This is a significant benefit, given that the HPMCAS plates did not break down, the API-loaded silica re mained immobilized and API could not be released from the silica. Therefore, the formation of seed crystals in SGF was prevented. Ultimately, this has a significant effect on the dissolution performance and provides an additional mechanism by which formulations with HPMCAS incorporated during the drug loading step can improve dis solution performance. In addition, it is interesting to observe that change in manufacturing process - without a change in the qualitative and quantitative composition of the formulation - can introduce new properties to the product. By incorporating the HMPCAS during the loading step rather than post-loading, premature release of the drug from the formulation was circumvented without the need to add extr excipients, coating processes or special capsules, which are typically otherwise required (Qiu and Lee, 2017). This property should be especially advantageous in the delivery of poorly soluble basic compounds, whose premature release and supersaturation in the stomach (due to ionization in acidic conditions) with subsequent precipitation in the intestine could be avoided. Although Van Speybroeck and colleagues described an improved oral absorption of itraconazole loaded silica in rats, they found that silica formulations with post-loading in corporation of HMPCAS were were unable to prevent the release of AP in the stomach and therefore absorption was reduce (Van Speybroeck, 2010). The potential for incorporation of HMPCAS during the drug loading step on the dissolution performance of poorly soluble weak base drugs should be further explored.

4.3. Co-incorporated formulations: just a solid dispersion?

Given the improvement of the formulation performance when HMPCAS was incorporated during the loading step rather than postloading, it was important to rule out that a simple solid dispersion was formed directly, and that the silica in the formulation plays an important role in the dissolution enhancement. EDX indicates that the drug is localized in the silica particles and on the HPMCAS plate when the polymer is incorporated during the loading step (Fig. 3, bottom panel), but is distributed freely throughout the entire sample when no silica is present (Fig. 5). The results suggest that drug is confined within the mesoporous silica particles, which are in turn were immobilized in the polymer platelets when HPMCAS is incorporated in the drug loading step. Without the nanoconfinement effects of the silica (Ditzinger, 2018), the drug can re-crystallize, as observed in the XRPD (Fig. 7). Ultimately, this resulted in the control sample showing no improvement in FaSSIF dissolution versus crystalline API (Fig. 6). Furthermore, if a portion of the sample was able to remain amorphous in the polymer platelets, the absence of drug-polymer interaction (as shown in the solid-state NMR spectra) would reduce the precipitation inhibition effect of the polymer (Fig. 10)

\section{Conclusions}

A novel co-incorporated formulation of glibenclamide and the pre cipitation inhibitor, HPMCAS, onto mesoporous silica is described. By co-incorporating the precipitation inhibitor, the formulation significantly outperformed the commonly applied simple physical blend, regarding improved supersaturation and dissolution in both singlemedium FaSSIF and transfer dissolution assays. Furthermore, the coincorporation approach allows the removal of a time-consuming and inefficient blending step. To provide a physical mechanistic basis is for the improved performance the co-incorporated formulation, a range of spectroscopic tools were utilized. It was concluded that the improved dissolution performance is a synergistic effect related to two key fac tors: formation of drug-polymer interactions in the solid state, and lack of release and premature precipitation under gastric conditions due to the immobilization of API-loaded silica particles within the enteric HPMCAS plates. Crucially, both of these properties are absent in a simple HPMCAS blend. Ultimately, the co-incorporation of precipita tion inhibitors with the API on mesoporous silica formulations has the potential to improve both the process and formulation efficiency in the development of poorly soluble drugs.

\section{Acknowledgements}

The authors would like to thank Dr. Dieter Lubda, Dr Gudrun Birke and Dr. Finn Bauer for providing the Parteck SLC ${ }^{\oplus}$ and for helpful and constructive discussions and feedback.

This work has received funding from the European Union's Horizon 2020research and innovation programme under grant agreement No 674909 (PEARRL). www.pearrl.eu

\section{Supplementary materials}

Supplementary material associated with this article can be found, in the online version, at doi:10.1016/j.ejps.2019.105113.

Appendix 1: thermodynamic solubility values For glibenclamide

\begin{tabular}{|c|c|}
\hline $\begin{array}{l}\text { Appendix } \\
\text { Medium }\end{array}$ & $\begin{array}{l}\text { lubility values } \\
\text { Solubility }(\mu \mathrm{g} / \mathrm{mL})\end{array}$ \\
\hline $\begin{array}{l}\text { FasSIF } \\
\text { SGF }\end{array}$ & $\begin{array}{l}8.1 \pm 0.1 \\
<\text { LOD }^{*}\end{array}$ \\
\hline
\end{tabular}




\section{Thesis Publications}

D.J. Price, et al.

Appendix 2: Post-FasSIF dissolution XRPD

Appendix 2. XRPD patterns for post-FaSSIF dissolution residues for (a) glibenclamide loaded silica, (b) glibenclamide loaded silica + HPMCAS blend, (c) glibenclamide and HPMCAS co-incorporated silica and (d) glibenclamide and HPMCAS prepared by rotary evaporation (control)

(a)

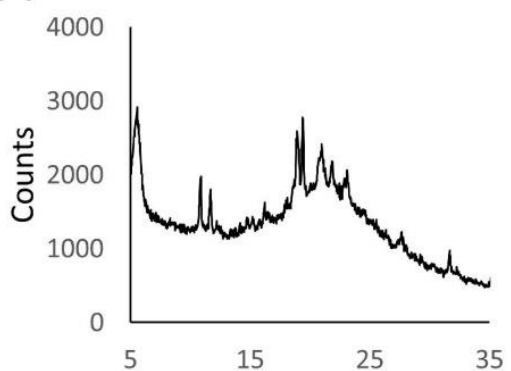

(c)

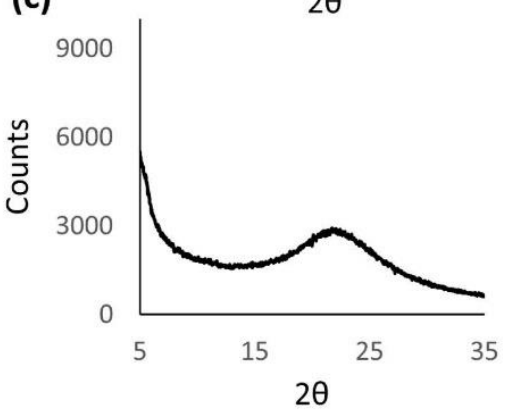

(b)

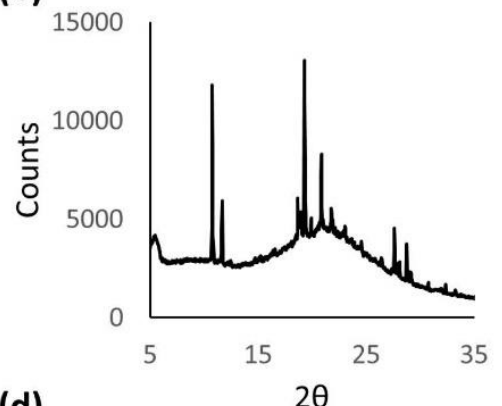

(d)

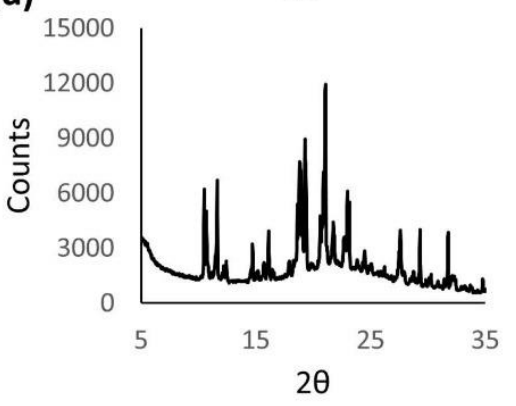

Appendix 3: Post-SGF transfer dissolution XRPD patterns

Appendix 3. XRPD patter for Glibenclamide loaded silica (a), glibenclamide loaded silica + HPMCAS blend (b) and GB/HPMCAS co-incorporated silica (c) residues post-SGF transfer dissolution Appendix 4. SS-NMR spectra

(a)

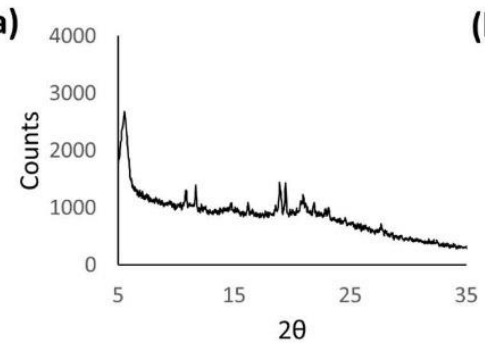

(b)

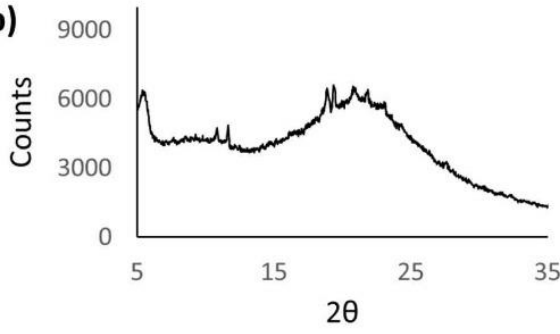

(c) 9000

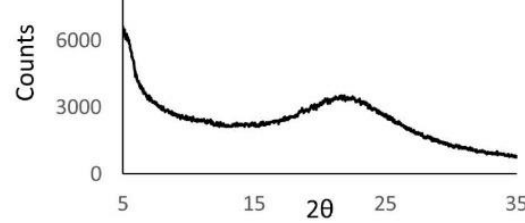




\section{Thesis Publications}

Appendix 4: Full solid-state NMR spectra for all samples showing peak shift in co-incorporated samples. The section highlighted corresponds to the section included in the main body of text

$\mathrm{xx}$

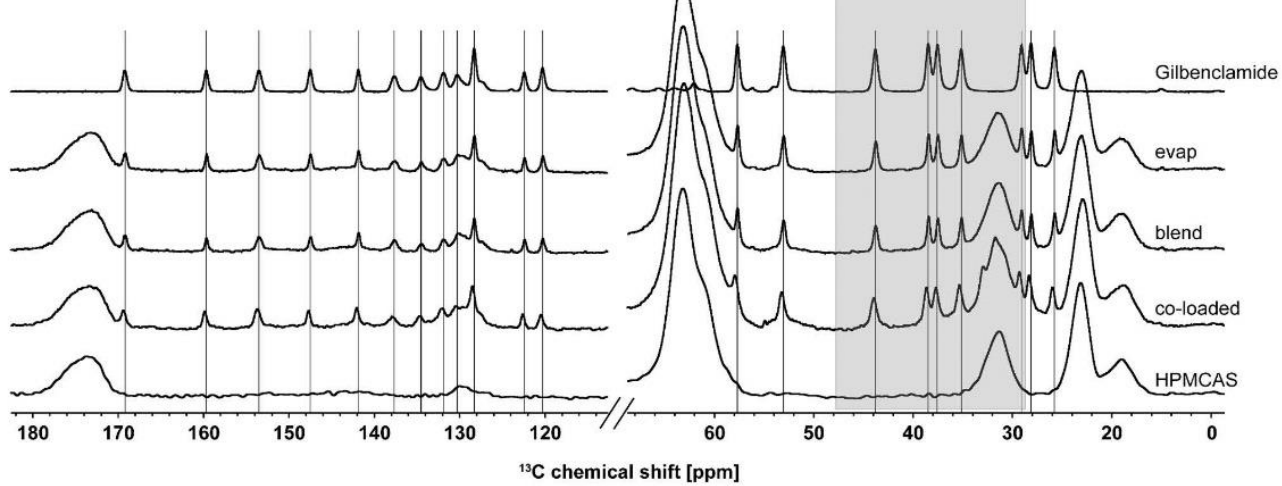

References

Dizzinger, F, et al., 2018. Lipophilicity and hydrophobicity considerations in bio-enabling oral formulations approaches-a PEARRL review. J. Pharm. Pharmacol Epub ahead of

print.
Galia, E., Nicolaides, E., Hörter, D., Löbenberg, R., Reppas, C., Dressman, J.B., 1998. Evaluation of various dissolution media for predicting in vivo performance of class

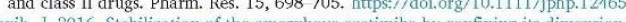
Mol. Pharm. 13 (4), 1308-1316.

Wrishnaiah, Y.S.R., 2010, Pharmaceurical technologies for enhancing oral bioavailability of poorly soluble drugs. J. Bioequivalence Bioavailability 2, 28-36,

aine, AL, et al., 2016. Fnhanced oral delivery of celecoxib via the development of a supersaturable amorphous formulation utilising mesoporous silica and co-loaded HPMCAS. Int. J. Pharm. 512 (1), 118-125. https://doi,org/10.1016/j.ijpharm. 2016

08.034.
Lof tsson, T., Brewster, ME., 2010. Pharmaceutical applications of cyclodextrins: basic science and product development. J Pharm. Pharmacol. 62, 1607-1621. hittps://doi. org/10.1111/2.2012-7158.2010.01030x.

McCarthy, CA., et al., 2016. Mesoporous silica formulation strategies for druy dissolution enhancement: a review. Exp. Op. Drug Deliv. 13, 93-108. https://doi.org/10.1517 (1)

O'Shea, J.P., et al., 2016. Mesoporous silica-based dosage forms improve release characteristics of poorly soluble drugs: case example fenofibrate. J. Pharm. Pharmacol. 68 (5), $634-645$

Price, 1.J., et al., 2018. Approaches to increase mechanistic understanding and aid in the selection of precipitation inhibitors for supersaturating formulations- A PEARRL re view. J. Pharm. Pharmacol. https://doi.org/10.1111/jphp.12927. epub ahead of

Price, DJ., et al, 2019. Calculation of drug-polymer mixing enthalpy as a new screening method of precipitation inhibitors for supersaturating pharmaceutical formulations.

u, Y., Lee, PI., 2017. Rational design of oral modified-release drug delivery systems. in developing solid oral dosage forms. Pharm. Theory Pract. 519-554.

Udevetoping solid oral dosage forms. Fharm. Hicory Pract. 519 554. (1)H NMR technique. Mol. Pharm. 12 (1), 1097 1101.

Van Speybroeci, M. et al., 2010. Combined use of ordered mesoporous silica and precipitation inhibitors for improved oral absorption of the poorly soluble weak base traconazole, Eur. J. Pharm. Biopharm. 75 (3), 351 365.

Warren, DB, et al., 2010. Using polymeric precipitation inhibitors to improve the at.sorption of poorly water-soluble drugs: A mechanistic basis for utility. J. Drug Tar.

Zheng, W., et al., 2012. Selection of oral bioavailability enhancing formulations during drug discovery. Drug Dev. Ind. Pharm 38 (2), 235247. 
Article

\title{
Opportunities for Successful Stabilization of Poor Glass-Forming Drugs: A Stability-Based Comparison of Mesoporous Silica Versus Hot Melt Extrusion Technologies
}

\author{
Felix Ditzinger ${ }^{1,2,+}$, Daniel J. Price ${ }^{3,4,+}$, Anita Nair ${ }^{3}$, Johanna Becker-Baldus ${ }^{5}$, \\ Clemens Glaubitz ${ }^{5}$, Jennifer B. Dressman ${ }^{4}$, Christoph Saal ${ }^{3}$ and Martin Kuentz ${ }^{2, *(B)}$ \\ 1 Department of Pharmaceutical Sciences, University of Basel, 4056 Basel, Switzerland; felix.ditzinger@fhnw.ch \\ 2 Institute of Pharma Technology, University of Applied Sciences and Arts Northwestern Switzerland, \\ 4132 Muttenz, Switzerland \\ 3 Merck KGaA, 64293 Darmstadt, Germany; daniel-joseph.price@merckgroup.com (D.J.P.); \\ anita.nair@merckgroup.com (A.N.);Christoph.Saal@merckgroup.com (C.S.) \\ 4 Institute of Pharmaceutical Technology, Goethe University, 60438 Frankfurt, Germany; \\ dressman@em.uni-frankfurt.de \\ 5 Institute for Biophysical Chemistry \& Centre for Biomolecular Magnetic Resonance, Goethe University, \\ 60438 Frankfurt, Germany; j.baldus@em.uni-frankfurt.de (J.B.-B.); Glaubitz@chemie.uni-frankfurt.de (C.G.) \\ * Correspondence: martin.kuentz@fhnw.ch; Tel.: +41-61-228-56-42 \\ + These authors contributed equally to this work.
}

Received: 25 September 2019; Accepted: 1 November 2019; Published: 4 November 2019

\begin{abstract}
Amorphous formulation technologies to improve oral absorption of poorly soluble active pharmaceutical ingredients (APIs) have become increasingly prevalent. Currently, polymer-based amorphous formulations manufactured by spray drying, hot melt extrusion (HME), or co-precipitation are most common. However, these technologies have challenges in terms of the successful stabilization of poor glass former compounds in the amorphous form. An alternative approach is mesoporous silica, which stabilizes APIs in non-crystalline form via molecular adsorption inside nano-scale pores. In line with these considerations, two poor glass formers, haloperidol and carbamazepine, were formulated as polymer-based solid dispersion via HME and with mesoporous silica, and their stability was compared under accelerated conditions. Changes were monitored over three months with respect to solid-state form and dissolution. The results were supported by solid-state nuclear magnetic resonance spectroscopy (SS-NMR) and scanning electron microscopy (SEM). It was demonstrated that mesoporous silica was more successful than HME in the stabilization of the selected poor glass formers. While both drugs remained non-crystalline during the study using mesoporous silica, polymer-based HME formulations showed recrystallization after one week. Thus, mesoporous silica represents an attractive technology to extend the formulation toolbox to poorly soluble poor glass formers.
\end{abstract}

Keywords: glass forming ability; hot melt extrusion; mesoporous silica; amorphous stability; supersaturation

\section{Introduction}

The increasing prevalence of poorly water-soluble drugs has driven the field of pharmaceutical technology to develop modern approaches for formulation development. A well-established technique is to formulate the drug in an amorphous form, which results in an increase in apparent solubility, dissolution performance, and subsequent oral bioavailability [1,2]. However, such an approach comes 


\section{Thesis Publications}

with difficulties related to thermodynamic instability of the amorphous state, which can lead to recrystallization and thus negation of the aforementioned formulation advantages [3].

To give guidance on the recrystallization tendency of drugs, Baird et al. developed a classification system based on a molecule's "glass forming ability" (GFA). The GFA is related to recrystallization behavior from super cooled melts [4-6]. Three classes of substances were defined: class one (GFA-I) drugs recrystallize upon cooling from the molten state, class two (GFA-II) drugs recrystallize after a heating-cooling-heating cycle, and class three (GFA-III) drugs remain amorphous throughout the entire experiment. Although the classification was developed for undercooled melts, which can be directly related to hot melt extrusion (HME), it has proven to be accurate for solvent evaporation processes as well [7]. This is a particularly relevant consideration for mesoporous silica systems, given that drug loading is driven by solvent penetration into pores and subsequent evaporation [8].

GFA-I compounds, poor glass formers, are particularly prone to recrystallization in amorphous formulations [9]. One strategy to tackle this instability is to combine the drug with a polymer in an amorphous solid dispersion. A very common technique to manufacture amorphous solid dispersions is IIME [10,11]. In this process, polymer and API are mixed in the molten state to form an extrusion strand, which is further processed into a solid dosage form, e.g., tablet or a capsule.

Another approach is to formulate GFA-I drugs with mesoporous silica. This is of particular interest due to the high stability of the amorphous API once it has been loaded into the porous network of the silica. This enhanced stability is related to nano-confinement in the meso-scale pores, which by definition range from $2-50 \mathrm{~nm}[12,13]$. Stability is further improved with complementary pore-API interactions that lower the free energy of the system [14]. Muller and co-workers demonstrated amorphous stability at ambient and accelerated conditions for 30 different mesoporous silica formulations [15]. One key consideration for mesoporous silica formulations is the location of the API within the sample. For GFA-I compounds, it is essential that the loading process is carried out carefully, avoiding oversaturation of the silica, to ensure the drug is loaded within the pores and not on the outer surface. GFA-I compounds adsorbed on the outer surface are prone to rapid crystallization, which can be observed with techniques such as differential scanning calorimetry (DSC) and powder X-ray diffraction (PXRD). However, upon successful loading of the drug in the internal porous network, silica formulations can provide a viable alternative for drugs that fail to form a stable amorphous formulation in classical solid dispersions [16-18]. Although amorphous stability in mesoporous silica has been previously described, there has been no comparison on stability of poor glass formers in HME and mesoporous silica technology published to date.

Certainly, solid-state stability is not the only important formulation consideration for poorly soluble drugs that are also poor glass formers. It is also essential to consider stability of the supersaturation generated upon dissolution. Indeed, recent work has demonstrated that, due to their high propensity for recrystallization, poor glass formers may also have issues with rapid onset of precipitation upon release, thus limiting their therapeutic potential [19]. This is in line with the well-established "Spring and Parachute" model [20], which identifies the need for additional excipients to sustain supersaturation of APIs in solution [21]. For polymer-based solid dispersions, the polymer may be able to meet both requirements: suspending the drug in an amorphous form and inhibiting precipitation from the supersaturated state. An example of such a polymer is polyvinyl alcohol that is interesting due to its low hygroscopicity and for which a special grade has been introduced recently for HME [22]. Unlike polymer-based solid dispersions, the ability of mesoporous silica to inhibit precipitation of the supersaturated API is limited. Therefore, it is often necessary to incorporate precipitation inhibitors into mesoporous silica formulations [23].

In this study, the amorphous stability of two model poor glass formers, haloperidol and carbamazepine, formulated as HME and with mesoporous silica, was investigated in line with ICH Q1 accelerated stability conditions [24] over three months. The stability was monitored by means of PXRD and underscored with DSC measurements of the samples before and at the end of the study. To the best of our knowledge, such a comparative study has not been reported previously. This 


\section{Thesis Publications}

stability comparison was complemented by non-sink release testing in biorelevant media $[25,26]$ to monitor drug release and dissolution throughout the study [23,27]. It is important to note that non-sink dissolution was not used as a direct comparison between the two formulations, as the mesoporous formulations do not inhibit precipitation upon release. Rather, the release curves are demonstrative of the decrease in dissolution performance that can be observed upon solid-state transformation. Finally, solid-state nuclear magnetic resonance (SS-NMR) spectroscopy was applied to investigate any qualitative changes drug-polymer spectra in HME formulations over the duration of the stability study [28].

From a practical perspective, both drugs have no thermal instability, which avoids the risk of heat-induced degradation during the HME process $[29,30]$. The drug load selected for the technology comparison was the highest amount that enabled initial amorphous loading for both HME and mesoporous silica formulations, so that a direct comparison between techniques could be attained.

It was hypothesized that mesoporous silica formulations of haloperidol and carbamazepine would show enhanced solid-state stability over time compared to solid dispersion obtained from HME.

\section{Materials and Methods}

\subsection{Materials}

Haloperidol, carbamazepine, HPLC grade acetonitrile, and HPLC grade methanol were purchased from MilliporeSigma (Darmstadt, Germany). Parteck MXP ${ }^{\circledR}$ (PVA) and Parteck SLC $\left.{ }^{(}\right)$were kindly provided by Merck KGaA (Darmstadt, Germany). FaSSGF/FaSSIF/FeSSIF powder to make biorelevant dissolution medium, Fasted Simulated Intestinal Fluid (FaSSIF), was obtained from Biorelevant (Biorelevant.com, London, UK).

\subsection{Methods}

\subsubsection{Thermodynamic Solubility Determination}

FaSSIF was prepared by weighing $45 \mathrm{mg}$ of FaSSGF/FaSSIF/FeSSIF powder into $45 \mathrm{~mL}$ of phosphate buffer ( $\mathrm{pH} 6.5)$ [31]. API (2-3 mg) was accurately weighed into a Uniprep ${ }^{(\mathbb{R}}$ syringeless filter $(5 \mathrm{~mL}$; $0.45 \mu \mathrm{m}) .2 \mathrm{~mL}$ of FaSSIF was added and the samples were agitated at $450 \mathrm{rpm}$ for $24 \mathrm{~h}$ at $37^{\circ} \mathrm{C}$. The $\mathrm{pH}$ was checked at $7 \mathrm{~h}$ and adjusted with $0.1 \mathrm{~N} \mathrm{NaOH}$ or $0.1 \mathrm{~N} \mathrm{HCl}$, if a deviation greater than $\pm 0.05 \mathrm{pH}$ units was observed. The final $\mathrm{pH}$ was also recorded after $24 \mathrm{~h}$.

Samples were filtered into the inner chamber of the Uniprep through the built-in PTFE $0.45 \mu \mathrm{m}$ Whatman filter after $24 \mathrm{~h}$. Filtrates were immediately diluted with acetonitrile and water $(1: 4 v / v)$ to avoid precipitation from the saturated solution. Samples were analyzed with UHPLC (Thermo Dionex Ultimate 3000, Thermo Fisher, Waltham, MA, USA) to determine the API concentration. API concentration was determined based on a standard calibration curve of nine standard concentrations $(50,30,10,5,3,1,0.5,0.3,0.1 \mu \mathrm{g} / \mathrm{mL})$. Three control samples of known concentrations $(30,3,0.3 \mu \mathrm{g} / \mathrm{mL})$ were prepared and used to check the robustness of the calibration curve. The determination was carried out in duplicate.

\subsubsection{Ultra-High Performance Liquid Chromatography (UHPLC)}

UHPLC analysis was performed using a Thermo Dionex Ultimate 3000 (Thermo Fisher, Waltham, MA, USA) equipped with a diode array detector at $282 \mathrm{~nm}$ for carbamazepine and $247 \mathrm{~nm}$ for haloperidol (Thermo Fisher, Waltham, MA, USA). The separation was achieved on an Acquity UPLC BEH column C8 $(2.1 \times 50 \mathrm{~mm}, 1.7 \mu \mathrm{m}$, Waters, Milford, MA, USA). The mobile phases A and B consisted of water:formic acid 999:1 $(v / v)$ and acetonitrile:formic acid 999:1 $(v / v)$, respectively. Gradient and flow rate is shown in Table 1. System management, data acquisition and processing were based on the Chromeleon ${ }^{\mathrm{TM}}$ software package, version 7.2 (Thermo Fisher, Waltham, MA, USA). 


\section{Thesis Publications}

Pharmaceutics $2019,11,577$

Table 1. UHPLC gradient and flow rates.

\begin{tabular}{|c|c|c|c|}
\hline Time (min) & Flow Rate $(\mathrm{mL} / \mathrm{min})$ & $\%(v / v)$ Mobile Phase A & $\%(v / v)$ Mobile Phase B \\
\hline 0.00 & 0.83 & 90 & 10 \\
\hline 0.83 & 0.83 & 10 & 90 \\
\hline 1.20 & 1.50 & 90 & 10 \\
\hline 2.00 & 1.50 & 90 & 10 \\
\hline 2.01 & 0.83 & 90 & 10 \\
\hline
\end{tabular}

2.2.3. Preparation of Hot Melt Extrudates

PVA was selected as an optimal polymer for hot-melt extrusion. This was based on three factors: (1) the grade of PVA was specifically designed for optimal HME due to particle size distribution and viscosity, (2) consideration of partial solubility parameters for both drugs, and PVA (3) low hygroscopicity of PVA to reduce water uptake in of the extrudates.

Binary mixtures of polyvinyl alcohol (PVA, Parteck $M X P^{\circledR}$ ) and drug at various drug loadings were mixed in a mortar and extruded on a ZE9 ECO twin-screw extruder by ThreeTec (Birren, Switzerland) with $9 \mathrm{~mm}$ diameter and $180 \mathrm{~mm}$ length co-rotating screws. A screw speed of $80 \mathrm{rpm}$ was applied at a temperature of $190^{\circ} \mathrm{C}$ through all three heating zones, which is in accordance with recommendation by the polymer manufacturer [32]. After extrusion, the extrudates were ground in a mortar, and the fraction retained between mesh sizes 150 and $425 \mu \mathrm{m}$ was retained for use in the study. The final extruded mixtures were cooled to room temperature and stored in falcon tubes. Mixing feasibility of the selected polymer for both drugs was verified by the Hansen solubility parameters [33,34], which were calculated using the quantitative structure property relationship (QSPR) method of the COSMOquick software (COSMOlogic, Leverkusen, Germany, Version 1.6) [35,36]. For the investigation of the formulations, a $7.5 \%(w / w)$ drug loading of haloperidol and $20 \%(w / w)$ was used for carbamazepine. This was selected on the basis of the highest drug load that was initially successful for both formulation technologies, and was the result of a formulation screening.

\subsubsection{Preparation of API-Loaded Silica Formulations}

Mesoporous silica formulations were prepared using the incipient wetness method [13]. API ( $\mathrm{g}$ ) was dissolved in acetone $(300 \mathrm{~mL} ; 10 \mathrm{mg} / \mathrm{mL})$, which was added drop-wise at a rate of $0.5 \mathrm{~mL} / \mathrm{min}$ to Parteck $\left.{ }^{(}\right)$SLC mesoporous silica $(7 \mathrm{~g})$, under constant stirring and heating at $60^{\circ} \mathrm{C}$. After complete addition of the concentrated API solution, the samples were dried overnight in a vacuum oven at $60^{\circ} \mathrm{C}$ to ensure complete removal of the solvent. The formulations were prepared at a drug loading of $7.5 \%$ $(w / w)$ for haloperidol and $20 \%(w / w)$ for carbamazepine.

\subsubsection{Storage of Samples for Stability Studies}

For storage of the samples in the stability study, each of the formulations was placed in a separate glass jar with a secure lid. A separate beaker containing saturated sodium chloride solution, also placed in the beaker, ensured a constant relative humidity of $75 \%$ in the surrounding environment [37]. This enclosed system was then placed in a stability cabinet set to $40^{\circ} \mathrm{C}$ to obtain storage conditions in accordance with ICH Q1.

\subsubsection{Powder X-Ray Diffraction (PXRD)}

Samples were prepared between $\mathrm{X}$-ray amorphous films and measured in transmission mode using Cu-K $\alpha 1$-radiation and a Stoe StadiP 611 KL diffractometer (STOE \& Cie GmbH, Darmstadt, Germany) in transmission mode equipped with Dectris Mythen1K PSD (DECTRIS Ltd, Baden-Daettwil, Switzerland). The measurements were evaluated with the software WinXPow 3.03 by Stoe (STOE \& Cie GmbH, Darmstadt, Germany), the ICDD PDF-4+ 2014 Database (ICDD, Newtown Square, PA, 


\section{Thesis Publications}

USA), and Igor Pro Version 6.34 (Wavemetrics Inc., Lake Oswego, OR, USA) Angular range: 1-40 20; PSD-step width: $2^{\circ} 2 \theta$; angular resolution: $0.015^{\circ} 2 \theta$ measurement time: $15 \mathrm{~s} / \mathrm{step}, 0.25$ h overall.

\subsubsection{Non-Sink-Mini-Dissolution in FaSSIF}

The equivalent of $5 \mathrm{mg}$ API of extrudate or API-loaded silica was weighed into a glass vial. Five milliliters of FaSSIF was added. The vials were agitated at $37^{\circ} \mathrm{C}$ and $450 \mathrm{rpm}$ in a shaker (IKA-Werke GmbH \& CO. KG, Staufen, Germany) for $120 \mathrm{~min}$. Samples of $0.3 \mathrm{~mL}$ were taken at 2, 15, 60, 90, and $120 \mathrm{~min}$, filtered (0.45 PTFE Whatman filters), diluted with acetonitrile and water, and analyzed by UHPLC. The mini-dissolution trials were conducted in duplicate for all samples.

\subsubsection{Scanning Electron Microscopy (SEM)}

Samples were prepared on carbon tape and imaged using a TM3000 Tabletop Scanning Electron Microscope (Hitachi, Tokyo, Japan), tungsten source, using low vacuum and accelerating voltage of 5 and $15 \mathrm{kV}$. A 4-Quadrant BSE detector was used, and imaging was at a magnification between $15 \times$ and $30,000 \times$.

\subsubsection{Differential Scanning Calorimetry (DSC)}

Samples were assessed by differential scanning calorimetry on a DSC 3 (Mettler Toledo, Greifensee, Switzerland). An amount of 5 to $9 \mathrm{mg}$ sample was placed in a $40 \mu \mathrm{L}$ aluminum pan with a pierced lid. A heating rate of $10^{\circ} \mathrm{C} / \mathrm{min}$ from 20 to $200^{\circ} \mathrm{C}$ was applied under nitrogen purging at $200 \mathrm{~mL} / \mathrm{min}$. The thermograms were analyzed with the STARe Evaluation-Software Version 16 (Mettler Toledo, Greifensee, Switzerland).

\subsubsection{Solid-State Nuclear Magnetic Resonance (SS-NMR) Spectroscopy}

SS-NMR experiments were conducted with magic-angle-sample (MAS) spinning using a Bruker $4 \mathrm{~mm}$ MAS HXY probe in double resonance mode with a Bruker Avance I $600 \mathrm{MHz}$ wide bore NMR spectrometer (Bruker, Rheinstetten, Germany) with a $4 \mathrm{~mm}$ rotor. The readout on the probe thermocouple was set to $290 \mathrm{~K}$. The sample spinning frequency was set to $10 \mathrm{kHz}$. All spectra were recorded with ${ }^{1} \mathrm{H}_{-}{ }^{13} \mathrm{C}$-cross polarization $(\mathrm{CP})$ using a contact time of $1 \mathrm{~ms} .100 \mathrm{kHz}$ high power proton decoupling following the SPINAL64 scheme was applied during acquisition. The recycle delay was $3 \mathrm{~s}$ The spectra were indirectly referenced to 4,4-dimethyl-4-silapentane-1-sulfonic acid (DSS) via the $\mathrm{CH}_{2}$ signal of Adamantane at $40.49 \mathrm{ppm}$.

\section{Results}

\subsection{Macro- and Microscopic Changes}

Qualitative macroscopic differences were observed between the fresh and one-week stressed samples of the hot-melt extrudates (Figure 1). Extrudates of carbamazepine and haloperidol were transparent immediately after manufacturing. This indicates the presence of molecularly dispersed API throughout the polymer in the amorphous form [11]. However, after only 7 days exposure to $40^{\circ} \mathrm{C}$ and $75 \% \mathrm{RH}$, both extrudates became opaque, indicating phase separation in the formulations [38]. This was in contrast to mesoporous silica formulations, in which no macroscopic differences were observed between the fresh and one-week stressed samples. Indeed, the appearance of mesoporous silica formulations remained consistent over the duration of the 3 month study.

Changes in the extrudate samples over time were also observed on a microscopic level in the SEM images. In freshly prepared formulations, the extrudates showed no heterogeneity but at the end of the stability study, after 90 days, phase separation and recrystallization were observed. API-loaded silica formulations, however, did not exhibit qualitative changes under either visual inspection or by SEM (Figure 2; Figure 3). 


\section{Thesis Publications}
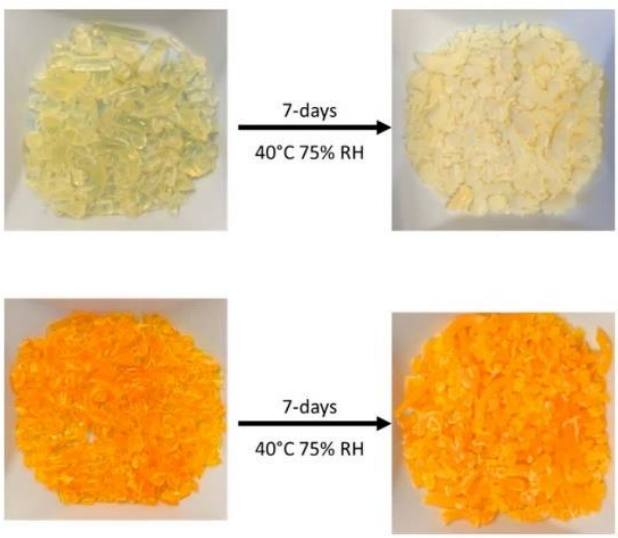

Figure 1. Haloperidol (top) and carbamazepine (bottom) hot melt extrusion (HME) before (left) and after (right) 7 days accelerated stability conditions as specified in the materials and methods section.

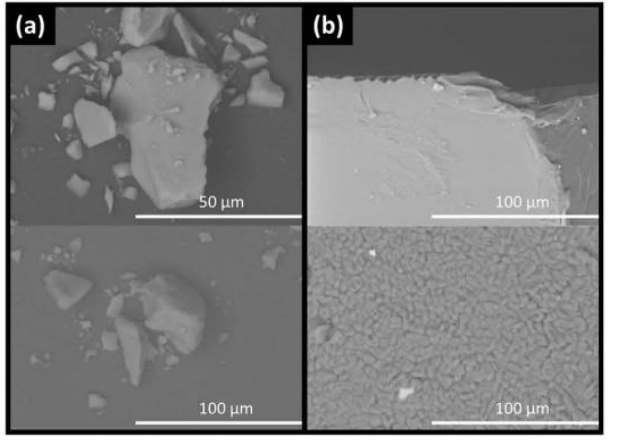

Figure 2. SEM images for carbamazepine loaded silica (a) and IIME (b) showing particle size and morphology at 0 days (top) and 90 days stability (bottom) as specified in the materials and methods section.

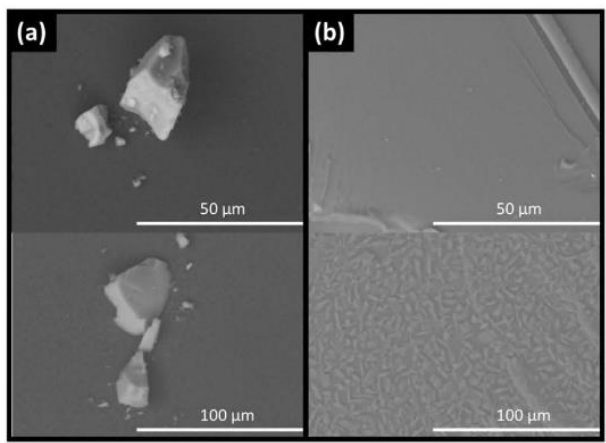

Figure 3. SEM images for haloperidol loaded silica (a) and HME (b) showing particle size and morphology at 0 days (top) and 90 days stability (bottom) as specified in the materials and methods section. 


\section{$\mathrm{X}$. Thesis Publications}

\subsection{Solid-State Stability of the Amorphous Form}

Both haloperidol and carbamazepine were crystalline before formulation with either HME or mesoporous silica. The outcome of the empirical loading approach is shown in Table2. For mesoporous silica, both APIs were successfully stabilized in the amorphous form at an initial concentration of $30 \%(w / w)$. However, HME was only successful in stabilizing amorphous API for carbamazepine at $20 \%(w / w)$ and haloperidol at $7.5 \%(w / w)$ (data not shown). At higher concentrations, the extrudates were crystalline upon cooling. Therefore, for the comparative accelerated stability study of the formulations a drug load of $20 \%(w / w)$ carbamazepine and $7.5 \%(w / w)$ haloperidol was selected for both the mesoporous silica and HME based solid dispersions. PXRD indicated that the initial form in both formulations was amorphous (Figure 4; Figure 5).

Table 2. Loading Capacities for Both Formulation Techniques.

\begin{tabular}{ccccc}
\hline \multirow{2}{*}{ Formulation } & \multicolumn{4}{c}{ Loading Content $(\%)(w / w)$} \\
\cline { 2 - 5 } & $\mathbf{3 0}$ & $\mathbf{2 0}$ & $\mathbf{1 5}$ & $\mathbf{7 . 5}$ \\
\hline Haloperidol HME & Crystalline & Crystalline & Crystalline & Amorphous \\
Haloperidol loaded silica & Amorphous & Amorphous & Amorphous & Amorphous \\
Carbamazepine HME & Crystalline & Amorphous & Amorphous & Amorphous \\
Carbamazepine loaded silica & Amorphous & Amorphous & Amorphous & Amorphous \\
\hline
\end{tabular}

Differences between silica-based formulations and PVA extrudates were apparent after one month of storage at elevated temperatures, with both HME formulations showing development of crystallinity. The crystalline percentage increased month by month over the duration of the study (Figure 4; Figure 5).

Conversely, both API-loaded mesoporous silica formulations remained amorphous for the duration of the three-month stability study, with no evidence of crystallinity in the PXRD patterns (Figure 4; Figure 5).

These findings were underscored by the absence of melting endotherms in the DSC thermograms of the silica-based formulations after 90 days [39]. By contrast, melting peaks were observed in both samples of the extruded formulations after 90 days, indicating the presence of drug crystallinity (data not shown).

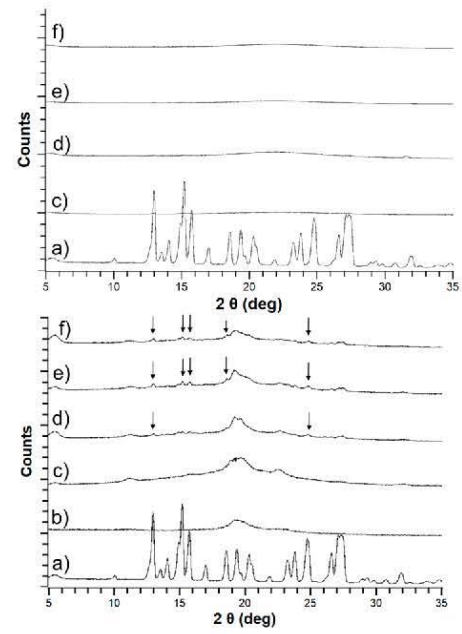

Figure 4. Powder X-ray (PXRD) patterns for carbamazepine loaded silica (top) and carbamazepine HME (bottom) showing crystalline carbamazepine (a), pure Parteck MXP ${ }^{\circledR}$ (PVA) (b), unstressed carbamazepine formulation (c) and stressed carbamazepine formulations at 30 (d), 60 (e), and 90 (f) days. The arrows indicate crystalline peaks in the diffractograms. 


\section{Thesis Publications}

Pharmaceutics 2019, 11, 577
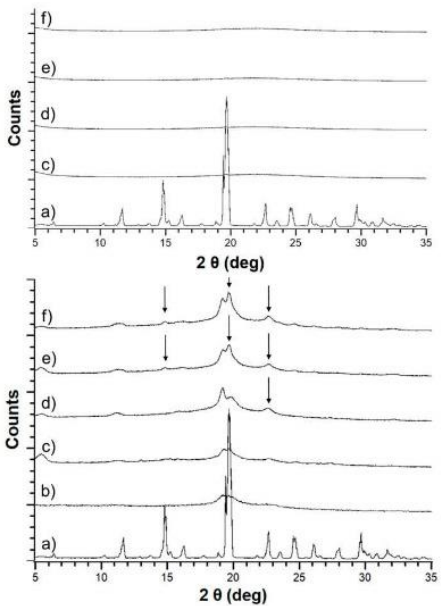

Figure 5. PXRD patterns for haloperidol loaded silica (top) and haloperidol HME (bottom) showing crystalline haloperidol (a), pure PVA (b), unstressed haloperidol formulation (c) and stressed haloperidol formulations at 30 (d), 60 (e), and 90 (f) days. The arrows indicate crystalline peaks in the diffractograms.

Although there were no pronounced drug-polymer interactions detectable in the SS-NMR spectroscopy for carbamazepine and haloperidol HME, it was possible to observe qualitative differences before and after storage of the samples at $45^{\circ} \mathrm{C} / 70 \%$ RH. Specifically, the freshly prepared samples had broad peaks in the spectra, related to the amorphous state of the sample. By contrast, an increased fine structure observed in the NMR-spectra at the end of the study indicated an increase in crystallinity. This was especially pronounced for haloperidol, with the stressed sample exhibiting peaks corresponding to the crystalline pure drug (Figure $6 \mathrm{c}$ ) at 118, 153, and 200 ppm in Figure 6a.

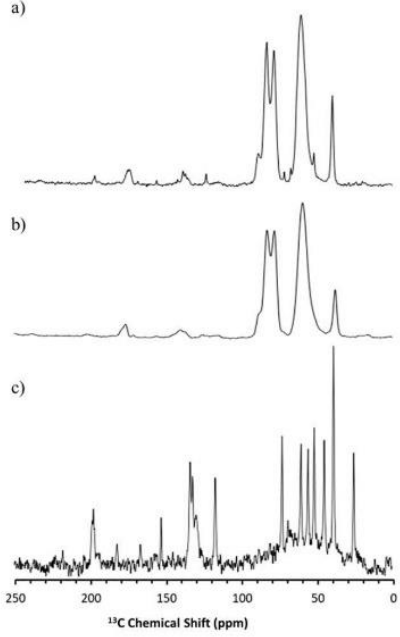

Figure 6. ${ }^{13} \mathrm{C}$ Solid-state nuclear magnetic resonance spectroscopy (SS-NMR) spectra for crystalline haloperidol (c), HME formulation at 0 days (b) and 90 days (a). 


\section{Thesis Publications}

For carbamazepine, the change was more subtle, because of overlapping peaks. However, it was obvious that the stressed sample exhibited crystalline peaks that were not observed in the freshly prepared samples e.g., at $131 \mathrm{ppm}$, as shown in Figure 7. The presence of sharper peaks in the stressed samples underscores the recrystallization in the formulations suggested by the $\mathrm{X}$-ray diffraction data.

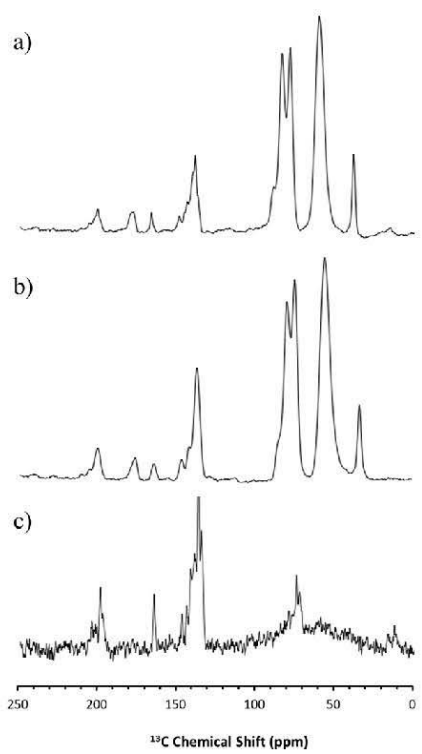

Figure 7. ${ }^{13}$ C SS-NMR spectra for crystalline carbamazepine (c) and carbamazepine HME formulation at 0 days (b) and 90 days (a).

\subsection{Stability of the Supersaturated State in FaSSIF}

The thermodynamic solubility of haloperidol and carbamazepine in FaSSIF was measured to be $259( \pm 1) \mu \mathrm{g} / \mathrm{mL}$ and $203( \pm 2) \mu \mathrm{g} / \mathrm{mL}$. Accordingly, the crystalline APIs showed a dissolution profile approaching these values over the course of the FaSSIF dissolution experiment.

Although both drugs have some solubility in FaSSIF, the dissolution was enhanced by both the mesoporous silica and HME formulations. For carbamazepine, a maximum supersaturation of 1.8 and 1.5-fold was generated for the silica and HME formulations, respectively. For haloperidol, a maximum supersaturation of about 2.0-fold was generated for both silica and HME.

The PVA in the HME formulation was able to sustain supersaturated concentrations for both APIs by inhibiting precipitation from aqueous medium. Mesoporous silica, on the other hand, was unable to inhibit drug precipitation from the supersaturated state and therefore, precipitation was observed for both APIs, with concentrations returning to the thermodynamic solubility. For mesoporous silica formulations, further development of the formulation would include a screening and a selection of a precipitation inhibitor to include in the formulation. The precipitation inhibitor would prevent the precipitation of the supersaturated API and could subsequently enhance oral absorption. However, although important, the precipitation inhibitor in a mesoporous silica formulation is not expected to impact on the solid-state stabilization of the API in the amorphous form. This is due to the fact that precipitation inhibitors are simply blended with the drug-loaded silica when the drug is already loaded onto the porous silica and stabilized in the solid state. Therefore, as the focus of this study was on the innate stabilization potential of mesoporous silica using poor glass formers, incorporation of a precipitation inhibitor was out of scope. 


\section{$\mathrm{X}$. Thesis Publications}

For both APIs, the dissolution profiles from mesoporous silica formulations were comparable throughout the duration of the entire accelerated stability study. Particularly notable was that the degree of supersaturation, or 'spring', remained consistent over the whole stability study (Figure 8; Figure 9). For HME formulations, the curves showed a decrease in supersaturation in each successive month of the stability study. After 30 days, the HME formulation containing carbamazepine was still able to generate supersaturation, but the profile was no longer stable; the carbamazepine concentration returned to the thermodynamic solubility within $60 \mathrm{~min}$. This difference between fresh and 30 day carbamazepine samples was indicative of the presence of seed crystals in the formulations. Such seeds foster crystallization in the formulation as well as in solution to most likely override the inhibition of precipitation by the polymer [40]. Furthermore, the release performance of carbamazepine HME declined even further at 60 and 90 days. By 90 days, no supersaturation was observed at any measured time point during the experiment, and the dissolution curve resembled that of crystalline carbamazepine more closely (Figure 8).
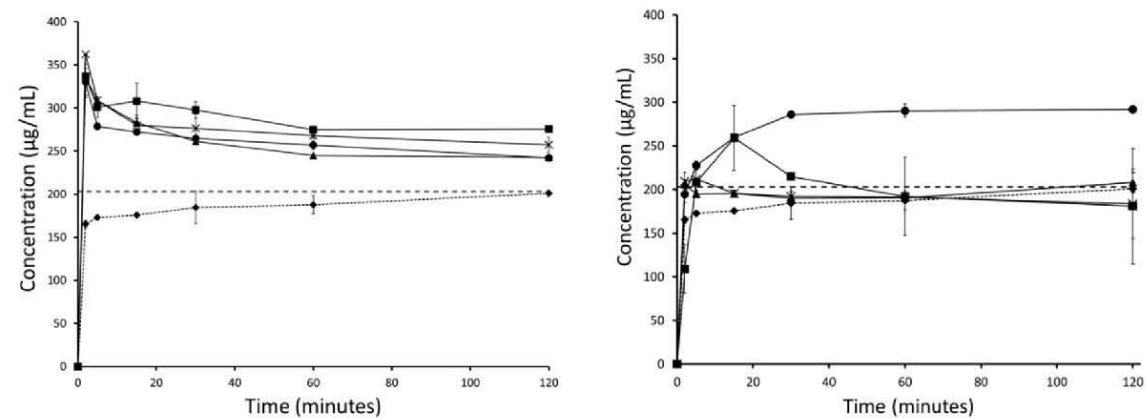

Figure 8. Fasted Simulated Intestinal Fluid (FaSSIF) mini-dissolution curves for carbamazepine loaded silica (left) and carbamazepine HME formulation (right) showing crystalline carbamazepine ( $\downarrow$ ), unstressed carbamazepine formulation (•), and stressed carbamazepine formulations at $30(\mathbf{\bullet}), 60(\mathrm{X})$, and $90(\mathbf{\Lambda})$ days.

Similar reductions in dissolution performance with storage were observed for the HME formulation of haloperidol. Although the dissolution performance of the haloperidol HME did not decline as quickly as that of the carbamazepine HME, its dissolution profile also resembled that of the crystalline API after 90 days (Figure 9 ).
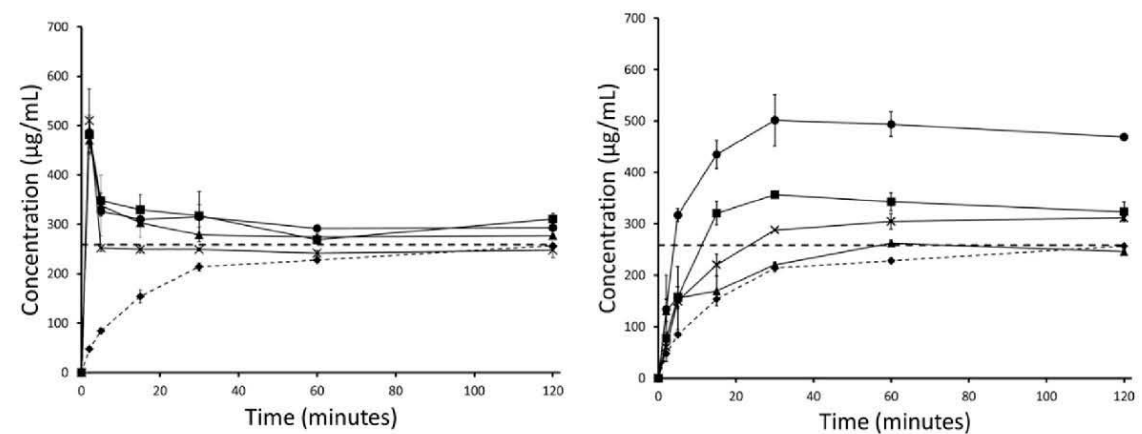

Figure 9. FaSSIF mini-dissolution curves for haloperidol loaded silica (left) and haloperidol HME formulation (right) showing crystalline haloperidol ( $\downarrow$ ), unstressed haloperidol formulation (•), and stressed haloperidol formulations at $30(\mathbf{0}), 60(X)$, and $90(\mathbf{\Lambda})$ days. 


\section{Thesis Publications}

\section{Discussion}

Development of amorphous solid dispersions requires a thorough understanding of the factors that influence stability in the amorphous form. One such factor is the GFA. According to the classification system proposed by Baird et al. [4], GFA-I drugs are especially challenging when developing amorphous formulations, due to the high propensity for recrystallization of such compounds. It is essential to demonstrate good physical stability in amorphous formulations to ensure that recrystallization does not occur over time, as this would reduce the shelf life of the product [41]. As crystallization is based on the stochastic nucleation process, a lack of physical stability could lead to variable product quality among batches. Any initial crystallization in the batch, or differences in the rate of crystallization, could lead to out-of-specification results based on insufficient drug product performance, for example in dissolution testing. Variability among batches would thus be problematic in terms of meeting regulatory and commercial requirements. Herein, it has been demonstrated that mesoporous silica can be used to successfully stabilize compounds of poor amorphous stability that are unsuitable for formulation in standard polymer-based amorphous solid dispersions.

Initial formulation development demonstrated the potential impact that such a poor amorphous stability could have on the viability of a formulation. For both HME formulations, significantly lower percentage drug loads were attainable in the initial formulation development. This is a crucial topic for the development of amorphous formulations. Taking the example of haloperidol, it was only possible to stabilize 7.5\% $(w / w)$ of the API in the amorphous form in the HME. Assuming a theoretical dose of $200 \mathrm{mg}$, one would require a tablet of approximately $2.6 \mathrm{~g}$ in weight to incorporate the entire dose in a single dosage form. Furthermore, this represents a very conservative estimation, as the actual API content would likely be reduced further to $3.75 \%$ when one considers that $50 \%$ of the tablet may consist of fillers, binders, glidants, and disintegrants. Ultimately, a low drug loading would be a substantial risk to the viability of the formulation, and could result in failure of the project. Mesoporous silica, however, was successful in stabilizing more reasonable drug loads in non-crystalline form.

Furthermore, the successful low drug loading HME formulations developed for carbamazepine and haloperidol were observed to be unstable during the ICH Q1 stability study (Figure 4; Figure 5). Instability in polymeric amorphous solid dispersions can be linked with increasing temperature and humidity. As temperature or water content in amorphous formulations increases, mobility of the drug within the polymer dispersion increases. Mehta and co-workers demonstrated this effect on model amorphous solid dispersions (ASDs). In their study, an increase in molecular mobility of all APIs in the polymer ASDs led to a decrease in recrystallization time [41,42]. As both studies by Mehta and colleagues investigated the physical stability of good to moderate glass formers (GFA-II/III), it is likely that the effect of moisture and temperature on increasing molecular mobility and subsequent physical instability would be even greater for GFA-I compounds. The observed instability of these poor glass formers is even more remarkable when one considers that, of the available polymers for HME, PVA has a substantially low hygroscopicity. This low hygroscopicity would have a stabilizing effect on the formulation due to a reduction in the uptake of water upon storage at humid conditions. However, this beneficial characteristic of PVA was not enough to prevent the poor glass formers from recrystallizing in the extrudates [32].

For mesoporous silica, however, molecular mobility is greatly reduced regardless of moisture or temperature. Brás and colleagues demonstrated that adsorption and nano-confinement of ibuprofen onto mesoporous silica resulted in a significant reduction of all known types of molecular mobility [43] Most interestingly, the Johari-Goldstein $\beta$ relaxation, a type of molecular mobility associated with recrystallization, was reduced. This was a crucial observation, as it has been shown that increased Johari-Goldstein relaxation is related to physical instability of the amorphous form [42,43]. Similar to the work by Mehta and colleagues, Brás et al. focused on good glass formers (GFA-III). Additional work demonstrated that a reduction in molecular mobility leads to successful stabilization of the very poor glass former menthol (GFA-I), which has a glass transition temperature of $-54.3^{\circ} \mathrm{C}$ [44]. This stabilization was related to a decrease in molecular mobility of both $\alpha$ (free transitional mobility in space) 


\section{Thesis Publications}

and the aforementioned Johari-Goldstein $\beta$ relaxations. Furthermore, a new type of molecular mobility, the S-type, was observed. S-type refers to mobility of a hindered molecule that is nano-confined within a single pore, and is much slower than standard molecular mobility events [44]. Based on these findings as well as those of our study (Figure 4; Figure 5), mesoporous silica may be a suitable way forward to stabilizing GFA-I glass formers under accelerated conditions.

There are only a handful of known GFA-I compounds that are also BCS II compounds and which would thus benefit from the apparent solubility increase of the amorphous form. In a recent review, Kawakami provided an overview of pharmaceutical compounds according to GFA classes [45]. Of the GFA-I compounds in the database only $29 \%$ were determined to be BCS II/IV, which is far lower than the commonly reported percentage of commercial compounds that fall into the poor solubility category $(60 \%)$ [8]. Hence, there appears to be a disconnect between the prevalence of compounds with poor solubility and the occurrence of poor glass formers on the market. This could be related to the difficulty in formulating such compounds, and the reduction in formulation performance related to physical instability.

For the two model BCS II drugs selected in this study, clear differences were observed in the non-sink release profiles of loaded silica and HME formulations. As expected from the literature, silica alone was not able to sustain supersaturated concentrations of API in solution resulting in precipitation [23]. Conversely, in the HME formulations, the API is sustained in solution by the polymer itself, which can function not only as a matrix polymer but also as a precipitation inhibitor during drug release. However, it was observed that dissolution of API loaded silica formulations remained consistent throughout the 3 month study (Figure 8; Figure 9), whereas the kinetic release of IIME formulations tended towards crystalline drug solubility (Figure 8; Figure 9). Here, we see the effect of phase separation and recrystallization on the dissolution performance of amorphous solid dispersions, with the presence of a crystalline phase reducing the achievable supersaturation and decreasing the dissolution performance of the compound [45]. Interestingly, both HME formulations retained some supersaturation after the first month of the stability study, indicating that full conversion from amorphous to crystalline had not yet occurred (Figure 8; Figure 9). However, the supersaturated solutions generated by the carbamazepine HME were less stable than those generated by the haloperidol HME, and precipitation occurred (Figure 8). This was related to the presence of seed crystals in the formulation, which sped up the rate of nucleation and reduced the ability of the polymer to prevent precipitation. Patel and co-workers demonstrated that a small amount of crystalline indomethacin significantly increased its recrystallization from the supersaturated state, even in the presence of precipitation inhibitors [40]. The present results support the view that GFA-I compounds may not be good candidates for formulation in polymeric amorphous solid dispersions, such as hot-melt extrudates, which have been investigated here. However, this is also expected by other formulations based on polymeric amorphous dispersions, e.g., spray dried dispersions or co-precipitates. Mesoporous silica, on the other hand, is an attractive formulation option for poorly soluble glass formers, generating consistent and supersaturated dissolution profiles.

\section{Conclusions}

The increasing prevalence of poorly soluble BCS II drug candidates in pharmaceutical development remains a challenging issue. Although polymer-based stabilization of the API in an amorphous form has been a common approach to their formulation for several decades. Such an approach may not be suitable for poorly soluble compounds that also show poor GFA. These compounds, which demonstrate both poor solubility and poor amorphous stability, are challenging for formulation with typical polymer-based technologies due to possible phase separation and recrystallization. Ultimately, these compounds may have an increased risk of failure during pharmaceutical development, as they constitute a risk from both a bioavailability and amorphous stability perspective. In this study, we demonstrated that poor glass forming (GFA-I) APIs have increased risk of recrystallization in polymer-based amorphous solid dispersions. By contrast, mesoporous silica was shown to provide 


\section{Thesis Publications}

optimal stabilization for such APIs. Therefore, mesoporous silica could be an attractive formulation technology to expand the formulation toolbox for APIs that are poor glass formers. More research in the future will clarify whether mesoporous silica should become a method of choice for oral delivery of poorly soluble GFA-I compounds.

Author Contributions: Conceptualization, F.D., D.J.P. and M.K.; Funding acquisition, J.B.D., C.S. and M.K. Investigation, F.D., D.J.P. and J.B.-B.; Methodology, F.D., D.J.P., A.N., C.S. and M.K.; Project administration, J.B.D. C.S. and M.K.; Resources, A.N. and C.S.; Software, F.D., D.J.P. and J.B.-B.; Supervision, A.N., C.G., C.S. and M.K Visualization, F.D. and D.J.P.; Writing - original draft, F.D. and D.J.P.; Writing - review \& editing, F.D., D.J.P., A.N., J.B.-B., C.G., J.B.D., C.S. and M.K

Funding: This project has received funding from the European Union's Horizon 2020 Research and Innovation Program under grant agreement No 674909.

Acknowledgments: The authors like to thank Dieter Lubda, Gudrun Birke, Finn Bauer and Thomas Kipping for providing the Parteck ${ }^{(B)}$ excipients, for helpful and constructive discussions and feedback.

Conflicts of Interest: Daniel J. Price, Anita Nair and Christoph Saal are full-time employees of Merck KGaA Otherwise, the authors report no conflict of interest. The studies were performed under the auspices of the EU grant, including all funding, and independent of the company.

\section{References}

1. Hancock, B.C.; Parks, M. What is the True Solubility Advantage for Amorphous Pharmaceuticals? Pharm. Res. 2000, 17, 397-404. [CrossRef]

2. Leuner, C; Dressman, J. Improving drug solubility for oral delivery using solid dispersions. Eur. J. Pharm. Biopharm. 2000, 50, 47-60. [CrossRef]

3. Rams-Baron, M.; Jachowicz, R.; Boldyreva, E.; Zhou, D.; Jamroz, W.; Paluch, M. Physical Instability: A Key Problem of Amorphous Drugs. In Amorphous Drugs; Springer International Publishing: Cham, Switzerland, 2018; pp. 107-157.

4. Baird, J.A.; Van Eerdenbrugh, B.; Taylor, L.S. A Classification System to Assess the Crystallization Tendency of Organic Molecules from Undercooled Melts. J. Pharm. Sci. 2010, 99, 3787-3806. [CrossRef] [PubMed]

5. Alhalaweh, A.; Alzghoul, A.; Mahlin, D; Bergström, C.A.S. Physical stability of drugs after storage above and below the glass transition temperature: Relationship to glass-forming ability. Int. I. Pharm. 2015, 495, 312-317. [CrossRef] [PubMed]

6. Wyttenbach, N.; Kirchmeyer, W.; Alsenz, J.; Kuentz, M. Theoretical Considerations of the Prigogine-Defay Ratio with Regard to the Glass-Forming Ability of Drugs from Undercooled Melts. Mol. Pharm. 2016, 13, 241-250. [CrossRef] [PubMed]

7. Van Eerdenbrugh, B.; Baird, J.A.; Taylor, L.S. Crystallization Tendency of Active Pharmaceutical Ingredients Following Rapid Solvent Evaporation-Classification and Comparison with Crystallization Tendency from Under cooled Melts. J. Pharm. Sci. 2010, 99, 3826-3838. [CrossRef] [PubMed]

8. Ditzinger, F.; Price, D.J.; Ilie, A.-R.; Köhl, N.J.; Jankovic, S.; Tsakiridou, G.; Aleandri, S.; Kalantzi, L.; Holm, R.; Nair, A.; et al. Lipophilicity and hydrophobicity considerations in bio-enabling oral formulations approaches-A PEARRL review. J. Pharm. Pharmacol. 2019,71, 464-482. [CrossRef]

9. Blaabjerg, L.I; Bulduk, B.; Lindenberg, E.; Löbmann, K.; Rades, T.; Grohganz, H. Influence of glass forming ability on the physical stability of supersaturated amorphous solid dispersions. I. Pharm. Sci. 2019, 108, 2561-2569. [CrossRef]

10. Wyttenbach, N.; Kuentz, M. Glass-forming ability of compounds in marketed amorphous drug products. Eur. J. Pharm. Biopharm. 2017, 112, 204-208. [CrossRef]

11. Repka, M.A.; Bandari, S.; Kallakunta, V.R.; Vo, A.Q.; McFall, H.; Pimparade, M.B.; Bhagurkar, A.M. Melt extrusion with poorly soluble drugs-An integrated review. Int. J. Pharm. 2018, 535, 68-85. [CrossRef]

12. Sliwinska-Bartkowiak, M.; Dudziak, G.; Gras, R.; Sikorski, R.; Radhakrishnan, R.; Gubbins, K.E. Freezing behavior in porous glasses and MCM-41. Colloids Surfaces A Physicochem. Eng. Asp. 2001, 187-188, 523-529. [CrossRef]

13. McCarthy, C.A.; Ahern, R.J.; Dontireddy, R.; Ryan, K.B.; Crean, A.M. Mesoporous silica formulation strategies for drug dissolution enhancement: A review. Expert Opin. Drug Deliv. 2016, 13, 93-108. [CrossRef] [PubMed] 


\section{Thesis Publications}

14. Azaïs, T.; Tourné-Péteilh, C.; Aussenac, F.; Baccile, N.; Coelho, C.; Devoisselle, J.-M.; Babonneau, F. Solid-State NMR Study of Ibuprofen Confined in MCM-41 Material. Chem. Mater. 2006, 18, 6382-6390. [CrossRef]

15. Müller, R.H.; Wei, Q.; Keck, C.M. CapsMorph: >4 Years Long-Term Stability of Industrially Feasible Amorphous Drug Formulations. In Proceedings of the Annual Meeting of the Controlled Release Society, Honolulu, HI, USA, 21-24 July 2013.

16. Mellaerts, R.; Houthoofd, K.; Elen, K.; Chen, H.; Van Speybroeck, M.; Van Humbeeck, J.; Augustijns, P.; Mullens, J.; Van den Mooter, G.; Martens, J.A. Aging behavior of pharmaceutical formulations of itraconazole on SBA-15 ordered mesoporous silica carrier material. Microporous Mesoporous Mater. 2010, 130, 154-161. [CrossRef]

17. Salonen, J.; Kaukonen, A.M.; Hirvonen, J.; Lehto, V.-P. Mesoporous Silicon in Drug Delivery Applications. J. Pharm. Sci. 2008, 97, 632-653. [CrossRef] [PubMed]

18. Williams, H.D.; Trevaskis, N.L.; Charman, S.A.; Shanker, R.M.; Charman, W.N.; Pouton, C.W.; Porter, C.J.H Strategies to address low drug solubility in discovery and development. Pharmacol. Rev, 2013, 65, 315-499.

19. Knopp, M.M.; Wendelboe, I.; Holm, R.; Rades, T. Effect of amorphous phase separation and crystallization on the in vitro and in vivo performance of an amorphous solid dispersion. Eur. J. Pharm. Biopharm. 2018, 130, 290-295. [CrossRef]

20. Guzmán, H.R.; Tawa, M.; Zhang, Z; Ratanabanangkoon, P.; Shaw, P.; Gardner, C.R.; Chen, H.; Moreau, J.; Almarsson, Ö.; Remenar, J.F. Combined Use of Crystalline Salt Forms and Precipitation Inhibitors to Improve Oral Absorption of Celecoxib from Solid Oral Formulations. J. Pharm. Sci. 2007, 96, 2686-2702. [CrossRef]

21. Price, D.J.; Ditzinger, F.; Koehl, N.J.; Jankovic, S.; Tsakiridou, G.; Nair, A.; Holm, R.; Kuentz, M.; Dressman, J.B.; Saal, C. Approaches to increase mechanistic understanding and aid in the selection of precipitation inhibitors for supersaturating formulations-A PEARRL review. J. Pharm. Pharmacol. 2019, 71, 483-509. [CrossRef]

22. Brough, C.; Miller, D.A.; Keen, J.M.; Kucera, S.A.; Lubda, D.; Williams, R.O. Use of Polyvinyl Alcohol as a Solubility-Enhancing Polymer for Poorly Water Soluble Drug Delivery (Part 1). AAPS PharmSciTech 2016 17, 167-179. [CrossRef]

23. Price, D.J.; Nair, A.; Kuentz, M.; Dressman, J; Saal, C. Calculation of drug-polymer mixing enthalpy as a new screening method of precipitation inhibitors for supersaturating pharmaceutical formulations. Eur. J. Pharm. Sci. 2019, 132, 142-156. [CrossRef] [PubMed]

24. ICH Expert Working Group. ICH Guideline Q1A(R2) Stability Testing of New Drug Substances and Products. International Conference on Harmonization. 2003. Available online: https:/www.ema.europa.eu/en/documents/scientific-guideline/ich-q-1-r2-stability-testing-newdrug-substances-products-step-5_en.pdf (accessed on 25 September 2019)

25. Vertzoni, M.; Fotaki, N.; Nicolaides, E.; Reppas, C.; Kostewicz, E.; Stippler, E.; Leuner, C.; Dressman, J. Dissolution media simulating the intralumenal composition of the small intestine: Physiological issues and practical aspects. J. Pharm. Pharmacol. 2004, 56, 453-462. [CrossRef] [PubMed]

26. Dressman, J.B.; Amidon, G.L.; Reppas, C.; Shah, V.P. Dissolution testing as a prognostic tool for oral drug absorption: Immediate release dosage forms. Pharm. Res. 1998, 15, 11-22. [CrossRef] [PubMed]

27. Edueng, K.; Mahlin, D.; Gråsjö, J.; Nylander, O; Thakrani, M; Bergström, C.A.S. Supersaturation Potential of Amorphous Active Pharmaceutical Ingredients after Long-Term Storage. Molecules 2019, 24, 2731. [CrossRef] [PubMed]

28. Paudel, A.; Geppi, M.; Mooter, G. Van den Structural and Dynamic Properties of Amorphous Solid Dispersions: The Role of Solid-State Nuclear Magnetic Resonance Spectroscopy and Relaxometry. J. Pharm. Sci. 2014, 103, 2635-2662. [CrossRef]

29. Lee, H.L.; Vasoya, J.M.; Cirqueira, M.D.L.; Yeh, K.L.; Lee, T.; Serajuddin, A.T.M. Continuous Preparation of 1:1 Haloperidol-Maleic Acid Salt by a Novel Solvent-Free Method Using a Twin Screw Melt Extruder. Mol. Pharm. 2017, 14, 1278-1291. [CrossRef]

30. Djuris, J.; Nikolakakis, I.; Ibric, S.; Djuric, Z.; Kachrimanis, K. Preparation of carbamazepine-Soluplus ${ }^{(\bar{B})}$ solid dispersions by hot-melt extrusion, and prediction of drug-polymer miscibility by thermodynamic model fitting. Eur. J. Pharm. Biopharm. 2013, 84, 228-237. [CrossRef]

31. Galia, E.; Nicolaides, E.; Hörter, D.; Löbenberg, R.; Reppas, C.; Dressman, J.B. Evaluation of various dissolution media for predicting in vivo performance of class I and II drugs. Pharm. Res, 1998, 15, 698-705. [CrossRef] 


\section{$\mathrm{X}$. Thesis Publications}

32. Zheng, M.; Bauer, F.; Birk, G.; Lubda, D. Polyvinyl Alcohol in Hot Melt Extrusion to Improve the Solubility of Drugs. Available online: https:/www.sigmaaldrich.com/content/dam/sigma-aldrich/0/content/pdf/PSPVA-HME-Improve-Solubility-03-2017_EN_MS.pdf (accessed on 2 May 2019).

33. Forster, A.; Hempenstall, J.; Tucker, I.; Rades, T. Selection of excipients for melt extrusion with two poorly water-soluble drugs by solubility parameter calculation and thermal analysis. Int. J. Pharm. 2001, 226,147-161. [CrossRef]

34. Laitinen, R.; Priemel, P.A.; Surwase, S.; Graeser K.; $_{\text {; }}$ Strachan, C.J.; Grohganz, H.; Rades, T. Theoretical Considerations in Developing Amorphous Solid Dispersions. In Amorphous Solid Dispersions; Springer: New York, NY, USA, 2014; pp. 35-90.

35. Niederquell, A; Wyttenbach, N.; Kuentz, M. New prediction methods for solubility parameters based on molecular sigma profiles using pharmaceutical materials. Int. J. Pharm. 2018, 546, 137-144. [CrossRef]

36. Loschen, C.; Klamt, A. COSMO quick: A Novel Interface for Fast o-Profile Composition and Its Application to COSMO-RS Solvent Screening Using Multiple Reference Solvents. Ind. Eng. Chem. Res. 2012, 51, 14303-14308. [CrossRef]

37. Greenspan, L. Humidity fixed points of binary saturated aqueous solutions. J. Res. Natl. Bur. Stand. Sect. A Phys. Chem. 1977, 81A, 89-96. [CrossRef]

38. Thiry, J.; Krier, F; Evrard, B. A review of pharmaceutical extrusion: Critical process parameters and scaling-up. Int. J. Pharm. 2015, 479, 227-240. [CrossRef] [PubMed]

39. Kissi, E.O.; Ruggiero, M.T.; Hempel, N.-J.; Song, Z.; Grohganz, H.; Rades, T.; Löbmann, K. Characterising glass transition temperatures and glass dynamics in mesoporous silica-based amorphous drugs. Phys. Chem. Chem. Phys. 2019. [CrossRef]

40. Patel, D.D.; Anderson, B.D. Effect of Precipitation Inhibitors on Indomethacin Supersaturation Maintenance: Mechanisms and Modeling. Mol. Pharm. 2014, 11, 1489-1499. [CrossRef]

41. Mehta, M.; Suryanarayanan, R. Accelerated Physical Stability Testing of Amorphous Dispersions. Mol. Pharm. 2016, 13, 2661-2666. [CrossRef]

42. Mehta, M.; Kothari, K.; Ragoonanan, V.; Suryanarayanan, R. Effect of Water on Molecular Mobility and Physical Stability of Amorphous Pharmaceuticals. Mol. Pharm. 2016, 13, 1339-1346. [CrossRef]

43. Brás, A.R.; Fonseca, I.M.; Dionísio, M.; Schönhals, A.; Affouard, F.; Correia, N.T. Influence of Nanoscale Confinement on the Molecular Mobility of Ibuprofen. J. Phys. Chem. C 2014, 118, 13857-13868. [CrossRef]

44. Cordeiro, T.; Castiñeira, C; Mendes, D.; Danède, F.; Sotomayor, J.; Fonseca, I.M.; Gomes da Silva, M.; Paiva, A.; Barreiros, S.; Cardoso, M.M; et al. Stabilizing Unstable Amorphous Menthol through Inclusion in Mesoporous Silica Hosts. Mol. Pharm. 2017, 14, 3164-3177. [CrossRef]

45. Kawakami, K. Crystallization Tendency of Pharmaceutical Glasses: Relevance to Compound Properties, Impact of Formulation Process, and Implications for Design of Amorphous Solid Dispersions. Pharmaceutics 2019, 11, 202. [CrossRef] (CC BY) license (http://creativecommons.org/licenses/by/4.0/). 


\section{Lipophilicity and hydrophobicity considerations in bio-enabling oral formulations approaches - a PEARRL review}

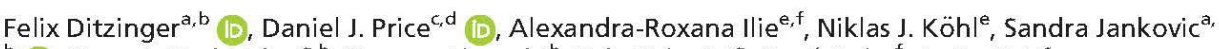
${ }^{b}$ (D), Georgia Tsakiridou ${ }^{g, h}$, Simone Aleandrib ${ }^{b}$ Lida Kalantzi ${ }^{g}$, René $\mathrm{Holm}^{f}$, Anita Nair ${ }^{c}$, Christoph Saal ${ }^{c}$, Brendan Griffin ${ }^{e}$ (D) and Martin Kuentz $z^{b}$ (D)

aDepartment of Pharmaceutical Sciences, University of Basel, Basel, "b Institute of Pharma Technology, University of Applied Sciences and Arts Northwestern Switzerland, Muttenz, Switzerland, "Analytics Healthcare, Merck KGaA, Darmstadt, "Goethe University, Frankfurt, Germany, "Echool of Pharmacy, University College Cork, Cork, Ireland, ${ }_{2}^{f}$ Drug Product Development, Janssen Research and Development, Johnson and Johnson, Beerse, Belgium, gProduct Design \& Evaluation, Pharmathen SA, Athens and ${ }^{\mathrm{h}}$ Faculty of Fharmacy, National and Kapodistrian University of Athens, Athens, Greece

Keywords

crystal lattice energy; hydrophobicity: lipophilicity; modern formulation

approaches; poorly water-soluble drug

Correspondence

Martin Kuentz, Institute of Pharma

Technology, University of Applied Sciences

and Arts Northwestern Switzerland,

Gründenstrasse 40,4132 Muttenz,

Switzerland.

E-mail: Martin.Kuentz@fhnw.ch

Received March 8, 2018

Accepted June 27, 2018

do: 10.111 1/jphp. 12984

\begin{abstract}
Objectives This review highlights aspects of drug hydrophobicity and lipophilicity as determinants of different oral formulation approaches with specific focus on enabling formulation technologies. An overview is provided on appropriate formulation selection by focussing on the physicochemical properties of the drug. Key findings Crystal lattice energy and the octanol-water partitioning behaviour of a poorly soluble drug are conventionally viewed as characteristics of hydrophobicity and lipophilicity, which matter particularly for any dissolution process during manufacturing and regarding drug release in the gastrointestinal tract. Different oral formulation strategies are discussed in the present review, including lipid-based delivery, amorphous solid dispersions, mesoporous silica, nanosuspensions and cyclodextrin formulations.

Summary Current literature suggests that selection of formulation approaches in pharmaceutics is still highly dependent on the availability of technological expertise in a company or research group. Encouraging is that, recent advancements point to more structured and scientifically based development approaches. More research is still needed to better link physicochemical drug properties to pharmaceutical formulation design.
\end{abstract}

\section{Introduction}

The use of modern drug discovery approaches, such as combinatorial chemistry and high-throughput screening as well as structural understanding of drug-target binding by $\mathrm{X}$-ray diffraction and molecular modelling, has resulted in an increasing percentage of highly potent lead compounds. However, these compounds present increasing issues for formulation development as they often have high-melting points $\left(T_{\mathrm{m}}\right)$ and high octanol-water partition coefficients $(\log P)^{[1}{ }^{14]}$ While $T_{\mathrm{m}}$ is a characteristic of crystal lattice energy, $\log P$, as a partition coefficient, denotes a solvation tendency or a lack of the same. These properties are in the chemical space of poorly soluble drugs often associated with hydrophobicity and lipophilicity, respectively. ${ }^{[5]}$ Most importantly, high values of $T_{\mathrm{m}}$ and $\log P$ limit aqueous solubility and as a consequence thereof often bioavailability when administered orally in conventional dosage forms. ${ }^{[3,4,6]}$ These limiting factors of drug solubility can be assigned to separate idealised dissolution processes. A molecule first has to overcome crystal lattice energy to interact with solvent molecules in the consecutive process of solvation. Figure la depicts the crystal structure of a model compound (cinnarizine), which is used to highlight molecular interactions in the solid state. Figure $1 \mathrm{~b}$ shows a molecule dissolved in water that requires interactions for the solvation step; hence, the ease of this hydration depends on the solute affinity to water.

The general chemical meaning of the word hydrophobicity is the physical characteristic of a molecule that it is 


\section{$\mathrm{X}$. Thesis Publications}

repelled by water. Such a rather broad definition of hydrophobicity indudes different reasons that cause a molecule to behave in this way. This umbrella use of hydrophobicity leads to some overlap with lipophilicity. A more specific use of hydrophobicity focuses on pharmaceutical compounds that exhibit limited aqueous solubility due to high crystal lattice energy. ${ }^{[5]}$

This has become a widespread convention in pharmaceutical sciences when poorly soluble drugs are considered. Hydrophobicity in this context has to be clearly differentiated from lipophilicity. Thus, lipophilicity as a scale is conventionally viewed as relative in the given chemical space For example, values of $\log P<2$ mark a low range of lipophilicity for poorly soluble drugs, whereas a high regimen of lipophilicity starts in this chemical space beyond about $\log P>6$. Such ranges are tentatively assigned and can be subject to change.

In line with these considerations, $T_{\mathrm{m}}$ and $\log P$ are generally seen as two important properties of biopharmaceutical drug profiling. ${ }^{[7]}$ Such profiling is required to predict biopharmaceutical issues early on and to propose adequate formulation strategies. The design, development and characterisation of bio-enabling formulations are among the
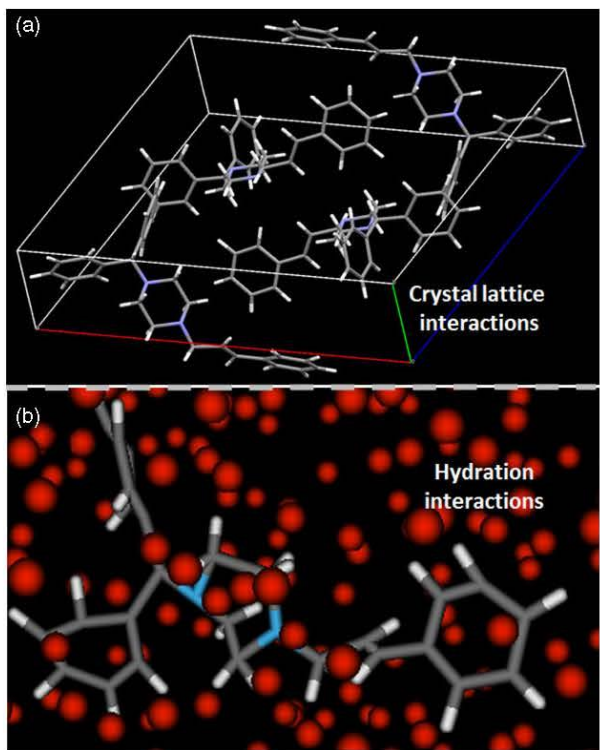

Figure 1 (a) Model poorly soluble compound (cinnarizine) is shown as the unit cell of the crystal lattice and upon (b) acueous solvation (water molecules are shown without hydrogens for clarity of presentation). core objectives of PEARRL (Pharmaceutical Education And Research with Regulatory Links), a European Network, focused on pharmaceutical education and research for faster patient access to breakthrough therapies. This study addresses the need to review oral bio-enabling formulation approaches from a specific viewpoint of compound lipophilicity and hydrophobicity characteristics.

\section{Theoretical Aspects of Lipophilicity and Hydrophobicity}

The molar solubility of a compound $x_{\text {eq }}$ can be obtained as a function of temperature, $T$, by considering the solidliquid equilibrium, which can be expressed as follows: ${ }^{[8,9]}$

$$
\begin{aligned}
\operatorname{In} x_{\mathrm{eq}}= & \frac{\Delta H_{\mathrm{f}}}{R}\left(\frac{1}{T_{\mathrm{m}}}-\frac{1}{T}\right)-\frac{1}{\mathrm{RT}} \int_{T_{\mathrm{m}}}^{T} \Delta C_{\mathrm{p}} \mathrm{d} T+\frac{1}{R} \int_{T_{\mathrm{m}}}^{T} \frac{\Delta C_{\mathrm{p}}}{T} \mathrm{~d} T \\
& -\ln \gamma_{\mathrm{eq}}
\end{aligned}
$$

where $\Delta H_{\mathrm{f}}$ is a compound's enthalpy of fusion, $R$ is the gas constant and $\Delta C_{\mathrm{p}}$ represents the heat capacity difference between the supercooled melt and the solid. The activity coefficient of the solute in the saturated solution is given by $\gamma_{\mathrm{eq}}$ and estimation of this property is a central task of various thermodynamic theories. A classical and rather simple approach is the regular solution theory of Scatchard-Hildebrand, ${ }^{[10]}$ which has been used by Jain and Yalkowsky ${ }^{[1]]}$ to describe drug solubility in octanol, $S_{0}$. Such solubility estimation of an organic molecule in an organic solvent, such as octanol, appears to be simpler than a direct estimation of a drug's water solubility, $S_{\mathrm{w}}$. The two properties are linked via the partition coefficient $\log P$ as approximated by:

$$
\log S_{\mathrm{w}}=\log S_{\circ}-\log P
$$

Use of the regular solution theory was complemented with further simplifications (for example Walden's assumption of a constant entropy change upon fusion, $\Delta S_{f}$ ) to obtain the general solution equation (GSE) for non-electrolytes: $[11]$

$$
\log S_{w}=0.5-0.01\left(T_{\mathrm{m}}-25\right)-\log P .
$$

The above GSE has particular importance for the present review because $\log S_{\mathrm{w}}$ is a function of a compound's crystal lattice energy and lipophilicity as expressed by $T_{\mathrm{m}}$ in $\mathrm{K}$ and $\log P$, respectively. This GSE approach (Equation 3) allows an estimation of how a drug's lattice energy (conventionally seen as hydrophobicity for poorly soluble drugs) and lipophilicity determine solubility. Compounds with high $T_{\mathrm{m}}$ values and moderate or $\operatorname{low} \log P$ values are often called 


\section{Thesis Publications}

'brick-dust', whereas low $T_{\mathrm{m}}$ and high $\log P$ values often give molecules the properties of so-called grease balls. While the brick-dust compounds exhibit a solid-state limited solubility with breakdown of the crystal lattice as most difficult step, the grease balls have the solvation step in water as the main hurdle for drug dissolution. ${ }^{[5,6]}$ The GSE can provide initial estimates of aqueous drug solubility, but for more precise values, it has been suggested to assume a non-constant entropy change between the solid and a supercooled melt. ${ }^{[12]}$

Hydrophobicity and lipophilicity are not only important characteristics for the different approaches to predict solubility ${ }^{[13]}$ but they are also interesting regarding further pharmaceutical aspects. Accordingly, one field of interest is about which molecular features or moieties lead to brick dust or grease ball characteristics. It has, for example, been observed that structural features related to rigidity or aromaticity were correlated with restricted solubility due to stable crystal structures and thus hydrophobicity. ${ }^{[14}$ Regarding solvation-limited solubility, it makes sense to consider the molecular features that lead to high partitioning into an apolar phase, for example as inferred from the Abraham solvation predictors. ${ }^{[15,16]}$ This approach has recently been used to obtain a better understanding of the molecular drug characteristics that drive solubilisation in biorelevant media. ${ }^{[17]}$ It is clear that an improved molecular understanding of solubility limitations would be of great help in the drug discovery phase when designing and selecting drug candidates. General developability criteria, such as Lipinski's rule of $5{ }^{[18]}$ could be further refined so there is clearly more research to be performed in this field. However, the current review approaches hydrophobicity and lipophilicity primarily from a formulator's perspective and provides guidance regarding the selection of excipients and bio-enabling formulation approaches.

\section{Lipophilicity and Hydrophobicity Regarding Different Formulation Approaches}

\section{Lipid-based formulations}

The utility of lipid-based formulations (LBF) as a bioenabling formulation approach for poorly water-soluble drugs is well recognised and has fostered the development of several marketed drug products. ${ }^{[4]}$ Oral LBFs are defined as delivery systems, which present the drug in a mixture of excipients consisting of triglyceride oils, partial glycerides, surfactants or cosurfactants and cosolvents. ${ }^{[19]}$ The key advantage of most marketed LBF's as a bio-enabling strategy is the ability to enhance drug solubilisation in vivo as the drug is generally dissolved in lipids. ${ }^{[4]}$ Following oral administration, the drug is ideally maintained in the solubilised/supersaturated state in the course of lipid dispersion and digestion. There is a risk of drug precipitation from LBFs, which is especially pronounced with formulations containing high percentages of cosolvents.

The thermodynamic factors governing drug solubility in lipids are illustrated in Figure 2. A drug sublimation helps to emphasise the crystal lattice hurdle and this idealised step comes with the Gibbs free energy change of $\Delta G_{\text {crystal-gasphase. }}^{0}$ This is followed by a second idealised step of drug solvation in the lipid vehicle with the free energy change of $\Delta G^{0}$ [20]

In terms of predicting which classes of drugs will be soluble in lipids, grease ball molecules are considered advantageous for LBFs because of their dominant lipophilic characteristics and relatively weak crystal lattice energy, which both favour solubilisation in lipids. In the case of brick-dust molecules, drug solubility in lipids will be limited by a high crystal lattice energy. However, compounds with medium hydrophobicity and lipophilicity are not categorisable as clear brick-dust or grease balls, as there is often a mixture of solid-state and solvation-limited solubility. Within the chemical space of compounds emerging from drug discovery, there is a need of better understanding how lipophilic and/or hydrophobic properties predict drug solubility in LBFs.

Regarding crystal lattice energy, it has been reported that compounds with $T_{\mathrm{m}}$ values below $423.15 \mathrm{~K}$ mostly show a reasonable solubility in glycerides. ${ }^{[21,22]}$ These studies further showed a clear trend towards reduced solubility in lipid excipients with increased $T_{\mathrm{m}}$ values of the drug. However, $T_{\mathrm{m}}$ is only one characteristic of the crystal lattice and equals to the ratio of changes in $\Delta H_{\mathrm{f}}$ and entropy of fusion $\left(\Delta S_{f}\right)$. More refined consideration of the solid state can therefore be advantageous.

Accordingly, Persson et al. ${ }^{[21]}$ reported that the accuracy of solubility predictions in lipids slightly increased, when

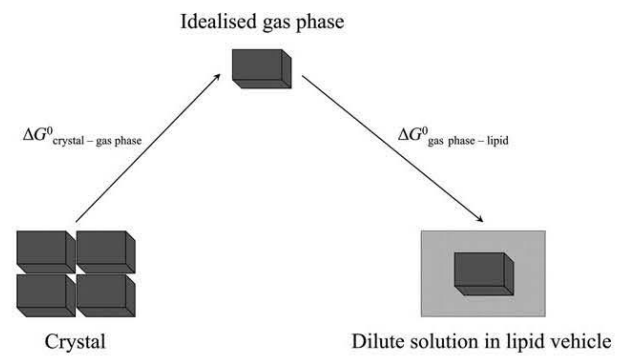

Figure 2 Factors that drive drug solubility in lipids as idealised steps of sublmation from cystal lattice and solvation in lipid vehicle. Further details and definitions of variables are given in the text. Adapted from Rane and Anderson. ${ }^{\left[{ }^{20]}\right.}$ 


\section{$\mathrm{X}$. Thesis Publications}

$T_{\mathrm{m}}$ and $\Delta \mathrm{S}_{\mathrm{f}}$ were both considered. More recently, Gautschi et al. ${ }^{[23]}$ similarly found that $T_{\mathrm{m}}$ alone could not reliably predict drug solubility in C-8 and C-10 triglycerides (TGs). Consequently, solubility estimations for lipids solely based on $T_{m}$ appear to describe only a part of data variability and may only be valid for a rather narrow chemical space. Interestingly, as illustrated in Figure 3, there is a large variability in $T_{m}$ of drugs approved as LBFs by the FDA, ranging from approximately 330.15 to $623.15 \mathrm{~K}$. However, $T_{\mathrm{m}}$ values should still be considered as an important descriptor in solubility models and a guiding predictor for decisions in formulation design. Therefore, a thorough solid-state characterisation is important to understand the factors that govern drug suitability in LBFs. For example, a compound with a high $T_{\mathrm{m}}$ combined with a low $\Delta S_{\mathrm{f}}$ is less favourable to dissolve in pure TGs and therefore unlikely to be suitable for a LBF. ${ }^{[22]}$ However, it is also worth noting that there are further limitations reported on the use of $T_{\mathrm{m}}$ in models to estimate the solubility in ethoxylated excipients, which are commonly used excipients in LBFs. ${ }^{[21,22\rfloor}$ Additiona work seems to be required to clarify such effects of individual excipient classes.

Apart from overcoming the crystal lattice energy, a favourable solvation process is crucial for the solubility in any solvent. ${ }^{[7]}$ In the case of drug solubility in LBFs, this is generally associated with a high $\log P$ value. It has been suggested that a $\log P$ of $>4$ would be desirable to achieve adequate solubility in pure TGs, while an intermediate $\log P$, between 2 and 4, may still show suitable solubility in LBF mixtures containing TGs, surfactants and cosolvents. ${ }^{.19]}$ These considerations of lipophilicity can provide initial formulation guidance but aspects of crystal lattice energy as well as dose strength must be also taken into account Recently, an analysis of $36 \mathrm{LB}$ s (26 different drugs) approved by the FDA showed that the range of $\log P$ was 0.8-7.5 with a median of 4.9. ${ }^{19]}$ The distribution of $\log P$ for the 26 drugs is illustrated in Figure 3. Moreover,

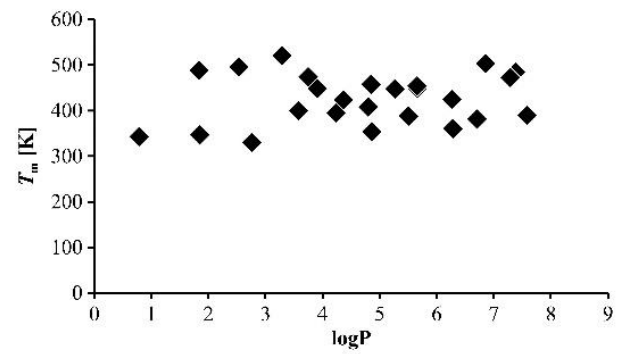

Figure $3 T_{m}$ vs $\log P$ of drugs in FDA approved LBFs. Only three compounds have a $\log P$ below 2 , however, a high variability for $\log P$ as well as $T_{\mathrm{m}}$ can be observed. LBFs, lipid-based formulations.
Figure 4 highlights the distribution of molecular weight (MW), $\log P$ and $T_{m}$ of the FDA approved LBFs. The majority of drugs have a $\log P$ above 2 which underpins the above-mentioned lipophilicity considerations.

Delving further into exploring the relationship between $\log P$ and lipid solubility, Figures 5 and 6 present the lipid solubility as a function of $\log P$ for a range of poorly watersoluble drugs from Alskär et al. ${ }^{[22]}$ (halofantrine omitted). Among the 34 model drugs included, there was a subtle trend of increased solubility in both medium chain (Captex 355) and long chain TGs (Soybean oil) between a $\log P$ of $2-5$. However, the situation is more complex for $\log P$ values larger than 5 , where solubility appears to decrease (Figures 5 and 6). A larger data set is probably needed to draw final conclusions on the relationship between $\log P$ and lipid solubility.

In the framework of this solubility analysis in lipids, it should be noted that poor aqueous solubility is only one rationale for LBFs. Also practical aspects such as, for example, high potency, hygroscopicity or instability can be reasons to select this formulation strategy. When a biopharmaceutical rationale is given, solubility in a LBF is only one aspect to consider, while it is also important how a drug solubilises upon dispersion/digestion as well as how the absorption step is influenced. $\log P$ has also been used to predict further biopharmaceutical properties of drugs including the propensity for lymphatic transport, intestinal permeability, potential presystemic clearance, susceptibility to cfflux pumps or further drug disposition. ${ }^{[24,25]}$ However, such a broad scope was not intended for the current review, but this should be kept in mind to avoid any dogmatic rules of formulation selection that are based solely on drug solubility in LBF.

Besides the drug characteristics, also the choice of excipients merits further discussion. In general, it was shown in two studies that the solubility of a data set of 10 and 35 structurally diverse compounds followed a general solubility ranking of long-chain $\mathrm{TG}<$ medium-chain TG $<$ surfactant. ${ }^{\mid 22,26]}$ LBFs can be classified depending on the oil, surfactant and cosolvent content, ${ }^{19,27 \mid}$ with four proposed classification types. Type I contains solely oils as TGs, diglycerides (DG), monoglycerides (MG) or a mixture thereof, whereas Type IV formulations do not contain lipids but are mixtures of surfactants and cosolvents. Type II, IIIA and IIIB contain different kinds of lipids, surfactants and cosolvents. ${ }^{[27]}$ The more lipophilic a drug molecule is, the more likely it will dissolve in Type I, II and III formulations. On the other hand, hydrophobic drug molecules would be more suited for Type IIIB or IV formulation given the more polar characteristics. ${ }^{[27]}$

Furthermore, several excipient properties can be used to predict and guide the choice of lipids, surfactants and cosolvents. ${ }^{28]}$ It was shown that the polarity expressed as the relative permittivity, hydrophilic and lipophilic balance 


\section{Thesis Publications}
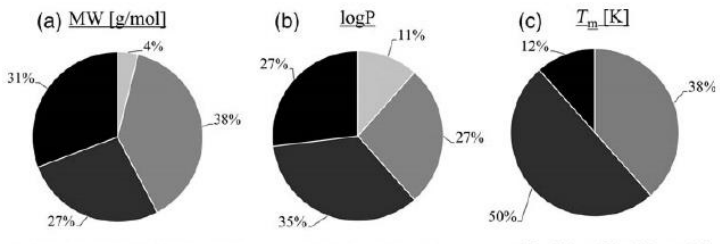

$\because<200=200-400=400-500 \backsim>500$

$=<2=2-4=4-6 \cdot>6$

$=300-400 \cdot 400-500 \cdot>500$

Figure 4 Physicochemical drug properties of drugs in FDA approved LEFs. The different panels are explained in the text. LBFs, lipid-based formulations.

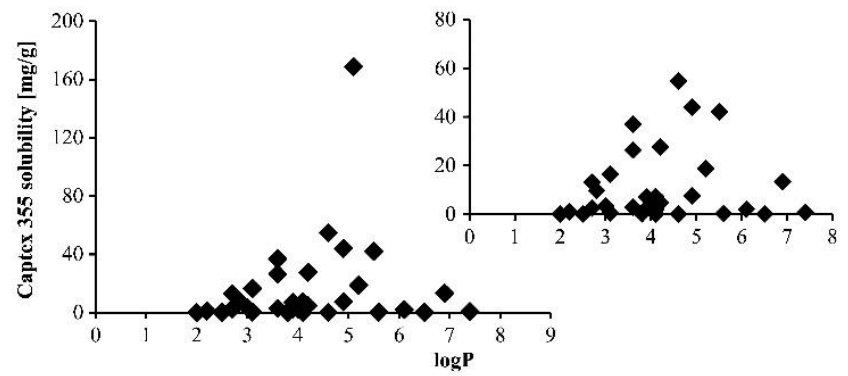

Figure 5 Solubility in Captex 355 plotted against the logP. Dataset from Alskär et a! $!^{[22]}$ Insert: Close up with fenofibrate omitted. The $T_{m}$ values of all used drugs were below $423.15 \mathrm{~K}$.

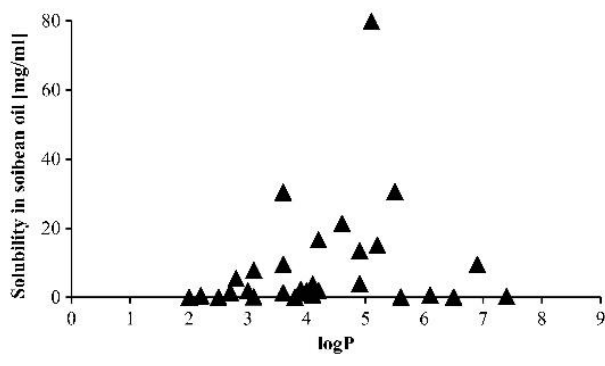

Figure 6 Solubility in soybean oil (SBO) plotted against the logP. Dataset from Alskär et af..$^{1221}$ The $T_{m}$ values of all used drugs were below $423.15 \mathrm{~K}$.

(HLB), ester concentration (in TGs), solubility parameter, surface tension, $\log P$, chain length, MW and molecular volume can be helpful to guide excipient selection. ${ }^{[28,29]}$ The polarity describes the capability of a drug or excipient to interact through dipole interactions and possibly also through hydrogen bonding. These molecular interactions are different from the dispersive interactions of the more apolar parts of molecules. In formulation design, it is critical for the excipient selection to know if the compound of interest needs to form polar and/or non-polar interactions with a given excipient. Such interactions of a drug molecule and an excipient can be visualised in molecular dynamics simulations to improve the understanding of where a drug molecule resides in a LBF and in the complex mixture after aqueous dispersion/digestion. ${ }^{[23,30]}$ As an example, these studies can be used to explain why high solubility in LBFs may not entail high solubility after dispersion or digestion in the intestinal media. It was, for example, observed for medium chain TGs that a $C 8$ chain length resulted in the best LBF solubilisation for several drugs, whereas the corresponding digestion phases yielded best solubilisation in favour of the $\mathrm{C} 10$ chain length TGs. ${ }^{[23]}$ These observations were similar to a previous study exploring lipid chain length and drug solubilisation. ${ }^{[31]}$ More data is needed to draw final conclusions on drug solubility effects in lipids while keeping in mind that lipid solubility is only one factor of adequate formulation design.

In an effort to overcome inherent solubility limitations for challenging drug candidates, a number of advanced LBF technologies have been investigated. Widespread is the concept of self-nanoemulsifying drug delivery systems (SNEDDS), which relies on the combination of drug, surfactant, oil and a coemulsifier resulting in the formation of a nanoemulsion upon aqueous dispersion. More recently, this approach has been advanced to so-called superSNEDDS that are supersaturated LBFs. ${ }^{[32]}$ This type of 


\section{Thesis Publications}

formulation is suitable for highly hydrophobic drugs and offers an option to solubilise more active pharmaceutical ingredient (API) than conventional LBF. Formulation of a super-SNEDDS involves heating of the drug-lipid mixture, to overcome the drug's crystal lattice energy. After cooling, the drug is maintained in a supersaturated state within the lipid vehicle and such formulations have been found to be kinetically stable, for drugs such as simvastatin or fenofibrate. ${ }^{[33,34]}$ Further studies are required to assess whether the kinetic stability is maintained throughout the commercial shelf life of super-SNEDDS formulations.

A recent approach proposed to use lipophilic counter ions to form a more lipophilic salt. ${ }^{[35]}$ This approach increases drug lipophilicity and due to the nature of the specific counter ion, the salt formation could reduce crystal lattice energy and $T_{\mathrm{m}}$ of the free drug. Furthermore, the higher lipophilicity promotes the solute-solvent interaction. For example, the solubility of the lipid salt of atazanavir in an unoptimised LBF vehicle increased several fold compared with the free base. ${ }^{[35]}$ Therefore, formation of ionic liquids (as defined by $T_{\mathrm{m}}<373.15 \mathrm{~K}$ for the resulting salt) has the potential to increase drug solubility of hydrophobic compounds in LBFs. ${ }^{[36]}$ Ionic liquids were synthesised using bulky and highly lipophilic counter ions. In the case of itraconazole, a 72 -fold solubility increase with a dioctyl sulfosuccinate salt in a longchain LBF was observed. ${ }^{[36]}$ In addition to the improved solubility in the formulation, the lipophilic salt can also promote drug absorption in the gastrointestinal tract. These approaches therefore broaden the applicability of LBFs, but for future market formulations, the toxicological and regulatory acceptability of the used counter ions must be assured. ${ }^{[37]}$

An alternative strategy is to employ lipophilic prodrugs to improve lipophilicity. While the use of lipophilic prodrug strategies is more commonly employed to improve biopharmaceutical properties such as increased intestinal permeability and lymphatic transport, the increased lipophilicity is often favourable regarding solubility in LBF. ${ }^{[3]}$ A study with a range of prodrugs for an anticancer drug (SN38) showed a significant increase in solubility in Soybean oil of 11 - 444 -fold. ${ }^{[38]}$ However, increasing lipophilicity via a prodrug approach also introduces risks to altered stability and pharmacokinetics, which must be adequately assessed. For example, while a TG ester prodrug of mycophenolic acid showed significant improvement in lymphatic transport and drug concentrations in lymphocytes, an alkyl ester prodrug showed poor metabolic stability and low permeability. ${ }^{[39]}$

While much focus to date has been on the use of lipid solutions as a bio-enabling strategy for challenging drugs, it has been reported that lipid suspensions may confer similar advantages in terms of improved biopharmaceutica performance. ${ }^{[40,41]}$ Thomas et al. ${ }^{[34]}$ reported, for example, that a lipid suspension of fenofibrate displayed similar oral bioavailability to a comparable lipid solution. However, the number of studies employing lipid suspension is relatively few and further studies are needed to assess reliability and reproducibility of this formulation approach.

In summary, lattice energy and lipophilicity considerations are important for the choice of the lipid delivery approach and for the selection of excipients. While $T_{\mathrm{m}}$ has proven useful in predicting lipid solubility of drugs, $\log P$ does not generally represent a reliable quantitative predictor of solubility. Therefore, the hydrophobic, or solid-state properties of a drug, appear to be crucial relative to their lipophilicity. The presented analysis of drugs marketed as LBFs suggests that poor hydrophobicity alone may not limit commercial development. There are a number of emerging formulation approaches such as ionic liquids, lipophilic prodrugs or lipid suspensions that may offer promise in addressing unfavourable hydrophobicity characteristics. Future work may provide more complex structural and physicochemical factors for guiding the design and development of LBFs.

\section{Amorphous solid dispersions}

The formulation approach of amorphous solid dispersion (ASD) was introduced by Chiou and Riegelmann in $1969^{[42]}$ because of an increasing number of poorly water-soluble compounds that required a new formulation perspective. Since then, this approach has been common practise for solubility enhancement of hydrophobic or lipophilic drugs. ${ }^{[43]}$

From a thermodynamic viewpoint, an amorphous state has generally a higher Gibbs free energy than a crystal. Therefore, an amorphous state is either labile or metastable with a considerable risk of crystallisation in non-stabilised ASDs.

Figure 7 emphasises the differences in Gibbs Energy between amorphous and crystalline material. ${ }^{[4]}$ Moreover, it shows the changes in this energy compared with increasing temperature, going from an amorphous state to the final transition in the liquid molten state, respectively. The two temperatures in Figure 7 mark the transition of an amorphous to the crystalline state. The glass transition temperature $\left(T_{g}\right)$ represents the alteration between an amorphous state and a supercooled liquid. The other change in the solid state can be observed at $T_{\mathrm{m}}$, when the crystals melt and become liquid.

Furthermore, the difference in Gibbs free energy shown in Figure 7 also results in an enhanced apparent solubility compared to the crystalline form. ${ }^{[45]}$ This so-called amorphous solubility advantage was coined by Hancock and Parks, who associated the difference in free energy with the 


\section{Thesis Publications}

difference in apparent solubility according to Equation $4 .^{[46]}$

$$
\Delta G_{T}^{a, c}=-R T \ln \left(\frac{\sigma_{T}^{a}}{\sigma_{T}^{c}}\right)
$$

The different solubilities of the amorphous and the crystalline state at a fixed temperature are represented by $\sigma_{T}^{a}$ and $\sigma_{T}^{c}$, respectively. Consequently, $\Delta G_{T}^{a, c}$ is the difference in free energy at a given temperature. A higher difference in free energy can be associated with a larger solubility advantage of the amorphous form over the crystalline form. Such increased apparent solubility has to be balanced with the general drawback of potential physical instability of an amorphous state. A possible crystallisation to the original crystal form or any other polymorph has to be monitored by adequate solid-state analysis.

Due to the high relevance of identification and quantification of amorphous drug in a solid dispersion, there are several methods commonly applied. A widely used tech nique is X-ray powder diffraction (XRPD), where an amorphous form shows a halo and absence of any Bragg peaks. ${ }^{[4]}$ This result underlines the lack of long-range order in the solid state. ${ }^{[48]}$ Another common method is differential scanning calorimetry (DSC), where the absence of a melting endotherm and the presence of a $T_{\mathrm{g}}$ indicate an amorphous state. The spectrum of further characterisation methods is very broad including also spectroscopic methods such as Raman or infrared spectroscopy as well as solid-state nuclear magnetic resonance spectroscopy. However, XRPD and DSC are still the most common methods,

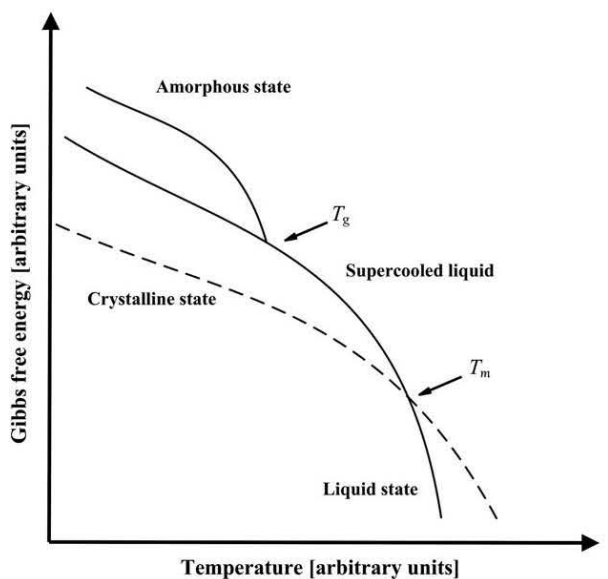

Figure 7 Gibbs Free Energy of amorphous and crystalline materia. Adapted from Hancock and Shamblin. ${ }^{[44]}$ because of their rather simple handling and good reproducibility.

Solid dispersions can be categorised according to the physical state of the given phases. ${ }^{[49]}$ The first dispersions were often eutectic mixtures, which were miscible in the molten state. A disadvantage of the eutectic systems is that recrystallisation occurs at the characteristic eutectic temperature, which typically takes place during the cooling process. Pioneer solid dispersions were prepared with a water-soluble carrier such as citric acid, and a poorly water-soluble drug (e.g. griseofulvin). ${ }^{[42]}$ Depending on the individual composition, it is possible to obtain an amorphous solid solution, where a compound is dispersed molecularly in the amorphous carrier. ${ }^{[50]}$ Leuner and Dressman pointed out that solid solutions can be continuous vs discontinuous or substitutional vs interstitial. Systems with an amorphous carrier are generally called glasses where glass solutions can be differentiated from glass suspensions depending on the physical state of the drug and whether one or two phases are present in the system. ${ }^{[50]}$

As ASDs have a long tradition, different generations of formulation types have been in use. Those different generations were described in detail by Vo et al. ${ }^{[51]}$ Main differences are given in the types of excipients selected during the pharmaceutical development of solid dispersions (Figwre 8).

In the first generation of solid dispersions, crystalline carriers (i.e. mostly small molecular additives) were used for dispersing the drug homogeneously in the solid state, which had the disadvantage that a rather fast drug precipitation upon aqueous dispersion was often observed. Therefore, a second generation of improved formulations was proposed that were based on polymeric carriers, which were advantageous regarding the biopharmaceutical fate of the drug. Such ASDs typically showed a dissolution rate that was widely controlled by the hydration and dissolution of the polymeric matrix. ${ }^{[52]}$ The third-generation solid dispersions consisted mainly of polymeric carriers combined either with each other or with surfactants to improve the aqueous dispersion following oral administration. Interesting is here a combined functionality such as, for example, the BASF polymer Soluplus, which represents a polymer (polyvinyl caprolactam-polyvinyl acetate-polyethylene glycol graft copolymer) with significant amphiphilic characteristics of a surfactant.

The production of the formulations mentioned above can be mainly divided into melt-based and solvent-based methods. ${ }^{[45]}$ This is critical as the preparation has a substantial effect on the physicochemical characteristics, stability and therefore performance of ASDs. ${ }^{[53]}$ Considering the marketed solid dispersions, it is interesting to see that a rather limited number of polymeric carriers and 


\section{Thesis Publications}

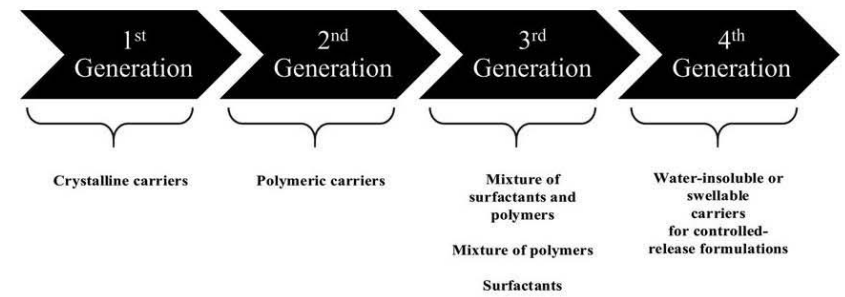

Figure 8 Different generations of solid dispersions. Adapted from Vo et at. ${ }^{[51]}$

production techniques have been used. ${ }^{[54]}$ While the choice of the formulation components is generally based on physical and chemical considerations and long series of experiments during development, the production methods are often more arbitrarily selected depending on available technological knowledge and equipment. ${ }^{[55]}$ Selection of the manufacturing method based upon the physicochemical drug properties is therefore desirable. Such an approach also may accelerate process development and should finally result in a robust manufacturing of drug product.

Different melt and fusion techniques represent the classical methods to prepare ASDs. ${ }^{[55,56]}$ For the melting of the API and carrier, temperatures should be above the $T_{\mathrm{m}}$ of the API ${ }^{[57]}$ Raising the temperature above the $T_{\mathrm{g}}$ of the mixture creates adequate molecular mobility for the API to be incorporated in the carrier. ${ }^{[5]}$ Although a variety of method modifications have been introduced throughout the years, ASDs with APIs presenting high $T_{\mathrm{m}}$ values (e.g. quercetin) typically encounter issues of lacking temperature stability of the carrier. These high-melting APIs therefore only have a limited range of available polymers that can be used at the needed process temperatures. Moreover, high shear forces in a process of hot melt extrusion may facilitate, besides the vigorous mixing and the desirable dispersion of the API in the carrier, the removal of oxygen and moisture. ${ }^{[51,58]}$ This enables the incorporation and usage of APIs that are sensitive to oxidation. However, high shear forces may also compromise the stability of thermo-sensitive APIs, due to possible local high temperatures. ${ }^{[58]}$

An alternative to any melt method is to prepare a solution of drug and carrier in a solvent. The fate of the solution may vary, from solvent evaporation to amorphous precipitation. The solvent evaporation method include first the dissolution of API and carrier in a common organic solvent (or solvent mixture) and the subsequent removal of the solvent by heating, spray drying or freezedrying. ${ }^{[59]}$ The choice of a common solvent for the APIcarrier systems may prove to be limiting, as it is challenging to identify a solvent for combinations that vary significantly in polarity. ${ }^{[59]}$ Generally, thermal degradation is not a common limitation in the solvent evaporation methods, as temperatures are kept low. Specifically for thermo-labile compounds, a freeze-drying method is of interest, where the API-carrier solution is frozen and the solvent or solvent mixture is sublimated at temperatures below the $T_{\mathrm{g}}$ of the mixture. ${ }^{[55]}$ A sublimation above this critical temperature comes with increased molecular mobility that can facilitate recrystallisation. Consequently, APIs with extremely low $T_{\mathrm{g}}$ 's may not be suitable for this method. In addition, during the removal of the solvent by heating, molecular mobility is critical, as elevated temperatures (above $T_{g}$ ) may facilitate API diffusion from the carrier, thereby creating a phase separation and subsequent crystallisation. This suggests that this method may be less suitable for APIs with a $T_{g}$ below the boiling point of common organic solvents (e.g. methanol, ethanol, acetone $\sim 60-70{ }^{\circ} \mathrm{C}$ ).

It seems that API lipophilicity has barely been investigated for its effect on different ASD manufacturing methods. Due to the fact that solubility is governed either by lipophilicity $(\log P$ ) or by the crystal lattice energy (hydrophobicity), ${ }^{[14]}$ it is expected that these molecular properties are relevant for selection and manufacturing of solid dispersions. Finally, the ratio of $T_{\mathrm{m}} / T_{\mathrm{g}}$ merits consideration because it is an indicator of glass-forming ability that can again be related to molar volume and $\Delta S_{f}{ }^{[60]}$ The latter entropy change can be estimated from chemical structure. Such in silico estimations of glass-forming ability are of high interest, not only for drug discovery, but also in development because $T_{g}$ is not easy to measure with fast crystallizing compounds. ${ }^{[60]}$

In line with these considerations of solid dispersion developability, Friesen et al. introduced the classification of several drugs according to the ratio of $T_{\mathrm{m}}$ and $T_{\mathrm{g}}$ plotted against $\log P$. Different groups of active substances were introduced. ${ }^{[61]}$ For drugs with $T_{\mathrm{m}} / T_{\mathrm{g}}$ ratio of maximum 1.25 (group 1), it was considered possible to form an amorphous state. Group 2 with $T_{\mathrm{m}} / T_{\mathrm{g}}$ ratios of $1.25-1.40$ was estimated to have a limited drug load of $35-50 \%(\mathrm{w} / \mathrm{w})$ due to lower amorphous stability. Most challenging compounds in terms of stability exhibit a $T_{\mathrm{m}} / T_{\mathrm{g}}$ range of higher 


\section{Thesis Publications}

than 1.4 and consequently, a drug load of maximum 35\% $(w / w)$ was proposed. These drugs with a $\log P$ smaller than 6 were assigned to group 3, whereas all other lipophilic drugs with $\log P$ higher than 6 were viewed as generally problematic regarding dissolution and assigned into group 4 regardless of their $T_{\mathrm{m}} / T_{\mathrm{g}}$ ratios.

For this review, the described categorisation approach was applied to drugs for which formation of a stable ASD was reported in the literature. ${ }^{[60,62,63]}$ The distribution of the MW, the $\log P$ and $T_{\mathrm{m}}$ values of these drugs are displayed in Figure 9

Figure 9a suggests that most substances successfully formulated in an amorphous state have a MW between 200 and $400 \mathrm{~g} / \mathrm{mol}$. This is in accordance with the publication of Edueng et al..$^{[5]}$ and Mahlin et al. ${ }^{[64]}$ who also found a limited occurrence of very small molecules, as MWs above $300 \mathrm{~g} / \mathrm{mol}$ are more optimal for good glass-forming ability The pie chart (Figure 9b) of the $\log P$ value reveals a broad distribution with a lowest fraction of highly lipophilic drugs. Furthermore, $T_{\mathrm{m}}$ (Figure $9 \mathrm{c}$ ) is mostly between 400 and $500 \mathrm{~K}$. These distributions of physicochemical properties cannot only be explained by ASD rationales as they are likely biased by the natural occurrence of $\mathrm{MW}, \log P$ and $T_{\mathrm{m}}$ among available drugs.

A novelty compared to the data gathered by Friesen et al. ${ }^{[61]}$ is the inclusion of melt-based amorphisation techniques. If only the subset of marketed drugs in amorphous products is considered, which are not included in Fig ure 10 , a challenging $T_{\mathrm{m}} / T_{\mathrm{g}}>1.4$ was only observed with etravirine and vemurafenib. ${ }^{[65]}$ This can be compared to glass-forming ability, which can be determined by means of a DSC method. ${ }^{[62]}$ Vemurafenib was verified as a fast crystallizing compound, while this is hard to demonstrate for etravirine due to thermal decomposition. ${ }^{[54]}$ Interestingly, etravirine as well as vemurafenib are manufactured in their market products by a solvent-based method. Apart from these drugs, other market compounds are in group 4 due to their high $\log P>6$, which is the case for itraconazole and everolimus. While Figure 10 shows the compounds found in scientific publications, it emphasises that more melt-based manufacturing methods have been reported in group 4. However, there are also reports on drugs for which melt-based as well as solvent-based techniques were used. Also in group 3, where the majority of the compounds is located, there is not a clear dominance of either the melt or solvent-based method, so clear trends of a preferred method are missing. There is also currently no established molecular rationale for the selection of a manufacturing method and this choice is greatly influenced by the available expertise of individual formulation scientists.

For a better evaluation of hydrophobicity and lipophilicity influences, the graph was modified to show $T_{\mathrm{m}}$ vs $\log P$ (Figure 11). Furthermore, the graph represents the drugs differentiated by their amorphisation method in accordance with Figure 10

Figure 11 indicates that melt-based methods were primarily used in research for compounds with $\log P>2$. Moreover, there are not many substances that have a $T_{m}>500 \mathrm{~K}$ and substances of $\log P>6$. The distribution of data points shows an accumulation between $\log P$ values of 2 and about 5, which may not be specific for the selection of solid dispersion technology, but also reflects the natural distribution of drug lipophilicity for the compounds studied.

In summary, Figure 11 as a plot of lipophilic and hydrophobic drugs does not reveal pronounced tendencies with respect to the process technique. It seems that melt-based methods are limited regarding high $T_{m}$, because of possible degradation issues of drug and excipients. However, also a solvation step can be clearly limited by excessive hydrophobicity. A more recently introduced solvent-based method is the microprecipitated bulk powder, where amorphous coprecipitation occurs with a polymer, which is based on ionisable groups to facilitate drug-excipient interactions. ${ }^{[66]}$ This broadens the applicability of ASDs for APIs with high $T_{m}$ values. Currently, there is only one drug (i.e. vemurafenib) formulated in this way on the market, but this can change in the future. At least for ionisable compounds, the possibilities for
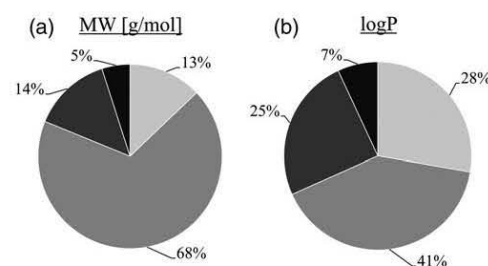

$1=<200=200-400=400-500=500 \quad=<2 \quad=2-4=4-6=>6$

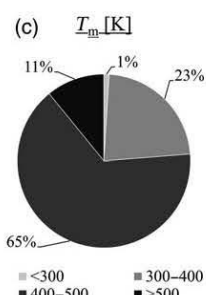

Figure 9 Properties of APIs formulated aS ASD. The different panels are explained in the text. API, active pharmaceutical ingredient; ASD, amorphous solid dispersion. 


\section{$\mathrm{X}$. Thesis Publications}

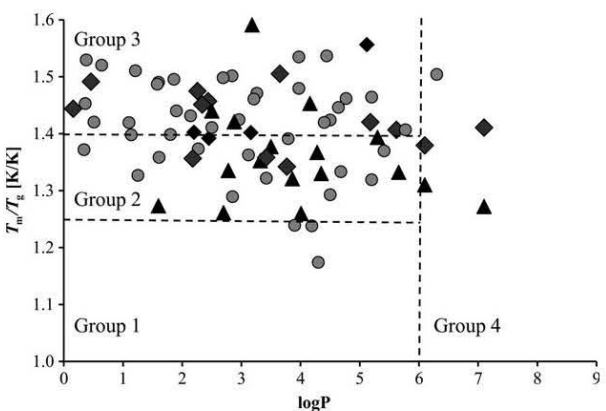

Figure 10 APIs gathered from the literature ${ }_{i}^{[60,62,63]}$ for which solid dispersions were successfully formulated, labelled by their amorphisation method. Diamonds represent melt-based amorphisation. Dot stand for sovent-based amorphisation and triangles are drugs that were formulated by both approaches. The lines indicate the different groups according to Friesen et al. ${ }^{[61]} \mathrm{AP}$, active pharmaceutical ingredient.

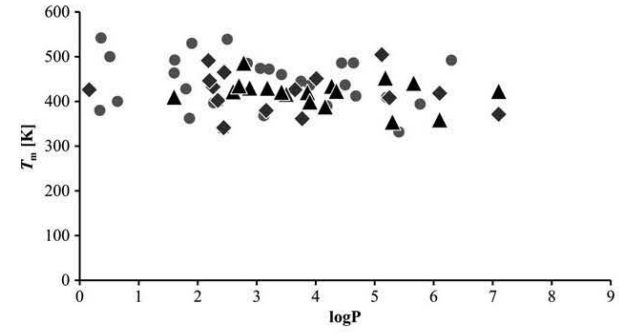

Figure 11 Scatter plot of drug melting temperature vs the log $P$ values for further evaluation of hydrophobicity (data taken from the literature $\left.{ }^{[60.62 .63]}\right)$. The colours and makers are analogues to Figure 10 .

solvent-based approaches can be broadened for compounds with very high $T_{\mathrm{m}}$ values.

This section approached amorphous systems from the viewpoint of hydrophobicity and lipophilicity, but further molecular aspects are relevant for the different quality attributes of amorphous systems. This has become a developing field of research and more information on molecular descriptors applicable to ASD attributes can be inferred from recent publications. ${ }^{[5,67]}$

\section{Mesoporous silica formulations}

Mesoporous silica refers to any number of a variety of materials synthesised to produce a $\mathrm{SiO}_{2}$ mesoporous structure. ${ }^{[68]}$ Mesoporous silica can be ordered or nonordered. ${ }^{[69,70]}$ The former include structures such as SBA-
15 and MCM-41, ${ }^{[71]}$ whilst the latter include novel, proprietary excipients manufactured by drug delivery specialists, such as the excipients Parteck SLC (Merck Millipore) ${ }^{[72,73]}$ and Neusilin (Fuji Chemical). ${ }^{[74]}$ Such systems have a wide range of applications, including: tissue engineering, ${ }^{[75]}$ catalysis, ${ }^{[76]}$ chromatography, ${ }^{[77]}$ adsorbents in environmental modelling ${ }^{[78]}$ and drug delivery systems for poorly soluble APIs. ${ }^{[79]}$ For the latter, it has been widely reported that mesoporous silica can act as a solubility enhancer by 'trapping' API in non-crystalline form within the mesoporous network. ${ }^{[72,73,80}$ 83]

For this purpose, there are various methods of loading crystalline API onto mesoporous silica, which can be grouped into three broad categories: solvent-based, ${ }^{[72]}$ mechanical activation ${ }^{[84]}$ and vapour-mediated. ${ }^{[85]}$ A thorough overview of these loading methods of poorly soluble API onto mesoporous silica is beyond the scope of this review, but the interested reader is referred to a number of publications that provide more details on the different routes to API-loaded silica. $\left.{ }^{[68,84,86} 88\right]$

Although a rather broad variety of methods is present in the literature; the solvent-based approach is most commonly employed. This can be attributed to the poor loading efficiencies and time-consuming processes involved in the solvent-free loading methods such as melt and supercritical fluid-based processes. ${ }^{[84]}$ The solvent approaches can be grouped into two main categories: solvent impregnation and incipient wetness. During the solvent impregnation loading approach, API is dissolved in organic solvent and added to mesoporous silica. Adsorption of API onto the silica is then initiated through mechanical agitation or sonication of the slurry. Finally, the solvent is removed, which can be achieved using a number of methods including vacuum drying, spray drying, lyophilisation or rotary evaporation. ${ }^{[68,72,89,90]}$ The second approach, incipient wetness, involves the steady addition of small volumes of concentrated API solution onto the silica, so the full amount of solvent is adsorbed into the network and then rapidly evaporated, which leaves the API within the pores. ${ }^{[73,82]}$ Both methods result in an API-loaded silica, in which the previously crystalline API is now amorphous or molecularly dispersed. This can be confirmed with analytical methods such as DSC or XRPD.

In general, the theoretical maximum drug content that can be loaded onto mesoporous silica is dependent on the surface area, ${ }^{[91]}$ pore volume ${ }^{[92]}$ and pore geometry of the silica ${ }^{[93]}$ with values as high as $75 \%$ reported in the literature. ${ }^{[89]}$ However, such very high loads are experimentally barely achievable and residual crystallinity is often observed in drug-loads exceeding $50 \%(\mathrm{w} / \mathrm{w}) .{ }^{[89]}$ Most cases of APIloaded silica in the literature do not exceed $40 \%$ loading.

The loading process can be considered from an energetic perspective of drug adsorption onto the carrier, but there is 


\section{Thesis Publications}

currently insufficient information available. Some hypotheses include the following: hydrogen bond interactions, ${ }^{[94,95}$ hydrophobic interactions, ${ }^{[83]}$ capillary action ${ }^{[89]}$ and ionic interactions, ${ }^{[96,97]}$ although the latter depends on the silanol groups on the surface of the silica to be deprotonated and negative.

One of the potential benefits of mesoporous silica relative to alternative amorphous formulations is the high stability that is achievable. This is due to the very small environment of the mesoporous network (so-called nanoconfinement) ${ }^{[98]}$ and complimentary interactions (yet to be fully resolved) with the silica surfaces, which lower the free energy of the system further. ${ }^{[99]}$ API-loaded silica can often be stored in open containers and at elevated temperatures and pressures, although this can be API-dependent as well. For example, Muller and coworkers demonstrated stability of an amorphous form at ambient and accelerated conditions for 30 different formulations of API-loaded silica, exceeding the requirements for regulatory stability studies. ${ }^{[100]}$ This could be particularly used for compounds that have high tendency to recrystallise, e.g. low $T_{g}$, where otherwise classical solid dispersions may fail to provide stable formulations. ${ }^{[31,71,101]}$

Following dispersion in an aqueous medium, such as in the gastrointestinal tract, the API is displaced from the mesoporous network and has the potential to generate supersaturation. The release mechanism can be considered using diffusion kinetics. However, due to the complexity of the porous network and multiple phases, a more refined view will likely emerge in the future when more research has focused on the topic. Simplified, upon administration to an aqueous environment, there is a diffusion of water into the porous network via capillary action. This in turn solubilises the loaded API, which generates a concentration gradient between the inside of the silica and the outside medium. This gradient drives the release of API via diffusion along a concentration gradient, generating supersaturation and potentially increased absorption. ${ }^{\text {[83] }}$

Such a release behaviour can be approximated by classical matrix diffusion (Equation 5), which was proposed by Higuchi in $1961^{[102]}$ to describe API release from thin ointment films:

$$
M_{t}=A \sqrt{2 c_{\text {ini }} D c_{s} t},
$$

where $M_{t}$ is the amount of drug released from the inorganic carrier; $A$ is the surface area available for release; $c_{i n}$ is initial drug concentration in the carrier; $D$ the diffusion coefficient of drug in the insoluble carrier and $c_{\mathrm{s}}$ is the maximum (saturation) concentration of the drug in the carrier.

The approach can also be expanded to describe the release of any API adsorbed or entrapped within an inorganic and insoluble matrix into an external medium via diffusion. ${ }^{[103]}$

When one considers the above Higuchi equation, the release of API from mesoporous carriers can be described as a diffusion-driven process. Similarly, to loading efficiency, drug release is further dependent on pore surface area, pore volume and pore morphology. However, there have been a number of examples in the literature, where Higuchi diffusion kinetics successfully modelled release of for example, ibuprofen from mesoporous materials. ${ }^{[104}$ 106]

There is surely more research needed to more specifically model release from mesoporous materials and to better understand molecular interactions of the loaded API with the porous carrier.

After the compound is released, a high energy state of drug supersaturation is typically obtained. Therefore, mesoporous formulations are often coupled with so-called precipitation inhibitors such as polymers such as poly (vinylpyrrolidone), which should inhibit a potential crystallisation. This maintenance of initial drug supersaturation forms the basis of the parachute in the spring and parachute model first proposed by Guzman, ${ }^{[107]}$ and is more generally used in formulations that generate supersaturation.

Mesoporous silica is a relatively novel formulation option for poorly soluble drugs, with no examples of commercially available formulations so far, and limited proof of concept studies in man that are available in public domain. ${ }^{[108]}$ However, there has been a dramatic increase in the interest for mesoporous silica-based formulations in the past decade. A summary of drug properties reported in mesoporous silica formulations is shown in Figure 12.

For MW, there might exist a 'sweet spot'. For very small molecules, there is efficient and deep penetration within the porous network, but microcrystalline domains can rise (extra space for critical nuclei formation), ${ }^{[98]}$ while release can often occur too quickly (high risk of precipitation). ${ }^{[80]}$ On the other hand, molecules that are too large and bulky may have problems accessing the pores efficiently, with only impractically low loading efficiencies being obtainable. Another factor of loading efficiency using larger molecules is the pore geometry of the porous network, with open 3D pore structures such as Parteck SLC likely being more accessible than the classical ordered 2D mesoporous silica such as MCM-41. Therefore, in the case of MW, it makes sense that the majority of compounds investigated are in the 'middle ground' of 300-500 Da. Further investigation into very small $(<200 \mathrm{Da})$ and very large $(>500 \mathrm{Da})$ molecules would offer interesting insights based on future work Such additional work may study different quality attributes of amorphous systems. A previous study highlighted, for example, the importance of $\mathrm{MW}$ and $T_{\mathrm{m}}$ for the amorphous solubility advantage, which could be further investigated specifically for mesoporous systems. ${ }^{[109]}$ 


\section{Thesis Publications}
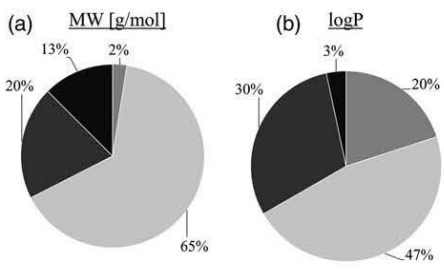

(c) $\underline{T}_{\underline{m}} / T_{\underline{g}}$
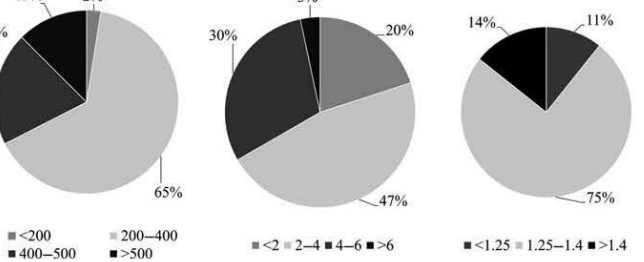

$\boldsymbol{-}<1.25=1.25-1.4 \mathbf{n}>1.4$

Figure 12 Properties of APIs formulated with mesoporous silica in the literature. The different panels are explained in the text. API, active pharmaceutical ingredient.

For $\log P$, and the hydrophobicity of the molecule, the retrospective analysis of reported mesoporous formulations is less clear. It is likely that the hydrophobicity of a molecule is of great importance in terms of with how it interacts with the surface of the mesoporous silica, and in turn how it is loaded and released. For example, not only polar but also dispersive interactions between API and the silica are expected to play an important role. However, in line with previous considerations of solid dispersions, a very high $\log P$ value is problematic with respect to drug dissolution. The $\log P$ data of mesoporous formulations show that most of the formulated poorly water-soluble compounds fall in the $\log P$ range of 3-5. Thus, in line with the previous comment surrounding MW, future studies on very lipophilic and hydrophobic molecules can only add to the growing understanding of mesoporous silica, and will significantly help towards creating a more rational selection criteria of the most 'suitable' candidates for this purpose.

Another parameter that can be considered is again the ratio $T_{\mathrm{m}} / T_{\mathrm{g}}$. A higher $T_{\mathrm{m}} / T_{\mathrm{g}}$ ratio indicates decreased glassforming ability and decreased stability, which comes with higher propensity for recrystallisation. ${ }^{[61]}$

Previously, it was highlighted that mesoporous silica can enhance shelf life stability via nano-confinement in combination with complimentary API-pore interactions, making them suitable even for fast crystallisers as critical compounds in class 3 and 4 of the Friesen plot mentioned above. However, it seems based on $T_{\mathrm{m}} / T_{\mathrm{g}}$ values that not many more of these difficult drugs have been formulated using mesoporous silica as compared to other solid dispersions. Thus, current literature trials with mesoporous silica have been carried out with the so-called 'usual suspects', that being the compounds that are known to work well with other amorphous formulations, such as those obtained by spray drying. This represents a gap in the literature More work should be carried out to identify a chemical space, where mesoporous silica has particular advantages compared to existing technologies, such as spray-dried dispersions and hot melt extrudates. It is possible that mesoporous silica could be an especially attractive formulation option for critical compounds with $T_{\mathrm{m}} / T_{\mathrm{g}}>1.4$. Further studies with such compounds would be helpful to map which technical option is the most promising to obtain an optimal amorphous system for a given drug linked to its physicochemical properties.

In line with the previous consideration of hydrophobicity (in terms of $T_{\mathrm{m}}$ ), Figure 14 highlights the observation that the commonly used APIs are interestingly confined to within a rather limited chemical space.

This leads to the initial impression from Figures 13 and 14 that, there is currently no pronounced difference to the other solid dispersion approaches even though mesoporous silica is an exciting prospect to add to the formulator's toolbox when considering poorly soluble API. As it stands, more work is required in key areas to better understand: the loading process, the interaction between API and porous surfaces and the release process. More research in this area will allow a more rational selection of mesoporous silica as a viable formulation option, based on a thorough understanding of the chemical drug space that is optimal for this formulation approach. This would avoid some unnecessary formulation screening and lead to a more focused drug development process. It is only when this understanding is established that there will be a shift away from the one-size-fits-all approach in terms of solid dispersions towards more tailored formulation techniques.

\section{Nanosuspensions}

The previous sections outlined the different strategies of how to solubilise poorly water-soluble drugs in the dosage form or how to convert their crystalline solid form, into an amorphous state. An alternative approach is to formulate such challenging drugs as nanosuspensions. ${ }^{[110]}$ This formulation technique is of particular interest when high crystal lattice energy is limiting the alternative solubilisation in, for example, LBF. This high lattice energy can be so pronounced that drug candidates might be neither soluble in 


\section{Thesis Publications}

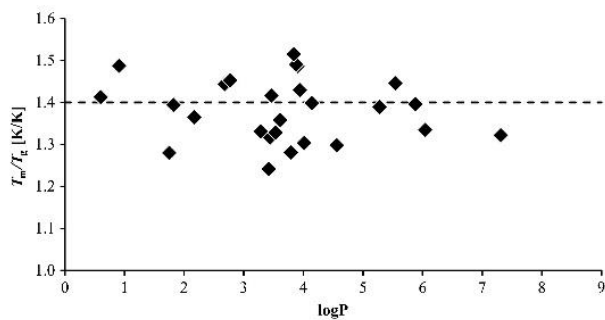

Figure $13 T_{\mathrm{m}} / T_{\mathrm{g}}$ vs $\log P$ for APIs formulated with mesoporous silica The dotted line indicates the fragileboundary, above with stability issues can be expected with the amorphous form. API, active pharmaceutical ingredient

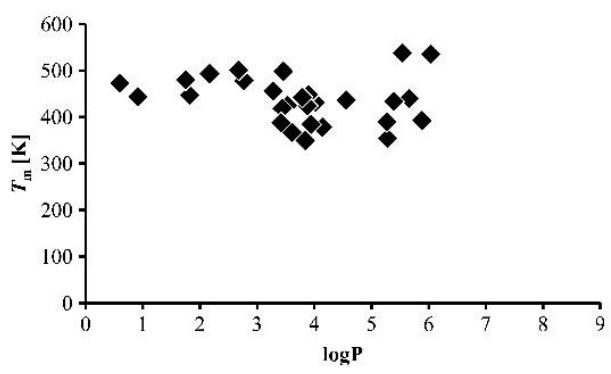

Figure $14 \log P$ as a function of $T_{\mathrm{m}}$ of compounds in formulations containing mesoporous silica.

polar nor apolar solvents or excipients. ${ }^{[111]}$ Nanosuspen sions can be formed by breaking larger micron-sized particles down (i.e. top-down approach) and stabilised by a mixture of polymer/surfactant, as in a wet milling tech nique. ${ }^{[12]}$ Several examples such as Rapamune (Wyeth), Emend (Merck), TriCor (Abbott Laboratorics) and Triglide (Skye Pharma) are already on the market. ${ }^{[1.3]}$ In carlie phases of drug development, nanosuspensions are often used to formulate poorly soluble compounds because high doses are administered in toxicological studies. It is here an advantage that wet milling is a rather versatile method to enable nanosuspension production with the majority of compounds. However, many of them do not achieve sufficient physical stability and therefore fail with their critical quality attributes of the nanosuspension. According to current literature, poorly soluble drugs with high $\mathrm{MW}$, high $T_{\mathrm{n}}$ values, and with a surface energy comparable to that of a given stabiliser, are able to form stable nanosuspensions. ${ }^{[14]}$ Specific interactions between the functional groups of stabilisers and drug particle surfaces in the stabilisation process have been demonstrated. ${ }^{[15]}$ However despite extensive efforts, most formulation scientists still evaluate their suspensions in a trial and error screening of stabilisers, even though some notable guidance has been reported by George et al. ${ }^{[16]}$ The main goal of their study was to correlate the characteristics of the drugs $(\log P$ and $\left.\Delta H_{f}\right)$ with feasibility of forming a stable nanosuspension. According to the data presented, the best candidates were drugs with a high $\Delta I I_{\Gamma}$ (or $T_{\mathrm{rn}}$ so high lattice energy) and high lipophilicity $(\log P)$, which can be stabilised either electrostatically or sterically. Thus, a wide range of stabilizing surfactants may result in stable nanosuspensions. On the other hand, drugs with comparatively low $\Delta H_{\mathrm{f}}$ (or $T_{\mathrm{m}}$ ) and rather low lipophilicity are poor candidates, irrespective of the stabiliser used. High $\Delta H_{\mathrm{f}}$ drugs with rather $\operatorname{low} \log P$ values may require an ionic surfactant as stabiliser. Drugs with lower lattice energies, which were more lipophilic (high $\log P$ ), were proposed to be stabilised by an ionic surfactant with rather high IILB value like sodium lauryl sulphate. This formulation guidance can provide some initial help but more research is needed to rationally design pharmaceutical nanosuspensions. Future research should address mechanisms of stabilisation. Until a more educated approach is available, it seems that the presented considerations of lipophilicity and lattice energy offer at least a starting point for nanosuspension development.

\section{Cyclodextrin formulations}

Enzymes belonging to the group cyclodextrin cycloglycosyltransferases (GCRase, EC 2.4.1.19) convert starch into cyclic oligosaccharides, known as cyclodextrins (CDs). These structures are composed of $\alpha$ - $(1,4)$ linked glucopyranose subunits. The $\alpha, \beta$, or $\gamma$ CDs, consisting of six, seven, or eight glucose units, respectively, are the most investigated CDs. ${ }^{[17,118]}$ The CD molecule has the shape of a torus due to the chair conformation of the glucopyranose units. Furthermore, the CD molecule has a spatial distribution of polar hydroxyl groups on the outer rim and apolar (relative to water) glucoside oxygens and hydrogens in the cavity. This is why the CD molecule has a hydrophilic outside and a hydrophobic cavity. $\Lambda$ s a consequence of this structure, CDs have the ability to form inclusion complexes through molecular encapsulation with a wide range of organic compounds. ${ }^{[17,118]}$ This special characteristic makes CDs valuable in a number of disciplines, including pharmaceutics, where the increased solubility of complexes can be used to increase the apparent solubility of poorly soluble drugs. ${ }^{119-1321}$

While natural $\alpha$ - and $\gamma \mathrm{CD}$ have good aqueous solubility, natural $\beta C D$ only has an aqueous solubility of $18.5 \mathrm{~g} / \mathrm{l}$, which is believed to be a reflection of the very rigid structure formed through $\mathrm{H}$-bonding of the C2-hydroxyl of one glucopyranose unit with the C-3 hydroxyl of an adjacent unit. ${ }^{[133]}$ In the $\beta C D$ molecule, a complete set of seven 


\section{$\mathrm{X}$. Thesis Publications}

intramolecular $\mathrm{H}$-bonds can be formed, effectively lowering the thermodynamic driving force for interaction with the solvent. ${ }^{[134]}$ Morcover, CDs can be chemically modified by e.g. hydroxylation, alkylation or sulfoalkylation, thereby breaking this belt of $\mathrm{H}$-bonds around the $\mathrm{CD}$ ring, which for $\beta C D$ increases the solubility significantly. Different chemically modified CDs with improved physical-chemical properties have been prepared and commercialised, including, 2-hydroxypropyl $\beta$-cyclodextrin (HP(3CD), 2-hydroxypropyl $\gamma$-cyclodextrin, methyl $\beta C D$ $(\mathrm{m} \beta \mathrm{CD})$, and sulfobutylether $\beta$-cyclodextrin. Due to the low toxicity of the CDs, they can be used for all administration routes (with the exception of parenteral applications of natural $\beta C D$ ). Kurkov and Loftsson recently made a comparative analysis of the more than 30 known CD containing commercial formulations across these administration routes and reported that the most frequently used $\mathrm{CD}$ was natural $\beta C D$ followed by HP $\beta C D .^{[13(1)]}$ In total, natural and modified $\beta C D$ s werc included in $83.4 \%$ of the formulations.

Cyclodextrins inclusion complexes are molecular complexes, which are formed by a reversible complexation that can be described by the following equilibrium;

$$
m \cdot D+n \cdot \mathrm{CD} \stackrel{K_{m: a}}{\rightleftharpoons} D_{m} \mathrm{CD}_{n}
$$

where $m$ is the number of drug $(D)$ molecules associated with $n$ molecules of CDs to form a complex with the stoichiometry of $m: n$ and $K_{m: n}$ is the stability constant. As the stoichiometry of the complex is often a $1: 1$ the stability constant can normally be written as;

$$
K=\frac{[D-\mathrm{CD}]}{[D] \cdot[\mathrm{CD}]}
$$

The total solubility in the presence of a CD is therefore the intrinsic solubility plus the drug fraction complexed with the CD. Across different routes of administration, the compounds included in commercial formulations containing CDs displayed an average MW of $369 \pm 130 \mathrm{~g} / \mathrm{mol}$. The distribution in MW was not associated with the type of CD used in the formulation, i.e. the lower MW molecules were not predominantly formulated with $\alpha \mathrm{CD}$ and the larget compounds with $\gamma \mathrm{CD}$. The $\log p$ of the molecules were $3.2 \pm 1.8$ with a distribution from -0.4 to 7.8 , see Figure $15 b$, with most of the compounds having a $\log P$ of $2-4$. This places the compounds used with CDs in the mid-range of the $\log P$ field, i.e. there is not a trend that this solubilising approach is used for the real grease balls among drug substances.

The compounds formulated with CDs had an average $T_{\mathrm{m}}$ of $436 \pm 82 \mathrm{~K}$ distributed from $194 \mathrm{~K}$ for nicotine to higher than $633 \mathrm{~K}$ for mitomycin, with most of the compounds having a $T_{\mathrm{m}}$ in the range of $473-573 \mathrm{~K}$.

When investigating $\log P$ as a function of $T_{\mathrm{m}}$ (Figure 16), it is clear that the compounds formulated with $\mathrm{CD}$ s tend to have a $\log \mathrm{P}$ from $2-4.5$ and a $T_{\mathrm{m}}$ between 473 and $573 \mathrm{~K}$. Based upon these data, compounds in the mid-range of the spectrum of these limits seem to provide most successful formulations with CDs.

The discriminating power between $\log P$ and the $T_{\mathrm{m}}$ revealed some value as a predictor for the useful application of CDs in a formulation. Important for the excipient functionality is the stability constant of inclusion complexation. ${ }^{[130]}$ The observed values for stability constants are usually reported to be between 50 to $2000 \mathrm{M}^{1}$. Therefore, an important element is also to define how the molecular usage of the CDs can be optimised, that is as high a

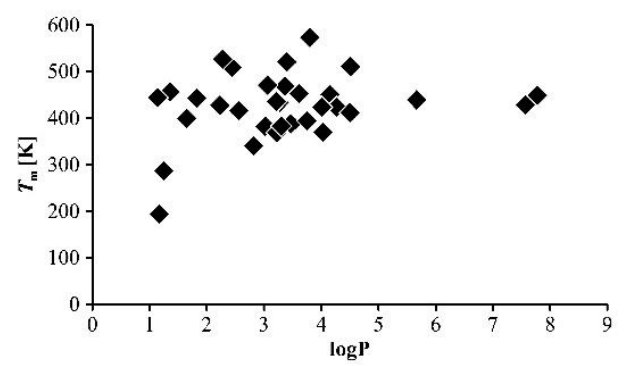

Figure $16 \log P$ as a function of $T_{\mathrm{m}}$ of compounds included in commercial formulations containing cyclodextrins.
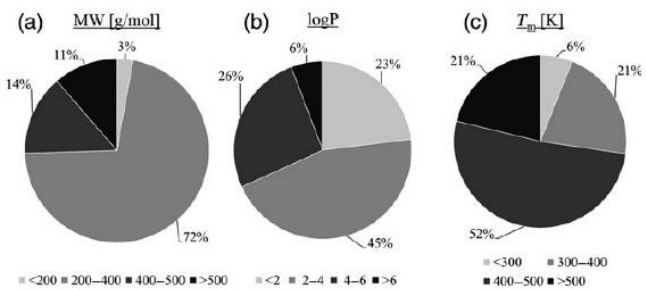

Figure 15 Properties of compounds included in commercial formulations containing cyclodextrins. The different panels are explained in the text. 


\section{Thesis Publications}

complexation efficacy as possible. ${ }^{[132]}$ As the complexation efficacy is defined as a function of the stability constant multiplied with the intrinsic aqueous solubility, different influences on these factors (e.g. ionisation of the drug, addition of cosolvents, addition of polymers, etc.) could be used to affect the complexation efficacy. ${ }^{[130]}$ When investigating the Yalkowsky equation presented in the theoretical section (Equation 3), it is clear that both $\log P$ and the $T_{\mathrm{m}}$ are part of the equation controlling the aqueous solubility. ${ }^{[11]}$ Consequently, molecules with a relatively low values of $\log P$ and $T_{\mathrm{m}}$ would naturally seem to be good candidates to obtain a high complexation efficacy in CDs. However Chari et al. ${ }^{[135]}$ concluded with a training set of 258 ligands that the most important molecular descriptor to describe the interaction with CDs was primarily $\log P$. Li et al..$^{[136]}$ reported the parameters of VAMP polarisation YY and VAMP dipole Y component, representing a semi-empirical molecular orbital package in the TSAR software, $\log P$, Balaban topological index, and cosmic electrostatic energy, as the five most important structural parameters influencing the binding constant between drugs and $\mathrm{BCD}$. Hence, there seems to be a general agreement that $\log P$ is a fair descriptor of potential interaction between drug molecules and CDs. Interestingly, $T_{\mathrm{m}}$ has not been mentioned in any of the studies focusing on molecular descriptors. It therefore seems that rather solvation-limited solubility than a high crystal energy of drug candidates would favour the choice of $\mathrm{CD}$ formulations. Moreover, geometric molecular fit requirements must be given drugs to achieve successful complexation with CDs. ${ }^{[137]}$

\section{Conclusions}

As lipophilicity is conventionally understood as a relative scale for given compounds, it should be clearly distinguished from hydrophobicity as a solubility limitation for poorly water-soluble drugs. The properties $T_{\mathrm{m}}$ (or $\Delta H_{\mathrm{f}}$ ) and $\log P$ have to be considered for the selection of promising bio-enabling oral formulation. It is for example reasonable to assume that very high $T_{\mathrm{m}}$ values can lead to issues of solubility in any solvent-based process and any LBF. While this can indeed give a first orientation about the formulation strategy, one should refrain from any absolute statements. Recent developments in LBF were discussed that may cope with quite hydrophobic drug bases. Also in the field of solid dispersions and mesoporous silica, a consideration of $T_{\mathrm{m}}$ or $T_{\mathrm{m}} / T_{\mathrm{g}}$ is important to guide initial experiments and to assess the stability of an amorphous state, but such simple approaches have their limits. It seems particularly difficult to obtain clear guidance on the selection of the manufacturing method based on available literature data. Novel approaches such as the mesoporous formulations or coprecipitated amorphous formulations are likely to become more important in the future. More work is also expected with respect to molecular modelling that can highlight effects of molecular size and specific interactions for example in cyclodextrin formulations or regarding stability in nanosuspensions. It can be expected that considerations of hydrophobicity and lipophilicity will also be of importance in the future but only for a rough assessment of the formulation strategy because more refined considerations require additional molecular properties for a rationale and structured development of bioenabling formulations.

\section{Declarations}

\section{Conflict of interest}

The authors declare that they have no conflict of interest to disclose.

\section{Funding}

This project has received funding from the European Union's Horizon 2020 Research and Innovation Programme under grant agreement No 674909 .

\section{References}

1. Porter CJH et al. Lipids and lipidbased formulations: optimizing the oral delivery of lipophilic drugs. $\mathrm{Nat}$ Rev Drug Discov 2007; 6: 231-248.

2. $\mathrm{Mu} \mathrm{H}$ et al. Lipid-based formulations for oral administration of poorly water-soluble drugs. Int $J$ Pharm 2013; 453: 215-224.

3. Feeney OM et al. 50 years of ora lipid-based formulations: provenance, progress and future perspectives. Adv Drug Deliv Rev 2016; 101: 167 194.

4. Savla $\mathrm{R}$ et al. Review and analysis of FDA approved drugs using lipidbased formulations. Drug Dev Ind Pharm 2017; 43: 1-16. https://doi. org/ $10.1080 / 03639045.2017 .1342654$.

5. Edueng $\mathrm{K}$ et al. The need for restructuring the disordered science of amorphous drug formulations. Pharm Res 2017; 34: 1754-1772.
6. Bergström CAS et al Poorly soluble marketed drugs display solvation linited solubility. J Med Chem 2007 50: 5858-5862.

7. Bergström CAS et al. Computationa prediction of formulation strategies for beyond-rule-of -5 compounds. Adv Drug Deliv Rev 2016; 101: 6-21.

8. Nordström FL, Rasmuson AC. Determination of the activity of a molecular solute in saturated solution. $J$ Chem Thermodyn 2008; 40: 1684-1692. 


\section{Thesis Publications}

9. Nordström FL, Rasmuson ÅC. Prediction of solubility curves and melting properties of organic and pharmaceutical compounds. Eur J Pharm Sci 2009; 36: 330-344.

10. Hildebrand J, Scott R. The Solubility of Nonelectrolytes. New York, NY: Reinhold, 1950.

11. Jain N, Yalkowsky SH. Estimation of the aqueous solubility I: application to organic nonelectrolytes. I Pharm Sci 2001; 90: 234-252.

12. Wassvik $\mathrm{CM}$ et al. Contribution of solid-state properties to the aqueous solubility of drugs. Eur I Pharm Sc 2006; 29(3-4 SPEC. ISS.): 294-305.

13. Elder D, Holm R. Aqueous solubility: simple predictive methods (in silico, in vitro and bio-relevant approaches). Int J Pharm 2013; 453: 3-11.

14. Wassvik CM et al. Molecular characteristics for solid-state limited solubility. I Med Chem 2008; 51: 3035-3039.

15. Abraham $\mathrm{MH}$ et al. Determination of olive oil-gas and hexadecane-gas partition coefficients, and calculation of the corresponding olive oil-water and hexadecane-water partition coefficients. I Chem Soc Perkin Trans 1987; 11. http://pubs.rsc.org/-/conte nt/articlepdf/1987/p2/p29870000797 (accessed 10 July 2017)

16. Abraham MH, Le J. The correlation and prediction of the solubility of compounds in water using an amended solvation energy relationship. J Pharm Sci 1999; 88: 868-880.

17. Niederquell A, Kuentz M. Biorelevant drug solubility enhancement modeled by a linear solvation energy relationship. I Pharm Sci 2017; 107: 503-506.

18. Lipinski $C A$ et al. Experimental and computational approaches to estimate solubility and permeability in drug discovery and development. Adv Drug Deliv Rev 2001; 46: 3-26.

19. Pouton CW. Lipid formulations for oral administration of drugs: nonemulsifying, self-emulsifying and "self-microemulsifying" drug delivery systems. Eur I Pharm Sci 2000; 11 (Suppl 2): S93-\$98.
20. Rane SS, Anderson BD. What determines drug solubility in lipid vehicles: is it predictable? Adv Drug Deliv $\operatorname{Rev} 2008$; 60: 638-656.

21. Persson LC et al. Computational prediction of drug solubility in lipid based formulation excipients. Pharm Res 2013; 30: 3225-3237.

22. Alskär LC et al. Tools for early prediction of drug loading in lipid-based formulations. Mol Pharm 2016; 13: 251-261.

23. Gautschi N et al. Rapid determination of drug solubilization versus supersaturation in natural and digested lipids. Int I Pharm 2016; 513: 164-174.

24. Constantinides PP, Wasan KM. Lipid formulation strategies for enhancing intestinal transport and absorption of P-glycoprotein (P-gp) substrate drugs: in vitro/in vivo case studies. $J$ Pharm Sci 2007; 96: 235-248.

25. Caliph SM et al. Effect of short-, medium-, and long-chain fatty acidbased vehicles on the absolute oral bioavailability and intestinal lymphatic transport of halofantrine and assessment of mass balance in lymph-cannulated and non-cannulated rats. I pharm Sci 2000; 89: 1073-1084.

26. Thi TD et al. Formulate-ability of ten compounds with different physicochemical profiles in SMEDDS. Eur J Pharm Sci 2009; 38: 479-488.

27. Pouton CW. Formulation of poorly water-soluble drugs for oral administration: physicochemical and physiological issues and the lipid formulation classification system. Eur J Pharm Sci 2006; 29: 278-287.

28. Dumanli I. Mechanistic studies to elucidate the role of lipid vehicles on solubility, formulation and bioavailability of poorly soluble compounds. Open Access Diss 2002 (Paper 171). http://digitalcommons.uri.edu/oa_d iss $/ 171$.

29. Hauss D. Oral Lipid-Based Formulations: Enhancing the Bioavailability of Poorly Water-Soluable Drugs (Drugs and the Pharmaceutical Sciences). Boca Raton: CRC Press, 2007.
30. Birru WA et al. Computational mod els of the gastrointestinal environment. 1. The effect of digestion on the phase behavior of intestinal fluids. Mol Pharm 2017; 14: 566-579.

31. Williams HD et al. Strategies to address low drug solubility in discov ery and development. Pharmacol Rev 2013; 65: 315-499.

32. Thomas $\mathrm{N}$ et al. In vitro and in vivo performance of novel supersaturated self-nanoemulsifying drug delivery systems (super-SNEDDS). I Control Release 2012; 160: 25-32.

33. Thomas $\mathrm{N}$ et al. Supersaturated selfnanoemulsifying drug delivery systems (Super-SNEDDS) enhance the bioavailability of the poorly watersoluble drug simvastatin in dogs. AAPS J 2013; 15: 219-227.

34. Thomas $\mathrm{N}$ et al. In vitro lipolysis data does not adequately predict the in vivo performance of lipid-based drug delivery systems containing fenofibrate. AAPS J 2014; 16: 539549.

35. Morgen $\mathrm{M}$ et al. Lipophilic salts of poorly soluble compounds to enable high-dose lipidic SEDDS formulations in crug discovery. Eur I Pharm Biopharm 2017; 117: 212-223.

36. Sahbaz $Y$ et al. Transformation of poorly water-soluble drugs into lipophilic ionic licuids enhances oral drug exposure from lipid based formulations. Mol Pharm 2015; 12: 1980-1991.

37. Williams $\mathrm{HD}$ et al. Transformation of biopharmaceutical classification system class I and III drugs into ionic liquids and lipophilic salts for enhanced developability using lipid formulations. J Pharm Sci 2017; 107: 203-216. https://oi.org/10.1016/j. xphs.2017.05.019.

38. Bala $\mathrm{V}$ et al. Lipophilic prodrugs of SN38: synthesis and in vitro characterization toward oral chemotherapy. Mol Pharm 2016; 13: 287-294.

39. Han $S$ et al. Targeted delivery of a model immunomodulator to the lymphatic system: comparison of alkyl ester versus triglyceride mimetic lipid prodrug strategies. I Control Release 2014; 177: 1-10. 


\section{Thesis Publications}

40. Larsen A et al. Lipid-based formulations for danazol containing a digestible surfactant, Labrafil M2125CS in vivo bioavailability and dynamic in vitro lipolysis. Pharm Res 2008 25: 2769-2777.

41. Dahan A, Hoffman A. The effect of different lipid based formulations on the oral absorption of lipophilic drugs: the ability of in vitro lipolysis and consecutive ex vivo intestinal permeability data to predict in vivo bioavailability in rats. Eur I Pharm Biopharm 2007; 67: 96-105.

42. Chiou WL, Riegelman S. Preparation and dissolution characteristics of several fast-release solid dispersions of griseofulvin. J Pharm Sci 1969; 58: 1505-1510.

43. Goldberg AH et al. Increasing dissolution rates and gastrointestinal absorption of drugs via solid solutions and eutectic mixtures II. J Pharm Sci 1966; 55: 482-487.

44. Hancock BC, Shamblin SL. Molecular mobility of amorphous pharmaceuticals determined usin differential scanning calorimetry. Thermochim Acta 2001; 380: 95-107.

45. Gupta $\mathrm{P}$ et al. Physical stability and solubility advantage from amorphous celecoxib: the role of thermodynamic quantities and molecular mobility. Mol Pharm 2004; 1: 406-413.

46. Hancock BC, Parks M. What is the true solubility advantage for amorphous pharmaceuticals? Pharm Res 2000; 17: 397-404.

47. Bragg WL. Diffraction of short electromagnetic waves by a crystal. Proc Camb Philos Soc 1913; 17: 43-57.

48. Klug HP, Alexander LE. X-Ray Diffraction Procedures for Polycrystalline and Amorphous Materials. Hoboken: John Wiley \& Sons, 1974

49. Chiou WL, Riegelman S. Pharmaceutical applications of solid dispersion systems. J Pharm Sci 1971; 60: 12811302.

50. Leuner C, Dressman J. Improving drug solubility for oral delivery using solid dispersions. Eur J Pharm Biopharm 2000; 50: 47-60.

51. Vo CL-N et al. Current trends and future perspectives of solid dispersions containing poorly watersoluble drugs. Eur J Pharm Biopharm 2013; 85: 799-813.

52. Chen $\mathrm{Y}$ et al. Initial drug dissolution from amorphous solid dispersions controlled by polymer dissolution and drug polymer interaction. Pharm Res 2016; 33: 2445-2458.

53. Patterson JE et al. Preparation of glass solutions of three poorly water soluble drugs by spray drying, melt extrusion and ball milling. Int Pharm 2007; 336: 22-34

54. Wyttenbach N, Kuentz M. Glassforming ability of compounds in marketed amorphous drug products. Eur J Pharm Biopharm 2017; 112: 204208.

55. Meng $\mathrm{F}$ et at. Classification of solic dispersions: correlation to (i) stability and solubility (ii) preparation and characterization techniques. Drug Dev Ind Pharm 2015; 41: 1401-1415.

56. Gurunath $S$ et al. Amorphous solid dispersion method for improving oral bioavailability of poorly watersoluble drugs. J Pharm Res 2013; 6: $476-480$.

57. Sekiguchi K, Obi N. Studies on absorption of eutectic mixture. I. A comparison of the behavior of eutec tic mixture of sulfathiazole and that of ordinary sulfathiazole in man Chem Pharm Bull (Tokyo) 1961; 9 : 866-872.

58. Repka MA et al. Melt extrusion with poorly soluble drugs - an integrated review. Int J Pharm 2018; 535: 68-85.

59. Paudel A et al. Manufacturing of solid dispersions of poorly water soluble drugs by spray drying: formulation and process considerations. Int $J$ Pharm 2013; 453: 253-284.

60. Wyttenbach $\mathrm{N}$ et al. Theoretical considerations of the prigogine-defay ratio with regard to the glass-formin ability of drugs from undercooled melts. Mol Pharm 2016; 13: 241-250.

61. Friesen DT et al. Hydroxypropyl methylcellulose acetate succinatebased spray-cried dispersions: an overview. Mot Pharm 2008; 5: 1003 1019.

62. Baird JA et al. A classification system to assess the crystallization tendency of organic molecules from uncercooled melts. J Pharm Sci 2010; 99: 3787-3806.

63. Alhalaweh A et al. Physical stability of drugs after storage above and below the glass transition temperature: relationship to glass-forming ability. Int J Pharm 2015; 495: 312317.

64. Mahlin D, Bergström CAS. Early drug development predictions of glass-forming ability and physical stability of drugs. Eur J Pharm Sci 2013; 49: 323-332.

65. Page $S$ et al. Structured development approach for amorphous systems. In: Williams RO III et al., eds. Formulating Poorly Water Soluble Drugs, Vol. 22. Basel: Springer International Publishing, 2016: 329-382.

66. Shah $\mathrm{N}$ et al. Development of novel microprecipitated bulk powder (MBP) technology for manufacturing stable amorphous formulations of poorly soluble drugs. Int $J$ pharm 2012; 438: 53-60.

67. DeBoyace K, Wildfong PLD. The application of modeling and prediction to the formation and stability of amorphous solid dispersions. J Pharm Sci 2018; 107: 57-74

68. Limnell $\mathrm{T}$ et al. Drug delivery formulations of ordered and nonordered mesoporous silica: comparison of three drug loading methods. J Pharm Sci 2011; 100: 3294-3306.

69. Barbé C et al. Silica particles: a novel drug-delivery system. Adv Mater 2004; 16: 1959-1966.

70. Kresge CT et al. Ordered mesoporous molecular sieves synthesized by a liquid-crystal template mechanism. Nature 1992; 359: 710-712.

71. Mellaerts $\mathrm{R}$ et al. Aging behavior of pharmaceutical formulations of itraconazole on SBA-15 ordered mesoporous silica carrier material. Microporous Mesoporous Mater 2010; 130: 154-161.

72. Lainé A-L et al. Enhanced oral delivery of celecoxib via the development of a supersaturable amorphous formulation utilising mesoporous silica and co-loaded HPMCAS. Int $J$ Pharm 2016; 512: 118-125. 


\section{Thesis Publications}

73. O'Shea IP et al. Mesoporous silicabased dosage forms improve bioavailability of poorly soluble drugs in pigs: case example fenofibrate. I Pharm Pharmacol 2017; 69: 1284-1292.

74. Khanfar M, Al-Nimry S. Stabilization and amorphization of lovastatin using different types of silica. AAPS PharmSciTech 2017; 18: 23582367.

75. Vallet-Regí M. Ordered mesoporous materials in the context of drug delivery systems and bone tissue engineering. Chemistry 2006; 12: 5934-5943.

76. Saad A et al. Triazole/triazine-functionalized mesoporous silica as a hybrid material support for palladium nanocatalyst. Langmuir 2017; 33: 7137-7146.

77. Majors RE. High-performance liquid chromatography on small particle silica gel. Anal Chem 1972; 44: 17221726.

78. Bhatnagar A, Sillanpää M. Utilization of agro-industrial and municipa waste materials as potential adsorbents for water treatment - a review. Chem Eng J 2010; 157: 277-296.

79. Wang S. Ordered mesoporous materials for drug delivery. Microporous Mesoporous Mater 2009; 117: 1-9.

80. Van Speybroeck $M$ et at. Enhanced absorption of the poorly soluble drug fenofibrate by tuning its release rate from ordered mesoporous silica. Eur J Pharm Sci 2010; 41: 623-630.

81. Vialpando $\mathrm{M}$ et al. Potential of ordered mesoporous silica for oral delivery of poorly soluble drugs. Ther Deliv 2011; 2: 1079-1091.

82. Dressman JB et al. Mesoporous silicabased dosage forms improve release characteristics of poorly soluble drugs: case example fenofibrate. I Pharm Pharmacol 2016; 68: 634-645.

83. Xu W et al. Mesoporous systems for poorly soluble drugs. Int $I$ pharm 2013; 453: 181-197.

84. Qian KK, Bogner RH. Spontaneous crystalline-to-amorphous phase transformation of organic or medicinal compounds in the presence of porous media, part 1 : thermodynamics of spontaneous amorphization. J Pharm Sci 2011; 100: 2801-2815.

85. Zhang $\mathrm{Z}$ et al. Loading amorphous Asarone in mesoporous silica SBA-15 through supercritical carbon dioxide technology to enhance dissolution and bioavailability. Eur J Pharm Biopharm 2015; 92: 28-31.

86. McCarthy CA et al. Mesoporous silica formulation strategies for drug dissolution enhancement: a review. Expert Opin Drug Deliv 2016; 13: 93-108.

87. Watanabe $\mathrm{T}$ et al. Solid state radical recombination and charge transfer across the boundary between indomethacin and silica under mechanical stress. I Solid State Chem 2002; 164: $27-33$.

88. Hillerström A et al. Solvent strategies for loading and release in mesoporous silica. Colloia Interface Sci Commun 2014; 3: 5-8.

89. Wei $Q$ et al. Oral hesperidin-amorphization and improved dissolution properties by controlled loading onto porous silica. Int J Pharm 2017; 518 : 253-263.

90. Meer $\mathrm{T}$ et al. Solubility modulation of bicalutamide using porous silica. J Pharm Investig 2013; 43: 279-285.

91. Vallet-Regí $M$ et al. Drug confinement and delivery in ceramic implants. Drug Metab Lett 2007; 1: 37-40.

92. Yang $\mathrm{Z}$ et al. Microstructure of an immiscible polymer blend and its stabilization effect on amorphous solid dispersions. Mol Pharm 2013; 10: $2767-2780$.

93. Heikkilä $\mathrm{T}$ et al. Evaluation of mesoporous TCPSi, MCM- 41 , SBA-15, and TUD-1 materials as API carriers for oral drug delivery. Drug Deliv 2007; 14: 337-347.

94. Wang F et al. Oxidized mesoporous silicon microparticles for improved oral delivery of poorly soluble drugs. Mol Pharm 2010; 7: 227-236.

95. Kinnari $\mathrm{P}$ et al. Comparison of mesoporous silicon and non-ordered mesoporous silica materials as drug carriers for itraconazole. Int $J$ Pharm 2011; 414: 148-156.
96. Atkin $\mathrm{R}$ et al. Mechanism of cationic surfactant adsorption at the solidaqueous interface. Adv Colloid Interface Sci 2003; 103: 219-304.

97. Turku I et al. Thermodynamics of tetracycline adsorption on silica. Environ Chem Lett 2007; 5: 225-228.

98. Sliwinska-Bartkowiak $M$ et al. Freezing behavior in porous glasses and MCM-41. Colloids Surf A Physicochem Eng Asp 2001; 187-188: 523529.

99. Azaïs T et al. Solid-state NMR study of ibuprofen confined in MCM-41 material. Chem Mater 2006; 18: 6382-6390.

100. Müller RH et al. CapsMorph: $>4$ years long-term stability of industrially feasible amorphous drug formulations. In: Int. Symp. Control. Rel. Bioact. Mater. 40. Honolulu/ Hawai, 2013.

101. Salonen I et al. Mesoporous silicon in drug delivery applications. I Pharm Sci 2008; 97: 632-653.

102. Higuchi T. Rate of release of medicaments from ointment bases containing drugs in suspension. J Pharm Sci 1961; 50: 874-875.

103. Siepmann I, Peppas NA. Higuchi equation: derivation, applications, use and misuse. Int $J$ Pharm 2011; 418: $6-12$.

104. Andersson $\mathrm{J}$ et al. Influences of material characteristics on ibuprofen drug loading and release profiles from ordered micro- and mesoporous silica matrices. Chem Mater 2004; 16: 4160-4167.

105. Horcajada $\mathrm{P}$ et al. Influence of pore size of MCM-41 matrices on drug delivery rate. Microporous Mesoporous Mater 2004; 68: 105-109.

106. Zhao $\mathrm{W}$ et al. Uniform rattle-type hollow magnetic mesoporous spheres as drug delivery carriers and their sustained-release property. Adv Funt Mater 2008; 18: 27802788.

107. Guzmán HR et al. Combined use of crystalline salt forms and precipitation inhibitors to improve oral absorption of celecoxib from solid oral formulations. J Pharm Sci 2007; 96: $2686-2702$. 


\section{Thesis Publications}

108. Bukara $\mathrm{K}$ et al. Ordered mesoporous silica to enhance the bioavailability of poorly water-soluble drugs: proof of concept in man. Eur J Pharm Biopharm 2016; 108: 220-225.

109. Kuentz M, Imanidis G. In silico prediction of the solubility advantage for amorphous drugs - are there property-based rules for drug discovery and early pharmaceutical development? Eur I Pharm Sci 2013; 48: 554562.

110. Chen $\mathrm{H}$ et al. Nanonization strategies for poorly water-soluble drugs. Drug Discov Today 2011; 16: 354-360.

111. Rabinow BE. Nanosuspensions in drug delivery. Nat Rev Drug Discov 2004; 3: 785-796.

112. Van Eerdenbrugh B et al. Top-down production of drug nanocrystals: nanosuspension stabilization, miniaturization and transformation into solid products. Int I Pharm 2008 364: 64-75.

113. Merisko-Liversidge E, Liversidge GG. Nanosizing for oral and parentera drug delivery: a perspective on for mulating poorly-water soluble compounds using wet media milling technology. Adv Drug Deliv Rev 2011; 63: 427-440.

114. Lee $\mathrm{J}$ et al. Characteristics of polymers enabling nano-comminution of water-insoluble drugs. Int $J$ Pharm 2008; 355: 328-336.

115. Lee MK et al. Hydrophilic and hydrophobic amino acid copolymers for nano-comminution of poorly soluble crugs. Int J Pharm 2010;384 173-180.

116. George M, Ghosh I. Identifying the correlation between drug/stabilizer properties and critical quality attributes (CQAs) of nanosuspension formulation prepared by wet media milling technology. Eur J Pharm Sci 2013; 48: 142-152.

117. Szejtli J. Cyclodextrin Technology. Dordrecht: Springer Netherlands, 1988. https://doi.org/10.1007/978-94015-7797-7.

118. Szejtli J. Chemistry, physical and bio logical properties of cyclodextrins. In: Szejtli J, Osa T, eds. Cyclodextri nes. Amsterdam: Elsevier Science Ltd., 1996: 189-204.

119. Carrier RL et al. The utility of cyclodextrins for enhancing oral bioavailability. I Control Release 2007; 123: 78-99.

120. Davis ME, Brewster ME. Cyclodextrin-based pharmaceutics: past, present and future. Nat Rev Drug Discov 2004; 3: 1023-1035.

121. Brewster ME, Loftsson T. Cyclodextrins as pharmaceutical solubilizers. Adv Drug Deliv Rev 2007; 59: 645-666.

122. Loftsson $\mathrm{T}$ et al Role of cyclodextrins in improving oral drug delivery Am J Drug Deliv 2004; 2: 261-275.

123. Szejtli J. Medicinal applications of cyclodextrins. Med Res Rev 1994; 14 353-386.

124. Rajewski RA, Stella VJ. Pharmaceutical applications of cyclodextrins. 2 . In vivo drug delivery. $I$ Pharm Sci 1996; 85: 1142-1169.

125. Uekama $\mathrm{K}$, Otagiri $\mathrm{M}$. Cyclodextrin in drug carrier systems. Crit Rev Ther Drug Carrier Syst 1987; 3: 1-40.

126. Challa $\mathrm{R}$ et al. Cyclodextrins in drug delivery: an updated review. AAPS PharmSciTech 2005; 6: E329-E357,

127. Arima $\mathrm{H}$ et al. Recent aspects of cyclodextrin-based pharmaceutical formulations. Recent Res Dev Chem Pharm Sci 2002; 2: 155-193.

128. Thompson DO. Cyclodextrins enabling excipients: their present and future use in pharmaceuticals. Crit
Rev Ther Drug Carrier Syst 1997; 14: $1-104$.

129. Uekama K. Design and evaluation of cyclodextrin-based drug formulation. Chem Pharm Bull (Tokyo) 2004; 52: 900-915.

130. Kurkov SV, Loftsson T. Cyclodextrins. Int J Pharm 2013; 453: $167-$ 180 .

131. Loftsson T, Brewster ME. Cyclodextrins as functional excipients: methods to enhance complexation efficiency. I Pharm Sci 2012; 101: 3019-3032.

132. Jambhekar SS, Breen P. Cyclodextrins in pharmaceutical formulations II: solubilization, binding constant, and complexation efficiency. Drug Discov Today 2016; 21: 363-368.

133. Saenger $W$ et al. Flip-flop hydrogen bonds in $\beta$-cyclodextrin - a generally valid principle in polysaccharides? Angew Chem Int Ed Engl 1983; 22: 883-884.

134. Naidoo KJ et al. Molecular properties related to the anomalous solubility of $\beta$-cyclodextrin. J Phys Chem $B$ 2004; 108: 4236-4238.

135. Chari $\mathrm{R}$ et al. Development of improved empirical models for estimating the binding constant of a beta-cyclodextrin inclusion complex. Pharm Res 2009; 26: 161-171.

136. Li $\mathrm{H}$ et al. Structure-based in silico model profiles the binding constant of poorly soluble drugs with $\beta$-cyclodextrin. Eur J Pharm Sci 2011; 42: 55-64.

137. Hazai $\mathrm{E}$ et al. Cyclodextrin KnowledgeBase a web-based service managing CD-ligand complexation data. J Comput Aided Mol Des 2010; 24: 713-717. 


\section{Application of the solubility parameter concept to assist with oral delivery of poorly water-soluble drugs - a PEARRL review}

Sandra Jankovic ${ }^{a, b}$ (D), Georgia Tsakiridou, ${ }^{c, d}$, Felix Ditzinger ${ }^{a, b}(D)$, Niklas J. Koehl ${ }^{e}$, Daniel J. Price ${ }^{f, g}$ (D), Alexandra-Roxana Ilie ${ }^{e, h}$, Lida Kalantzi ${ }^{c}$, Kristof Kimpe, René Holm ${ }^{h}$, Anita Nair ${ }^{f}$, Brendan Griffin ${ }^{e}$ (DD, Christoph Saal ${ }^{f}$ and Martin Kuentz ${ }^{b}$ (D)

${ }^{a}$ Department of Pharmaceutical Sciences, University of Basel, Basel, bInstitute of Pharma Technology, University of Applied Sciences and Arts Northwestern Switzerland, Muttenz, Switzerland, 'Pharmathen SA, Product Design \& Evaluation, ${ }^{d}$ Faculty of Pharmacy, National and Kapodistrian University of Athens, Athens, Greece, e'school of Pharmacy, University College Cork, Cork, Ireland, ${ }^{\mathrm{f}}$ Merck Group, Molecule Characterisation,

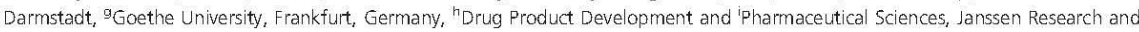
Development, Johnson \& Johnson, Beerse, Belgium

Keywords

enabling formulation; in silico prediction: poorly water-soluble drug; solubility parameter

\section{Correspondence}

Martin Kuentz, Institute of Pharma

Technology, University of Applied Sciences and Arts Northwestern Switzerland,

Gründenstrasse 40, 4132 Muttenz,

Switzerland.

E-mail: Martin.Kuentz@fhnw.ch

Received February 21, 2018

Accepted May 28, 2018

do: $10.1111 /$ jphp. 12948

\begin{abstract}
Objectives Solubility parameters have been used for decades in various scientific fields including pharmaceutics. It is, however, still a field of active research both on a conceptual and experimental level. This work addresses the need to review solubility parameter applications in pharmaceutics of poorly water-soluble drugs. Key findings An overview of the different experimental and calculation methods to determine solubility parameters is provided, which covers from classical to modern approaches. In the pharmaceutical field, solubility parameters are primarily used to guide organic solvent selection, cocrystals and salt screening, lipid-based delivery, solid dispersions and nano- or microparticulate drug delivery systems. Solubility parameters have been applied for a quantitative assessment of mixtures, or they are simply used to rank excipients for a given drug.

Summary In particular, partial solubility parameters hold great promise for aiding the development of poorly soluble drug delivery systems. This is particularly true in early-stage development, where compound availability and resources are limited. The experimental determination of solubility parameters has its merits despite being rather labour-intensive because further data can be used to continuously improve in silico predictions. Such improvements will ensure that solubility parameters will also in future guide scientists in finding suitable drug formulations.
\end{abstract}

\section{Introduction}

Solubility parameters have received much attention and numerous applications have been reported in diverse scientific fields. ${ }^{[1]}$ Pharmaceutics has been a prime discipline for applying solubility parameters to formulation design. Previous studies have, for example, reported the use of solubility parameters to predict suitable solvents for solutes, select polymer blends, and to describe surface and adhesion phenomena. ${ }^{[1]}$ It would be interesting to have an overview of such pharmaceutical applications with a particular emphasis on the development of poorly soluble drug formulations.
Development of new formulations requires the use of different tools to predict and analyse the physiochemical properties and interactions of dosage form components. $^{[2,3]}$ For prediction of material properties and their interactions, for example, solubility parameters are routinely used with high levels of success. ${ }^{[2]}$ Historically, this strategy has been employed in drug development for the selection of solvents for coating. Since then, further applications, as well as more robust thermodynamic methods for solubility parameter calculations, have also been reported. Specifically, such thermodynamic methods can be used for study and 


\section{$\mathrm{X}$. Thesis Publications}

prediction of the physical and chemical properties of compounds together with their effects on mixtures and dosage forms. ${ }^{[2]}$

The definition of solubility parameter was first coined by Hildebrand and Scott in $1950 .{ }^{[1]}$ An important theoretical development was then proposed later in 1967 with introduction of the so-called Hansen solubility parameter (HSP). ${ }^{[1]}$ This concept divides the total solubility parameter $\left(\delta_{t}\right)$ into three different contributions: polar, nonpolar and hydrogen bonding, and it is therefore more versatile than the original one-dimensional solubility parameter defined by Hildebrand, which does not account for these specific contributions. ${ }^{[2]}$

Solubility parameters can be derived experimentally from heat of vaporisation, internal pressures, surface tensions and other material characteristics as outlined by Hildebrand and Scott. ${ }^{[4]}$ Since then, a number of researchers have reported new methods to more accurately predict solubility parameter values, considering, for example, purely acidic or basis compounds ${ }^{[5]}$ for a specific process technique. ${ }^{[6]}$ More recent predictions of solubility parameters rely on molecular dynamics simulations or on the conductor-like screening model (COSMO), and these computational methods have been compared by Diaz et al. ${ }^{[7]}$ Important in this context is the research of Panayiotou and coworkers who contributed to the theoretical concept of solubility parameter and proposed a modern quantitative structure-property relationship. ${ }^{[8]}$ Furthermore the recent article by Louwerse et al broadly summarised limitations of the solubility parameter concept and proposed theoretical improvements. ${ }^{[9]}$

Previously, various authors ${ }^{[2,10,11]}$ have shown the practical importance of solubility parameters, but a general overview of their applications in the pharmaceutical field is missing. This article addresses the particular need to review the use of solubility parameters in pharmaceutics with respect to formulation of poorly soluble drugs. The latter oral delivery systems are central to the PEARRL research consortium that is deals with the design of such formulations and new tools for their biopharmaceutical assessment.

\section{Theory and experimental aspects of the solubility parameter concept}

\section{Introduction to the solubility parameter concept}

The principle of 'like dissolves like' is a well-known term within chemical and pharmaceutical sciences, which can be more generally described as 'like seeks like'. The usefulness of such approaches depends of course on the ability to assign a numerical value to molecular similarity or dissimilarity. Such a quantitative number is provided by the solubility parameter, which is a rather simple but very powerful approach.

For a better understanding of the solubility parameter concept, it is helpful to discuss its historical origins that are linked to the theory of nonideal solutions. For such solutions, the activity of a solute, $\alpha_{2}$, is the product of the concentration $X_{2}$ (mole fraction) and the activity coefficient, $\gamma_{2}$ (Equation 1):

$$
\alpha_{2}=X_{2} \gamma_{2}
$$

It is a central task of thermodynamic theories to predict $\gamma_{2}$ and a classical approach is that by Scatchard ${ }^{[12]}$ and by Hildebrand and Wood: ${ }^{[13]}$

$$
\ln \left(\gamma_{2}\right)=\left(w_{22}+w_{11}-2 w_{12}\right) \frac{V_{2} \varphi_{1}^{2}}{R T}
$$

where $V_{2}$ is the volume of the solute and $\varphi_{1}$ is the volume fraction of the solvent and $R$ and $T$ are the gas constant and temperature, respectively (Equation 2). The term $w_{11}$ denotes the energy needed to remove solute molecules from the bulk, while $w_{22}$ equals to the idealised removal of solvent molecules to generate a cavity in the solvent for the molecule to dissolve. Such an idealised transfer of the molecule would lead to gained insertion energy in the solvent or release of solvation energy of $-2 w_{12}$. It was an important idea to approximate the interaction term $w_{12}$ by the square root of the product of $w_{11}$ and $w_{22}$ so that the following equation is obtained:

$$
\ln \left(\gamma_{2}\right)=\left[\left(w_{11}\right)^{0.5}-\left(w_{22}\right)^{0.5}\right]^{2} \frac{V_{2} \varphi_{1}^{2}}{R T}
$$

The advantage of having only pure component properties of the solvent and solute in Equation 3 is that they can be listed for the different chemicals without the need to additionally determine a specific interaction parameter like $w_{12}$. Hildebrand and $S$ cott ${ }^{[4]}$ coined the notion of the solubility parameter $\delta_{x}$ that is here given for the solute as follows (Equation 4):

$$
\delta_{2}=\left(w_{22}\right)^{0.5}
$$

The solubility parameter is the square root of energy per volume, and it is often named as square root of cohesive energy density. Units can also be expressed in $\mathrm{MPa}^{0.5}$ and hence can also be viewed as an internal pressure.

Equations (3) and (4) show that in the absence of differences in the solubility parameters, the activity coefficient $\gamma_{2}$ becomes unity so that ideal solubility is reached and activity and concentration are equal. Regular solution theory does not consider more complex nonideal solutions that may lead to an activity coefficient of smaller than unity. Even though regular solution theory is limited in scope, it marked the birth of the solubility parameter concept that is more broadly applicable. 


\section{Thesis Publications}

The solubility parameter, for example, can be applied to any mixing process. According to Hildebrand and Scott, the enthalpy of a mixing process is proportional to the square difference in solubility parameters for the mixture components, $\delta_{1}$ and $\delta_{2}$ (Equation 5$)^{[4]}$ :

$$
\Delta H_{M}=\varphi_{1} \varphi_{2} V_{M}\left(\delta_{1}-\delta_{2}\right)^{2}
$$

where $\varphi_{1}$ and $\varphi_{2}$ are the volume fractions of the mixing components that can be, for example, a drug and polymer.

Apart from mixing enthalpy, a solubility parameter can be linked to any thermal property or to any other molecular interaction parameter. A latter example is the well-known Flory chi parameter $\chi_{12}$ that can be expressed in terms of Equation 6 for mixtures that involve a polymer ${ }^{[1,14]}$ :

$$
\chi_{12}=\frac{V\left(\delta_{1}-\delta_{2}\right)^{2}}{R T}+\beta
$$

where $\beta$ is an entropy correction term and $V$ is the molar volume of a solvent or drug in mixture with a polymer. It seems that $\beta$ may not be required for essentially nonpolar systems, but in other cases, such an empirical correction of $\beta$ may be needed to avoid under prediction of $\chi_{12}{ }^{[1,14]}$ These examples show that particular care is needed when the solubility parameter is used for quantitative conversion to other physicochemical properties or parameters.

The solubility parameter approach is comparatively simple but the art lies in correct application for each given system. The total solubility parameter is, for example, primarily useful to describe apolar components, whereas Hansen introduced the more versatile concept of partial solubility parameters. ${ }^{[1,15]}$ As previously mentioned, the basic idea in this approach is to split the total cohesion energy $\left(E_{\text {tot }}\right)$ into different parts that originate from separate molecular interactions. The dispersive energy $\left(E_{d}\right)$ stems from atomic nonpolar forces, i.e. dispersive Van der Waals interactions, whereas forces between molecules of permanent dipoles constitute a polar energy contribution $\left(E_{p}\right)$. Due to the specific nature of hydrogen bonding, this energy contribution is considered separately $\left(E_{h}\right)$. These partial cohesion energies $E_{d}, E_{p}$ and $E_{h}$ are divided by molar volume to result in the corresponding total and partial solubility parameters according to Equations $(7)$ and $(8)^{[1]}$.

$$
\begin{aligned}
& \frac{E_{t o t}}{V}=\frac{E_{d}}{V}+\frac{E_{p}}{V}+\frac{E_{h}}{V} \\
& \delta_{t}^{2}=\delta_{d}^{2}+\delta_{p}^{2}+\delta_{h}^{2}
\end{aligned}
$$

Equation 8 shows that a three dimensional (3-D) version of the solubility parameter is obtained by consideration of the different partial contributions to cohesive energy density. Figure 1 depicts a series of solvents in this space of dispersive, polar and hydrogen bonding contribution to the
HSP. The invention of partial solubility parameters has certainly advanced the original cohesive energy density approach and opened the field to diverse potential applications wherever molecular interactions of the type like seeks like' play a critical role. We will in the following part first describe the different ways to obtain total or partial solubility parameters and will then discuss the different applications in the pharmaceutical field. Finally, gaps and current trends will be discussed.

\section{Experimental and in silico determination of solubility parameters}

\section{Introduction to solubility parameter determination}

Solubility parameters can be evaluated directly by vaporisation of solvents (or sublimation of solids) as described in the original definition. This, however, is only feasible for materials that can be either vaporised or sublimated (in case of solids), which is often not possible. Many pharmaceutically relevant materials, such as coating polymers, polymers for amorphous solid dispersions, drug compounds or surfactants, require other methods. Therefore, indirect methods have been widely used in the literature for determination of solubility parameters, which are based upon relationships between diverse physicochemical properties and cohesion energy. Solubility parameters can be deduced from measurements of other substance properties than vaporisation. Some of the different methods to obtain a solubility parameter are schematically depicted in Figure 2, and for further reference, see the comprehensive review on this topic by Barton. ${ }^{[16]}$ Values for solubility parameters determined using these various methods may vary based upon method set-up and/or material. This section describes the methods that have or could be used to characterise pharmaceutical compounds.

\section{Classical determination of solubility parameter}

Classical approach via latent heat of evaporation. As mentioned earlier, the (total) solubility parameter, was introduced by Hildebrand and Scott, and was defined as the root of the cohesive energy density (CED). The cohesive energy density was in turn defined as the energy needed to break all attractive interactions in one mole of a solvent divided by the molar volume according to Equation 9:

$$
\delta_{t}=(C E D)^{\frac{1}{2}}=\left(\frac{\Delta E_{v}}{V_{m}}\right)^{1 / 2}
$$

where $\Delta E_{v}$ is the latent energy of evaporation and $V_{m}$ is the molar volume of the solvent. This molar cohesive energy can be further expressed as follows: 


\section{Thesis Publications}

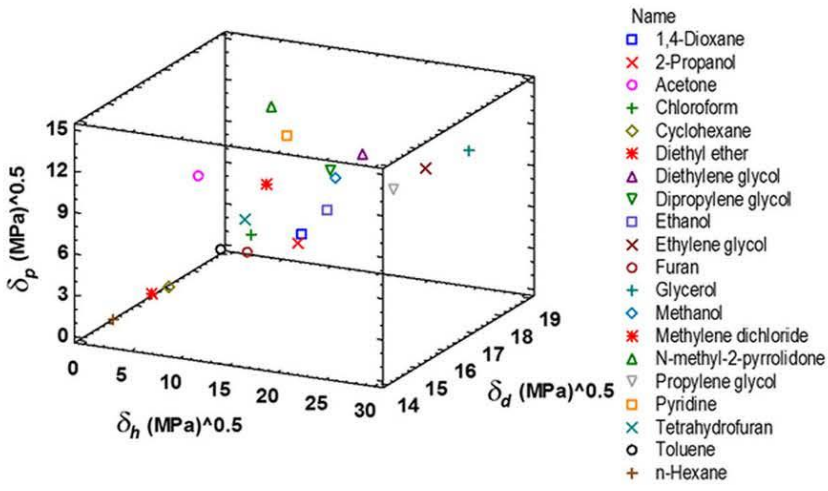

Figure 1 Common solvents are displayed according to their Hansen solubility parameter (HSP) with dispersive $\left(\delta_{d}\right)$, polar $\left(\delta_{p}\right)$ and hydrogen bonding $\left(\delta_{n}\right)$ contribution. Other excipients or also drugs can be represented in this Hansen space and a close proximity of substances suggests their miscibility (for colour codes please refer to the online version of the article)

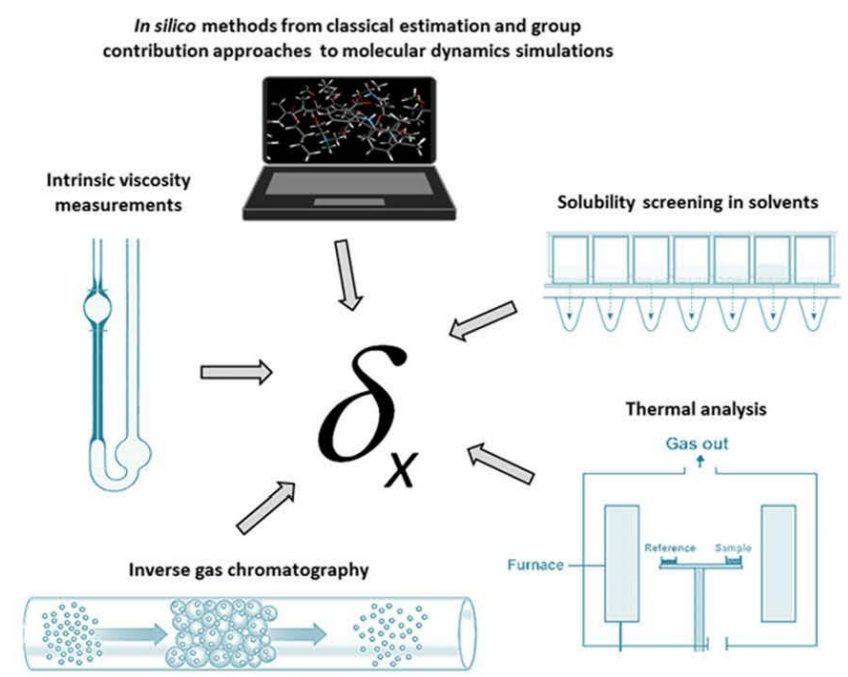

Figure 2 Different methods to obtain one or three dimensional solubility parameter $\left\langle\delta_{x}\right\rangle$

$$
\Delta E_{\psi}=\Delta H_{\psi}-R T
$$

Equation 10 can be expressed in terms of latent heat of vaporisation $\Delta H_{v}$ (in case of solvents) the universal gas constant $R$ and the absolute temperature $T . \Delta H_{v}$ can be obtained from calorimetric or vapour-pressure data to finally determine $\delta_{t}$.

Hansen expanded the total solubility parameter into three components, $\delta_{d}, \delta_{p}$ and $\delta_{h}$, as discussed for
Equation 8. This means for nonpolar molecules that $\delta_{p}$ and $\delta_{h}$ are zero, and hence, $\delta_{t}$ equals $\delta_{d}$. For polar molecules, the situation is more difficult and calculation methods were developed to assess the partial solubility parameters based on accessible physical properties.

Calculation of the nonpolar dispersion solubility parameter. The energy of evaporation can be divided into a polar and nonpolar part using the homomorph approach. $\left.{ }^{[17} 19\right]$ 


\section{Thesis Publications}

The homomorph of a polar molecule is a nonpolar molecule with nearly the same size and shape as that of the polar molecule of interest. The dispersion energy part of evaporation of a polar molecule is therefore approached by the energy of evaporation of the homomorph at the same reduced temperature, $T_{r}$. The reduced temperature $T_{r}$ is defined as follows:

$$
T_{r}=\frac{T}{T_{c}}
$$

Equation $\mathbf{1 1}$ includes the absolute temperature $T$ and the critical temperature $T_{c}$ which can be estimated based upon the Lydersen group contributions. ${ }^{[1]}$ Corrections to this approach are required for molecules containing atoms which are significantly greater than carbon, e.g. chlorine, bromine, sulfur, but not for oxygen and nitrogen. ${ }^{[1]}$ For instance, when the evaporation energy of the nonpolar carbon tetrachloride is compared with the nonpolar homomorph 2,2 dimethyl propane, a difference of $1580 \mathrm{cal} / \mathrm{mol}$ $(6610 \mathrm{~J} / \mathrm{mol})$ is found. This difference is divided by four to obtain a correction factor for a chlorine atom in a molecule. ${ }^{[20]}$ Dividing by the molar volume and then taking the square root (see Equation 9) gives the dispersion solubility parameter. $^{[1]}$

Calculation of the polar solubility parameter. The polar parameter of a polar molecule was originally calculated by Hansen and Skaarup ${ }^{[20]}$ using the Böttcher equation and expressed in $\mathrm{cal} / \mathrm{cm}^{3}$ (Equation 12):

$$
\delta_{p}^{2}=\frac{12108}{V_{m}^{2}} \frac{\varepsilon-1}{2 \varepsilon+n_{D}^{2}}\left(n_{D}^{2}+2\right) \mu^{2}
$$

where $\mu$ is the dipole moment, in Debye, $V_{m}$ the molar volume in $\mathrm{cm}^{3} /$ mole, $n_{D}$ the refractive index using the sodium $D$ line and $\varepsilon$ the dielectric constant. Later a more simplified equation was used by Hansen and Beerbower ${ }^{[21]}$ according to Equation 13 in $(\mathrm{MPa})^{1 / 2}$ :

$$
\delta_{p}=\frac{37.4 \mu}{V_{m}^{1 / 2}}
$$

Calculation of the hydrogen bond solubility parameter. Hansen and Skaarup ${ }^{[20]}$ used data from infrared spectroscopy to assign an energy of evaporation of $5000 \mathrm{cal} /$ mol for the $\mathrm{OH}-\mathrm{O}$ hydrogen bond; hence, for a solvent containing hydroxyl groups, the hydrogen solubility parameter can be calculated utilising Equation 14:

$$
\delta_{h}=\sqrt{\frac{5000 N}{V_{m}}}
$$

where $N$ is the number of alcohol groups in the solvent molecule. More often, $\delta_{h}$ is calculated by subtracting the dispersion and polar contributions from the total solubility parameter according to Equation $15^{[1,22]}$ :

$$
\left(\delta_{h}\right)^{2}=\left(\delta_{t}\right)^{2}-\left(\delta_{p}\right)^{2}-\left(\delta_{d}\right)^{2}
$$

As hydrogen bonding has a profound effect on solubility, attempts have been undertaken to further expand the hydrogen bond solubility parameter. A hydrogen bond comprises a hydrogen bond donor and a hydrogen bond acceptor, and molecules can therefore be classified as ${ }^{[16]}$ (1) proton donor, e.g. trichloromethane; (2) proton acceptor, e.g. ketones, aldehydes, esters, ethers, tertiary amines, aromatic hydrocarbons, alkenes; (3) proton donor/acceptor, e.g. alcohols, carboxylic acids, water, primary and secondary amines; and (4) proton nondonor/nonacceptor, e.g. alkanes, carbon disulfide and tetrachloromethane. From a qualitative point of view, miscibility can be predicted if hydrogen bonds are formed upon mixing, while demixing occurs when hydrogen bonds are destroyed (e.g. water mixed with alkanes).

An extension of the hydrogen bond solubility parameter to account for hydrogen bond donor and acceptor properties has been suggested by Small ${ }^{[23]}$ and others (Sorenson et $a l^{[24]}$, using Equation 16 :

$$
\delta_{h}^{2}=2 \delta_{a} \delta_{b}
$$

where $\delta_{\mathrm{a}}$ is the acidic solubility parameter (the donor proton of the hydrogen bond, which is the electron acceptor in the Lewis acid framework) and $\delta_{b}$ is the basic solubility parameter (Lewis base, electron donor). Beerbower and Martin ${ }^{[21]}$ developed the concept to estimate the solubility of naphthalene, benzoic acid, $p$-hydroxybenzoic acid and methyl $p$-hydroxybenzoate in different sol vents. ${ }^{[25]}$ However, improvement in accurate miscibility prediction was limited (e.g. benzoic acid: $63 \%$ acceptable - i.e. less than $30 \%$ error vs observed - predictions using four parameters vs $60 \%$ acceptable predictions with the three Hansen solubility parameters).

\section{Determination of partial solubility parameters using solvent solubility data}

Determination of solubility parameters for drug substances and polymers typically cannot be accomplished through the measurement of the energy of evaporation (because of their nonvolatility and due to the fact that evaporation frequently is accompanied by degradation) and therefore only indirect methods can be used. Solubility determination of a solid in a series of selected solvents, which cover the solubility design space in terms of $\delta_{d} \delta_{p}$ and $\delta_{b}$, is a frequently used technique to achieve this goal. ${ }^{[1,2632]}$

Typically, 10-40 solvents are used for the solubility measurements, which can be conducted as, e.g. a classical shake-flask approach where the thermodynamic solubility 


\section{Thesis Publications}

Table 1 Overview of experimentally determined HSP for pharmaceutical polymers

\begin{tabular}{|c|c|c|c|c|c|}
\hline Polymer & $\delta_{d}(\mathrm{MPa})^{1 / 2}$ & $\delta_{p}(\mathrm{MPa})^{1 / 2}$ & $\delta_{h}(\mathrm{MPa})^{1 / 2}$ & References & Remarks \\
\hline HPMC 2906, F4M & 18.2 & 16.5 & 15.5 & [34] & 34 solvents \\
\hline HPMC 2208, K4M & 18.0 & 15.3 & 19.4 & [34] & 34 solvents \\
\hline HPMC 2910 E4M & 17.4 & 14.9 & 19.3 & [34] & 34 solvents \\
\hline HPMC 2910 E15 & 18.8 & 9.4 & 11.8 & [194] & $\begin{array}{l}29 \text { solvents, calculation using HSPiP } \\
\text { software }{ }^{[67]}\end{array}$ \\
\hline HPMC 2910 E5 & 18.7 & 9.8 & 11.6 & [194] & $\begin{array}{l}29 \text { solvents, calculation using HSPiP } \\
\text { software }{ }^{[67]}\end{array}$ \\
\hline Methylcellulose A4M & 18.0 & 15.3 & 19.4 & [34] & 17 solvents \\
\hline HPC, Klucel H Dow data & 17.2 & 9.8 & 13.5 & [34] & \\
\hline $\begin{array}{l}\text { HPC, Klucel } H \text {, solubility } \\
\text { data from Exxon Dow } \\
\text { calculation }\end{array}$ & 17.6 & 10.2 & 15.4 & [34] & \\
\hline HPMCAS 716 (L grade) & 17.7 & 11.87 & 10.19 & Dow technical information & Not mentioned \\
\hline HPMCAS 912 (M-grade) & 16.73 & 12.37 & 10.33 & Dow technical information & Not mentioned \\
\hline HPMCAS 126 (H grade) & 18.09 & 12.76 & 9.67 & Dow technical information & Not mentioned \\
\hline HPMCAS MG/Agoat MG & 16.2 & 10.9 & 9.0 & {$[40]$} & $\begin{array}{l}\text { Jansen analysis using HSPIP software of } \\
\text { the solubility data of } 110 \text { solvents }{ }^{[57]}\end{array}$ \\
\hline HPMCAS L grade & 18.9 & 12.4 & 9.0 & [194] & $\begin{array}{l}29 \text { solvents, calculation using HSPiP } \\
\text { software }{ }^{[6]]}\end{array}$ \\
\hline HPMC phthalate HP 50 & 20.4 & 10.2 & 11.7 & [194] & \\
\hline PVP K30 & 18.3 & 12.9 & 11.4 & [194] & \\
\hline
\end{tabular}

is determined or only kinetic solubility values are estimated by continuously adding small drug amounts to solvents with optical detection of residual drug. Generally, the solvents are selected such that the compound of interest has good solubility in at least half of the solvents screened. The solubility parameters can then be calculated with multiple regression analysis based on mole fractions solubilised in the various solvents ${ }^{[27,28]}$ or with more modern computer software that uses an iterative method for improving drug solubility parameter estimates in a Cartesian coordinate system (Figure 1) of $\delta_{d}, \delta_{p}, \delta_{k}$. The distances between the sample reference point to point representations are calculated for solvents. Then the iterative method adjusts the partial solubility parameters such that the distance between compatible and incompatible solvents are minimised and maximised, respectively. This iterative minimisation/maximisation process can then determine a more reliable solubility parameter as recently done by Howell and coworkers. ${ }^{[33]}$

Drug substances as well as polymers can be used as samples. For example, HPMC and HPMCAS are frequently used polymers in stabilised amorphous solid dispersions and with the 'like dissolves like' approach the solubility parameter seems as a feasible way to provide a first guess on the active pharmaceutical ingredient (API) miscibility in these systems. An overview of experimentally determined HSP using the solvent method is provided in Table 1. For HPMCAS, the values were consistent between three independent determinations (see references given in Table 1). For HPMC, a difference was noted for the polarity and hydrogen bonding parameters between Janssen and
Archer. ${ }^{[34]}$ This may be attributed both to (1) substantial difference in molecular weight, (2) the usage of a limited number of solvents, e.g. 10 and (3) the usage of water as solvent by Archer, ${ }^{[34]}$ which is generally not recommended due to the complexity of the solvent. ${ }^{[1]}$

Solubility parameters obtained via the solvent approach have also been reported for characterisation of drug molecules and solid excipients such as, preservatives. $\left.{ }^{[25} 27,32,35,36\right]$ These studies use about $10-25$ different solvents to obtain an accurate determination of the partial solubility parameters, which seems to be reasonable given the number of unknowns in the regression analysis. Verheyen and coworkers ${ }^{[27]}$ investigated five chemically related molecules and reported that the method was sufficient enough to capture the differences between molecules. Barra et al. ${ }^{[26]}$ reported a similar finding by investigating a number of free acids and their associated sodium salts and finally, as well as those results published by Kitak and coworkers ${ }^{[32]}$ could find differences between two ibuprofen salts and the free acid. The method is therefore generally perceived as sufficiently precise to determine accurate partial solubility parameters by solubility screening in a number of organic solvents or mixtures of these.

\section{Determination of partial solubility parameters} using intrinsic viscosity measurements

The use of viscosity measurements is a frequently used technique to determine the solubility parameter for 


\section{Thesis Publications}

polymers. ${ }^{[1,37}{ }^{46]}$ In this approach, the intrinsic viscosity of the polymer of interest is determined in a number of solvents. ${ }^{[1,37,39]}$ Intrinsic viscosity, $[\eta]$, is given by:

$$
[\eta]=\lim _{c \rightarrow 0}\left(\frac{\eta_{s}-\eta_{0}}{\eta_{0} c}\right)
$$

Equation 17 can be expressed in terms of the solution viscosity $\left(\eta_{s}\right)$ or the solvent viscosity $\left(\eta_{0}\right)$ where $c$ is the solution concentration. The conformation of a polymer in solution is dependent on its interactions with the solvent. In so-called good solvents with many interactions, the polymers can swell to some extent, which increases of solutions viscosity. By contrast, in solvents with limited interactions (i.e. a 'poor solvent') the polymer will reduce the contact area to the solvent by shrinking. An interesting approach is to normalise intrinsic viscosities for a polymer in a solvent that provides the highest viscosity value. These normalised data can be used to determine the HSP according to the Equations below $18-20^{[39]}$ :

$$
\begin{aligned}
\delta_{D 2} & =\sum\left(\delta_{D i} \times[\eta]_{i}\right) / \sum[\eta]_{i} \\
\delta_{P 2} & =\sum\left(\delta_{P i} \times[\eta]_{i}\right) / \sum[\eta]_{i} \\
\delta_{H 2} & =\sum\left(\delta_{H i} \times[\eta]_{i}\right) / \sum[\eta]_{i}
\end{aligned}
$$

where the subscript 2 indicates the polymer and ' $i$ ' the solvents. The intrinsic viscosity of the $i$-th solvent is described by the parameter $[\eta]_{i}$. The solvents that are most compatible have the highest intrinsic viscosities and are closest to the geometric centre of a sphere in the Hansen space that encloses good solvents. ${ }^{[1]}$ The separating distance from the centre of the sphere to a last still compatible solvent provides the interaction sphere radius $R_{0}$ and is defined according to Equation 21:

$$
R_{0}^{2}=4\left(\delta_{d i}-\delta_{d p}\right)^{2}+\left(\delta_{p i}-\delta_{p p}\right)^{2}+\left(\delta_{h i}-\delta_{h p}\right)^{2}
$$

where $\delta_{d i}, \delta_{p i}$ and $\delta_{h i}$ are partial solubility parameters of the last still compatible solvent, whereas $\delta_{d p}, \delta_{p p}$ and $\delta_{h p}$ denote the partial solubility parameters of the sample polymer. Analogues to Equation 21, is possible to calculate the distance between the sample polymer and any solvent, which is called 'solubility parameter distance', $R_{a}$. This distance parameter is of more general use and is not restricted to viscosimetry to determine solubility parameters.

An alternative method to determine the partial solubility parameters via intrinsic viscosity was developed by $\mathrm{Miecz}$ kowski. ${ }^{[47,48]}$ In this method, the values of the volume fraction of a solvent $\left(\varphi_{s}\right)$ were determined for three mixtures of solvents at which the maximum intrinsic viscosity was found. These fractions were then inserted into to Equation 22:

$$
\sum_{=0}^{3} p_{i}\left(a_{i}-b_{i}\right)-\left[\varphi_{s} \sum_{i=1}^{3}\left(a_{i}-b_{i}\right)^{2}+\sum_{i=1}^{3} b_{i}\left(a_{i}-b_{i}\right)\right]
$$

where $p_{i}$ is the component of the solubility parameter of the polymer, $a_{i}$ is the component of the first solvent and $b_{i}$ is the component of the second solvent. Using this method, the HSPs for PEO (polyethylene oxide) 2000 were determined as $\delta_{d}: \quad 17.3 \pm 2 \mathrm{MPa}^{1 / 2}, \quad \delta_{p}$ : $3.0 \pm 1 \mathrm{MPa}^{1 / 2}$ and $\delta_{h}: 9.4 \pm 0.5 \mathrm{MPa}^{1 / 2}$.

Furthermore, Bustamante et al. ${ }^{[43]}$ also employed viscosity to determine the partial solubility parameter of HPMC with 28-30\% methoxy and 7-12\% hydroxypropyl content and an approximate molecular weight of $86 \mathrm{kDa}$ (equivalent to Dow E4M). The experimental results were fitted according to following regression model (Equation 23):

$$
\begin{aligned}
\ln [\boldsymbol{\eta}]= & C_{0}+C_{1} \delta_{1 d}+C_{2} \delta_{1 d}^{2}+C_{3} \delta_{1 p}+C_{4} \delta_{1 p}^{2}+C_{5} \delta_{1 h} \\
& +C_{6} \delta_{1 h}^{2}
\end{aligned}
$$

whereas the subscript 1 refers to solvent and the subscript 2 in the following Equations $24-26$ to the polymer:

$$
\begin{aligned}
& \delta_{2 d}=-\left(\frac{C_{1}}{2 C_{2}}\right) \\
& \delta_{2 p}=-\left(\frac{C_{3}}{2 C_{4}}\right) \\
& \delta_{2 d}=-\left(\frac{C_{5}}{2 C_{6}}\right)
\end{aligned}
$$

An extended regression model was used to allow the determination of the Lewis acid $\left(\delta_{a}\right)$ and base $\left(\delta_{b}\right)$ solubility parameters according to Equation 27 :

$$
\begin{aligned}
\ln [\boldsymbol{\eta}]= & C_{0}+C_{1} \delta_{1 d}+C_{2} \delta_{1 d}^{2}+C_{3} \delta_{1 p}+C_{4} \delta_{1 p}^{2}+C_{5} \delta_{1 a} \\
& +C_{6} \delta_{1 b}+C_{7} \delta_{1 a} \delta_{1 b}
\end{aligned}
$$

Solubility parameters of HPMC for dispersion, polarity and hydrogen bonding were reported to be $14,16.8$ and $31 \mathrm{MPa}^{1 / 2}$, respectively. As can be seen in Table 1 , the data obtained by Bustamante ${ }^{[43]}$ for $\delta_{d}$ and $\delta_{p}$ were in line with the values obtain by Archer $^{[34]}$ while there was a substantial difference for the $\delta_{h}$ solubility parameter. This difference may be attributed to the use of different Hansen solubility parameters for water. Bustamante used 15.5, 15.95 and 42.34 $\mathrm{MPa}^{1 / 2}$, while Archer used 19.5, 17.8 and $17.6 \mathrm{MPa}^{1 / 2}$ for dispersion, polar and hydrogen bonding respectively. Additionally, Madsen and coworkers ${ }^{[4]}$ employed viscosity to determine the solubility parameter for the pharmaceutical 


\section{$\mathrm{X}$. Thesis Publications}

polymers, PLGA and polycaprolactone (PCL). The authors demonstrated that the solubility parameter could be correlated with protein release from the two polymer systems. This example underpins again, how broadly the solubility parameter approach can be applied.

\section{Determination of partial solubility parameters of liquids using inverse gas chromatography}

The HSP for pharmaceutical liquids are difficult to determine experimentally using 'solubility' methods. Additionally, many pharmaceutical substances including, for example, polymers, polysorbates, ethoxylated oils and vitamin E TPGS are complex mixtures without defined chemical structure and hence are difficult to approach theoretically. In such cases, the determination of the HSP by inverse gas chromatography, is a valuable option. Inverse gas chromatography has been applied to investigate the solubility parameters of polymers, ${ }^{[40,49}$ 52] surfactants, ${ }^{[53,54]}$, epoxidised soybean oil, ${ }^{[55]}$ triglycerides ${ }^{[56]}$ and liquid crystal systems. ${ }^{[57}{ }^{59]}$ For measurement of solids, it is recommended for the samples to contain some amorphous fraction, so that the probe gases can enter the bulk. Otherwise, only surface interactions could skew the results for estimated solubility parameters.

Using the inverse gas chromatography method, the material of interest is the stationary phase and is placed into a column. It is then characterised using diverse volatile probes (i.e. volatile solvents with known HSPs). The HSP of the material can then be calculated based on the retention data of the solvent probes. ${ }^{[54]}$ First, the retention times of the solvent probes are converted to specific retention volumes $\left(V_{g}\right)$. These are then used to calculate the Flory-Huggins interaction parameter between the solvent and the sample using the following equation (Equation 28) (60 $^{[62]}$ :

$$
\begin{aligned}
\chi_{1,2}^{\infty}= & \ln \left(\frac{273 \cdot 15}{M_{1} V_{g}^{\circ} P_{1}^{\circ}} \cdot R\right)-\frac{P_{1}^{\circ}}{R \cdot T}\left(B_{11}-V_{1}^{\circ}\right)+\ln \left(\frac{\rho_{1}}{\rho_{2}}\right) \\
& -\left(1-\frac{V_{1}^{\circ}}{V_{2}^{\circ}}\right)
\end{aligned}
$$

where $\chi_{1,2}^{\infty}$ is the Flory-Huggins interaction parameter between the material of interest and the solvent probe, $M_{1}$ is the molecular mass, $P_{1}^{\circ}$ is the vapour pressure of the solvent probe at the measurement temperature calculated using the Antoine equation, $V_{1}^{\circ}$ is the molar volume of the probe, $V_{2}^{\circ}$ is the molar volume of the examined material, $B_{11}$ is the second virial coefficient of the solvent probe calculated according to Voelkel and Fall, ${ }^{[63]} \rho_{1}$ and $\rho_{2}$ are the densities of the solvent probe and material, respectively. The total solubility parameter is then calculated using Equation 29 ${ }^{[50,53,64,65]}$ :

$$
\frac{\delta_{1}^{2}}{R T}-\frac{\chi_{1,2}^{\infty}}{V_{i}}=\frac{2 \delta_{2}}{R T} \delta_{1}-\left(\frac{\delta_{2}^{2}}{R T}+\frac{\chi_{s}^{\infty}}{V_{i}}\right)
$$

where $\delta_{1}$ is the total solubility parameter of the consecutive test solutes and $\delta_{2}$ the total solubility parameter of the material of interest, $\chi_{S}^{\infty}$ is the entropic part of the Flory-Huggins interaction constant and is usually estimated in the range $0.2-0.4$ or $0.6 .^{[52]}$ By plotting the left hand side of the equation vs $\delta_{1}$, a straight line is obtained of which the slope and intercept are used to calculate $\delta_{2}$.

Voelkel and Janas ${ }^{[66]}$ extended this original concept, introduced by Price and Shillcock ${ }^{[57]}$ to obtain partial solubility parameters from the linear relationships for solvent groups ( $n$-alkanes for $\delta_{2 d}$, polar solvents for $\delta_{2 p}$ and hydrogen bonding solvents for $\delta_{2 h}$, while $S$ is indicated as the value for the slope) according to Equations $30-32$ :

$$
\begin{gathered}
\delta_{2 d}=\frac{S_{n-\text { alkanes }}}{2} R T \\
\delta_{2 p}=\frac{\left(S_{\text {polars }}-S_{n-\text { alkanes }}\right)}{2} R T \\
\delta_{2 h}=\frac{\left(S_{h-\text { bonds }}-S_{n-\text { alkanes }}\right)}{2} R T
\end{gathered}
$$

Using this approach Adamska and Voelkel ${ }^{[52]}$ determined the HSPs of di- $n$-butyladipat (Cetiol), caprylocaproyl macrogol-8 glycerides (Labrasol, Gattefosse) and polysorbate 80 (Tween 80 ) using inverse gas chromatography. Packing of columns with semi-solids and liquid compounds often require special preparation and here, the excipients were dissolved in a suitable solvent to coat on a solid support by slow solvent evaporation. By comparison, an alternative method with fewer assumptions has been used for some of the same excipients investigated by Adamska and Voelkel ${ }^{[54]}$ as described in Table 2. The method used has some parallels with the solubility approach described above. In this method the three partial parameters were iteratively and systematically changed to give the best fit between predicted and experimental values for the interaction parameter, $\chi_{1,2}^{\infty}$, for all the solvent probes tested; hence, no solvent probe families were needed. The predicted interaction constant was calculated according to Equation 33:

$$
\begin{aligned}
\chi_{1,2}^{\infty}= & C_{1}+V_{m} \cdot C_{2} \\
& \cdot\left[4\left(\delta_{1 d}-\delta_{2 d}\right)^{2}+\left(\delta_{1 p}-\delta_{2 p}\right)^{2}+\left(\delta_{1 h}-\delta_{2 h}\right)^{2}\right]
\end{aligned}
$$

where $C_{1}$ and $C_{2}$ are constants and $V_{m}$ is the molar volume of the solvent probe. This approach is adopted in the software package 'Hansen Solubility Parameters in 


\section{Thesis Publications}

Table 2 Hansen solubility parameters obtained for materials using inverse gas chromatography compared using different data treatment

\begin{tabular}{|c|c|c|c|c|c|}
\hline Material & $\delta_{d}(\mathrm{MPa})^{1 / 2}$ & $\delta_{p}(\mathrm{MPa})^{1 / 2}$ & $\delta_{h}(\mathrm{MPa})^{1 / 2}$ & Remark & References \\
\hline Cetiol & 16.5 & 1.4 & 4.8 & \multirow[t]{4}{*}{ Inverse gas chromatography } & \multirow{3}{*}{ [54] } \\
\hline Labrasol & 18.0 & 0.8 & 3.2 & & \\
\hline Tween 80 & 19.3 & 0.9 & 2.8 & & \\
\hline PEG 2000 (at $85^{\circ} \mathrm{C}$ ) & 19.4 & 1.6 & 1.2 & & [52] \\
\hline Labrasol & 18.3 & 5.8 & 7.2 & \multirow{4}{*}{$\begin{array}{l}\text { Inverse gas chromatography } \\
\text { followed by iterative } \\
\text { calculation using H5PP }\end{array}$} & \multirow[t]{4}{*}{ Internal Janssen data } \\
\hline PEG 400 & 19.7 & 8.3 & 8.8 & & \\
\hline Tween 80 & 19 & 5.3 & 5.6 & & \\
\hline Olive oil & 16.9 & 0.6 & 4.2 & & \\
\hline
\end{tabular}

Practice', HSPiP. ${ }^{[67]}$ This software is helpful as it provides tools for many processes such as determining HSP from solubility data and, estimating HSP from the chemical structure as well as databases, which include HSP values for many polymers and excipients. The values obtained for polysorbate 80 and caprylocaproyl macrogol-8 glycerides (Labrasol, Gattefosse) were somewhat different than the ones obtained by Adamska and Voelkel. ${ }^{[54]}$ This is possibly due to the use of different sample preparation methods and column packing material, which influences the retention volume determination. However, it should be also noted that it may be problematic to determine a solubility parameter for surfactants and other amphiphilic substances (e.g. some copolymers) by the use of only a single set of partial solubility parameters. Superstructures such as liquid-crystalline phases are typically formed and probe gas molecules can interact with different microdomains, which is an issue of sample heterogeneity.

$\mathrm{Klar}$ and Urbanetz ${ }^{[40]}$ investigated the solubility parameter for hypromellose acetate succinate (granular type $\mathrm{M}$ HPMCAS) by turbidimetric titration, inverse gas chromatography and seven different calculation methods. The total solubility parameter determined by turbidity was $24.7 \pm 3.2 \mathrm{MPa}^{1 / 2}$ for moderately hydrogen bonded solvents and $24.4 \pm 0.3 \mathrm{MPa}^{1 / 2}$ when determined by inverse gas chromatography. Furthermore, the partial HSPs that were determined by inverse gas chromatography and obtained data $\left(17.69,12.06\right.$ and $11.7 \mathrm{MPa}^{1 / 2}$ for dispersion, polar and hydrogen bonding respectively) showed good accordance with the value generated by the calculation methods, especially those calculated via the Hoy and $\mathrm{Te}$ Nijenhuis method, using experimentally determined molecular volumes, ${ }^{[40]}$ as well as the Janssen and Dow values determined from solubility data, see Table 1 .

In summary, even though the practice and the theory to determine solubility parameters by inverse gas chromatography has a long tradition, there are new theoretical and practical developments. ${ }^{[56,68]}$ For future applications, it is interesting to evaluate different calculation methods for inverse gas chromatography. The use of the Flory-Huggins interaction parameters is quite abundant but the theory was developed initially for polymers so that small molecules may be better described by, for example, the Bragg-Williams interaction parameters. ${ }^{[53]}$

\section{Other experimental methods to determine solubil-} ity parameter

Besides the typical methods described in the section above, a number of other analytical techniques used to determine the solubility parameter have been reported. These include swelling, $\left.{ }^{[69} 74\right]$ turbidity, ${ }^{[40,7477]}$ ultraviolet spectroscopy, and differential scanning calorimetry (DSC) as well as differential scanning microcalorimetry ( $\mu$ DSC) ${ }^{[78]}$

Gee described ${ }^{[79]}$ the swelling of rubber in various liquids to derive its solubility parameter, an approach which has also been applied in more recent studies. For example, Eroglu et al. ${ }^{[73]}$ and Cavuş et al. ${ }^{[69]}$ measured the weight of a polymer before and after addition of different solvent systems with known solubility parameters by gravimetry and then defined the solubility at the point where optimal swelling was observed. Furthermore, Cavus et al. ${ }^{[69]}$ also investigated the swelling of PVC and found good correlations to the theoretical calculation of the solubility parameter based upon the van Krevelen-Hoftyzer and Hoy methods. ${ }^{[69]}$ The simplicity of the method is highly attractive but the swelling must be of course detectable.

For turbidimetric titration, a polymer (or another relevant excipient) is dissolved in an appropriate solvent. $\mathrm{A}$ second solvent that is miscible with the first solvent but act as anti-solvent for the polymer is then added until precipitation occurs. Shu and coworkers ${ }^{[75,76]}$ developed a method whereby the solubility parameter could be determined from these data. As described above Klar and Urbanetz ${ }^{[40]}$ showed good correlation between the solubility parameter for HPMCAS determined by inverse gas chromatography and turbidity titration. In addition, Schenderlein et al. ${ }^{[74]}$ reported the turbidity titration to be more precise relative to the swelling approach when investigating different proportions of lactide to glycolide for poly(d,l-lactide-co-glycolide) (PLGA). 


\section{Thesis Publications}

Carvalho et al. ${ }^{[78]}$ used several methods to determine the solubility parameter for naphthalene, phenanthrene and pyrene and reported UV-vis as the most suited method for a wider molar mass range. For compounds with a low molar mass determination of the vapour enthalpy by DSC was argued to be better based on the similar results and a lower quantity of compound consumption, whereas the $\mu D S C$ method still required some optimisation. Miecszkowski ${ }^{[46]}$ used refractive index in several solvents to determine the partial solubility parameter for polystyrene and compared these experimental results together with calculations according to the Van Krevelen approach. Ravindra et al ${ }^{[38]}$ derived the solubility parameter of chitosan from intrinsic viscosity, surface tension and the dielectric constant for data comparison including different group contribution calculations. The average of the calculation methods yielded $43.1 \mathrm{MPa}^{1 / 2}$ for chitosan, while the experimental data provided $41.5,39.8$ and $37.0 \mathrm{MPa}^{1 / 2}$ when determined by viscosity, surface tension and dielectric constant, respectively. This demonstrates the variation that might be seen across methods. All of the mentioned approaches have their specific assumptions and limitations both with experimental design and data evaluation.

\section{Group contribution methods to calculate partial solubility parameters}

The partial solubility parameters describe the ability of a molecule to interact with another molecule via intermolecular forces. Given that molecules contain several structura fragments/groups that can contribute to such molecular forces and volume, group contribution methods are meaningful to estimate solubility parameters based only on the chemical structure. The most frequently applied methods are the approaches described by Hoy, ${ }^{[80]}$ Fedors, ${ }^{[81]}$ and Van Krevelen and Hoftyzer, ${ }^{[82]}$ however newer approaches have also been reported, e.g. by Stefanis and Panayiotou. ${ }^{[5,83]}$ An example of solubility parameter calculation is given for lacidipine according to Hoftyzer/Van Krevelen (Table 3). Group contribution calculations are attractive as they require pure in silico approach, which demands less computation time compared to, for example, molecular dynamics simulations.

A solubility parameter from calculations or modelling is typically a bulk property of a liquid or of a supercooled melt. For that reason, it is essential to know whether a compound or polymer is completely amorphous or contains crystalline parts, which may affect precision of the calculation. Computational concepts assume values for atomic or group contributions to the total cohesive energy. Most of the computational methods are related to the molar volume $V_{m}$ of a substance, thus $V_{m}$ has to be either known or calculated. Therefore, some researchers published values of
Table 3 Example of the parameters required for solubility parameter calculation for lacioipine according to Hotfyzer and Van Krevelen

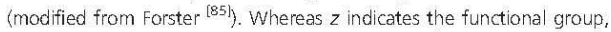
$F_{d i}$ is the group dispersion component giving $\delta_{d i} F^{2}{ }_{o i}$ is the group polar component, $E_{n j}$ is the hydrogen bonding component, and $V$ is the molar volume

\begin{tabular}{lrrrc}
\hline Group & \multicolumn{1}{c}{$F_{d i}$} & \multicolumn{1}{c}{$F_{p i}^{2}$} & \multicolumn{1}{c}{$E_{R i}$} & $\Sigma^{2} \mathrm{~V} / \mathrm{cm}^{3} \mathrm{~mol}^{-1}$ \\
\hline (7) $\mathrm{CH}_{3}$ & 2940 & 0 & 0 & 234.5 \\
(2) $\mathrm{CH}_{2}$ & 540 & 0 & 0 & 32.2 \\
(1) $\mathrm{NH}$ & 160 & 210 & 3100 & 4.5 \\
(1) $\mathrm{C}$ & -70 & 0 & 0 & -19.2 \\
(1) $\mathrm{CH}$ & 80 & 0 & 0 & -1.0 \\
(4) $\mathrm{C}=$ & 280 & 0 & 0 & -22 \\
(1) $\mathrm{Phenylene}$ & 1270 & 110 & 0 & 52.4 \\
(2) $\mathrm{HC}=$ & 400 & 0 & 0 & 27 \\
(3) $\mathrm{COO}$ & 1170 & 1470 & 21000 & 54 \\
(1) Ring (6) & 190 & & & 16 \\
$\Sigma$ & 6960 & 1790 & 24100 & 378.4 \\
\hline
\end{tabular}

group contributions to $V_{m}$, as in some cases, reliable values of solubility parameters can be calculated best, when using two complementary methods. Whereas, other concepts, e.g. the concept by Hoy, are suited to be combined $V_{m}$ ascertained by experimental techniques. ${ }^{\left[{ }^{[0]}\right.}$ Especially for high molecular materials as polymers, experimental determined $V_{m}$ can provide more precise values for solubility parameters, although an experimental determined $V_{m}$ is not recommended in all cases. For example, the method proposed by Fedors can only yield concise results when using the correlated calculation concept for $V_{m}$, as $V_{m}$ of Fedors' method is often different to other calculations or experimentally determined $V_{m}$. Based on such differences it would be a pragmatic approach to use different in silico methods to work with the mean of these predictions. ${ }^{[32,38]}$

Based on these group contribution components the partial solubility parameters can be calculated;

$$
\begin{gathered}
\delta_{d}=\frac{\sum F_{d i}}{V}=\frac{6960}{378.4}=18.4 \mathrm{MPa}^{1 / 2} \\
\delta_{p}=\frac{\sqrt{\sum F_{p i}^{2}}}{V}=\frac{\sqrt{1790}}{378.4}=0.11 \mathrm{MPa}^{1 / 2} \\
\delta_{h}=\sqrt{\frac{\sum E_{h i}}{V}}=\sqrt{\frac{24100}{378.4}}=7.98 \mathrm{MPa}^{1 / 2}
\end{gathered}
$$

From which the total solubility parameter can be calculated:

$$
\begin{aligned}
\delta_{t} & =\sqrt{\delta_{d}^{2}+\delta_{p}^{2}+\delta_{h}^{2}}=\sqrt{18.4^{2}+0.11^{2}+7.98^{2}} \\
& =20.1 M P a^{1 / 2}
\end{aligned}
$$

The ease of the calculations has seen group contribution approaches being widely adopted, e.g. Shah and Agrawal ${ }^{[84]}$ used the Van Krevelen and Hoftyzer method to estimate 


\section{$\mathrm{X}$. Thesis Publications}

the partial solubility parameters for a number of lipid excipients, including Dynasan 114, Capmul MCM, Migloyol $812 \mathrm{~N}$ and others. This approach has also been used for the calculation of solubility parameters for drug substances, ${ }^{[26,27,32,35,85]}$ which are often compared to experimentally obtained data. Besides the classical group contribution methods it is in principle also possible to employ, for example, the COSMO-RS ${ }^{[86]}$ approach, molecular dynamics simulations, ${ }^{[87]}$ or quantitative structure-property relationships. Future research may compare such a modern approach to classical group contribution methods for pharmaceutical dataset.

\section{Applications of solubility parameters in pharmaceutics}

\section{Organic solvent selection}

Knowledge of solubility in pure solvents and solvent mixtures is crucial for designing the crystallisation process of drug substances. The first step in finding the optimal crystallisation conditions is usually a solvent screening. To minimise time and resource investment, it would be desirable to conduct in silico screenings using solubility parameters or other modelling approaches to reach an educated first guess. ${ }^{[88]}$ For this purpose, the use of solubility parameters is simpler than more advanced and complex theoretical models such as COSMO-RS (conductor-like screening model for real solvents), which is computation intensive, and PC-SAFT (perturbed-chain statistical associating fluid theory), which requires extensive parameter determination. ${ }^{[88,89]}$ Thus, in silico solubility parameter determination is not the only way to rank solvents for further experiments, however it is significantly more straightforward, especially when considering group contribution methods.

Furthermore, solvent selection can be essential when considering these processes. For example, Rogers and Marangoni ${ }^{[90]}$ reported that changes in solvent chemistry affected nucleation and crystal growth events and therefore defined the physical properties of obtained crystals. In addition, solvent selection can change, solvent-gelator compatibility, affecting the degree of undercooling, chemical potential, kinetics of gelation and crystal morphology. These parameters are all interrelated and can be correlated to partial solubility parameters. Liu et al. ${ }^{[59]}$ studied crystallisation behaviour of two anhydrous as well as the monohydrate form of piroxicam. Interestingly, additives with a similar hydrogen bonding pattern to piroxicam facilitated crystallisation of anhydrous form I. It was argued that such additives would affect the formation of the different molecular clusters in the supersaturated solution. As a consequence, these additives had a higher ability to influence the nucleation of the different polymorphs. These data suggested that the HSP could indeed be used for preliminary screening of additives for the solid form control of piroxicam during the crystallisation processes. ${ }^{[59]}$ Zhu et al. ${ }^{[91]}$ used the partial solubility parameters to select the optimum solvent system for aerogels, which are open-porous predominantly mesoporous solids with a wide range of applications, including oil-spill clean-up and $\mathrm{CO}_{2}$ capture. A HSP based search method was used to target a specific gel system and because the solvent in this case had a functional influence, its selection was of crucial importance. ${ }^{[1]}$ Solubility parameters can also be used for defining extraction processes, as described by Masurel et al. ${ }^{[92]}$ for the removal of tar from flue gas, but also other publication focus on the use of solubility parameters for liquid extraction, ${ }^{93}$ 96] even though the applications are not necessarily pharmaceutical. In particular, the partial solubility parameters seem to have several applications within the space of organic solvent selection for either crystallisation or extraction. These processes are especially important for the final steps of drug substance manufacture and even though a solubility parameter approach, however more can be done to harness the power of the HSP in the area of pharmaceutical solvent selection.

\section{Cocrystal and salt screening}

Solubility as well as dissolution rate can be significantly influenced by the selection of a suitable solid-state form. Generally high solubility and fast dissolution rate is realised by usage of an amorphous solid-state form. ${ }^{[97]}$ However, a direct use of the amorphous form, e.g. in a capsule or tablet, bears the risk of uncontrolled conversion to the crystalline solid-state form, which has inferior solubility and dissolution behaviour. Therefore, amorphous APIs are not normally used directly but stabilised by suitable formulation techniques, ${ }^{[98]}$ which will not be discussed in this section. Instead, we focus on optimisation of solubility and dissolution by choosing advantageous crystalline solid-state forms. Generally, these comprise pharmaceutical salts, polymorphs - including pseudopolymorphs such as hydrates and solvates - and cocrystals. Optimisation of aforementioned properties by polymorph selection comes with the same risks as discussed for the amorphous form. Selection of metastable polymorphs increases solubility, but bears the risk of uncontrolled conversion to the thermodynamically stable form. Therefore, a thermodynamically stable polymorph is typically chosen for clinical development, and only in rare cases or when there is limited knowledge of the APIs polymorph landscape, is the metastable form selected. Examples of cases where metastable polymorphs have been used in clinical development can be found in the literature. $\left.{ }^{[99} 101\right]$ Additionally, compared to 


\section{Thesis Publications}

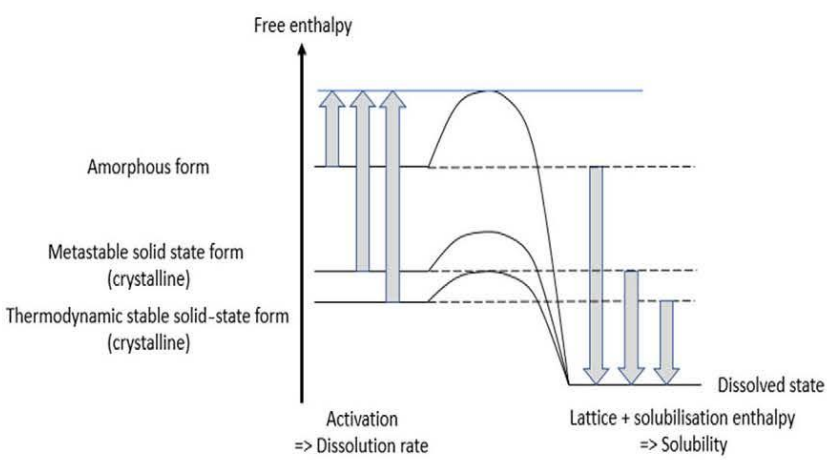

Figure 3 Enthalpic processes involved in the dissolution process.

the amorphous state, metastable polymorphs provide a much lower increase in solubility, which is why they are less attractive to develop. A schematic summary of the enthalpic processes involved in the dissolution process is given in Figure 3.

For this reason, optimisation of solubility, dissolution rate and consequently bioavailability can be best realised by selecting an appropriate pharmaceutical salt or cocrystal. Even though salt selection is well established in the pharmaceutical industry, cocrystals have also emerged during recent years as part of more educated solid form engineering.

A comprehensive summary of salt selection is given by Stahl ${ }^{[102]}$ as well as Neau. ${ }^{[103]}$ Historically, there have also been a few publications which summarise the use of pharmaceutical salts. ${ }^{[104}{ }^{108]}$ From such analysis, it is clear that hydrochlorides historically represented the most frequently used pharmaceutical salts for basic APIs and the same was true for sodium if acidic APIs were used. However, in recent decades, it has been clearly recognised that these 'one-fits-all' counterions did not always lead to the desired properties intended by the salt formation. This is in line with the observation that new drug candidates entering clinical development, in addition to newly approved drugs, exhibit significantly lower solubility (BCS class II and IV) and require more individualised solutions to optimise their physicochemical behaviour. This does not only include above-mentioned solubility and dissolution aspects, but also further parameters such as hygroscopicity, melting point, chemical and physical stability as well as crysta habit. Several examples of optimising such properties can be found in the literature. ${ }^{[109}{ }^{112]}$ In many cases, sulfonate salts such as mesylates, tosylates, napsylates and edisilates showed far better performance with regards to increased solubility and dissolution rate ${ }^{[113]}$ compared to other salt forms. Lower dissolution rate of the parent (either free base or free acid) compared to pharmaceutical salts can be easily explained by the presence of ions in the crystal lattice of a pharmaceutical salt, leading to stronger interactions between the crystal lattice and water as the dissolution medium. However, a similar explanation as to why, for example, sulfonate salts generally show excellent dissolution rate so far is not available. This phenomenon remains unexplained at least by simple chemical reasoning.

However, there are other more systematic approaches to improve solubility by usage of pharmaceutical salts, which are based on mechanistic understanding: as solubility depends on crystal lattice energy, and high lattice energy will generally result in low solubility, reducing the lattice energy represents a means to improve solubility. For pharmaceutical salts, Coulomb interactions contribute with a major part to the lattice energy. Use of large, 'noncoordinating' counterions with low charges (one positive charge or one negative charge) are useful in designing such pharmaceutical salts with improved solubility. ${ }^{[14]}$ In the extreme case, such pharmaceutical salts do not even represent solid APIs anymore, but become liquids. $\left.{ }^{[15}{ }^{118}\right]$ As stability can be more challenging for such ionic liquids compared to solids due to increased mobility, this certainly has to be assessed critically. Moreover, toxicological assessment and regulatory acceptance represent the major hurdles for the use of such noncoordinating ions in humans, as none of these salt formers are currently used in human beings or 'generally regarded as safe' (GRAS) by the FDA. ${ }^{[119]}$ Therefore, this promising conceptual work on noncoordinating ions has not yet led to approved drugs on the market nor in clinical development.

From a more practical standpoint, several aspects must be considered for selection of pharmaceutical salts or cocrystals. The process steps include salt- or coformer screening and physicochemical characterisation with a typical focus on solubility and dissolution rate but also regarding chemical and physical stability while considering 


\section{Thesis Publications}

toxicological aspects of the selected salts or coformers. This screening stage represents the starting points for the selection of a salt or cocrystal, respectively. Earlier, such screening was carried out as a typical synthesis process in lowe gram-scale. Today salt or coformer screening is conducted on the lower mg-scale which saves API and allows conductance of such screens earlier during the research process. For this purpose, different screening approaches exist in the pharmaceutical industry. A salt or cocrystal screening might be carried out in an automated way using robotic systems that employ different solvents and solvent mixtures according to a fixed protocol. Alternatively, salt or cocrystal screens can be based on a rationale, nonautomated process where small-scale crystallisation trials are performed and crystallisation is closely observed. This allows information to be obtained sequentially from one experiment to another. Even though for a single trial, larger amounts of API are required compared to the automated approach a repetition of unsuccessful experiments is avoided. A useful comparison of different screening approaches can be found in the literature for salt selection. ${ }^{[120]}$

Following initial screening of possible salt and cocrystal formation, characterisation of solid-state properties, including melting point, hygroscopicity and stability is important; as well as analysis of solubility, dissolution and supersaturation. The aforementioned steps to salt and cocrystal formation offer different opportunities to use solubility parameters. One application is certainly the selection of organic solvents or antisolvents for solid form screening, which is in line with the previous section. Other applications of partial solubility parameters are selection of ionic liquids even though they are still currently quite toxic or not sufficiently characterised leading to regulatory hurdles in pharmaceutical development. More important therefore are applications to the field of cocrystals where it has been attempted to predict formation based on HSP. ${ }^{[121]}$ A recent study conducted cocrystal search of itraconazole during which the HSP was used to rank different amino acids as potentially suitable coformers. ${ }^{[122]}$ In spite of such reports, there are also typical limitations of solubility parameters because they cannot account for specific aspects of the crystal lattice. There are currently methods in structural informatics that can use the Cambridge Structural Database to assist in silico screening of the energy landscape of possible crystals. These approaches together with other computational methods used in pharmaceutical solid-state chemistry have been recently reviewed in an excellent book. ${ }^{[123]}$ Therefore, partial solubility parameters can be applied successfully in the field of salt and cocrystal screening, however in cases where details of a crystal lattice energy landscape should be considered, alternative methods of solid-state modelling are recommended.

\section{Solubility parameter concept in lipid-based formulations}

Lipid-based formulations have been an important part of the toolbox to formulate low water-soluble compounds for over 50 years. Additionally, an increased prevalence of such poorly soluble drug candidates in the last two decades has also intensified the interest in lipid-based drug delivery systems (LBDDS). The biopharmaceutical advantages of LBDDS include the potential to enhance bioavailability, decrease pharmacokinetic variability as well as food effects, promote lymphatic absorption and support controlled drug release. ${ }^{[124}$

A key element of LBDDS is that the drug substance is dissolved within the lipid excipients before administration, hereby presenting the drug in a presolubilised state in the gastrointestinal tract. The level of solubility in the LBDDS is, hence, important to achieve the above-mentioned biopharmaceutical advantages. Therefore, options to predict and rank drug solubility in lipid excipient mixtures are of high interest to guide LBDDS formulation development. Generally, the solubility in lipid excipients is determined experimentally; however, these experiments are costly, time-consuming, resource-intensive and the experimental protocols vary substantially between different research groups. ${ }^{[125]}$ Also, LBDDS are often mixtures of different excipients, eg. lipids, surfactant and cosolvents, so the number of typical solubility studies required can be very extensive. ${ }^{[125,126]}$ Recently, the utility of empirical models to predict drug solubility in lipids has been explored, using multivariate data analysis with several molecular descriptors. ${ }^{[125}{ }^{127]}$ A disadvantage of any such multivariate approach is that the size and quality of the calibration dataset highly influences the accuracy of the prediction. Thus, an alternative to complex computational models, is the HSP approach that has been proposed to guide lipid-excipient selection. Dumanli et al..$^{[128]}$ used the HSP to rank compound solubility and miscibility in/with lipids. The required solubility parameters can be obtained either from experiments or from calculations as previously outlined in Section 2. For lipid-based excipients, vapour pressure and boiling point determinations, miscibility of reference liquids (with known cohesive energy), inverse gas chromatography and calculations using group contribution methods are of particular interest to determine HSP. ${ }^{[129]}$ Solubility parameters have been commonly employed for this purpose, especially for characterisation and release of raw materials for cosmetics ${ }^{[30}{ }^{132]}$ and in the oil and textile industry. ${ }^{[133]}$ However, there are not many applications of solubility parameters for lipid-based systems in the pharmaceutical field. Most studies determine the solubility parameter using theoretical group contribution approaches 


\section{Thesis Publications}

and often only consider simple mixtures, i.e. compound $A$ in oil $\mathrm{B} .{ }^{[84,134]}$

De La Peña-Gil and coworkers ${ }^{[135]}$ investigated methods to predict and calculate HSP of complex lipidic mixtures (i.e. vegetable oils) using the HSPiP software. ${ }^{[67]}$ Two approaches were used to determine HSPs. In one approach it was assumed that each functional group (fatty acids, fatty acids + glycerol and fatty acid methyl esters) present in triglycerides, had an additive contribution to the dispersion, dipole-dipole and hydrogen bonding interactions. Therefore, the composition of triglycerides was divided into different functional groups and each component of the total HSP $\left(\delta_{t}\right)$ was calculated (i.e. $\delta_{d}, \delta_{p}$ and $\delta_{h}$ ). The second approach assumed that vegetable oils are mixtures of simple triacylglycerols in the same mass fractions as the fatty acids. ${ }^{[135]}$

Two studies by Shah and Agrawal ${ }^{[84,136]}$ investigated the utility of HSP as a predictor for optimal carrier and solvent system selection: ${ }^{[136]}$ firstly to describe the solubility behaviour of a drug (ciprofloxacin $\mathrm{HCl}$ ) and lipid excipients and secondly, for the design of solid lipid nanoparticles (SLNs). ${ }^{[84]}$ Both studies used the Van Krevelen/Hoftyze group contribution approach to calculate the HSP for different solvents, polymers and lipids using the Molecular Modeling Pro software. Calculation of the solubility parameter was based on at single repeating polymeric unit for the polymers whilst, for lipids consisting of mixture of glycerides the calculation was carried out based on an average predominant ratio. ${ }^{[136]}$ With the Van Krevelen approach, the molar attraction constant $(F)$ was calculated and all the extended HSPs determined. The calculated values of the total HSP were matched between different lipids, organic solvents and drug compounds and when similar values were observed then the systems were considered miscible. Shah and Agrawal ${ }^{[84]}$ validated the mathematical model used by experimental testing of drug solubility in the selected excipients and solvents. Both studies ${ }^{[8,136]}$ succeeded in qualitatively predicting the solubility of ciprofloxacin $\mathrm{HCl}$ in different lipids and subsequently the solubility of lipids in various organic solvents using the HSP determination/calculation. This approach resulted in identifying the most promising lipid candidates for maximum drug loading in SLN formulation consisting of glyceryl monocaprylate and glycerol monostearate 40-55. These studies demonstrate the possibility of using the HSP for optimal selection of excipients in designing SLNs and qualitative prediction of excipient-drug compatibility.

Another application of the HSP was suggested by $\mathrm{Li}$ et al. ${ }^{[137]}$ to estimate the compatibility between materials to facilitate the design of polymer-lipid hybrid nanoparticles (PLN). Specifically, the enthalpies of mixing for a drugpolymer complex (i.e. verapamil in dextran-sulfatesodium) and 15 different lipids including triglycerides, fatty acids, glycerol esters and mixtures of glycerol esters were predicted by accounting for the volume fractions of components in the mixture. The study also used the Van Krevelen/Hoftyzer group contribution approach to calculate HSP and showed the suitability of the HSP theory in the screening of lipid carriers for PLN design of verapamil. ${ }^{[137]}$

While useful to determine how a lipid or lipid mixtures may behave as solvent(s) and the possibility to predict how some compounds solubilise in lipids, the HSP concept has also some limitations. Firstly, the concept only considers the energies derived from direct contact of components and does not account for the entropy effects and the free volume of, for example, amorphous solids. ${ }^{[84]}$ The free volume is defined as an empty space in a solid or liquid that is not occupied by the molecules. Generally, amorphous solids are inefficiently packed and present a considerable amount of free volume compared to ordered materials. Additionally, it was mentioned before as a disadvantage that a given crystalline solid-state form is not accounted for. By contrast, experimental HSP determinations are generally conducted with the most stable polymorph. Studies have suggested that HSP calculations based on group contribution methods provide accurate predictions if materials with similar structures are compared, as the calculation does not account for the dependencies on conformation, concentration and specific intermolecular interactions that might occur in binary mixtures. ${ }^{[134]}$ Finally, another limitation of the theoretical model is that it disregards specific self-association of molecules, which is also the case with further thermodynamic approaches other than SAFT (statistical associating fluids theory) calculations.

The HSP approach within LBDDS has only been investigated to a limited extend, potentially due to the variability and complexity of the lipid excipient composition and the multitude of drug-excipient interactions. However, there are further pharmaceutical applications that are to some extent linked to lipids in general such as intestinal drug absorption, ${ }^{[138]}$ skin penetration of topically applied drugs, ${ }^{[139]}$ and prediction of drug-nail interactions. ${ }^{[140]}$ The available studies suggest that partial solubility parameters have the potential to be a useful tool in early development of LBDDS.

\section{Solid dispersions}

\section{Amorphous solid dispersions}

Modification of the solid drug form to increase solubility and dissolution rate has been mentioned before (Cocrystal and salt screening section). In this endeavour, the high energy that the amorphous state can provide is especially interesting. ${ }^{[2,141}{ }^{143]}$ However, the amorphous state is thermodynamically unstable and tends to revert to a crystalline 


\section{Thesis Publications}

polymorph. The amorphous drug state can be stabilised by solid dispersion, ${ }^{[144,145]}$ complexation with large (e.g. cyclodextrins) ${ }^{[146]}$ or small molecules, ${ }^{[147]}$ and spatial confinement (e.g. absorption on silica). ${ }^{[148,149]}$

Solid dispersions involving the immobilisation of amorphous API in polymer have been studied extensively in the

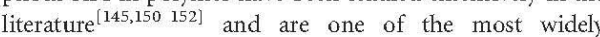
employed approaches to formulate an amorphous drug form. Amorphous solid dispersions (ASD) is an umbrella term for different types, which can be glass solution, glass suspension and solid solutions of drug in the carrier. ${ }^{[145,153]}$ Drug-polymer miscibility is a key parameter to consider when formulating solid dispersions ${ }^{[154,155]}$ and to obtain a single phase system, both the API and the carrier have to be miscible. Such miscibility can be calculated using advanced thermodynamic models, such as the previously mentioned PC-SAFT and COSMO-RS theories or the simpler FloryHuggins model may be applied. ${ }^{[156]}$ The advantages of suitable prediction accuracy of advanced thermodynamic approaches have to be balanced against drawbacks like either extensive computation time or the need for parameters that have to be based on extensive experimentation. This makes the simpler solubility parameter approach very attractive and either the total Hildebrand solubility parameter can be considered or partial solubility parameters. Thus, HSP has been extensively used to identify drug-excipient miscibility with a general rule of thumb that molecules with similar $\delta$ values are assumed to be miscible. ${ }^{[32,121,155,157]}$ This method considers the dispersion and polar interactions of a system as well as the hydrogen bond contributions of the test molecules. We emphasise again that HSP hereby addresses the main limitation of the conventional Hildebrand solubility parameter, which does not discriminate the different partial contributions to cohesive energy density. ${ }^{[1]}$

However, ASD formulation miscibility has been investigated with both the Hildebrand ${ }^{[158]}$ as well as the Hansen $\left.{ }^{[85,159} 161\right]$ approach. It was proposed by Greenhalgh and coworkers ${ }^{[155]}$ that systems with a difference in solubility parameters from 1.6 to $7.0 \mathrm{MPa}^{0.5}$ present no miscibility problems. However, substances with a difference of 10.8 to 18.0 $\mathrm{MPa}^{0.5}$ were considered immiscible. Further practical measurements showed that systems with a difference of 1.6 to $7.5 \mathrm{MPa}^{0.5}$ could be considered miscible in the molten stage. Systems with a difference of 7.4 to $15 \mathrm{MPa}^{0.5}$ were slightly immiscible in the liquid state. Total immiscibility was observed in systems with differences greater than $15 \mathrm{MPa}^{0.5}$ [155]

With the Hansen approach similar observations were made when testing the miscibility of 2 drugs in 11 excipients. ${ }^{[85]}$ Systems that were predicted to be miscible using the Greenhalgh values formed glass solution via hot melt extrusion while those combinations that were predicted to be immiscible formed solid dispersions in which the amorphous drugs was dispersed in crystalline carriers. On the other hand, when the Hildebrand approach was utilised in combination with $\log P, \mathrm{p} K_{a}$ and $T_{g}$ considerations, amorphous miscibility between additives and polymers failed to be predicted for systems in which acid-base interactions took place. ${ }^{[158]}$ Systems which were predicted to be immiscible, but showed acid-base interactions, still resulted in ASD formulations. Contrastingly, this approach accurately predicted miscibility and ASD formation for systems produced via a solvent evaporation method without acid-base interactions. This underlines again that the Hildebrand concept with a single value for total solubility parameter cannot adequately account for specific or hydrogen bonding interactions.

As mentioned previously, the HSP concept offers a 3-D solubility parameter concept that can be visualised in the Hansen space (Figure 1). A way to project a 3-D solubility parameter in a two-dimensional plane was proposed by Bagley et al. ${ }^{[162]}$ It was argued that the thermodynamic contribution to the solubility parameter of the polar and dispersion interactions are often similar, and therefore, a combined solubility parameter $\left(\delta_{v}{ }^{2}\right)$ can be derived from Equation 8 to yield the following Equation ${ }^{[162]}$ :

$$
\delta_{d}^{2}+\delta_{p}^{2}=\delta_{v}^{2}
$$

The plot of $\delta_{v}$ vs $\delta_{b}$, simplifies the 3-D Hansen space into the 2-D plane, which is referred to as a Bagley plot. Analogues to the previously discussed Hansen space, it is assumed that molecules in vicinity of each other are more likely to be miscible. ${ }^{[32,154]}$ The Bagley plot has been utilised to evaluate and predict miscibility between molecules using the $R_{a(v)}$ parameter, which gives an idea of the 'area of miscibility' around a molecule, and should be $\leq 5.6 \mathrm{MPa}^{1 / 2}$ for miscibility. This parameter is analogous to the $R_{a}$ in Equation 21 but uses the simplification of Equation 39 to a drug-polymer system:

$$
R_{a}(v)^{2}=\left[4\left(\delta_{v 2}-\delta_{v 1}\right)^{2}+\left(\delta_{h 2}-\delta_{h 1}\right)^{2}\right]
$$

Bagley plots are highly versatile and have been used to investigate the miscibility of polymers in solvents, ${ }^{[82]}$ drugs in excipients ${ }^{[32,154,157]}$ and polymers in polymers. ${ }^{[163]}$ It is important to consider the selected method when calculating solubility parameters, as different methods can give rise to different Bagley plots, which was highlighted by Meaurio and colleagues. ${ }^{[163]}$

These theoretical considerations were applied to ASD formations by different authors. ${ }^{[154,157]}$ One study tested 84 drug molecules for their miscibility in PEG and demonstrated a good correlation between the group of drugs forming ASDs with PEG with the plot region around PEG derived from theoretical solubility parameter calculation. ${ }^{[154]}$ 


\section{Thesis Publications}

Alhalaweh et ai. ${ }^{[157]}$ compared experimentally derived miscibility data of indomethacin and excipients with predicted miscibility data. The Bagley plots of these data sets were almost identical and the predicted data showed a good correlation to the ASD formation.

Another prerequisite for the formation and stability of successful ASDs is that the change in the free energy of the system upon mixing should be negative. Because ideal mixing increases the entropy of the system, the entropy contribution should facilitate mixing. Consequently, it is the enthalpy contribution that may prevent free mixing energy $\Delta G_{m}$ to be negative. In drug-polymer systems the FloryHuggins equation has been adapted to align with the lattice-based Equation (40) used for the dissolution of

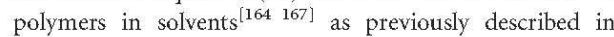
Equation 5.

$$
\Delta H_{m}=V_{d p} \varphi_{d} \varphi_{p}\left(\delta_{d}-\delta_{p}\right)^{2}
$$

where subscript $d$ and $p$ describe drug and polymer respectively, while $\Delta H_{m}$ can, also, be given by the van Laar expression according to Equation 42 :

$$
\Delta H_{m}=\chi_{d p} \varphi_{d} \varphi_{p} R T
$$

From Equation 42 it can be seen that the value of the drug-polymer interaction parameter, $\chi_{d p}$, could be a surrogate for the enthalpy of the system, for given conditions. By combining Equations (40) and (41), the drug-polymer interaction parameter can be given by Equation 42 (neglecting $\beta$ ) which is in line with the previously discussed Equation 6:

$$
\chi_{d p}=\frac{V_{d p}\left(\delta_{d}-\delta_{p}\right)^{2}}{R T}
$$

where $V_{d p}$ is volume of mixture, $\varphi_{d}$ and $\varphi_{p}$ are volume fractions of drug and polymer, and $\delta_{d}$ and $\delta_{p}$ are solubility parameters of drug and polymer.

Similar to the total (or Hildebrand) solubility parameter, the HSP has also been linked to the drug-polymer interaction parameter $\left(\chi_{d p}\right)$ by consideration of partial contributions to cohesive energy density. ${ }^{[82]}$

The drug-polymer interaction parameter has been used widely for the investigation of binary mixtures especially, miscibility based on a negative $\Delta G_{m}$. Although this parameter can be determined by melting point depression, $\left.{ }^{[165,167} 169\right]$ there are also some studies that use the 3-D solubility parameter approach ${ }^{[154,167,169]}$ to investigate drug-excipient miscibility in a more quantitative way. Such construction of phase diagrams show the differentiation of single and two phase system at equilibrium (binodal line) and kinetic decomposition of metastable mixtures (spinodal line) can be estimated. ${ }^{[170,171]}$ It is attractive to construct entire phase diagrams but this objective is more ambitious than use of the solubility parameters to simply rank excipient selection based on rules of thumb, such as based on the proximity of drug and excipient representations in the Hansen space or Bagley plot. A key strength of the solubility parameter is that such approaches are in general rather simple. A more ambitious quantitative calculation of entire phase diagrams can suffer from the simplifications of the thermodynamic approach as well as from lacking prediction of solubility parameter estimates. Phase diagrams constructed via solubility parameters might therefore be better considered as a first approximation only.

\section{Mesoporous silica}

Recent developments suggests mesoporous silica as a new and promising material to formulate poorly water-soluble drugs with the intent to increase dissolution rate and solubility. $\left.{ }^{[49,172} 176\right]$

Loaded mesoporous silica is prepared via adsorption of API from a concentrated organic solution followed by evaporating the solvent. This adsorption into the porous network stabilises the API in the amorphous state, due to steric effects. Upon administration, the amorphous API is then released and the dissolution is increased. ${ }^{[173]}$

Utilisation of the solubility parameter in the development of amorphous mesoporous silica is still largely unemployed without commercial formulations that are globally on the market. This represents a key gap in the literature, as solubility parameters can guide the selection of suitable solvent to maximise the penetration efficiency of API into mesoporous silica, which was demonstrated by Hideo Hata and coworkers. ${ }^{[177]}$ They showed that, of six different solvents used to incorporate taxol onto mesoporous silica, only two resulted in effective loading of the API. They concluded that solubility parameter was a key factor in this observation; where solvents that interacted strongly with taxol resulted in the most effective loading, whereas those with weak interaction showed poor loading efficiency. Such interaction can be determined using solubility parameter approach. ${ }^{[177]}$ However, the range of solvents suitable for use with formulation development is limited. Those solvents that show the strongest solubilisation power are often seen as 'no-go' solvents based on ICH guidelines. ${ }^{[18]}$ Therefore, most instances of mesoporous silica use in the literature are carried out with either ethanol, acetone or dichloromethane, with even the latter being less utilised due to toxicity considerations. ${ }^{[149]}$ The ICH list certainly limits solvent selection but not to the extent as it is currently reflected in the literature on mesoporous silica so there is a potential to access a wider range of solvents by employing a rank-order-based protocol based on in silico solubility parameter calculation. 


\section{$\mathrm{X}$. Thesis Publications}

Amorphous solid dispersions systems have proved to be a useful formulation tools in battling the solubility issues of pharmaceutical molecules. However, because of their limitations in stability, miscibility of the system is of crucial importance for a successful formulation. This makes the identification of tools to evaluate miscibility in the development phase, not only useful, but imperative. Herein the application of solubility parameter concepts to assess miscibility has been reviewed and discussed in the previous section. It should be emphasised again that a consideration of partial solubility parameters is preferred to a simple comparison of total (i.e. Hildebrand) solubility parameters. Moreover, the need of a complete database for partial solubility parameter either for drug and polymer was highlighted when using HSP. A limited number of data is not only available with respect to experimentally determined values but also for group contributions as used for in silico prediction of solubility parameters. The latter limitation can be especially troublesome in case of HSP estimation for more complex drug molecules. Application of the modified solubility parameter approach was applied by Piccinni ${ }^{[179]}$ and even though the usefulness for early excipient ranking was recognised, it was also emphasised that physical screening tests should not be replaced by the in silico method until more robust prediction methods are available. This supports what was discussed in the previous sections that solubility parameters can be calculated in silico to rank excipients for a subsequent experimental screening. Such screening phase can therefore be designed in a more focused and cost-effective way rather that be entirely replaced.

\section{Application of solubility parameters in the formulation of nano- and microparticulate systems}

One of the most used methods for dissolution improvement of poorly water-soluble drugs is particle size reduction. The decrease in size increases the surface to volume ratio, which accelerates dissolution kinetics. Especially interesting here are sizes well below $1 \mu \mathrm{m}$, which have the highest potential increase the kinetic drug solubility ${ }^{[180,181]}$ in addition to the dissolution rate enhancement. Thus, by bringing particle size to the nanoscale, solvation pressure increases and facilitates the disruption of the intrasolute bonds thus promoting solubilisation. ${ }^{[182]}$

Micronisation and nanosizing methods can be grouped into two categories: the top-down methods that entails size reduction of bigger particles by the use of shear forces and the bottom-up methods that involve the isolation of drug particles after recrystallisation from a highly supersaturated drug solution. ${ }^{[181]}$ Although traditional micronisation techniques, such as dry-milling, are still being used, bottom-up approaches are becoming increasingly sophisticated, gaining merit as techniques that can circumvent typical process limitations. ${ }^{[183]}$ These limitations may involve unwanted amorphisation, disruption of the crystal lattice and unpredictable particle size distributions. ${ }^{[184]}$ However, bottomup approaches also face their individual limitations. Due to the fact that they rely on the controlled precipitation of particles from supersaturated solutions, miscibility and solubility considerations should be taken into account for the optimal choice of formulation combinations.

Moreover, technical developments in both top-down (e.g. 'pearl' milling and high-pressure homogenisation) and bottom-up techniques (e.g. new-generation spray dryers, supercritical fluid technology and freeze drying) have broadened the availability of nanosizing approaches. As nanoparticles exhibit high surface cohesive energies, they are especially prone to aggregation, ${ }^{[185,186]}$ making their stabilisation indispensable. The use of various stabilisers, often surfactants and/or polymers, has been reported. It is proposed that they work by sterically or ionically stabilising the surface of nanoparticles, to limit aggregation. ${ }^{[187]}$ The choice of appropriate stabilisers seems to greatly depend on the physical characteristics of the surface of drug molecules. ${ }^{[188]}$ Consequently, the need for reliable tools to estimate possible candidates for nanoparticle formulations is evident.

Several attempts for nano- and micronised formulations have been reported in literature using a variety of materials including proteins, lipids and polymers. ${ }^{[189]}$ We considered here not only the dassical top-down and bottom-up approaches but more broadly reviewed also micro- and nanoparticle formation. Calculated solubility parameters are used eventually here as an early evaluation of miscibility of solvent-solute combinations as well as miscibility between molecules. However, evidence of using solubility parameters later during formulation development is very limited. To the best of our knowledge, only few relevant literature references exist, i.e. where a solubility parameter approach has been used for choosing the appropriate lipids in drug-polymerlipid nanoparticles, ${ }^{[137]}$ for choosing the drug-polymer combinations for the construction of polymeric micelles and for predicting drug loading. ${ }^{[190,191]}$ Furthermore, Mahmud et al. ${ }^{[191]}$ and Dwan' Isa et al. ${ }^{[190]}$ used the HSP to calculate the Flory-Huggins interaction parameter of drug-polymer combinations $\left(\chi_{i 0}\right)$ and considered it as a measure of miscibility. ${ }^{[192]}$ Dwan'Isa et al. tested 19 drugs for their interactions with a diblock copolymer (MePEG- $b$-(PCL-co-TMC)), while Mahmud et al. studied the interactions of one anticancer drug (curcubitacin I) against a variety of core-forming polymers. In both studies, the calculation of the $\chi_{d p}$ parameter was able to reveal the optimal drug-polymer combinations, as well as to predict the drug loading capacity of the polymer micelle formulations. Even though the calculation of the interaction parameter provided a reasonably accurate 


\section{Thesis Publications}

method for the qualitative prediction of drug solubilisation in the polymeric micelles, a definite rank order of combination miscibility among the variations tested could not be achieved, which may be attributed to the limitations of the Flory-Huggins theory as well as lacking accuracy of estimating the interaction parameter. Simplifications included the disregard of polymer molecular weight and polymer solvent interactions. ${ }^{[192]}$ Nevertheless, the calculations of the HSP and the Flory-Huggins interaction parameter have been proven to be adequate tools for the primary selection of potentially miscible nano- and microparticulate systems, which helps in reducing the number of early screening experiments.

\section{Conclusions}

Solubility parameters have proven to be useful in diverse scientific fields, and this review has outlined the different applications concerning oral delivery of poorly water-soluble compounds. These compounds typically require bioenabling formulation for successful development and solubility parameters can help, for example with ranking of solvents and excipients to achieve a more focused formulation development. Herein, predictions based on chemical structure are particularly interesting as only limited compound is available in early development. Despite the usefulness of applying solubility parameters in pharmaceutics, there are also some gaps. In particular, drugs in a solid crystalline state or also rather complex molecules in general may result in erratic predictions of solubility parameters. However, it is promising that new theoretical developments have been reported that present conceptual improvements. Moreover, experimental methods such as high-throughput solubility testing or greater availability of inverse gas chromatography will help in generating more data. Important is to obtain more comparative data of experimental and computational methods to better learn about variability of results. This can in turn help to further improve in silico predictions of solubility parameters, and a just accepted article has been following this research direction. ${ }^{[193]}$ Therefore, it can be expected that solubility parameters will also in the future rank among the mostly used thermodynamic approaches in pharmaceutics.

\section{Declarations}

\section{Conflict of interest}

The Authors declare that they have no conflict of interests to disclose.

\section{Funding}

This project has received funding from the European Union's Horizon 2020 Research and Innovation Programme under Grant Agreement No 674909 (PEARRL).

\section{References}

1. Hansen CM. Hansen Solubility Parameter a User's Handbook, 2nd edn. Boca Raton, FL: CRC Press, 2007.

2. Hancock B et al. The use of solubility parameters in pharmaceutical dosage form design. Int $J$ Pharm 1997; 148: $1-21$.

3. Kuentz $\mathrm{M}$ et al. Methodology of oral formulation selection in the pharmaceutical industry. Eur $J$ Pharm Sci 2016; 87: 136-163.

4. Hildebrand $\mathrm{JH}$, Scott RL. Solubility of Nonelectrolytes, 3rd edn. New York, NY: Dover, 1964

5. Stefanis E, Panayiotou C. Prediction of hansen solubility parameters with a new group-contribution method. Int $J$ Thermophys $2008 ; 29: 568-585$.

6. Just $S$ et al. Improved group contribution parameter set for the application of solubility parameters to melt extrusion. Eur $J$ Pharm Biopharm 2013; 85(3 Part B): 1191-1199.
7. Díaz I et al. Comparison between three predictive methods for the calculation of polymer solubility parameters. Fluid Phase Equilib 2013 337: 6-10.

8. Panayiotou $\mathrm{C}$ et al. Redefining solubility parameters: bulk and surface properties from unified molecular descriptors. J Chem Thermodyn 2017; 111: 207-220.

9. Louwerse $\mathrm{M} T$ et al. Revisiting Hansen solubility parameters by including thermodynamics. ChemPhysChem 2017; 18: 2999-3006.

10. Bustamante $\mathrm{P}$ et al. The modified extended Hansen method to determine partial solubility parameters of drugs containing a single hydrogen bonding group and their sodium derivatives: benzoic acid/Na and ibuprofen/Na. Int JPharm 2000; 194: 117-124.

11. Bashimam M. Hansen solubility parameters: a quick review in pharmaceutical aspect. I Chem Pharm Res 2015; 7: 597-599.
12. Scatchard G. Equilibria in non-electrolyte solutions in relation to the vapor pressures and densities of the components. Chem Rev 1931; 8: 321-333.

13. Hildebrand JH, Wood SE. The derivation of equations for regular solutions. J Chem Phys 1933; 1: 817.

14. Biroa $I$ et al. Prediction of the $x$ parameter by the solubility parameter and corresponding states theories. Macromolecules 1971; 4: 30-35.

15. Hansen CM. The Three Dimensional Solubility Parameter and Solvent Diffusion Coefficient. Their Importance in Suface Coating Formulation. Danish Technical Press, 1967. Available at: https:/hansen-solubility.com/contents/ HSP1967-OCR.pdf.

16. Barton A. Handbook of Solubility Parameters and Other Cohesion Parameters, 2nd edn. Boca Raton, FL: CRC Press, 1991.

17. Brown HC, Grayson M. Homomorphs of 2,6-dimethyl-t-butylbenzene. $J \mathrm{Am}$ Chem Soc 1953; 75: 20-24. 


\section{Thesis Publications}

18. Brown $\mathrm{HC}$ et al. Strained homomorphs. ${ }^{1} 14$. General summary. Am Chem Soc 1953; 75: 1-6.

19. Blanks RF, Prausnitz JM. Thermodynamics of polymer solubility in polar and nonpolar systems. Ind Eng Chem Fundam 1964; 3: 1-8.

20. Hansen CM, Skaarup K. Independent calculation of the parameter components. J Paint Technol 1967; 37: 511515.

21. Hansen CM, Beerbower CH. Solubility parameters. Kirk-Othmer Encycl Chem Technol 1971; 75: 889-910.

22. Hansen CM. 50 Years with solubility parameters-past and future. Prog Org Coat 2004; 51: 77-84.

23. Small PA. Some factors affecting the solubility of polymers. J Chem Technol Biotechnol 1953; 3: 71-80.

24. Sorenson P. Application of the acid/ base concept describing the interaction between pigments, binders, and solvents. J Paint Technol 1975; 47: 31.

25. Beerbower A et al. Expanded solubility parameter approach I: naphthalene and benzoic acid in individual solvents. J Pharm Sci 1984; 73: 179-188.

26. Barra $J$ et al. Proposition of group molar constants for sodium to calculate the partial solubility parameters of sodium salts using the van Krevelen group contribution method. Eur $J$ Pharm Sci 2000; 10: 153-161.

27. Verheyen $S$ et al. Determination of partial solubility parameters of five benzodiazepines in incividual solvents. Int I Pharm 2001; 228: 199 207.

28. Bustamante $P$ et al A modification of the extended Hildebrand approach to predict the solubility of structurally related drugs in solvent mixtures. I Pharm Pharmacol 1993; 45 253-257.

29. Jouyban A, Acree WE. Comments on "Prediction of Drug Solubility in Lipid Mixtures from the Individua Ingredients". AAPS PharmSciTech 2014; 15: 83-85.

30. Vay $\mathrm{K}$ et al. Application of Hansen solubility parameters for understanding and prediction of drug distribution in microspheres. Int J Pharm 2011 416: 202-209.
31. Muela $S$ et al. Influence of temperature on the solubilization of thiabendazole by combined action of solid dispersions and co-solvents. Int $J$ Pharm 2010; 384: 93-99.

32. Kitak $\mathrm{T}$ et al. Determination of solubility parameters of ibuprofen and ibuprofen lysinate. Molecules 2015 20: 21549-21568.

33. Howell $\mathrm{J}$ et al. A functional approach to solubility parameter computations. I Phys Chem B 2017; 121: 4191-4201.

34. Archer WL. Determination of Hansen solubility parameters for selected cellulose ether derivatives. Ind En Chem Res 1991; 30: 2292-2298.

35. Bustamante $P$ et al. Partial solubility parameters of piroxicam and niflumic acid. Int J Pharm 1998; 174: 141-150.

36. Martin A et al. Extended Hansen solubility approach: naphthalene in individual solvents. J Pharm Si 1981 70: 1260-1264.

37. Van Dyk JW et al. Solubility, solvency, and solubility parameters. Ind Eng Chem Prod Res Dev 1985; 24 473-478.

38. Ravindra $\mathrm{R}$ et al. Solubility parame ter of chitin and chitosan. Carbohyd Polvm 1998; 36: 121-127.

39. Segarceanu $O$, Leca $M$. Improved method to calculate Hansen solubility parameters of a polymer. Prog Org Coat 1997; 31: 307-310.

40. Klar F, Urbanetz NA. Solubility parameters of hypromellose acetate succinate and plasticization in cry coating procedures. Drug Dev Ind Pharm 2016; 42: 1621-1635.

41. Kent DJ, Rowe RC. Solubility studies on ethyl cellulose used in film coating. J Pharm Pharmacol 1978; 30: 808-810

42. Han KH et al. Prediction of solubility parameter from intrinsic viscosity Ind Eng Chem 2013; 19: 1130-1136.

43. Bustamante $P$ et al. A new method to determine the partial solubility parameters of polymers from intrinsic viscosity. Eur J Pharm Si 2005; 24 229-237.

44. Madsen CG et al. Simple measurements for prediction of drug release from polymer matrices - solubility parameters and intrinsic viscosity. Eur J Pharm Biopharm 2015; 92: 1-7.
45. Weerachanchai $\mathrm{P}$ et al. Determination of solubility parameters of ionic liquids and ionic liquid/solvent mixtures from intrinsic viscosity. ChemPhysChem 2014; 15: 3580-3591.

46. Mieczkowski R. The determination of the solubility parameter components of polystyrene by partial specific volume measurements. Eur Polym J 1988; 24: 1185-1189.

47. Mieczkowski R. The determination of the solubility parameter components of polystyrene. Eur Polym I 1989; 25: 1055-1057.

48. Mieczkowski R. Solubility parameter components of some polyols. Eur Polym J 1991; 27: 377-379.

49. Huang I-C. Methods to determine solubility parameters of polymers at high temperature using inverse gas chromatography. I Appl Polym Sci 2004; 94: 1547-1555.

50. DiPaola-Baranyi G, Guillet JE. Estimation of polymer solubility parameters by gas chromatography. Macromolecules 1978; 11: 228-235.

51. Ito $\mathrm{K}$, Guillet JE. Estimation of solubility parameters for some olefin polymers and copolymers by inverse gas chromatography. Macromolecules 1979; 12: 1163-1167.

52. Adamska K, Voelkel A. Hansen solubility parameters for polyethylene glycols by inverse gas chromatography. I Chromatogr A 2006; 1132: 260-267.

53. Choi $\mathrm{P}$ et al. Measurement of threedimensional solubility parameters of nonyl phenol ethoxylates using inverse gas chromatography. J Colloid Interface Sci 1996; 180: 1-8.

54. Adamska K, Voelkel A. Inverse gas chromatographic determination of solubility parameters of excipients. Int J Pharm 2005; 304: 11-17.

55. Wang $Q$ et al. Determination of the solubility parameter of epoxidized soybean oil by inverse gas chromatography. I Macromot Sci Part B 2013; 52: 1405-1413.

56. Adamska $\mathrm{K}$ et al. New procedure for the determination of Hansen solubility parameters by means of inverse gas chromatography. $J$ Chromatogr $A$ 2008; 1195: 146-149. 


\section{Thesis Publications}

57. Price GJ, Shillcock IM. Inverse gas chromatographic measurement of solubility parameters in liquid crystalline systems. J Chromatogr A 2002; 964: 199-204.

58. Cakar $\mathrm{F}$ et al. Physicochemical characterization of 5-cecyloxy-2-[[[4-hexyloxyphenyl] imino] methyl] phenol liquid crystal by inverse gas chromatography. Optoelectron Adv Mater Commin 2008; 2: 871-875.

59. Liu $\mathrm{G}$ et al. Crystallization of piroxicam solid forms and the effects of additives. Chem Eng Technol 2014; 37: 1297-1304.

60. Langer SH et al. Gas-chromatographic study of the solution thermodynamics of hydroxylic derivatives and related compounds. I Phys Chem 1982; 86: 4605-4618.

61. Coca J et al. Thermodynamic properties of some organic compounds with tetrachloroterephthaloyl oligomers by gas chromatography. $J$ Chem Eng Data 1989; 34: 280-284.

62. Voelkel A et al. Characterization of the interactions in polymer/silica systems by inverse gas chromatography. Macromol Symp 2001; 169: 45-55.

63. Voelkel A, Fall J. Influence of predic tion method of the second virial coefficient on inverse gas chromatographic parameters. I Chromatogr A 1996; 721: 139-145.

64. Price GJ et al. Measurement of solubility parameters by gas-liquid chromatography. J Chromatogr A 1986; 369: 273-280.

65. Voelkel A, Grześkowiak T. Properties of zirconate modificates of silica gel as examined by inverse gas chromatography. Macromol Symp 2001. 169: 35-44

66. Voelkel A, Janas J. Solubility parameters of broad and narrow distributed oxyethylates of fatty alcohols. J Chromatogr A 1993; 645: 141-151.

67. Abbott $S$ et al. Hansen Solubility Parameters in Practice, HSPiP, a package of software, datasets and eBook. 2017. Available at: http:// www. hansen-solubility.com/HSPiP accessed 05-June-2017.

68. Panayiotou CG. Inverse gas chromatography and partial solvation parameters. I Chromatogr A 2012; 1251: 194-207.

69. Çavus $S$ et al. Solvent dependent swelling behaviour of poly $(N$-vinylcaprolactam) and poly ( $N$-vinylcaprolactam-co-itaconic acid) gels and determination of solubility parameters. Chem Pap 2015; 69: 1367-1377.

70. Bristow GM, Watson WF. Cohesive energy densities of polymers. Part 1. Cohesive energy densities of rubbers by swelling measurements. Trans Faraday Soc 1958; 54: 1731-1741.

71. Bristow GM, Watson WF. Cohesive energy densities of polymers. Part 2.-Cohesive energy densities from viscosity measurements. Trans Faraday Soc 1958; 54: 1742-1747.

72. Aharoni SM. The solubility parameters of aromatic polyamides. I Appl Polym Sci 1992; 45: 813-817.

73. Eroglu MS et al Determination of solubility parameters of poly (epichlorohydrin) and poly(glycidyl azide) networks. Polymer (Guildf) 1997; 38: 1945-1947.

74. Schenderlein $S$ et al. Partial solubility parameters of poly(d, l-lactide-co-glycolide). Int J Pharm 2004; 286: 19-26.

75. Suh KW, Corbett IM. Solubility parameters of polymers from turbicimetric titrations. J Appl Polym Sci 1968; 12: 2359-2370.

76. Suh KW, Clarke DH. Cohesive energy densities of polymers from turbidimetric titrations. I Polym Sci Part A-1 Polym Chem 1967; 5: 1671-1681.

77. Lin $\mathrm{X}$ et al. Hansen solubility parameters of coal tar-cerived typical PAHs using turbidimetric titration and an extended Hansen approach. I Chem Eng Data 2017; 62: 954-960.

78. Carvalho SP et al. Determining Hildebrand solubility parameter by ultraviolet spectroscopy and microcalorimetry. J Braz Chem Soc 2013; 24: 1998-2007.

79. Gee $\mathrm{G}$. The interaction between rubber and liquids. III. The swelling of vulcanized rubber in various liquids. Rubber Chem Technol 1943; 16: 263-267.

80. Hoy KL. New values of the solubility parameters from vapor pressure data. J Paint Technol 1970; 42: 76-118.

81. Fedors RF. A method for estimating both the solubility parameters and molar volumes of liçuids. Polym Eng Sci 1974; 14: 147-154

82. van Krevelen DW. Properties of Polymers: Their Correlation with Chemical Structure; Their Numerical Estimation and Prediction from Additive Group Contributions, 4th edn. Amsterdam, The Netherlands: Elsevier, 2009.

83. Stefanis E, Panayiotou C. A new expanded solubility parameter approach. Int J Pharm 2012; 426: 29-43.

84. Shah M, Agrawal Y. High throughput screening: an in silico solubility parameter approach for lipids and solvents in SLN preparations. Pharm Dev Technol 2013; 18: 582-590.

85. Forster $\mathrm{A}$ et al. Selection of excipients for melt extrusion with two poorly water-soluble drugs by solubility parameter calculation and thermal analysis. Int I Pharm 2001; 226: 147-161.

86. Klamt A. COSMO-RS: From Quantum Chemistry to Fluid Phase Thermodynamics and Drug Design, 1st edn. Amsterdam, The Netherlands: Elsevier, 2005.

87. Gupta J et al. Prediction of solubility parameters and miscibility of pharmaceutical compounds by molecular dynamics simulations. $J$ Phys Chem $B$ 2011; 115: 2014-2023.

88. Ruether F, Sadowski G. Modeling the solubility of pharmaceuticals in pure solvents and solvent mixtures for drug process design. I Pharm Sci 2009; 98: 4205-4215.

89. Jouyban A et al. Comment on "Mea surement and Correlation of Solubilities of (Z)-2-(2-Aminothiazol-4-yl) 2-methoxyiminoacetic Acid in Different Pure Solvents and Binary Mixtures of Water + (Ethanol, Methanol, or Glycol)". J Chem Eng Data 2012; 57: 1344-1346.

90. Rogers MA, Marangoni AG. Kinetics of 12-hydroxyoctadecanoic acid SAFiN crystallization rationalized using hansen solubility parameters. Langmuir 2016; 32: 12833-12841.

91. Zhu $\mathrm{Z}$ et al. Superinsulating polyisocyanate based aerogels: a targeted search for the optimum solvent system. ACS Appl Mater Interfaces 2017; 9: $18222-18230$ 


\section{Thesis Publications}

92. Masurel E et al. Screening method for solvent selection used in $\operatorname{tar}$ removal by the absorption process. Environ Technol 2015; 36: 2556-2567.

93. Cascant MM et al. A green analytical chemistry approach for lipid extraction: computation methods in the selection of green solvents as alternative to hexane. Anal Bioanal Chem 2017; 409: 3527-3539.

94. Laboukhi-Khorsi $S$ et al. Efficient solvent selection approach for high solubility of active phytochemicals: application for the extraction of an antimalarial compound from medicinal plants. ACS Sustain Chem Eng 2017; 5: 4332-4339.

95. Sánchez-Camargo AP et al. Application of Hansen solubility approach for the subcritical and supercritical selective extraction of phlorotannins from Cystoseira abies-marina. RSC Adv 2016; 6: 94884-94895.

96. Fardi $\mathrm{T}$ et al. Artwork conservation materials and Hansen solubility parameters: a novel methodology towards critical solvent selection. J Cult Herit 2014; 15: 583-594.

97. Pudipeddi $M$, Serajuddin ATM. Trends in solubility of polymorphs. Pharm Sci 2005; 94: 929-939.

98. Liu R. Water-Insoluble Drug Formulation, 2nd edn. Bosa Roca, USA: Taylor \& Francis Inc, 2008

99. Bauer $J$ et al. Ritonavir: an extraordinary case of conformational polymorphism. Pharm Res 2001; 18: 859-866.

100. Dinnebier RE et al. Structural char acterization of three crystalline modifications of telmisartan by single crystal and high-resolution $\mathrm{X}$-ray powder diffraction. $J$ pharm Sci 2000; 89: 1465-1479.

101. Cimarosti $Z$ et al. Development of drug substances as mixture of polymorphs: studies to control form 3 in casopitant mesylate. Org Process Res Dev 2010; 14: 1337-1346.

102. Stahl P, Wermuth CG. Handbook of Pharmaceutical Salts, 2nd edn. Zurich: Wiley-VCH, 2011.

103. Neau SH. Pharmaceutical salts. In: Liu R, ed. Water-Insoluble Drug Formulation, 2nd edn. Bosa Roca, USA Taylor \& Francis Inc., 2008.
104. Gould PL. Salt selection for basic drugs. Int I Pharm 1986;33: 201-217.

105. Paulekuhn GS et al. Trends in active pharmaceutical ingredient salt selec tion based on analysis of the orange book database. I Med Chem 2007 50: 6665-6672.

106. Saal C, Becker A. Pharmaceutical salts: a summary on doses of sal formers from the Orange Book. Eu J Pharm Sci 2013; 49: 614-623.

107. Berge SM et al. Pharmaceutical salts J Pharm Sci 1977; 66: 1-19.

108. Haynes DA et al. Occurrence of pharmaceutically acceptable anion and cations in the Cambridge structural database. $J$ Pharm Sci 2005; 94 2111-2120.

109. Paulekuhn GS et al. Salt screening and characterization for poorly soluble, weak basic compounds: case study albendazole. Pharmazie 2013 68: 555-564.

110. Nechipadappu SK, Trivedi DR. Pharmaceutical salts of ethionamide with GRAS counter ion donors to enhance the solubility. Eur I Pharm Sci 2017; 96: 578-589.

111. Fini $\mathrm{A}$ et al. Diclofenac salts, Part 7 are the pharmaceutical salts with aliphatic amines stable? I Pharm Sc 2012; 101: 3157-3168.

112. Surov $A O$ et al. New solid forms of the antiviral drug arbidol: crysta structures, thermodynamic stability and solubility. Mol Pharm 2015; 12 4154-4165.

113. Elder DP et al. The utility of sul fonate salts in drug development. J Pharm Sci 2010; 99: 2948-2961.

114. Saal C, Weber K-DF. Composition of anions and cations with pharmacological activity. 2016

115. Balk A et al. Ionic liquid versus prodrug strategy to address formulation challenges. Pharm Res 2015; 32: 2154-2167.

116. Frizzo CP et al. Pharmaceutical salts solids to liquids by using ionic liquid design. In: Kadokawa J, ed. Ionic Liq uids: New Aspects for the Future. Rijeka: InTech, 2013: Ch. 21.

117. Kumar V, Malhotra SV. Ionic liquids as pharmaceutical salts: a historica perspective. In: ACS Symposium series.
American chemical Society, ed. Ionic Liquid Applications: Pharmacenticals, Therapeutics, and Biotechnology, 2010: $1-12$.

118. Hough WL et al. The third evolution of ionic licuids: active pharmaceutical ingredients. New I Chem 2007; 31: 1429-1436.

119. FDA. List of Generally Regarded as Safe Ingredients. Available at: http:// www.fda.gov/Food/IngredientsPackag ingLabeling/GRAS/. Accessed November $21,2017$.

120. Fernández Casares $A$ et al. An evaluation of salt screening methodologies. J Pharm Pharmacol 2015; 67: $812-822$.

121. Mohammad MA et al. Hansen solubility parameter as a tool to predict cocrystal formation. Int $I$ pharm 2011; 407: 63-71.

122. Shetea $A$ et al. Cocrystals of itraconazole with amino acids: screening, synthesis, solid state characterization, in vitro drug release and antifungal activity. J Drug Deliv Sci Technol 2015; 28: 46-55.

123. Abramov YA. Computational Pharmaceutical Solid State Chemistry, 1st edn. Hoboken, NJ: Wiley Online Library, 2016.

124. Rane SS, Anderson BD. What determines drug solubility in lipid vehicles: Is it predictable? Adv Drug Deliv Rev 2008; 60: 638-656.

125. Persson LC et al. Computational prediction of drug solubility in lipid based formulation excipients. Pharm Res 2013; 30: 3225-3237.

126. Patel SV, Patel S. Prediction of the sol ubility in lipidic solvent mixture: investigation of the modeling approach and thermodynamic analysis of solubility Eur J Pharm Si 20 15; 77: 161-169.

127. Alskär LCC et al. Tools for early prediction of drug loading in lipidbased formulations. Mol Pharm 2016; 13: 251-261

128. Dumanli I. Mechanistic studies to elucidate the role of lipid vehicles on solubility,formulation and bioavailability of poorly soluble compounds. 2002.

129. Shah $\mathrm{NH}$ et al. Oral Lipid-Based Formulations - Enhancing the 


\section{Thesis Publications}

Bioavailability of Poorly Water-Soluble Drugs (Hauss DJ, ed.). London, UK: Informa Healthcare, 2007

130. Vaughan CD. Solubility parameters for characterizing new raw materials. Cosmet Toilet 1993; 108: 57-64.

131. Van Loon . Van Loon Chemical Innovations Brochure. Van Loon Chem Innov Brochure. Available at: http:// www.in-cosmetics.com/_novadocume $\mathrm{nts} / 326978 ? \mathrm{v}=636220595266800000$. Accessed November 21, 2017

132. Yamamoto DH. Hansen Solubility Parameter (HSP) and Allergens for Cosmetics. Hansen Solubility Param Allergens Cosmet. Available at: https: / www.pirika.com/NewHP/PirikaE/Alle rgens.html. Accessed November 21, 2017.

133. Vaughan $\mathrm{CD}$. Using solubility parameters in cosmetics formulation. J Soc Cosmet Chem 1985; 36: 19-333.

134. Huynh $\mathrm{L}$ et al. Computational approaches to the rational design of nanoemulsions, polymeric micelles, and dendrimers for drug delivery. Nanomedicine 2012; 8: 20-36.

135. De La Peña-Gil A et al. Simplifying Hansen solubility parameters for complex edible fats and oils. Food Biophys 2016; 11: 283-291.

136. Shah M, Agrawal Y. Ciprofloxacin hydrochloride-loaded glyceryl monostearate nanoparticle: factorial design of Lutrol F68 and Phospholipon 90G. JMicroencapsul 2012; 29: 331-343.

137. Li Y et al. Screening of lipid carriers and characterization of drug-poly mer-lipid interactions for the rational design of polymer-lipid hybrid nanoparticles (PLN). Pharm Res 2006; 23: $1877-1887$.

138. Breitkreutz J. Prediction of intestinal drug absorption properties by threedimensional solubility parameters. Pharm Res 1998; 15: 1370-1375.

139. Abd E. Targeted Skin Delivery of Topically Applied Drugs by Optimised Formulation Design. 2015.

140. Hossin B et al. Application of Hansen solubility parameters to predict drug-nail interactions, which can assist the design of nail medicines. Eur I Pharm Biopharm 2016; 102: $32-40$.
141. Kawabata $\mathrm{Y}$ et al. Formulation design for poorly water-soluble drugs based on biopharmaceutics classification system: basic approaches and practical applications. Int J Pharm 2011; 420 1-10.

142. Einfalt $\mathrm{T}$ et al. Methods of amorphization and investigation of the amorphous state. Acta Pharm 2013. 63: 305-334.

143. Leuner C. Improving drug solubility for oral delivery using solid dispersions. Eur J Pharm Biopharm 2000; 50: $47-60$.

144. Serajuddin ATM. Solid dispersion of poorly water-soluble drugs: early promises, subsequent problems, and recent breakthroughs. I Pharm Sci 1999; 88: 1058-1066.

145. Chiou WL, Riegelman S. Pharmaceutical applications of solid dispersion systems. J Pharm Sci 1971; 60: 12811302.

146. Zhang J, Ma PX. Cyclodextrin-based supramolecular systems for drug delivery: recent progress and future perspective. Adv Drug Deliv Rev 2013; 65: 1215-1233.

147. Hoppu $\mathrm{P}$ et al. Characterisation of blends of paracetamol and citric acid. $J$ Pharm Pharmacol 2007; 59:373-381.

148. Simovic $S$ et al. Silica materials in drug delivery applications. Curr Drug Discov Technol 2011; 8: 250-268.

149. Xu W et al. Mesoporous systems for poorly soluble drugs. Int I Pharm 2013; 453: 181-197.

150. Huang Y, Dai W-G. Fundamental aspects of solid dispersion technology for poorly soluble drugs. Acta Pharm Sin B 2014; 4: 18-25.

151. Karavas $\mathrm{E}$ et al. Investigation of the release mechanism of a sparingly water-soluble drug from solid dispersions in hydrophilic carriers based on physical state of drug, particle size distribution and drug-polymer interactions. Eur J Pharm Biopharm 2007; 66: 334-347.

152. Baghel $\mathrm{S}$ et al. Polymeric amorphous solid dispersions: a review of amorphization, crystallization, stabilization, solid-state characterization, and aqueous solubilization of biopharmaceutical classification system class II drugs. J Pharm Sci 2016; 105: 25272544.

153. Sekiguchi K, Obi N. Studies on absorption of eutectic mixture. I. A comparison of the behavior of eutectic mixture of sulfathiazole and that of ordinary sulfathiazole in man. Chem Pharm Bull 1961; 9: 866-872.

154. Thakral S, Thakral NK. Prediction of drug-polymer miscibility through the use of solubility parameter based flory-huggins interaction parameter and the experimental validation: $\mathrm{PEG}$ as model polymer. J Pharm Sii 2013; 102: 2254-2263.

155. Greenhalgh DI et al. Solubility parameters as predictors of miscibility in solid dispersions. J Pharm Sci 1999; 88: 1182-1190.

156. DeBoyace $K$, Wildfong PLD. The application of modeling and prediction to the formation and stability of amorphous solid dispersions. I Pharm Sci 2017; 107: 57-74.

157. Alhalaweh A et al. Data mining of solubility parameters for computational prediction of drug-excipient miscibility. Drug Dev Ind Pharm 2014; 40: 904909.

158. Yoo $S$ et al. Miscibility/stability considerations in binary solid dispersion systems composed of functional excipients towards the design of multi-component amorphous systems. I Pharm Sci 2009; 98: 4711-4723.

159. Diuris $J$ et al. Preparation of carbamazepine-Soluplus ${ }^{(\mathbb{b})}$ solid dispersions by hot-melt extrusion, and prediction of drug-polymer miscibility by thermodynamic model fitting. Eur J Pharm Biopharm 2013; 84: 228-237.

160. Sarode AL et al. Hot melt extrusion (HME) for amorphous solid dispersions: predictive tools for processing and impact of drug-polymer interactions on supersaturation. Eur I Pharm Sci 2013; 48: 371-384.

161. Meng $\mathrm{F}$ et al. Investigation and correlation of drug polymer miscibility and molecular interactions by various approaches for the preparation of amorphous solid dispersions. Eur J Pharm Sci 2015; 71: 12-24.

162. Bagley $\mathrm{EB}$ et al. Three-dimensional solubility parameters and their 


\section{Thesis Publications}

relationship to internal pressure measurements in polar and hydrogen bonding solvents. I Paint Technol 1971; 43: 35-42.

163. Meaurio $\mathrm{E}$ et al. Predicting miscibility in polymer blends using the Bagley plot: blends with poly (ethylene oxide) Polymer (Guildf) 2017; 113: 295-309.

164. Arrighi V et al. Miscibility. In: Ency clopedia of Polymer Science and Tech nology, 3rd edn. Hoboken, NJ: John Wiley \& Sons, Inc., 2002

165. Paudel A et al. Theoretical and experimental investigation on the solid solubility and miscibility of naproxen in poly(vinylpyrrolidone) Mol Pharm 2010; 7: 1133-1148.

166. Qian F et al. Solution behavior of PVP-VA and HPMC-AS-based amorphous solid dispersions and their bioavailability implications. Pharm Res 2012; 29: 2766-2776.

167. Tian $Y$ et al. Construction of drugpolymer thermodynamic phase diagrams using Flory-Huggins interaction theory: identifying the relevance of temperature and drug weight fraction to phase separation within solid dispersions. Mol Pharm 2013; 10: 236-248.

168. Marsac PJ et al. Theoretical and practical approaches for prediction of drug-polymer miscibility and solubility. Pharm Res 2006; 23: 2417-2426.

169. Zhao $Y$ et al. Prediction of the thermal phase diagram of amorphous solid dispersions by Flory-Huggins theory. Pharm Sci 2011; 100: 3196-3207.

170. Koningsveld $\mathrm{R}$ et al. Polymer Phase Diagrams: A Textbook. Oxford, USA Oxford University Press, 2001

171. Friesen DT et at. Hydroxypropyl methylcellulose acetate succinate-based spray-dried dispersions: an overview. Mol Pharm 2008; 5: 1003-1019.

172. O'Shea JP et al. Mesoporous silicabased dosage forms improve bioavailability of poorly soluble drugs in pigs: case example fenofibrate. I Pharm Pharmacol 2017; 69: 1284-1292.

173. Lainé A-L et al. Enhanced oral delivery of celecoxib via the development of a supersaturable amorphous for mulation utilising mesoporous silica and co-loaded HPMCAS. Int Pharm 2016; 512: 118-125.

174. Van Speybroeck $M$ et al. Enhanced absorption of the poorly soluble drus fenofibrate by tuning its release rate from ordered mesoporous silica. Eu J Pharm Sci 2010; 41: 623-630

175. Dressman JB et al. Mesoporous sil ica-based dosage forms improve release characteristics of poorly soluble drugs: case example fenofibrate J Pharm Pharmacol 2016; 68: 634645.

176. McCarthy CA et al. Mesoporous silica formulation strategies for drug dissolution enhancement: a review. Expert Opin Drug Deliv 2016; 13: 93-108.

177. Hata $\mathrm{H}$ et al. Adsorption of taxol into ordered mesoporous silicas with various pore diameters. Chem Mater 1999; 11: 1110-1119.

178. ICH Expert Working Group. Impuri ties: Guideline for Residual Solvents. 2016. Available at: https:/www.ich org/fileadmin/Public Web Site/ICH Products/Guidelines/Quality/Q3C/ Q3C_R6_Step_4.pdf. Accessed July 31, 2017.

179. Piccinni $\mathrm{P}$ et al. Solubility parameter-based screening methods for early-stage formulation developmen of itraconazole amorphous solid dispersions. J Pharm Pharmacol 2016 68: 705-720.

180. Sun J et al. Effect of particle size on solubility, dissolution rate, and ora bioavailability: evaluation using coenzyme $\mathrm{Q}_{10}$ as naked nanocrystals. Int $J$ Nanomed 2012; 7: 5733-5744.

181. Williams HD et al. Strategies to adcress low drug solubility in discovery and development. Pharmacol Rev 2013; 65: 315-499.

182. Junghanns J-UAH, Müller RH Nanocrystal technology, drug delivery and clinical applications. Int Nanomed 2008; 3: 295-309.

183. Khadka $\mathrm{P}$ et al. Pharmaceutical particle technologies: an approach to improve drug solubility, dissolution and bioavailability. Asian J Pharm Sci 2014; 9: 304-316.

184. Rasenack N, Müller BW. Micron-size drug particles: common and nove micronization techniques. Pharm Dev Technol 2004; 9: 1-13.

185. Kundu S. Silk Biomaterials for Tissue Engineering and Regenerative Medicine, 1st edn. Amsterdam, Boston: Woodhead Publishing, 2014.

186. Singh AK. Engineered Nanoparticles: Structure, Properties and Mechanisms of Toxicity. Carnbridge, MA: Academic Press, 2015.

187. Merisko-Liversidge EM, Liversidge GG. Drug nanoparticles: formulating poorly water-soluble compounds. Tox icol Pathol 2008; 36: 43-48.

188. Date AA, Patravale VB. Current strategies for engineering drug nanoparticles. Curr Opin Colloid Interface Sci 2004; 9: 222-235.

189. Wu L et at. Physical and chemical stability of drug nanoparticles. Adv Drug Deliv Rev 2011; 63: 456-469.

190. Latere Dwan'Isa JP et al. Prediction of drug solubility in amphiphilic diblock copolymer micelles: The role of polymer-drug compatibility. Pharmazie 2007; 62: 499-504.

191. Mahmud A et al. Self-associating poly(ethylene oxice)- $b$-poly $(\alpha$-cholesteryl carboxylate- $\varepsilon$-caprolactone) block copolymer for the solubilization of STAT-3 inhibitor cucurbitacin I. Biomacromol 2009; 10: 471-478.

192. Flory PJ. Principles of Polymer Chem istry, 3rd edn. Ithaca, NY: Cornell University Press, 1953.

193. Niederquell $\mathrm{A}$ et al. New prediction methods for solubility parameters based on molecular sigma profiles using pharmaceutical materials. Int Pharm 2018; 546: 137-144.

194. Janssen. Development of a semi-automated system for the determination of the Hansen solubility parameters of pharmaceutical excipients. Unpublished data 2015. https:// doi.org/10.1111/jphp.12948 
International Journal of Pharmaceutics 512 (2016) 118-125

\begin{tabular}{cc}
\hline Contents lists available at ScienceDirect \\
\hline ELSEVIER & International Journal of Pharmaceutics \\
\hline
\end{tabular}

\section{Enhanced oral delivery of celecoxib via the development of a supersaturable amorphous formulation utilising mesoporous silica and co-loaded HPMCAS}

A.-L. Lainéa,*, D. Price ${ }^{a}$, J. Davis ${ }^{a}$, D. Roberts ${ }^{a}$, R. Hudson ${ }^{a}$, K. Back ${ }^{\mathrm{b}}$, P. Bungay ${ }^{\mathrm{a}}$, N. Flanagan ${ }^{a}$

a Pfizer-Neuroscience and Pain Research Unit, Granta Park, Cambridge CB21 6CS, United Kingdom ${ }^{\mathrm{b}}$ Pfizer, Discovery Park, Ramsgate Rd, Sandwich CT13 9ND, United Kingdom

\section{ARTICLE INFO}

Article history:

Received 27 May 2016

Received in revised form 10 August 2016

Availate online 16 August 2016

\section{Keywords:}

Amorphous formulatio

Pharmacokinetic study

Mesoporous material

In vitro $\mathrm{pH}$ shift assay
A B S T RACT

Stabilization of amorphous formulations via mesoporous silica has gained considerable attention for ora delivery of poorly soluble drugs. The release of the drug from the silica is expected to generate supersaturation which is often associated with subsequent precipitation. The aim of the study was hence to develop a novel supersaturable amorphous formulation through the co-loading of a BCS class II drug Celecoxib (CXB) with a precipitation inhibitor hydroxypropyl methylcellulose acetate succinate (HPMCAS) onto the silica. The addition of HPMCAS did not hamper the adsorption but on the contrary promoted the complete solid state conversion of the drug as proved by DSC analysis. In an in vitro pH shift assay, the CXB-HPMCAS co-loaded silica achieved a 5 -fold solubility increase over the crystalline CXB and over the CXB-loded silica blender with HPMCAS which did not show any enhanement The drug coover the CXB-loaded silica blended with HPMCAS which did not show any enhancement. The drug coloaded silica was then suspended in an aqueous vehicle facilating the dosing to animals. The CXBHPMCAS co-loaded silica suspension achieved 15 -fold solubility increase in vitro over the crystalline counterpart which translated in 1,35-fold Cmax increase in vivo after oral dosing in rats. This approach represents a novel formulation strategy to maximize in vivo exposure of poorly soluble drugs critical for discovery studies.

(6) 2016 Elsevier B.V. All rights reserved.

\section{Introduction}

Oral delivery of poorly soluble drugs remains one of the greatest challenges that formulation scientists face during drug discovery process. With the emergence of combinatorial chemistry and high throughput screening, new chemical entities often show suboptimal physicochemical properties such as high lipophilicity and low aqueous solubility leading to poor bioavailability (Lipinski et al., 2001 ). The identification of a suitable drug delivery system is therefore critical to achieve sufficient in vivo exposure. Several strategies (Kalepu and Nekkanti, 2015; Leucuta, 2014; Williams et al., 2013) have been developed in that regard such as particles size reduction (Dizaj et al., 2015), salt formation (Serajuddin, 2007), nanoparticles (Merisko-Liversidge and Liversidge, 2008), self-emulsifying drug delivery systems (SEDDS) (Pouton, 2000),

* Corresponding author at: Medimmune-AstraZeneca, Aaron Klug Building, Granta Park, Cambridge, CB21 6GH, United Kingdom.

E-mail address: lainean@medimunne.com (A. -L. Lainé).

0.1016/jijpharm 201608.034

0378-5173/@ 2016 Elsevier B.V. All rights reserved.

lipid formulation (Pouton and Porter, 2008; Hauss, 2007) and cocrystal (Elder et al., 2013). More recently, amorphous materials (Van den Mooter, 2012; Newman et al., 2015) have gained considerable attention for solubility enhancement as they can generate several-fold higher solution concentration (called supersaturation) compared to the corresponding crystalline form due to the lack of long range order and high free energy. However, a disadvantage of the high energy state of amorphous solids is the inherent metastability driving recrystallization under storage. Additionally, when the glassy solid is introduced into an aqueous medium, it often rapidly generates a transient supersaturated state immediately followed by a steep concentration decrease due to precipitation triggered by thermodynamic force. This dissolution profile, known as the spring effect (Guzman et al., 2007), creates a limited supersaturation window that may not offer a bioavailability increase after in vivo dosing. Accordingly, to benefit from the amorphous form and design a suitable drug product, the stability of the glassy form needs to be addressed (Grohganz et al., 2014; Laitinen et al., 2013). The most common way to stabilize the amorphous drug is by formulating it within an inert polymeric 


\section{Thesis Publications}

matrix referred to as a solid dispersions (Newman et al, 2015) Besides its function of amorphous stabilizer, the polymer also plays the role of precipitation inhibitor (PI) helping to maintain supersaturation state after dissolution (spring and parachute effect). The most common manufacturing processes include hot melt extrusion (Lakshman et al., 2008; Wilson et al., 2012) and spray-drying (Paudel et al., 2013) which require specific equipment and are not always appropriate for low volume laboratory scale production. Alternatively, in the past decade, mesoporous silica (Shen et al., 2013; Vialpando et al., 2011; Xu et al., 2013) has shown appealing characteristics as an amorphous drug stabilizer and carrier due to high pore volume and surface area contributing to high drug loading capacity. The adsorption in the confined and restricted space of the pores prevents the compound from crystallizing. Additionally, silica is widely used in tablet manufacture and is generally regarded as orally biocompatible (Rowe et al., 2006). Whereas numerous in vitro studies have demonstrated the ability of drug loaded silica to increase the solubility of poorly soluble drugs, only a few have shown in vivo absorption improvement as recently highlighted by Van Speybroeck (Van Speybroeck et al., 2010). The probable cause for the lack of correlation is that unlike solid dispersion, the silica cannot act as precipitation inhibitor once the compound is released from its pores. Consequently, the addition of a polymer is required to maintain the supersaturated solubility which is critical for enhanced in vivo oral delivery. Van Speybroeck et al. (2010) were then the first to combine Itraconzaole loaded silica with a PI and showed successful in vivo translation of the in vitro solubility increase. Based on this result, the aim of the present study was to develop a novel supersaturable amorphous formulation through the co-loading of a precipitation inhibitor and the drug onto the silica. Celecoxib (CXB), a nonsteroidal anti-inflammatory drug (Frampton and Keating, 2007) widely prescribed for treatment of osteoarthritis, rheumatois arthritis and acute pain, was used as an example of poorly soluble compound. Belonging to the BCS class II substance, CXB is highly lipophilic and poorly water soluble with a good permeability. The absorption of CXB has been reported to be limited by its dissolution (Paulson et al., 2001). As a consequence, the clinical dosage form, marketed under the trade name Celebrex ${ }^{\circledR}$, was designed to enhance the oral absorption of CXB through particle size reduction (D90 below $25 \mu \mathrm{m}$ ) and the addition of a wetting agent (sodium lauryl sulfate) (Gao et al., 2000). Due to its dissolution limited absorption characteristic Celecoxib has been the subject of many solubility enhancing formulation attempts including, salt formation (Guzman et al., 2007), nanoparticles (Morgen et al., 2012), complexation with cyclodextrins (Rawat and Jain, 2004) and supersaturating SEDDS (Song et al., 2014). As the mechanism of adsorption onto the silica is mainly governed by hydrogen-bond interaction, CXB is a good model drug for this approach since it contains both hydrogen bond donor and acceptors. The mesoporous material utilised in the present work was a non-ordered silica named Parteck SLC ${ }^{\text {(8) }}$ (developed by Merck Milipore) and hydroxypropyl methylcellulose acetate succinate (HPMCAS) was evaluated as PI. The performance of the resulting CXB-HPMCAS co-loaded silica was assessed firstly in an in vitro transfer dissolution assay aiming to simulate physiological $\mathrm{pH}$ change conditions and, then in vivo in a rat pharmacokinetic (PK) study. Its behavior was compared with that of CXB loaded silica blended with HPMCAS

\section{Materials and methods}

2.1. Materials

Ethanol, DMSO and acetonitrile were purchased from Sigma Aldrich (Sigma-Aldrich, St. Louis, MO, USA). Celecoxib, Celebrex
$200 \mathrm{mg}$ capsules (composition: lactose monohydrate, Sodium Lauryl Sulfate (SLS), povidone, croscarmellose sodium, magnesium stearate) and HPMCAS-HF were supplied through Pfizer centra stores (Pfizer, Groton, CT, USA). Parteck SLC ${ }^{(B)}$ (non ordered silica with mesopores size ranging from 2 to $7 \mathrm{~nm}$ ) was kindly provided by Merck Millipore ${ }^{\circledR}$ (Merck Millipore, Darmstadt, Germany) Simulated Intestinal Fluid (SIF) was purchased from Biorelevant (Biorelevant, Surrey, UK). Methocel A4M (methycellulose) and Tromethamine was provided by Dow chemical company (Midland, USA) and Sigma-Aldrich (St Louis, USA), respectively. All water used was purified using the Millipore Milli- $Q$ Integral water purification system (Merck Millipore, Darmstadt, Germany).

\subsection{Thermodynamic solublity}

The thermodynamic solubility of CXB was determined in three media: $0.01 \mathrm{M} \mathrm{HCl}(\mathrm{pH} 2)$, phosphate buffer (pH 6.5) and FaSSIF (pH 6.5). Approximately $1 \mathrm{mg}$ of $\mathrm{CXB}$ was accurately weighed into an Eppendorf tube and $400 \mu \mathrm{L}$ of medium pre-heated to $37^{\circ} \mathrm{C}$ was added. The tubes were shaken for $18 \mathrm{~h}$ at $37^{\circ} \mathrm{C}$. The resultant dispersions were then double centrifuged at $37^{\circ} \mathrm{C}$ for 15 and $30 \mathrm{~min}$. The supernatant was then diluted in acetonitrile/water. 20/80, v/v and quantified for API concentration via HPLC against a standard calibration curve.

\subsection{HPLC analysis}

Two stock solutions of CXB, referred to as a standard stock solution and a quality control $(\mathrm{OC})$ stock solution, were prepared in DMSO at $1 \mathrm{mg} / \mathrm{mL}$. A calibration curve ranging from 0.1 to $50 \mu \mathrm{g}$ / $\mathrm{mL}$ was obtained using serial dilutions in Acetonitrile/Water $(20 /$ $80 ; \mathrm{v} / \mathrm{v}$ ) from the standard stock. Serial dilutions were also done from the QC stock solution to prepared QC samples $(0.3,3$ and $30 \mu \mathrm{g} / \mathrm{mL}$ ).

HPLC analysis was performed using an Agilent 1200 series (Agilent technologies, Santa Clara, USA) system equipped with a UV detector (detection wavelength: $220 \mathrm{~nm}$ ). Chromatographic separation was achieved on a Luna C18 column $(50 \times 4.6 \mathrm{~mm}$, $3 \mu \mathrm{m}$ particle size, $100 \AA$ Phenomenex, California, USA). The mobile phases consisted of 90:10 water: $\mathrm{ACN}$ and $0.1 \%$ trifluoroacetic acid (TFA) for mobile phase $A$ and $100 \% \mathrm{ACN}+0.1 \%$ TFA for mobile phase $B$ and a linear gradient method was applied from $95 \%$ of mobile phase A to $95 \%$ of mobile phase B at a flow rate of $1 \mathrm{~mL} / \mathrm{min}$. System management and data acquisition and processing were performed via the Empower ${ }^{(i)}$ software package (Waters, Milford MA).

\subsection{Loading procedure}

2.4.1. Single drug loading procedure

CXB-Loaded Silica was prepared using the solvent impregnation rotary evaporator method developed in-house. Briefly, a solution $(10 \mathrm{mg} / \mathrm{mL})$ of CXB in ethanol was added to Parteck SLC (1:2 API Parteck SLC $\left.{ }^{\circledR}\right)$ under magnetic stirring for $15 \mathrm{~min}$. The suspension was then transferred to a rotary evaporator in order to remove the solvent under reduced pressure at $45^{\circ} \mathrm{C}$. After observing the complete removal of solvent, the powder was left to dry under reduced pressure for $2 \mathrm{~h}$.

\subsubsection{CXB- HPMCAS co-loading procedure}

In order to co-load the hydroxypropyl methylcellulose acetate succinate (HPMCAS) and the CXB onto the silica (CXB: silica 1:2) the polymer was firstly homogenized using an ultraturax mixer in the concentrated API solution prior to addition of Parteck SLC ${ }^{\circledR}$. The subsequent steps were identical to the single loading method. The resulting powder was ground with a mortar and pestle to breakinto 


\section{Thesis Publications}

finer particles. The targeted ratio CXB/HPMCAS was $10 / 3 \mathrm{w} / \mathrm{w}$ and the theoretical loading of CXB was $30 \% \mathrm{w}_{\mathrm{CXB}} / \mathrm{w}_{\text {SIIICa }}+\mathrm{HPMCAS}+\mathrm{CXB}$.

2.4.3. Physical blend CXB co-loaded silica and HPMCAS

For the preparation of the physical blend, the two components of CXB loaded silica and HPMCAS were combined using a pestle and mortar to achieve an overall ratio of 10:3 CXB: HPMCAS.

\subsection{Preparation of the buffers and $\mathrm{CXB}$ formulations}

2.5.1. Preparation of silica suspension

The drug loaded silica was suspended in an aqueous vehicle composed of $0.5 \%$ w $/ v$ Methocel (MC) A4M and $0.5 \%$ w/v HPMCAS in Tris Buffered water. The stock solution of the aqueous vehicle was firstly prepared and stored at $4{ }^{\circ} \mathrm{C}$. Briefly, $0.24 \% \mathrm{w} / \mathrm{v}$ tromethamine and $0.5 \%$ w/v HPMCAS-HF were added in purified water and stirred until fully dissolved. The solution was then heated to $70^{\circ} \mathrm{C}$ and $0.5 \%$ Methocel A4M was added gradually under vigorous stirring. Once the addition of Methocel completed, the heating was stopped and the solution was kept under magnetic stirring until cooling down to room temperature. The $\mathrm{pH}$ was subsequently adjusted to 7.4 .

The CXB-HPMCAS co-loaded silica suspension was then prepared at $1.6 \mathrm{mg} / \mathrm{mL}$ in the $(0.5 \%$ w/v Methocel (MC) A4M/ $0.5 \%$ w/v HPMCAS in Tris buffered water) vehicle by homogenization with a pestle in a mortar.

In order to assess and monitor the release kinetic and solid state conversion rate of the silica suspension over time, the formulation was kept at room temperature over 2 days and samples were taken regularly $(0,1 \mathrm{~h}, 4 \mathrm{~h}, 55 \mathrm{~h}$ post preparation), over the course of the assay. Each sample was centrifuged twice. The residue was assessed via XRPD and PLM for solid state evaluation and the supernatant was quantified via HPLC for free CXB concentration.

2.5.2. Preparation of the $\mathrm{CXB}$ solution

A CXB solution was prepared in $40 \% \mathrm{v} / \mathrm{V}$ PEG $400 / 60 \% \mathrm{v} / \mathrm{v}(20 \%$ w/v SBE-cyclodextrin in water) at $1.6 \mathrm{mg} / \mathrm{mL}$. Briefly, CXB was firstly dissolved in PEG 400 to which (20\%w/v SBE-cyclodextrin in water) component was added and mixed.

2.5.3. Preparation of concentrated FaSSIF

Concentrated Fasted state simulated intestinal fluid (FaSSIF) $\mathrm{pH}$ 7.0 was prepared based on Biorelevant com guidance but with a 3.4 factor applied to all the excipient contents. The concentration factor was due to the subsequent 3.4 dilution factor in the transfer dissolution assay. Briefly, $\mathrm{NaOH}(29.6 \mathrm{mM}), \mathrm{NaH}_{2} \mathrm{PO}_{4}(96.6 \mathrm{mM})$ and $\mathrm{NaCl}(360.1 \mathrm{mM})$ were dissolved in $90 \%$ of purified water. The $\mathrm{pH}$ was then adjusted to 7.0 with $0.1 \mathrm{M} \mathrm{HCl}$ prior to making to final volume with purified water. To prepare the concentrated FaSSIF, $0.76 \%$ w/v of SIF-v1 powder (3.4-fold higher than recommended by biorelevant) was added to the concentrated phosphate buffer $\mathrm{pH}$ 7.0 and stirred. The final composition of the concentrated FaSSIF was $10.2 \mathrm{mM}$ of sodium taurocholate and $2.55 \mathrm{mM}$ of lecithin

\subsection{Characterization}

26.1. Crystallinity assessment: $X$-ray powder diffraction (XRPD)

2-4 $\mathrm{mg}$ of powder was placed on a XRPD disc and transferred to the D2 Phaser (Bruker, Billerica) with Cu-Ka radiation $(\lambda=1.54 \AA$ ) at $30 \mathrm{kV}$ and $10 \mathrm{~mA}$. Data were collected over $10 \mathrm{~min}$ from 3 to $55^{\circ} 20$.

2.6.2. Differential scanning calorimetry (DSC) analysis

Approximately $1-3 \mathrm{mg}$ of powder was accurately weighed into an aluminium Tzero pan. This was sealed with a Tzero lid and crimped shut. The sample was run on a TA Instruments Discovery differential scanning calorimeter against an empty reference pan of the same type, using a method that heated the sample from $25^{\circ} \mathrm{C}$ to $200^{\circ} \mathrm{C}$ at $10^{\circ} \mathrm{C} /$ minute, with a nitrogen purge gas.

\subsubsection{Loading quantification}

Approximately $10 \mathrm{mg}$ of loaded silica were accurately weighed in triplicate to which $5 \mathrm{~mL}$ of DMSO were added and the samples were vortex mixed. $3 \times 1 \mathrm{~mL}$ were then taken, double centrifuged, and the supernatant diluted (1:50) with 20:80 acetonitrile (ACN): water and analysed for $\mathrm{CXB}$ content against known standards using HPLC. Percentage loading was then calculated.

\subsubsection{Non-sink transfer dissolution}

The transfer dissolution assays were performed firstly on dry powder samples (simulating a readily disintegrating tablet administration) and then on samples suspended in an aqueous vehicle $(0.5 \% \mathrm{w} / \mathrm{v} \mathrm{MC} / 0.5 \% \mathrm{w} / \mathrm{v}$ HPMCAS in tris buffered water). For the powder samples, crystalline CXB, CXB loading silica, CXBHPMCAS-co-loading silica or CXB loaded silica blended with HPMCAS weighings were made in order to obtain $4 \mathrm{mg}$ of CXB active drug. $2.5 \mathrm{~mL}$ of water was added to this. For the suspensions

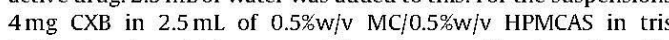
buffered water vehicle was used. Samples were then transferred to a water bath at $37^{\circ} \mathrm{C}$ and kept under magnetic stirring. $300 \mu \mathrm{L}$ of $37^{\circ} \mathrm{C}$ pre-heated $0.1 \mathrm{M} \mathrm{HCl}$ was then added. $200 \mu \mathrm{L}$ samples were taken ( 15 and $30 \mathrm{~min}$ ), double centrifuged and $50 \mathrm{uL}$ of supernatant diluted in $A C N / \mathrm{H}_{2} \mathrm{O}$ for HPLC analysis. At $30 \mathrm{~min}, 1 \mathrm{~mL}$ of concentrated FaSSIF solution was added to the vial to give a final $\mathrm{pH}$ of 6.8 . The remaining samples were then taken at a cumulative time of $42,55,70,90,120$ and $180 \mathrm{~min}$. The samples were analysed by HPLC against a calibration curve. The temperature was maintained at $37^{\circ} \mathrm{C}$ throughout and the $\mathrm{pH}$ checked at various time points.

After the end of the dissolution, a sample was taken from the dissolution vials and centrifuged. The solid residue was transferred to the D2 XRPD and analysed as previously described.

\subsection{In vivo pharmacokinetic study}

All experimental procedures were conducted in accordance with UK Animals (Scientific Procedures) Act 1986 and followed the guidelines under the International Association for the Study of Pain.

Male CD rats (Charles River Sprague Dawley) were allowed to acclimatize for at least one week prior to the study. Water and food were available ad libitum throughout the experiment. The animals ( $n=3$ per group) were dosed by oral gavage with $5.8 \mathrm{mg} / \mathrm{kg} \mathrm{CXB}$ at $3.6 \mathrm{~mL} / \mathrm{kg}$ as either crystalline suspension, amorphous CXBHPMCAS co-loaded silica suspension or in solution in $40 \% \mathrm{v} / \mathrm{V}$ PEG400/60\% v/v (20\%w/v SBE-cyclodextrin in water). Blood samples $(20 \mu \mathrm{L})$ were taken from the tail vein at $0.5 \mathrm{~h}, 1 \mathrm{~h}, 2 \mathrm{~h}$, $4 \mathrm{~h}, 8 \mathrm{~h}$ and $24 \mathrm{~h}$ post-dose and collected onto dry blood spot (DBS) cards.

DBS samples, together with the calibration and QC samples, were cut using a $3 \mathrm{~mm}$ punch into a 96 well plate. $10 \mu \mathrm{L}$ tolbutamide $(375 \mathrm{ng} / \mathrm{mL})$ in $25 \%$ acetonitrile were added as an internal standard (except for control blank). Samples were extracted by the addition of $300 \mu \mathrm{L}$ methanol for approximately $10 \mathrm{~min}$ on a plate shaker. All samples (excluding paper spots) were then transferred to a clean 96 well plate and evaporated under liquid nitrogen at $40^{\circ} \mathrm{C}$. Following evaporation, samples were reconstituted in $200 \mu \mathrm{L}$ 25: 75 acetonitrile: water and injected $(80 \mu \mathrm{L})$ into an HPLC-MS/MS system with resolution on a Kinetex $\mathrm{XB}-\mathrm{C} 1850 \times 3 \mathrm{~mm} 2.6 \mu \mathrm{m}$ column. For MS/MS, $\mathrm{m} / \mathrm{z} \mathrm{Q} 1 / \mathrm{Q} 3$ transitions were $382.1 / 206.7$ for celecoxib and $271.2 / 172.1$ for tolbutamide (internal standard). Quantitation was performed by 


\section{Thesis Publications}

reference to peak area ratio of celecoxib: internal standard over a calibration range of $5-2000 \mathrm{ng} / \mathrm{mL}$.

\subsection{Statistical analysis}

The statistical comparison of the rate and extent of CXB absorption (AUC and Cmax) obtained with the various formulations was performed on the log-transformed data using a one way analysis of variance (ANOVA) with the InVivoStat software (version 3.0). The significance level was set a $\mathrm{P}$ values of $<0.05$.

\section{Result and discussion}

\subsection{Celecoxib thermodynamic solubility}

The thermodynamic solubility of celecoxib was generated at pH 2 in $0.01 \mathrm{M} \mathrm{HCl}$ (stomach pH simulating medium), in phosphate buffer (PBS) pH 6.5 and in FaSSIF (Fasted state simulating intestinal fluid pH6.5) (Table 1).

As CXB is a neutral molecule, the solubility at $\mathrm{pH} 2$ and $\mathrm{pH} 6.5$ (PBS) appeared similar as expected and very low (3.7 and $2.4 \mu \mathrm{g}$ / $\mathrm{mL}$ respectively). A 20 -fold increase solubility was observed in FaSSIF in comparison to PBS which was attributed to solubilisation within the micelles formed by the phospholipids and bile salts present in the medium.

\subsection{CXB loaded silica characterization}

The preparation method involved impregnation of silica with a concentrated drug solution in ethanol followed by evaporation of the solvent leading to drug entrapment within the pores. The entire process was relatively rapid taking $3 \mathrm{~h}$ in total to obtain a dry powder. The DSC thermograms of crystalline CXB, CXB loaded silica and CXB-HPMCAS-co-loaded silica are shown in Fig. 1. The melting point of crystalline CXB appears at $162^{\circ} \mathrm{C}$. Whereas XRPD turned out not to be sensitive enough to detect any signs of crystallinity in the two silica batches, DSC revealed an endothermic event in the single CXB loaded silica thermogram at $164^{\circ} \mathrm{C}$ corresponding to the CXB melting point. This suggests that a small portion of drug remained in the crystalline form. Interestingly, the co-loading with HPMCAS seemed to promote the complete conversion of CXB in an amorphous state. It is worth mentioning that according to the literature, HPMCAS is one of the most effective polymers at preventing drug crystallization and hence maintaining supersaturation (Curatolo et al., 2009; Ueda et al., 2013). Finally, the HPLC analysis confirmed a CXB loading of $31.1 \% \mathrm{w} / \mathrm{w}$ demonstrating that the co-loading by solvent impregnation rotary evaporator method developed in-house was successful.

\subsection{In vitro transfer dissolution assay}

The dissolution performance of CXB-HPMCAS co-loaded silica was evaluated through an in vitro non sink transfer dissolution(TD) model. This assay was designed to simulate the journey of a drug from the stomach environment to the intestine compartment and hence try and predict the behavior of the formulation performance in vivo. As such, all volumes used were scaled down from physiological estimates resulting in a non-sink dissolution

Table 1

Thermodynamic solubility of Celecoxib.

\begin{tabular}{cccl}
\hline Medium & $\begin{array}{l}0.01 \mathrm{M} \mathrm{HCl} \mathrm{pH} \mathrm{2} \\
(\mu \mathrm{g} / \mathrm{mL})\end{array}$ & $\begin{array}{l}\text { Phosphate Buffer } \mathrm{pH} 6.5 \\
(\mu \mathrm{g} / \mathrm{mL})\end{array}$ & $\begin{array}{l}\text { FassIF pH 6.5 } \\
(\mu \mathrm{g} / \mathrm{mL})\end{array}$ \\
\hline Celecoxib & $3.7 \pm 0.1$ & $2.4 \pm 0.4$ & $43.3 \pm 0.7$ \\
\hline
\end{tabular}

experiment. Whereas most of the $\mathrm{pH}$ shift dissolution assay in the literature are carried out under sink conditions, non-sink conditions utilized here are critical to capture any potential precipitation event.

The various $\mathrm{CXB}$ formulations (crystalline $\mathrm{CXB}$, Celebrex ${ }^{\circledR}, \mathrm{CXB}$ loaded silica, CXB-loaded silica blended with HPMCAS and CXBHMPCAS co-loaded silica) were initially introduced in the TD assay as powder simulating a tablet (Fig. 2). As expected the crystalline CXB solubility profile closely correlated to the aforementioned thermodynamic solubility for each medium with an increase in solubility in FaSSIF. In comparison to the bulk CXB, the marketed formulation Celebrex reached a higher solubility plateau in FaSSIF most likely due to the presence of the SLS surfactant and confirming the hypothesis that CXB is influenced by micellar solubilisation. The loading of $\mathrm{CXB}$ onto the silica did not improve its dissolution profile but conversely generated a lower solubility in FaSSIF medium. This unexpected effect could be due to readsorption of the CXB onto the mesoporous material resulting in a gradual decrease of the concentration.

When CXB loaded silica was blended with HPMCAS, the presence of the polymer seemed to reduce the readsorption of CXB onto the silica once released. The co-loading of HPMCAS with $\mathrm{CXB}$ onto the silica led to a slower release in FaSSIF resulting in a 4 fold solubility increase over the crystalline CXB which was sustained over $1.5 \mathrm{~h}$. These data suggest that the addition of HPMCAS through co-loading proved to be more efficient to generate and maintain supersaturation than introduction via physical blending.

The post transfer dissolution residue was assessed via PXRD and PLM for the various formulations (Supplementary information). Al samples showed crystalline residue at the end of the assay demonstrating that the amorphous formulation partly converted back to crystalline CXB parent.

It was also noteworthy that for all the loaded silica, no major release was observed in the acidic medium over $30 \mathrm{~min}$ as indicated by the concentrations measured at 15 and $30 \mathrm{~min}$ (Table 2). The increase in solubility appeared only upon addition of FaSSIF. It is commonly hypothesized that the drug is released upon exposure with an aqueous media due to competition for the hydrophilic silica surface with water molecules displacing the drug from its confinement. However, this present finding seems to suggest that the drug desorption is most likely to happen in a medium promoting solubilisation such as the micelle containing FaSSIF medium. This observation is in accordance with previous data published in the literature where a non-release in acid medium was also shown for Indomethacin and glibenclamide (van Speybroeck et al., 2011).

Interestingly, this new understanding of the release mechanism offers alternative use of the silica. While the drug loaded silica is commonly dosed to animals via capsules to avoid release before dosing, the possibility of dosing the silica as a suspension can be considered and was evaluated in this work. Thus, CXB-HPMCAS coloaded silica was suspended in an aqueous vehicle (0.5\% HPMCAS $0.5 \% \mathrm{MC}$ in TRIS buffer $\mathrm{pH} 7.4$ ) at $1.6 \mathrm{mg} / \mathrm{mL}$ and aimed to be dosed in vivo. This vehicle corresponds to the vehicle used to prepare extemporaneous suspension in a typical first-in human clinical trial and was chosen to reinforce the parachute effect via the presence of HPMCAS. A stability study was performed on the suspension in order to assess and monitor the release kinetic and solid state conversion rate. The suspension was maintained at room temperature over 2 days and samples were taken regularly over the course of the assay (Table $3:$ X-ray diffractograms in Supplementary information). The fraction solubilized reached up to $20 \%$ of the formulation concentration $(1.6 \mathrm{mg} / \mathrm{mL})$ in $2 \mathrm{~h}$ and maintained that level for 2 days. Additionally no conversion to crystalline parent was observed as determined via polarized light 


\section{Thesis Publications}

122

A.-L. Lainé et al./International Journal of Pharmaceutics 512 (2016) 118-125

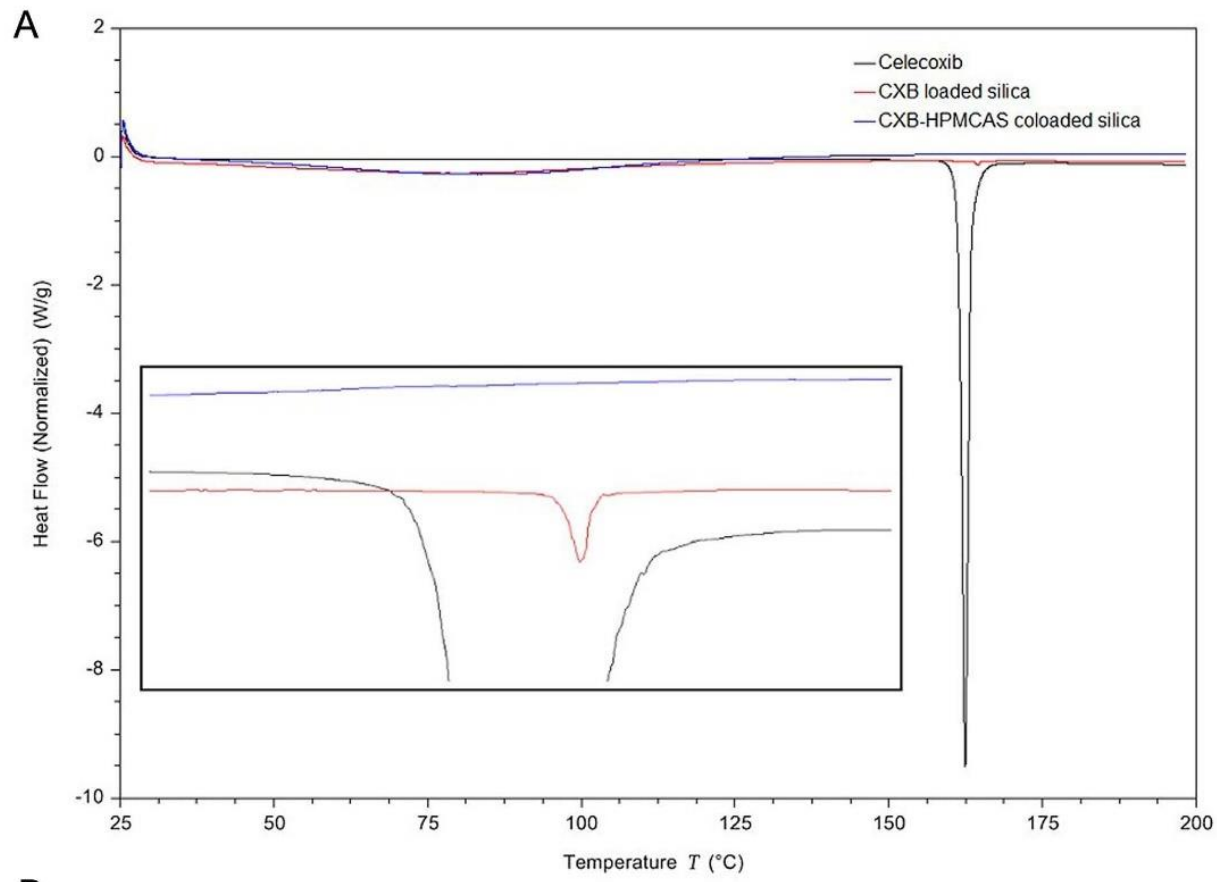

B

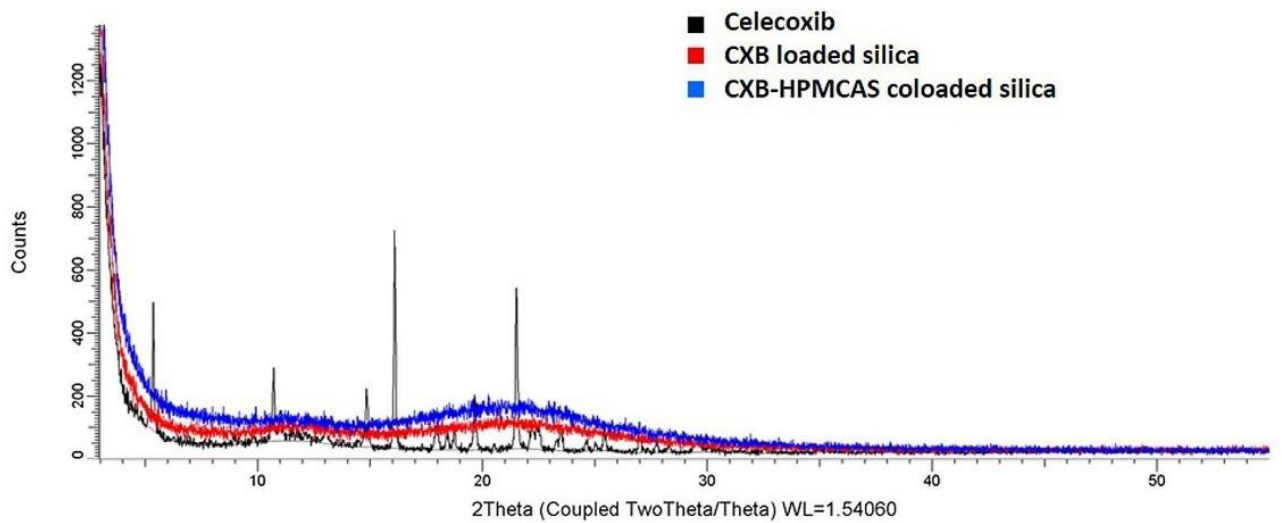

Fig. 1. Solid state characterization of CXB, CXB loaded silica and CXB-HPMCAS co-loaded silica via DSC (A) and XRPD (B). For the CXB trace on the DSC curve (A), there is a single endotherm with an onset of $161^{\prime} \mathrm{C}$. For the CXB loaded silica, there is a single endotherm with an onset of $163.9^{\prime} \mathrm{C}$. The inset box shows a higher magnification view of the endotherm in the CXB loaded silica.

microscopy (PLM). Despite the release, CXB seemed to stay in solution likely due to the high HPMCAS content.

Collectively, these data suggested that dosing the CXB-HPMCAS loaded silica as a suspension was a viable option. In conjunction with the TD data demonstrating the most promising dissolution performance enhancement, only the co-loaded formulation was selected for a pharmacokinetic study. Before running the in vivo

experiment, in vitro transfer dissolution assay was carried out with the CXB-HPMCAS co-loaded silica suspension in comparison to a crystalline CXB suspension to predict its performance. The amorphous CXB suspension achieved 15-fold solubility increase compared to the crystalline CXB suspension (Fig. 3 ) and a 3-fold increase over that seen previously with the powder in Fig. 2. This significant increase is most likely due to the presence of HPMCAS in 


\section{Thesis Publications}

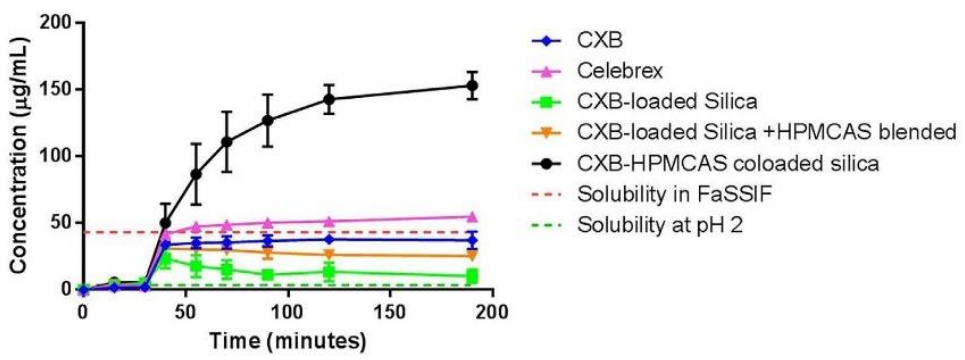

Fig. 2. Concentration vs time profile of crystalline CXB, Celebrex, CXB loaded silica, CXB loaded silica blended with HPMCAS, CXB-HPMCAS co-loaded silica obtained through the in vitro transfer dissolution assay $(n=4)$.

Table 2

Concentrations of the various $\mathrm{CXB}$ formulations at $15 \mathrm{~min}$ and $30 \mathrm{~min}$ in the acidic medium during the transfer dissolution assay.

\begin{tabular}{lccccc}
\hline & $\begin{array}{l}\text { CXB } \\
(\mu \mathrm{g} / \mathrm{mL})\end{array}$ & $\begin{array}{l}\text { Celebrex } \\
(\mu \mathrm{g} / \mathrm{mL})\end{array}$ & $\begin{array}{l}\text { CXB loaded silica } \\
(\mu \mathrm{g} / \mathrm{mL})\end{array}$ & $\begin{array}{l}\text { CXB loaded silica +HPMCAS Blend } \\
(\mu \mathrm{g} / \mathrm{mL})\end{array}$ & \multicolumn{2}{c}{$\begin{array}{l}\text { CXB-HPMCAS co-loaded silica } \\
(\mu \mathrm{g} / \mathrm{mL})\end{array}$} \\
\hline $\mathrm{T} 15 \mathrm{~min}$ & 1.9 & 3.2 & 3.8 & 4.1 & 5.8 \\
$\mathrm{~T} 30 \mathrm{~min}$ & 1.8 & 3.1 & 4.0 & 3.9 & 5.6 \\
\hline
\end{tabular}

Table 3

Physical stability of CXB-HPMCAS co-loaded silica suspension (PL.M: polarized light microscopy).

\begin{tabular}{lcc}
\hline Time point & XRPD/PLM & Solubilised fraction $(\mu \mathrm{g} / \mathrm{mL})$ \\
\hline T0 & Absence of crystallinity & 157 \\
$1 \mathrm{~h}$ & Absence of crystallinity & 283 \\
$4 \mathrm{~h}$ & Absence of crystallinity & 305 \\
$55 \mathrm{~h}$ & Absence of crystallinity & 316 \\
\hline
\end{tabular}

the vehicle which is crucial to prevent precipitation and maintain supersaturation as confirmed by the amorphous residue obtained post assay (Supplementary information).

3.4. In vivo pharmacokinetic comparison

The CXB-HPMCAS co-loaded silica suspension was then assessed through a PK study in rats in comparison to the crystalline $\mathrm{CXB}$ suspension. The dose and dose volume were selected to correspond to clinical practice. As the limiting factor for $\mathrm{CXB}$ absorption is the dissolution rate, $\mathrm{a} \mathrm{CXB}$ solution was dosed as a positive control to overcome this parameter. To ensure a reliable positive control, the $\mathrm{CXB}$ solution performance was assessed in the in vitro transfer dissolution aiming to predict any precipitation upon dosing. The profile of the solution maintained at $100 \%$ solubility over the whole course of the assay including stomach and intestine compartment indicating a low risk of precipitation in vivo (data not shown). The three formulations were subsequently dosed and the resulting systemic exposure compared altogether (Fig. 4, Table 4).

In this study, crystalline $\mathrm{CXB}$ suspension displayed a reasonable oral absorption profile in spite of its low solubility. It is worth pointing out that the experiment was carried out on fed animals with food available ad libitium, so that $\mathrm{CXB}$ exposure may have been promoted by a food effect, as previously demonstrated in dogs (Paulson et al., 2001). Nevertheless, despite a similar bioavailability between the formulations (represented by AUC) the plasma concentration vs. time profile of the crystalline suspension was distinct, with a slower rate of absorption than the solution and the CXB-HPMCAS co-loaded silica suspensions groups. Significantly higher Cmax were indeed observed for the amorphous suspension and the solution compared to the crystalline suspension (1.35 and 1.44-fold increase respectively, $\mathrm{p}<0.005$ ). The data demonstrate that $\mathrm{CXB}$ co-loaded silica suspension effectively produced the same profile of a solution dosed in rat at $5.8 \mathrm{mg} / \mathrm{kg}$ proving that the silica technology can overcome poor dissolution rate. Additionally, the possibility of dosing the silica in a suspension rather than in capsules as conventionally administered rends the technology more suitable and accessible for preclinical studies.

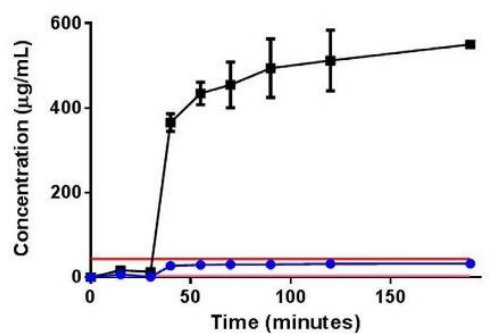

$\rightarrow$ Crystalline CXB suspension

- CXB -HPMCAS coloaded silica suspension

- Solubility in FaSSIF

- Solubility at $\mathrm{pH} 2$

Fig. 3. In vitro transfer dissolution profile of crystalline CXB suspension $(n=2)$ and CXB-HPMCAS co-loaded silica suspension ( $n=4)$. 


\section{Thesis Publications}

124

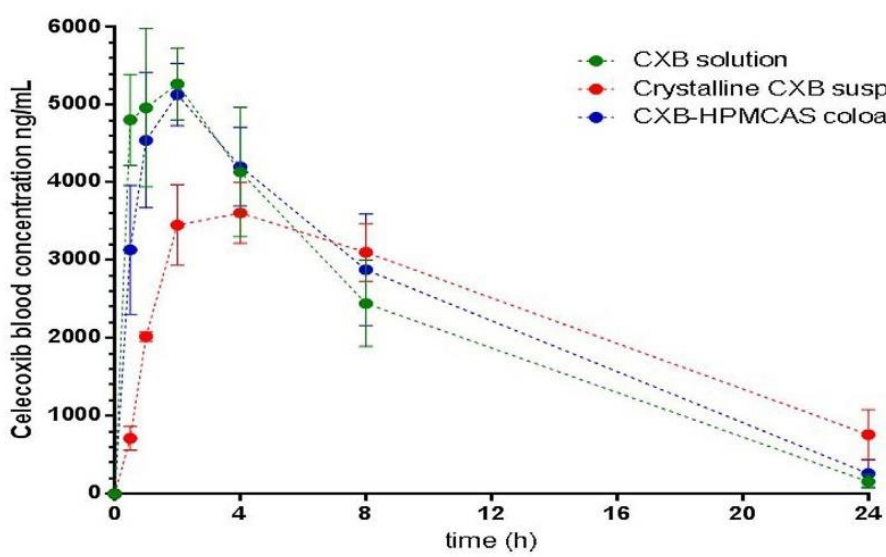

Fig. 4. Plasma concentration vs time profile of crystalline CXB suspension, CXB solution and CXB- HPMCAS co-loaded silica suspension after oral dosing in rats + table with pk parameters.

Table 4

Pharmacokinetic parameters after oral absorption of various CXB formulations.

\begin{tabular}{|c|c|c|c|c|c|c|}
\hline & \multicolumn{2}{|c|}{ Solution } & \multicolumn{2}{|c|}{ Crystalline CXB suspension } & \multicolumn{2}{|c|}{ CXB-HPMCAS co-loaded silica suspension } \\
\hline & mean & sd & mean & $\mathrm{Sd}$ & mean & sd \\
\hline AUC $\mathrm{nghmL}^{-1}$ & 50977 & 6689 & 54790 & 6891 & 55383 & 11493 \\
\hline Cmax ng/mL & 5507 & 438 & 3817 & 276 & 5133 & 402 \\
\hline
\end{tabular}

\section{Conclusion}

A supersaturable amorphous suspension was developed through a quick one-step process based on the co-loading of a precipitation inhibitor (HPMCAS) and the drug (Celecoxib) onto the mesoporous silica. A simple in vitro transfer dissolution test was effective in showing the enhanced performance of different formulations for selection in subsequent in vivo studies. The coloaded formulation has demonstrated a sustained supersaturation in vitro and enhanced in vivo absorption rate of the poorly soluble CXB after oral delivery. Whereas further work is required to better understand the release mechanism, this technology represents a novel easy preclinical formulation approach to maximize in vivo exposure critical for discovery studies.

\section{Acknowledgement}

The authors would like to thank Merck Millipore for providing the Parteck ${ }^{\mathbb{R}}$ SLC and for their technical support.

\section{Appendix A. Supplementary data}

Supplementary data associated with this article can be found, in the online version, at http://dx.doi.org/10.1016/j. ijpharm.2016.08.034.

\section{References}

Curatolo, W., Nightingale, J.A., Herbig, S.M., 2009. Utility of

hydroxypropylmethylcellulose acetate succinate (HPMCAS) for initiation and maintenance of drug supersaturation in the Gl milieu. Pharm. Res. 26 (6), 14191431.

Dizaj, S.M., Vazifehasi, Z., Salatin, S., Adibkia, K., Javadzadeh, Y., 2015. Nanosizing of drugs: effect on dissolution rate. Res. Pharm. Sci. 10 (2), 95-108.
Elder, D.P., Holm, R., Diego, H.L., 2013. Use of pharmaceutical salts and cocrystals to address the issue of poor solubility. Int. J. Pharm. 453 (1), 88-100.

Frampton, J.E., Keating, G.M., 2007. Celecoxib: a review of its use in the management (16) 2433-2472.

Go, D., Hlinak, A.J. Mazhary, A.M., Truelove, J.F., Vaughn, M.B.W., 2000 . Celecoxil K Nielsen L H. Latinen R. Mullertz A etal. 2014. Refining stability and dissolution rate of amorphous drug formulations. Expert Opin. Drug Deliv. 11 (6), 977-989. Guzman, H.R., Tawa, M., Zhang, Z., Ratanabanangkoon, P., Shaw, P., Gardner, C.R., et
al., 2007. Combined use of crystalline salt forms and precipitation inhibitors to improve oral absorption of celecoxib from solid oral formulations. J. Pharm. Sci

Hauss, D.J., 2007. Oral lipid-based formulations. Adv. Drug Deliv. Rev. 59 (7), 667-

676.
Kalepu, S., Nekkanti, V., 2015. Insoluble drug delivery strategies: review of recent advances and business prospects. Acta Pharm. Sin. B 5 (5), 442-453.

Laitinen, R., Lobmann, K.., Strachan, C..., Grohganz, H., Rades, T., 2013. Emerging akshman, J.P., Cao, Y., Kowalski, J., Serajuddin, A.T., 2008. Application of melt extrusion in the development of a physically and chemically stable high-energy amorphous solid dispersion of a poorly water-soluble drug. Mol. Pharm. 5 (6) 994-1002.

Leucuta, S.E., 2014. Selecting oral bioavailability enhancing formulations during drug discovery and development. Expert Opin. Drug Discov. 9 (2), 139-150. ipinski, C.A., Lombardo, F., Dominy, B.W., Feeney, P.J., 2001. Experimental and computational approaches to estimate solubility and permeability in drug discovery and development settings. Adv. Drug Deliv. Rev. $46(1-3), 3-26$. poorly water-soluble compounds. Toxicol. Pathol. 36 (1), 43-48. Morgen, M., Bloom, C., Beyerinck, R., Bello, A., Song, W., Wilkinson, K., et al., 2012. Polymeric nanoparticles for increased oral bioavailability and rapid absorption using celecoxib as a model of a low-solubility, high-permeability drug. Pharm. Res. 29 (2), 427-440.

Newman, A., Nagapudi, K., Wenslow, R., 2015. Amorphous solid dispersions: a robust platform to address bioavailability challenges. Ther. Deliv. 6(2), 247-26 Paudel, A., Worku, Z.A., Meeus, J., Guns, S., Van den Mooter, G., 2013. Manufacturing of solid dispersions of poorly water soluble drugs by spray drying: formulation

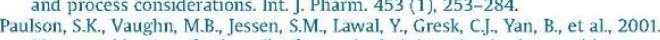
Pharmacokinetics of celecoxib after oral administration in dogs and humans: 


\section{Thesis Publications}

Pouton. CW., Porter, C.J., 2008. Formulation of lipid-based delivery systems for oral administration: materials, methods and strategies. Adv. Drug Deliv. Rev. 60 (6) $625-637$.

Pouton, C.W. 2000. Lipid formulations for oral administration of drugs: nonemulsifying, self-emulsifying and 'self-microemulsifying' drug delivery systems. Eur. J. Pharm. Sci. 11 (Suppl. 2), S93-\$98.

Rawat, S., Jain, S.K., 2004. Solubility enhancement of celecoxib using $\beta$-cyclodex trin inclusion complexes. Eur. J. Pharm. Biopharm. 57 (2), 263-267.

we, R.C., Sheskey, PJ., Owen, S.C., American Pharmacists Association, 2006. Handbook of Pharmaceutical Fxcipients Pharmaceutical Press

Serajuddin, A.T., 2007. Salt formation to improve drug solubility. Adv. Drug Deliv. Rev. 59 (7),

LS. Dong. Y.C. Tan. R. B., 2013. Applications of mesoporous materials as excipients for innovative drug delivery and formulation. Curr. Pharm. Des. 19 (35), 6270-6289.

Song, W.H., Yeom, D.W., Lee, D.H., Lee, K.M., Yoo, H.J., Chae, B.R, et al., 2014. In situ intestinal permeability and in vivo oral bioavailability of celecoxib in supersat

Jeda, K., Higashi, K., Yamamoto, K., Moribe, K., 2013. Inhibitory effect of hydroxypropyl methylcellulose acetate succinate on drug recrystallization from a supersaturated solution assessed using nuclear magnetic resonance measurements. Mol. Pharm. $10(10), 3801-381$
Van Speybrceck, M. Mols. R. Mellaerts, R. Thi. T. D. Martens, JA. Van Humbeeck J. et al.. 2010. Combined use of ordered mesoporous silica and precipitatio
inhibitors for improved oral absorption of the poorly soluble weak base itraconazole. Eur. J. Pharm. Biopharm. 75 (3). 354-365.

Van den Mooter, G., 2012. The use of amorphous solid dispersions: a formulation strategy to overcome poor solubility and dissolution rate. Drug Discov. Today Technol. 9 (2), e71-e174.

van Speybroeck, M., Mellaerts, R., Thi, T.D, Martens, J.A., Van Humbeeck, J., Annaer P., et al. 2011. Preventing release in the acidic environment of the stomach via occlusion in ordered mesoporous silica enhances the absorption of poorly soluble weakly acidic drugs. J. Pharm. Sci. 100 (11), 4864-4876.

Valpando, M., Martens, J.A., Van den Mooter, G., 2011. Potential of ordered illiams

askis, N.L., Charman, S.A., Shanker, R.M., Charman, W.N., Pouton, CW., et al, 2013. Strategies to address low drug solubility in discovery and

development. Pharmacol. Rev. 65 (1), 315 -499.
Wilson, M., Williams, M.A., Jones, D.S., Andrews, G.P., 2012. Hot-melt extrusion technology and pharmaceutical application. Ther. Deliv. 3 (6), 787-797.

$\mathrm{Xu}, \mathrm{W}$, Rikonen, J., Lehto, V.P., 2013. Mesoporous systems for poorly soluble drugs. Int. J. Pharm. 453 (1), 181-197. 
1

2

3

4 Niklas J. Koehl, Laura J. Henze ${ }^{I}$, Harriet Bennett-Lenane ${ }^{I}$, Daniel J. Price ${ }^{2,3,}$

5 René Holm ${ }^{4,5}$, Martin Kuentz ${ }^{6}$, Brendan T. Griffin ${ }^{1^{*}}$

6

$7{ }^{1}$ School of Pharmacy, University College Cork; College Road, Cork, T12 YN60 Ireland

$8 \quad{ }^{2}$ Merck KGaA, Darmstadt, Germany

$9 \quad{ }^{3}$ Goethe University Frankfurt, Frankfurt am Main, Germany

$10{ }^{4}$ Drug Product Development, Janssen Research and Development, Johnson \& Johnson,

11 Turnhoutseweg 30, 2340 Beerse, Belgium

$12{ }^{5}$ Department of Science and Environment, Roskilde University, 4000 Roskilde, Denmark

$13{ }^{6}$ University of Applied Sciences and Arts Northwestern Switzerland, Institute of Pharma

14 Technology, Muttenz, Switzerland

15

$16 *$ Corresponding author:

17 Brendan Griffin, University College Cork, School of Pharmacy, Cavanagh Building, College

18 Road, Cork, Ireland

19 Phone: +353 (0) 21490 1657, Fax: +353 (0) 21490 1656, e-mail: Brendan.Griffin@ucc.ie 


\section{Thesis Publications}

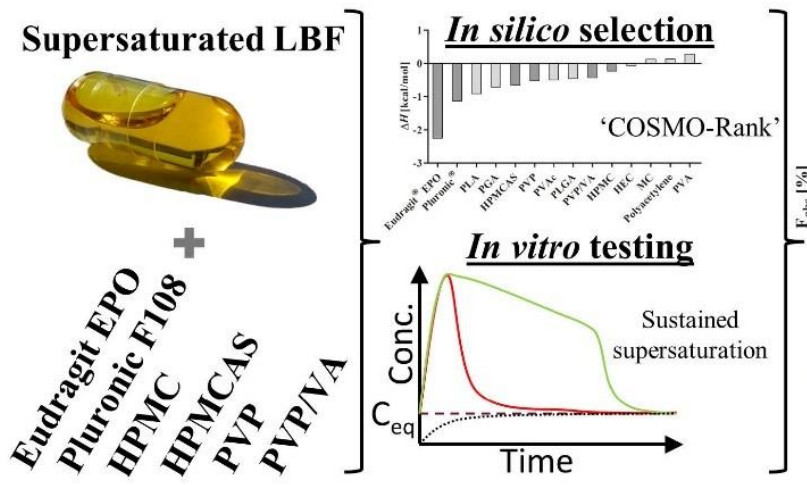

\section{In vivo evaluation}

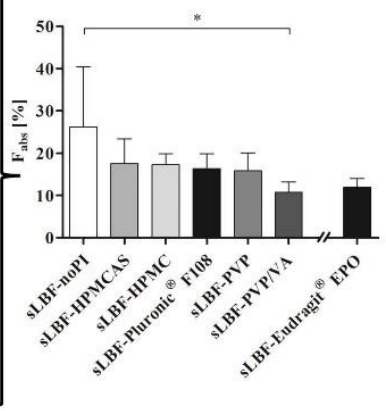

\section{ABSTRACT}

26 The concept of using precipitation inhibitors (PIs) to sustain supersaturation is well established for amorphous formulations, but less in the case of lipid-based formulations (LBF). This study applied a systematic in silico-in vitro-in vivo approach to assess the merits of incorporating PIs in supersaturated LBFs (sLBF) using the model drug venetoclax. SLBFs containing HPMC, HPMCAS, PVP, PVP/VA, Pluronic ${ }^{(i)}$ F108 and Eudragit ${ }^{10}$ EPO were assessed in silico calculating a drug-excipient mixing enthalpy, in vitro using a PI solvent shift test and finally bioavailability was assessed in vivo in landrace pigs. The estimation of pure interaction enthalpies of drug and excipient were deemed useful in determining the most promising PIs for

34 venetoclax. The sLBF alone (i.e. no PI present) displayed high initial concentration in the

35 aqueous phase during in vitro screening. SLBF with Pluronic ${ }^{\text {to }}$ F108 displayed the highest venetoclax concentration in the aqueous phase and sLBF with Eudragit ${ }^{\mathbb{B}}$ EPO the lowest. In vivo, the sLBF alone showed the highest bioavailability of $26.3 \pm 14.2 \%$. Interestingly, a trend

38 towards a decreasing bioavailability was observed for sLBF containing PIs, with PVP/VA

39 being significantly lower compared to SLBF alone. In conclusion, the ability of a sLBF to 


\section{Thesis Publications}

40 generate supersaturated concentrations of venetoclax in vitro was translated to increased

41 absorption in vivo. While in silico and in vitro PI screening suggested benefits in terms of

42 prolonged supersaturation, addition of a PI did not increase in vivo bioavailability. The findings

43 of this study are of particular relevance to pre-clinical drug development, where the high in vivo

44 exposure of venetoclax was achieved using a SLBF approach and despite a perceived risk of

45 drug precipitation from a SLBF, including of a PI may not be merited in all cases.

KEYWORDS:

49 Precipitation inhibitor, Lipid based formulation, Venetoclax, SEDDS, SNEDDS, SMEDDS,

50 Lipid suspension, Polymers, Super-SNEDDS, supersaturation, Super-SMEDDS,

51 Supersaturating Drug Delivery Systems

52

53 ABBREVIATIONS

54 COSMO, COnductor like Screening Model; LBF, lipid-based formulation; PI, precipitation 55 inhibitor; sLBF, supersaturated lipid-based formulation; sLBF-PI, supersaturated lipid-based 56 formulation with incorporated precipitation inhibitor; sLBF-aqPI, supersaturated lipid-based

57 formulation with pre-dissolved precipitation inhibitor in the dispersion media 


\section{Thesis Publications}

\section{INTRODUCTION}

Favourable solubility in the gastrointestinal fluids and intestinal permeability is a prerequisite for a high oral bioavailability of any drug. However, drug discovery approaches such as high throughput screenings, modifications during lead optimization as well as the noticeable therapeutic target shift towards intracellular targets deliver more drugs displaying low aqueous solubility and beyond Rule-of-Five properties. ${ }^{1-3}$ These drug candidates display sub-optimal biopharmaceutical properties and create a need for bio-enabling formulation approaches. The design of such formulations includes strategies to generate and maintain high concentrations or supersaturation in intraluminal fluids. Prominent examples of such supersaturating formulations are amorphous solid dispersions and the use of lipid-based formulations (LBF). ${ }^{4}$ ${ }^{5}$ In particular, drugs with a low aqueous solubility, a high lipophilicity and/or bioavailability that is increased by co-ingestion of fatty meals, LBFs can offer particular formulation advantages. ${ }^{4-6}$ The most convenient and conventional LBFs are lipid solutions, where the drug is dissolved in the lipid vehicle, and hence most widely applicable for drugs that show high lipid solubility. As the drug is dissolved in the LBF solution, this circumvents the dissolution process and provides an enhanced intestinal solubilisation for lipophilic drugs in the gastrointestinal environment. ${ }^{7}$ Upon digestion of the lipid excipient, the changing nature of the intraluminal colloids potentially generates supersaturated drug concentrations in the gastrointestinal fluids effectively promoting absorption. ${ }^{7}$ Alternatively, for drugs which display low solubility in lipid vehicles, strategies to increase dose loading in the lipid vehicles may be required such as lipid suspensions, ${ }^{8-10}$ supersaturated LBFs (sLBF), ${ }^{11-14}$ lipophilic salts, ${ }^{15-17}$ or lipid-hybrid systems. ${ }^{18-20}$

sLBFs can be beneficial in delivering drug candidates of this type and interest in their application has increased over the past number of years. The most common method to generate 


\section{Thesis Publications}

84 drug supersaturation in lipids is by heating excess drug in the lipids, followed by cooling, ${ }^{13}$

85 whereupon the amount of dissolved drug in the lipid excipients may exceed the thermodynamic solubility (at lower temperatures) and the LBF becomes supersaturated. A supersaturated formulation is considered kinetically stable, and SLBFs have successfully been applied to improve oral bioavailability of a number of poorly water-soluble drugs such as cinnarizine, simvastatin and halofantrine. ${ }^{11,12,21-23}$ An SLBF formulation approach offers a number of advantages particularly in early stages of development. These advantages include the ease of preparation, suitability for ease of dosing in pre-clinical models and the ability to prepare prototype formulations on a small scale while keeping the development costs low at times where the attrition rate is usually high. Thomas et al. developed a sLBF of simvastatin at $150 \%$ of the saturation solubility and assessed bioavailability in dogs. ${ }^{11}$ Oral bioavailability of the sLBF was 1.8 -fold higher when compared to the same dose of a LBF solution at $75 \%$ of the saturation solubility. ${ }^{11}$ Recently, our group has reported that a sLBF of venetoclax (containing venetoclax at $350 \%$ of the apparent saturation solubility) increased oral bioavailability 2.1 -fold when compared to a control LBF (i.e. lipid suspension) and 3.8-fold when compared to the crude drug powder. ${ }^{14}$ Additionally, the venetoclax study demonstrated during in vitro lipolysis testing of the SLBF that the drug concentration of venetoclax in the aqueous digest phase were in the range of the reported amorphous solubility in fasted state simulated intestinal fluid (FaSSIF). ${ }^{14}$ With such elevated aqueous phase concentrations reached by a sLBF, the risk of precipitation is deemed high and hence it was hypothesized that incorporation of a precipitation inhibitor (PI) may be beneficial.

While the incorporation of PIs in a supersaturated drug delivery system has been widely explored for solid solutions, to the best of our knowledge, to date no study has reported the incorporation of PIs in sLBFs testing in vivo. A limited number of studies have explored the 


\section{Thesis Publications}

use of PIs to reduce the risk of drug precipitation upon dispersion and digestion of classical LBFs. ${ }^{24-27}$ Gao and co-workers developed an undersaturated self-emulsifying LBF for paclitaxel with hydroxypropyl methylcellulose (HPMC) as PI. ${ }^{26}$ The HPMC concentration was $50 \mathrm{mg} / \mathrm{mL}$ and a 10 -fold higher oral bioavailability was observed by incorporating HPMC compared to a HPMC-free formulation in rats. ${ }^{26}$ Gao and colleagues described such formulations as 'supersaturable' referring primarily to the ability of many LBFs to generate supersaturated drug concentrations on dispersion/digestion in intestinal fluids. However, it is worth noting that supersaturable LBFs explored by Gao et al. contain drug below saturation solubility and are distinct from sLBFs even though this nomenclature is not consistently used throughout the literature. A subsequent study examined the effect of incorporating HPMC in LBFs for the investigational drug PNU-91325. The study confirmed that the use of HPMC as a PI resulted in a higher aqueous phase concentration in vitro as well as the increases in bioavailability in dogs. ${ }^{25}$ The LBFs explored in these studies contained $70-100 \%(w / w)$ cosolvents and surfactants (i.e. type IV of the lipid formulation classification system (LFCS) ${ }^{28}$, ${ }^{29}$ ), which in general present a greater risk of drug precipitation on dispersion. Similarly, Suys et al. recently reported that the utility of PIs to prolong supersaturation was more evident for a type IV (50\% co-solvent) and type IIIB (25\% co-solvent and $25 \%$ surfactants) formulation, whereas for a type IIIA formulation (no co-solvent, 35\% surfactant), the PIs studied had no impact on prolonging supersaturation during in vitro digestion. ${ }^{27}$ Collectively, these studies demonstrate the merits of incorporating PIs in a LBF where there is a perceived high risk of precipitation on dispersion/digestion due to for example a rapid co-solvent depletion. However, to date no study has explored the utility of PIs to maintain supersaturation in sLBFs, which the present study aimed to investigate. 


\section{Thesis Publications}

133 Venetoclax is a highly lipophilic drug $\left(\log P\right.$ of $\left.5.5^{30}\right)$ with a high molecular mass of 868.44

134

135 $\mathrm{g} / \mathrm{mol}^{30}$ and a melting point of $139^{\circ} \mathrm{C}$ (Table S 1 ) represents a recently licenced drug with properties in the beyond Rule-of-Five space. The drug is classified as a BCS class IV drug based on low aqueous solubility and permeability. The commercial formulation Venclyxto ${ }^{\circledR}$ displays a pronounced food dependent oral bioavailability with a 3.4-fold increase in oral bioavailability after a low-fat meal and a 5 -fold increase after a high fat meal compared to the fasted state. ${ }^{30}$

This study explored the merits of PIs on the oral bioavailability of an oil-based SLBF of venetoclax. A range of promising PIs have been identified based on calculated excess enthalpy of mixing (COSMOquick software), which served to estimate molecular excipient interaction in the more complex aqueous dispersions. The ability of the selected PIs to maintain supersaturation of venetoclax in biorelevant media was tested in vitro and the impact of incorporating PIs into the sLBF in comparison to the separate addition (pre-dissolved) of the PIs in the simulated intestinal fluids was evaluated. A subsequent in vivo study examined the impact of incorporated PIs in SLBFs on the oral bioavailability of venetoclax in landrace pigs.

\section{MATERIALS AND METHODS}

Chemicals and materials

Venetoclax was purchased from Kemprotec Ltd. (UK) (Batch \# 1810004). Olive oil, highly refined and low acidity, taurodeoxycholic acid (NaTDC) and pancreatic lipase ( $8 \times$ USP) were ordered from Sigma-Aldrich (Ireland). Lipoid ${ }^{\circledR}$ E PC S was obtained from Lipoid GmbH (Germany) and Eudragit ${ }^{\circledR}$ EPO was obtained from Evonik (Germany). Hydroxypropyl methylcellulose acetate succinate (HPMCAS) (AQOAT (HPMCAS-MF)) was purchased from ShinEtsu (Japan) and Pluronic ${ }^{(B)}$ F108, hydroxypropyl methylcellulose (HPMC), 


\section{Thesis Publications}

158 Polyvinylpyrrolidone (PVP) were purchased from MilliporeSigma (St Louis, MO, USA).

159 Kollidon $^{\circledR}$ VA 64 (PVP/VA) was kindly donated by BASF (Germany). Capmul $\mathrm{MCM}^{\circledR}$ and

160 Captex $^{\circledR} 1000$ were kindly donated by Abitec corporation. A sample of Peceol ${ }^{\circledR}$ was kindly

161 donated by Gattefossé (France) and SIF powder Version 1 was kindly donated by

162 biorelevant.com (UK). Water was purified by a MilliQ ${ }^{\circledR}$ water system. All other chemicals and

163 solvents were of analytical or high-performance liquid chromatography (HPLC) grade and were purchased from Sigma-Aldrich (Ireland) and used as received. Apparent solubility

167 Apparent solubility was determined in olive oil, Captex ${ }^{\circledR} 1000$, Peceol $^{\circledR}$ and Capmul MCM ${ }^{\circledR}$. 168 In brief, an excess of venetoclax was added to $2 \mathrm{~mL}$ of the excipients and stirred at $200 \mathrm{rpm}$ 169 (25\% power) (Mixdrive $15,2 \mathrm{MAG}$, Germany) at $37^{\circ} \mathrm{C}$. Solid excipients were melted at $50^{\circ} \mathrm{C}$ and cooled to $37^{\circ} \mathrm{C}$ prior to venetoclax addition. Samples were taken after $24 \mathrm{~h}, 48 \mathrm{~h}, 72 \mathrm{~h}$ and centrifuged at $21,380 \mathrm{~g}$ and $37^{\circ} \mathrm{C}$ for $15 \mathrm{~min}$ (Mikro $200 \mathrm{R}$, Andreas Hettich GmbH \& Co. KG,

172 Germany). The supernatant was transferred to a new sample tube and centrifuged again using 173 identical conditions. To solubilise the oily excipient, the supernatant was diluted in acetonitrile:ethyl acetate $(1: 3 \mathrm{v} / \mathrm{v})$. Followed by further $1: 10(\mathrm{v} / \mathrm{v})$ dilution with acetonitrile:ethyl acetate $(3: 1 \mathrm{v} / \mathrm{v})$. The obtained samples were diluted appropriately with mobile phase before analysis by reverse phase HPLC as described below. All samples were run

177 in triplicates.

Biorelevant solubility

180 FaSSIF and fed state simulated intestinal fluids (FeSSIF) were prepared according to the 181 instructions by biorelevant.com. FeSSIF was used directly, whereas FaSSIF was left at room

182 temperature for $2 \mathrm{~h}$ prior to usage. Excess venetoclax was added to $2 \mathrm{~mL}$ of biorelevant media 


\section{Thesis Publications}

and placed in a water bath shaker at 200 shakes/min (GLS400, Grant Instruments, UK) and $37^{\circ} \mathrm{C}$. Samples were taken after $3 \mathrm{~h}, 6 \mathrm{~h}$ and $24 \mathrm{~h}$ and centrifuged at $21,380 \mathrm{~g}$ and $37^{\circ} \mathrm{C}$ for 15 min (Mikro 200 R, Andreas Hettich GmbH \& Co. KG, Germany). The supernatant was transferred to a new sample tube and centrifuged again under identical conditions. Subsequently, the supernatant was diluted with a mobile phase before analysis by HPLC.

The samples were analysed using an Agilent 1200 series HPLC system (Agilent Technology Inc., US) that comprised a binary pump, degasser, autosampler and variable wavelength detector. Data were analysed using the software EZChrom Elite ${ }^{\mathbb{B}}$ version 3.2. Venetoclax was separated from the sample matrix with a Zorbax ${ }^{\circledR}$ Eclipse Plus-C18 column (5 $\mu \mathrm{m}, 4.6 \mathrm{~mm} x$ $150 \mathrm{~mm}$ ) including a Zorbax ${ }^{\circledR}$ Eclipse Plus-C18 guard column $(5 \mu \mathrm{m}, 4.6 \mathrm{~mm} \times 12.5 \mathrm{~mm})$ at $40{ }^{\circ} \mathrm{C}$. The mobile phase consisted of (a) acetonitrile with $0.5 \%$ trifluoroacetic acid (TFA) and (b) water with $0.5 \%$ TFA at a ratio of $53: 47(\mathrm{a}: \mathrm{b} \mathrm{v} / \mathrm{v})$ and was used at a flow rate of $1 \mathrm{~mL} / \mathrm{min}$. The injection volume was $20 \mu \mathrm{L}$ and the detection wavelength was set to $316 \mathrm{~nm}$. The limit of detection (LOD) was $20 \mathrm{ng} / \mathrm{mL}$ and the limit of quantification (LOQ) $65 \mathrm{ng} / \mathrm{mL}$ determined using the standard error of $y$-intercept according to International Council for Harmonisation (ICH) Q2 guideline. ${ }^{31}$

\section{Formulations for in vivo and in vitro studies}

In the in vivo study and in vitro PI screen, the supersaturated lipid solution (sLBF) was prepared as previously reported with a lower temperature to reduce the thermal impact on the drug and excipient. ${ }^{14}$ In brief, $300 \mathrm{mg}$ venetoclax were added to $6 \mathrm{~mL} \mathrm{Peceol}^{\circledR}$ and dispersed at $600 \mathrm{rpm}$ (Stuart CD162 heat-stir, Cole-Parmer, UK) and sealed with parafilm. A continuous nitrogen stream into the vial removed oxygen throughout the preparation. After suspending the drug particles, the obtained suspension was slowly heated to $55{ }^{\circ} \mathrm{C}$ (Stuart CD 162 heat-stir, Cole- 


\section{Thesis Publications}

208 Parmer, UK). The mixture was kept at $55^{\circ} \mathrm{C}$ for $10 \mathrm{~min}$ and cooled to $25^{\circ} \mathrm{C}$ while continuously

209 stirring at $600 \mathrm{rpm}$. Subsequently, the mixture was heated a second time under the same conditions as stated above and cooled to room temperature to obtain the final sLBF. The absence of crystals was confirmed using cross-polarized light microscopy. For the in vivo study,

212 sLBF was administered in hard gelatine capsules size 00EL (Licaps ${ }^{\circledR}$, Capsugel, Lonza Group Ltd.) with $1 \mathrm{~mL} /$ capsule.

For the preparation of the sLBF with PI, HPMC, HPMCAS, Pluronic ${ }^{\circledR}$ F108, Eudragit ${ }^{\circledR}$ EPO, PVP and PVP/VA were added to the sLBF at a drug:PI ratio of $1: 1(w / w)$. At the given PI concentration $(50 \mathrm{mg} / \mathrm{mL})$ Pluronic ${ }^{\circledR}$ F108, Eudragit ${ }^{\circledR}$ EPO, PVP and PVP/VA were soluble in Peceol ${ }^{\circledR}$ at $37^{\circ} \mathrm{C}$, while HPMC and HPMCAS resulted in a suspension. In the case of the soluble PIs, the Peceol ${ }^{\circledR}$-PI-solution was used to prepare the PI containing SLBF using the method described above. In the case of HPMC and HPMCAS, the SLBF was prepared as described above and HPMC and HPMCAS were added and dispersed ad hoc into the sLBF before in vitro and in vivo experiments. The amount of PI in the lipid vehicle $(50 \mathrm{mg} / \mathrm{mL})$ was based on previous work by Gao et al. ${ }^{24,25}$

The absence of crystalline material in the supersaturated solutions was confirmed by means of cross-polarised light microscopy using an Olympus BX51 with an Olympus SC100 camera operated by Olympus Stream ${ }^{\circledR}$ essentials 2.3.3. The light was polarized using the polarizer UPOT and analysed with the analyser U-ANT. The absence of crystals was assumed, if no birefringence was observed. 


\section{Thesis Publications}

\section{In vitro evaluation: drug solubilization during formulation dispersion and digestion} In vitro lipolysis was performed using a pH-stat apparatus (Metrohm AG, Herisau, Switzerland) comprising a Titrando ${ }^{\circledR} 907$ stirrer, 804 Ti-stand, a pH electrode (Metrohm AG, Herisau, Switzerland) and two 800 Dosino ${ }^{\circledR}$ dosing units coupled to a $20 \mathrm{~mL}$ autoburette. The system was operated by the Tiamo ${ }^{\circledR} 2.2$ software. The in vitro protocol was used as previously reported. ${ }^{32}$ In brief, the buffer contained $2 \mathrm{mM}$ TRIS maleate, $150 \mathrm{mM} \mathrm{NaCl}, 1.4 \mathrm{mM} \mathrm{CaCl} 2 \cdot 2 \mathrm{H}_{2} \mathrm{O}$, adjusted to $\mathrm{pH}$ 6.5. For the digestion experiments the buffer was supplemented with $3 \mathrm{mM}$ NaTDC and $0.75 \mathrm{mM}$ PC (digestion buffer) and stirred for $12 \mathrm{~h}$ before further usage. The pancreatin extract was prepared freshly by adding $5 \mathrm{~mL}$ of $5{ }^{\circ} \mathrm{C}$ digestion buffer to $1 \mathrm{~g}$ of porcine pancreatic enzymes ( $8 \mathrm{x}$ USP), which was vortexed thoroughly. The mixture was centrifuged for $15 \mathrm{~min}$ at $5{ }^{\circ} \mathrm{C}, 2800 \mathrm{~g}$ (Rotina $380 \mathrm{R}$, Andreas Hettich $\mathrm{GmbH} \& \mathrm{Co} . \mathrm{KG}$, Germany) and $4 \mathrm{~mL}$ of supernatant was recovered and stored at $2-8^{\circ} \mathrm{C}$ before further usage. The pancreatic extract had a pancreatic lipase activity of $\sim 10000 \mathrm{TBU} / \mathrm{mL}$ (to provide approximately $1000 \mathrm{TBU}$ per $\mathrm{mL}$ of digest), where $1 \mathrm{TBU}$ represents the amount of enzyme that liberates $1 \mu \mathrm{mol}$ of FA from tributyrin per min. ${ }^{33}$

For the in vitro lipolysis experiment $1.075 \mathrm{~g}$ of lipid formulation was dispersed into $39 \mathrm{~mL}$ of digestion buffer for $10 \mathrm{~min}$. Three $1 \mathrm{~mL}$ samples were taken at $2.5,5$ and $10 \mathrm{~min}$ from the middle of the vessel. $\mathrm{pH}$ of the media was adjusted and maintained at 6.5 using $0.2 \mathrm{M} \mathrm{NaOH}$. To the remaining $36 \mathrm{~mL}(1.0 \mathrm{~g}$ lipid formulation) dispersion $4 \mathrm{~mL}$ of pancreatin extract was added to initialize digestion. After $60 \mathrm{~min}$ the released non-ionized free fatty acids were determined by a $\mathrm{pH}$ increase of the buffer to $\mathrm{pH} 9$. The stirring speed throughout dispersion and digestion was set at $450 \mathrm{rpm}$. 


\section{Thesis Publications}

256 Samples of $1.0 \mathrm{~mL}$ were taken at 5, 10, 15, 30, 45 and 60 min during the digestion experiment from the middle of the vessel. In each sample and after 60 min the enzymes were inhibited by the addition of $1 \mathrm{M} 4$ - bromophenylboronic acid in methanol (5 $\mu \mathrm{L}$ per $\mathrm{mL}$ sample). Additionally, to each $1 \mathrm{~mL}$ sample during digestion a $100 \mu \mathrm{L}$ sample was taken and added to $900 \mu \mathrm{L}$ of acetonitrile and mixed. This sample was used to quantify the total drug recovery, which allowed adjustment of inhomogeneous samples. All samples were centrifuged at $37^{\circ} \mathrm{C}$ and $21,000 \mathrm{~g}$ for $30 \mathrm{~min}$ using a benchtop centrifuge of the type Hettich Micro $200 \mathrm{R}$ (Andreas Hettich GmbH \& Co. KG, Germany).

The excess enthalpy of mixing between venetoclax and the polymeric PI was calculated using the COSMOquick software (COSMOlogic, Germany, Version 1.6). The software is based on the Conductor like Screening Model for Real Solvents ${ }^{34,35}$ that combined quantum chemical surface charge calculations with statistical thermodynamics. The COSMOquick approach in particular allows for a fast estimation of these surface charge densities for further calculations. this study approximated as trimers. The drug:PI ratio was set at a stoichiometric ratio of $1: 1$ to ${ }^{36}$ Venetoclax and the polymers were entered in smiles notation. As the quantum chemical calculations cannot capture the full complexity of the polymers, the polymer structures were in represent the formulations used in vitro and in vivo and the temperature was set to $37^{\circ} \mathrm{C}$. In line with previous applications of COSMOquick for co-former screening in co-crystal selection, ${ }^{37}$ drug solubility estimations in glycerides ${ }^{38}$ and polymer screening for supersaturated formulations, ${ }^{39}$ a more negative value of calculated excess enthalpy ranks the strength of molecular drug-excipient interaction. This is just an approximation, as the presence of an aqueous phase is not considered in the calculations nor the complexity of any biorelevant medium. Therefore, the results should be understood as a first in silico estimation of relative 


\section{Thesis Publications}

excipient comparison. This has previously been referred to as a higher or lower 'COSMORank’. ${ }^{39}$

\section{In vitro precipitation inhibitor testing}

The in vitro PI testing for venetoclax was done by means of a solvent shift. The effect of a fully hydrated PI (dissolved in FaSSIF) was compared against the PI in the formulation. Thus, the employed PIs HPMC, HPMCAS, Eudragit ${ }^{\mathbb{B}}$ EPO, PVP, PVP/VA and Pluronic ${ }^{(B)}$ F108 were dissolved in either FaSSIF or either suspended or dissolved in the supersaturated lipid solution. For the hydrated PI test, $5 \mathrm{mg}$ PI was dissolved in $5 \mathrm{~mL}$ FaSSIF (prepared according to biorelevant.com) and $5 \mathrm{mg}$ of venetoclax dissolved in either DMSO $(100 \mathrm{mg} / \mathrm{mL})$ or Peceol ${ }^{\circledR}$ (sLBF $50 \mathrm{mg} / \mathrm{mL}$ ) was added. For the evaluation of the PI in the lipid formulation, $100 \mu \mathrm{L}$ of the $\mathrm{SLBF}(50 \mathrm{mg} / \mathrm{mL}$ venetoclax and $50 \mathrm{mg} / \mathrm{mL}$ PI) was added to $5 \mathrm{~mL}$ FaSSIF. The vials were sealed and placed in a water bath shaker at 200 shakes/min and $37{ }^{\circ} \mathrm{C}$ (GLS400, Grant Instruments, UK). After $2,5,10,15,30,60,120,180 \min 250 \mu \mathrm{L}$ samples were taken. The samples were filtered with a $0.2 \mu \mathrm{m}$ syringe filter (Whatman ${ }^{\circledR}$ Spartan $13 / 0.2$ ) and samples that contained lipids were additionally centrifuged for $30 \mathrm{~min}$ at $21,380 \mathrm{~g}$ and $37^{\circ} \mathrm{C}$ (Mikro $200 \mathrm{R}$, Hettich GmbH, Germany). The aqueous phase was collected, and one part was diluted 1:10 $(\mathrm{v} / \mathrm{v})$ with acetonitrile (including $0.5 \%(\mathrm{v} / \mathrm{v})$ TFA). A pure (non-diluted) and diluted sample was analysed by HPLC as described above.

Additionally, the solubility of venetoclax was determined in FaSSIF with dissolved PI. Excess venetoclax was added to $5 \mathrm{~mL}$ FaSSIF with $5 \mathrm{mg}$ PI. $250 \mu \mathrm{L}$ samples were taken at $3 \mathrm{~h}, 6 \mathrm{~h}$, $24 \mathrm{~h}$ and filtered through a $0.2 \mu \mathrm{m}$ syringe filter (Whatman ${ }^{\circledR}$ Spartan 13/0.2), diluted with acetonitrile (containing $0.5 \%(\mathrm{v} / \mathrm{v})$ TFA) and analysed by HPLC as described above. 


\section{Thesis Publications}

\section{In vivo study}

All experiments were approved and conducted with licences issued by the Health Product Regulatory Authority, Ireland (project licence AE 19130/P058) as directed by the EU Statutory instruments of the EU directive 2010/63/EU (Protection of Animals used for Scientific Purposes). Local ethical approval was granted by University College Cork Animal Experimentation Ethics Committee (AEEC). In order to test all PIs two bioavailability studies had to be conducted. The first study was with 5 pigs and a 6-way crossover and the second study with 3 pigs and a 4-way crossover. Both studies were randomised and conducted in male landrace pigs $(15-17 \mathrm{~kg}$ ) and each pig received a single dose of $100 \mathrm{mg}$ venetoclax. Pigs were fed approximately $175 \mathrm{~g}$ of standard weanling pig pellet feed twice daily. In the fasted study legs the final feed of $175 \mathrm{~g}$ was given $24 \mathrm{~h}$ prior to dosing. As part of the study design any remaining food was removed $16 \mathrm{~h}$ before dosing, however, no food remained at this point in any of the groups. On day 1, an indwelling intravenous catheter was inserted from the ear vein into the jugular vein under general anaesthesia, which was used for repeated blood sampling throughout the study. In the first study, on day 3 , following an overnight fast of $16 \mathrm{~h}$, pigs were administered either sLBF or sLBF with PI, respectively. The tested PIs included HPMC, HPMCAS, PVP, PVP/VA, Pluronic ${ }^{\circledR}$ F108. In the second study, on day 3, following an overnight fast of $16 \mathrm{~h}$, pigs were administered either a reference capsule with venetoclax powder, a venetoclax Peceol ${ }^{\circledR}$ suspension, a sLBF or a sLBF including Eudragit ${ }^{\circledR}$ EPO, respectively. The results of the venetoclax powder, Peceol ${ }^{\circledR}$ suspension and sLBF have previously been reported ${ }^{14}$ and are not further described in the current study. In both studies all formulations were administered with the aid of a dosing gun followed by $50 \mathrm{~mL}$ of water via a syringe. In order to control the water intake with the dosage forms, the water availability was restricted for $3 \mathrm{~h}$ post dosing. At all other times water was available ad libitum. To facilitate handling during the oral administration, an intramuscular dose of ketamine $(5 \mathrm{mg} / \mathrm{kg})$ and 


\section{Thesis Publications}

331 xylazine $(1 \mathrm{mg} / \mathrm{kg})$ was administered in both studies. Blood samples were collected after $0.5,1$,

332

333
$1.5,2,3,4,5,6,7,8,9,10,12,24 \mathrm{~h}$ in heparinised tubes. Upon collection, blood samples were immediately centrifuged at $3000 \mathrm{~g}, 4{ }^{\circ} \mathrm{C}$ for $5.5 \mathrm{~min}$ (Eppendorf $5702 \mathrm{R}$, Rotor A-4-38, Eppendorf Ltd., UK). The supernatant plasma was harvested and stored at $-20^{\circ} \mathrm{C}$ until further analysis. A 6-day washout period was maintained between the study legs.

\section{Bioanalysis}

The plasma concentrations of venetoclax were determined by reversed phase HPLC. The Agilent 1260 series HPLC system (Agilent Technology Inc., US) comprised a binary pump, degasser, temperature controlled autosampler, column oven and diode array detector. The system was operated, and the data analysed with EZChrom Elite ${ }^{\circledR}$ version 3.3.2. A Zorbax ${ }^{\circledR}$ Eclipse Plus-C18 column $(5 \mu \mathrm{m}, 4.6 \mathrm{~mm} \times 150 \mathrm{~mm})$ with a Zorbax ${ }^{\circledR}$ Eclipse Plus-C18 guard column $(5 \mu \mathrm{m}, 4.6 \mathrm{~mm} \times 12.5 \mathrm{~mm})$ was used for the separation of venetoclax. The mobile phase consisted of water and acetonitrile with $0.5 \%(\mathrm{v} / \mathrm{v})$ TFA at a ratio of 47:53 (v/v) and was used at a flow rate of $1.0 \mathrm{~mL} / \mathrm{min}$. The sample and column temperature were set at $5{ }^{\circ} \mathrm{C}$ and $40{ }^{\circ} \mathrm{C}$, respectively, and the detection wavelength was set to 250,290 and $316 \mathrm{~nm}$. Venetoclax was extracted from the plasma samples by liquid-liquid extraction as reported previously. ${ }^{14}$ In brief, venetoclax was extracted from $500 \mu \mathrm{L}$ plasma using acetonitrile and ethyl acetate. Vemurafenib was used as internal standard. The extraction solvents were dried under a nitrogen stream at $60{ }^{\circ} \mathrm{C}$ and the residues were reconstituted in $100 \mu \mathrm{L}$ mobile phase (excluding TFA) followed by centrifugation at $25^{\circ} \mathrm{C}, 11,500 \mathrm{~g}$ for $5 \mathrm{~min}$ (Mikro $200 \mathrm{R}$, Andreas Hettich GmbH \& Co. KG, Germany). The injection volume used for HPLC analysis of the supernatant was $50 \mu \mathrm{L}$. 


\section{Thesis Publications}

\section{Data Analysis}

Prior to statistical analysis the Bartlett's test was used to check for equal variances. A one-way analysis of variance (one-way ANOVA) was performed for the lipolysis data using a Tukey post-hoc test to compare the different formulation performances. While the concentration time profiles of the in vitro PI test were analysed using a two-way ANOVA with the Bonferroni post-test, the area under the curve (AUC) was analysed using a one-way ANOVA with a Tukey post-hoc test. The pharmacokinetic parameters were calculated using Microsoft ${ }^{(\mathbb{B}}$ Excel $^{(\mathbb{B}}$ by means of the trapezoidal rule. The plasma concentration profiles were analysed by noncompartmental analysis. The statistical analysis for the in vivo parameters of the formulations within the same crossover study was performed using a one-way ANOVA after using the Bartlett's test to check for equal variances. The pairwise comparison of the groups was done using Tukey's multiple comparison test. All statistical analyses were carried out using GraphPad Prism ${ }^{\circledR} 5$.

\section{RESULTS}

Apparent solubility of venetoclax in lipid excipients

Solubility screening in pure lipid excipient indicated that venetoclax showed a higher apparent solubility in long chain than medium chain based excipients (Figure $1 \mathrm{~A}$ ). Within the long chain and medium chain excipients, a higher apparent solubility was observed for the monoglycerides compared to the triglycerides. Subsequently, biorelevant solubility of venetoclax was determined (Figure $1 \mathrm{~B}$ ). Solubility in FaSSIF was $6.8 \pm 1.8 \mu \mathrm{g} / \mathrm{mL}$ translating to only $1.7 \%$ of a $100 \mathrm{mg}$ dose venetoclax in $250 \mathrm{~mL}$ of gastrointestinal fluid. In the fed state the solubility increased 3.9-fold to $6.7 \%$ of the $100 \mathrm{mg}$ venetoclax dose, which in general is in agreement with the observation in increased bioavailability for venetoclax reported from clinical studies. 30 


\section{Thesis Publications}

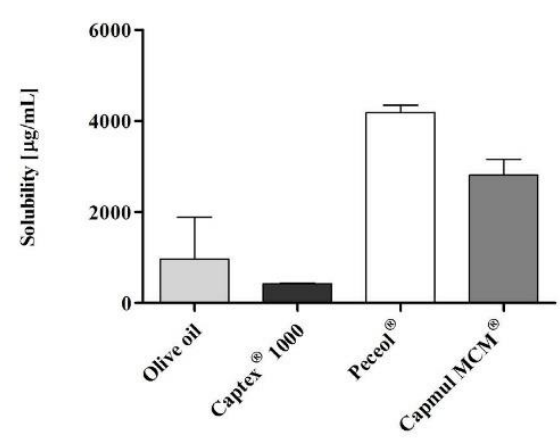

B

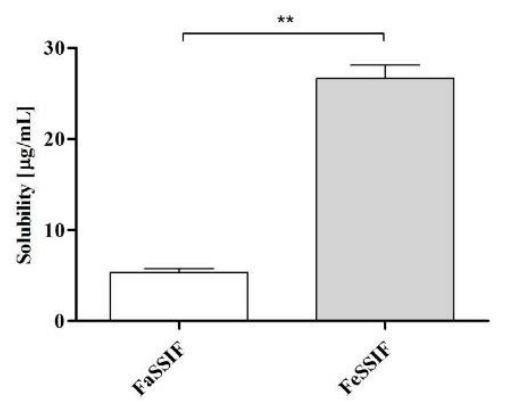

380

381

sLBF achieves high aqueous drug concentration during in vitro dispersion and digestion.

Previous studies by our group developed a venetoclax sLBF using a preparation method at 70 ${ }^{\circ} \mathrm{C}$. ${ }^{14}$ Process optimisation in this study focused on improving the thermally-induced supersaturation process by lowering the overall energy input while maintaining the desired level of supersaturation. $\mathrm{sLBFs}$ were prepared at the target dose load of $50 \mathrm{mg} / \mathrm{mL}$ by heating a LBF suspension $(50 \mathrm{mg} / \mathrm{mL})$ to $55^{\circ} \mathrm{C}$. The (s)LBF was kept at $55{ }^{\circ} \mathrm{C}$ for $10 \mathrm{~min}$ to dissolve venetoclax before cooling the solution to room temperature. In this study, the obtained sLBF had a 11.9-fold (1193.7\%) higher drug load compared to the apparent saturation solubility. The venetoclax concentrations in the aqueous phase during dispersion and digestion are shown in Table 1 and Figure S 1. 


\section{Thesis Publications}

Table 1. Venetoclax concentration in the in vitro lipolysis experiment after $10 \mathrm{~min}$ of dispersion and after $5 \mathrm{~min}$ and $60 \mathrm{~min}$ of digestion. Venetoclax was formulated as $\mathrm{sLBF}(50 \mathrm{mg} / \mathrm{mL})$, Peceol ${ }^{\circledR}$ suspension $(50 \mathrm{mg} / \mathrm{mL})$ and aqueous suspension $(50 \mathrm{mg} / \mathrm{mL})$. All experiments were run with $\mathrm{n}=3$ and results are shown as mean \pm SD.

Venetoclax concentration $(\mu \mathrm{g} / \mathrm{mL})$ in aqueous phase (AP) during $\mathrm{LBF}$ dispersion and digestion

\begin{tabular}{llll}
\cline { 2 - 3 } Formulation & $\begin{array}{l}\text { AP } \\
(10 \mathrm{~min})\end{array}$ & $\begin{array}{l}\mathrm{AP} \text { Digersion } \\
(5 \mathrm{~min})\end{array}$ & $\begin{array}{l}\text { AP } \\
(60 \mathrm{~min})\end{array}$ \\
\hline $\mathrm{sLBF}^{\mathrm{a})}$ & $18.7 \pm 0.0$ & $37.5 \pm 3.2$ & $73.8 \pm 6.4$ \\
LBF suspension $^{\mathrm{b}, \mathrm{c})}$ & $1.0 \pm 0.1$ & $1.7 \pm 0.3$ & $2.9 \pm 0.5$ \\
aqueous suspension $^{c)}$ & $3.0 \pm 0.1$ & $9.1 \pm 0.2$ & $9.4 \pm 0.3$
\end{tabular}

a) SLBF drug loading was $1194 \%$ of determined apparent solubility

401

b) LBF drug loading was $100 \%$ solubilised $+158 \%$ suspended of determined apparent solubility ${ }^{c)}$ Data as previously reported. ${ }^{14}$

As the drug needs to be dissolved prior to absorption by the body, the solubilised drug in the aqueous phase of the in vitro lipolysis test is often used as a guide to formulation performance in vivo. The sLBF displayed relatively high concentrations in the aqueous phase throughout in vitro lipolysis, with an average venetoclax concentration of $18.6 \pm 1.3 \mu \mathrm{g} / \mathrm{mL}$ during dispersion and $73.8 \pm 6.4 \mu \mathrm{g} / \mathrm{mL}$ after $60 \mathrm{~min}$ of digestion. Interestingly, the final concentration at the end of the digestion, was equivalent to $6.4 \pm 0.5 \%$ of the venetoclax dose used in the experiment and was 2.8-fold higher when compared to the apparent solubility of venetoclax in FeSSIF. Additionally, the measured concentration was higher than the reported amorphous solubility in FeSSIF of $26.4-54.6 \mu \mathrm{g} / \mathrm{mL} .{ }^{40}$ Most of venetoclax was recovered in the lipid phase with an average of $98.5 \pm 2.8 \%$ of the venetoclax dose during dispersion and $88.6 \pm 0.2 \%$ after $60 \mathrm{~min}$ of digestion (Figure S 1). The lipid phase can act as a reservoir for the drug, which gradually 


\section{Thesis Publications}

415 diffuses into the aqueous phase during the transit through the gastrointestinal tract. Additionally,

416

417

418 the lipid phase is digested during the transit releasing venetoclax. In the case of the sLBF the lowest amount of venetoclax was recovered in the solid phase. SLBF showed no precipitation during dispersion and after $60 \mathrm{~min}$ of digestion only $6.2 \pm 0.6 \%$ of the venetoclax dose was precipitated. These results are in line with previous studies showing a superior performance (high aqueous phase concentration, low solid phase concentration) of the SLBF in vitro relative to a lipid-based suspension and aqueous suspension. ${ }^{14}$ The optimised processing method, therefore, yielded comparable formulation performance.

\section{COSMO-RS as a preliminary in silico screening tool for PI selection}

To identify the most promising PIs, reduce the extent of in vitro testing and to evaluate the utility of this computational tool in the formulation design of sLBFs, the interactions between venetoclax and various PIs were evaluated in silico. Based on the COSMO-RS theory ${ }^{34,41}$ that combines quantum chemistry and statistical thermodynamics, the COSMOquick software was utilized to predict the PI-drug interactions similarly to previous reports where this screening technique of PI selection was utilised for silica formulations. ${ }^{39}$ The COSMOquick software predicts the interactions of two molecules based on equilibrium thermodynamics and the predicted chemical potential in liquids. The stronger the interaction between venetoclax and the PI, the more negative the calculated excess enthalpy of interaction. A simplified view assigns to a PI that has a more negative excess enthalpy, a higher 'COMSO-Rank' with respect to its suitability as PI. While this tool may be beneficial to reduce in vitro testing by identifying the most promising PI-drug interactions as shown for other formulation approaches, ${ }^{39}$ it must be acknowledged that the in silico calculations did not consider the presence of water nor other formulation excipients, potentially limiting the applicability of this tool for the more complex bio-enabling formulation approach of LBFs. In the present study, the excess enthalpy of mixing 


\section{Thesis Publications}

440 for a variety of PIs was calculated, and PIs with varying negative enthalpy of mixing were

441 selected. The chosen PIs had the following 'COSMO-Rank': Eudragit ${ }^{(\mathbb{R}}$ EPO $>$ Pluronic ${ }^{\circledR}>$

442 HPMCAS $\geq$ PVP $\geq$ PVP/VA $>$ HPMC as shown in Figure 2.

443

444

445

446

447

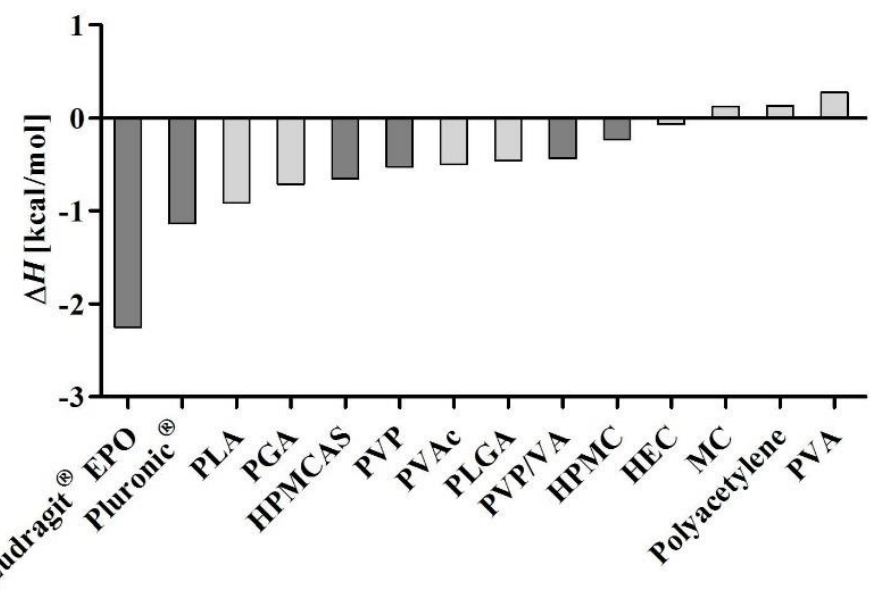

Figure 2. COSMOquick screen. Calculated excess enthalpy of interaction between venetoclax and the PIs. The dark grey PIs were selected for further in vitro and in vivo evaluation. The calculation was based on a 1:1 ratio of drug to PI. PLA: Polylactic acid, PGA: Polyglycolic acid, HPMCAS: Hydroxypropyl methylcellulose acetate succinate, PVP: Polyvinylpyrrolidone, PVAc: Polyvenyl acetate, PLGA: Poly lactic-co-glycolic acid, PVP/VA: Polyvinylpyrrolidoneco-vinyl acetate, HPMC: Hydroxypropyl methylcellulose, HEC: Hydroxypropyl cellulose, MC: Methylcellulose, PVA: Polyvinyl alcohol

\section{In vitro testing for PIs for prolonging supersaturation}

The selected PIs from the in silico screening were further evaluated experimentally in vitro to assess the PI performance (aqueous phase concentration) under simulated conditions and with

Page 20 


\section{Thesis Publications}

456 LBFs. The in vitro PI test was performed by means of solvent shift by dispersing the formulation in (i) FaSSIF or (ii) FaSSIF with pre-dissolved PI (FaSSIF-PI). In FaSSIF-PI was dispersed (a) venetoclax dissolved in DMSO $(100 \mathrm{mg} / \mathrm{mL})$, as a positive control and (b) sLBF (sLBF-aqPI). In FaSSIF was dispersed (c) SLBF without PI (sLBF-noPI) and (d) sLBF with incorporated PI (sLBF-PI). Polymers that were soluble at the tested concentration of $50 \mathrm{mg} / \mathrm{mL}$ were dissolved in SLBF (PVP, PVP/VA, Pluronic ${ }^{\circledR}$ F108, Eudragit ${ }^{\circledR}$ EPO) and those that were not soluble in the sLBF were therefore ad hoc suspended in the sLBF (HPMC, HPMCAS). Additionally, the solubility of venetoclax in FaSSIF-PI was measured. The results of the PI testing are shown in Figure 3 and Table 2. In all cases, the venetoclax solubility in FaSSIF-PI was higher compared to the solubility in FaSSIF with an increase in solubility from 1.3-fold to 3.4-fold. In order of decreasing solubility in FaSSIF-PI the ranking was Eudragit ${ }^{\circledR}$ EPO, HPMC, Pluronic ${ }^{\circledR}$ F108, PVP/VA, HPMCAS and PVP. In general, upon dispersion of the DMSO solution in FaSSIF-PI HPMC, Pluronic ${ }^{\circledR}$ F108, PVP/VA, HPMCAS and PVP venetoclax concentrations reached FaSSIF-PI solubility,

471 which was maintained throughout the experiment. In the case of Eudragit ${ }^{\mathbb{B}}$ EPO a decrease below the FaSSIF-PI solubility was observed. 


\section{Thesis Publications}
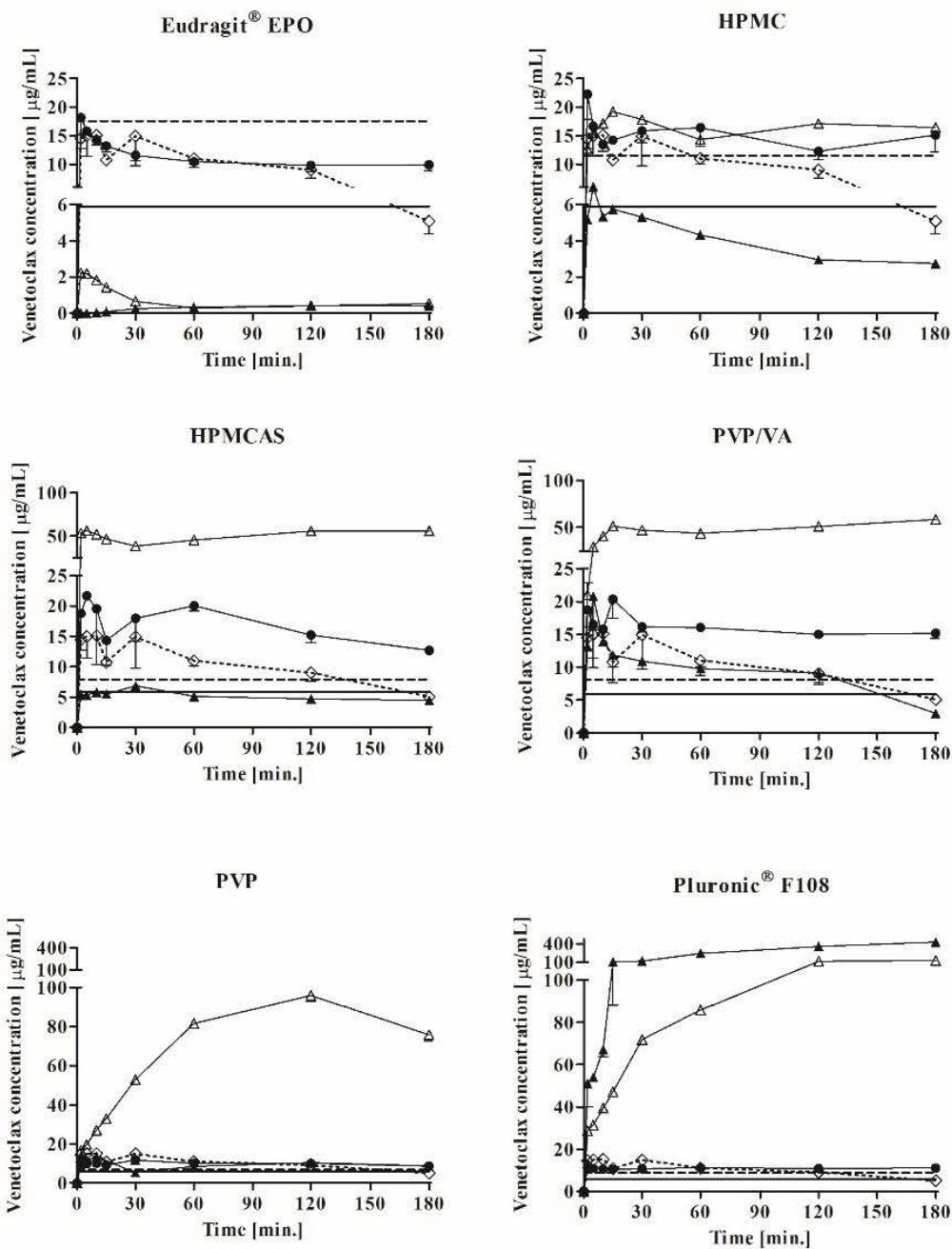

Figure 3. In vitro evaluation of venetoclax concentration profile during dispersion of sLBFs in

FaSSIF (mean \pm SD with $n=3$ ). The dashed line represents the apparent venetoclax solubility in FaSSIF with pre-dissolved PI (FaSSIF-PI) and the solid line represents FaSSIF solubility.

478 The dotted line $(\diamond)$ represents the sLBF alone dispersed in FaSSIF (sLBF-noPI). Venetoclax dissolved in DMSO dispersed in FaSSIF-PI $(\bullet)$, sLBF dispersed in FaSSIF-PI (sLBF-aqPI) ( $\Delta)$, sLBF with PI (sLBF-PI) dispersed in FaSSIF $(\Delta)$. 


\section{Thesis Publications}

Table 2. Area under the curve (AUC) of the in vitro PI testing for sLBF dispersed in FaSSIF-PI (sLBF-aqPI), sLBF with PI incorporated in the

482 formulation (sLBF-PI) and dispersed in FaSSIF, venetoclax dissolved in DMSO dispersed in FaSSIF-PI (DMSO) and sLBF alone dispersed in FaSSIF

483 (sLBF-noPI), \% solubilised venetoclax and apparent supersaturation ratio for sLBF-aqPI and sLBF-PI as well as FaSSIF-PI solubility (mean \pm SD,

$484 \mathrm{n}=3)$

\begin{tabular}{|c|c|c|c|c|c|c|c|c|}
\hline \multirow[b]{2}{*}{ PI } & \multicolumn{3}{|c|}{$\mathrm{AUC}[\mathrm{mg} * \mathrm{~min} / \mathrm{mL}]$} & \multirow[b]{2}{*}{$\begin{array}{l}\text { Apparent FaSSIF-PI } \\
\text { solubility }[\mu \mathrm{g} / \mathrm{mL}]\end{array}$} & \multicolumn{2}{|c|}{ solubilised venetoclax $[\%]^{\text {a) }}$} & \multicolumn{2}{|c|}{$\begin{array}{c}\text { apparent } \\
\text { supersaturation ratio }\end{array}$} \\
\hline & DMSO & sLBF-aqPI & sLBF-PI & & sLBF-aqPI & sLBF-PI & sLBF-aqPI & sLBF-PI \\
\hline Eudragit ${ }^{\hat{E}} \mathrm{EPO}$ & $1.9 \pm 0.3$ & $0.1 \pm 0.004$ & $0.1 \pm 0.02$ & $17.6 \pm 0.8$ & $0.03 \pm 0.002$ & $0.1 \pm 0.01$ & $<\mathrm{LOQ}$ & $0.3 \pm 0.03$ \\
\hline HPMC & $2.6 \pm 0.5$ & $0.7 \pm 0.1$ & $2.9 \pm 0.3$ & $11.5 \pm 2.4$ & $0.4 \pm 0.04$ & $1.6 \pm 0.2$ & $0.9 \pm 0.2$ & $2.3 \pm 0.2$ \\
\hline HPMCAS & $3.0 \pm 0.3$ & $0.9 \pm 0.03$ & $9.1 \pm 0.4$ & $7.9 \pm 1.6$ & $0.5 \pm 0.02$ & $5.1 \pm 0.2$ & $0.8 \pm 0.1$ & $8.3 \pm 0.3$ \\
\hline PVP & $1.8 \pm 0.3$ & $1.5 \pm 0.2$ & $13.5 \pm 0.1$ & $6.7 \pm 0.2$ & $0.9=0.1$ & $7.5 \pm 0.04$ & $1.7 \pm 0.2$ & $2.9 \pm 0.4$ \\
\hline $\mathrm{PVP} / \mathrm{VA}$ & $2.8 \pm 0.2$ & $1.6 \pm 0.2$ & $8.8 \pm 0.3$ & $8.1 \pm 1.4$ & $0.9 \pm 0.1$ & $4.9 \pm 0.2$ & $3.0 \pm 1.6$ & $4.3 \pm 0.3$ \\
\hline Pluronic ${ }^{\sqrt{8}} \mathrm{~F} 108$ & $2.0 \pm 0.4$ & $50.4 \pm 2.4$ & $17.5 \pm 0.6$ & $8.9 \pm 0.2$ & $28.1 \pm 1.3$ & $9.8 \pm 0.3$ & $7.9 \pm 0.2$ & $4.6 \pm 0.2$ \\
\hline sLBF-noPI & & $1.8+0.3$ & & & 1.0 & 0.2 & & 0.5 \\
\hline
\end{tabular}

Page 23

a) \% solubilised was calculated by dividing the AUC of the concentration versus time profiles by the maximum AUC, i.e. representing $100 \%$ solubilised,

over the same period of time.

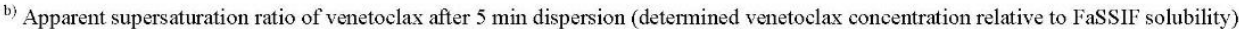




\section{Thesis Publications}

In the case of the sLBF-noPI, a high initial concentration was observed $(14.5 \pm 1.7 \mu \mathrm{g} / \mathrm{mL})$, which was above the FaSSIF solubility $(5.2 \pm 0.4 \mu \mathrm{g} / \mathrm{mL})$. In addition, the initial venetoclax concentrations of SLBF-noPI was above FaSSIF-PI in the case of HPMC, PVP/VA, HPMCAS, PVP and Pluronic ${ }^{\circledR}$ F108 indicating that the investigated PI-free sLBF-noPI was able to generate supersaturation, i.e. a spring effect upon dispersion. The observed initial venetoclax concentrations, i.e. spring effect, of the SLBF-noPI were also similar to all the initial aqueous phase concentrations of the positive control with DMSO, which had the PIs pre-dissolved in the media. Relative to the FaSSIF solubility, an apparent supersaturation ratio of $2.2 \pm 0.5$ after 5 min of dispersion was observed for the sLBF-noPI (Table 2). The degree of supersaturation from the sLBF-noPI gradually decreased to $1.3 \pm 0.2$ after $2 \mathrm{~h}$, whereas at $3 \mathrm{~h}$ the drug concentration had dropped to FaSSIF solubility. However, while the SLBF-noPI was not able to maintain supersaturated concentrations throughout the experiment, the use of PIs in the positive control maintained supersaturation ratios $>1$ for up to $3 \mathrm{~h}$. As the function of the PIs was intended as a 'parachute' to prolong supersaturation, the results indicated a beneficial precipitation inhibitory effect of PIs on the formulation performance.

Interstingly, the combination of PIs in SLBF (sLBF-PI) yielded a higher spring concentration in the case of HPMCAS, PVP, PVP/VA and Pluronic ${ }^{\circledR}$ F 108 when compared to sLBF-noPI and the positive control with DMSO. While the SLBF-PI with HPMC showed an initial venetoclax concentration similar to sLBF-noPI, sLBF-PI with Eudragit ${ }^{\circledR}$ EPO resulted in a statistically significant lower venetoclax concentration in the media when compared to SLBF-noPI and DMSO $(p<0.05)$. In fact, the venetoclax concentration was below FaSSIF and FaSSIF-PI solubility when Eudragit ${ }^{\circledR}$ EPO and lipid were present simultaneously. In the case of dispersing sLBF into FaSSIF-PI (sLBF-aqPI), an increased initial venetoclax concentration was only 


\section{Thesis Publications}

532 In the case of incorporating HPMC, HPMCAS, PVP, PVP/VA and Pluronic ${ }^{\circledR}$ F108 within the 533 sLBF higher amount of venetoclax was solubilised compared to sLBF-noPI. Furthermore, the

evident for Pluronic ${ }^{\circledR}$ F108 when compared to sLBF-noPI. All other PIs resulted in a similar or decreased initial concentration.

The impact of polymer-sLBF combinations on the ability to solubilise venetoclax and prolong supersaturation across six different PIs is summarised in Table 2 and Figure 3. In the case of HPMC, HPMCAS and Eudragit ${ }^{\circledR}$ EPO, the SLBF-aqPI resulted in a lower amount of solubilised venetoclax throughout the experiment compared to sLBF-noPI. Similarly, in comparison to DMSO controls the sLBF-aqPI resulted in a lower AUC in the case of Eudragit ${ }^{\mathbb{R}}$ EPO, HPMC, HPMCAS and PVP/VA. As an example, the amount of the solubilised venetoclax dose was $0.033 \pm 0.002 \%$ for Eudragit ${ }^{\circledR}$ EPO in the case of sLBF-aqPI. In addition, sLBF-aqPI resulted in the case of Eudragit ${ }^{\circledR}$ EPO, HPMC, HPMCAS, PVP and PVP/VA in a similar or lower apparent supersaturation ratio compared to SLBF-noPI and the respective DMSO controls. In the case of Pluronic ${ }^{\circledR}$ F108, the amount of venetoclax solubilised and the apparent supersaturation ratio was increased compared to the sLBF-noPI and DMSO control. Nevertheless, overall the results of the sLBF-aqPI suggested that a pre-dissolved PI in FaSSIF was less effective at prolonging venetoclax supersaturation, indicating that concomitant administration (i.e. chase dosing) a PI with a SLBF would not potentially offer benefits in terms of sustained high concentrations for absorption in vivo. sLBF-PIs resulted in a higher amount of solubilised venetoclax in comparison to the SLBFaqPI. In addition, higher apparent supersaturation ratios (after $5 \mathrm{~min}$ of dispersion) were observed for sLBF-PI relative to sLBF-noPI. In the case of HPMC, HPMCAS, PVP and PVP/VA the apparent supersaturation ratios were higher compared to the sLBF-aqPI indicating

Page 26 


\section{Thesis Publications}

that these sLBF-PI combinations achieved a balance between generating and maintaining supersaturated concentrations. However, Pluronic ${ }^{\circledR}$ F108 and Eudragit ${ }^{\circledR}$ EPO did not follow this trend. Pluronic ${ }^{\circledR}$ F108 showed a higher supersaturation ratio and \% solubilised venetoclax for the sLBF with pre-dissolved Pluronic ${ }^{\circledR}$ F108 (sLBF-aqPI), compared to the incorporation into the sLBF (sLBF-PI). Such high venetoclax concentrations and supersaturation ratios in the presence of Pluronic ${ }^{\circledR}$ F108 may be attributed to the surfactant nature of the polymer, which can increase the solubilisation capacity of the test media, especially when pre-dissolved. In the case of Eudragit ${ }^{\mathbb{R}}$ EPO, a significantly decreased amount of solubilised venetoclax and supersaturation ratios $<1$ in all cases of lipid excipient-PI combinations were observed.

Based on the amount of venetoclax solubilised in the in vitro PI test, the general performance ranking was Pluronic ${ }^{\circledR} \mathrm{F} 108>\mathrm{PVP}>\mathrm{HPMCAS} \geq \mathrm{PVP} / \mathrm{VA}>\mathrm{HPMC}>$ Eudragit $^{\circledR}$ EPO in the case of sLBF-PI and Pluronic ${ }^{\circledR}$ F108 $>$ PVP/VA $\geq$ PVP $\geq$ HPMCAS $\geq$ HPMC $>$ Eudragit $^{\circledR}$ EPO in the case of sLBF-aqPI. While this performance ranking was not in line with the in silico calculated COSMO-Rank, it should be acknowledged that Eudragit ${ }^{\circledR}$ EPO was not able to generate supersaturated venetoclax concentrations in the presence of the lipid excipient. Therefore, this particular PI, may not be suitable to enhance the sLBF performance. Subsequently, the relationship between the in vitro determined solubilised venetoclax and the in silico calculated COSMO-Rank was reassessed without Eudragit ${ }^{\circledR}$ EPO. The results for sLBF-PI and sLBF-aqPI are summarised in Figure 4. It was evident that there is a trend that a higher COSMO-Rank, i.e. higher interaction between venetoclax and the PIs resulted in a higher supersaturation ratio in the aqueous phase of the in vitro test in the case of sLBF-PI. From the in silico screen and in vitro experiments a relationship between COSMO-Rank and venetoclax solubilisation and supersaturation was less evident for sLBF-aqPI (no relationship was observed after $180 \mathrm{~min}$ ). The results of both approaches (in silico and in vitro) indicated 


\section{$\mathrm{X}$. Thesis Publications}

563 that for lipid systems an overly strong interaction between the drug and the PI may be less

564 favourable and in the case of Eudragit ${ }^{\mathbb{R}}$ EPO resulted in a depletion of aqueous venetoclax

565 concentrations

566
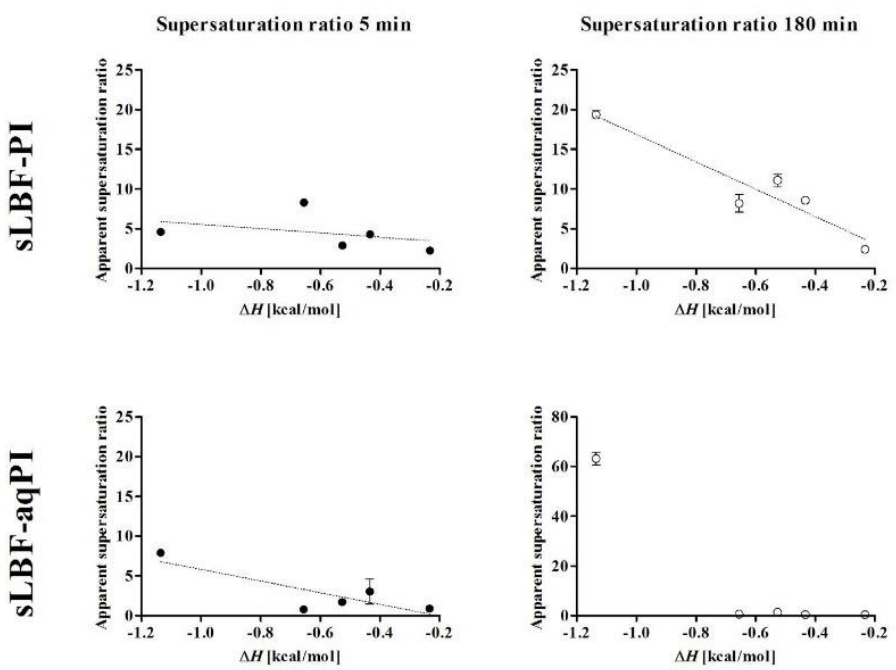

567

Figure 4. Relationship between excess enthalpy of mixing calculating in silico using

COSMOquick and the in vitro determined apparent supersaturation ratio after 5 min and 180 min, respectively for sLBF with incorporated PI added to FaSSIF (sLBF-PI) and sLBF that was added to FaSSIF-PI (sLBF-aqPI). Eudragit ${ }^{\mathbb{R}}$ EPO was excluded from the dataset due to the inability to generate supersaturation. Data is presented as mean $\pm \mathrm{SD}, \mathrm{n}=3$.

575 The aim of the in vivo study was to explore whether the in vitro observations would translate to in vivo using sLBF-PI across six different PIs. Venetoclax formulations $(50 \mathrm{mg} / \mathrm{mL})$ were prepared as a supersaturated Peceol ${ }^{\mathbb{(})}$ solution alone (sLBF-noPI) or in combination with HPMC, 


\section{Thesis Publications}

HPMCAS, PVP, PVP/VA, Pluronic ${ }^{\sqrt{18}}$ F108, Eudragit ${ }^{\mathbb{R}}$ EPO at a ratio of 1:1, respectively. PVP, PVP/VA, Pluronic ${ }^{\mathbb{B}}$ F108 and Eudragit ${ }^{(\mathbb{B}}$ EPO were readily soluble in the sLBF, whereas HPMC and HPMCAS were not soluble in the sLBF and were therefore mixed in the sLBF prior to oral administration. An oral dose of $100 \mathrm{mg}$ venetoclax was used. Absolute bioavailability was determined with reference to intravenous data that has been previously reported for landrace pigs. The absolute bioavailability as a function of each formulation is shown in Figure 5 and the associated pharmacokinetic parameters presented in Table 3. Individual plasma profiles for all tested formulations are shown in Figure S 2 and Figure S 3 in the supporting information. While it was only feasible to conduct a full six by six way cross-over study in this experiment facility, a seventh study leg using Eudragit ${ }^{\mathbb{B}}$ EPO was performed in a separate group of three pigs. In this case only mean comparisons were possible, whereas for all other groups formulation differences within each pig were performed.
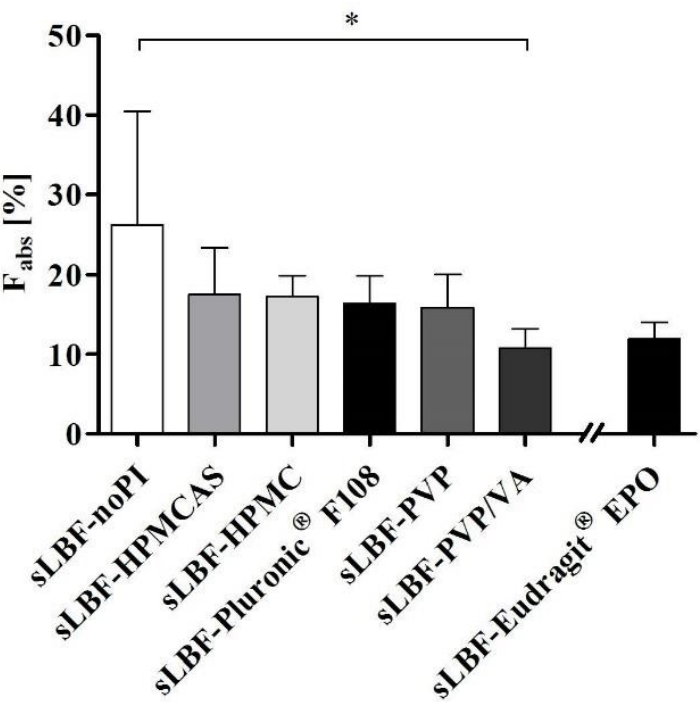


\section{Thesis Publications}

592 Figure 5. Absolute bioavailability $\left(\mathrm{F}_{\text {abs }}\right)$ in landrace pigs for $100 \mathrm{mg}$ venetoclax as a 6-way crossover with sLBF-noPI, sLBF-HPMC, sLBF-HPMCAS, sLBF-PVP, sLBF-PVP/VA, sLBFPluronic ${ }^{\circledR}$ F108 and an additional study including sLBF-Eudragit ${ }^{\circledR}$ EPO. All data is presented as mean $\pm \mathrm{SD}$, where $\mathrm{n}=5$ (except $\mathrm{sLBF}$ - Eudragit ${ }^{\circledR} \mathrm{EPO}$, where $\mathrm{n}=3$ ).

The sLBF-noPI showed the highest mean bioavailability $(26.3 \pm 14.2 \%)$ compared to all other tested lipid formulations. An increased bioavailability was not observed by incorporation of PIs but rather a trend towards a decreased oral bioavailability, albeit only the sLBF-PVP/VA formulation displayed statistically significant lower bioavailability relative to the sLBF-noPI $(\mathrm{p}<0.05)$. The rank order of the oral bioavailability was $\mathrm{sLBF}-\mathrm{noPI} \geq \mathrm{sLBF}-\mathrm{HPMCAS} \geq \mathrm{sLBF}-$ HPMC $\geq$ sLBF-Pluronic ${ }^{\circledR}$ F $108 \geq$ aLBF-PVP $\geq$ sLBF-Eudragit ${ }^{\circledR}$ EPO $\geq$ sLBF-PVP/VA. The observed bioavailability for SLBF-noPI is in agreement with previously reported venetoclax bioavailability in fasted pigs $(17.3 \pm 5.5 \%)$. The time to reach the maximum plasma concentrations $\left(t_{\max }\right)$, the mean residence time (MRT) and mean absorption time (MAT) tended to increase with lower oral bioavailability, however, this observation is not statistically significant. Overall the results showed that the use of PIs did not enhance the sLBF formulation performance in vivo. 


\section{Thesis Publications}

609 Table 3. Pharmacokinetic parameters of the follow up study of venetoclax. Oral administration of $100 \mathrm{mg} / \mathrm{pig}$ to male landrace pigs. Venetoclax was

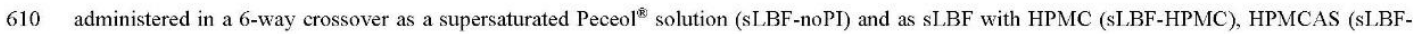
611 HPMCAS), PVP (sLBF-PVP), PVP/VA (sLBF-PVP/VA), Pluronic ${ }^{\sqrt{6}} \mathrm{~F} 108$ (sLBF- Pluronic ${ }^{\sqrt{6}} \mathrm{~F} 108$ ) and in a 2-way crossover including Eudragit ${ }^{\sqrt{\mathbb{E}}}$ 612 EPO (sLBF- Eudragit $\left.{ }^{\sqrt{D}} \mathrm{EPO}\right)$, respectively. $t_{\max }, \mathrm{MAT}$ and MRT are given as median (range), all other parameters as mean $\pm \mathrm{SD}(\mathrm{n}=5$, except $\mathrm{sLBF}-$

613 Eudragit ${ }^{\text {B }} \mathrm{EPO}$, where $\mathrm{n}=3$ )

\begin{tabular}{|c|c|c|c|c|c|c|c|}
\hline \multicolumn{8}{|c|}{ Pharmacokinetic parameters } \\
\hline & sLBF-noPI & sLBF-HPMC & SLBF-HPMCAS & sLBF-PVP & sLBF-PVP/VA & $\begin{array}{l}\text { sLBF-Pluronic }^{80} \\
\text { F108 }\end{array}$ & $\begin{array}{l}\text { sLBF-Eudragit }{ }^{\text {s }} \\
\text { EPO }^{\text {b) }}\end{array}$ \\
\hline$c_{\max }[\mu \mathrm{g} / \mathrm{mL}]$ & $1.38 \pm 0.84$ & $0.92 \pm 0.24$ & $0.83 \pm 0.20$ & $0.81 \pm 0.19$ & $0.48 \pm 0.67$ & $0.80 \pm 0.20$ & $0.51 \pm 0.28$ \\
\hline$t_{\max }[\mathrm{h}]$ (range) & $6(2-10)$ & $6(6-8)$ & $8(7-10)$ & $8(6-8)$ & $9(8-10)$ & $9(6-10)$ & $10(7-10)$ \\
\hline $\begin{array}{l}\text { AUC } 0 \mathrm{~h}-\text { inf. } \\
{\left[\mu \mathrm{g}^{*} \mathrm{~h} / \mathrm{mL}\right]}\end{array}$ & $11.40 \pm 6.15$ & $7.39 \pm 1.40$ & $7.69 \pm 2.53$ & $6.90 \pm 1.83$ & $4.73 \pm 1.08$ & $7.19 \pm 1.50$ & $5.23 \pm 1.60$ \\
\hline MRT [h] (range) & $8.64(7.41-14.20)$ & $10.17(9.43-15.23)$ & $10.96(9.69-12.03)$ & $10.44(9.49-11.71)$ & $11.48(8.67-11.73)$ & $10.62(8.54-12.58)$ & $13.67(10.82-15.62)$ \\
\hline MAT [h] (range) & $4.76(3.53-10.32)$ & $6.29(5.55-11.35)$ & $7.08(5.81-8.15)$ & $6.56(5.62-7.83)$ & $7.61(4.79-7.86)$ & $6.74(4.66-8.70)$ & $9.79(6.94-11.74)$ \\
\hline $\mathrm{F}_{\text {rel }}[\%]^{\text {a) }}$ & 100 & $76.62 \pm 32.34$ & $78.86 \pm 37.81$ & $72.22 \pm 30.56$ & $52.95 \pm 29.23$ & $80.24 \pm 42.16$ & $68.87 \pm 21.01^{\mathrm{e})}$ \\
\hline
\end{tabular}

Page 31

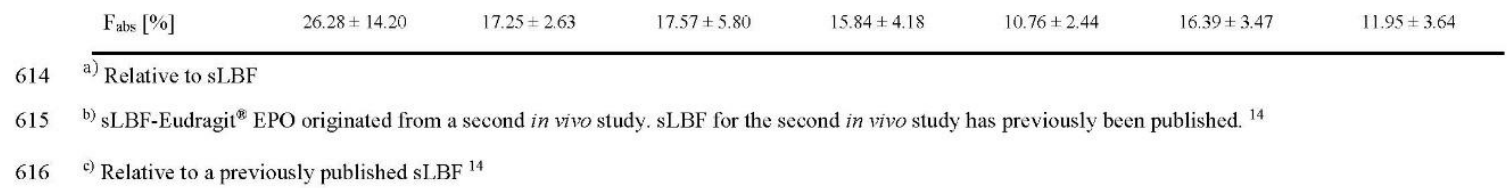

Page 32 


\section{Thesis Publications}

617

618

619

640 In recent years, LBF approaches have been mechanistically described as 'supersaturable' drug

641 delivery systems, on the basis of generating supersaturation following dispersion/digestion in

\section{DISCUSSION}

Bio-enabling formulations that enhance the extent of oral drug absorption are increasingly required to meet the challenge of poor water-soluble properties of drugs emerging from discovery pipelines. Drug candidates which fail to meet Lipinski's Rule-of-Five criteria are a common target for such bio-enabling formulation strategies ${ }^{2}$ due to poor biopharmaceutical properties, resulting from low aqueous solubility and/or poor permeability. Ideally, a formulation design that rapidly generates elevated drug concentrations in the gastrointestinal fluids, i.e. that generates the so-called 'spring effect' to increase concentration above the saturation solubility in gastrointestinal fluids and therein promote absorption. In order to maintain such high drug concentrations, PIs have been used to delay the onset of precipitation, i.e. the so-called parachute. ${ }^{42,43}$ This fundamental advantage of prolonged supersaturated drug concentrations in the intraluminal environment is assumed to be beneficial for absorption in vivo. ${ }^{42,44,45}$ However, the choice of suitable PIs can be complicated ${ }^{24}$ as the inhibitory effect is reported to be drug specific and there are gaps in our understanding which PIs are suited for a particular drug type. ${ }^{27,42}$ While various in vitro screening tests have been reported, to date the PI selection is mostly empirical and there is a lack of comprehensive studies comparing across all the various PI types. ${ }^{42}$ Attempts have been made to increase the mechanistic understanding to aid with the selection of PIs to streamline formulation development, ${ }^{42}$ however, it is often not known how effective an in vitro tool is in estimating the impact on in vivo absorption, especially for rather complex bio-enabling formulations. In addition, given the complexity of polymer-drug interactions, there is also a need for more computational tools to guide excipient selection.

Page 33 


\section{Thesis Publications}

642 the intraluminal environment. Indeed, it is widely recognised that dispersion/digestion can 643 present a key risk to prolonged supersaturation, where there is a lower solubilisation capacity 644 of the post digestive milieu. In particular, LBFs that contain high \% of co-solvents (such as 645 LFCS type IV systems) are considered to be at greatest risk of drug precipitation in the gastrointestinal tract. It has been shown for such type IIIB/IV LBF systems that the addition of an excipient to prolong supersaturation can lead to enhanced bioavailability. ${ }^{24-27}$ The present study, therefore, aimed to explore the utility of PIs to enhance performance for supersaturated lipid solutions (sLBFs). Such systems are being explored particularly for poorly water-soluble drugs when a high drug load and exposure is needed for example in pre-clinical development studies. Our group and others have previously shown that SLBFs enhance oral bioavailability of poorly water-soluble drugs, albeit there is a perceived risk of drug precipitation due to the systems being considered merely kinetically stabile. To address this, in the current study, the utility of several PIs to enhance formulation performance of a sLBF of venetoclax has been evaluated in vitro and in vivo. We further calculated the excess enthalpy of mixing of the drug and various polymers to approximate the interaction between venetoclax and the PIs in a more complex aqueous environment. This approach facilitated a rationalisation of in vitro testing, which was based on a solvent shift to assess the precipitation inhibitory effect. The in vitro lipolysis confirmed the ability of SLBF to generate supersaturated aqueous phase concentrations of venetoclax during dispersion and digestion, which exceeded the experimentally determined apparent solubility in FaSSIF (Figure 1) and the reported values for the experimentally determined amorphous solubility in FaSSIF $(20.7 \pm 0.5 \mu \mathrm{g} / \mathrm{mL}$, pH 5.3 ; $33.7 \pm 13.5 \mu \mathrm{g} / \mathrm{mL}, \mathrm{pH} 6.9)$ and FeSSIF $(26.4 \pm 0.2 \mu \mathrm{g} / \mathrm{mL}, \mathrm{pH} 5.3 ; 54.6 \pm 2.0 \mu \mathrm{g} / \mathrm{mL}, \mathrm{pH} 6.9)$. and therefore confirm the ability of SLBF to generate supersaturated drug concentrations on 


\section{Thesis Publications}

dispersion/digestion in intestinal fluids. Additionally, as a class III glass former ${ }^{14}$ and due to the high molecular weight, venetoclax tends to crystallise more slowly. Moreover, venetoclax has been reported to undergo liquid-liquid-phase separation at concentrations above the amorphous solubility and it was assumed that supersaturation is maintained for a duration that is physiologically promising $(24 \mathrm{~h}) .{ }^{40}$ While in the case of the sLBF the physical state of the drug in such a separated drug-rich phase is unknown it can potentially serve as a reservoir of absorbable drug. ${ }^{40}$ The co-existence of a drug rich phase and an aqueous phase at amorphous solubility (which were not separated) may explain the amount of venetoclax measured above the amorphous solubility in this test setup.

The in vitro PI screening method revealed that the incorporation of the PI into the sLBF resulted in higher venetoclax concentration in the aqueous phase compared to the addition of sLBF to the media containing pre-dissolved PI. Interestingly, the sLBF formulation demonstrated an initial supersaturated venetoclax concentration, which was maintained for up to $2 \mathrm{~h}$ even in the absence of a PI (i.e. sLBF-noPI). These findings are in line with the observations during in vitro lipolysis and confirm the ability of SLBF approaches to generate supersaturation, i.e. to act as a spring. The incorporation into the sLBF (sLBF-PI) of PIs: PVP/VA, HPMCAS, PVP, Pluronic $^{\circledR}$ F108 and HPMC proved beneficial for venetoclax resulting in prolonged supersaturation in vitro (apparent supersaturation ratio $>2.4$ for all polymers after $180 \mathrm{~min}$ of dispersion). However, the incorporation of Eudragit ${ }^{\circledR}$ EPO resulted in a decrease in venetoclax concentration in the aqueous phase, to below FaSSIF solubility (0.08-fold reduction relative to FaSSIF solubility). It was also noticeable that the sLBF-Eudragit ${ }^{\circledR}$ EPO was poorly dispersible in FaSSIF, forming a two-phase system with drug rich agglomerates dispersed in buffer (Figure S 4). In contrast, all the sLBF-noPI and sLBFs containing PIs dispersed consistently in FaSSIF to form a homogenous dispersion on mixing. One possible explanation for the significant lower 


\section{Thesis Publications}

692

693

694

venetoclax concentrations observed for the sLBF-Eudragit ${ }^{\circledR}$ EPO system may reflect the poor dispersion in FaSSIF. This observation suggested that venetoclax may have remained within the lipid-rich agglomerates and was not released from the formulation into the aqueous phase. Additionally, Eudragit ${ }^{\circledR}$ EPO (in the unionised form) and venetoclax are relatively lipophilic and interact strongly with each other, as indicated by the calculated negative excess enthalpy of interaction with the in silico tool, which may have further promoted the drug retention in the lipid phase. Another aspect is that the unionised Eudragit ${ }^{\circledR}$ EPO was not expected to swell in aqueous medium and such a swelling of a more hydrophilic polymer is likely to contribute to the performance of a PI. This complexity in aqueous medium was not captured by the simple mixing enthalpy calculations of binary drug-polymer systems.

The in vivo study demonstrated that the highest oral bioavailability was obtained with the sLBFnoPI. The sLBF-PI formulations showed a trend towards a decreased oral bioavailability, when compared to sLBF-noPI. While the overall bioavailability of $26.3 \pm 14.2 \%$ is in line with previous reports of venetoclax bioavailability in large animal models. ${ }^{14,46}$ However, the results of the present study were unexpected since previously published studies exploring the inclusion of PIs with LBF resulted in an increased bioavailability. ${ }^{24-26}$ However, in the previous reported studies LFCS type IIIB/IV LBFs systems were used, which contained high amounts of cosolvents and exhibit a high risk of precipitation due to the dilution effect upon dispersion. ${ }^{27}$ Therefore, this confirmed that the ability of an oil-only sLBF to generate supersaturated concentrations of venetoclax in vitro was translated to increased absorption in vivo and that the duration of supersaturation for the SLBF was sufficient to obviate the inclusion of a PI. This observation that a PI was not required may be specific for venetoclax, given that the drug as a class III glass former has a low tendency to crystallise. ${ }^{40}$ Hence, for other drugs such as poor glass formers as well as SLBF containing higher proportions of co-solvents further studies are 


\section{Thesis Publications}

717

required to assess whether $\mathrm{SLBF}$ approaches with PIs are needed to maximise absorption and mitigate a perceived risk of precipitation in vivo.

The median MAT for the sLBF-PI formulations was higher among all PI containing formulations relative to SLBF-noPI (Table 3). Overall, given the variability in absorption rate of venetoclax in each group, no statistically significant differences were observed. However, it would appear that the inclusion of a PI may present a risk of a delayed absorption that may reflect a delay of drug release from the formulation. An explanation for this observation might be a combined effect of the extent of drug-polymer interaction and a reduced polymer swelling. The incorporation of the PI into the sLBF may have reduced polymer swelling (which normally happened in the aqueous media) and caused trapping of the drug. In addition, the interaction between the PIs and the drug reduces diffusion of both PI and drug and hence the partitioning of venetoclax and PIs from the SLBF to the aqueous phase. The partitioning may be further reduced due to an increased viscosity of the SLBF-PI formulations, which further decreases diffusion, but also dispersibility and subsequently digestibility. A less dispersed LBF exhibits a lower surface area, which potentially leads to less drug released from the sLBF. However, further studies are needed to explore the effect of viscosity on SLBF performance in vivo.

From the in vivo data it appears that the solubility of the polymer in the sLBF may have impacted the bioavailability. In the cases of PIs being soluble in the sLBF, i.e. Pluronic ${ }^{\circledR}$ F108, PVP, PVP/VA and Eudragit ${ }^{\mathbb{B}}$ EPO dissolved completely in the sLBF, a lower bioavailability was observed when compared to the PIs that showed a lower solubility in the SLBF, i.e. HPMC and HPMCAS formed suspensions in the SLBF. One explanation for this observation may be that a PI that is soluble in the lipid vehicle can, in combination with a high drug affinity of the PI, lead to a drug retention in the vehicle instead of showing a more favourable PI functionality 


\section{Thesis Publications}

of reducing precipitation once the $\mathrm{SLBF}$ has dispersed in the intraluminal fluids. On the other hand, a more hydrophilic polymer that is suspended in the sLBF may lead to polymer swelling upon aqueous dispersion to allow for drug release and the intended PI functionality. Further studies exploring polymer solubility in SLBF and its effect on drug release may therefore be merited to fully predict the impact of PIs on in vivo performance.

Overall, a relationship between the in silico calculated excess enthalpy of mixing and the in vitro determined supersaturation ratios and amounts of venetoclax solubilised was established in the case of PIs that generated supersaturated concentrations. This study showed that with increasing 'COSMO-Rank' (i.e. lower excess enthalpy of mixing), higher apparent supersaturation ratios and higher amounts of solubilised venetoclax were obtained. Since Eudragit $^{\circledR}$ EPO resulted in undersaturated aqueous solutions (venetoclax concentration below FaSSIF saturation solubility) due to a lower release from the formulation, the PI was not considered in the analysis of the relationship between in silico and in vitro. However, the result of Eudragit ${ }^{\mathbb{B}}$ EPO showed a simple consideration of high negative excess enthalpy for a more lipophilic polymer in SLBF should be interpreted with care regarding PI performance.

The deviation of the in vivo results from the in silico calculations by the COSMOquick software may reflect an oversimplification of calculated parameters. The calculations considered the interactions between the drug and the PI, but the interaction between the drug or the PI with formulation excipients, water or the components of gastrointestinal fluids such as bile salts and phospholipids were not taken into account. While this might have not been crucial for an in vitro dispersion experiment, the digestion of the lipid excipients in vivo further increased the complexity of the gastrointestinal fluids, by releasing fatty acids and other digestion products in the gastrointestinal environment. It is, hence, unclear whether a drastic simplification to 


\section{Thesis Publications}

767

768

769

solely the drug and PI by the selected COSMOquick approach is applicable for LBFs to define PIs. Nevertheless, the current in silico approach may be useful for type IV LBFs/sLBFs that contain less digestible excipients, i.e. co-solvents, and may be a quick screening tool to reduce the initial PI choice to a reasonable number, which subsequently can be tested in vitro and in vivo. Furthermore, the complementing in vitro and in silico techniques used in the present study may be helpful in understanding the formulation behaviour of sLBFs in vivo.

A lack of PI impact on increasing bioavailability may also reflect that the in vitro model was poorly predictive of the in vivo situation. The reasons for the in vitro test not being predictive of a reduce overall drug absorption in the presence of PIs can be manifold such as (a) more complex intestinal conditions in vivo, ${ }^{47}$ (b) a lack of absorptive sink in vitro ${ }^{48}$ or (c) a venetoclax specific effect. The employed in vitro test exhibited a high drug and lipid load as well as higher hydrodynamics compared to in vivo. In addition, a solvent shift may meet the industry need for a fast screening tool, however, in the case of LBFs it is not physiologically relevant. A combined dispersion-digestion setup ${ }^{27}$ or a dispersion-digestion setup with absorptive sink ${ }^{49-51}$ may provide more mechanistic, albeit lower throughput, results. A further limitation of the study may have been the incorporation of the PI into the sLBF. While this decision was guided by in vitro data, in light of the in vivo results it is unclear whether separating the PI and sLBF may have conferred advantages in vivo.

\section{CONCLUSION}

The formulation approach of using PIs to prolong supersaturation is well recognised for amorphous formulations, but less well explored for sLBFs. The present study, applied in silico, in vitro and in vivo models to extend the concept of PIs in an oil-only sLBF. An in silico tool was successfully used for an initial PI selection and aided in explaining the low free venetoclax 


\section{Thesis Publications}

792 concentration in vitro in case of the sLBF with Eudragit ${ }^{\circledR}$ EPO. It was found that the strong

793 predicted interaction between the drug and the polymer may have led to an overall reduction in

794 drug release from the formulation. In addition, the in vitro PI screening tool showed that the

795 incorporation of the PI into the sLBF yielded higher free drug concentrations compared to the

796 separate addition of the PI. While the in vitro screening showed prolonged supersaturated

797 concentrations in the presence of PIs in five out of six cases, in vivo a trend towards a lower

798 overall bioavailability was observed for PI containing formulations, indicating that

799 incorporating a PI into the SLBF was not necessary. The oral bioavailability of venetoclax was

800 highest for the PI free sLBF-noPI, which suggests that reduced oral absorption due to

801 precipitation from an oil-only sLBF was low.

802

804 N.J. Koehl, L.J. Henze, D.J. Price, B.T. Griffin, R. Holm and M. Kuentz are part of the 805 PEARRL European Training network, which has received funding from the Horizon 2020 Marie Sklodowska-Curie Innovative Training Networks programme under grant agreement No. 674909. 


\section{Thesis Publications}

809

81

812 2. DeGoey, D. A.; Chen, H. J.; Cox, P. B.; Wendt, M. D. Beyond the Rule of 5: Lessons

813 Learned from AbbVie's Drugs and Compound Collection. J Med Chem 2018, 61, (7), 2636-

8142651.

815 3. Di, L.; Fish, P. V.; Mano, T. Bridging solubility between drug discovery and

816 development. Drug Discov Today 2012, 17, (9-10), 486-95.

817 4. Porter, C. J. H.; Pouton, C. W.; Cuine, J. F.; Charman, W. N. Enhancing intestinal drug

818 solubilisation using lipid-based delivery systems. Adv Drug Deliver Rev 2008, 60, (6), 673-691.

819 5. Porter, C. J. H.; Trevaskis, N. L.; Charman, W. N. Lipids and lipid-based formulations:

820 optimizing the oral delivery of lipophilic drugs. Nat Rev Drug Discov 2007, 6, (3), 231-248.

821 6. Kuentz, M. Lipid-based formulations for oral delivery of lipophilic drugs. Drug Discov

822 Today Technol 2012, 9, (2), e71-e174.

823 7. Feeney, O. M.; Crum, M. F.; MeEvoy, C. L.; Trevaskis, N. L.; Williams, H. D.; Pouton,

824 C. W.; Charman, W. N.; Bergstrom, C. A.; Porter, C. J. 50years of oral lipid-based formulations:

825 Provenance, progress and future perspectives. Adv Drug Deliv Rev 2016, 101, 167-94.

826 8. Koehl, N. J.; Holm, R.; Kuentz, M.; Griffin, B. T. New Insights into Using Lipid Based

827 Suspensions for "Brick Dust' Molecules: Case Study of Nilotinib. Pharm Res-Dordr 2019, 36, 828 (4).

829 9. Dahan, A.; Hoffman, A. The effect of different lipid based formulations on the oral 830 absorption of lipophilic drugs: the ability of in vitro lipolysis and consecutive ex vivo intestinal 831 permeability data to predict in vivo bioavailability in rats. Eur J Pharm Biopharm 2007, 67, (1), $83296-105$. 


\section{Thesis Publications}

833 10. Koehl, N. J.; Holm, R.; Kuentz, M.; Griffin, B. T. Chase dosing of lipid formulations

834 to enhance oral bioavailability of nilotinib in rats. Pharm Res 2020, accepted.

835 11. Thomas, N.; Holm, R.; Garmer, M.; Karlsson, J. J.; Mullertz, A.; Rades, T.

836 Supersaturated self-nanoemulsifying drug delivery systems (Super-SNEDDS) enhance the

837 bioavailability of the poorly water-soluble drug simvastatin in $\operatorname{dogs}$. Aaps $J \mathbf{2 0 1 3}, 15,(1), 219$ -

83827.

839 12. Thomas, N.; Holm, R.; Mullertz, A.; Rades, T. In vitro and in vivo performance of 840 novel supersaturated self-nanoemulsifying drug delivery systems (super-SNEDDS). $J$ Control 841 Release 2012, 160, (1), 25-32.

842 13. Ilie, A. R.; Griffin, B. T.; Kolakovic, R.; Vertzoni, M.; Kuentz, M.; Holm, R. 843 Supersaturated lipid-based drug delivery systems - exploring impact of lipid composition type 844 and drug properties on supersaturability and physical stability. Drug Dev Ind Pharm 2020, 1-9.

845 14. Koeh1, N. J.; Henze, L. J.; Kuentz, M.; Holm, R.; Griffin, B. T. Supersaturated lipid846 based formulations to enhance oral bioavailability of venetoclax Pharmaceutics 2020, (under 847 review).

848 15. Morgen, M.; Saxena, A.; Chen, X. Q.; Miller, W.; Nkansah, R.; Goodwin, A.; Cape, J.; 849 Haskell, R.; Su, C.; Gudmundsson, O.; Hageman, M.; Kumar, A.; Chowan, G. S.; Rao, A.; 850 Holenarsipur, V. K. Lipophilic salts of poorly soluble compounds to enable high-dose lipidic 851 SEDDS formulations in drug discovery. Eur J Pharm Biopharm 2017, 117, 212-223.

852 16. Williams, H. D.; Ford, L.; Han, S.; Tangso, K. J.; Lim, S.; Shackleford, D. M.; Vodak, 853 D. T.; Benameur, H.; Pouton, C. W.; Scammells, P. J.; Porter, C. J. H. Enhancing the Oral 854 Absorption of Kinase Inhibitors Using Lipophilic Salts and Lipid-Based Formulations. Mol 855 Pharm 2018.

856 17. Williams, H. D.; Ford, L.; Lim, S.; Han, S.; Baumann, J.; Sullivan, H.; Vodak, D.; 857 Igonin, A.; Benameur, H.; Pouton, C. W.; Scammells, P. J.; Porter, C. J. H. Transformation of 


\section{Thesis Publications}

Biopharmaceutical Classification System Class I and III Drugs Into Ionic Liquids and Lipophilic Salts for Enhanced Developability Using Lipid Formulations. J Pharm Sci 2018, $107,(1), 203-216$.

18. Maghrebi, S.; Prestidge, C. A.; Joyce, P. An update on polymer-lipid hybrid systems for improving oral drug delivery. Expert Opin Drug Deliv 2019, 16, (5), 507-524.

19. Simovic, S.; Heard, P.; Hui, H.; Song, Y.; Peddie, F.; Davey, A. K.; Lewis, A.; Rades, T.; Prestidge, C. A. Dry hybrid lipid-silica microcapsules engineered from submicron lipid droplets and nanoparticles as a novel delivery system for poorly soluble drugs. Mol Pharm $\mathbf{2 0 0 9}$, $6,(3), 861-72$.

20. Tan, A.; Simovic, S.; Davey, A. K.; Rades, T.; Prestidge, C. A. Silica-lipid hybrid (SLH) microcapsules: a novel oral delivery system for poorly soluble drugs. $J$ Control Release $\mathbf{2 0 0 9 ,}$ 134, (1), 62-70.

21. Thomas, N.; Richter, K.; Pedersen, T. B.; Holm, R.; Mullertz, A.; Rades, T. In vitro lipolysis data does not adequately predict the in vivo performance of lipid-based drug delivery systems containing fenofibrate. Aaps $J$ 2014, 16, (3), 539-49.

22. Siqueira Jorgensen, S. D.; Al Sawaf, M.; Graeser, K.; Mu, H.; Mullertz, A.; Rades, T. The ability of two in vitro lipolysis models reflecting the human and rat gastro-intestinal conditions to predict the in vivo performance of SNEDDS dosing regimens. Eur J Pharm Biopharm 2018, 124, 116-124.

23. Siqueira, S. D.; Mullertz, A.; Graeser, K.; Kasten, G.; Mu, H.; Rades, T. Influence of drug load and physical form of cinnarizine in new SNEDDS dosing regimens: in vivo and in vitro evaluations. Aaps $J$ 2017, 19, (2), 587-594.

24. Gao, P.; Akrami, A.; Alvarez, F.; Hu, J.; Li, L.; Ma, C.; Surapaneni, S. Characterization and Optimization of AMG 517 Supersaturatable Self-Emulsifying Drug Delivery System (SSEDDS) for Improved Oral Absorption. J Pharm Sci-Us 2009, 98, (2), 516-528. 


\section{Thesis Publications}

883

884

885

886

25. Gao, P.; Guyton, M. E.; Huang, T.; Bauer, J. M.; Stefanski, K. J.; Lu, Q. Enhanced oral bioavailability of a poorly water soluble drug PNU-91325 by supersaturatable formulations. Drug Dev Ind Pharm 2004, 30, (2), 221-9.

26. Gao, P.; Rush, B. D.; Pfund, W. P.; Huang, T.; Bauer, J. M.; Morozowich, W.; Kuo, M. S.; Hageman, M. J. Development of a supersaturable SEDDS (S-SEDDS) formulation of paclitaxel with improved oral bioavailability. J Pharm Sci 2003, 92, (12), 2386-98.

27. Suys, E. J. A.; Chalmers, D. K.; Pouton, C. W.; Porter, C. J. H. Polymeric Precipitation Inhibitors Promote Fenofibrate Supersaturation and Enhance Drug Absorption from a Type IV Lipid-Based Formulation. Mol Pharm 2018, 15, (6), 2355-2371.

28. Pouton, C. W. Formulation of poorly water-soluble drugs for oral administration: physicochemical and physiological issues and the lipid formulation classification system. Eur JPharm Sci 2006, 29, (3-4), 278-87.

29. Pouton, C. W. Lipid formulations for oral administration of drugs: non-emulsifying, self-emulsifying and 'self-microemulsifying' drug delivery systems. Eur J Pharm Sci 2000, 11, (Suppl 2), 93-8.

30. FDA Venclexta (Venetoclax) Clinical pharmacology and biopharmaceutics review(s) application number: 208573 Orig $1 \mathrm{~s} 000$. $\underline{\text { https://www.accessdata.fda.gov/drugsatfda docs/nda/2016/2085730rig1s000ClinPharmR.pdf }}$ (January 23rd),

31. ICH, G. u. i. d. e. 1. i. n. e. In Validation of analytical procedures: text and methodology Q2 (RI), International conference on harmonization, Geneva, Switzerland, 2005; ICH harmonised tripartite guideline.

32. Koehl, N. J.; Holm, R.; Kuentz, M.; Jannin, V.; Griffin, B. T. Exploring impact of surfactant type and digestion: Highly digestible surfactants improve oral bioavailability of nilotinib. Mol Pharmaceut 2020, under review. 


\section{Thesis Publications}

33. Sek, L.; Porter, C. J.; Charman, W. N. Characterisation and quantification of medium chain and long chain triglycerides and their in vitro digestion products, by HPTLC coupled with in situ densitometric analysis. J Pharm Biomed Anal 2001, 25, (3-4), 651-61. 34. Klamt, A.; Eckert, F. COSMO-RS: a novel and efficient method for the a priori prediction of thermophysical data of liquids. Fluid Phase Equilibria 2000, 172, (1), 43-72. 35. Hornig, M.; Klamt, A. COSMOfrag: a novel tool for high-throughput ADME property prediction and similarity screening based on quantum chemistry. $J$ Chem Inf Model 2005, 45, (5), 1169-77.

36. Loschen, C.; Klamt, A. COSMOquick: A Novel Interface for Fast sigma-Profile Composition and Its Application to COSMO-RS Solvent Screening Using Multiple Reference Solvents. Ind Eng Chem Res 2012, 51, (43), 14303-14308.

37. Abramov, Y. A.; Loschen, C.; Klamt, A. Rational coformer or solvent selection for pharmaceutical cocrystallization or desolvation. J Pharm Sci 2012, 101, (10), 3687-97.

38. Alsenz, J.; Kuentz, M. From Quantum Chemistry to Prediction of Drug Solubility in Glycerides. Mol Pharm 2019, 16, (11), 4661-4669.

39. Price, D. J.; Nair, A.; Kuentz, M.; Dressman, J.; Saal, C. Calculation of drug-polymer mixing enthalpy as a new screening method of precipitation inhibitors for supersaturating pharmaceutical formulations. Eur J Pharm Sci 2019, 132, 142-156.

40. Emami Riedmaier, A.; Lindley, D. J.; Hall, J. A.; Castleberry, S.; Slade, R. T.; Stuart, P.; Carr, R. A.; Borchardt, T. B.; Bow, D. A. J.; Nijsen, M. Mechanistic Physiologically Based Pharmacokinetic Modeling of the Dissolution and Food Effect of a Biopharmaceutics Classification System IV Compound-The Venetoclax Story. J Pharm Sci 2018, 107, (1), 495502.

41. Klamt, A. The COSMO and COSMO-RS solvation models. WIREs Computational Molecular Science 2011, 1, (5), 699-709. 


\section{Thesis Publications}

933 42. Price, D. J.; Ditzinger, F.; Koehl, N. J.; Jankovic, S.; Tsakiridou, G.; Nair, A.; Holm, R.;

934 Kuentz, M.; Dressman, J. B.; Saal, C. Approaches to increase mechanistic understanding and

935 aid in the selection of precipitation inhibitors for supersaturating formulations - a PEARRL

936 review. J Pharm Pharmacol 2019, 7I, (4), 483-509.

937 43. Xu, S.; Dai, W. G. Drug precipitation inhibitors in supersaturable formulations. Int $J$

938 Pharm 2013, 453, (1), 36-43.

939 44. Gao, P.; Shi, Y. Characterization of supersaturatable formulations for improved

940 absorption of poorly soluble drugs. Aaps $J$ 2012, 14, (4), 703-13.

941 45. Brouwers, J.; Brewster, M. E.; Augustijns, P. Supersaturating drug delivery systems:

942 the answer to solubility-limited oral bioavailability? J Pharm Sci 2009, 98, (8), 2549-72.

943 46. Choo, E. F.; Boggs, J.; Zhu, C.; Lubach, J. W.; Catron, N. D.; Jenkins, G.; Souers, A.

944 J.; Voorman, R. The role of lymphatic transport on the systemic bioavailability of the Bcl-2

945 protein family inhibitors navitoclax (ABT-263) and ABT-199. Drug Metab Dispos 2014, 42,

946 (2), 207-12.

947 47. Griffin, B. T.; Kuentz, M.; Vertzoni, M.; Kostewicz, E. S.; Fei, Y.; Faisal, W.; Stillhart,

948 C.; O'Driscoll, C. M.; Reppas, C.; Dressman, J. B. Comparison of in vitro tests at various levels

949 of complexity for the prediction of in vivo performance of lipid-based formulations: Case

950 studies with fenofibrate. Eur J Pharm Biopharm 2014, 86, (3), 427-437.

951 48. Stillhart, C; Imanidis, G.; Griffin, B. T.; Kuentz, M. Biopharmaceutical Modeling of

952 Drug Supersaturation During Lipid-Based Formulation Digestion Considering an Absorption

953 Sink. Pharm Res-Dordr 2014, 31, (12), 3426-3444.

954 49. Keemink, J.; Martensson, E.; Bergstrom, C. A. S. Lipolysis-Permeation Setup for

955 Simultaneous Study of Digestion and Absorption in Vitro. Mol Pharm 2019, 16, (3), 921-930. 


\section{Thesis Publications}

956 50. Alvebratt, C.; Keemink, J.; Edueng, K.; Cheung, O.; Stromme, M.; Bergstrom, C. A. S.

957 An in vitro dissolution-digestion-permeation assay for the study of advanced drug delivery

958 systems. Eur J Pharm Biopharm 2020, 149, 21-29.

959 51. O'Dwyer, P.; Box, K.; Koehl, N. J.; Bennett-Lenane, H.; Reppas, C.; Holm, R.; Kuentz,

960 M.; Griffin, B. T. A Novel Biphasic Lipolysis Method to Predict In Vivo Performance of Lipid

961 Based Formulations. Mol Pharmaceut 2020, under review.

962

963

Page 47 
Supporting information

\section{In silico, in vitro and in vivo evaluation of}

\section{precipitation inhibitors in supersaturated lipid-}

\section{based formulations of venetoclax}

Niklas J. Koehl, Laura J. Henze ${ }^{I}$, Harriet Bennett-Lenane, Daniel J. Price ${ }^{2,3}$

René Holm ${ }^{4,5}$, Martin Kuentz ${ }^{6}$, Brendan T. Griffin ${ }^{{ }^{*}}$

${ }^{1}$ School of Pharmacy, University College Cork; College Road, Cork, T12 YN60 Ireland

${ }^{2}$ Merck KGaA, Darmstadt, Germany

${ }^{3}$ Goethe University Frankfurt, Frankfurt am Main, Germany

${ }^{4}$ Drug Product Development, Janssen Research and Development, Johnson \& Johnson,

Turnhoutseweg 30, 2340 Beerse, Belgium

${ }^{5}$ Department of Science and Environment, Roskilde University, 4000 Roskilde, Denmark

${ }^{6}$ University of Applied Sciences and Arts Northwestern Switzerland, Institute of Pharma

Technology, Muttenz, Switzerland

\begin{tabular}{lc} 
Content & Page \\
\hline In vitro lipolysis: Venetoclax concentrations in the different phases (Figure S 1) & 2 \\
Individual plasma concentration time profiles, study 1 (Figure S 2) & 3 \\
Individual plasma concentration time profiles, study 2 (Figure S 3) & 3 \\
PI test samples of sLBF-PI with HPMC, Eudragit® EPO and HPMCAS (Figure S 4) & 4 \\
Thermal properties of venetoclax (Table S 1) & 4 \\
\hline
\end{tabular}




\section{Thesis Publications}

A

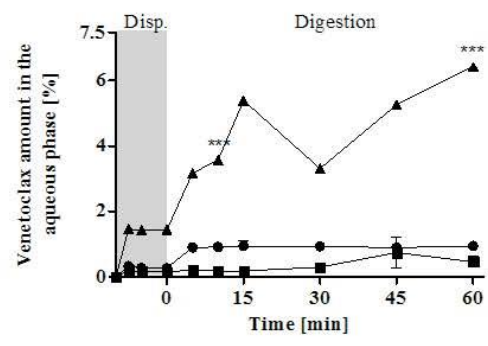

C

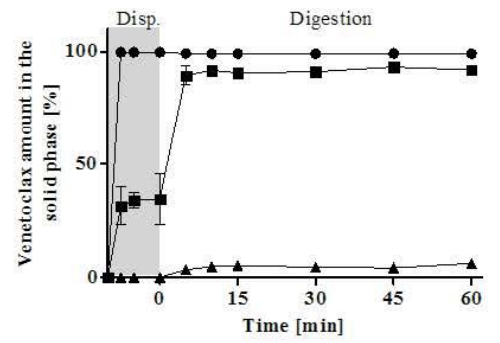

B
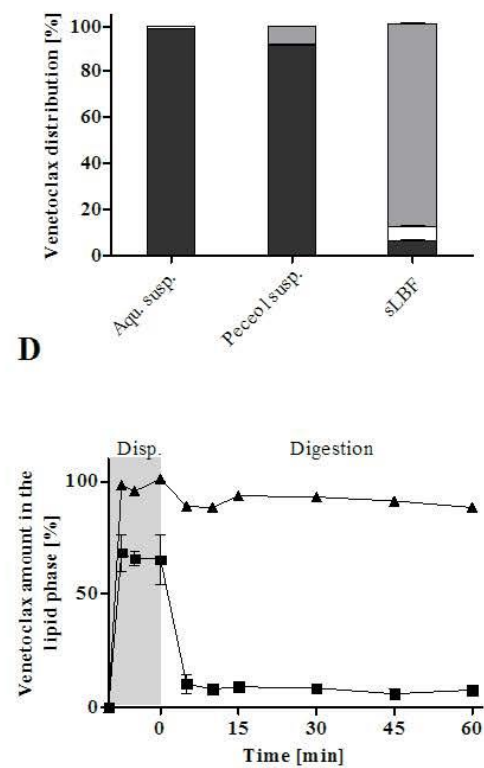

981

982 Figure S 1. In vitro lipolysis of venetoclax formulated as supersaturated Peceol ${ }^{\circledR}$ solution

$983(\mathrm{sLBF})(\boldsymbol{\Delta})$, as well as previously reported aqueous suspension $(\bullet)$, Peceol ${ }^{\mathbb{Q}}$ suspension $(\boldsymbol{\bullet}){ }^{14}$

984 A: \% of venetoclax in the aqueous phase, B: Distribution of venetoclax into the different phases

985 after 60 min of lipolysis, C: \% of venetoclax in the solid phase, D: \% of the venetoclax in the

986 lipid phase. All experiments were run with $\mathrm{n}=3$ and results are shown as mean $\pm \mathrm{SD}$. 


\section{Thesis Publications}
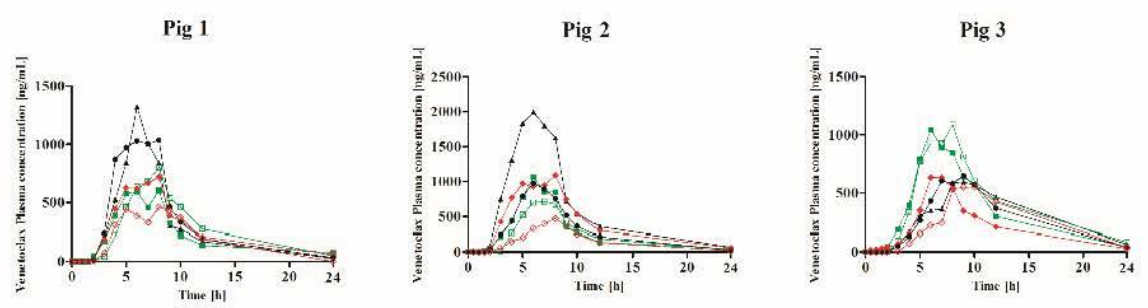

988
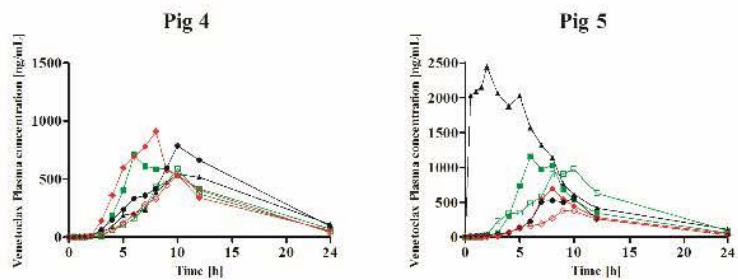

989 Figure S 2. Individual plasma concentration profile from $0-24 \mathrm{~h}$ of $100 \mathrm{mg}$ venetoclax in

990 landrace pigs for the tested formulations. sLBF-noPI ( $\mathbf{\Delta})$, sLBF-HPMC ( $\boldsymbol{\square}$ - green), sLBF-

991 HPMCAS ( $\square$ - green), sLBF-PVP $\left(\bullet-\right.$ red), sLBF-PVP/VA $\left(\diamond-\right.$ red), sLBF-Pluronic ${ }^{\circledR}$ F108 $(\bullet)$

Pig 1

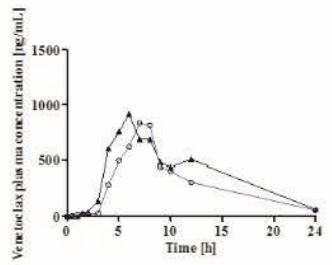

Pig 2

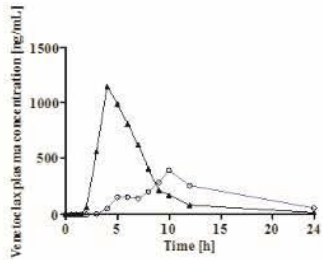

Pig 3

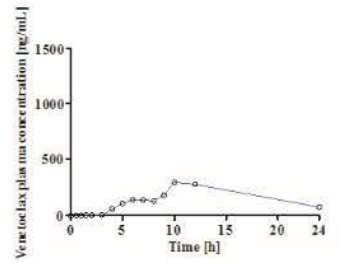

992 


\section{Thesis Publications}

999

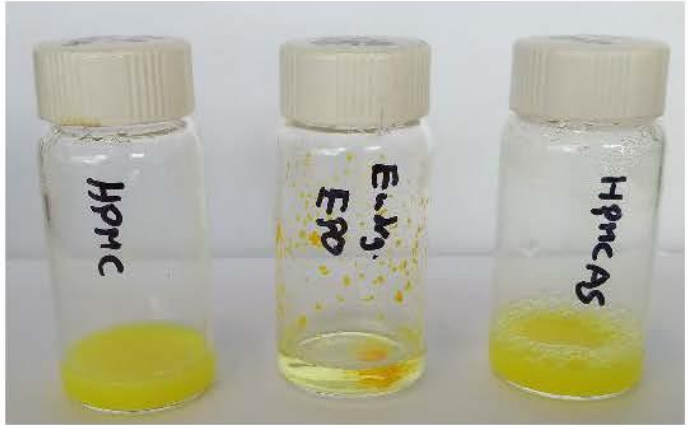

1000 Figure S 4. PI test samples of sLBF-PI with HPMC, Eudragit ${ }^{\circledR}$ EPO and HPMCAS after 180

1001 min in FaSSIF. Agglomeration of Eudragit ${ }^{\circledR P O}$ can be observed.

1002

1003 Table $\mathbf{S}$ 1. Thermal properties of venetoclax. Melting point $\left(T_{\mathrm{m}}\right)$, Enthalpy of fusion $\left(\Delta H_{\mathrm{fus}}\right)$,

1004 Entropy of fusion $\left(\Delta S_{\text {fus }}\right)$.

\begin{tabular}{lccc} 
& $T_{\mathrm{m}}\left[{ }^{\circ} \mathrm{C}\right]$ & $\Delta H_{\text {fus }}[\mathrm{kJ} / \mathrm{mol}]$ & $\begin{array}{c}\Delta S_{\text {fus }} \times 10^{-2} \\
{[\mathrm{~kJ} / \mathrm{mol} / \mathrm{K}]}\end{array}$ \\
\hline $\begin{array}{l}\text { Venetoclax batch } \\
1810004\end{array}$ & $139.1 \pm 0.03$ & $19.2 \pm 0.03$ & $4.7 \pm 0.01$ \\
\hline
\end{tabular}

1005

Page 51 